Portland State University

PDXScholar

\title{
Achieving Operational Seismic Performance of RC Bridge Bents Retrofitted with Buckling-Restrained Braces
}

Ramiro Andrés Gabriel Bazáez Gallardo

Portland State University

Follow this and additional works at: https://pdxscholar.library.pdx.edu/open_access_etds

Part of the Structural Engineering Commons

Let us know how access to this document benefits you.

\section{Recommended Citation}

Bazáez Gallardo, Ramiro Andrés Gabriel, "Achieving Operational Seismic Performance of RC Bridge Bents Retrofitted with Buckling-Restrained Braces" (2017). Dissertations and Theses. Paper 3476.

https://doi.org/10.15760/etd.5360

This Dissertation is brought to you for free and open access. It has been accepted for inclusion in Dissertations and Theses by an authorized administrator of PDXScholar. Please contact us if we can make this document more accessible: pdxscholar@pdx.edu. 
Achieving Operational Seismic Performance of RC Bridge Bents

Retrofitted With Buckling-Restrained Braces

by

Ramiro Andrés Gabriel Bazáez Gallardo

A dissertation submitted in partial fulfillment of the requirements for the degree of

Doctor of Philosophy

in

Civil and Environmental Engineering

\author{
Dissertation Committee: \\ Peter Dusicka, Chair \\ Franz Rad \\ Thomas Schumacher \\ William Wood
}

Portland State University

2017 
(C) 2017 Ramiro Andrés Gabriel Bazáez Gallardo 


\begin{abstract}
Typical reinforced concrete (RC) bridges built prior to 1970 were designed with minimum seismic consideration, leaving numerous bridges highly susceptible to damage following an earthquake. In order to improve the seismic behavior of substandard RC bridges, this study presents the seismic performance of reinforced concrete bridge bents retrofitted and repaired using Buckling-Restrained Braces (BRBs) while considering subduction zone earthquake demands. In order to reflect displacement demands from subduction ground motions, research studies were conducted to develop quasi-static loading protocols and then investigate their effect on structural bridge damage. Results suggested that subduction loading protocols may reduce the displacement ductility capacity of RC bridge columns and change their failure mode. The cyclic performance of reinforced concrete bridge bents retrofitted and repaired using BRBs was experimentally evaluated using large-scale specimens and the developed loading histories. Three BRB specimens were evaluated with the aim of assessing the influence of these components on the overall performance of the retrofitted and repaired bents. Additionally, subassemblage tests were conducted in an effort to study the response of these elements and to allow for refined nonlinear characterization in the analysis of the retrofitted and repaired systems. The results of the large-scale experiments and analytical studies successfully demonstrated the effectiveness of utilizing buckling-restrained braces for achieving high displacement ductility of the retrofitted and repaired structures, while also controlling the damage of the existing vulnerable reinforced concrete bent up to an operational performance level.
\end{abstract}


To my family 


\section{ACKNOWLEDGMENTS}

I would like to express my sincere gratitude to my advisor, Dr. Peter Dusicka, for all his guidance and support throughout the duration of my PhD studies at Portland State University. I also would like to thank my doctoral committee members, Dr. Franz Rad, Dr. Thomas Schumacher, and Dr. William Wood, for taking the time to review my dissertation and provide valuable guidance and comments.

I also wish to thank the members of the Department of Civil Engineering and my fellow graduate students and friends in the iSTAR Laboratory, in particular Dr. Arlindo Lopes, Alvaro Lopez, Ilya Palnikov, Selamawit Mehary, Anwer Mohammed, Marshall Stokes, Stuart Garth, and Stephen Smithson for invaluable advice and fruitful discussions.

The financial assistance provided by the Oregon Department of Transportation (ODOT) is gratefully acknowledged. I would like to thank Star Seismic and CoreBrace for providing the buckling-restrained braces used for the completion of my research project. I also would like to thank the Universidad Técnica Federico Santa María (UTFSM) and

CONICYT PAI/INDUSTRIA 72140358 for the grants provided to stay at Portland State University.

I would also like to extend my deepest gratitude to my parents, grandparents, brother, parents-in-law and relatives for their continuous support and encouragement. Above all, I wish to thank my wife, Karol, and son, Isaac, for their love, encouragement, understanding and support during the years. 


\section{TABLE OF CONTENTS}

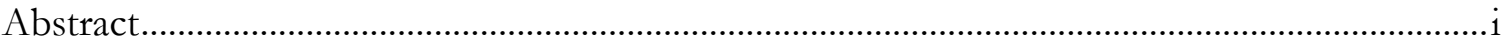

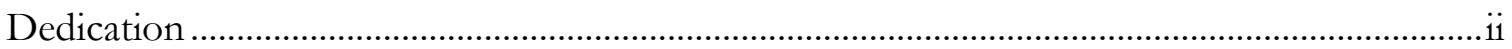

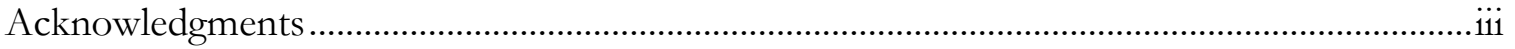

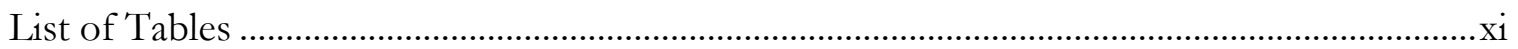

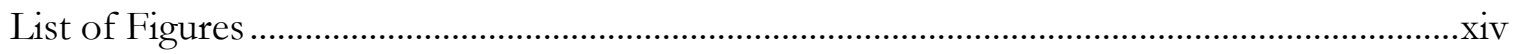

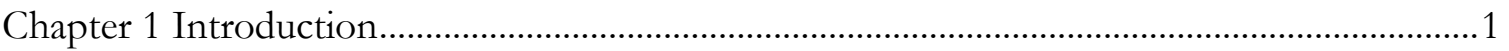

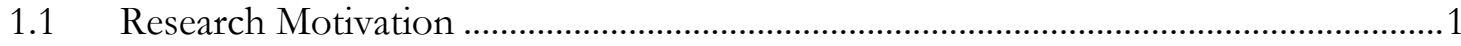

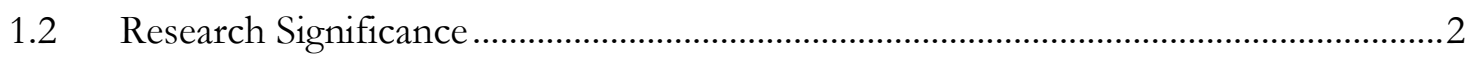

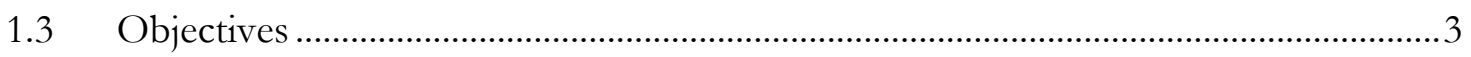

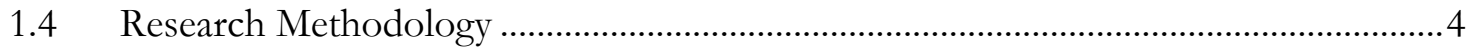

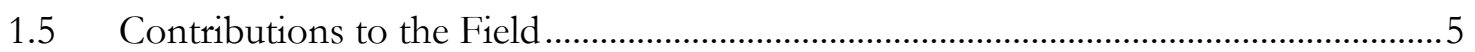

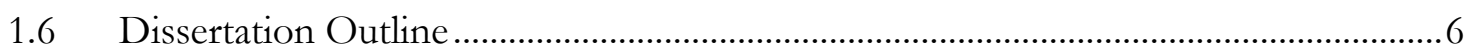

1.7 Main Components of a Typical RC Bridge............................................................. 8

Chapter 2 Cyclic Loading Protocol Considering Subduction Zone Earthquake Demands...... 9

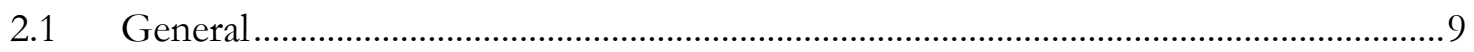

2.2 Implications from Past Research.........................................................................11

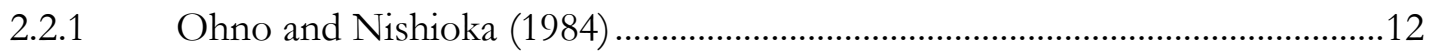

2.2.2 Takemura and Kawashima (1997) …..................................................................12

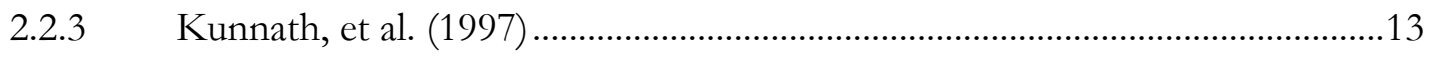

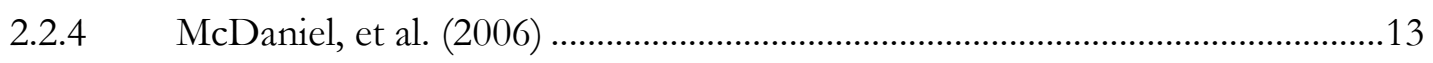




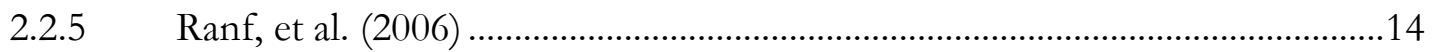

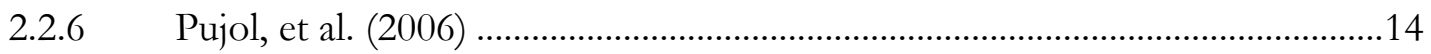

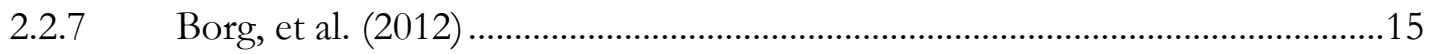

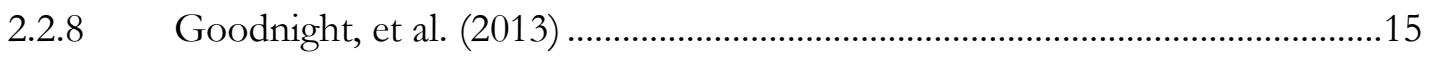

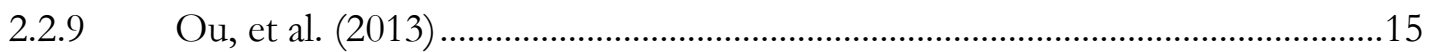

2.3 Selection of Earthquake Ground Motions ..............................................................16

2.4 Hysteresis Model and Target Response ..................................................................19

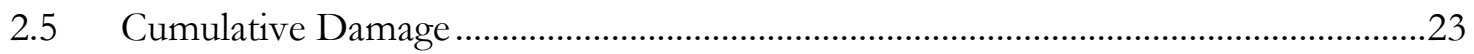

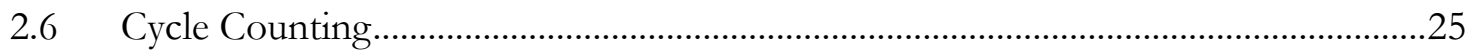

2.7 Cyclic Counting Results and Protocol Development................................................28

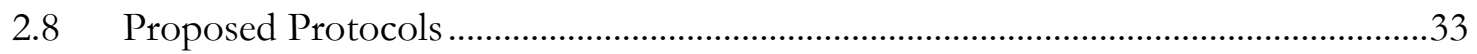

2.9 Comparison to Conventional Protocols .............................................................................

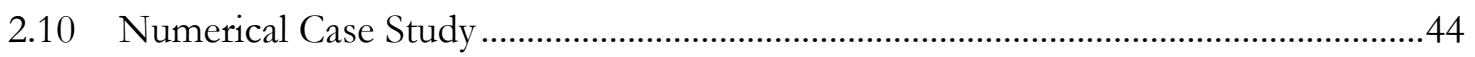

2.11 Effect of Loading Protocol Based on Experimental Results ......................................50

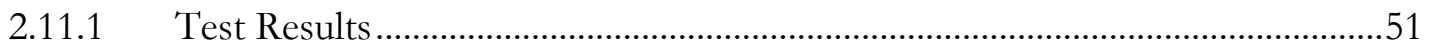

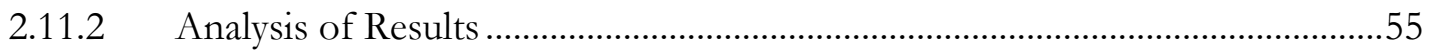

2.11.3 Improved Numerical Modeling Based on Experimental Results ....................64

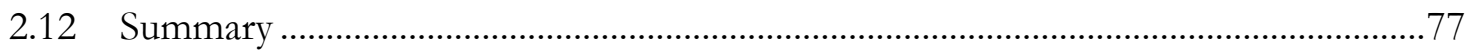

Chapter 3 State-of-the-Art on Retrofit Measures for RC Multi-Column Bridge Bents ..........81

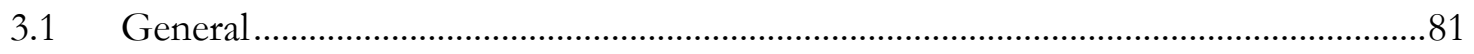

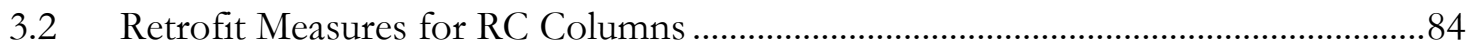

3.2.1 Concrete Jacketing (Concrete Overlays)............................................................. 84 


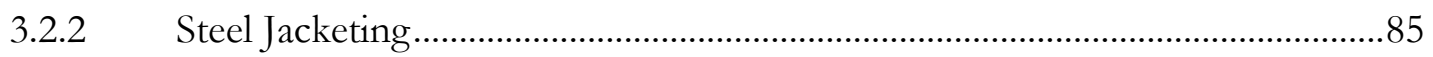

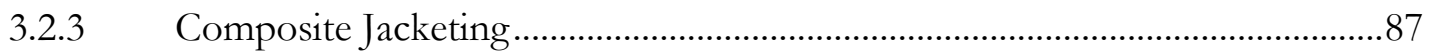

3.2.4 External Prestressing Steel.............................................................................. 90

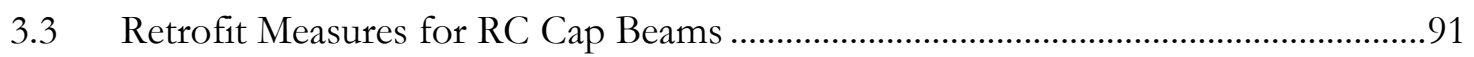

3.4 Retrofit Measures for RC Bridge Bents .................................................................92

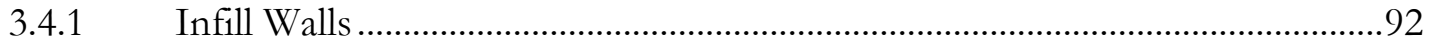

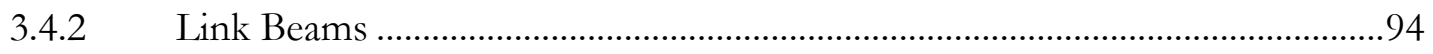

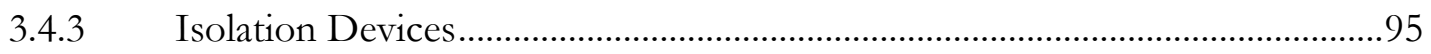

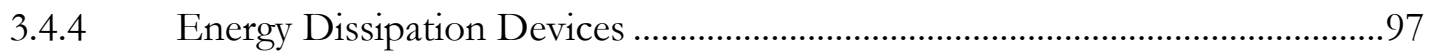

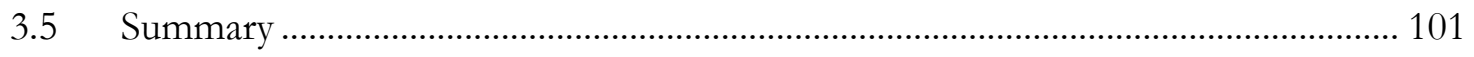

Chapter 4 Seismic Retrofit and Repair of Multi-Column RC Bridge Bents Utilizing Buckling-

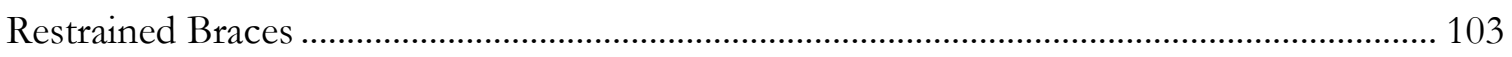

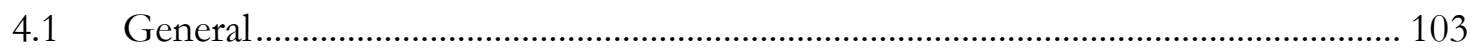

4.2 Actual Seismic Design and Retrofit Philosophy ....................................................... 104

4.2.1 Ground Motion Hazard and Seismic Retrofit Categories.............................. 105

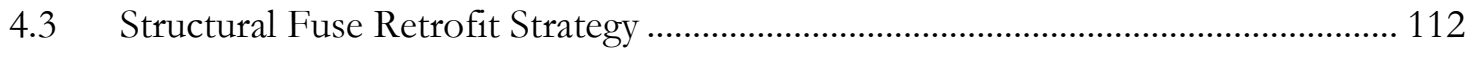

4.4 Design and Implementation of Structural Fuses ................................................... 114

4.4.1 General Design and Implementation ........................................................... 114

4.4.2 Step1: Preliminary Assessment of the As-built RC bent................................. 115

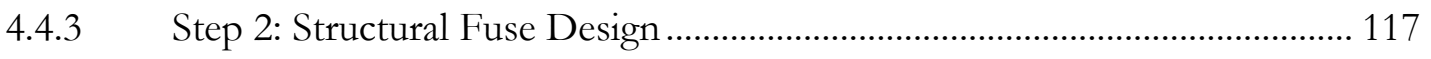

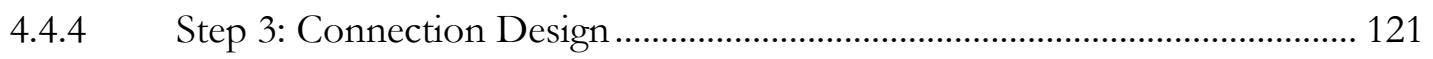

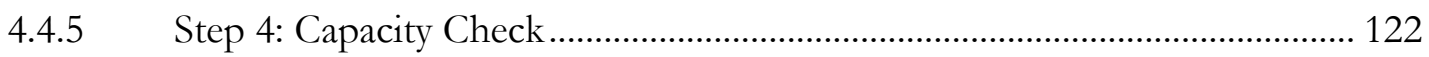


4.5 BRBs as Structural Fuse for Multi-Column RC Bridge Bents

4.5.1 Implementation of the Structural Fuse Concept Using BRBs

4.6 Implementation on Substandard RC Bridges

4.6.1 Multi-Column Prestressed Concrete Stringer/Girder (MPCG) Bridge Bent 130

4.6.2 Multi-Column Continuous Concrete Stringer/Girder (MCCG) Bridge Bent 138

4.6.3 BRB Design 141

4.7 Post-Earthquake Damage Repair of RC Bents using BRBs 143

4.8 Summary 147

Chapter 5 Experimental Evaluation 149

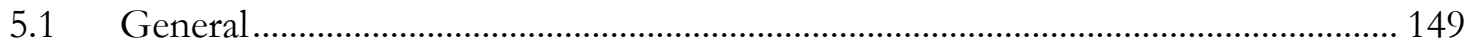

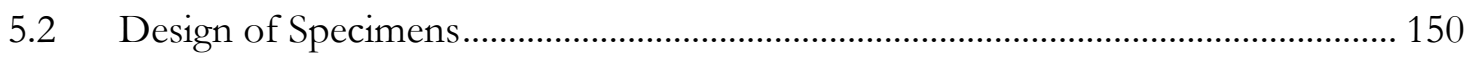

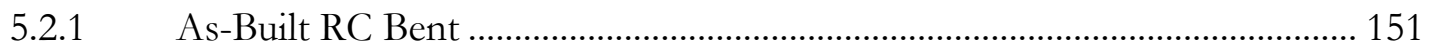

5.2.2 Buckling-Restrained Braces for Retrofitted Bent........................................... 155

5.2.3 Buckling-Restrained Brace for Repaired Bent .............................................. 158

5.2.4 Brace-to-Bent Connection............................................................................ 159

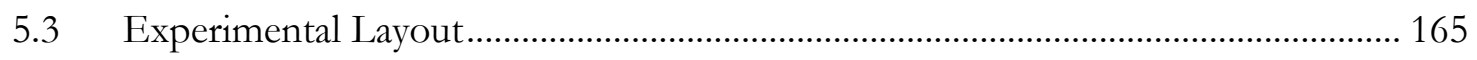

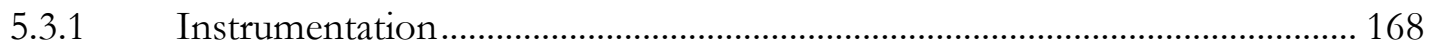

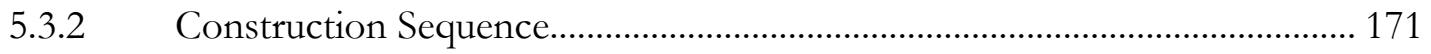

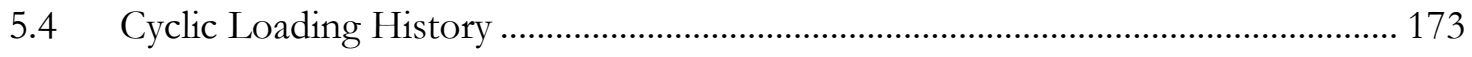

5.5 First Stage - Experimental Evaluation of Retrofitted Bent .................................. 175 vii 
5.5.1 Cyclic Behavior and Damage Progression ....................................................... 175

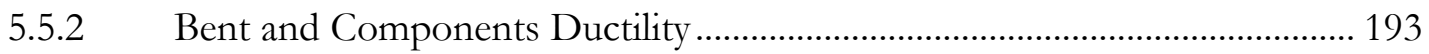

5.5.3 Backbone Curve Comparison ....................................................................... 198

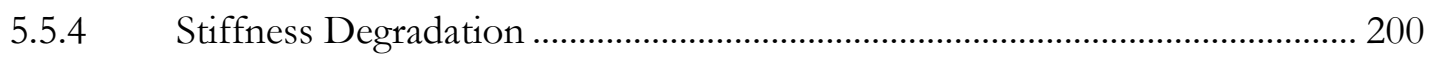

5.5.5 Energy Dissipation and Equivalent Viscous Damping .................................. 201

5.5.6 Comparison to Analytical Displacement Demands........................................ 203

5.5.7 Performance Assessment Based on Strain Measurements ............................. 204

5.6 Second Stage - Experimental Evaluation of Repaired Bent .................................. 219

5.6.1 Cyclic Behavior and Damage Progression .................................................. 219

5.6.2 Yield Displacement and Ductility .................................................................. 225

5.6.3 Backbone Curve Comparison ......................................................................... 229

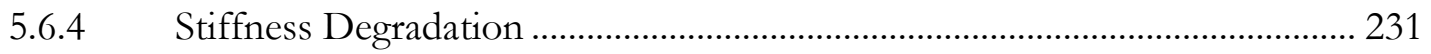

5.6.5 Equivalent Viscous Damping....................................................................... 232

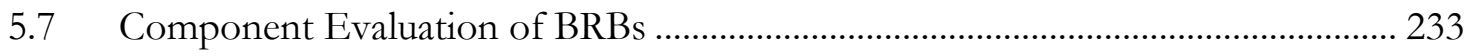

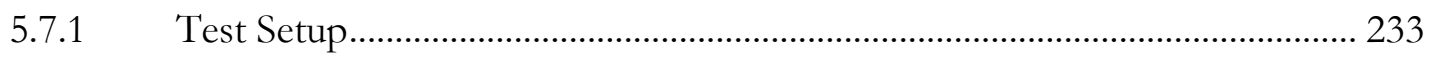

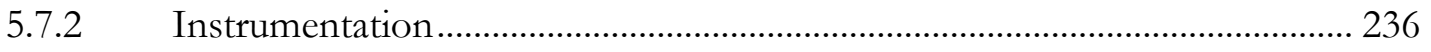

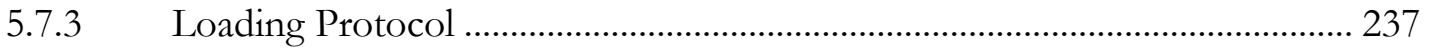

5.7.4 Cyclic Behavior and Damage ……............................................................... 239

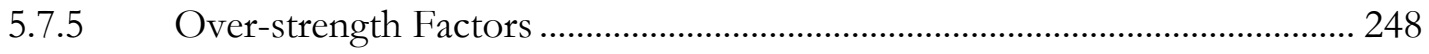

5.7.6 Cumulative Inelastic Deformation and Energy Dissipation........................... 250

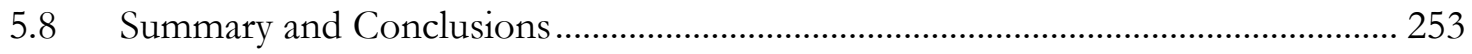

Chapter 6 Nonlinear Characterization Based on Experimental Results ............................... 256 


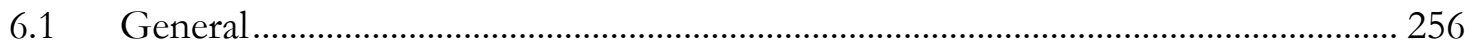

6.2 Numerical Model of Buckling-Restrained Braces..................................................... 257

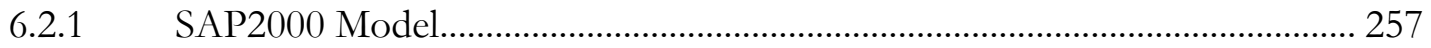

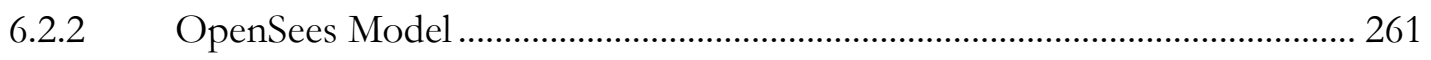

6.3 Numerical Model of Large-Scale Bridge Bents ..................................................... 265

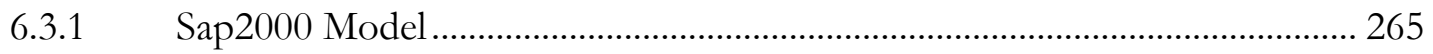

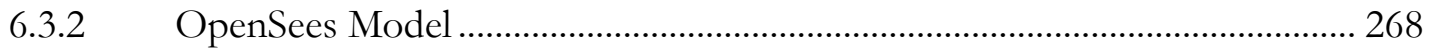

6.4 Modeling of Multi-Column Prestressed Concrete Stringer/Girder Bridge ........... 271

6.4.1 Overview of As-built Numerical Model............................................................. 271

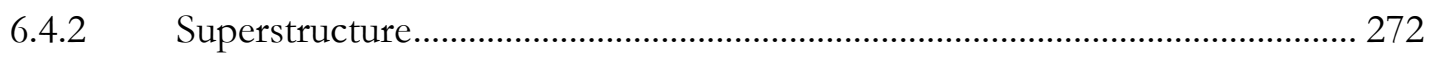

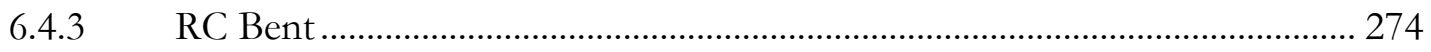

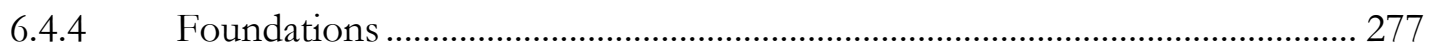

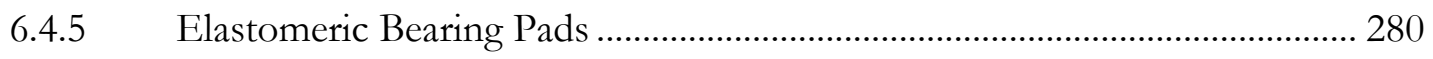

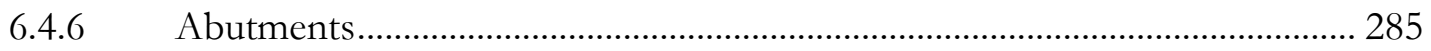

6.4.7 Nonlinear Time History Analysis (NLTHA) ……......................................... 290

6.5 Modeling of Multi-Column Concrete Continuous Stringer/Girder Bridge .......... 294

6.5.1 Overview of As-built Numerical Model............................................................ 295

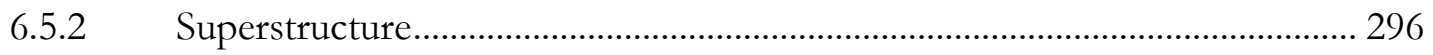

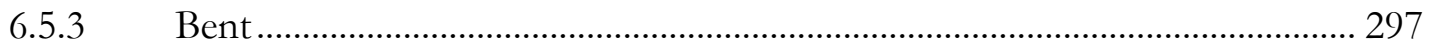

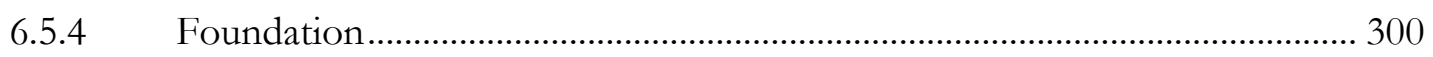

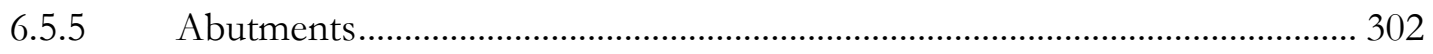

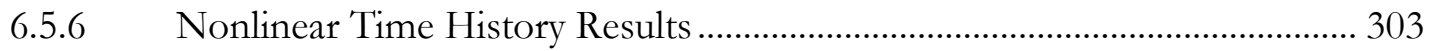




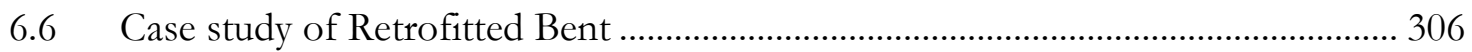

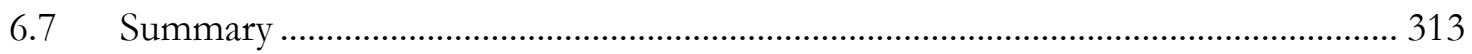

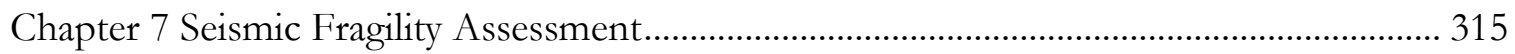

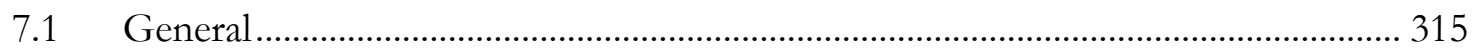

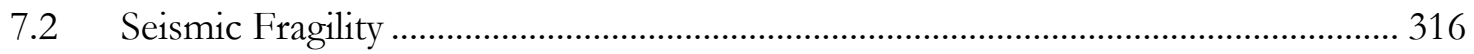

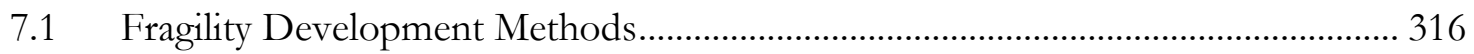

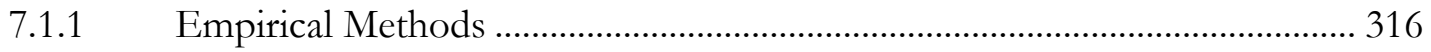

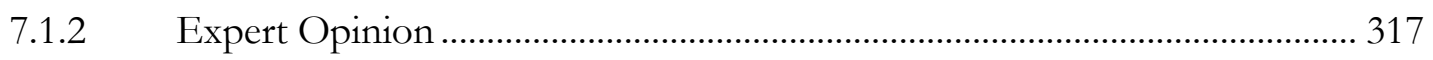

7.1.3 Experimental Methodology .......................................................................... 317

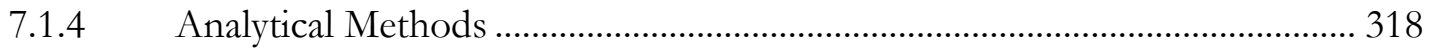

7.1.5 Fragility Curves for Retrofitted Bridges ........................................................ 320

7.2 Fragility Curves for Representative Bridge Bent................................................... 321

7.2.1 Strong Ground Motions ……………............................................................ 322

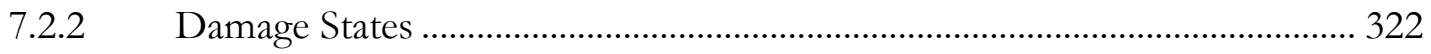

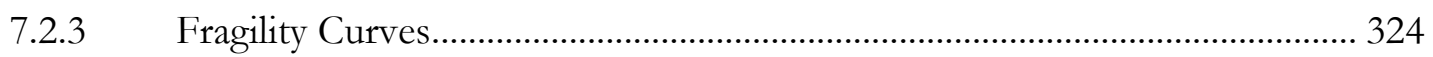

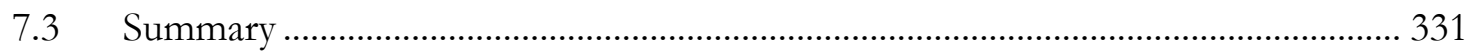

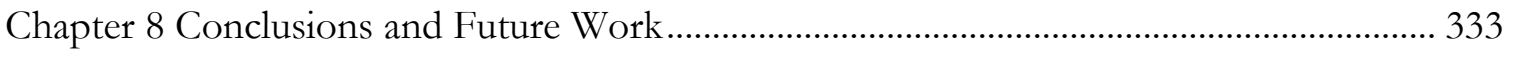

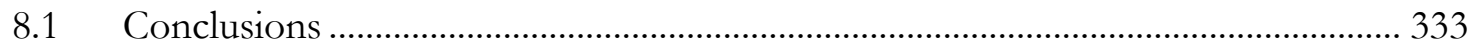

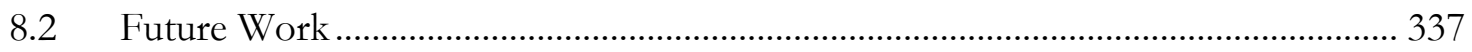

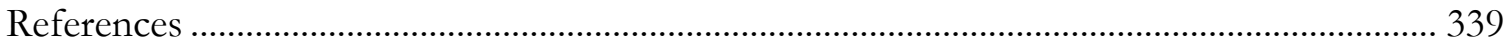

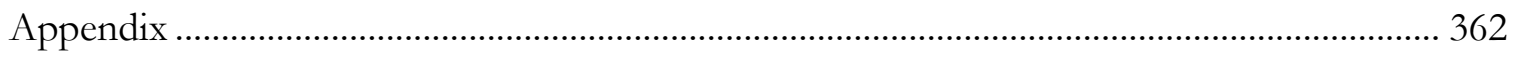




\section{LIST OF TABLES}

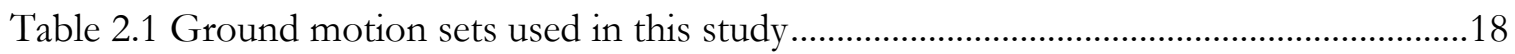

Table 2.2 Degrading model parameters ....................................................................................21

Table 2.3 Comparison of pre-peak and all excursion demands ...............................................33

Table 2.4 Target and proposed demands.......................................................................................

Table 2.5 Proposed loading protocols utilizing Pre-peak approach ...........................................37

Table 2.6 Exponential coefficients to obtain cycle amplitudes. .................................................39

Table 2.7 Comparison of conventional protocols and proposed protocols..............................42

Table 2.8 Column properties .....................................................................................................46

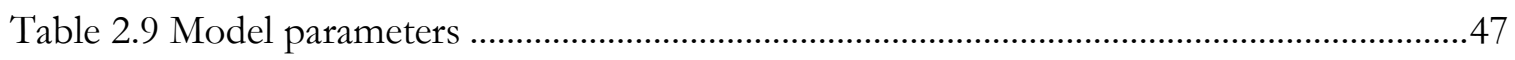

Table 2.10 Measured concrete properties for square RC columns .............................................51

Table 2.11 Maximum bar force transferred in the splice region ..................................................57

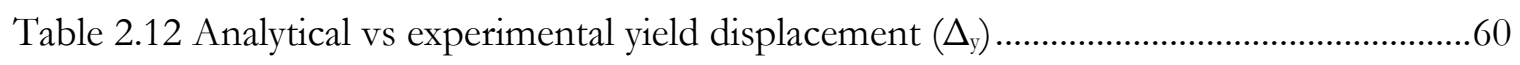

Table 2.13 Parameters for the lumped-plasticity model .............................................................67

Table 4.1 Minimum performance levels for expected retrofitted bridges in Oregon ........... 106

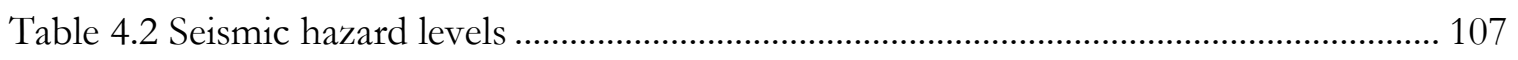

Table 4.3 Seismic retrofit categories, adapted for bridges in Oregon ..................................... 107

Table 4.4 Evaluation methods for existing structures ........................................................... 108

Table 4.5 Seismic design code considerations, seismic hazard adopted by ODOT, and number of bridges built over time, adapted from Nako et al. (2009)...................................... 129

Table 4.6 Displacement demand for the MPCG Bridge …..................................................... 134

Table 4.7 Displacement capacities for the MPCG bridge bent ........................................... 138

Table 4.8 Displacement demand for the MCCG bridge bent ................................................ 141 
Table 4.9 Displacement capacities for the MCCG bridge bent 141

Table 4.10 Modification factors for damaged RC components 145

Table 5.1 Dimension of representative bridge and test specimen. 152

Table 5.2 Compressive strength of concrete cylinders for RC bents 154

Table 5.3 Reinforcing steel properties 154

Table 5.4 Results of BRB designs for Bent/BRB1 and Bent/BRB2 157

Table 5.5 Results of BRB design for repaired bent. 159

Table 5.6 Capacity-to-demand ratios for the gusset plate 162

Table 5.7 Displacement ductility of retrofitted bents 198

Table 5.8 Quantitative limit states in terms of strains 205

Table 5.9 Component Performance Levels adapted from Hose \& Seible 218

Table 5.10 As-built Bent 1 Performance Parameters (Limit States)..... 218

Table 5.11 Displacement ductility of repaired bent 226

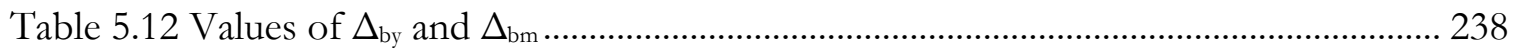

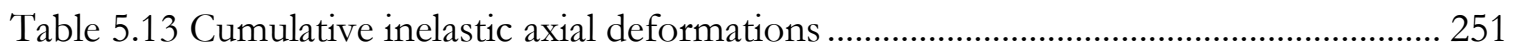

Table 6.1 Initial parameters for nonlinear modeling of BRBs................................................. 259

Table 6.2 Improved parameters for nonlinear modeling of BRBs ........................................ 260

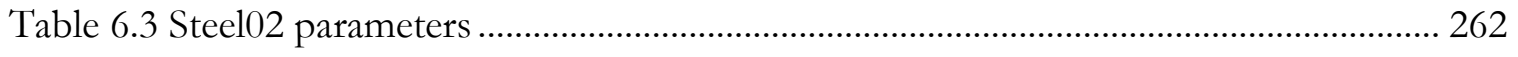

Table 6.4 Superstructure modeling parameters for MPCG Bridge ....................................... 274

Table 6.5 Superstructure modeling parameters for MCCG Bridge ......................................... 297

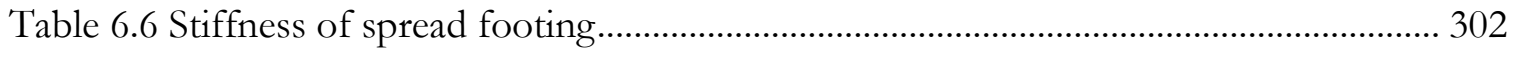

Table 6.7 Ground motion records used in the case study...................................................... 309

Table 7.1 Selected earthquake ground motion records ............................................................ 323 
Table 7.2 Damage states for fragility curve development .................................................... 323

Table 7.3 Fragility curve values for representative RC bridge bent ......................................... 328

Table 7.4 Fragility curve values for retrofitted RC bridge bent............................................... 328

Table 7.5 Fragility curve values for representative RC bridge bent ....................................... 331

Table 7.6 Fragility curve values for retrofitted RC bridge bent............................................. 331 


\section{LIST OF FIGURES}

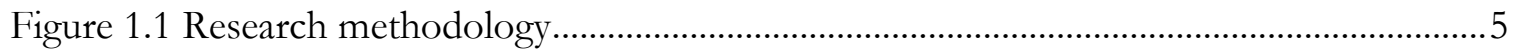

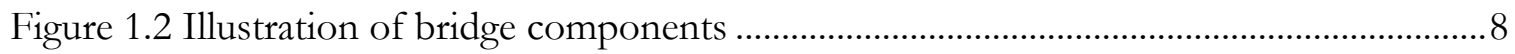

Figure 2.1 Conventional loading protocols used on RC column assessment. (a) New Zealand

Protocol (Cheung, Pauley and Park 1991), (b) Modified New Zealand Protocol (Priestley,

Seible and Hines 2002), (c) ACI374 unidirectional protocol (ACI 374.2R-13 2013)..............12

Figure 2.2 Difference in ground motion duration for selected ground motions......................19

Figure 2.3 Hysteretic behavior - Degrading Model. Adapted from Ibarra et al. (2005a)........20

Figure 2.4 Flowchart of target ductility design procedure ..........................................................22

Figure 2.5 Illustration of target ductility design procedure ........................................................23

Figure 2.6 Illustrative procedure for simplified rainflow counting: (a) inelastic response

history; (b) pre-peak inelastic response history; (c) ordered cycles including mean effect; (d)

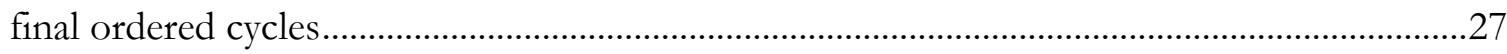

Figure 2.7 Number of inelastic cycles for different component ductilities $(\mu)$ :

(a) Pre-Peak excursions; (b) All excursions. ..................................................................................

Figure 2.8 Cumulative Displacement Ductility (CDD) for different component ductilities. .32

Figure 2.9 Demand parameters for different component ductilities.

(a) Number of Inelastic Cycles; (b) Cumulative Displacement Ductility (CDD) .....................33

Figure 2.10 Proposed loading protocols for component ductilities $(\mu)=2,4$ and 8 .

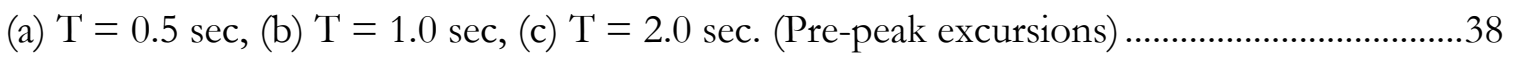

Figure 2.11 Comparison of protocols for ductility 8 and period $0.5 \mathrm{sec}$..................................39

Figure 2.12 Comparison of cumulative ductility demands for period of $0.5 \mathrm{sec} . . . \ldots \ldots . . . . . . . . . . . .43$ 
Figure 2.13 Comparison of cumulative distribution for structures of period $0.5 \mathrm{sec}$ and different component ductilities. .43

Figure 2.14 Cross section of a typical square RC column in Oregon, USA..............................45

Figure 2.15 Effect of loading protocol and model parameters on column response. .49

Figure 2.16 Load vs. displacement curve of column C-7 .52

Figure 2.17 Load vs. displacement curve of column S-7 .53

Figure 2.18 Load vs. displacement curve of column S-17 .55

Figure 2.19 Experimental vs analytical envelope curves. .58

Figure 2.20 Deformation components for RC columns .59

Figure 2.21 Backbone curves of square RC bridge columns .....................................................61

Figure 2.22 Stiffness degradation of square RC bridge columns ..............................................63

Figure 2.23 Illustration of energy dissipated through hysteretic behavior................................63

Figure 2.24 Cumulative energy dissipated by square RC bridge columns .................................64

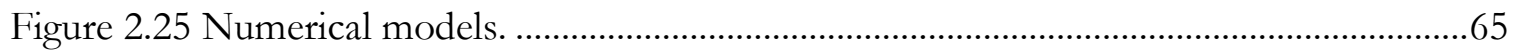

Figure 2.26 Degrading model with pinched hysteretic response .............................................67

Figure 2.27 Experimental vs numerical results - Lumped-Plasticity model..............................68

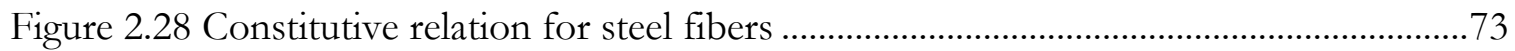

Figure 2.29 Constitutive relation for concrete fibers .................................................................. 73

Figure 2.30 Experimental vs numerical results - Distributed-Plasticity model. .......................74

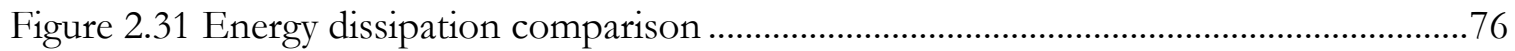

Figure 3.1 Poorly Confined Column in 1971 San Fernando Earthquake ..................................82

Figure 3.2 Collapsed Cypress street viaduct in Oakland during the 1989 Loma Prieta

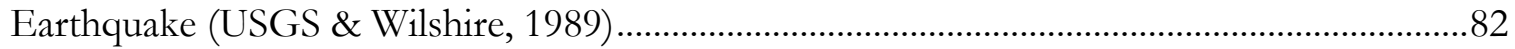


Figure 3.3 Damage in RC columns on the Simi Valley Freeway during the 1994 Northridge Earthquake (Teng, 1994) .83

Figure 3.4 Damage in Nakasone viaduct during 2011 Japan earthquake (Kawashima, et al., 2011)

Figure 3.5 Concrete Jacketing. (a) Concrete overlay (FHWA, 2006), (b) Concrete jacketing of a column in Illinois (Poplar Street Complex)

Figure 3.6 Steel Jacketing for: (a) circular bridge columns in Seattle (photo: Lucia Bill), (b) rectangular bridge column in San Francisco. Left: retrofitted column. Right: As-built column

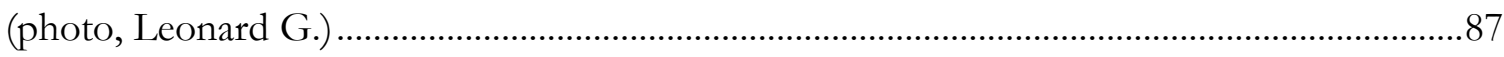

Figure 3.7 Installation of composite jacketing on a seismically deficient RC column .............88

Figure 3.8 Results of an experimental study using CFRP wrapping on a seismically deficient

RC column (Mehary, et al., 2014)

Figure 3.9 External prestressing steel retrofitting of columns in Illinois. .91

Figure 3.10 Retrofit measures for cap beams and beam-column joints. (a) FRP wrapping (Pantelides \& Gergely, 2008). (b) Prestressing rods. (c) Steel jackets (Wright, et al., 2011)....92

Figure 3.11 Effect of different retrofit measure for RC frames. (Sugano, 1981)

Figure 3.12 Infill wall. (a) Schematic, (b) Installation on West Lake Sammish Parkway (photo: L.M. Marsh) .94

Figure 3.13 Application of link beams in San Rafael Viaduct, California. .95

Figure 3.14 Effect of seismic isolation. ..................................................................................

Figure 3.15 Lead rubber bearing (LRB) (Robinson, 1982) ......................................................97

Figure 3.16 Friction dampers. (a) Pall damper (Pall \& Marsh, 1982), (b) FDD (Mualla \&

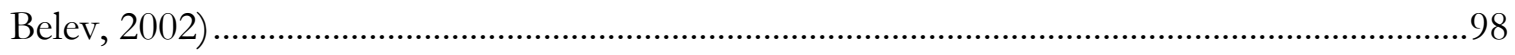


Figure 3.17 Fluid viscous damper. (Constantinou \& Symans, 1993).....

Figure 3.18 Hysteretic dampers (metallic devices): (a) BRBs (Clark, et al., 1999), (b) EBFs (Mazzolani, et al., 2009), (c) U-Shaped dampers (Bagheru, et al., 2015), (d) EDBs (Dolce, et

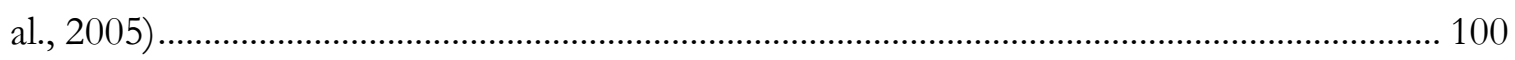
Figure 3.19 Hysteretic behavior of a BRB. (Clark, et al., 1999) ................................................ 101 Figure 4.1 Visualization of PBSD (after Moehle and Deierlein 2004), adaptation to ODOT's

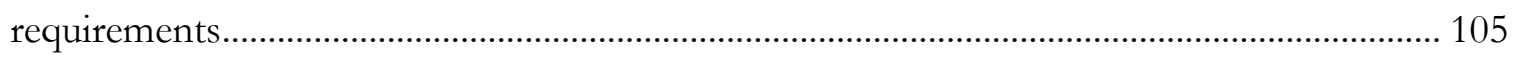

Figure 4.2 Representative response spectrums ........................................................................ 109

Figure 4.3 CSZ 2014 full rupture acceleration values for (a) PGA, (b) $0.2 \mathrm{sec}$, (c) $1.0 \mathrm{sec} . .110$ Figure 4.4 Response spectrum used in this study vs deterministic response spectrum for the 500-year event. (a) Map of Oregon (Google) (b) Portland, (c) Astoria, (d) Newport........... 111 Figure 4.5 Structural fuse design implementation. .................................................................. 115

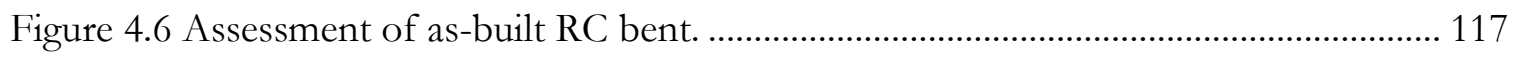

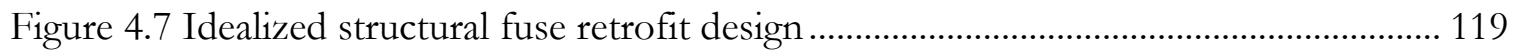

Figure 4.8 Configuration of a typical buckling-restrained brace. .......................................... 123

Figure 4.9 BRB retrofit configuration. (a) Structural fuse schematic. (b) Diagonal

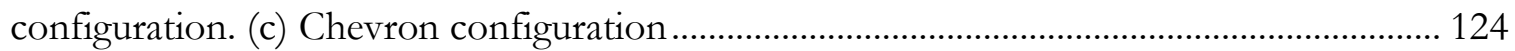

Figure 4.10 Toward operational performance using BRBs.................................................... 125

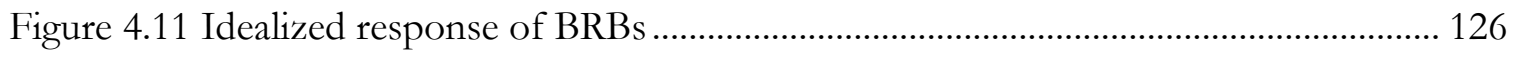

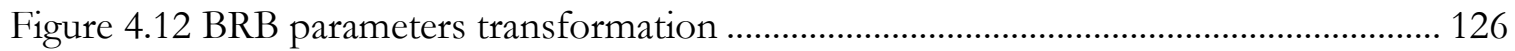

Figure 4.13 Typical MPCG bridge. (a) 3D schematic, (b) Elevation...................................... 131

Figure 4.14 Typical RC bent used in MPCG bridges.............................................................. 132

Figure 4.15 SAP2000 model of the MPCG Bridge .................................................................... 134

xvii 
Figure 4.16 Modeling approach in the transverse direction..................................................... 136

Figure 4.17 Response of lap-spliced columns. Adapted from Priestley et al. (1996) ............ 137

Figure 4.18 Pushover analysis of the MPCG bent in the transverse direction ....................... 137

Figure 4.19 Typical MCCG bridge. (a) 3D schematic, (b) Elevation ..................................... 139

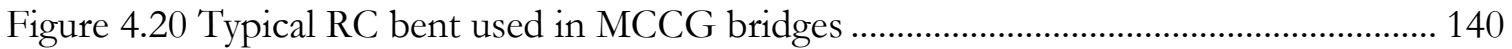

Figure 4.21 SAP2000 model of the MCCG Bridge.................................................................... 141

Figure 4.22 Modeling of damaged component. Adapted from FEMA306 ............................ 145

Figure 4.23 BRBs as seismic repair for damaged RC bents ................................................... 147

Figure 5.1 Geometry and reinforcement of RC Bridge bent specimens................................ 153

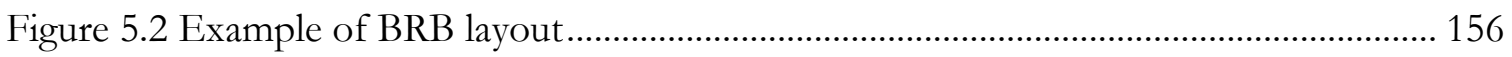

Figure 5.3 Expected load vs displacement behavior of retrofitted bent specimens.............. 158

Figure 5.4 Expected load vs displacement behavior of the repaired bent specimen ............ 159

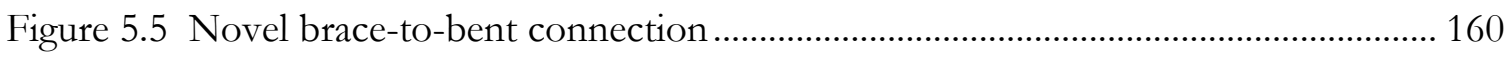

Figure 5.6 Free body diagram used in the design of the gusset plate-to-bent connection.. 162

Figure 5.7 Anchor rods design example (top connection) .................................................. 163

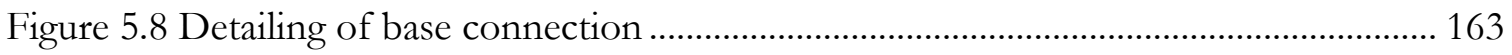

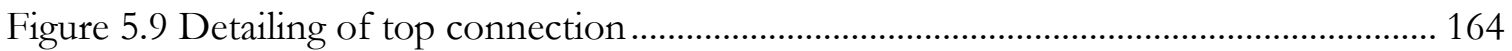

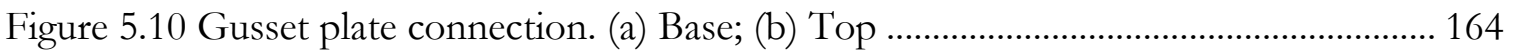

Figure 5.11 Schematic and actual of test setup configuration ................................................. 166

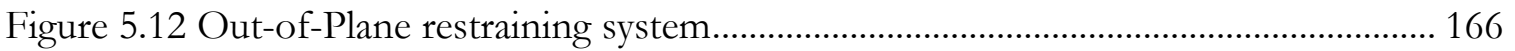

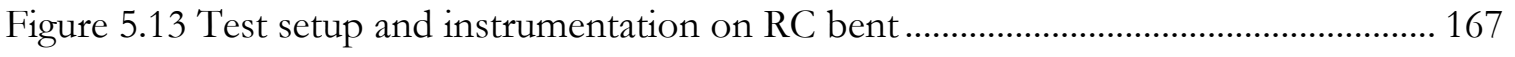

Figure 5.14 Additional instrumentation on brace-to-bent connection................................... 168

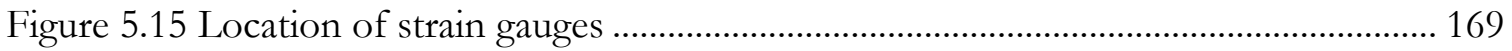

xviii 
Figure 5.16 Installation of strain gauges. (a) Strain gauges on longitudinal reinforcement and

dowel, (b) Strain gauges with protective coating. ....................................................................... 170

Figure 5.17 Load cells to monitor axial load ........................................................................ 170

Figure 5.18 6-DOF load cell used in the experimental program............................................. 171

Figure 5.19 Construction sequence of RC bents ..................................................................... 173

Figure 5.20 Post-installed anchors for gusset plates .............................................................. 173

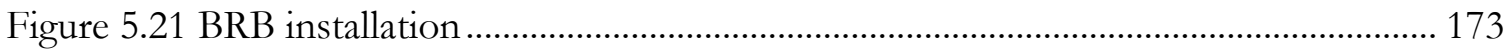

Figure 5.22 Cyclic loading histories. (a) Retrofitted bents, (b) As-built and repaired bents. 175

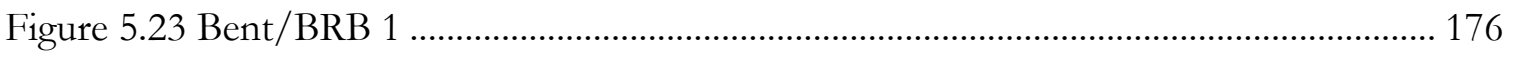

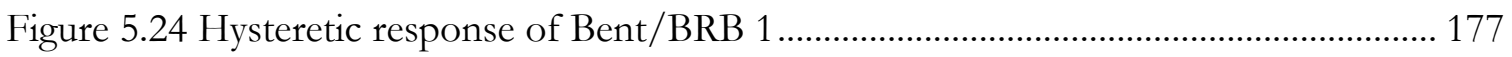

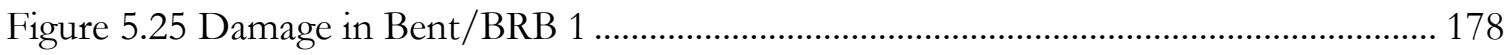

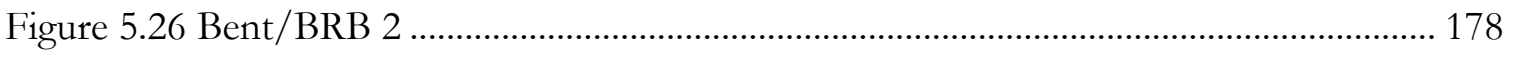

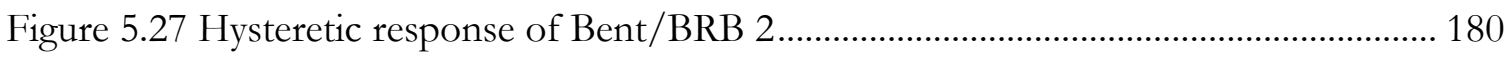

Figure 5.28 Damage in Bent/BRB 2. (a) Horizontal cracks, (b) Spalling of concrete.......... 181

Figure 5.29 Hysteretic response of As-built Bent 1 ............................................................... 182

Figure 5.30 Damage in As-built Bent 1; (a) Overall damage, (b) Buckling of steel, (c) Rupture

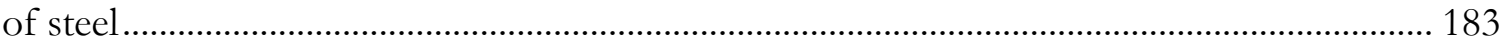

Figure 5.31 BRB elongation in Bent/BRB 1. (a) Top and Bottom, (b) West and East........ 184

Figure 5.32 BRB elongation in Bent/BRB 2. (a) Top and Bottom, (b) West and East....... 184

Figure 5.33 Buckling-restrained brace response. (a) Bent/BRB 1; (b) Bent/BRB 2............ 186

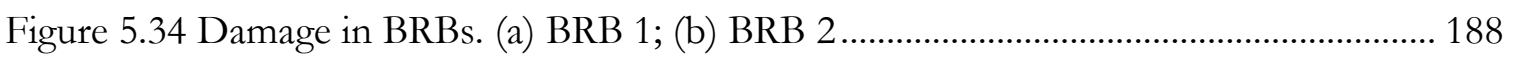

Figure 5.35 Internal column axial load. (a) Bent/BRB 1; (b) Bent/BRB 2; (c) As-built Bent 1. 
Figure 5.36 Plastic hinge damage in columns. (a) C1-Base; (b) C1-Top; (c) C2-Base; (d) C2-

Top 192

Figure 5.37 Plastic hinge damage in cap beam

Figure 5.38 Average curvature profiles in columns. (a) Bent/BRB 1; (b) Bent/BRB2; ....... 196 Figure 5.39 Average curvature profiles in cap beam. (a) Bent/BRB 1; (b) Bent/BRB2; (c) Asbuilt 1 . 197

Figure 5.40 Backbone curves comparison ( $1^{\text {st }}$ stage $)$ 199

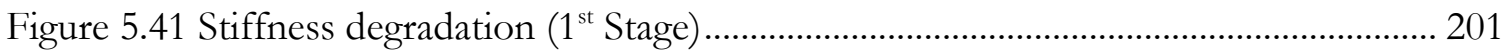

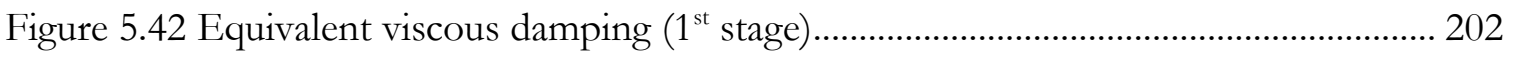

Figure 5.43 Comparison between experimental results and analytical demand; ................... 204

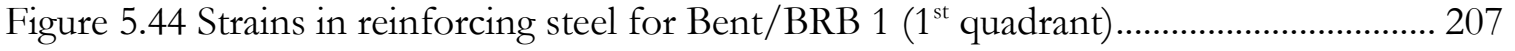

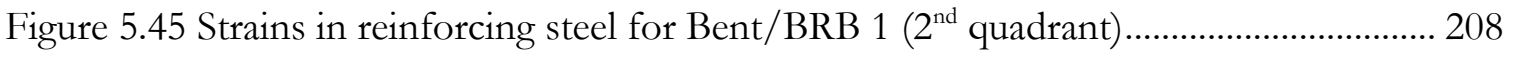

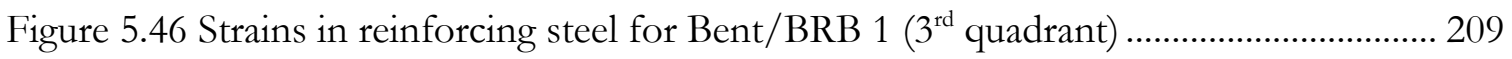

Figure 5.47 Strains in reinforcing steel for Bent/BRB 1 ( $4^{\text {th }}$ quadrant $)$................................. 210

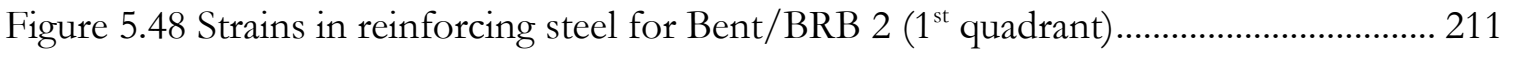

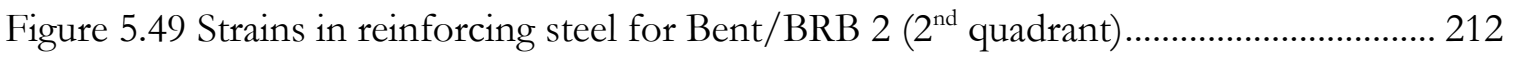

Figure 5.50 Strains in reinforcing steel for Bent/BRB 2 ( $3^{\text {rd }}$ quadrant $)$................................. 213

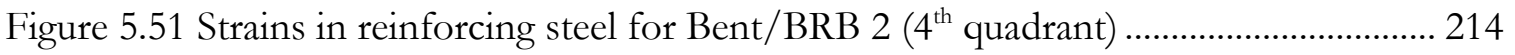

Figure 5.52 Rebar strains in cap beam. (a) Bent/BRB1; (b) Bent/BRB2 ............................. 215

Figure 5.53 Linear strain profile for a circular section............................................................. 216

Figure 5.54 Compression strains in concrete (a) Bent/BRB1; (b) Bent/BRB2 ................... 217

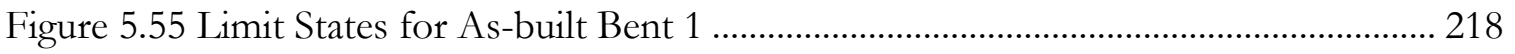

Figure 5.56 Hysteretic response of As-built Bent 2 ................................................................. 220 
Figure 5.57 Damage in As-built Bent 2; (a) Damage in cap beam, (c) Significant spalling in columns, 221

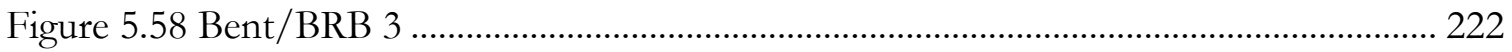

Figure 5.59 Hysteretic response of Bent/BRB 3 ................................................................... 223

Figure 5.60 Damage in Bent/BRB3; (a) Overall damage, (b) Buckling of steel, .................. 225

Figure 5.61 BRB deformation in Bent/BRB 3. (a) Top and Bottom, (b) Total deformation

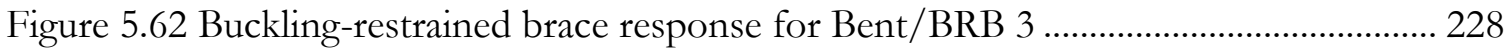

Figure 5.63 Internal column axial load. (a) As-built Bent 2; (b) Bent/BRB 3...................... 229

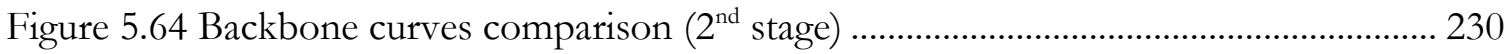

Figure 5.65 Backbone curves comparison for all specimens .................................................. 230

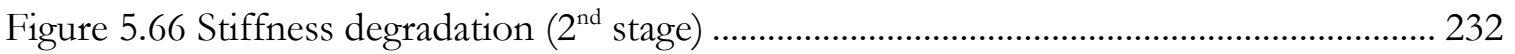

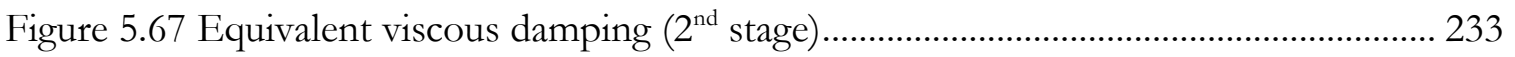

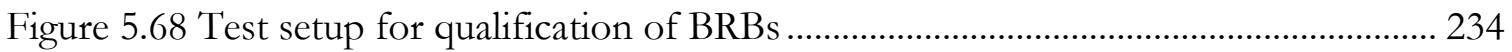

Figure 5.69 Out-of-plane mechanism in subassemblage tests.................................................. 235

Figure 5.70 Top gusset plate for qualification of BRBs ......................................................... 235

Figure 5.71 Bottom gusset plate for qualification of BRBs ................................................... 236

Figure 5.72 Instrumentation on subassemblage tests (a) Base, (b) Top, (c) String pots....... 237

Figure 5.73 Loading protocol for evaluation of BRBs ......................................................... 238

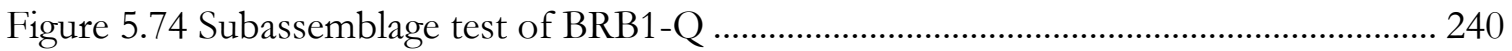

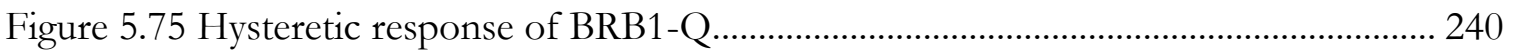

Figure 5.76 Distribution of axial deformation on BRB1-Q .................................................. 241

Figure 5.77 Damage in BRB1-Q. (a) Damage in steel casing, (b) Damage in concrete,....... 242 
Figure 5.78 Subassemblage test of BRB2-Q

Figure 5.79 Hysteretic response of BRB2-Q.

Figure 5.80 Distribution of axial deformation on BRB2-Q................................................... 244

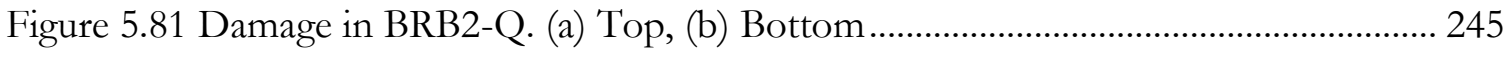

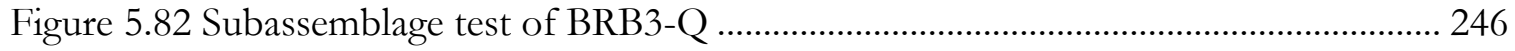

Figure 5.83 Hysteretic response of BRB3-Q........................................................................ 247

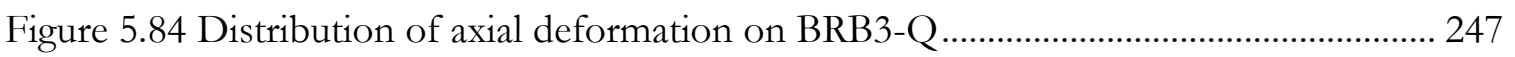

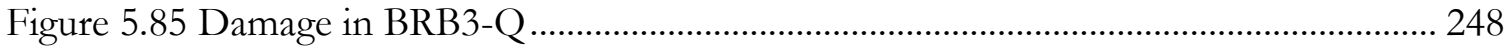

Figure 5.86 Tension adjustment factor. (a) BRB1-Q, (b) BRB2-Q, (c) BRB3-Q ................. 249

Figure 5.87 Compression adjustment factor. (a) BRB1-Q, (b) BRB2-Q, (c) BRB3-Q ......... 250

Figure 5.88 Dissipated energy. (a) BRB1-Q, (b) BRB2-Q, (c) BRB3-Q ................................. 252

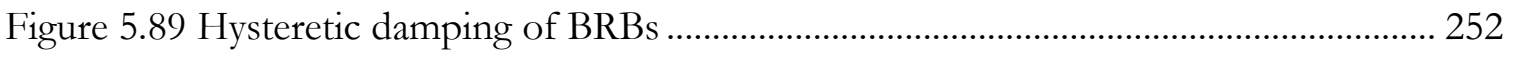

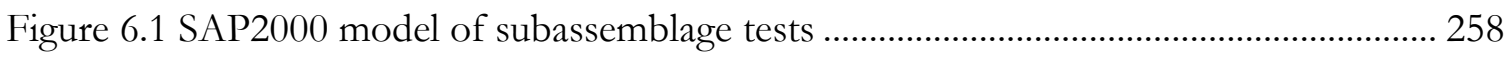

Figure 6.2 Preliminary SAP2000 results. (a) BRB1-Q, (b) BRB2-Q, (c) BRB3-Q ................ 260

Figure 6.3 SAP2000 models based on experimental results. (a) BRB1-Q, (b) BRB2-Q, (c)

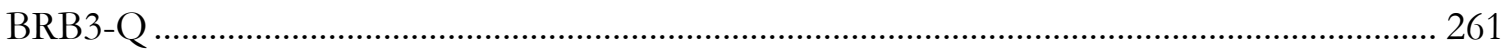

Figure 6.4 OpenSees simulations based on experimental results............................................ 264

Figure 6.5 Model schematic for nonlinear static analysis in SAP2000 ................................. 266

Figure 6.6 SAP2000 models based on experimental results. (a) Bent/BRB1, (b) Bent/BRB2,

Figure 6.7 Numerical model of tested RC bent. (a) As-built, (b) Retrofitted and repaired

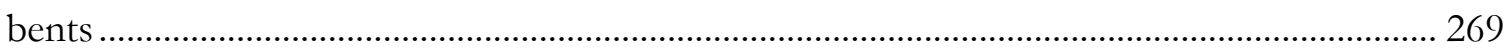

Figure 6.8 OpenSees simulations based on experimental results............................................ 270

xxii 
Figure 6.9 General layout of a MPCG bridge (a) 3D Model (b) Elevation... 272

Figure 6.10 Typical superstructure used in MPCG bridges ...................................................... 273

Figure 6.11 Numerical model for MPCG bridge bents ........................................................... 276

Figure 6.12 Expected force-displacement behavior.............................................................. 276

Figure 6.13 Typical foundations used in MPCG bridges (a) 3D schematic (b) Elevation ... 278

Figure 6.14 Numerical models for pile foundations used in this study.................................. 279

Figure 6.15 Elastomeric bearing pad configuration used in MPCG bridges.......................... 281

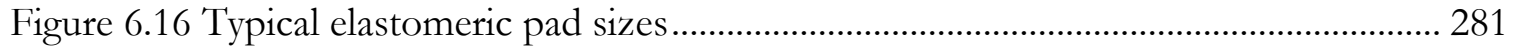

Figure 6.17 Hysteretic behavior of elastomeric pad............................................................... 283

Figure 6.18 Hysteretic behavior of a steel dowel.................................................................... 284

Figure 6.19 Hysteretic behavior of elastomeric bearings.................................................... 284

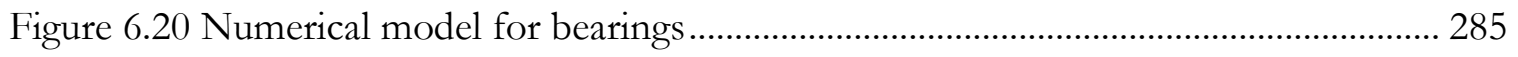

Figure 6.21 Typical abutments used in MPCG bridges (a) 3D schematic (b) Elevation...... 286

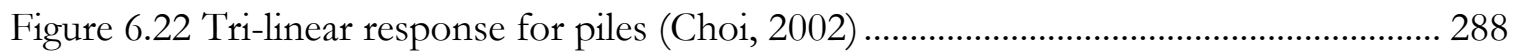

Figure 6.23 Hyperbolic response for abutment backwall (Shamsabadi, et al., 2007) ........... 289

Figure 6.24 Numerical model for abutments ......................................................................... 290

Figure 6.25 Ground motions used in NLTHA of 3D bridge models.................................... 291

Figure 6.26 Results of NLTHA for MCCG bridge with fixed supports. ............................... 292

Figure 6.27 Results of NLTHA for MCCG bridge with additional mass at abutments....... 293

Figure 6.28 Results of NLTHA for MCCG bridge with spring foundations......................... 294

Figure 6.29 General layout of a MCCG bridge (a) 3D Model (b) Elevation........................... 295

Figure 6.30 General layout (a) 3D Model (b) Elevation ......................................................... 297

Figure 6.31 Numerical model for MCCG bridge bent ............................................................. 299

xxiii 
Figure 6.32 Expected force-displacement behavior. 300

Figure 6.33 Typical foundations used in MCCG bridges (a) 3D schematic (b) Elevation... 300 Figure 6.34 Typical abutments used in MCCG bridges (a) 3D schematic (b) Elevation ..... 303

Figure 6.35 Results of NLTHA for MCCG bridge with fixed supports. ............................... 304

Figure 6.36 Results of NLTHA for MCCG bridge with additional mass at abutments....... 305

Figure 6.37 Results of NLTHA for MCCG bridge with spring foundations.......................... 306

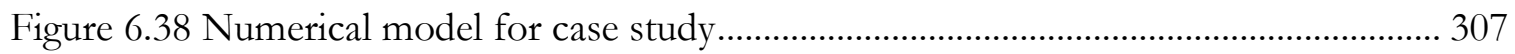

Figure 6.39 Ground motion records and response spectrum for 5\% damping..................... 309

Figure 6.40 Results of nonlinear analysis. (a) As-built bent, (b) Retrofitted bent.................. 311

Figure 6.41 Response of BRBs in the nonlinear analysis ...................................................... 312

Figure 6.42 Toward Operational performance level......................................................... 313

Figure 7.1 Capacity vs demand spectra showing uncertainty in structural behavior and

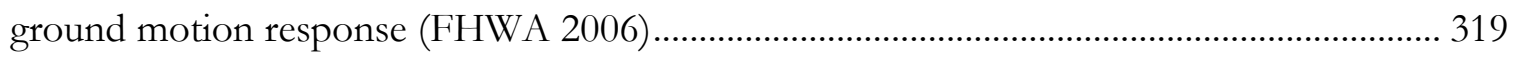

Figure 7.2 Response spectra for the 30 time histories used in this study (5\%damping) ...... 322

Figure 7.3 Performance displacement of as-built RC bridge bent ......................................... 324

Figure 7.4 Performance displacement of retrofitted RC bridge bent .................................... 325

Figure 7.5 Fragility curves for representative as-built RC bridge bent for damage state: (a)

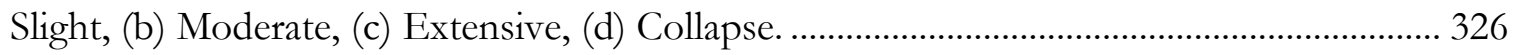

Figure 7.6 Fragility curves for retrofitted RC bridge bent for damage state: (a) Slight, (b)

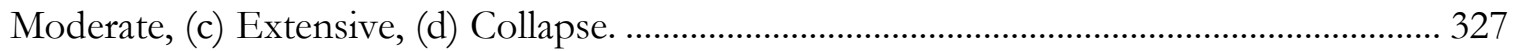

Figure 7.7 Fragility curves. (a) As-built bent, (b) Retrofitted bent......................................... 327

Figure 7.8 Comparison of median values of PGA for representative RC bridge bent......... 328

Figure 7.9 Methodology for seismic risk assessment of RC bridge bents .............................. 330

xxiv 
Figure 7.10 Fragility curves. (a) As-built bent, (b) Retrofitted bent...................................... 331 


\section{CHAPTER 1}

\section{INTRODUCTION}

\subsection{RESEARCH Motivation}

The Cascadia subduction zone (CSZ) is over $1000 \mathrm{~km}$ long boundary between the Juan de Fuca and North American plates. The seismic hazard posed by the CSZ in the Pacific Northwest of the United States had been largely underestimated due to the believe that the Cascadia Subduction zone was a quiet fault. However, with the studies carried out by Atwater et al. (2005) and Goldfinger et al. (2003) we now know that a megathrust subduction earthquake is likely to strike the coasts of the Pacific Northwest. Research studies (Goldfinger, et al. 2008) have shown that major subduction earthquake events have occurred in the Cascadia subduction zone in the past 3000 years including a M9.0 earthquake in 1700 (Atwater, et al., 2005). The recent occurrence of highly devastating subduction megathrust earthquakes of long duration, 2010 Maule, Chile and 2011 Tohoku, Japan have raised researchers' interest in how earthquake duration and number of cycles may affect structural response and overall performance of structural components subjected to subduction zone earthquakes. Capturing the mega subduction effect is of vital importance in the assessment of reinforced concrete $(\mathrm{RC})$ bridge components located in the Pacific Northwest coast of the United States since typical multi-column reinforced concrete bridge bents constructed in the 1950 to mid-1970 in that area were designed and built with minimum seismic considerations. This resulted in inadequate detailing within plastic hinge 
zones, leaving numerous reinforced concrete bents highly susceptible to damage following an earthquake. This high level of seismic hazard creates a need for a more thorough understanding of the differences in structural response resulting from large subduction zone earthquakes, and consequently plan retrofit measures to mitigate the potential damage that these type of earthquakes poses in seismically vulnerable RC bridges.

\subsection{RESEARCH SIGNIFICANCE}

Current seismic retrofitting manuals and guidelines for existing highway structures in the United States are based on a performance-based design methodology, which uses a dual level approach to performance criteria with two seismic hazards (FHWA 2006), (ODOT 2015). Therefore, engineers designing retrofit measures for improving the seismic resistance of existing bridge substructures need to ensure that the structure remains operational under a moderate earthquake and that life safety is preserved after a large earthquake. Conventional seismic retrofit measures including steel jacketing and fiber composite wrapping have been typically utilized to improve the ductility and shear resistance of reinforced concrete substructures, and their implementation have effectively prevented the collapse of bridges during major earthquakes (Kawashima, et al. 2011), (Hoshikuma and Guangfeng 2013). However, these measures neither prevent damaging of columns nor excessive displacements, which in some cases could result in not meeting the intended operational performance level. In order to overcome this problem, this research presents and experimentally validates the option of using Buckling-Restrained Braces (BRBs) as a retrofit and repair measure for reinforced concrete bridges in an effort to successfully demonstrate the effectiveness of utilizing buckling-restrained braces for achieving high displacement ductility of the retrofitted and repaired structure, while also controlling the damage of the existing 
vulnerable reinforced concrete bents. The study of this retrofit technique will contribute to the knowledge of the structural engineering field and would be of great help for bridge design professionals in order to comply with performance-based criteria used to assess typical retrofit and repair options.

\subsection{OBJECTIVES}

The main objectives of the study are as follow:

Develop a rational quasi-static loading protocol capable of simulating the demands imposed on reinforced concrete bridge columns subjected to strong motions of long duration from subduction megathrust earthquakes. In order to achieve this objective, the results from non-linear time history analyses, which considered numerous subduction ground motions, and a simplified rainflow counting procedure (ASTM E1049-85, 2005) were used.

$\square \quad$ Propose design implementation guidelines, perform experimental evaluation and analytical studies on retrofitting deficient RC bridge bents subjected to subduction zone earthquake demands with the aim of achieving an operational performance level. The retrofit measure to be studied comprises steel braces in the form of Buckling-Restrained Braces (BRBs).

$\square \quad$ Assess and experimentally evaluate the option of repairing earthquake-damaged RC bridge bents through the inclusion of BRBs in an effort to restore the strength and stiffness of the damaged structure and improve its energy dissipation capacity. Propose and validate refined numerical models based on experimental results in an effort to aid researchers and designers in performing nonlinear analyses. 
$\square \quad$ Develop seismic fragility curves that describe the conditional probability of exceeding a level of direct or indirect bridge damage for a given level of seismic hazard. Nowadays, fragility curves have emerged as an important decision tool to prioritize bridge retrofitting and estimate potential losses during and after a major earthquake.

\subsection{Research Methodology}

The research was divided into four main parts. First, a cyclic loading protocol was developed in order to reflect the displacement demands that subduction zone earthquakes impose on RC bridge substructures. Second, a literature review was carried out to examine current retrofit measures for RC bridge components. Third, buckling-restrained braces as retrofit and repair measure were analytically assessed and experimentally evaluated with the aim of achieving operational performance level. Finally, nonlinear modeling of the as-built and retrofitted RC bent will be carried out in order to characterize the system, validate the experimental results, and obtain seismic fragility curves. Figure 1.1 shows the schematic of the research methodology. 


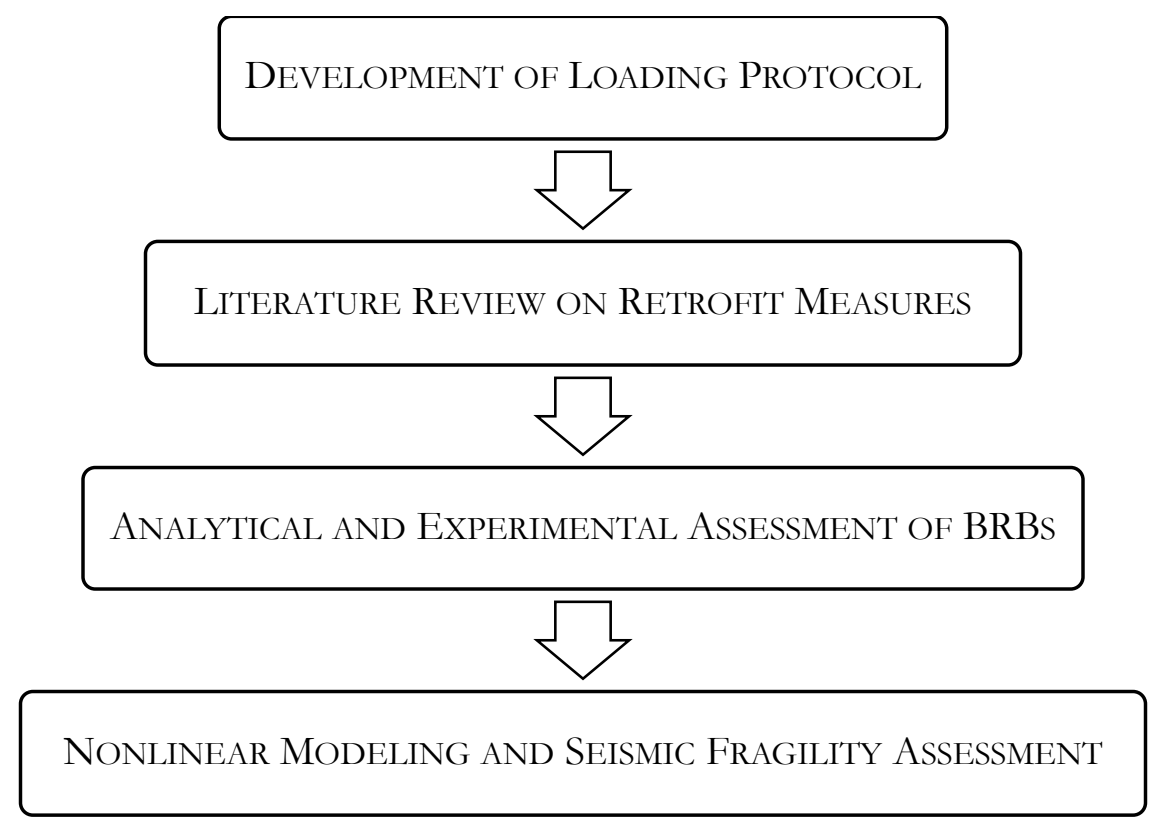

Figure 1.1 Research methodology

\subsection{CONTRIBUtions TO THE FIELD}

The main contributions of this $\mathrm{PhD}$ dissertation research can be summarized as follows:

$\square$ Reflect subduction zone earthquake demands in the form of a stepwise quasi-static loading protocol, which may be utilized in laboratory and numerical evaluations of RC bridge components.

口 Present and analyze the first large-scale experiments utilizing BRBs for the retrofit and repair of multi-column reinforced concrete bridge bents.

$\square \quad$ Propose, analyze and effectively implement a novel gusset plate detailing to connect BRBs to concrete elements.

$\square \quad$ Produce design guidelines for the BRB/RC bent system and brace-to-concrete connection in order to comply with performance criteria required by bridge codes. 
Evaluate the seismic fragility of a representative bridge bent built prior 1970 in the State of Oregon in its as-built and retrofitted condition.

\subsection{Dissertation OUtLine}

The dissertation is organized into eight main chapters with the following contents:

Chapter 1 discusses the motivation of the study, scope of work, objectives and research methodology.

Chapter 2 presents the development of a rational quasi-static loading protocol capable of simulating the demands imposed on bridge structures in an effort to advance the seismic assessment of reinforced concrete bridge columns subjected to subduction zone earthquakes of long duration. In this chapter, numerical and experimental results of seismically deficient square reinforced concrete columns constructed before 1971 in the State of Oregon are also presented with the aim of assessing the effect of the proposed loading histories on substandard RC columns.

Chapter 3 presents a literature review on conventional and emerging retrofit measures capable of improving the seismic resistance of deficient RC bridge substructures.

Chapter 4 describes the option of using sacrificial elements also referred to as structural fuses as a retrofit measure for reinforced concrete bridges in an effort to satisfy a dual level performance criteria, which states that a structure should remain operational under a moderate earthquake and that life safety is preserved after a large earthquake. In particular, Chapter 4 presents the design implementation of buckling-restrained braces for retrofitting and repairing substandard RC bridge bents.

Chapter 5 presents experimental evaluation of using BRBs for retrofitting and repairing $\mathrm{RC}$ bridge bents using cyclic loading protocols that aim to represent displacement 
demands imposed in bridges by subduction zone mega earthquakes. With that aim, five large-scale RC bridge bent specimens are tested in their as-built, retrofitted and repaired states. Moreover, subassemblage tests of three BRBs are performed in an effort to evaluate BRBs at a component level.

Chapter 6 presents numerical characterization and simulations of RC bridge bents based on the experimental results presented in Chapter 5. Numerical simulations of the BRB/RC bent system are conducted using SAP2000 and OpenSees.

Chapter 7 presents fragility curves for the as-built and retrofitted condition of a representative bridge bent. Thus, the effects of subduction zone earthquakes and retrofit measures can be better understood. 


\subsection{Main Components OF A TYPICAL RC BRIDGE}

This section briefly describes the main components of a typical RC bridge since those terms are used throughout this dissertation. An illustration of the main components is shown in Figure 1.2.

Superstructure: The portion of a bridge that is directly subjected to live loads such as pedestrian traffic, trucks, etc., is referred to as the superstructure. The superstructure may consist of a bridge deck, longitudinal beams also referred to as girders or stringers, transverse beams or diaphragms, and barriers or parapets.

Substructure: Part of a bridge that supports the superstructure and has the function of transferring the loads from the superstructure to the foundation. The substructure includes: abutments, footings and RC bridge bents (or piers). The latter comprise RC columns and cap beams (bent cap) and are the focus of this study.

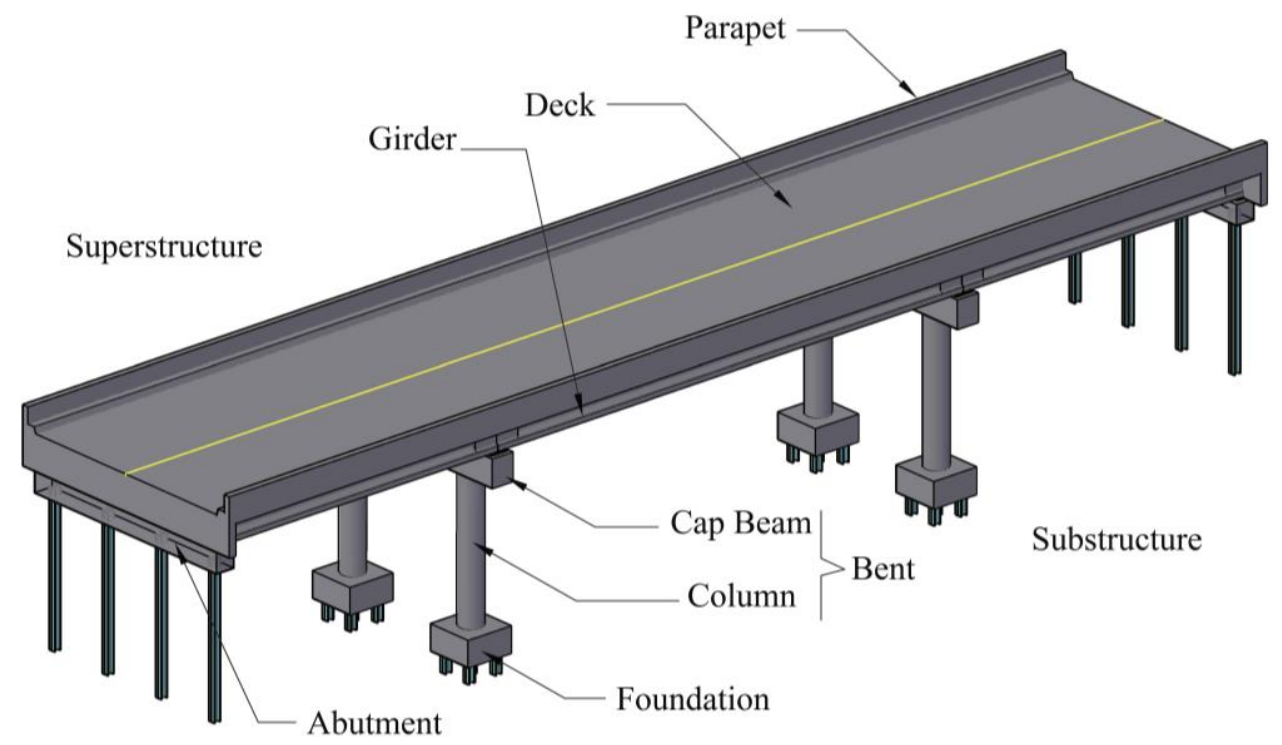

Figure 1.2 Illustration of bridge components 


\section{CHAPTER 2}

\section{CYCLIC LOADING PROTOCOL CONSIDERING SUBDUCTION ZONE EARTHQUAKE DEMANDS}

\subsection{GENERAL}

All structural components have limited capacity. For that reason, understanding their behavior under strong ground motion excitations has always been a major objective of earthquake engineering. One method to assess the performance of structural components is via experimental evaluations utilizing quasi-static cyclic loading. The relatively slow application of the load in quasi-static tests allows experimentalists to relate structural metrics such as top displacement, chord rotation, drift, strains, etc. to visual damage of specimens (e.g., first cracking, spalling of the concrete, buckling of longitudinal reinforcement). Current earthquake design procedures for structural components have been established based on experimental results utilizing quasi-static cyclic tests. Moreover, design codes are trending to a relatively new design methodology called "performance-based seismic design" (PBSD). In this methodology, a number of performance levels, which are frequently defined in terms of acceptable levels of damage, need to be satisfied under different levels of seismic hazards. Under this design methodology the assessment of different structural components plays a fundamental role.

Numerous experimental and analytical studies have been conducted in order to assess structural components, define limit states and acceptance criteria to be used in performance-based seismic design [Hose and Seible (1999), FEMA356 (2000), 
ASCE/SEI41-06 (2007)]. Many protocols have been developed and utilized to assess structural and non-structural components, e.g. Krawinkler et al. (1983), Cheung et al. (1991), ATC-24 (1992), Krawinkler et al. (2000a), (2000b), Richard and Uang (2006), FEMA461 (2007), Hutchinson and Wood (2013), ACI374.2R-13 (2013). Despite these efforts, past loading protocol developments had not considered subduction ground motions because a scarcity of this type of record. However, the recent occurrence of highly devastating subduction megathrust earthquakes of long duration, 2010 Maule, Chile and 2011 Tohoku, Japan, have raised researchers' interest in how earthquake duration and number of cycles affect structural response, collapse assessment and overall performance of structural components subjected to subduction zone earthquakes. The occurrence of these seismic events suggests that large magnitude ground motions of long duration have the potential of significantly increase the number of inelastic excursions and consequently incur more extensive structural damage compared to ground motions with similar elastic spectral displacement demands but less duration (Dusicka \& Knoles, 2012), (Raghunandan \& Liel, 2013), (Chandramohan, et al., 2013). This effect is mostly attributed to the rate of structural strength and stiffness deterioration due to an increase in load reversals imposed for large magnitude and long duration ground motions. This aspect is particularly relevant in subduction zones due to the fact that larger magnitude earthquakes are associated with strong motions of long duration (Dobry, 1978), (Midorikawa, et al., 2012). The increase of inelastic demands creates a critical necessity to improve current loading protocols, which predominantly have been developed using crustal ground motions of moderate to high magnitude. 
Capturing the mega subduction effect is of vital importance in the assessment of RC bridge components located in the Pacific Northwest coast of the United States which lies near the Cascadia subduction zone and where a mega thrust earthquake of long duration forms a major component of the seismic risk. This chapter discusses the development of rational quasi-static loading protocols capable of simulating the demands imposed on reinforced concrete bridge columns and its effect is investigated both numerically and experimentally.

\subsection{IMPLICATIONS FROM PAST RESEARCH}

Limited experimental data can be found on reinforced concrete columns subjected to long duration protocols that try to simulate subduction zone earthquakes since most of the seismic assessment of RC bridge columns has been carried out using conventional cyclic loading protocols, such as those shown in Figure 2.1. These conventional protocols have been developed to reflect seismic cumulative demands of short period structures that are not representative of long period structures (Cheung, et al., 1991), (Priestley, et al., 2002), (ACI 374.2R-13, 2013) and do not represent the demands imposed by subduction zone earthquakes.

Experimental studies have shown that the displacement capacity and the failure mode of structural components is influenced by the loading history applied to them. In this section relevant experimental research studies with regard to the loading history effect on RC columns are briefly described. 


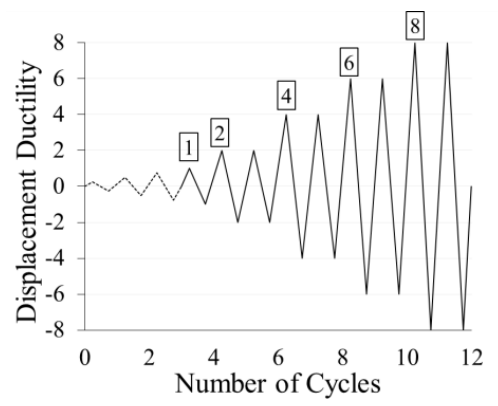

(a)

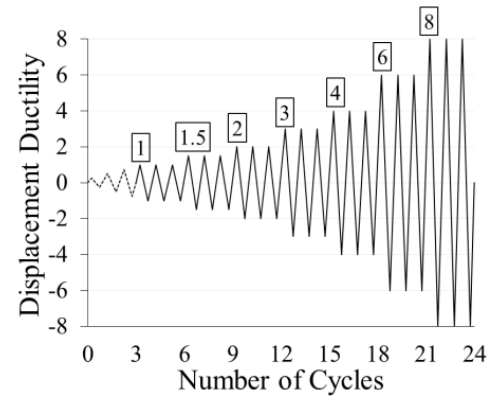

(b)

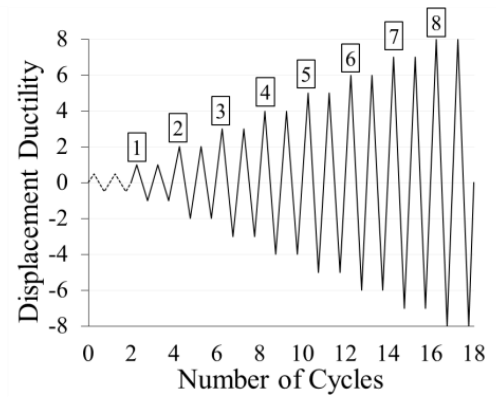

(c)

Figure 2.1 Conventional loading protocols used on RC column assessment. (a) New Zealand Protocol (Cheung, Pauley and Park 1991), (b) Modified New Zealand Protocol (Priestley, Seible and Hines 2002), (c) ACI374 unidirectional protocol (ACI 374.2R-13 2013).

\subsubsection{Ohno and Nishioka (1984)}

Ohno and Nishioka conducted an experimental research to investigate the energy dissipation capacity of reinforced concrete bridge columns under repeating cyclic loading. The experimental program consisted of five RC columns tested under four different loading histories. The columns were representative of bridge columns having shear-span ratio about 4. The results of this study showed that the displacement capacity and the cumulative energy dissipation of RC members is clearly affected by the number of loading cycles. However, they also concluded that the total energy dissipation capacity of RC columns is not affected by the total number of loading cycles.

\subsubsection{Takemura and Kawashima (1997)}

A relevant research study was carried out by Takemura and Kawashima (1997) to study the influence that different loading histories has on the ductility capacity of reinforced concrete bridge piers. In Takemura's research six nominally identical specimens were tested under different loading protocols resulting in six different responses. They concluded that a lower value of drift is achieved when a larger number of loading cycles is utilized. 


\subsubsection{Kunnath, et al. (1997)}

Another relevant research was carried out by Kunnath, et al. (1997) to investigate the cumulative seismic damage in circular reinforced concrete bridge columns, which were mostly controlled by flexural behavior. With that purpose, twelve columns were tested to quarter-scale. Test results led to the conclusion that the load path highly influences the failure mode of RC columns. This study found that columns subjected to cycles of low amplitude were likely to present a failure in the confinement rather than in the longitudinal bars. On the other hand, under cycles of high amplitude the failure mode was controlled by rupture in the longitudinal reinforcement. The study also revealed that conventional loading protocols commonly used in experimental testing tend to replicate unrealistic drift demands because numerous large inelastic reversals are imposed in the component. This is not representative of typical earthquakes demands, which usually imposed several inelastic cycles of low amplitude and just a few large inelastic cycles. They also demonstrated that the number and amplitude of inelastic cycles are very important in order to predict damage.

\subsubsection{McDaniel, et al. (2006)}

Similarly, using the concept of low-cycle fatigue and the cumulative damage model employed in the research carried out by Kunnath, experimental tests were performed at the Washington State University in order to investigate the performance of pre-1975 concrete bridges subjected to subduction earthquakes (McDaniel, et al., 2006). In this research, eight circular lightly confined reinforced concrete columns were tested using different displacement history to represent the demands imposed by subduction zone ground motions of long duration. Loading protocols with cycles of constant amplitude were performed to simulate those demands. The columns showed three different modes of failure depending on 
the load protocol employed. The first mode of failure was an interaction of shear and flexure when the applied protocol consisted of large initial inelastic cycles; the second failure mode was buckling of the longitudinal reinforcement when the column was subjected to many small inelastic cycles. The final mode of failure was slipping of the longitudinal reinforcement under a considerable amount of small amplitude inelastic cycles followed by large ones. Thus, these results, as well as those obtained by Kunnath (1997), showed that the failure mode of RC columns depends on the displacement history applied to them.

\subsubsection{Ranf, et al. (2006)}

Ranf, et al. (2006) tested six nominally identical lightly confined circular RC bridge columns under six different loading protocols in order to evaluate the effect of cycling on the performance of such columns. The lightly confined columns were representative of typical bridge columns constructed before the mid-1970s in the State of Washington. The results led to the conclusion that the maximum top displacement preceding failure can decrease by $35 \%$ when the number of cycles at the same displacement amplitude increased from one to fifteen.

\subsubsection{Pujol, et al. (2006)}

Six flexural-dominated reinforced concrete columns were tested under different lateral displacement histories and constant axial load with the aim of studying the effect of displacement history. In this study special attention was put in columns subjected to relatively high nominal shear stresses. The main conclusion of Pujol's study was that the drift capacity and stiffness deterioration were sensitive to displacement history and are functions of the amplitude and number of cycles. 


\subsubsection{Borg, et al. (2012)}

In this work, six reinforced concrete columns, three square cross sections and three rectangular cross section, were tested using step-wise loading histories having different number of cycles at each displacement level. Same axial load ratios was used for both cross sections. The results of this study showed that the nonlinear response is influenced by the displacement history and the number of cycles.

\subsubsection{Goodnight, et al. (2013)}

In this work, eight nominally identical well-confined circular RC bridge columns were tested under various unidirectional loading histories. The displacement histories comprised conventional laboratory cyclic loadings as those shown in Figure 2.1 and displacement responses from nonlinear time history analysis of crustal and subduction ground motions. Test results showed that the buckling of the longitudinal reinforcing steel was affected by the applied load history. They also concluded that the three-cycle-set load history (Modified New Zeland Protocol) was more severe than the loading history recreated from the displacement response caused by ground motions because of the high number of inelastic cycles at high displacement amplitudes imposed by conventional protocols.

\subsubsection{Ou, et al. (2013)}

Well-detailed rectangular reinforced concrete bridge columns were tested applying two different loading protocols to investigate the influence of the number of cycles on bridge columns. Test results showed that columns under a long duration protocol behave significantly different in terms of strength and stiffness degradation than those columns under conventional protocols showing that on high levels of damage the strength and 
stiffness degradation of the specimen would increase markedly under long duration earthquakes of large magnitude.

Thus, research studies in conjunction with the occurrence of devastating subduction zone mega earthquakes demonstrate that the development of loading protocols reflecting the increase in the number of inelastic demands posed by ground motions of large magnitude and long duration is needed to improve the assessment of RC bridge columns through experimental evaluations.

\subsection{SELECTION OF EARTHQUAKE GROUND Motions}

Regions of the Pacific Northwest Coast of the United States, which lies near the Cascadia subduction zone (also referred to as the Juan de Fuca subduction zone), may be affected by a megathrust earthquake of long duration similar to those occurred in Chile and Japan (Heaton \& Hartzell, 1986). Most of the loading protocols used in seismic assessment have been developed for a specific structural or nonstructural component by utilizing a set of crustal ground motions. These ground motions are often representative of the $10 \%$ probability of exceedance in 50 years (10/50) hazard level for Los Angeles conditions and were selected to avoid near-fault effects, at distances from fault rupture greater than $13 \mathrm{~km}$, and from crustal earthquakes of moment magnitudes $\left(\mathrm{M}_{\mathrm{w}}\right)$ varying between 6.7 and 7.3. (Krawinkler, et al., 2000a), (FEMA 461, 2007).

With the aim of developing representative loading protocols for bridge components, a selection of strong motion records was conducted in order to determine the inelastic demands imposed by subduction megathrust earthquakes as shown in Table 2.1. The subduction zone ground motion sets used in the development of loading protocols were chosen from the 1985 Valparaiso (COSMOS), 2007 Sumatra (COSMOS), 2010 Maule (U. 
Chile), and 2011 Tohoku (K-Net) earthquakes with distances to the epicenter greater than $100 \mathrm{~km}$ to reduce the number of records and ensure far field response. The subduction ground motion sets, in spite of being treated as a single set called Subduction, were divided into four sub-sets in order to observe the differences in the inelastic demands that could be generated by variations in earthquake characteristics or regional geology. Tohoku ground motions were further differentiated, referred to herein as Tohoku1 and Tohoku 2, because of the large amount of records available on the Kyoshin Network Database (K-Net) and in order to have one of the Tohoku sets with similar PGA range to the other ground motion sets. Only one pair of ground motions for the 2007 Sumatra earthquake was utilized due to the lack of strong motion records available.

A set of crustal ground motions was utilized to allow for demand comparisons. Crustal ground motions, referred to herein as Crustal set, were chosen from the FEMA P695 far-field record (FEMA P695, 2009), which is based on a representative set of twenty-two horizontal ground motions taken from the PEER database (PEER, 2006) with the following criteria: from sites located at distance greater than $10 \mathrm{~km}$ from the fault rupture, moment magnitude greater than 6.5, recorded from soils categorized as Site Class C and D, and originated from shallow crustal sources (strike-slip or reverse fault mechanisms). The number of strongest records was limited from each earthquake to two and thus avoid bias in the results.

For each ground motion recording two orthogonal horizontal records were treated separately, while the vertical ground motion components were not considered. Although, there is no general consensus on the definition and determination of the strong ground motion duration, in this study the duration was taken as the "Bracketed Duration", which is 
defined as the first to the last occurrence of an acceleration of $0.05 \mathrm{~g}$ (Bolt, 1969). The average duration of subduction ground motions was found to be at least twice that of the crustal set as summarized in Table 1 and depicted in Figure 2.2 for selected ground motions. Furthermore, the bracketed duration for the Tohoku sets is from 7 to 10 times higher than the duration for the crustal set. No scaling procedure was employed on the records. In this manner, the earthquake source variability was minimized since recent studies have demonstrated that scaling input records to a target spectral acceleration level $\left(\mathrm{S}_{\mathrm{a}}\right)$ can produce biased results (Luco \& Bazzurro, 2007).

Table 2.1 Ground motion sets used in this study

\begin{tabular}{c|c|c|c|c|c}
\hline Set & $\mathrm{M}_{\mathrm{w}}$ & $\begin{array}{c}\text { Site } \\
\text { Class }\end{array}$ & $\begin{array}{c}\text { PGA } \\
\text { Range (g) }\end{array}$ & $\begin{array}{c}\text { No. } \\
\text { Records }\end{array}$ & $\begin{array}{c}\text { Average } \\
\text { Duration (sec) }\end{array}$ \\
\hline Crustal & $6.5-7.6$ & $\mathrm{C} / \mathrm{D}$ & $0.21-0.82$ & 37 & 15 \\
\hline Valparaíso & $7.8^{2}$ & $\mathrm{~B} / \mathrm{D}$ & $0.11-0.71$ & 36 & 39 \\
\hline Sumatra & 7.9 & - & 0.13 & 2 & 48 \\
\hline Maule & 8.8 & $\mathrm{~B} / \mathrm{D}$ & $0.13-0.93$ & 31 & 53 \\
\hline Tohoku 1 & 9.0 & $\mathrm{~B} / \mathrm{C} / \mathrm{D}$ & $0.94-2.01$ & 27 & 153 \\
\hline Tohoku 2 & 9.0 & $\mathrm{D} / \mathrm{E}$ & $0.20-0.81$ & 166 & 110 \\
\hline
\end{tabular}

${ }^{2}$ Referred to Ms magnitude. Ms: Surface wave magnitude 


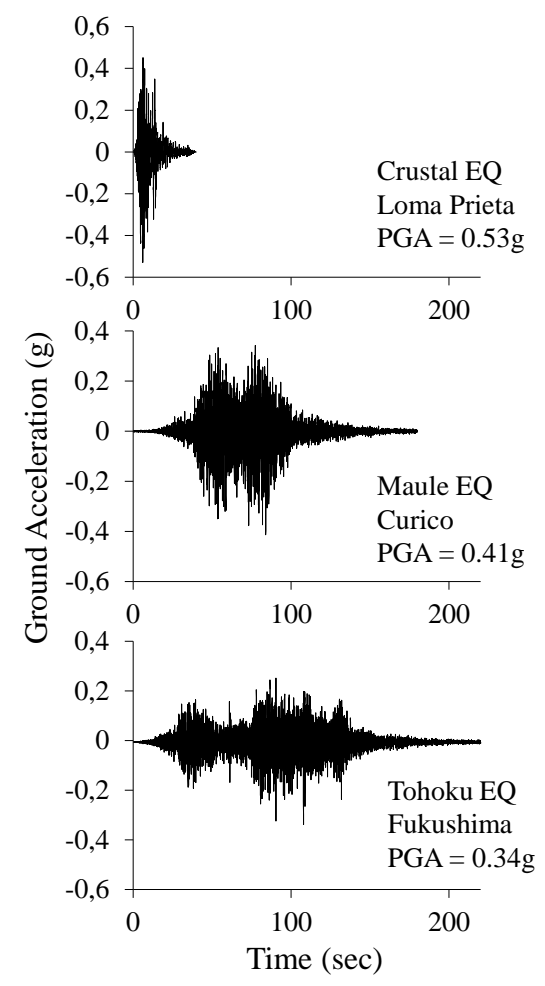

Figure 2.2 Difference in ground motion duration for selected ground motions

\subsection{Hysteresis MOdel AND TARget RESPONSE}

The main objective of a loading protocol is to assess the structural capacity of components. Since demand and capacity are not independent, it is reasonable to think that one should know the seismic excitation imposed on the component and its response before the development of any protocol.

In order to predict the damage that a structure undergoes during severe earthquakes, it is important to represent in a realistic way the behavior of structural components during loading reversals. In the case of reinforced concrete components, the Clough (1966) and Takeda (1970) hysteretic models are widely used. However, these models assume that the stiffness degradation is related to the maximum displacement of the system and not to the number of cycles. Since the analyses were expected to incorporate numerous large inelastic 
excursions, the peak oriented Ibarra-Medina-Krawinkler hysteretic model (Ibarra, et al., 2005a) was utilized. This model hereinafter referred to as "Degrading model" includes strength capping, residual strength, and strength and stiffness deterioration caused by load reversals as illustrated in Figure 2.3. This model was calibrated using test results of bridge columns dominated by flexural behavior. Column tests can be found in the Pacific Earthquake Engineering Research Center (PEER) structural performance database. This process allowed finding appropriate parameters to closely simulate load-deformation behavior of the components in study.

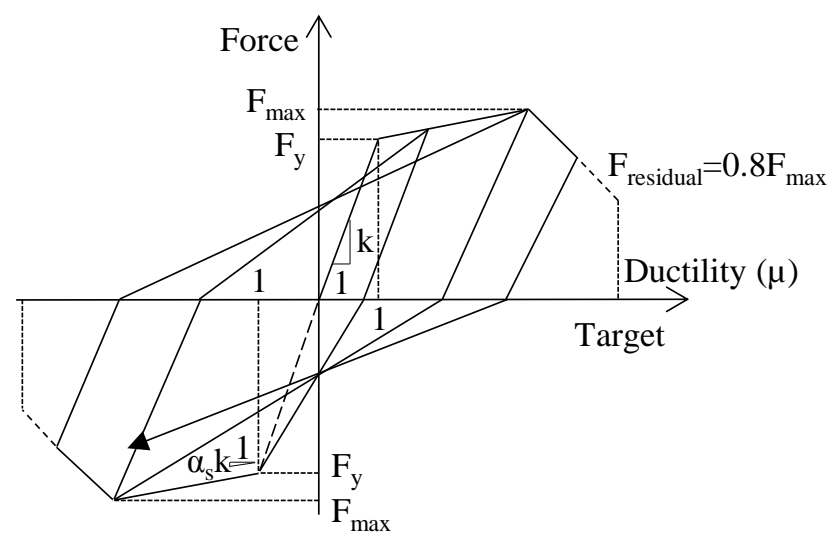

Figure 2.3 Hysteretic behavior - Degrading Model. Adapted from Ibarra et al. (2005a)

Results from nonlinear time-history analyses of single degree of freedom systems (SDOF) using OpenSees (2013) were utilized. In OpenSees each SDOF system was modeled as a zero length element. The model parameters were calibrated using test results of bridge columns dominated by flexural behavior. A strain hardening ratio $\left(\alpha_{\mathrm{s}}\right)$ of 0.0167 was found to fit well the hysteresis model with the experimental results. The capacity boundary parameters of the hysteresis model, such as the pre-capping rotation $\left(\theta_{p}\right)$ and the postcapping rotation $\left(\theta_{p c}\right)$, were defined based on FEMA 440A (2009) and the results of the calibration process. The ultimate rotation capacity $\left(\theta_{u}\right)$ was defined to be very large in order 
to aid the numerical computations. The cyclic deterioration parameter $(\lambda)$, which is at least somewhat dependent on the loading protocol, was defined so that no significant cyclic deterioration existed. A default value of 1 was used to represent the rate of strength deterioration $(c)$ and cyclic deterioration $(D)$. Parameters for the hysteresis model are shown in Table 2.2. A damping ratio of 5\% was set for the analysis. A wide range of structural fundamental periods were considered from 0.2 to 4.0 seconds by maintaining the mass of the system constant and varying the stiffness of the SDOF system.

Table 2.2 Degrading model parameters

\begin{tabular}{c|c|c|c}
\hline Parameter & Ductility 2 & Ductility 4 & Ductility 8 \\
\hline$k$ & $k=\frac{m}{(T / 2 \pi)^{2}}$ & $k=\frac{m}{(T / 2 \pi)^{2}}$ & $k=\frac{m}{(T / 2 \pi)^{2}}$ \\
\hline$\alpha_{s}$ & 0.0167 & 0.0167 & 0.0167 \\
\hline$\lambda$ & $50 F_{y}$ & $100 F_{y}$ & $200 F_{y}$ \\
\hline$c$ & 1.0 & 1.0 & 1.0 \\
\hline$\theta_{p}$ & $0.001\left(f_{y} / k\right)$ & $1.44\left(f_{y} / k\right)$ & $4.32\left(f_{y} / k\right)$ \\
\hline$\theta_{p c}$ & $2.5\left(f_{y} / k\right)$ & $3.4\left(f_{y} / k\right)$ & $3.56\left(f_{y} / k\right)$ \\
\hline$\theta_{u}$ & $100,000\left(f_{y} / k\right)$ & $100,000\left(f_{y} / k\right)$ & $100,000\left(f_{y} / k\right)$ \\
\hline$D$ & 1.0 & 1.0 & 1.0 \\
\hline Residual & 0.8 & 0.8 & 0.8 \\
\hline
\end{tabular}

Since the majority of modern seismic design codes for bridges (AASHTO, 2009), (Caltrans, 2013) rely on component ductilities, a constant ductility inelastic response approach (Ridell \& Newmark, 1979), (Krawinkler, 1996) was deemed suitable to perform the nonlinear analyses. This approach assumes that for each ground motion and at each period, the structural system is designed to reach the pre-determined ductility $(\mu)$ by finding the yield strength of the structure that produced the desired ductility value. Ductility is defined as the ratio of the maximum displacement to the yield displacement $\left(\mu=\delta_{\mathrm{u}} / \delta_{\mathrm{y}}\right)$, where the maximum ductility capacity was calculated when the force in the post-capping range degraded to not less than 80 percent of the maximum as illustrated in Figure 2.3. This 
assumption of $20 \%$ of loss in load carrying capacity was deemed suitable in the analysis since it has been a common definition used by experimentalists to determine the maximum available displacement from laboratory testing (Park, 1989). Consequently, recursive analyses were needed to design each system in order to reach pre-determined displacement ductilities of 2, 4 and 8 . The recursive process is presented through a flowchart in Figure 2.4 and illustrated in Figure 5.

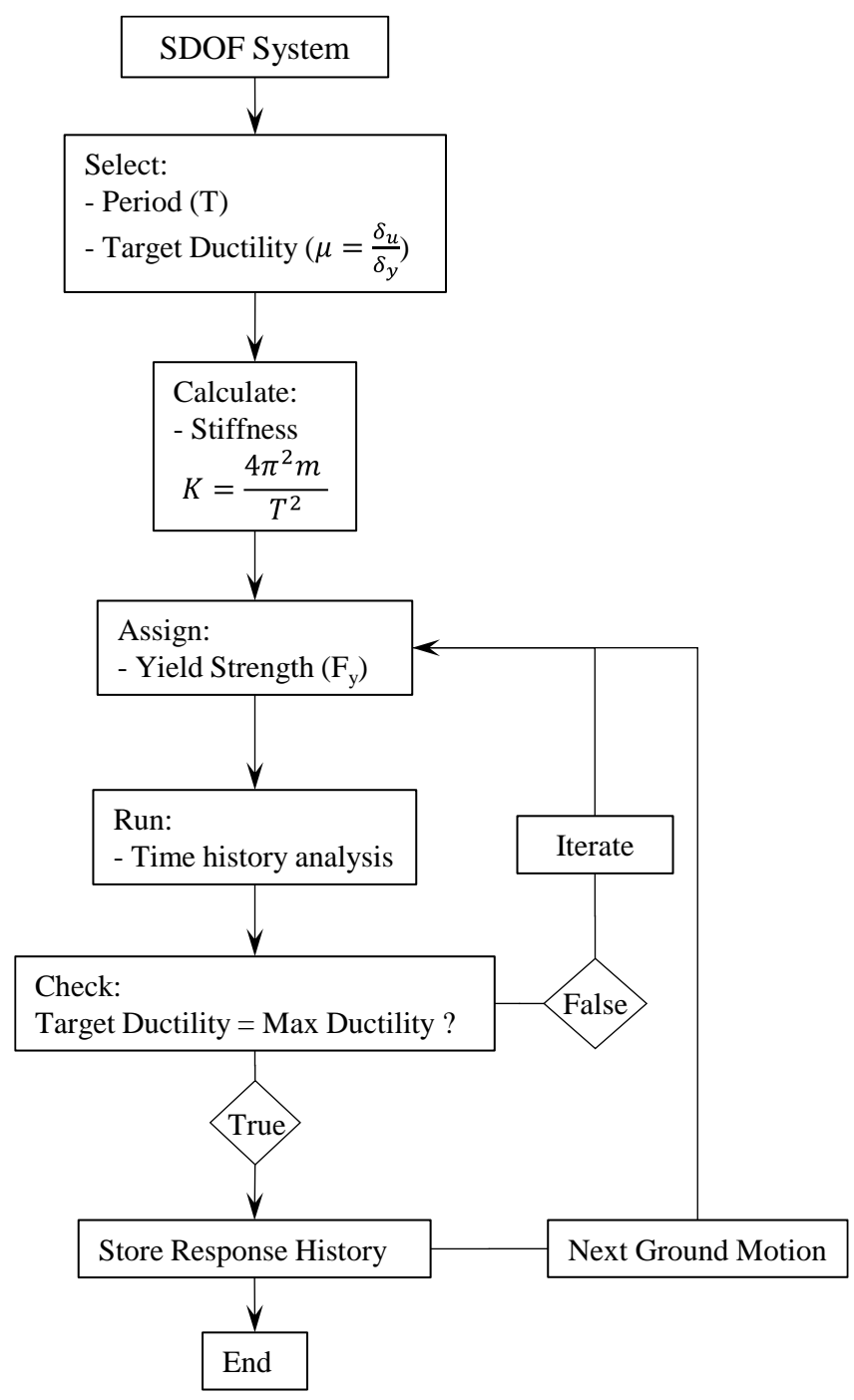

Figure 2.4 Flowchart of target ductility design procedure 


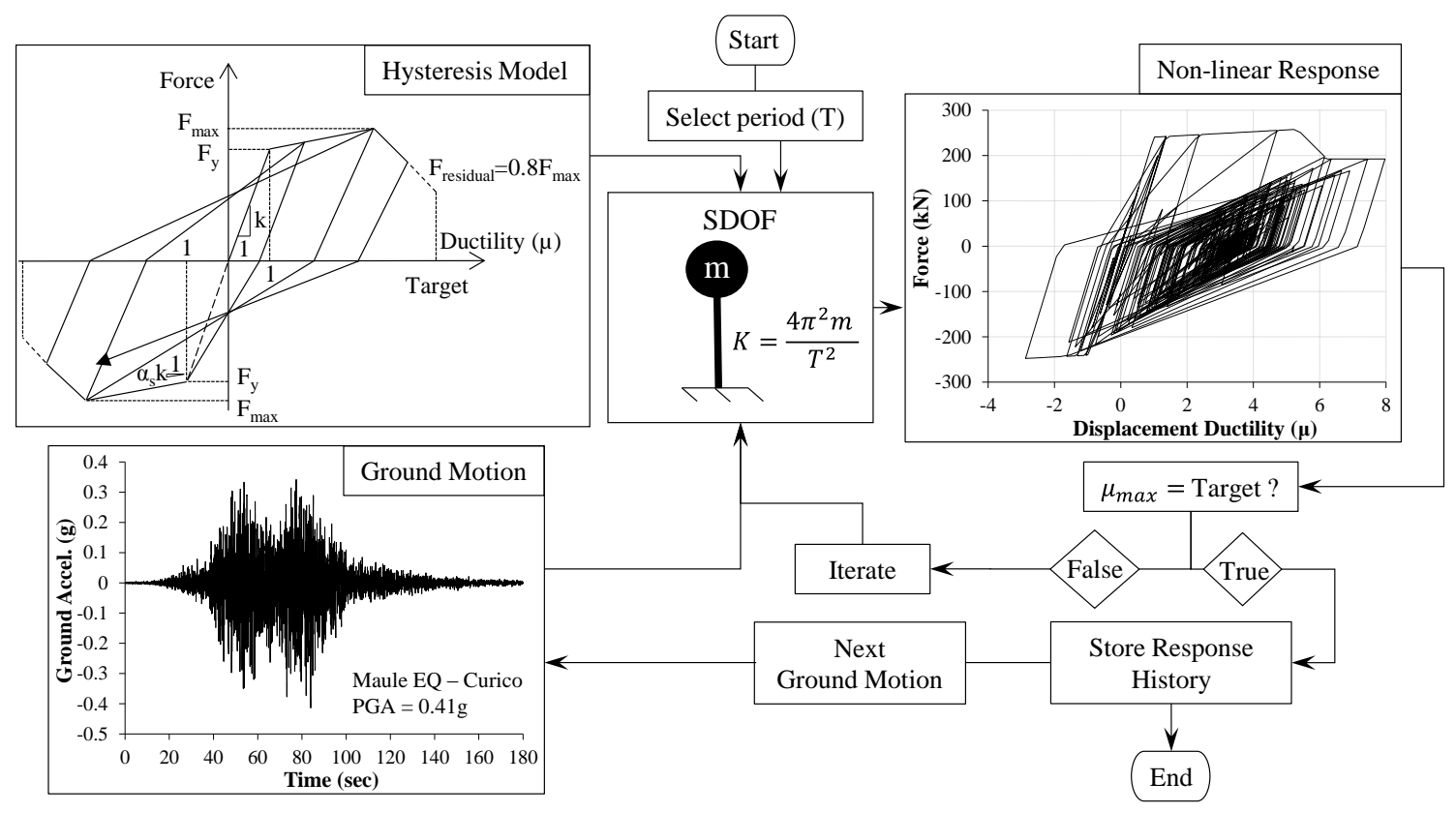

Figure 2.5 Illustration of target ductility design procedure

\subsection{Cumulative Damage}

Strength and deformation capacities of structural components depend on the cumulative damage that the system undergoes under load reversals imposed by seismic excitations. A direct relationship between the number of inelastic cycles and damage can be assumed (Krawinkler, et al., 1983), (Stephens \& Yao, 1987). Various models have been developed to quantify this relationship (Cosenza, et al., 1993) (Ghobarah, et al., 1999). In order to capture the behavior under ground motion excitations, most of current testing protocol developments and experimental studies have been conducted based on a general cumulative damage concept using the Coffin-Mason model and the Miner's rule of linear damage accumulation as a baseline (Krawinkler, et al., 1983). This concept implies that every excursion in the inelastic range will cause damage in the component, and this damage will be accumulated from excursion to excursion, where an excursion is defined as the path from one peak to the next peak in the time history response. Thus, the performance of structural 
components depends on the previous inelastic excursions alluding to a memory of past damaging events. In this approach, the damage is directly affected by the number of inelastic excursions $(N)$, the range of each plastic excursion $\left(\Delta \delta_{\mathrm{pi}}\right)$, and the sum of those ranges as shown in Eq. (2.1). C and c are structural performance parameters that depend on the type of component and failure mode. The parameter $\mathrm{c}$ is usually greater than 1.0 , which implies that large inelastic excursions cause more damage than low ones. This damage index has demonstrated good correlations between the quantitative and descriptive damage for steel structures (Krawinkler, et al., 1983) and reinforced concrete structures (Kunnath, et al., 1997).

$$
D=C \sum_{i=1}^{N}\left(\Delta \delta_{p i}\right)^{c}
$$

Another damage index used in reinforced concrete structures was formulated by Park and Ang (1985), which accounts that damage is caused by the maximum deformation and the cumulative dissipated energy as shown in Eq. (2.2).

$$
D_{p a}=\frac{\delta_{\max }}{\delta_{u}}+\frac{\beta}{Q_{y} \delta_{u}} \int d E
$$

Where $D_{p a}$ is the damage index; $\delta_{\max }$ is the maximum response deformation; $\delta_{u}$ is the ultimate deformation capacity under static load (monotonic response); $Q_{y}$ is the calculated yield strength; $d E$ is the incremental dissipated hysteretic energy; and $\beta$ is a factor that depends on several structural parameters that try to measure the duration effect on the hysteretic energy. However, in order to calculate the damage indices $D$ and $D_{p a}$, in a meaningful way, the parameters $\mathrm{c}, \mathrm{C}$ and $\beta$ have to be experimentally obtained and validated, which can lead to undesirable uncertainties in the development of the protocol since those parameters are directly influenced by the cyclic loading history. For that reason, in this study 
was employed another damage index also based on cumulative damage referred to as Cumulative Displacement Ductility Factor (CDD) (Park, 1989). This metric is calculated by accumulating the ratio of plastic displacement under an excursion $\left(\delta_{\mathrm{pi}}\right)$ to the yield displacement $\left(\delta_{\mathrm{y}}\right)$ as shown in Eq. (2.3).

$$
C D D=\sum_{i=1}^{N} \frac{\delta_{p i}}{\delta_{y}}=\sum_{i=1}^{N} \mu_{i}
$$

Caution is needed when assessing the effects of several loading cycles through the use of the CDD factor since two cyclic loading protocols can have the same CDD but different number of inelastic cycles, which can lead to a significant difference in damage. For that reason, the number of damaging cycles $(\mathrm{N})$ in conjunction with the CDD were considered the target demand parameters in the development of testing protocols for RC bridge columns. In this sense, a cycle is considered damaging when its amplitude is greater than the yield displacement.

\subsection{Cycle Counting}

The earthquake response exhibited by a structural component contains excursions that are not symmetric, and do not follow a consistent pattern from one ground motion to the next. Sequence effects, which are related to the fact that not all the inelastic excursions occur before the maximum one, have not been fully established through analytical and experimental studies. This is primarily because the sequence of inelastic cycles that a strong ground motion imposed on a structure does not follow a regular pattern. In order to overcome this effect in the development of loading protocols for structural components, researchers have been using the concept of pre-peak excursions (Krawinkler, et al., 2000a), (FEMA 461, 2007). Understanding as pre-peak excursion any excursion that occurs before 
either the maximum or minimum peak displacement. As discussed in Krawinkler, et al. (2000a), using all excursions may lead to an overestimation of cumulative damage because most of the damage sustained by a structural component occurs when excursions tend to widen the hysteresis response. This overestimation on cumulative damage is even more relevant in models that take into account stiffness and strength degradation since an excursion that occurs after the peak excursion would present significant degradation in stiffness and strength; consequently, adding little hysteretic energy dissipation and cumulative damage even if its amplitude is large. The development of the loading protocols in this study was primarily based on pre-peak excursions. However, given the long duration of the subduction records, both pre-peak as well as all excursions were considered for comparison purposes in order to analyze the implications of selecting one over the other.

To rationalize the development of the testing protocol and compare the demands imposed by different ground motions, the time history responses based on pre-peak excursions were converted into a series of cycles using the simplified rainflow counting methodology (ASTM E1049-85, 2005) as illustrated in Figure 2.6. This method of cyclecounting results in a deformation history composed only of full cycles due to the fact that the history response is re-arranged in a way that always starts with either the maximum or minimum peak.

The resultant excursions and cycles obtained after performing the rainflow counting are not symmetric with respect to the undeformed condition, also referred to as the mean effect, which could distort the cumulative damage of the structure. However, this effect is not considered in many practical cases because the effect of large mean deformations primarily influences the response in situations of near fault rupture (Krawinkler, et al., 
2000b). Thus, the proposed displacement protocols were developed with a stepwise increasing deformation of symmetric excursions instead of asymmetric excursions.
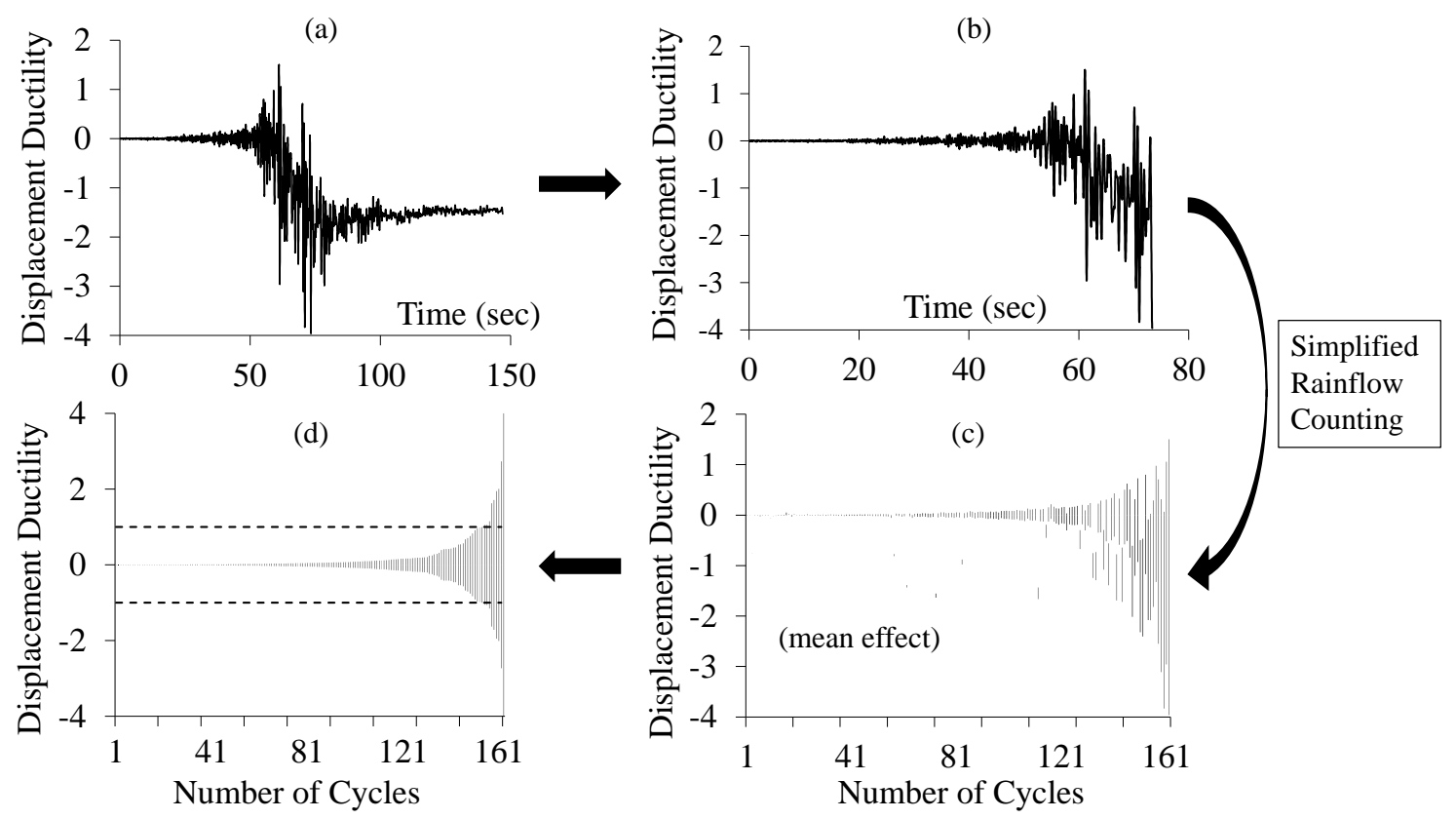

Figure 2.6 Illustrative procedure for simplified rainflow counting: (a) inelastic response history; (b) pre-peak inelastic response history; (c) ordered cycles including mean effect; (d) final ordered cycles

The rainflow counting procedure was employed in the non-linear time history response of individual SDOF systems across the range of fundamental periods. This generated a vast amount of data, which was statistically reduced to allow for comparisons in a rational way. The data that were extracted and post-processed from the rainflow counting procedure was the number of inelastic cycles and the cumulative ductility factor (CDD), where the number of inelastic cycles was equal to the number of cycles above ductility one $(\mu=1.0)$, as shown in Figure 2.6(d), and the CDD was obtained by using Eq. (3). A Matlab script was developed to perform the simplified rainflow counting procedure and the statistical analyses. 


\subsection{Cyclic Counting Results and Protocol Development}

Results from the three pre-determined ductilities and a wide range of structural fundamental periods were considered in the development of the protocols in order to represent a vast number of bridge structures. Moreover, the results obtained by using structures of different fundamental periods was specially considered since conventional protocols commonly used to assess the seismic performance of RC structures were developed to reflect demands on short period structures (FEMA 461, 2007), which are not often representative of bridge responses.

The number of inelastic cycles and the cumulative displacement ductility factor (CDD), which were obtained from performing the rainflow counting procedure, were found to be normally distributed. Therefore, in order to represent a reasonable and conservative estimate of the parameters, and also to bound the applicability of the resulting protocols, the parameters were represented by employing the 84 th percentile (mean +1 standard deviation) as a target value. From these results, the number of inelastic cycles and the CDD showed a high dependence on the fundamental period, as illustrated in Figure 2.7 and Figure 2.8. The figures represent the $84^{\text {th }}$ percentile of each set and clearly trend toward reduced demand with increase in period. Furthermore, the $84^{\text {th }}$ percentile of all the subduction records was utilized to develop the protocols since the results of each set of subduction ground motions showed a similar trend for structures with periods less than $2.0 \mathrm{sec}$. The demand parameters also showed that for structures with periods less than $2.0 \mathrm{sec}$ the demands imposed by subduction earthquakes are on average 100\% higher than those for crustal earthquakes. For periods above $2.0 \mathrm{sec}$, the results depended on the earthquake set. This effect is most evident for the Chilean earthquakes (Valparaiso and Maule sets) where the number of 
inelastic cycles and the CDD tend to plateau significantly above the crustal sets for fundamental periods over $2.0 \mathrm{sec}$. Meanwhile, the same parameters for the Indonesia and Tohoku sets tend to decrease with increasing period, approaching the demands under the action of the Crustal set. The results led to the conclusion that cumulative ductility in bridges of long fundamental periods $(\geq 2.0 \mathrm{sec}$ ) imposed by Chilean earthquakes (Maule and Valparaiso), are larger than those imposed by Tohoku and Indonesia earthquakes. This effect is more predominant for higher target ductilities and when all the excursions are considered.

The number of inelastic cycles and the CDD decrease as the period of the system increases, which implies that the critical demand occurs for structures with low fundamental periods. Multi span bridges that rely on columns in addition to abutments are unlikely to exhibit such short fundamental periods. For those reasons, three fundamental periods of 0.5 , 1.0 and 2.0 seconds were selected for cyclic load protocol development as a benchmark representation of expected bridge fundamental periods, referred to herein as short $(0.5 \mathrm{~s})$, medium (1.0s) and long (2.0s) fundamental periods. The consideration of different natural periods reflects the period dependency of the analysis results. Appropriate selection of resulting protocols may avoid overestimation of inelastic cycles that the structure undergoes or distortion in the assessment of the behavior during physical testing.

The influence of different target ductilities can also be compared. The increase in achieved ductility increases both the number of inelastic cycles $(N)$ and the cumulative displacement ductility (CDD). For the benchmark periods averaged over all sets, results have shown a nearly linear relation in the CDD for different ductilities as illustrated in Figure 2.9, which implies that for structures with other ductilities the cumulative demand may be found by linear interpolation of the values for ductilities 2,4 , and 8 . On the other hand, the 
number of inelastic cycles does not show a linear relation as the gradient reduces with increased period. This trend implies that linear interpolation between the selected benchmark periods would result in conservative estimate of inelastic cycles and could be a practical choice for protocol selection between the representative target ductilities used in this study.

Comparison of pre-peak and all excursion demands is summarized in Table 2.3 using a relative ratio. For structures of low ductility $(\mu=2)$ pre-peak excursions represent over $75 \%$ of the demands computed considering all excursions. Identical protocols, for both prepeak and all excursions, were proposed for low ductile structures since the demands between the two approaches were similar. On the other hand, the ratios between pre-peak excursion demands and all excursion demands decrease as the ductility of the structure increases, with ratios as low as $63 \%$ in the case of high ductile structures $(\mu=8)$. Therefore, using all excursions in the development of testing protocols may lead to more damage in moderate and high ductility structures. 


\begin{tabular}{|c|c|c|}
\hline - - Crustal & - - Maule & - Tohoku1 Tohoku2 \\
\hline
\end{tabular}
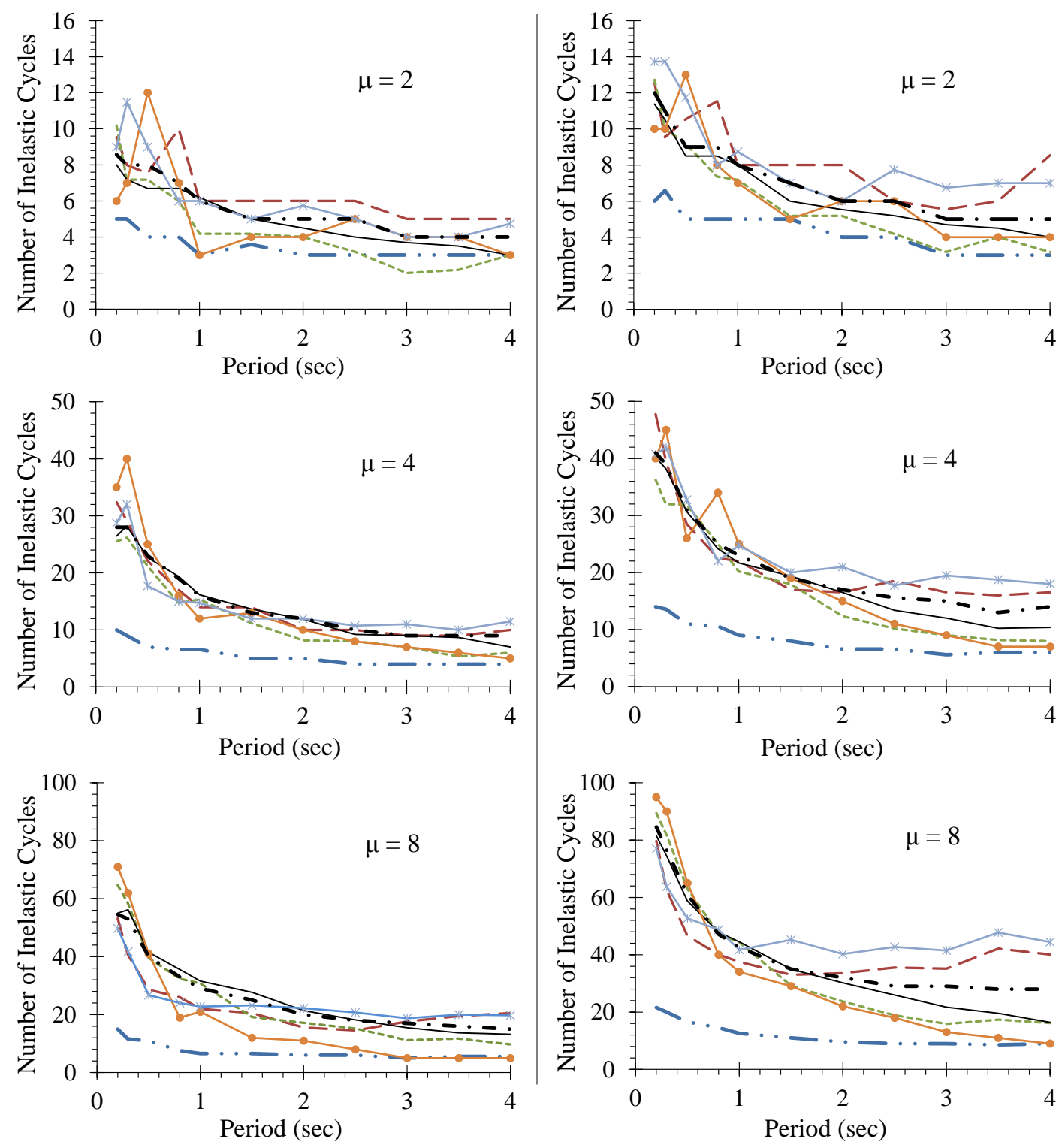

(a)

(b)

Figure 2.7 Number of inelastic cycles for different component ductilities $(\mu)$ :

(a) Pre-Peak excursions; (b) All excursions. 

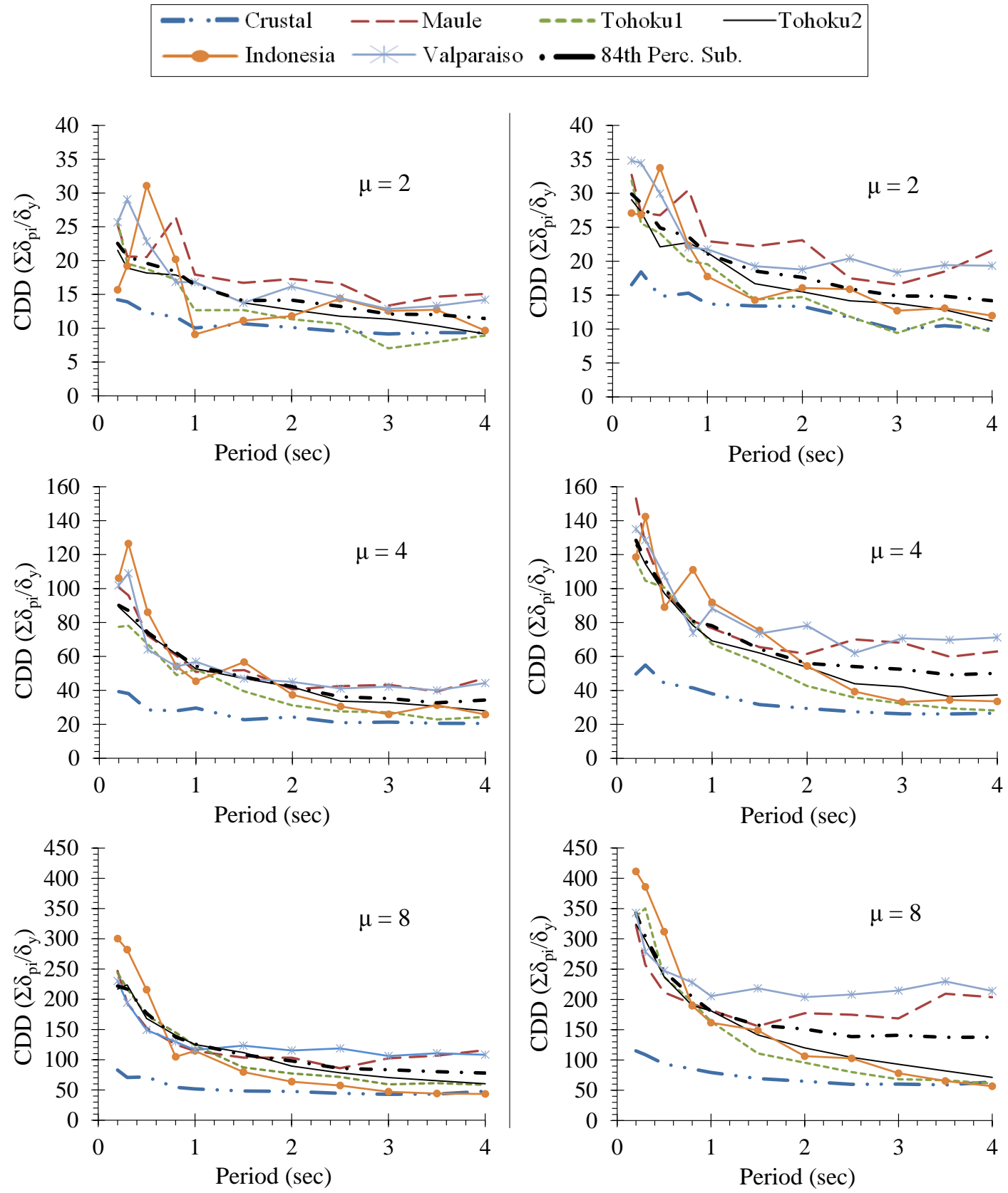

(a)

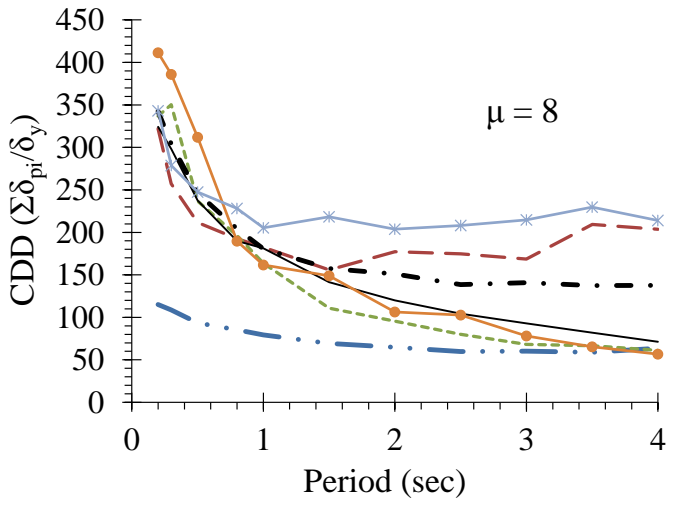

(b)

Figure 2.8 Cumulative Displacement Ductility (CDD) for different component ductilities.

(a) Pre-Peak excursions; (b) All excursions. 


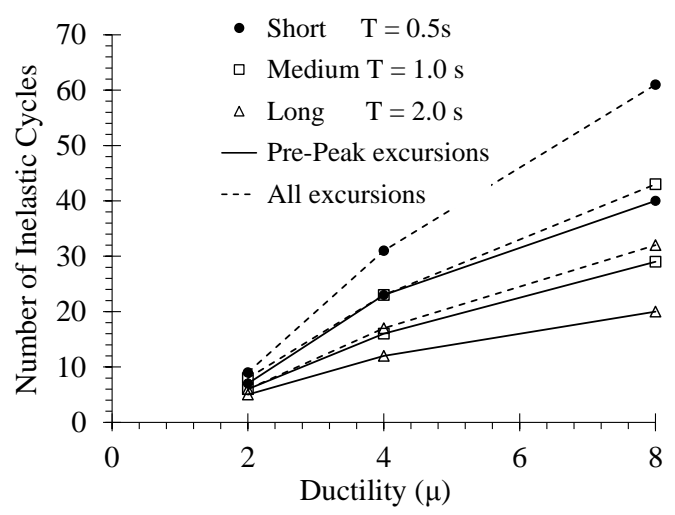

(a)

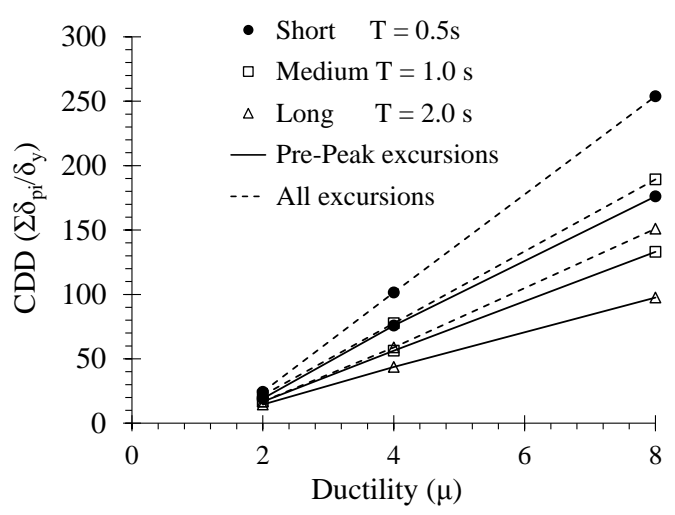

(b)

Figure 2.9 Demand parameters for different component ductilities.

(a) Number of Inelastic Cycles; (b) Cumulative Displacement Ductility (CDD)

Table 2.3 Comparison of pre-peak and all excursion demands

\begin{tabular}{c|c|c|c}
\hline \multicolumn{4}{c}{ Ratio Pre-Peak/All Excursions } \\
\hline $\begin{array}{c}\text { Period } \\
\mathrm{T}\end{array}$ & $\begin{array}{c}\text { Target } \\
\text { Ductility }\end{array}$ & $\mathrm{N}_{\text {cycles }}>\delta_{\mathrm{y}}$ & CDD \\
\hline \multirow{4}{*}{0.5} & 2 & $78 \%$ & $80 \%$ \\
\cline { 2 - 4 } & 4 & $74 \%$ & $75 \%$ \\
\cline { 2 - 4 } & 8 & $66 \%$ & $69 \%$ \\
\hline \multirow{4}{*}{1.0} & 2 & $75 \%$ & $77 \%$ \\
\cline { 2 - 4 } & 4 & $70 \%$ & $72 \%$ \\
\cline { 2 - 4 } & 8 & $67 \%$ & $70 \%$ \\
\hline \multirow{4}{*}{2.0} & 2 & $83 \%$ & $85 \%$ \\
\cline { 2 - 4 } & 4 & $71 \%$ & $74 \%$ \\
\cline { 2 - 4 } & 8 & $63 \%$ & $65 \%$ \\
\hline
\end{tabular}

\subsection{Proposed Protocols}

The analysis results led to differentiating the testing protocol in terms of ductility and period of the structure. In order to closely reflect the subduction zone demands the loading protocols were developed using the target values of the parameters summarized in Table 2.4 for pre-peak excursions. The proposed loading protocols consider two stages. The first stage 
consists of three cycles, in each of the following displacements (or loads), $0.25 \delta_{\mathrm{i}}\left(\mathrm{V}_{\mathrm{i}}\right), 0.5 \delta_{\mathrm{i}}$ $\left(\mathrm{V}_{\mathrm{i}}\right), 0.75 \delta_{\mathrm{i}}\left(\mathrm{V}_{\mathrm{i}}\right)$ and one cycle at $1.0 \delta_{\mathrm{i}}\left(\mathrm{V}_{\mathrm{i}}\right)$ in order to visualize low damage states (e.g. first cracking). Where, $\delta_{\mathrm{i}}$ is the theoretical yield displacement and $\mathrm{V}_{\mathrm{i}}$ is the theoretical strength at first yield. The second stage of inelastic cycles aims to replicate the demands imposed on concrete bridge columns by subduction zone earthquakes of long duration. The proposed loading histories, the amplitude of each inelastic cycle and number of cycles at those amplitudes are illustrated in Table 2.5 and Figure 2.10. In Figure 2.10 the dotted lines represent the first stage and the solid lines the second stage. Additionally, the cycle amplitudes of the second stage approximately follow the values obtained using Eq. (2.4) and the appropriate coefficients showed in Table 2.6, which were obtained through regression using an exponential curve on the statistical results obtained from the simplified rainflow counting procedure. For example, in order to obtain the amplitude for the sixth inelastic cycle $(\mathrm{N}=6)$ for a structural system of ductility 8 and period $0.5 \mathrm{~s}, \mathrm{f}(6)$ that represents the amplitude of the sixth inelastic cycle is equal to 1.06 times the yield displacement $\left(\delta_{\mathrm{y}}\right)$. The maximum number of cycles for each protocol is equal to the respective proposed value shown in Table 2.4. For additional comparison, Table 2.6 also shows values considering all excursions in case experimentalists decide those would be more appropriate for their application.

$$
f(N)=\left(a \cdot e^{b N}+c \cdot e^{d N}\right) \cdot \delta_{y}
$$

The proposed protocols were developed using the concept of pre-peak excursions. This approach was used since cycles that occur after the maximum displacement will cause less cumulative damage and should be considered separately (Krawinkler, et al., 2000a). For that reason, in cases when the specimen does not reach failure under the applied stepwise 
loading protocol, the test should continue under lower amplitude cycles also referred to as trailing cycles. Trailing cycles of amplitude $3 \delta_{\text {yield }}$ for moderate ductile column and $5 \delta_{\text {yield }}$ for highly ductile column were chosen. These values are based on member ductility demand requirements found in AASHTO Seismic Specifications (2009), which state that the maximum individual member displacement ductility demand for Seismic Design Category (SDC) C shall be equal to 3 and for SDC D shall be equal to 5 for cases of single-column bents. The number of trailing cycles was calculated from the analyses considering all excursions instead of only pre-peak. The number of trailing cycles is shown inside parentheses in Table 2.5. The notation (+ number of trailing cycles) represents the number of trailing cycles that are added following the completion of the entire loading protocol. The trailing cycles are at a set amplitude, which is shown in the first column of Table 2.5 and may be either 3 or 5 depending on the proposed protocol to be used. Experimentalists can modify the testing protocol for structures with different ductility in order to represent the target ductility demand appropriate for their desired application, in which case interpolation of the demand values presented in Table 2.4 is recommended.

Since the proposed protocols are based on increments of displacement ductility demand, determining the yield displacement of the specimen is essential and mirrors other cyclic protocols. A first estimate of the yield displacement may be found by performing a moment-curvature analysis of the column section based on measured material properties. In order to determine the yield displacement $\left(\delta_{\mathrm{y}}\right)$ to be used in experimental tests, researchers have employed two approaches. The first one consists of performing a monotonic pushover test before cyclic loading tests. This approach is used for three main reasons. Firstly, the yield displacement for future cyclic tests can be established based on the monotonic test. 
Secondly, it reflects that the structural response under seismic excitations usually shows an increase of deformations in only one direction, phenomenon denominated "ratcheting" of the response, which implies that cyclic deterioration under load reversals would diminish in the inelastic range close to the system collapse (Lignos \& Krawinkler, 2012). Finally, a monotonic test would provide the value of target ductility in cases when more refined cyclic loading protocols want to be performed.

Table 2.4 Target and proposed demands

\begin{tabular}{|c|c|c|c|c|c|c|c|c|c|}
\hline \multirow{3}{*}{$\begin{array}{c}\text { Period } \\
\mathrm{T}\end{array}$} & \multirow{3}{*}{$\underset{\mu}{\operatorname{Max}}$} & \multicolumn{4}{|c|}{$\mathbf{N}_{\text {cycles }}>\delta_{\mathrm{y}}$} & \multicolumn{4}{|c|}{$\mathrm{CDD}$} \\
\hline & & \multicolumn{2}{|c|}{ Pre-Peak } & \multicolumn{2}{|c|}{ All } & \multicolumn{2}{|c|}{ Pre-Peak } & \multicolumn{2}{|c|}{ All } \\
\hline & & $\begin{array}{l}\text { Target } \\
\text { Value }\end{array}$ & $\begin{array}{c}\text { Proposed } \\
\text { Protocol }\end{array}$ & $\begin{array}{l}\text { Target } \\
\text { Value }\end{array}$ & Proposed & $\begin{array}{l}\text { Target } \\
\text { Value }\end{array}$ & $\begin{array}{c}\text { Proposed } \\
\text { Protocol }\end{array}$ & $\begin{array}{l}\text { Target } \\
\text { Value }\end{array}$ & Proposed \\
\hline \multirow{3}{*}{0.5} & 2 & 7 & 9 & 9 & 9 & 19 & 24 & 24 & 24 \\
\hline & 4 & 23 & 23 & 31 & 31 & 76 & 76 & 101 & 103 \\
\hline & 8 & 40 & 40 & 61 & 61 & 176 & 177 & 254 & 253 \\
\hline \multirow{3}{*}{1.0} & 2 & 6 & 9 & 8 & 9 & 17 & 24 & 22 & 24 \\
\hline & 4 & 16 & 16 & 23 & 23 & 56 & 77 & 78 & 77 \\
\hline & 8 & 29 & 29 & 43 & 43 & 133 & 132 & 189 & 188 \\
\hline \multirow{3}{*}{2.0} & 2 & 5 & 6 & 6 & 6 & 14 & 17 & 17 & 17 \\
\hline & 4 & 12 & 12 & 17 & 17 & 44 & 43 & 59 & 59 \\
\hline & 8 & 20 & 20 & 32 & 32 & 98 & 97 & 151 & 151 \\
\hline
\end{tabular}

The second approach consists of determining the yield displacement during the progression of the cyclic test. Load control cycles are first employed based on percentages of the theoretical component strength $\left(\mathrm{V}_{\mathrm{i}}\right)$, usually $0.25 \mathrm{~V}_{\mathrm{i}}, 0.5 \mathrm{~V}_{\mathrm{i}}, 0.75 \mathrm{~V}_{\mathrm{i}}$, and $\mathrm{V}_{\mathrm{i}}$. The theoretical strength is determined dividing the first yield moment, obtained from a moment-curvature analysis, by the column cantilever length. Then the experimental yield displacement $\left(\delta_{\mathrm{y}}\right)$ is established by using the ratio of the theoretical force at which the concrete cover reaches a strain of 0.004 to the experimental elastic stiffness $\left(K_{e}\right)$, which is calculated as the ratio of the 
theoretical first yield force $\left(\mathrm{V}_{\mathrm{i}}\right)$ to the displacement measured experimentally $\left(\delta_{\mathrm{y}}{ }^{\prime}\right)$ (Priestley, et al., 2002).

Table 2.5 Proposed loading protocols utilizing Pre-peak approach

\begin{tabular}{|c|c|c|c|c|c|c|c|c|c|}
\hline \multirow{3}{*}{$\begin{array}{c}\text { Cycle } \\
\text { Amplitude } \\
\mathbf{x} \delta_{\text {yield }}\end{array}$} & \multicolumn{9}{|c|}{ Number of Inelastic Cycles (trailing cycles shown in parentheses) } \\
\hline & \multicolumn{3}{|c|}{ Ductility $(\mu=2)$} & \multicolumn{3}{|c|}{ Ductility $(\mu=4)$} & \multicolumn{3}{|c|}{ Ductility $(\mu=8)$} \\
\hline & $0.5 \mathrm{~s}$ & $1.0 \mathrm{~s}$ & $2.0 \mathrm{~s}$ & $0.5 \mathrm{~s}$ & $1.0 \mathrm{~s}$ & $2.0 \mathrm{~s}$ & $0.5 \mathrm{~s}$ & $1.0 \mathrm{~s}$ & $2.0 \mathrm{~s}$ \\
\hline 1.0 & 1 & 1 & 1 & 3 & 2 & 2 & 3 & 3 & 2 \\
\hline 1.1 & 2 & 2 & 1 & 3 & 2 & 1 & 3 & 3 & 2 \\
\hline 1.2 & 2 & 2 & 1 & 3 & 2 & 1 & 3 & 3 & 1 \\
\hline 1.3 & 1 & 1 & - & 2 & 1 & 1 & 3 & 2 & 1 \\
\hline 1.4 & - & - & 1 & 2 & 1 & 1 & 3 & 1 & 1 \\
\hline 1.5 & 1 & 1 & - & 1 & 1 & 1 & 3 & 1 & 1 \\
\hline 1.6 & - & - & - & 1 & 1 & - & 3 & 1 & 1 \\
\hline 1.7 & 1 & 1 & 1 & 1 & 1 & 1 & 2 & 1 & 1 \\
\hline 1.8 & - & - & - & 1 & - & - & 2 & 1 & 1 \\
\hline 1.9 & - & - & - & 1 & 1 & - & 2 & 1 & 1 \\
\hline 2.0 & 1 & 1 & 1 & 1 & 1 & 1 & 1 & 1 & 1 \\
\hline 2.1 & & & & - & - & - & - & 1 & - \\
\hline 2.2 & & & & 1 & - & 1 & 1 & 1 & 1 \\
\hline 2.4 & & & & - & - & - & 1 & 1 & - \\
\hline 2.6 & & & & 1 & 1 & - & 1 & 1 & 1 \\
\hline 2.8 & & & & - & - & - & 1 & 1 & - \\
\hline 3.0 & & & & $(+5)$ & $1(+4)$ & $1(+3)$ & 1 & 1 & 1 \\
\hline 3.2 & & & & 1 & - & - & 1 & - & - \\
\hline 3.5 & & & & - & - & - & 1 & 1 & 1 \\
\hline 4.0 & & & & 1 & 1 & 1 & 1 & 1 & - \\
\hline 4.5 & & & & & & & 1 & - & 1 \\
\hline 5.0 & & & & & & & $(+8)$ & $1(+6)$ & $(+5)$ \\
\hline 5.5 & & & & & & & 1 & - & - \\
\hline 6.0 & & & & & & & - & 1 & 1 \\
\hline 6.5 & & & & & & & 1 & - & - \\
\hline 8.0 & & & & & & & 1 & 1 & 1 \\
\hline
\end{tabular}


$\mu=2$
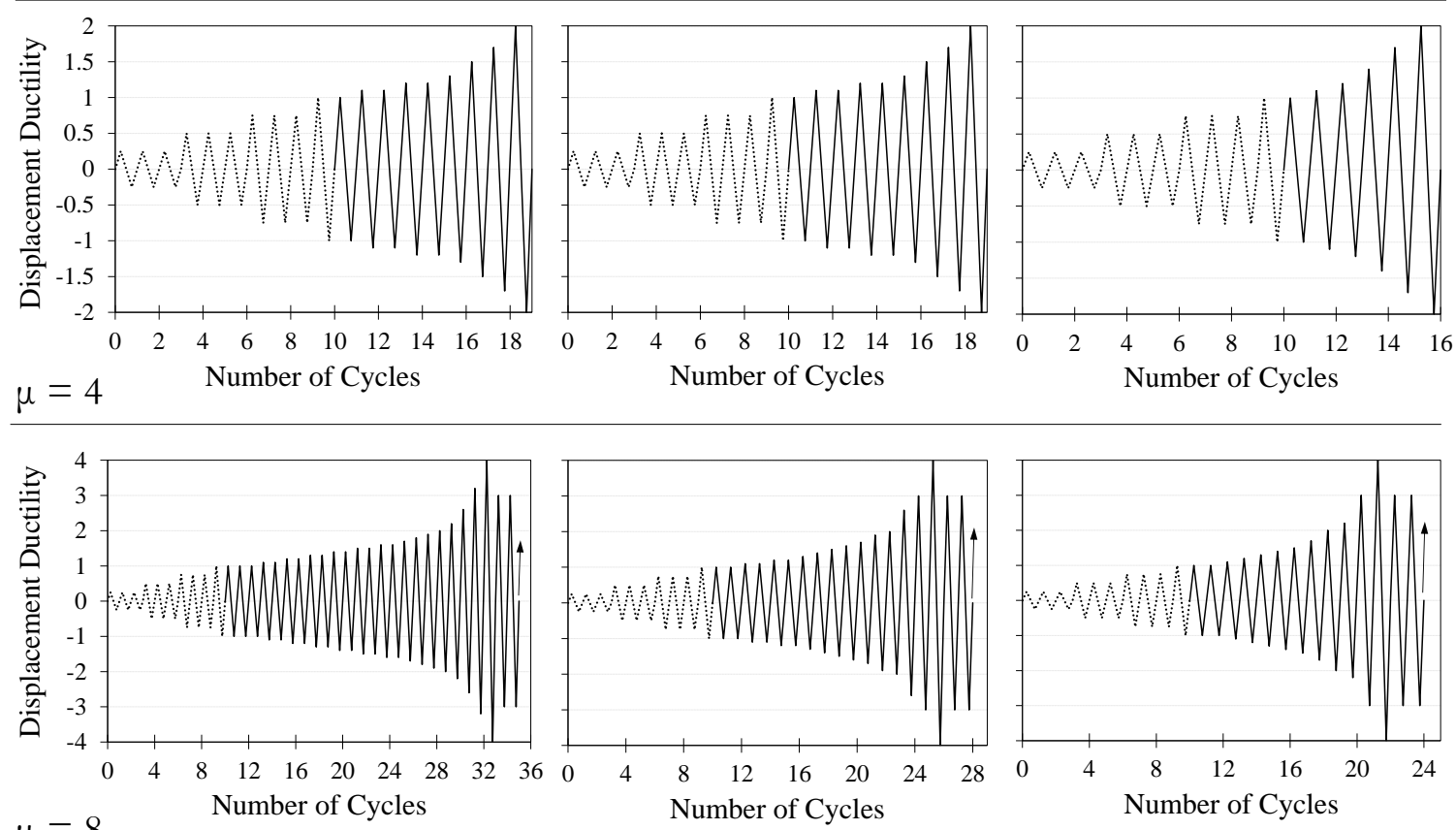

$\mu=8$

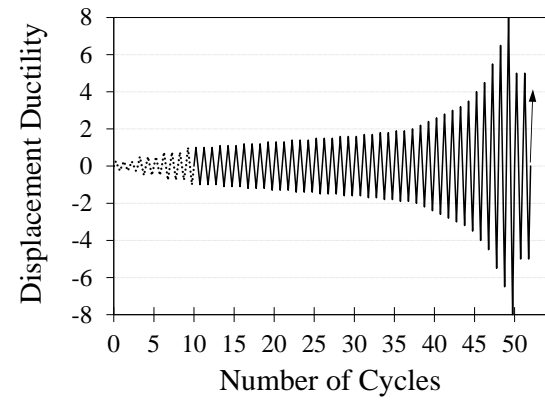

(a)

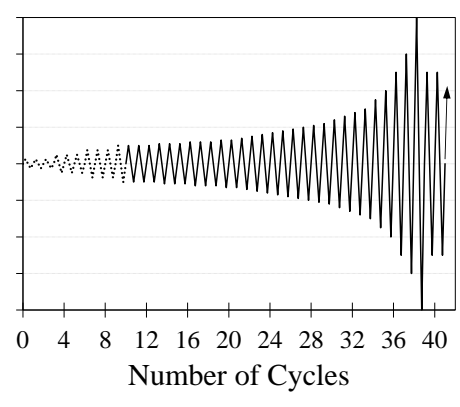

(b)

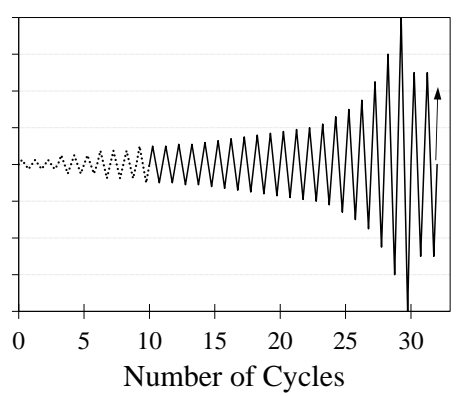

(c)

Figure 2.10 Proposed loading protocols for component ductilities $(\mu)=2,4$ and 8 . (a) $\mathrm{T}=0.5 \mathrm{sec}$, (b) $\mathrm{T}=1.0 \mathrm{sec}$, (c) $\mathrm{T}=2.0 \mathrm{sec}$. (Pre-peak excursions)

\subsection{Comparison to Conventional Protocols}

Three conventional protocols, which are commonly used to assess the capacity of reinforced concrete bridge columns, were selected in order to allow comparisons with the proposed protocols. The selected protocols (Figure 2.1) were the New Zealand protocol (Park, 1989), (Cheung, et al., 1991), the Modified N.Z. Protocol (Priestley, et al., 2002), and the ACI374 protocol (ACI 374.2R-13, 2013). An illustrative comparison of the three 
conventional cyclic protocols with the proposed protocol for a target ductility capacity of 8 and period $0.5 \mathrm{sec}$, is depicted in Figure 2.11.

Table 2.6 Exponential coefficients to obtain cycle amplitudes.

\begin{tabular}{|c|c|c|c|c|c|c|c|c|c|}
\hline \multirow[b]{2}{*}{ Coeff. } & \multicolumn{3}{|c|}{$\mu=2$} & \multicolumn{3}{|c|}{$\mu=4$} & \multicolumn{3}{|c|}{$\mu=8$} \\
\hline & $\begin{array}{c}\mathrm{T} \\
0.5 \mathrm{~s}\end{array}$ & $\begin{array}{c}\mathrm{T} \\
1.0 \mathrm{~s}\end{array}$ & $\begin{array}{c}\mathrm{T} \\
2.0 \mathrm{~s}\end{array}$ & $\begin{array}{c}\mathrm{T} \\
0.5 \mathrm{~s}\end{array}$ & $\begin{array}{c}\mathrm{T} \\
1.0 \mathrm{~s}\end{array}$ & $\begin{array}{c}\mathrm{T} \\
2.0 \mathrm{~s}\end{array}$ & $\begin{array}{c}\mathrm{T} \\
0.5 \mathrm{~s}\end{array}$ & $\begin{array}{c}\mathrm{T} \\
1.0 \mathrm{~s}\end{array}$ & $\begin{array}{c}T \\
2.0 \mathrm{~s}\end{array}$ \\
\hline \multicolumn{10}{|c|}{ Pre-Peak Excursions } \\
\hline $\mathrm{a}$ & 0.9934 & 0.9934 & 0.5215 & 0.9653 & 0.9557 & 0.9638 & 0.8695 & 0.9014 & 0.9869 \\
\hline $\mathrm{b}$ & 0.0221 & 0.0221 & -0.3778 & 0.0281 & 0.0439 & 0.0543 & 0.0336 & 0.0415 & 0.0444 \\
\hline c & 0.0137 & 0.0137 & 0.5674 & $1.15 \mathrm{E}-4$ & $8.91 \mathrm{E}-4$ & $2.72 \mathrm{E}-3$ & $1.913 \mathrm{E}-7$ & $2.83 \mathrm{E}-5$ & $1.09 \mathrm{E}-3$ \\
\hline d & 0.4511 & 0.4511 & 0.2066 & 0.4284 & 0.4853 & 0.5570 & 0.4252 & 0.417 & 0.4282 \\
\hline \multicolumn{10}{|c|}{ All Excursions } \\
\hline a & \multirow{4}{*}{\multicolumn{3}{|c|}{$\begin{array}{c}\text { Same as Pre-Peak } \\
\text { Excursions }\end{array}$}} & 0.9505 & 0.9335 & 0.936 & 0.8356 & 0.8659 & 0.8931 \\
\hline $\mathrm{b}$ & & & & 0.0245 & 0.0357 & 0.0485 & 0.0224 & 0.0314 & 0.0433 \\
\hline c & & & & $1.18 \mathrm{E}-5$ & $3.14 \mathrm{E}-5$ & $5.57 \mathrm{E}-5$ & $1.84 \mathrm{E}-8$ & $3.45 \mathrm{E}-7$ & $3.4 \mathrm{E}-6$ \\
\hline d & & & & 0.3875 & 0.478 & 0.613 & 0.3167 & 0.3816 & 0.4398 \\
\hline
\end{tabular}

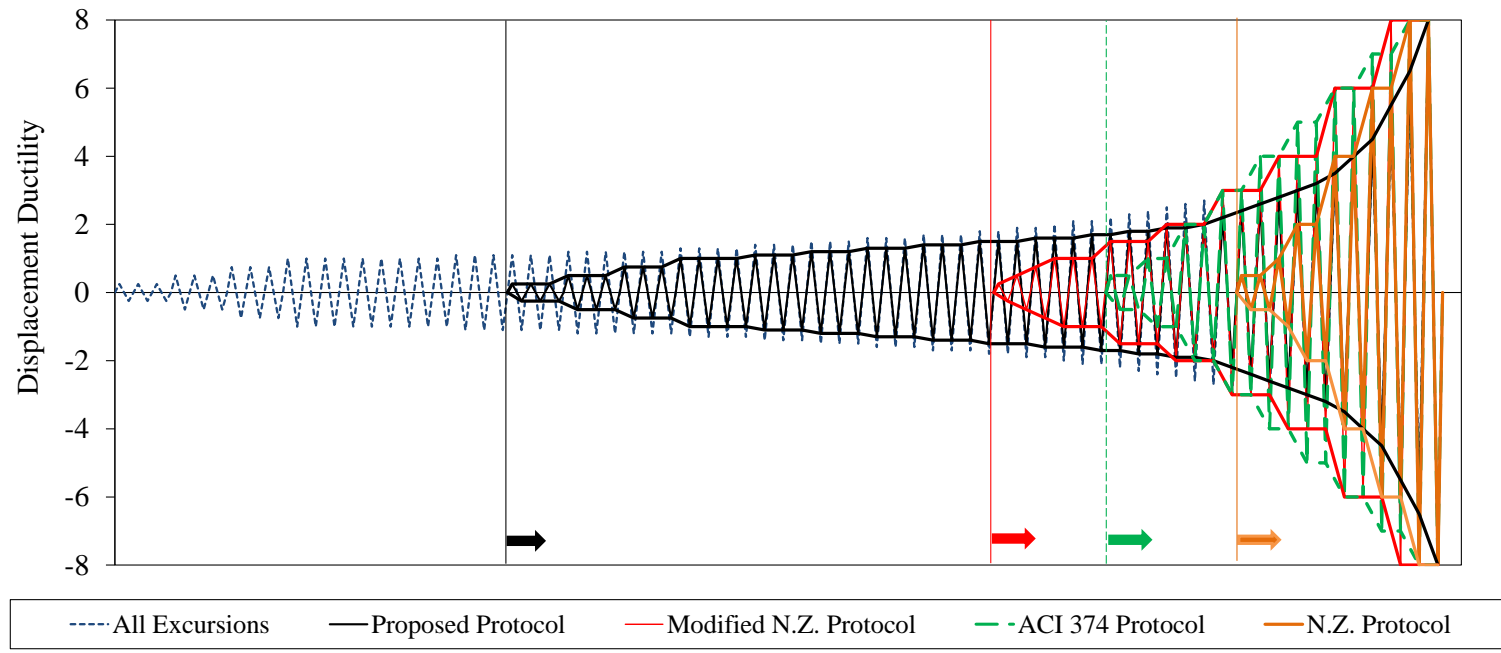

Figure 2.11 Comparison of protocols for ductility 8 and period $0.5 \mathrm{sec}$. 
For reference, a potential protocol considering all the excursions was also included. Since the different protocols culminate in the same ductility demand. This ordering of protocols more readily demonstrates the increased number of inelastic cycles for the proposed protocol at the lower amplitude and decreased number of inelastic cycles at the higher amplitudes.

Computed demand parameters for the three conventional protocols and the proposed are also compared in Table 2.7. The Modified N.Z. protocol is observed to be more demanding in terms of CDD than the proposed testing protocols for structures of low ductility capacity in the range of periods selected, and for structures of high ductility capacity with medium and long fundamental periods. The fact that the Modified N.Z. protocol is more demanding than the proposed protocols for structures with medium and long fundamental periods was not necessarily surprising since conventional protocols have been developed to reflect the demands in short period structures $(T=0.2-0.3 \mathrm{sec})$ and because their arrangement of cycles tends to overestimate the number of large inelastic cycles imposed by seismic excitations (FEMA 461, 2007), (ACI 374.2R-13, 2013). This overestimation is evident in that over $55 \%$ of the cumulative demand (CDD) using the conventional protocols came from cycles of large amplitude, as compared to $16 \%$ in the case of the proposed protocols for short period structures with high ductility capacity, and $44 \%$ in the case of long period structures with low ductility capacity. A visual comparison is shown in Figure 2.12 for the cumulative displacement ductility demand among the proposed protocols for period $0.5 \mathrm{sec}$, the conventional protocols and the results from selected ground motions shown in Figure 2.2. Proposed protocols closely resemble the cumulative displacement ductility demand and the number of inelastic cycles obtained by using the 
subduction ground motions, which in Figure 2.12 are referred to Maule-Curico and TohokuFukushima. This correlation verifies the appropriate selection of the discrete amplitudes for the proposed protocols. In the same way, the result from the crustal ground motion resembles the conventional protocols. A period of $0.5 \mathrm{sec}$ was selected as the closest period to make comparisons with the conventional protocols since those protocols were developed for structures of period equal to 0.2 seconds (ACI 374.2R-13, 2013). Longer periods may not be representative and could lead to incompatible comparisons. Despite the differences in short period definition, the proposed protocols considering the subduction megathrust earthquakes generally exhibit higher cumulative ductility demands than the conventional protocols when compared at the corresponding last cycle. This difference in demand could be even greater had the proposed protocol considered even shorter period than $0.5 \mathrm{sec}$ structural response. An exception occurs for a ductility demand of 2, where the Modified N.Z. protocol shows slightly greater CDD than the proposed protocol. The final CDD of the proposed protocols has a similar value than the Modified N.Z. However, results in Figure 2.12 show that in order to achieve similar CDD, the proposed protocols present a greater number of inelastic cycles than the Modified N.Z. protocol, which in consequence can affect the displacement capacity of the RC bridge column to be tested.

Moreover, Figure 2.13 depicts the cumulative distribution function (CDF) of the number of inelastic cycles at increments of displacement ductility of the proposed protocols, the loading protocols commonly used on RC columns, and the results from the statistical analyses. This figure shows that the conventional protocols used for the assessment of RC columns contain far fewer inelastic cycles at low displacement ductilities than the proposed protocol, and overestimate the number of large inelastic cycles at large ductilities. Despite 
the overestimation of large inelastic cycles in the conventional protocols, the proposed protocols for structures of moderate and high ductility capacity exhibit higher demands than the conventional protocols due to a substantial increase in the number of small inelastic cycles. This difference is due to the fact that the proposed protocols try to reflect the demands imposed by subduction earthquake excitations, which generally contain many small inelastic cycles and only a few large inelastic cycles.

Table 2.7 Comparison of conventional protocols and proposed protocols

\begin{tabular}{|c|c|c|c|c|c|c|}
\hline \multirow{2}{*}{ Demand Parameter } & \multirow{2}{*}{$\begin{array}{c}\text { N.Z. } \\
\text { Protocol }\end{array}$} & \multirow{2}{*}{$\begin{array}{l}\text { ACI } 374 \\
\text { Protocol }\end{array}$} & \multirow{2}{*}{$\begin{array}{c}\text { Modified } \\
\text { N.Z. } \\
\text { Protocol }\end{array}$} & \multicolumn{3}{|c|}{ Proposed Protocols } \\
\hline & & & & $\mathrm{T}=0.5 \mathrm{~s}$ & $\mathrm{~T}=1.0 \mathrm{~s}$ & $\mathrm{~T}=2.0 \mathrm{~s}$ \\
\hline \multicolumn{7}{|c|}{ Ductility $(\mu)=2$} \\
\hline No. Inelastic Cycles & 3 & 4 & 9 & 9 & 9 & 6 \\
\hline CDD & 10 & 12 & 27 & 24 & 24 & 17 \\
\hline$(\mu \geq 1.5) / \mathrm{CDD}^{1}$ & $80 \%$ & $67 \%$ & $78 \%$ & $43 \%$ & $43 \%$ & $44 \%$ \\
\hline \multicolumn{7}{|c|}{ Ductility $(\mu)=4$} \\
\hline No. Inelastic Cycles & 5 & 8 & 15 & 23 & 16 & 12 \\
\hline CDD & 26 & 40 & 69 & 76 & 56 & 43 \\
\hline$(\mu \geq 3) / \mathrm{CDD}$ & $62 \%$ & $70 \%$ & $61 \%$ & $19 \%$ & $25 \%$ & $33 \%$ \\
\hline \multicolumn{7}{|c|}{ Ductility $(\mu)=8$} \\
\hline No. Inelastic Cycles & 9 & 16 & 21 & 40 & 29 & 20 \\
\hline CDD & 82 & 144 & 153 & 177 & 132 & 97 \\
\hline$(\mu \geq 6) / \mathrm{CDD}$ & $68 \%$ & $58 \%$ & $55 \%$ & $16 \%$ & $21 \%$ & $29 \%$ \\
\hline
\end{tabular}

${ }^{1}$ Percentage of cumulative displacement ductility due to large inelastic cycles 

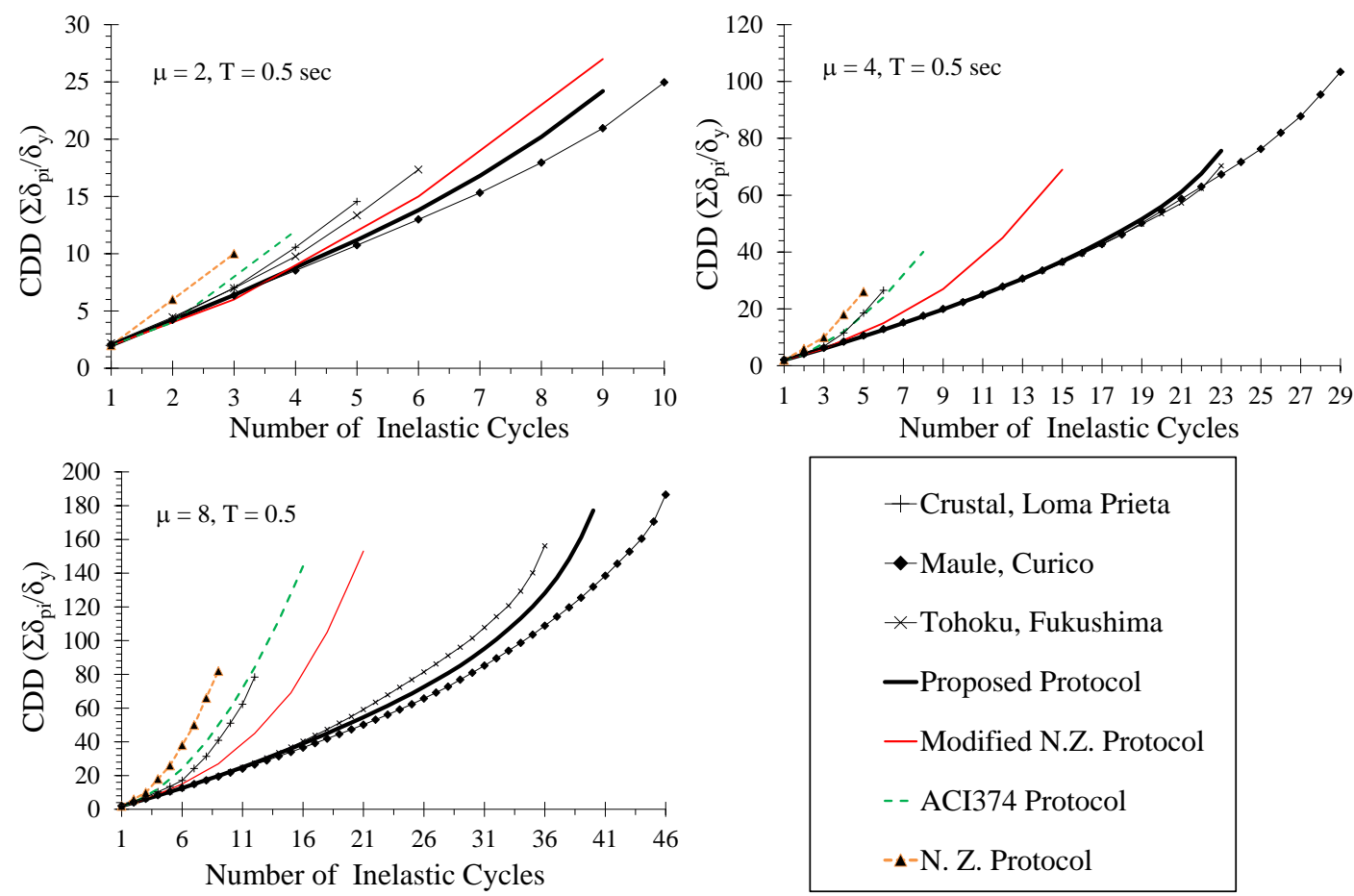

+ Crustal, Loma Prieta

- Maule, Curico

$*$ Tohoku, Fukushima

- Proposed Protocol

—Modified N.Z. Protocol

- - ACI374 Protocol

-N. Z. Protocol

Figure 2.12 Comparison of cumulative ductility demands for period of $0.5 \mathrm{sec}$.
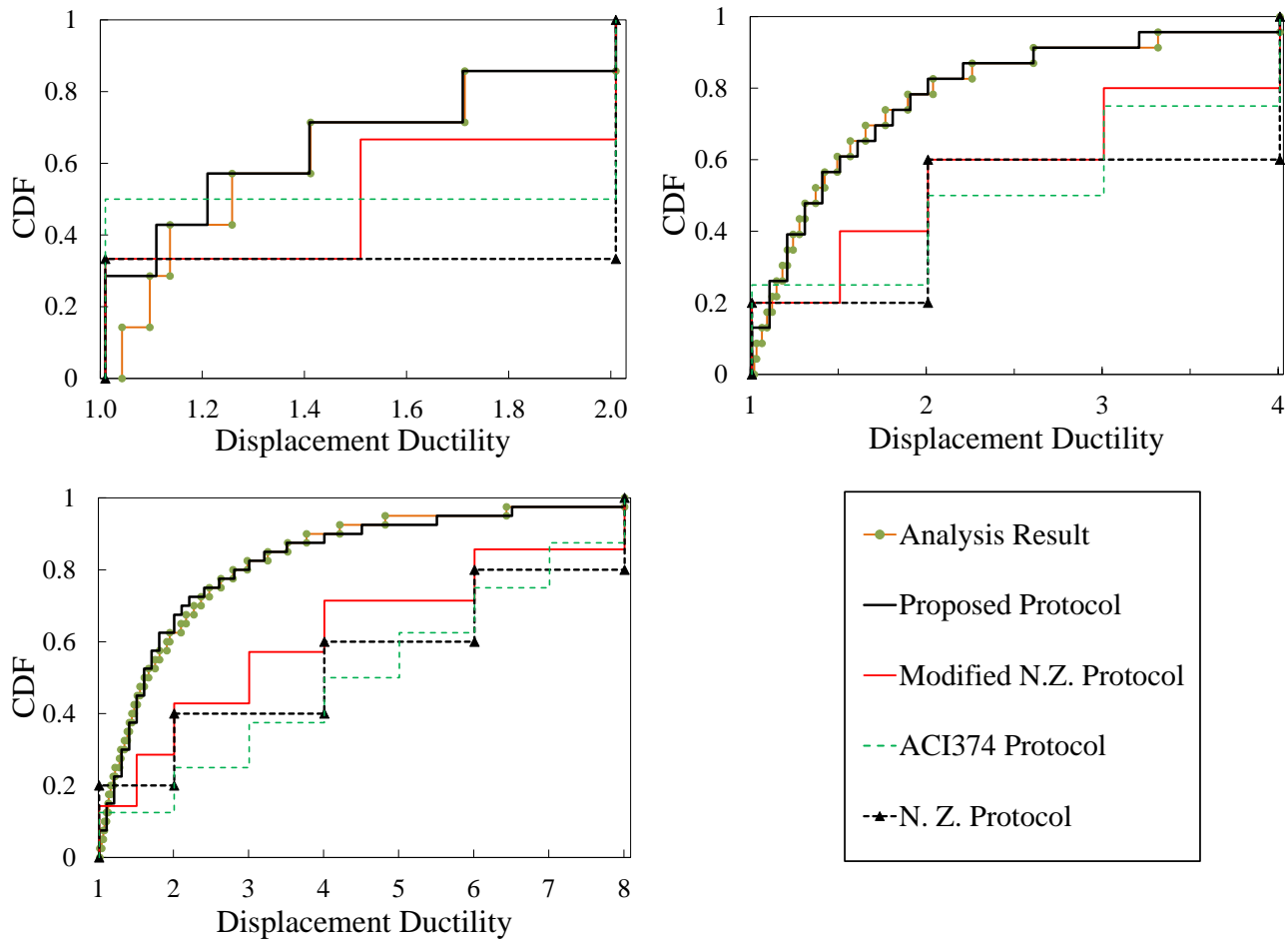

Figure 2.13 Comparison of cumulative distribution for structures of period $0.5 \mathrm{sec}$ and different component ductilities. 


\subsection{Numerical CASE STUdy}

As discussed in previous sections, the cumulative inelastic demands imposed by subduction zone earthquakes can increase as compared to crustal earthquake demands. This aspect is particularly relevant in the State of Oregon and the Northwest coast of North America due to their proximity to the Cascadia subduction zone. The Cascadia subduction zone is the over $1000 \mathrm{~km}$ long boundary between the Juan de Fuca and North American plates. Geological evidence has shown that 13 significant earthquakes have occurred in the past 3000 years (Goldfinger, et al., 2008). The most notable of which, the M9.0 earthquake of 1700, produced a tsunami large enough to reach Japan (Atwater, et al., 2005). Historical evidence combined with comparisons of the Cascadia fault to other subduction zones has led geologists to conclude that a megathrust earthquake in the Cascadia subduction zone is impending (Heaton \& Kanamori, 1984). Given such seismic hazard, the assessment of deficient RC bridge substructures has become crucial in order to prioritize the bridges that need to be retrofitted and to maintain the highway network operable after a major seismic event.

The case study presented in this section aims to assess the behavior of pre-1970 bridge columns located in the State of Oregon, USA. Such columns are commonly deficient in flexural ductility and shear strength as bridges were designed primarily for gravity loads without much consideration to lateral forces from seismic loading. As a result, columns lack sufficient transverse reinforcement to provide satisfactory performance in a major seismic event. Typically, No. 3 or No. 4 hoops at 12 inches on center were provided in columns regardless of the column cross-sectional dimensions. The stirrups were anchored by $90^{\circ}$ hooks with short extensions and intermediate ties were seldom used. Minimal restraint 
provided by the hoops can cause the longitudinal reinforcement to buckle once the concrete cover spalls. Furthermore, bridges built prior to 1970 have undesirable lap splices at the base of RC column. This lap splice detail can potentially be a cause for reduced column ductility and can result in rapid loss of flexural strength.

Numerical studies were performed considering a representative pre-1970 RC column from a multi-column bridge bent subjected to a conventional protocol and one of the proposed subduction protocols. The Modified New Zealand protocol (Figure 2.1) was used as the conventional protocol and the proposed protocol for structures of ductility 8 and fundamental period of $0.5 \mathrm{sec}$ was used as the subduction protocol. Protocols with that target ductility were used because the ductility obtained from moment-curvature analysis of the column in study was close to 8 . The cross-sectional dimensions and reinforcement of the representative column are depicted in Figure 2.14.

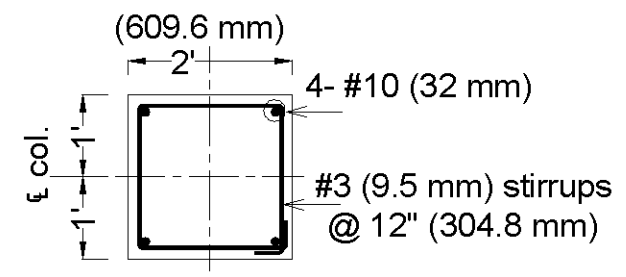

Figure 2.14 Cross section of a typical square RC column in Oregon, USA.

In order to model the inelastic behavior of the RC column a concentrated plasticity approach was utilized. The plastic hinge was modeled using the hysteretic model developed by Ibarra et al. (2005a), as was illustrated in Figure 2.3, and the software OpenSees (2013). Model parameters for column hinges, such as moment capacity and rotation capacity, were obtained from empirical equations based on a vast amount of column tests (Haselton, et al., 2008) (Biskinis \& Fardis, 2009). The column properties used in the numerical model are shown in Table 2.8 . 
Table 2.8 Column properties

\begin{tabular}{c|c|c|c|c|c|c|c|c|c|c|c}
\hline $\begin{array}{c}\mathrm{f}_{\mathrm{c}} \\
(\mathrm{ksi})\end{array}$ & $\begin{array}{c}\mathrm{f}_{\mathrm{ce}} \\
(\mathrm{ksi})\end{array}$ & $\begin{array}{c}\mathrm{f}_{\mathrm{y}} \\
(\mathrm{ksi})\end{array}$ & $\begin{array}{c}\mathrm{f}_{\mathrm{ye}} \\
(\mathrm{ksi})\end{array}$ & $\begin{array}{c}\text { Cantilever } \\
\text { Length } \\
(\mathrm{ft})\end{array}$ & $\begin{array}{c}\text { Width } \\
(\mathrm{in})\end{array}$ & $\begin{array}{c}\text { Depth } \\
(\mathrm{in})\end{array}$ & $\begin{array}{c}\text { Axial } \\
\text { Load } \\
(\mathrm{kips})\end{array}$ & $\begin{array}{c}\text { Axial } \\
\text { Load } \\
\text { Ratio } \\
(\%)\end{array}$ & $\begin{array}{c}\text { @T } \\
(\%)\end{array}$ & $\begin{array}{c}\text { @L } \\
(\%)\end{array}$ & $\begin{array}{c}\text { Lap } \\
\text { Splice } \\
\text { Length } \\
\left(\mathrm{d}_{\mathrm{b}}\right)\end{array}$ \\
\hline 3.3 & 4.29 & 60 & 68 & 9.25 & 24 & 24 & 160 & 8.4 & 0.094 & 0.88 & 28 \\
\hline
\end{tabular}

${ }_{1}$ Axial load ratio $=\mathrm{P} /\left(\mathrm{A}_{\mathrm{g}} \mathrm{f}_{\mathrm{c}}\right)$

Where,

$\mathrm{f}_{\mathrm{c}}$ : specified compressive strength of concrete at 28 days.

$\mathrm{f}_{\mathrm{ce}}$ : expected compressive strength of concrete. $\mathrm{f}_{\mathrm{ce}}=1.3\left(\mathrm{f}_{\mathrm{c}}\right)$

$\mathrm{f}_{\mathrm{y}}$ : specified yield stress of steel.

$\mathrm{f}_{\mathrm{ye}}$ : expected yield stress of steel. $\mathrm{f}_{\mathrm{ye}} \approx 1.1 \mathrm{f}_{\mathrm{y}}$

@т: transverse ratio of steel.

@L: Longitudinal ratio of steel.

$\mathrm{d}_{\mathrm{b}}$ : diameter of longitudinal steel reinforcement.

The hysteretic energy dissipation capacity plays a fundamental role in the assessment of bridge columns subjected to subduction zone ground motion. Haselton et al. (2008) has proposed equations to calculate this capacity $(\lambda)$, which according his equation depends on the amount of transverse reinforcement, shear capacity and axial load ratio. Another equation also proposed by Haselton is included in the PEER/ATC 72-1 (2010) report, in which the value of $\lambda$ only depends on the axial load ratio. The PEER/ATC report stated that for a typical column with seismic detailing, typical values of the parameter $\lambda$ are on the order of 10 to 20 . On the other hand, in the study carry out by Haselton (2008) values from 2 to 5 were employed for highly deteriorated components. This means that a lower $\lambda$ indicates that the element has a high rate of strength and stiffness deterioration and therefore less capacity 
to dissipate energy. Since pre-1970 columns were built without seismic detailing the behavior of these columns is expected to be represented by $\lambda$ values near 2 .

The model parameters using equations proposed by Haselton (2008), Biskinis (2009), and moment-curvature analysis are summarized in Table 2.9. The moment-curvature analysis was based on conventional reinforced concrete flexure theory following AASHTO Specifications (2009). It is worth mentioning that all the analyses utilized the expected material properties, where $\mathrm{f}_{\mathrm{ce}}=1.3 \mathrm{f}^{\mathrm{c}}$ and $\mathrm{f}_{\mathrm{ye}} \approx 1.1 \mathrm{fy}$.

Table 2.9 Model parameters

\begin{tabular}{|c|c|c|c|c|c|c|c|c|c|}
\hline Reference & $\begin{array}{c}\text { My } \\
\text { (kip-ft) }\end{array}$ & $\begin{array}{c}\mathbf{M}_{\mathrm{c}} / \\
\mathbf{M}_{\mathbf{y}}\end{array}$ & 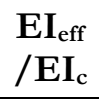 & $\begin{array}{c}\mathbf{M}_{\mathrm{r}} / \\
\mathbf{M}_{\mathrm{y}}\end{array}$ & $\begin{array}{c}\theta_{\mathrm{y}} \\
(\mathrm{rad})\end{array}$ & $\begin{array}{c}\theta_{\mathrm{p}} \\
(\mathrm{rad})\end{array}$ & $\begin{array}{c}\theta_{\mathrm{pc}} \\
(\mathrm{rad})\end{array}$ & $\begin{array}{c}\theta_{\mathrm{u}} \\
(\mathrm{rad})\end{array}$ & $\lambda$ \\
\hline $\begin{array}{c}\text { Theory } \\
\text { (AASHTO, 2009) }\end{array}$ & 401 & 1.07 & 0.29 & 0.8 & 0.006 & 0.043 & - & 0.049 & - \\
\hline $\begin{array}{l}\text { Haselton } \\
\text { (2008) }\end{array}$ & 401 & 1.13 & 0.20 & - & 0.009 & 0.019 & 0.033 & 0.062 & 42 \\
\hline $\begin{array}{l}\text { Biskinis } \\
(2009)\end{array}$ & 401 & - & 0.19 & - & 0.010 & 0.022 & - & 0.032 & - \\
\hline $\begin{array}{c}\text { PEER/ATC 72-1 } \\
\text { (2010) }\end{array}$ & 401 & 1.13 & 0.20 & 0.0 & 0.009 & 0.019 & 0.033 & 0.062 & 24 \\
\hline This study & 401 & 1.13 & 0.20 & 0.2 & 0.009 & 0.019 & 0.033 & 0.062 & $\begin{array}{c}42 \\
24 \\
2 \\
\end{array}$ \\
\hline
\end{tabular}

A shortcoming of the equations proposed by Haselton (2008) and Biskinis \& Fardis (2009) is that they do not include the effect of number of cycles on the column rotation capacity. Moreover, Haselton's equations do not account for the effect of lap-spliced rebars in expected plastic hinge locations. Despite this fact, Haselton's and Biskinis's equation lead to similar plastic rotation capacity $\left(\theta_{\mathrm{p}}\right)$.

Figure 2.15 shows the results using the model parameters summarized in Table 2.9. These plots show the effect of the conventional protocol and the subduction protocol for structures of ductility 8. Comparing the results from the two protocols it can be observed 
that for structures with high values of $\lambda$, i.e. low rate of strength and stiffness deterioration, the behavior of the column under both protocols is similar in terms of rotation capacity, which is considered as the rotation when a reduction in moment capacity of $20 \%$ has occurred. On the other hand, if a high rate of deterioration (low $\lambda$ ) is considered the column under the subduction protocol shows less rotation capacity and less strength as compared to the column under the conventional protocol. The reduction in strength was approximately $5 \%$ when $\lambda$ was equal to 2 . This implies that faster the rate of deterioration, more significant the expected effect of number of inelastic cycles on column behavior.

A high rate of deterioration is expected on pre-1970 columns due to the fact that they were built with insufficient transverse reinforcement and lap splices in plastic hinge regions. Therefore, the behavior of these columns would be highly influenced by subduction mega earthquakes. This result is consistent with experimental and numerical studies, e.g. Ibarra \& Krawinkler (2005), Ou et al. (2013), Chandramohan, et al. (2013). In those studies were concluded that structural components' capacity and collapse are influenced by the duration of ground motion and the number of inelastic cycles. 

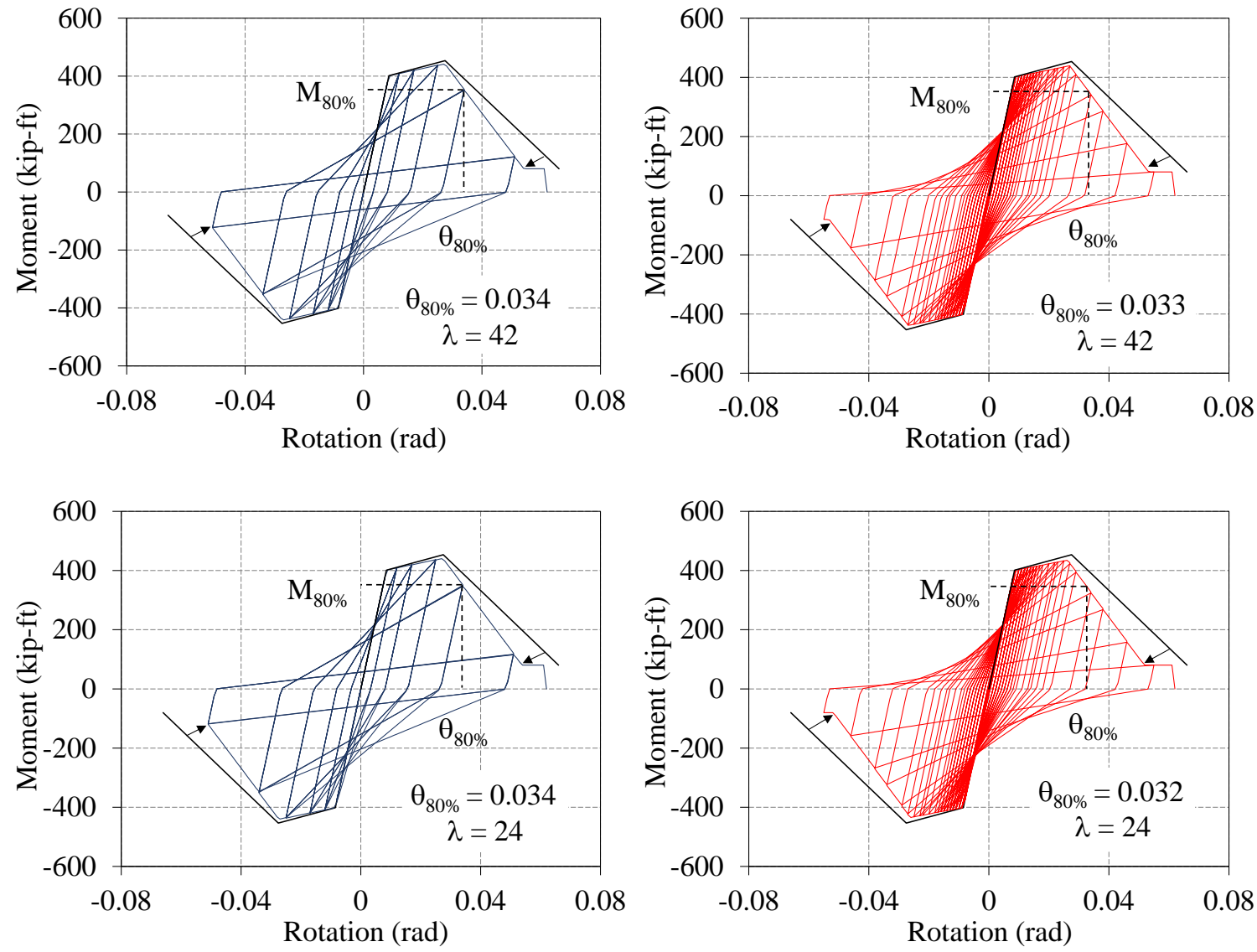

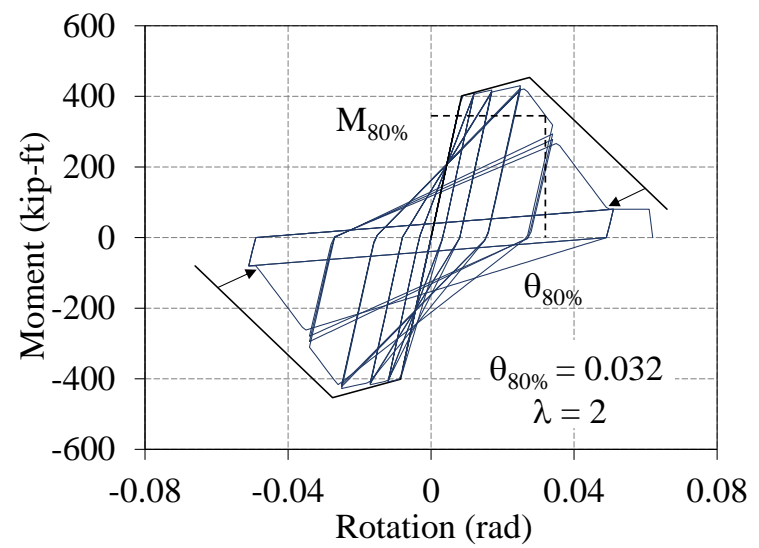

(a)

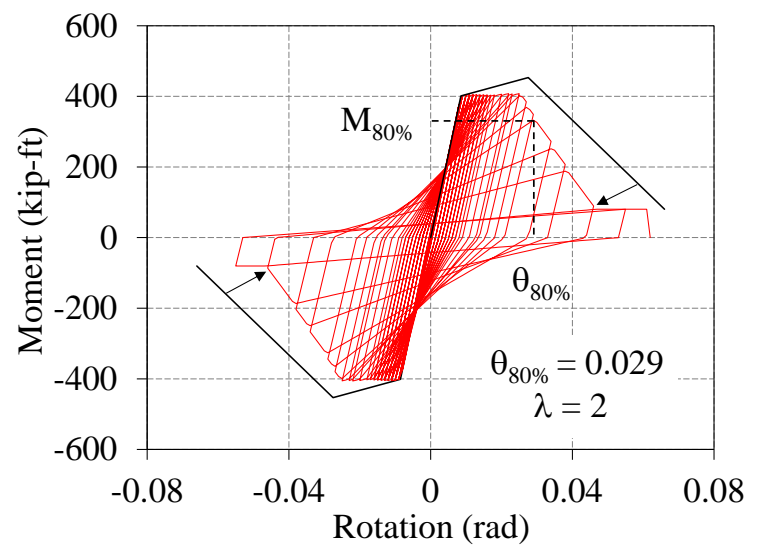

(b)

Figure 2.15 Effect of loading protocol and model parameters on column response.

(a) Conventional protocol. (b) Subduction protocol 


\subsection{EFFEct of LoAding Protocol BASEd on ExPERIMENTAL RESUlts}

In order to further investigate the effect of different loading histories on pre-1970 RC bridge columns, experimental tests were conducted by Mehary and Dusicka (2015) on full-scale square cross-section reinforced concrete bridge columns in the iSTAR Laboratory at Portland State University. The results of those tests are further analyzed in this section.

The experimental program carried out by Mehary and Dusicka (2015) consisted of

three specimens intended to represent full-scale models of a typical RC bridge column found in multi-column bents constructed in the 1950s to mid-1970s and one retrofitted specimen using carbon fiber polymers (CFRP) wrapping. In this study the retrofitted column was not considered since the testing variables to be analyzed are the effect of different cyclic loading protocols and axial load applied at the top of the columns. The Modified N.Z. loading history (Figure 2.1b) referred as to Conventional protocol for its wide use in experimental assessment of RC structures, and the proposed protocol for structures of ductility 8 and fundamental period of $0.5 \mathrm{sec}$ (Figure 2.10a) referred to as Subduction protocol were used.

The columns in the experimental program were given an identification that denoted the loading protocol $(\mathrm{S}=$ Subduction, $\mathrm{C}=$ Conventional $)$ and axial load utilized $(7 \%-17 \%)$. The identifications for the three columns in the experimental program were C-7, S-7 and S17. The specimens were nominally identical since they were constructed using the same material properties, cross-sectional dimensions and reinforcement ratios (Table 2.8). The longitudinal reinforcement consisted of four \#10 bars on each corner with \#3 stirrups spaced at 12 inches center to center as shown in Figure 2.14. Lap splices were located at the base of the test specimens through the incorporation of 4 dowels. The lap splice length was 36 inches, which corresponds to 28 times the diameter of the longitudinal steel 
reinforcement $\left(28 \mathrm{~d}_{\mathrm{b}}\right)$. Although pre-1970 RC columns were typically constructed using Grade 40 deformed bars, all reinforcing steel used to construct the test specimens consisted of Grade 60 deformed bar conforming to the American Society for Testing and Materials (ASTM) designation A615. The use of Grade 60 steel was caused by current rebar size limitation and availability of Grade 40 in the US. Measured reinforcing steel strengths were $70.3 \mathrm{ksi}$ and $100.9 \mathrm{ksi}$ for the yield and tensile strength, respectively. Normal weight concrete was used to construct the test specimens with a target 28-day strength of 3500 psi. The concrete cover was 2 inches. The average of standard compression testing of 6 -inch by 12 inch concrete cylinder the day of testing are shown in Table 2.10.

Table 2.10 Measured concrete properties for square RC columns

\begin{tabular}{c|c}
\hline Specimen & $\begin{array}{c}\text { Compressive Strength }\left(\mathbf{f}_{\mathbf{c}}\right) \\
\text { ksi }\end{array}$ \\
\hline C-7 & 4.41 \\
\hline S-7 & 4.17 \\
\hline S-17 & 4.64 \\
\hline
\end{tabular}

Axial load on the column was applied in an effort to simulate the dead loads from the superstructure. The applied axial loads were approximately 7 and 17 percent of the nominal axial strength $\left(\mathrm{A}_{\mathrm{g}} \mathrm{f}_{\mathrm{c}}\right)$. Actual axial load ratios based on measured properties were $5.9 \%, 6.2 \%$ and $13.1 \%$ for column C-7, S-7 and S-17, respectively.

\subsubsection{Test Results}

Results of the experimental program are briefly described hereafter. Details can be found in Mehary and Dusicka (2015). 


\subsubsection{Column C-7}

The hysteretic behavior of this specimen showed a moderate-ductile behavior by reaching a displacement ductility of 6.2 and 6.7 in the positive and negative direction, respectively. Column C-7 showed a hysteretic response typical of flexural behavior with slightly pinching behavior. As expected in the elastic range, minor strength degradation between cycles at the same displacement amplitude can be observed before the occurrence of the yield displacement. Cycles at the same amplitude after yielding showed notorious strength and stiffness degradation. The specimen exhibited a significant decrease in lateral strength after the peak lateral load because of noticeable crushing of concrete at the base of the column. Failure of the specimen was assumed when the applied lateral load dropped below $80 \%$ of the peak load. Displacements of 6 inches and -6.5 in were computed as the failure displacements in the positive and negative direction, respectively.

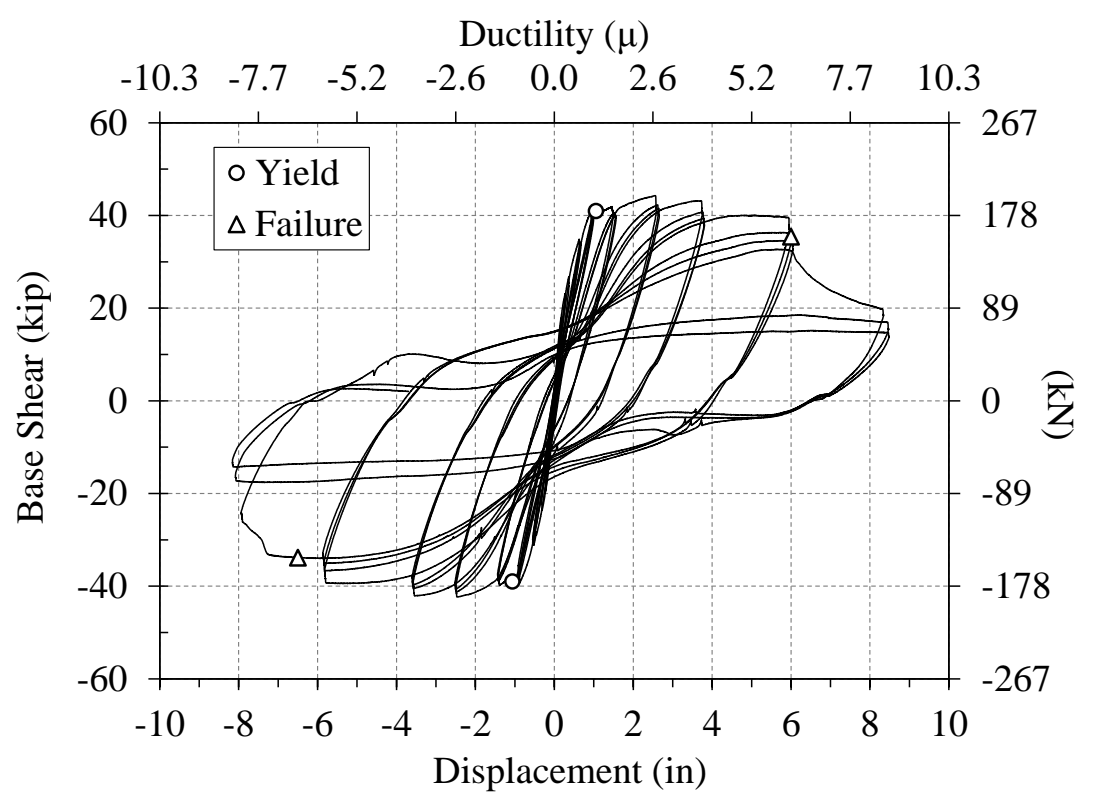

Figure 2.16 Load vs. displacement curve of column C-7 


\subsubsection{Column S-7}

The proposed protocol for structures of ductility 8 and fundamental period of 0.5 sec, as shown in Figure 2.10(a), was utilized to test Column S-7. Vertical cracks consistent with lap splice failure started showing at an early stage. The primary mode of failure was lap splice failure followed by crushing of concrete at the base of the column and buckling of dowels. The buckling of the dowels was observed once the cover of the concrete at the base of the column was completely crushed.

The lateral load vs. displacement hysteresis curve for the column S-7 is illustrated in Figure 2.17.

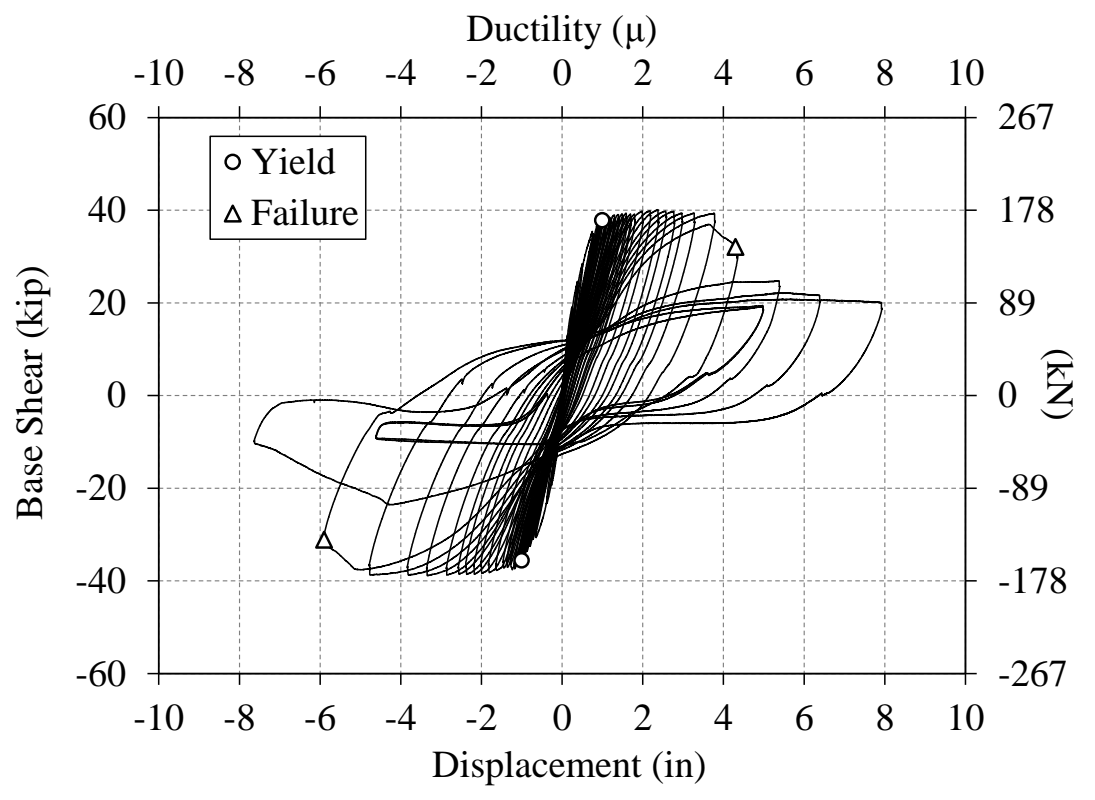

Figure 2.17 Load vs. displacement curve of column S-7

The hysteretic behavior of this specimen showed a moderate ductile behavior by reaching a displacement ductility of 4.1 and 5.7 in the positive and negative direction, respectively. Column S-7 showed an initial hysteretic response typical of flexural behavior. 
Minimum strength degradation was noticed up to the peak load. The specimen exhibited a significant decrease in lateral strength after both peak lateral loads because of a vertical crack that was attributed to lap splice failure. Failure of the specimen was assumed when the applied lateral load dropped below $80 \%$ of the peak load. Displacements of 4.3 inches and 5.9 in were computed as the failure displacements in the positive and negative direction, respectively.

\subsubsection{Column S-17}

Column S-17 was also tested using the subduction protocol developed for structures with a fundamental period close to $0.5 \mathrm{sec}$ and ductility 8 shown in Figure 2.10(a). However, in this case the axial load was increased from 150 kips to 350 kips (17\% of the nominal axial column strength) in an effort to investigate the effect of different axial loads acting on RC bridge columns.

Similar to specimens C-7 and S-7, horizontal cracks were observed at the column base. As the test progressed horizontal cracks were increasing in width and propagated up to mid-height of the column. Diagonal cracks attributed to shear behavior were also visible in this specimen. The emergence of diagonal cracking was a consequence of increasing the flexural capacity through an increase in the axial load applied to this specimen, which caused an evident flexion-shear interaction. At the last stage of testing the vertical crack in the lap splice region was evident and caused the failure of the specimen.

The lateral load vs. displacement hysteresis curve for the column S-17 is illustrated in Figure 2.18. The hysteretic behavior of this specimen showed a less-ductile behavior by reaching displacement ductilities of 4.0 and 3.5 in the positive and negative direction, respectively. Column S-17 showed an initial hysteretic response typical of flexural behavior. 
However, the failure mechanism was by lap-splice failure. Failure displacements of 4.8 inches and -4.4 in were computed when the applied lateral load dropped below $80 \%$ of the peak load in the positive and negative direction, respectively.

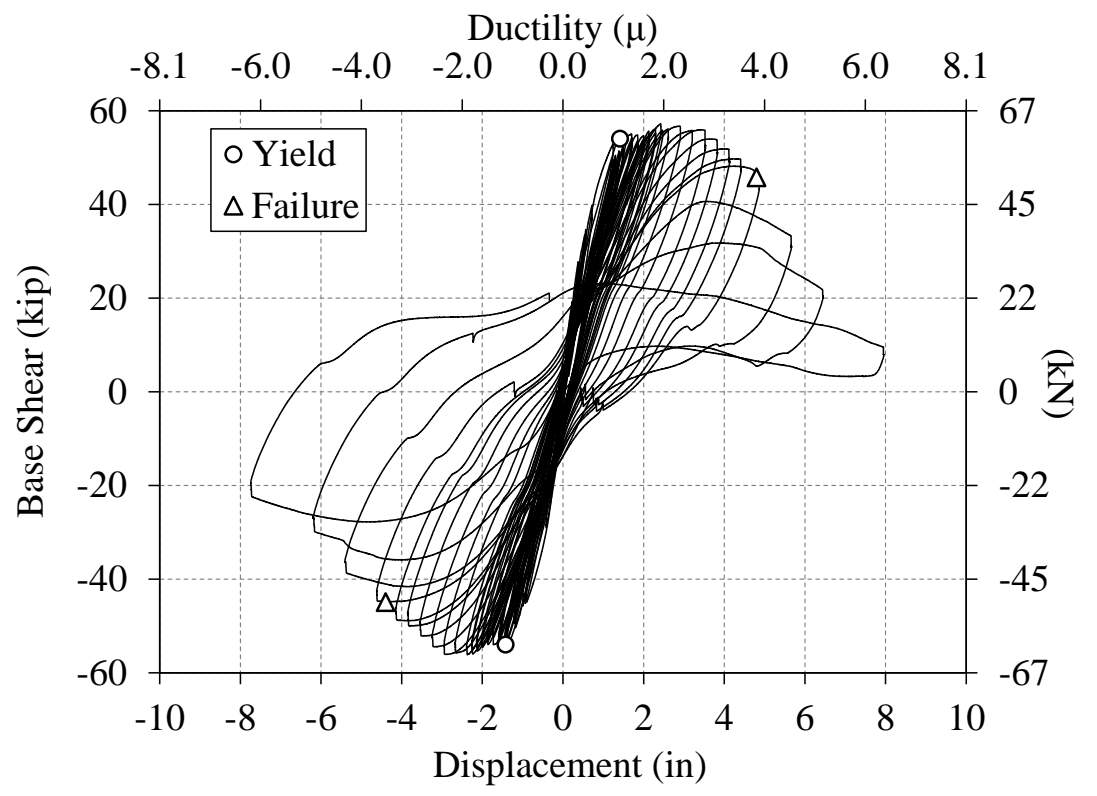

Figure 2.18 Load vs. displacement curve of column S-17

\subsubsection{Analysis of Results}

\subsubsection{Comparison to analytical results}

In this section the experimental results are compared to analytical results by means of conventional moment-curvature analysis. The analysis were performed using the Section Designer feature found in SAP2000 and Caltrans specifications (2013). The momentcurvature analysis was done assuming Mander (1988) parametric stress-strain curve to represent the unconfined and confined concrete, and Park parametric stress-strain curve to represent the reinforcing steel constitutive law. The conversion from moment-curvature to load-displacement was done by using a lumped plastic hinge model assuming a curvature and deflection relationship for a reinforced concrete cantilever column developed by Paulay and 
Priestley (1992). In this simple model for calculating displacements, the displacement at yield $\left(\Delta_{\mathrm{y}}\right)$ can be calculated using Eq. (2.5). Where, $\phi_{y}$ is the yield curvature of the section and $H$ is the effective height of the specimen. In this study, the idealized yield curvature, following Caltrans (2013) and AASHTO (2009) recommendations, was utilized to compute the yield displacement due to flexural behavior.

$$
\Delta_{y}=\frac{\phi_{y} \cdot H^{2}}{3}
$$

After yielding, the plastic displacement at the effective height is calculated using Eq. (2.6). Where, $L_{p}$ is the plastic hinge length and $\phi_{i}$ is the corresponding curvature.

$$
\Delta_{p}=\left(\phi_{i}-\phi_{y}\right) L_{p}\left(H-0.5 L_{p}\right)
$$

The length of the plastic hinge is calculated using Eq. (2.7). Where, $\mathrm{f}_{\mathrm{ye}-\mathrm{me}}$ is the measured yield stress of the longitudinal reinforcing steel, and $d_{b}$ is the diameter of the longitudinal rebar. This resulted in a plastic hinge length equal to 26.8 inches.

$$
L_{p}=0.08 H+0.15 f_{y-m e} d_{b} \geq 0.3 f_{y-m e} d_{b} \quad(k s i, \text { in })
$$

The comparison between experimental and analytical envelope curves is shown in Figure 2.19. The comparison shows that the strength for all columns was over-estimated by the analytical results. This effect was significant for column S-7 since the overestimation at the peak strength was $31 \%$. This overestimation is most likely caused by the assumption in the initial model that the full tensile capacity could be transferred in the lap splice region. However, it is well-known that the longitudinal reinforcement should not be spliced in plastic hinge regions in order to avoid under-development of the full tensile capacity of longitudinal rebars. Priestley et al. (1996) proposed Eq. (2.8) to estimate the total force 
transferred $\left(\mathrm{T}_{\mathrm{b}}\right)$ through a lap splice without considering any aid from the transverse reinforcement.

$$
T_{b}=A_{b} f_{s}=f_{t} p l_{s}
$$

Where, $A_{b}$ is the area of the longitudinal rebar, $f_{s}$ is the stress of the rebar, $f_{t}$ is the concrete tension stress, $\mathrm{p}$ is the concrete perimeter associated with each bar, and $l_{\mathrm{s}}$ is the length of the splice. Priestley recommended using a conservative value of $f_{t}=4 \sqrt{f_{c}{ }^{\prime}}$ based on experimental results, and a perimeter (p) equal to:

$$
p=\frac{s}{2}+2\left(d_{b}+c\right) \leq 2 \sqrt{2}\left(c+d_{b}\right)
$$

Where, $\mathrm{s}$ is the average spacing between spliced bars along the critical column face and $\mathrm{c}$ is the concrete cover. In this particular case, the spliced pairs of bars are widely spaced. Therefore, the upper limit is applicable. As a result, the total force that can be developed in the splice is presented in Table 2.11 for each column and is compared to the required force to develop yielding and tensile force in the longitudinal rebars. The comparison shows that the maximum force that can be developed in the splice region is close to the force to develop yielding of the longitudinal rebar. Moreover, these results also showed that the section will not be able to develop its full flexural strength. This is consistent with the results showed in Figure 2.19, where the maximum base shear recorded in the experiments was less than the analytical force calculated using moment-curvature analysis.

Table 2.11 Maximum bar force transferred in the splice region

\begin{tabular}{c|c|c|c}
\hline Specimen & $\begin{array}{c}\text { Maximum Bar Force } \\
\text { (kip) }\end{array}$ & $\begin{array}{c}\text { Yielding Force } \\
\text { (kip) }\end{array}$ & $\begin{array}{c}\text { Tensile Force } \\
\text { (kip) }\end{array}$ \\
\hline C-7 & 88.4 & 89.2 & 127.5 \\
\hline S-7 & 86.0 & 89.2 & 127.5 \\
\hline S-17 & 90.7 & 89.2 & 127.5 \\
\hline
\end{tabular}




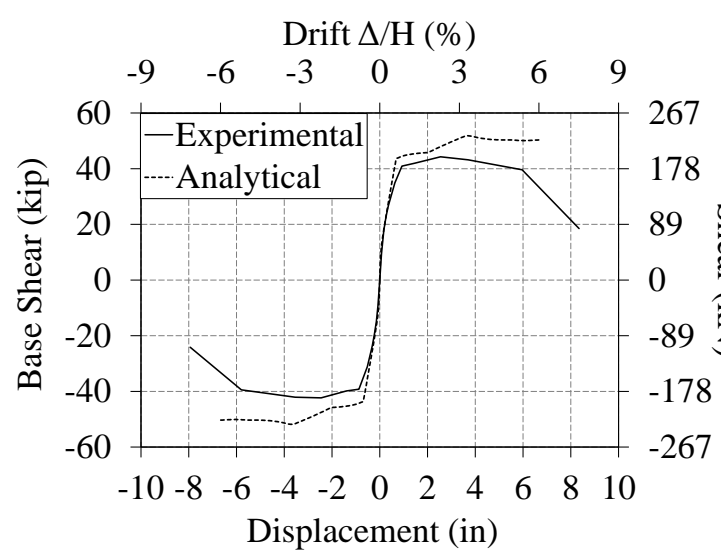

(a)

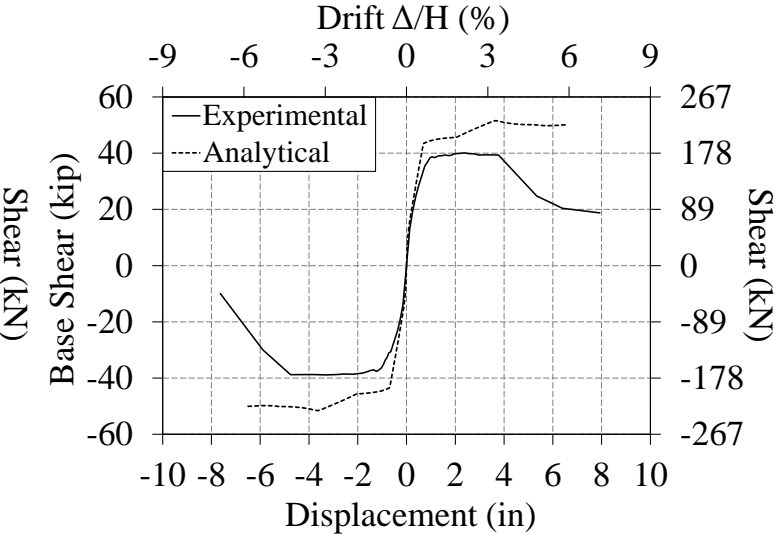

(b)

Drift $\Delta / \mathrm{H}(\%)$

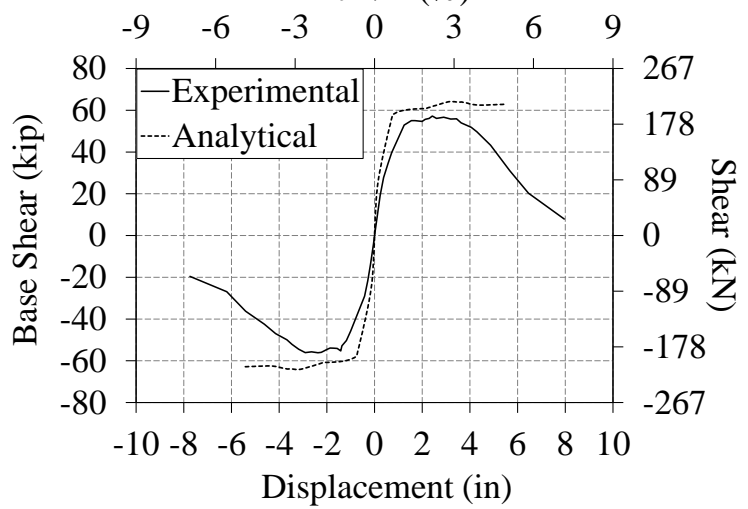

(c)

Figure 2.19 Experimental vs analytical envelope curves.

(a) column C-7, (b) column S-7, (c) column S-17

The aforementioned moment-curvature analysis considered the flexural behavior of the specimens. However, the deformations of a RC component are a combination of flexural, shear and bond slip deformations as shown in Figure 2.20. For that reason, the analytical yield displacement originally calculated using moment-curvature analysis was improved including the shear and bond slip contribution to the total displacement.

Shear deformations were estimated using Eq. (2.10), assuming isotropic and homogenous material with constant shear modulus. 


$$
\Delta_{v}=\frac{V H}{A_{e f f} G_{e f f}}
$$

Where, $A_{\text {eff }}$ is the effective shear cross-sectional area $(5 / 6$ of the gross area for rectangular sections), $\mathrm{G}_{\text {eff }}$ is the effective shear modulus, and $\mathrm{V}$ is the shear force. In this study, an effective shear modulus ( $\mathrm{G}_{\mathrm{eff}}$ ) equal to $0.2 \mathrm{E}_{\mathrm{c}}$ was considered following the recommendation of Elwood and Eberhard (2009).

The deformation at the top of the column that results from slip of the tensile longitudinal reinforcement was calculated using Eq. (2.11) (Elwood \& Eberhard, 2009). Where, $u$ is a uniform bond stress that typically ranges from $6 \sqrt{f_{c}{ }^{\prime}}$ to $12 \sqrt{f_{c}{ }^{\prime}}$ psi (Sozen, et al., 1992), (Lehman \& Moehle, 2000), (Sezen, 2002), fy-me is the measured yield stress of longitudinal reinforcing steel, $\mathrm{H}$ is the effective height of the specimen, $\mathrm{d}_{\mathrm{b}}$ is the diameter of the longitudinal rebar and $\phi_{\mathrm{y}}$ is the yield curvature.

$$
\Delta_{s l i p}=\frac{H d_{b} f_{y-m e} \phi_{y}}{8 u}
$$

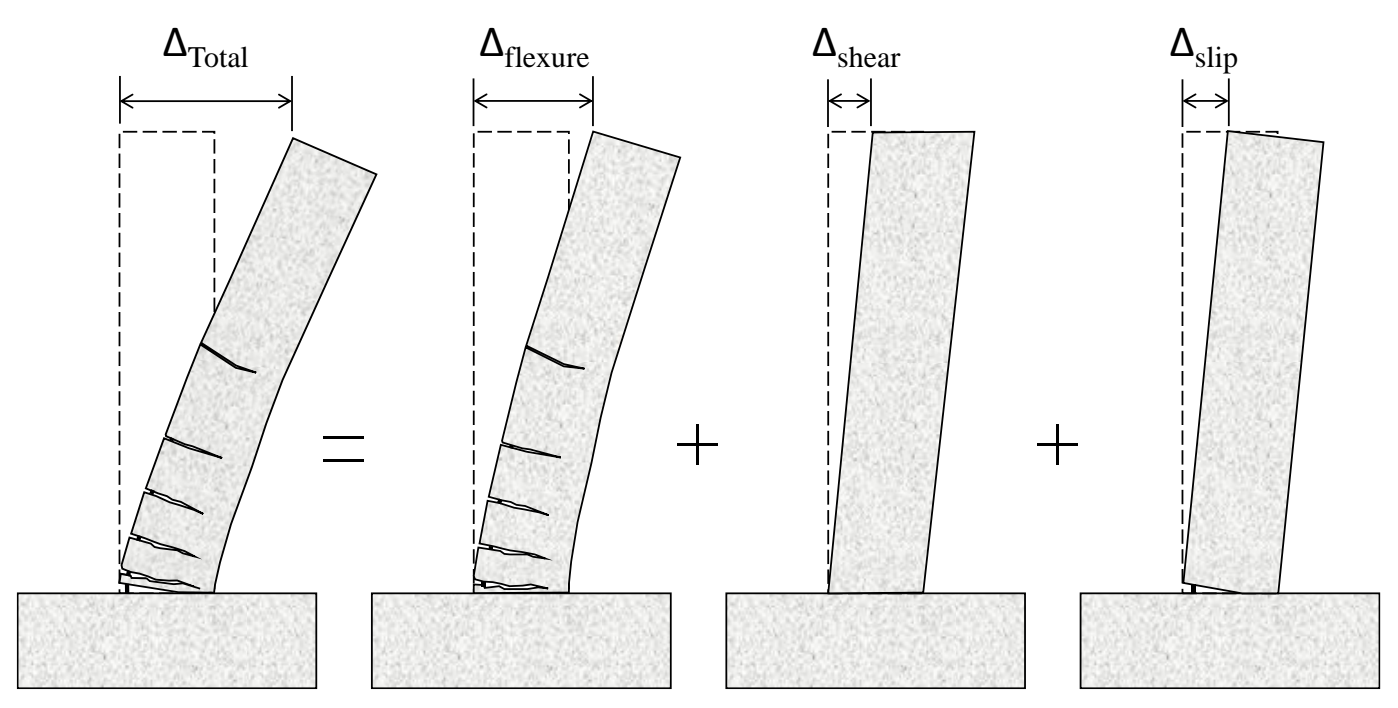

Figure 2.20 Deformation components for RC columns 
Table 2.12 shows a comparison between the yield displacement calculated analytically and experimentally. In this comparison, the uniform bond stress $(u)$ was taken as $12 \sqrt{f_{c}^{\prime}}$ psi following the recommendation of Lehman and Moehle (2000) and Sezen and Setzler (2008). The ratio of calculated to measured yield displacement shows a larger discrepancy for column S-17. The lower ratio for column S-17 may be caused by an under-estimation of the shear and bond slip contribution.

Table 2.12 Analytical vs experimental yield displacement $\left(\Delta_{\mathrm{y}}\right)$

\begin{tabular}{c|c|c|c|c}
\hline Specimen & $\begin{array}{c}\text { Analytical } \\
\text { Flexural (in) }\end{array}$ & $\begin{array}{c}\text { Analytical } \\
\text { Total (in) }\end{array}$ & $\begin{array}{c}\text { Experimental } \\
\text { (in) }\end{array}$ & $\begin{array}{c}\text { Ratio } \\
\text { Analytical/Experimental }\end{array}$ \\
\hline C-7 & 0.78 & 1.09 & 0.97 & 1.12 \\
\hline S-7 & 0.78 & 1.09 & 0.99 & 1.10 \\
\hline S-17 & 0.81 & 1.12 & 1.24 & 0.90 \\
\hline
\end{tabular}

\subsubsection{Backbone curves}

The column S-7 tested under the subduction loading protocol showed less strength and deformation capacity as compared to column C-7 tested under the conventional loading protocol as shown in Figure 2.21. Column S-17 showed more strength capacity than columns C-7 and S-7 because of the increased axial load. In terms of displacements, column S-17 showed similar displacement capacity than column S-7 but considerably less than column C-7. The fact that columns S-7 and S-17 had had less deformation capacity was attributed to the increasing number of low amplitude inelastic cycles that these columns underwent when using the subduction protocol. This increase in the number of small displacement inelastic cycles may be the cause of early emergence of vertical cracks that culminated in lap splice failures in columns S-7 and S-17. This result has direct implications in the design of retrofit measures for existing RC bridge columns since one would expect 
less capacity for columns subjected to subduction zone earthquakes and is in agreement with the numerical case study presented in the previous section and previous studies (Takemura \& Kawashima, 1997), (Kunnath, et al., 1997), (FEMA P440A, 2009), (Ou, et al., 2013). Moreover, the result implies that the common assessment of existing bridges by doing pushover analysis might overestimate the actual strength and deformation capacity of RC bridge substructures if the increased strength and stiffness degradation are not taken into consideration.

Despite the seismic detailing deficiencies of the specimens, the results showed that the columns had moderate ductility. The moderate-ductile performance is likely a result of low axial column loads $\left(0.07-0.17 \mathrm{f}_{\mathrm{c}} \mathrm{A}_{\mathrm{g}}\right)$ and a relatively long lap splice $\left(28 \mathrm{~d}_{\mathrm{b}}\right)$. Even though, this moderate ductility was unexpected, similar results were obtained by ElGawady et al. (2010) for typical deficient columns built prior 1970 in the State of Washington.

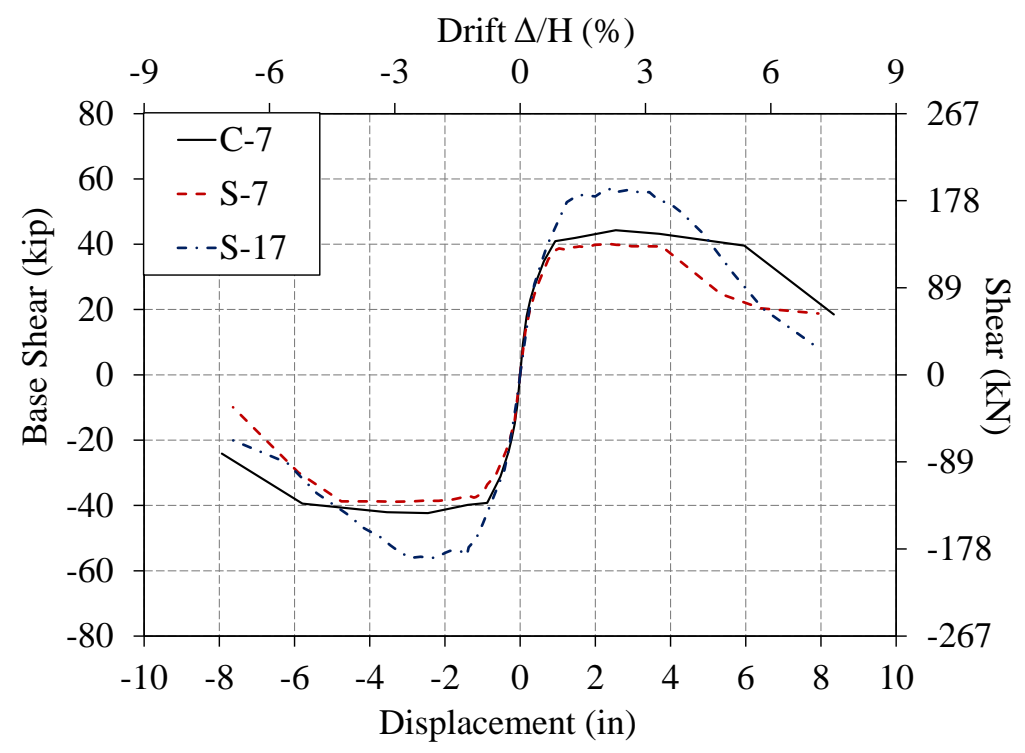

Figure 2.21 Backbone curves of square RC bridge columns 


\subsubsection{Stiffness degradation}

The stiffness degradation is an important property in $\mathrm{RC}$ bridges subjected to seismic events since it changes the effective natural period of the structure. Stiffness degradation can be attributed to concrete nonlinear behavior caused by flexural and shear cracking, slippage of steel reinforcement, etc. In this study, the normalized stiffness, represented as the secant stiffness $\left(\mathrm{k}_{\text {sec }}\right)$ divided by the yield stiffness $\left(\mathrm{k}_{\mathrm{y}}\right)$, at different displacement ductilities, was utilized as the stiffness degradation parameter. In terms of stiffness degradation, the test specimens showed similar stiffness degradation up to displacement ductility 2.5 , and there was no significant effect of the loading protocol applied on the stiffness degradation up to that ductility as depicted in Figure 2.22. However, for ductilities above 2.5, the columns S-7 and S-17 showed a higher stiffness degradation than column C-7, which is concordant with the increasing number of inelastic cycles imposed by the subduction zone protocol and the lap splice failure observed during the tests. Moreover, the results show that columns subjected to increased axial load have higher rate of strength deterioration.

Gulkan and Sozen (1974) proposed a relation between the displacement ductility $(\mu)$ and the secant stiffness $\left(\mathrm{k}_{\mathrm{sec}}\right)$ for $\mathrm{RC}$ columns. The test results are in agreement with the predictions obtained using Eq. (2.12) up to ductility 3.5. After that point, the stiffness

degradation of column C-7 matches the degradation rate calculated using the equation up to ductility 5.6. For ductilities above 5.6 the equation underestimates the rate of stiffness degradation for substandard columns.

$$
\frac{k_{\mathrm{sec}}}{k_{y}}=\frac{1}{\mu} \leq 1
$$




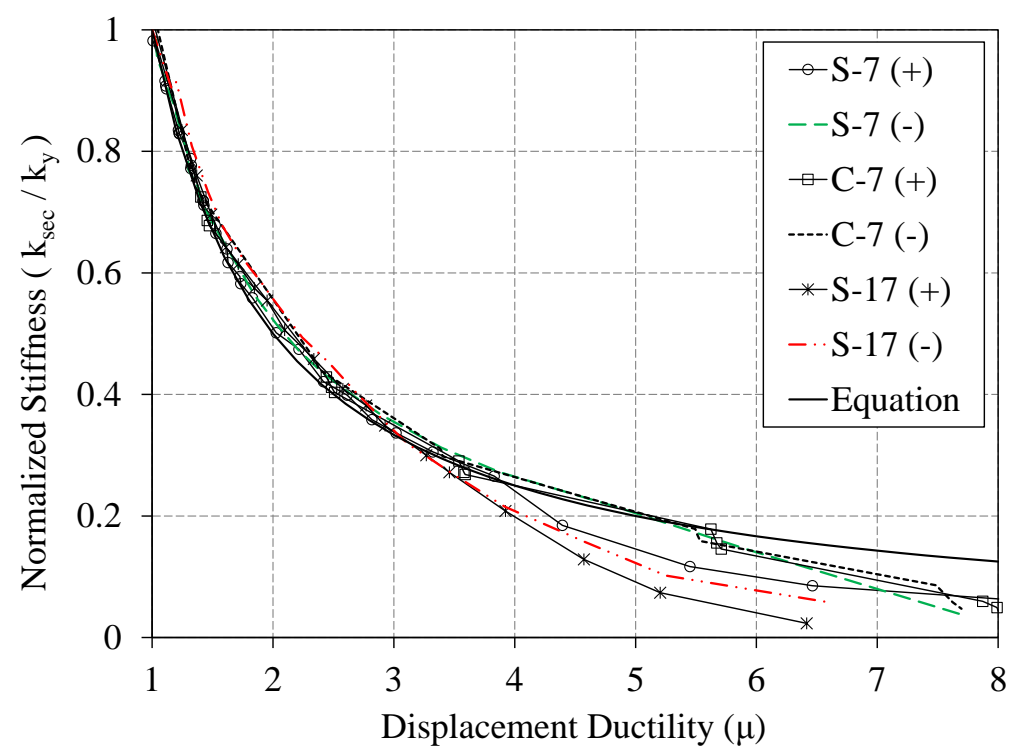

Figure 2.22 Stiffness degradation of square RC bridge columns

\subsubsection{Energy Dissipation}

The property of dissipating energy through hysteretic behavior is desirable in structures subjected to major seismic events. In this study the amount of energy that was dissipated by the specimens was calculated as the area $\left(A_{d}\right)$ enclosed by a full cycle as shown in Figure 2.23. The cumulative energy dissipated by the specimens on each cycle is shown in Figure 2.24. The results showed that the total cumulative energy dissipated is not affected by an increase in axial load and is not significantly affected by the number of loading cycles.

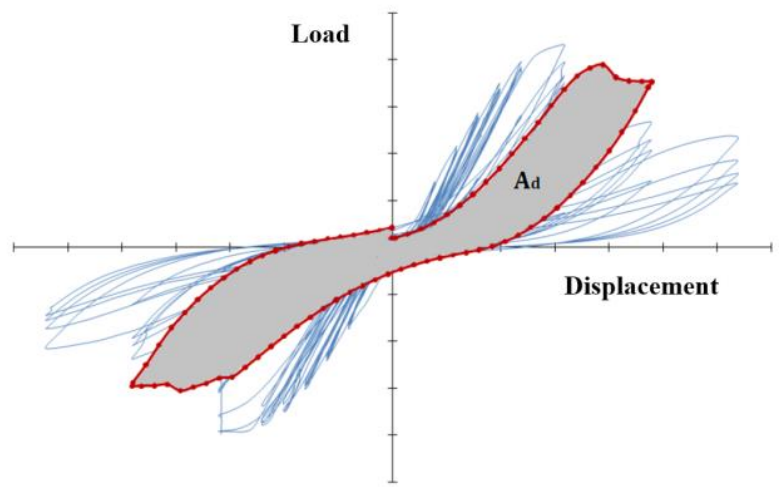

Figure 2.23 Illustration of energy dissipated through hysteretic behavior 


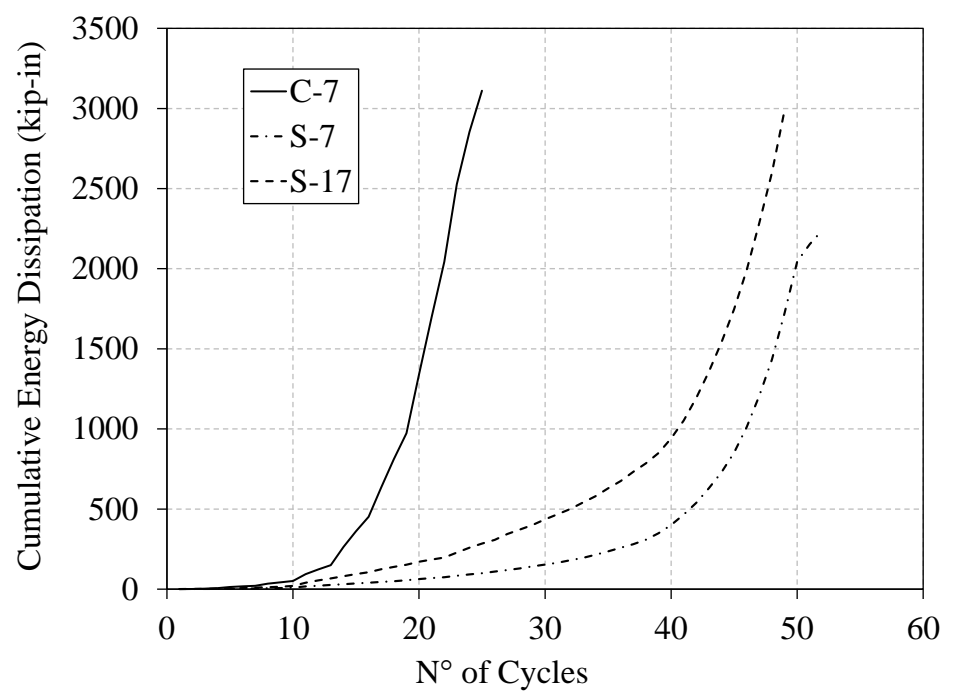

Figure 2.24 Cumulative energy dissipated by square RC bridge columns

\subsubsection{Improved Numerical Modeling Based on Experimental Results}

Two main numerical methods have been extensively used to characterize the behavior of RC columns, lumped-plasticity and distributed-plasticity (Taucer, et al., 1991), (Scott \& Fenves, 2006), (Berry \& Eberhard, 2008). In the lumped-plasticity method, all the nonlinear behavior is concentrated in a plastic hinge where the overall force-deformation response of the component is defined. In the distributed-plasticity method the nonlinear behavior is distributed along the length of the member through the use of discrete fiber sections and integration points. The RC bridge columns presented in this paper were modeled using these two methodologies with the aim of providing modeling parameters applicable for each method, and assess the effect of the loading protocol on those parameters and on the overall column behavior. OpenSees (2013) was utilized to carry out the numerical modeling for its vast library of available materials and elements to characterize the nonlinear response of structural components. 
Both model strategies are depicted in Figure 2.25. Four nodes were used to represent the column test setup. The bottom node represents the interface between the RC column and the footing. This node was constrained in all directions in order to represent a fixed support. The adjacent node was used in order to include the inelastic spring in the case of the lumped-plasticity model and the bond-slip contribution in the case of the distributedplasticity model. The axial load, P, and the lateral load, F, were applied at the very top node at a height of 113 in in order to mimic the actual test setup, where the axial and the lateral load were directly applied to the steel beam located on top of the columns. Further, the transfer steel beam was represented in the model through the use of an elastic element of length 13 in. The very top node was selected as the control node to record the lateral displacement of the column.

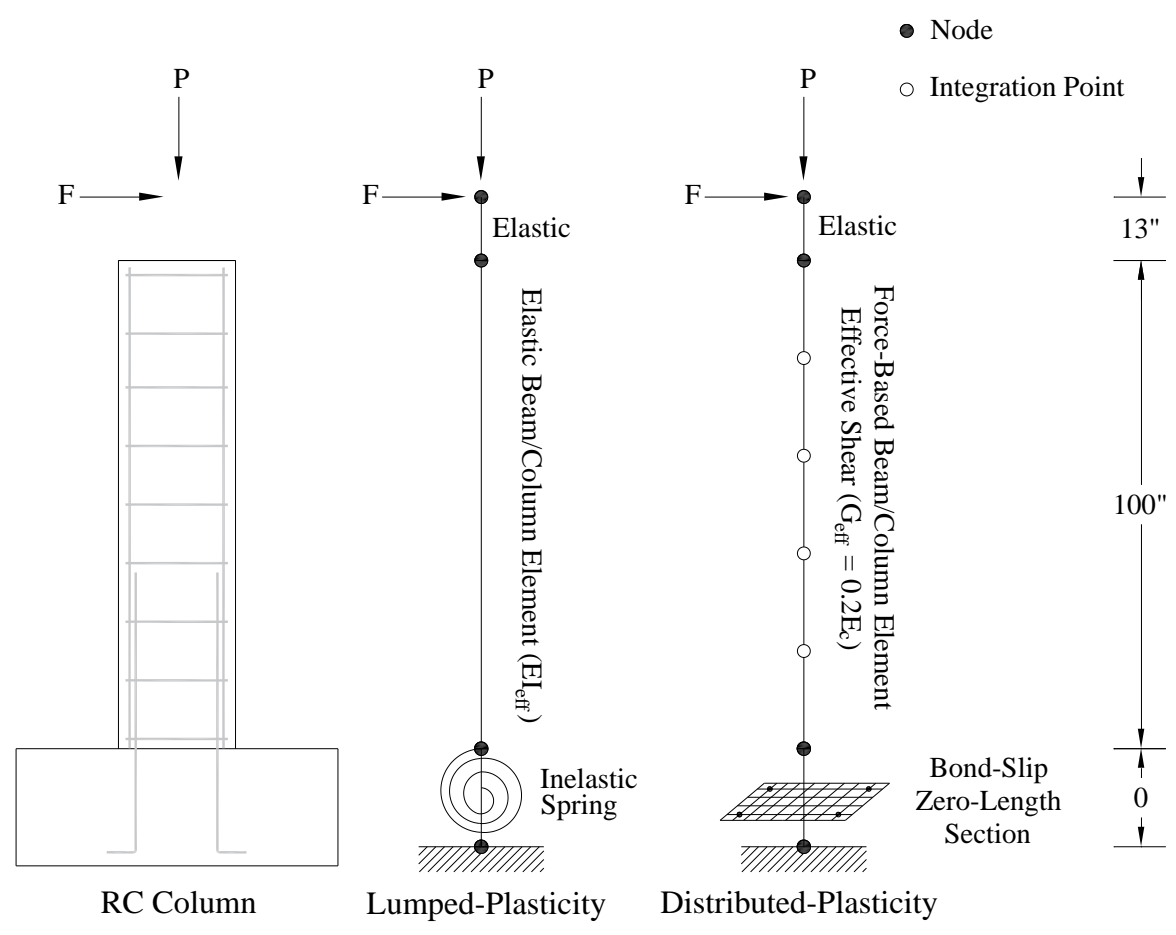

Figure 2.25 Numerical models. 


\subsubsection{Lumped-Plasticity model description}

The cyclic behavior of the lumped-plasticity model is controlled by the hysteretic behavior of the inelastic spring at the base of the column. This spring has the role of concentrating all the inelastic behavior due to flexure, shear and bond slip deformations as shown in Figure 2.20. In this study, the rotational spring was modeled using a zero length element and a uniaxial material given by the modified Ibarra-Medina-Krawinkler deterioration model with pinched hysteretic response (Ibarra, et al., 2005a), (OpenSees, 2013) as illustrated in Figure 2.26.This uniaxial material is capable of capturing strength and stiffness degradation in a more empirical manner. In addition to that, the model can capture the inherent pinching behavior of bond slip failures, which is typical on RC columns with poor detailing and lap splices in expected plastic hinge zones. The characteristic of being able to represent strength and stiffness degradation has made this uniaxial material one of the most used hysteretic models in the study of global collapse of RC structures (Haselton, et al., 2008), (PEER/ATC-72-1, 2010).

The initial behavior of the model is controlled by the parameters selected for the inelastic spring such as the elastic stiffness, $\mathrm{K}_{0}$, and the effective yield strength, $M_{y}$. The pinching behavior is controlled by the ratio of force at which reloading begins, $F_{p r}$, and the ratio of reloading stiffness, $A_{\text {pincb }}$. The stiffness and strength deterioration of the model is controlled by 8 parameters, $\lambda_{s}, \lambda_{c}, \lambda_{A}, \lambda_{K}, c_{s}, c_{s}, c_{A}, c_{K}$, where $\lambda$ is a cyclic deterioration parameter and $\mathrm{c}$ is the rate of deterioration, the subscripts $\mathrm{S}, \mathrm{C}, \mathrm{A}$, and $\mathrm{K}$ stand for strength deterioration, post-capping strength deterioration, reloading stiffness deterioration and unloading stiffness deterioration. Inelastic deformation capacity is controlled by the plastic rotation capacity, $\theta_{p}$, the post-capping rotation capacity, $\theta_{p c}$, the residual strength ratio, Res, 
and the ultimate rotation capacity, $\theta_{u}$. These parameters were selected with the aim of approximating the experimental results and are presented in Table 2.13. It is worth mentioning that a relatively large ultimate rotation capacity $\left(\theta_{u}=0.5 \mathrm{rad}\right)$ was used in order to facilitate convergence. Further, the parameter $\mathrm{c}$ that represents the rate of deterioration was set equal to 1 for all columns.

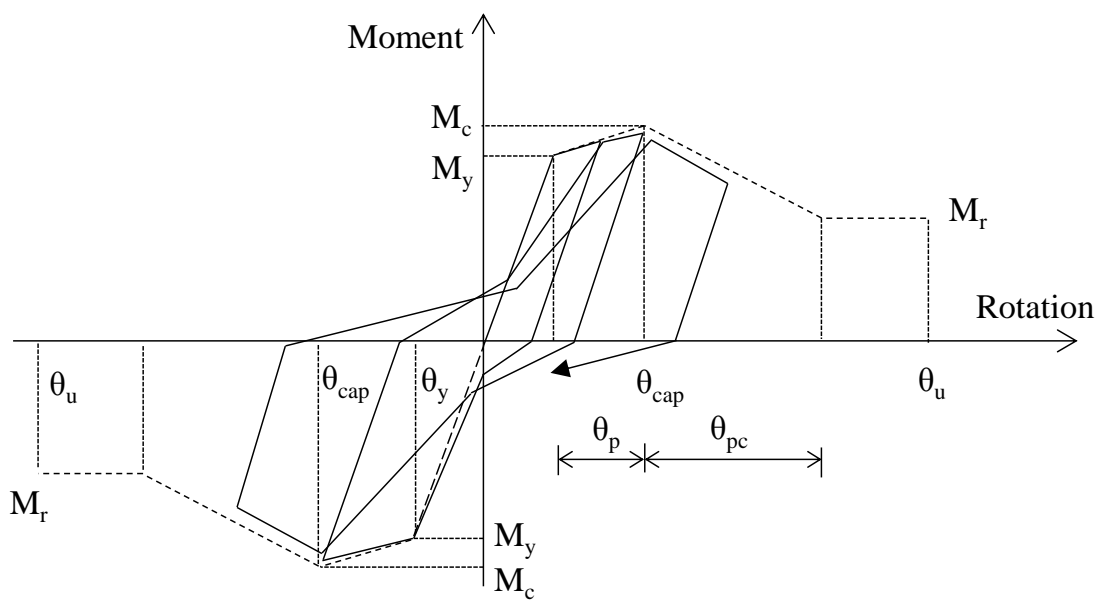

Figure 2.26 Degrading model with pinched hysteretic response

Table 2.13 Parameters for the lumped-plasticity model

\begin{tabular}{c|c|c|c|c|c|c|c|c|c|c|c}
\hline Column & $\begin{array}{c}\mathrm{K}_{0} \\
\text { kip-ft/rad }\end{array}$ & $\begin{array}{c}\mathrm{M}_{\mathrm{y}} \\
\text { kip-ft }\end{array}$ & $\alpha_{\mathrm{s}}$ & $\mathrm{F}_{\mathrm{pr}}$ & $\mathrm{A}_{\text {pinch }}$ & $\lambda_{\mathrm{s}}$ & $\lambda_{\mathrm{c}}$ & $\lambda_{\mathrm{K}}$ & $\theta_{\mathrm{p}}$ & $\theta_{\mathrm{pc}}$ & Res \\
\hline C-7 & 75,711 & 392 & 0.04 & 0.7 & 0.1 & 2 & 2 & 1 & 0.05 & 0.15 & 0.6 \\
\hline S-7 & 60,480 & 367 & 0.06 & 0.6 & 0.1 & 2 & 1.5 & 1 & $\begin{array}{c}0.03(+) \\
0.045(-)\end{array}$ & 0.10 & $\begin{array}{c}0.8(+) \\
0.6(-)\end{array}$ \\
\hline S-17 & 75,984 & 545 & 0.09 & 0.6 & 0.1 & 2 & 1.5 & 1 & 0.03 & 0.15 & $\begin{array}{c}0.6(+) \\
0.8(-)\end{array}$ \\
\hline
\end{tabular}




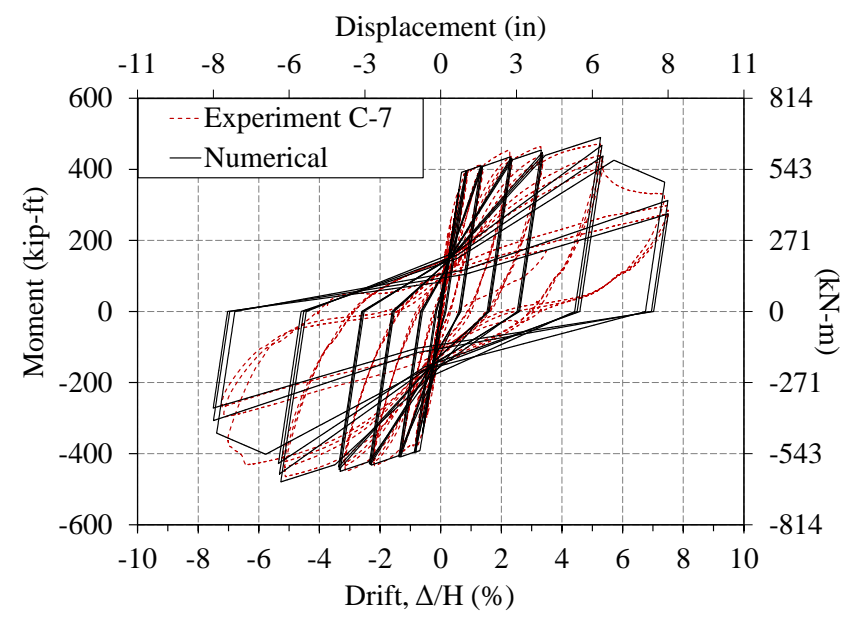

(a)

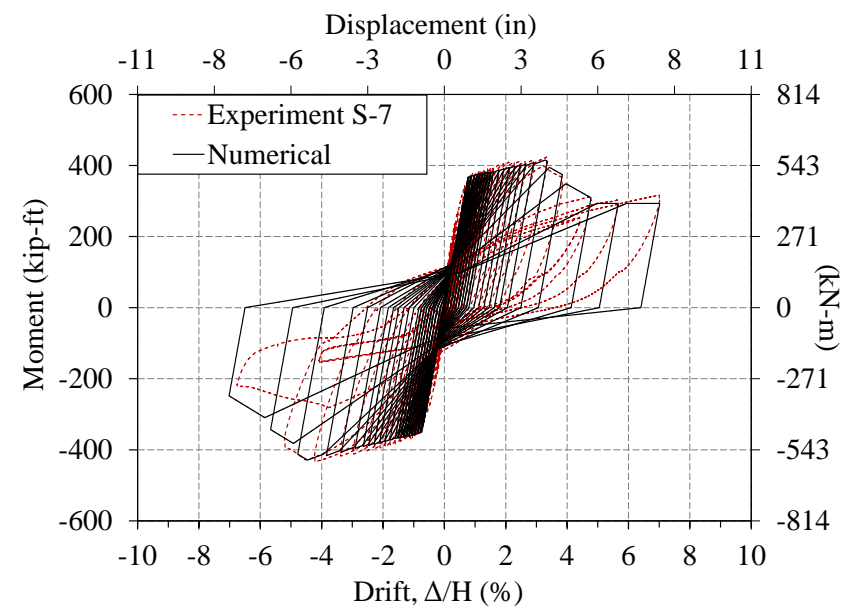

(b)

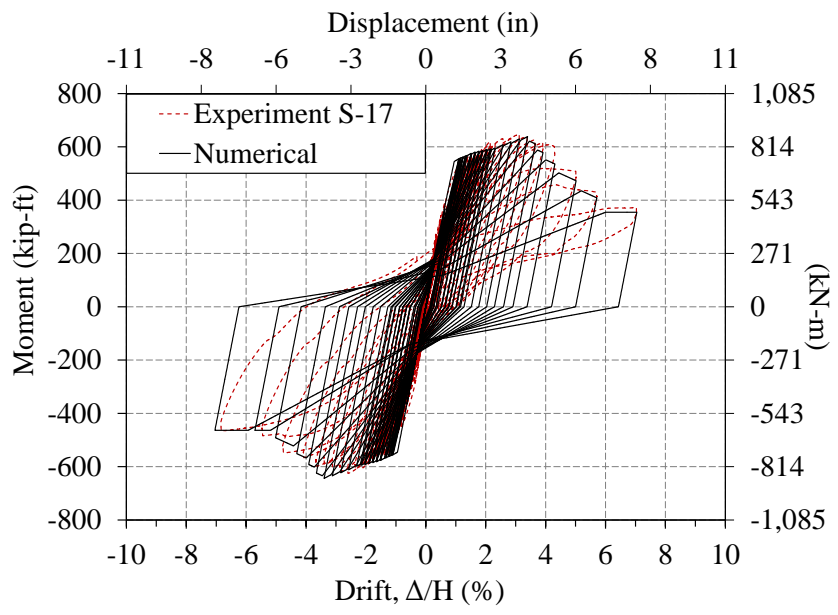

(c)

Figure 2.27 Experimental vs numerical results - Lumped-Plasticity model.

(a) column C-7; (b) column S-7, (c) column S-17 
Comparisons between the experimental and the numerical results using the lumpedplasticity approach are shown in Figure 2.27. Numerical results using the lumped-plasticity model are in agreement with the experimental results and captured reasonably well the incycle and cyclic strength and stiffness deterioration of the specimens. Understanding the incycle deterioration as the degradation in a cycle, and the cyclic deterioration as the degradation in strength and stiffness that occurs in subsequent cycles (FEMA P440A, 2009). However, the model presents deficiencies capturing the unloading stiffness of the specimens.

\subsubsection{Distributed-Plasticity model description}

In the distributed-plasticity model, discrete fiber sections were used throughout the length of the column. A force-based beam-column element with six integration points was used to represent the column. The number of integration points was selected following the recommendation of Berry and Eberhard (2008). The Gauss-Lobatto integration rule was selected as the plastic hinge integration method. This integration rule includes additional integration points at the element ends.

The column section was divided into three main fiber sections representing the core (confined) concrete, cover (unconfined) concrete and the reinforcing steel. The cover and core concrete were discretized into 24 and 20 strips in both directions, respectively. The Concrete02 with linear tension softening uniaxial material was used in this study to model both confined and unconfined concrete. In order to obtain the confined concrete parameters, the model proposed by Mander et al. (1988) was used. The longitudinal reinforcing steel was modeled using the uniaxial Hysteretic material. This material was selected because it can capture pinching of force and deformation, which is of vital 
importance to characterize bond slip failure in RC columns with lap splices. The parameters pinchx and pinchy were set equal to 1.0 in order to represent the observed pinching behavior of the columns. The hysteretic material is also capable of representing damage due to ductility and energy, and degraded unloading stiffness that can be caused by concrete crushing, splitting cracks, bar buckling and bar fracture. The damage parameter damage1, which is related to the damage due to ductility was set equal to 0.006 to account for the cyclic deterioration from one cycle to the following cycle observed in the specimens. The parameter damage2, which takes into account the damage due to energy, was set equal to 0.002 in an effort to capture the strength deterioration observed in the columns at a fixed displacement or strain amplitude. The degraded unloading stiffness parameter (beta) was set equal to 0.3 for all columns.

The stress-strain values for the reinforcing steel used in the numerical models represent the measured values from the experimental study and were obtained from tensile testing of a coupon. It is worth mentioning that the yield stress, $f_{y}$, and ultimate stress, $f_{u}$, for column S-7 were reduced by $12 \%$ from $f_{y}=70.3 \mathrm{ksi}$ and $f_{u}=101 \mathrm{ksi}$ to $f_{y}=67 \mathrm{ksi}$ and $f_{u}=95$ ksi in order to represent that this column did not reach its maximum flexural strength capacity. This lower flexural capacity was caused by the lap splice located at the base of the column, which inhibited the full development of the force transferred through the lap splice (Priestley, et al., 1996). This aspect is in accordance with the observation of early formation of vertical splitting cracks during the test that denotes the development of a bond slip failure mechanism. The effective bar stress $\left(f_{s}\right)$ to be used in the model can be computed using Eq. (2.13) (Priestley, et al., 1996). 


$$
f_{s}=\frac{f_{t} \cdot p \cdot l_{s}}{A_{b}}
$$

Where, $A_{b}$ is the area of the longitudinal rebar, $f_{t}$ is the concrete tension stress, $p$ is the concrete perimeter associated to the failure surface of each bar, and $l_{s}$ is the length of the splice. Priestley recommended using a conservative value of $f_{t}=4 \sqrt{f_{c}^{\prime}}$ psi based on experimental results, and a perimeter $(p)$ equal to $2 \sqrt{2}\left(c+d_{b}\right)$ for widely spaced lap-spliced bars, where $\mathrm{c}$ is the concrete cover and $d_{b}$ is the diameter of the longitudinal rebar.

Column deformations typically result from flexural, bond slip and shear behavior as shown in Figure 2.20. The flexural behavior was modeled with the force-based beam/column element as previously described. In order to add the shear behavior, a section aggregator was utilized. Shear deformations were idealized as an isotropic material with constant shear modulus. In this study, an effective shear modulus, $G_{e f f}$ equal to $0.2 E c$ was used following the recommendation of Elwood and Eberhard (2009). In order to model the additional flexibility that is introduced from the slip of the longitudinal reinforcement at the anchorage, the bond slip model proposed by Ghannoum (2007) was used. In this bond slip model, the slip behavior is modeled using a zero-length fiber section with the same discretization strategy used for the nonlinear beam/column element. However, in the zerolength section an equivalent stress-slip displacement relationship for the steel and concrete fibers is used instead of the stress-strain relationships as shown in Figure 2.28 for the steel fibers and Figure 2.29 for the concrete fibers. In the steel fibers the slip displacement at yield $\left(s_{y}\right)$ was calculated from the measurements of the LVDTs located at the base of the columns and then compared to the results obtained from Eq. (2.14) (Sezen, 2002). 


$$
s_{y}=\frac{\varepsilon_{y} f_{y} d_{b}}{8 \cdot u}
$$

Where, $\varepsilon_{y}$ is the strain at yielding, $d_{b}$ is the diameter of the longitudinal bar, and $u$ is an average uniform bond stress. Different values of uniform bond stress can be found in the literature. Typically $u$ varies in the range from $6 \sqrt{f_{c}{ }^{\prime}}$ to $12 \sqrt{f_{c}{ }^{\prime}}$ psi $\left(0.5 \sqrt{f_{c}{ }^{\prime}}\right.$ to $\left.1.0 \sqrt{f_{c}{ }^{\prime}} \mathrm{MPa}\right)$, (Sozen, et al., 1992), (Lehman \& Moehle, 2000), (Sezen, 2002). In this study, a uniform bond stress value of $6 \sqrt{f_{C}{ }^{\prime}}$ psi was found to fit well with the experimental results and is recommended in the case of columns with lap splices. Slip displacement at yield, $s_{y}$, of 0.07 in, 0.067 in, and 0.085 in were used in the distributed-plasticity model for columns S-7, C-7 and S-17, respectively. The Steel02, Giuffre-Mengotto-Pinto model was employed to represent the steel fiber in the bond slip zero-length section because its ease of implementation and convergence. The confined and unconfined concrete strains were also modified in the bond slip fiber section in order to avoid discontinuities in the steel stresses and neutral axis location between the fibers of the nonlinear beam/column element and the bond slip section (Ghannoum, 2007). This modification was accomplished by multiplying the concrete strains by a scale factor, $\mathrm{SF}_{\text {conc }}=s_{y} / \varepsilon_{y}$. This scale factor is dimensionally incorrect but allows an increase in the concrete strains to maintain compatibility between the fiber sections. Scale factors of 28, 34, and 35 were used for C-7, S-7 and S-17 respectively. Figure 2.28 and Figure 2.29 show the constitutive relationship for the steel and concrete fibers, respectively. 


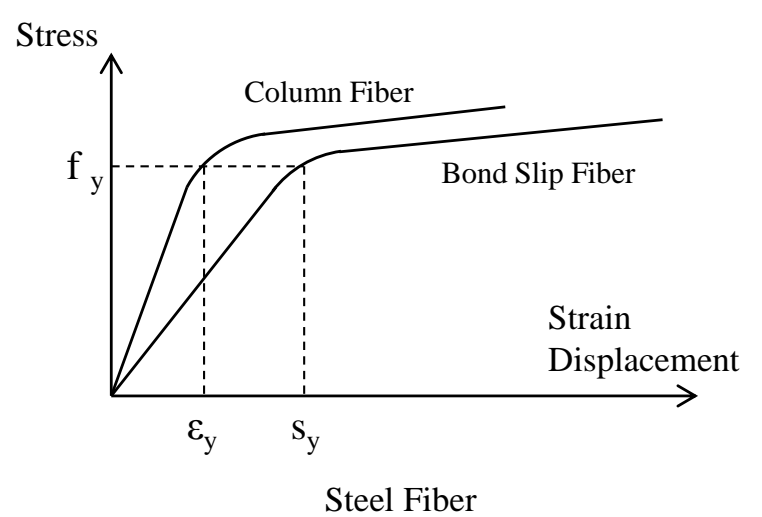

Figure 2.28 Constitutive relation for steel fibers

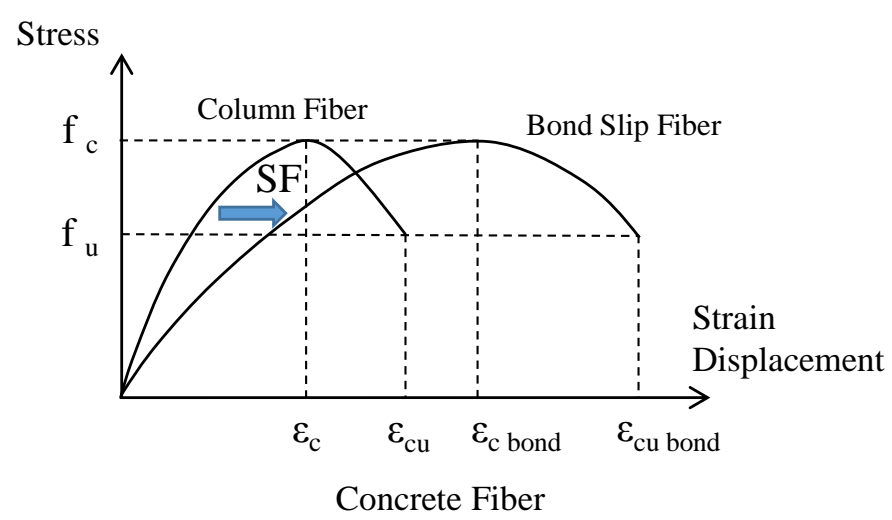

Figure 2.29 Constitutive relation for concrete fibers

Comparisons between the experimental and the numerical results using the distributed-plasticity approach are shown in Figure 2.30. The distributed-plasticity model shows a well agreement with the experimental results and captured reasonably well the incycle and cyclic deterioration of the specimens. 


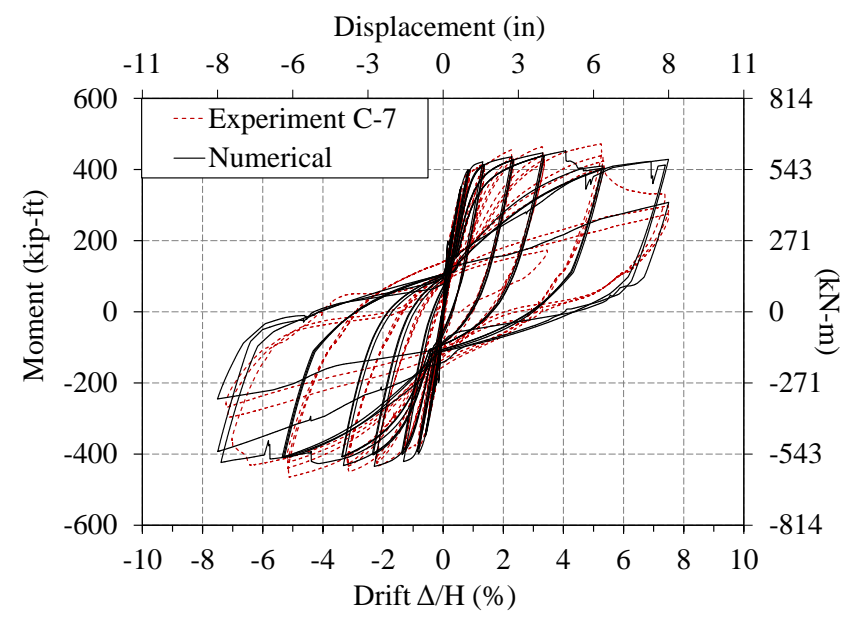

(a)

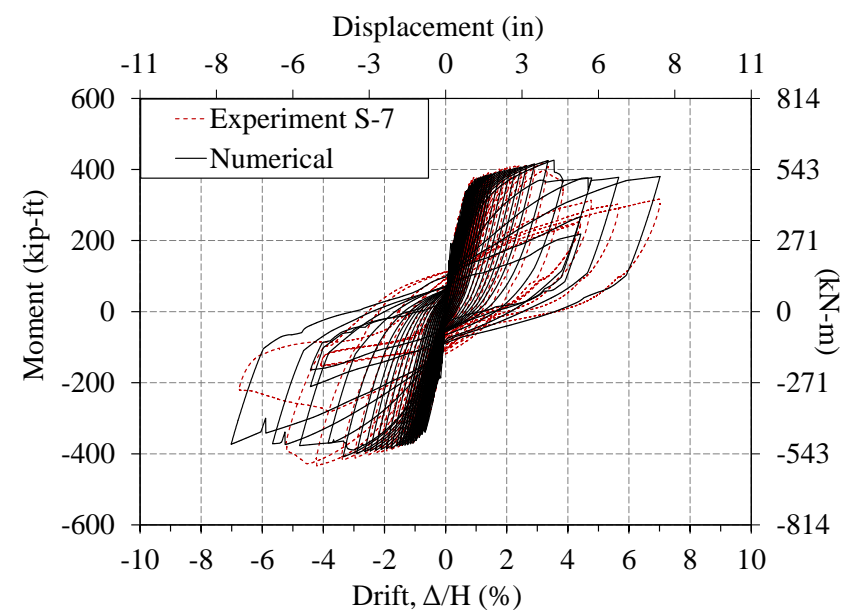

(b)

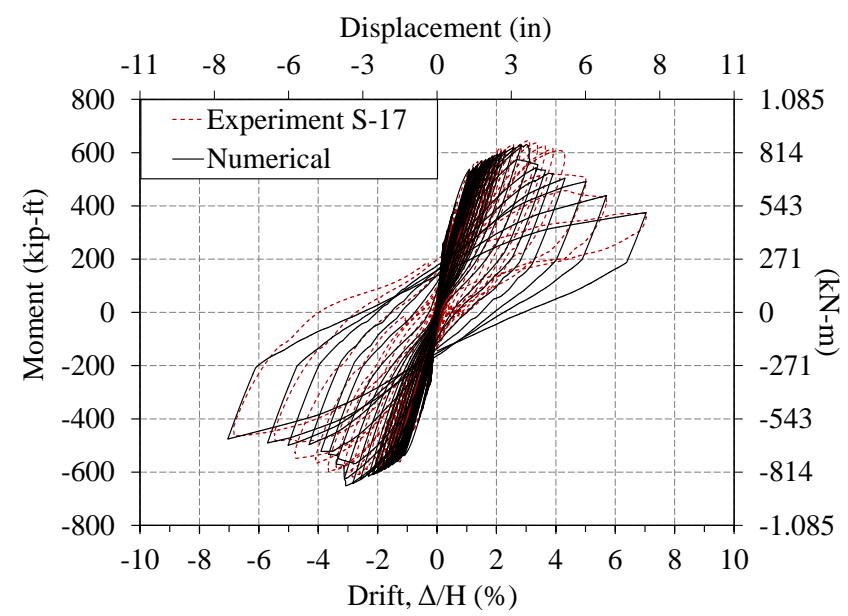

(c)

Figure 2.30 Experimental vs numerical results - Distributed-Plasticity model. (a) column C-7; (b) column S-7, (c) column S-17 


\subsubsection{Model validation and discussion}

Comparisons between the experimental and the numerical results using the lumpedplasticity and distributed-plasticity approaches are shown in Figure 2.27 and Figure 2.30, respectively. Both of the numerical models were able to characterize the behavior of the tested columns. Values of initial stiffness, strength and deformation capacities, pinching effect, and strength and stiffness degradation were closely represented by the models as shown in Figure 2.27 and Figure 2.30.

Figure 2.31 depicts the total dissipated energy in the experiments compared to the one calculated for the numerical models. The total energy dissipated was calculated adding the enclosed area of each cycle in the load vs displacement response as shown in Figure 2.23. Both numerical models tend to overestimate the total energy dissipated by the specimens. The results show that despite of using a degrading model with pinched hysteretic response the lumped-plasticity model overestimates the total dissipated energy more than the distributed-plasticity model. This is evident in the case of column S-17 that was tested with a higher axial load. In that case the overestimation was $72 \%$ with respect to the experimental value. This overestimation is primarily caused by the hysteretic rule limitation on properly characterizing the unloading stiffness. On the other hand, the distributed-plasticity model presents less overestimation in the total dissipated energy as compared to the experimental results with a maximum error of $23 \%$.

These results led to the conclusion that the distributed-plasticity strategy more closely represents the experimental results. This improved accuracy comes with a cost, which manifest in convergence issues caused by the sensitivity of the damaging parameters within the hysteretic material utilized to represent the reinforcing steel. Convergence can be greatly 
improved if the lumped-plasticity model is used since the inherent integration method used in distributed approaches is avoided. The distributed-plasticity model strategy may be used in cases of biaxial loads without major modifications, making it suitable for three dimensional modeling cases. On the other hand, if biaxial loads are considered the lumped-plasticity model would need the definition of another inelastic spring in a different axes in order to effectively capture the biaxial behavior of the RC column.

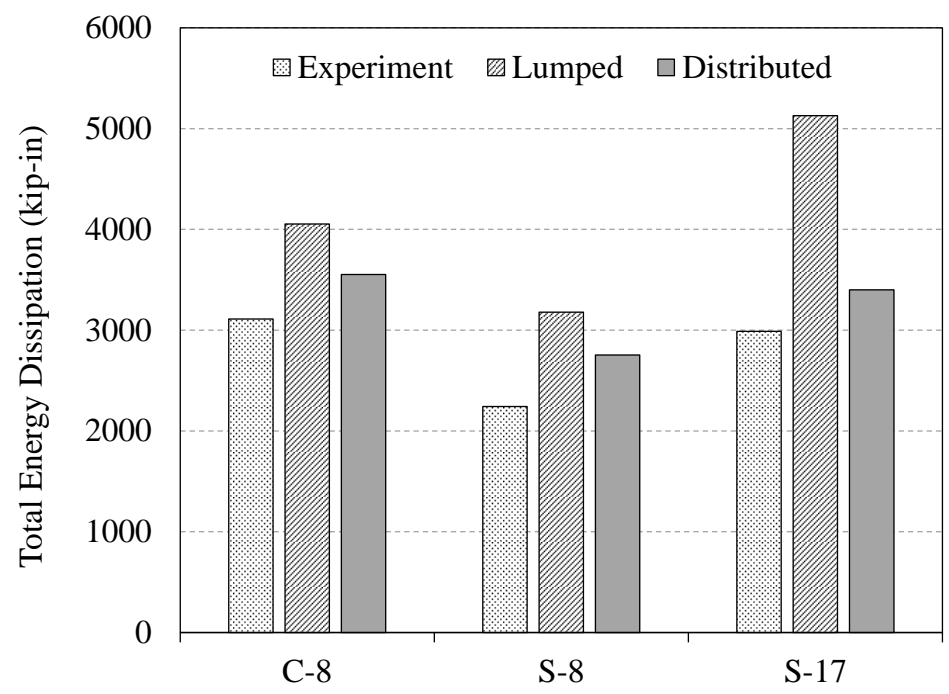

Figure 2.31 Energy dissipation comparison

Hysteretic cyclic deterioration plays a fundamental role in the assessment of bridge columns subjected to ground motions. In the case of the lumped plasticity, Haselton et al. (2008) proposed equations to predict the cyclic deterioration parameter ( $\lambda$ ). ). According to Haselton's study the cyclic deterioration parameter varies with the amount of transverse reinforcement, shear capacity and axial load ratio. Using Haselton's equation for the specimens tested leads to $\lambda$ values that ranges from 30 to 40 . Another equation also proposed by Haselton is included in the PEER/ATC 72-1 (2010) report, in which the value of $\lambda$ only depends on the axial load ratio. The PEER/ATC report stated that for a typical 
column with seismic detailing, typical values of the parameter $\lambda$ are on the order of 10 to 20 . Lower $\lambda$ values indicate that the element has a higher rate of strength and stiffness deterioration and therefore less capacity to dissipate energy. In this study, a $\lambda$ value of 2 was calibrated based on the experimental results and is recommended for characterizing the behavior of columns built prior to 1970 . Moreover, plastic rotation capacities $\left(\theta_{p}\right)$ for columns with low axial load and minimum confinement have been computed in previous studies (Haselton, Liel, Taylor, \& Deierlein, 2008), (PEER/ATC-72-1, 2010) to be close to 0.031, which agrees with the values indicated in Table 2.13. Also a slightly different pinching parameter $\left(F_{p r}\right)$ was used in the case of C-7 to represent the less pinched behavior of that column compared to the ones tested using the subduction protocol.

In the case of the distributed-plasticity model values of 0.006 and 0.002 were proposed to account for the deterioration due to ductility (damage1) and energy (damage2), respectively. The damage1 parameter reflects the higher cyclic deterioration from one cycle to the next caused by an increase on the number of small inelastic cycles. On the other hand, the damage 2 parameter reflects the increased stiffness and strength deterioration at a fixed cycle amplitude observed during the tests.

\subsection{SUMMARY}

The assessment of bridge columns through representative cyclic protocols could play a significant role in the future establishment of limit states and acceptance criteria to be applied in performance-based seismic design of bridge columns in areas subjected to subduction earthquake hazard. A range of protocols was developed with the aim of capturing more closely the inelastic demands of subduction megathrust earthquakes and, consequently, improving the seismic assessment of bridge columns through physical testing. 
The loading protocols were developed using the results from non-linear time history analyses considering numerous subduction ground motions imposed on structures with a wide range of structural periods and pre-determined ductilities (Dusicka \& Knoles, 2012) and further analyzed using a simplified rainflow counting procedure (ASTM E1049-85, 2005).

Throughout the analyses and development, a methodology similar to the one utilized for the development of a testing protocol for wood frame structures (Krawinkler, et al., 2000a) was utilized. Since strength and deformation capacities of structural components depend on the cumulative damage that the system undergoes under load reversals imposed by seismic excitations, a direct relationship between the number of inelastic cycles and damage is recognized (Krawinkler, et al., 1983), (Stephens \& Yao, 1987). Various models have been developed to quantify this relationship (Cosenza, et al., 1993), (Ghobarah, et al., 1999). Two well-known relationships describing the cumulative damage are the normalized cumulative ductility and the Coffin-Manson law for low-cycle fatigue in conjunction with the Miner's rule of linear damage accumulation. Both models show a direct relationship between structural damage and the number and amplitude of damaging cycles. Thus, the number of inelastic cycles and a cumulative damage demand were selected as the main parameters to develop the protocols. Observations based on these parameters showed that for structural periods below $2.0 \mathrm{~s}$, the subduction mega earthquakes produced significantly greater number of inelastic cycles as well as cumulative plastic displacements than crustal earthquakes. Statistical assessment of the demand parameters were used to develop quasi-static loading protocols. Due to the dependence of the results on structural ductility and natural period of vibration, different loading protocols were proposed for three column ductilities $(2,4$ and 8$)$ and for three different periods representing short, medium and long fundamental periods. 
The proposed loading protocols include a larger number of small amplitude inelastic cycles as compared to conventional protocols, revealing that conventional loading protocols commonly used in experimental testing tend to impose unrepresentative drift demands for subduction megathrust earthquakes through imposing numerous large inelastic reversals on the component. Despite the higher number of large inelastic cycles, the overall normalized cumulative plastic displacement demands were similar when compared to the proposed protocols.

A representative pre-1970 lightly reinforced and lap-spliced bridge column was numerically and experimentally studied to observe the effect of the proposed protocol on the behavior of seismically deficient reinforced concrete bridge columns. Observations based on numerical and experimental results of applying cyclic loading protocols on pre-1970 RC bridge columns can be summarized as follows:

The numerical case study showed the importance of modeling the rate of strength and stiffness deterioration in RC bridges. This is of paramount importance in regions susceptible to be struck by subduction zone mega earthquakes since the faster the rate of deterioration the more significant the expected effect of number of inelastic cycles on column behavior.

The experimental results of this study indicated that square columns present in bridges built before 1970 in the Pacific Northwest have unexpected deformation capacity. This moderately ductile performance was predominantly observed in columns with low axial load level $\left(0.07 \mathrm{f}_{\mathrm{c}} \mathrm{A}_{\mathrm{g}}\right)$ and low longitudinal steel reinforcement ratio $(\varrho=1.2 \%)$. 
The experimental study also demonstrated that subduction loading protocols may reduce the displacement ductility capacity of reinforced concrete columns and change their failure mode. The primary mode of failure for the columns tested under subduction loading protocol was by lap splice failure. On the contrary, column C-7, which was tested using a conventional cyclic loading protocol, failed showing crushing of concrete as a primary mode of failure.

Despite the fact that the conventional protocol contains a higher number of large inelastic excursion, results showed that the use of the subduction protocol can highly influence the response of deteriorating components. Therefore, the assessment of bridge columns through representative testing load protocols would play a key role in the future establishment of limit states and acceptance criteria to be applied in performance-based seismic design of bridge columns.

Both lumped and distributed-plasticity numerical models were able to reasonably reproduce the initial stiffness, and strength as well as the stiffness degradation of the specimens. This demonstrates the potential of these models to be used in the numerical assessment of reinforced concrete. Severe degradation parameters were needed to appropriately capture the damage on substandard columns. Since pre-1970 columns were built without seismic detailing, the behavior of these columns is expected to be represented by $\lambda$ values near 2 in the lumped-plasticity model. In the distributed-plasticity model, values of 0.006 and 0.002 for the damage1 and damage2, respectively, were suggested. These higher damage parameters should be incorporated in areas where subduction earthquakes are expected. 


\section{CHAPTER 3}

\section{STATE-OF-THE-ART ON RETROFIT MEASURES FOR RC MULTI-COLUMN BRIDGE BENTS}

\subsection{GENERAL}

Reinforced concrete bridge substructures constructed before 1970 are commonly deficient in flexural ductility and shear strength as those bridges were designed primarily for gravity loads with little or no design consideration to seismic resistance. RC bridge bents lack sufficient steel reinforcement to provide satisfactory performance in a major seismic event. Typically, No. 3 or No. 4 hoops at 12 inches on center were provided in columns regardless of the column cross-sectional dimensions. The stirrups were anchored by $90^{\circ}$ hooks with short extensions and intermediate ties were seldom used. RC bridges built prior to 1970 also have inadequate lap splices in potential plastic hinge zones since dowels were usually extended between 20 to 40 longitudinal bar diameters $\left(d_{b}\right)$ from the foundations. This detail may cause a considerable reduction on column ductility and a rapid loss of flexural and axial strength. Furthermore, the foundations constructed at that time were not design with a

capacity design in mind. As a result, foundations are highly susceptible to brittle flexural and shear failures since they do not contain neither top longitudinal reinforcement nor transverse reinforcement.

All these details contribute to bridge' deficiency and make these structures highly vulnerable to a major seismic event. This vulnerability of pre-1970 bridges was especially evident in the 1971 San Fernando, 1989 Loma Prieta earthquake, and the 1994 Northridge earthquake as 
shown in Figure 3.1, Figure 3.2 and Figure 3.3, respectively. Further, the recent occurrence of subduction zone mega earthquakes in Chile and Japan has demonstrated how vulnerable RC bridges are when subjected to major seismic events as shown in Figure 3.4.

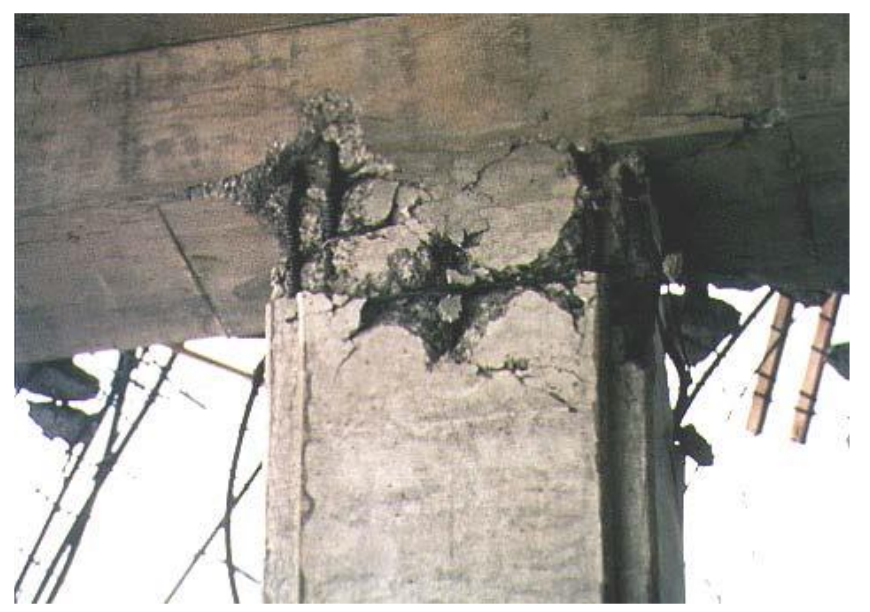

Figure 3.1 Poorly Confined Column in 1971 San Fernando Earthquake

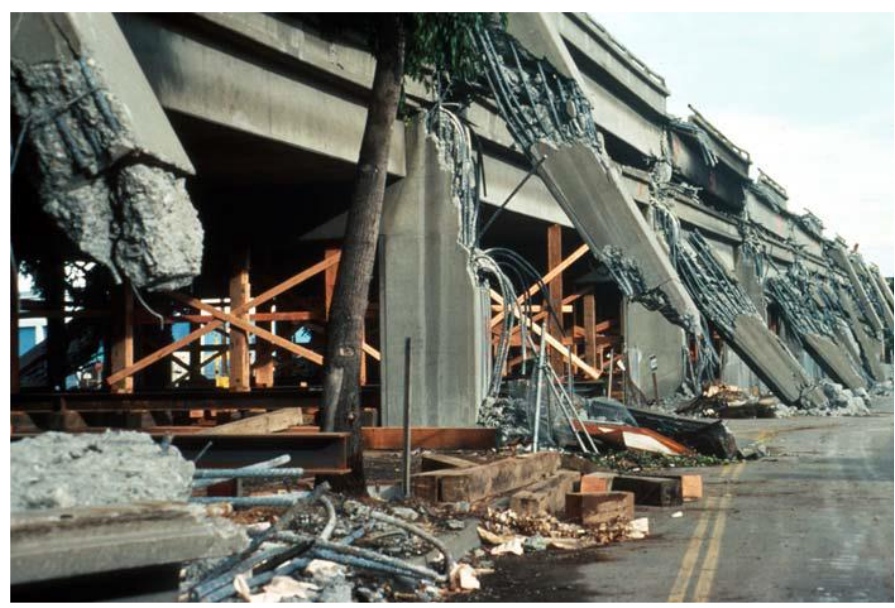

Figure 3.2 Collapsed Cypress street viaduct in Oakland during the 1989 Loma Prieta Earthquake (USGS \& Wilshire, 1989) 


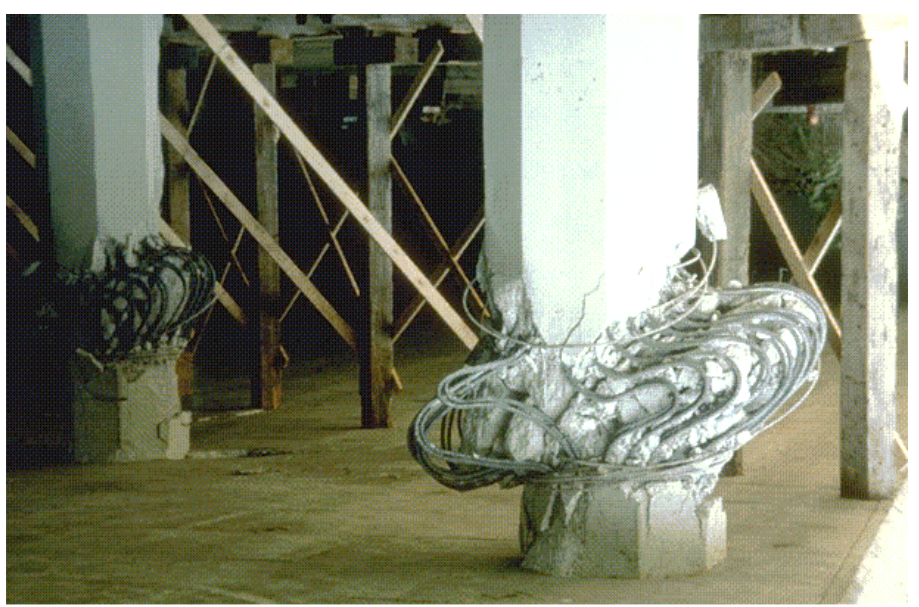

Figure 3.3 Damage in RC columns on the Simi Valley Freeway during the 1994 Northridge Earthquake (Teng, 1994)

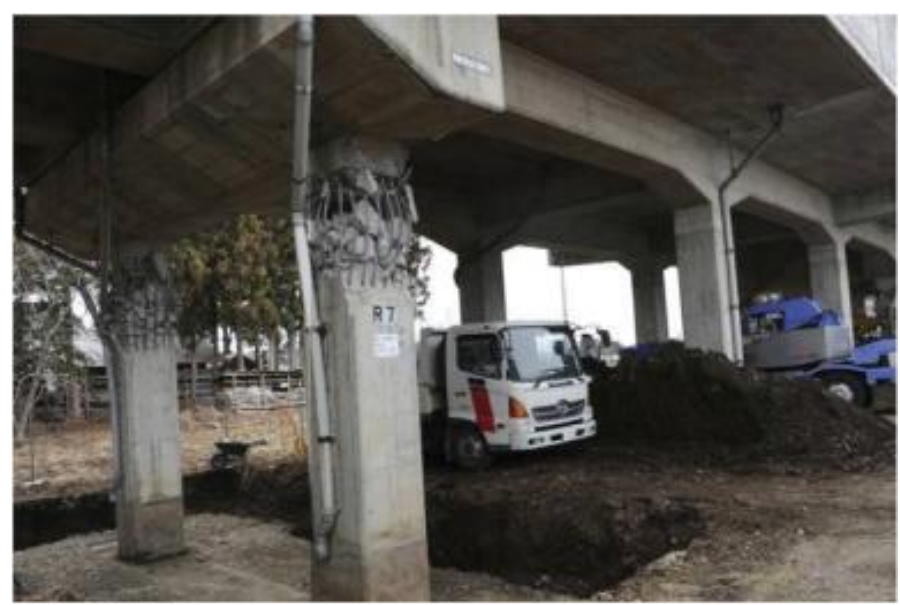

Figure 3.4 Damage in Nakasone viaduct during 2011 Japan earthquake (Kawashima, et al., 2011)

The ability of structures to achieve adequate deformation capacity plays a significant role in the prevention of structural failures in seismic events. The main goal of any seismic retrofit measure is to minimize structural collapse, while meeting certain performance requirements. Nowadays, the number of available retrofit measures has increased markedly as a result of extensive analytical and experimental studies. For example, the deformation capacity of existing bridges can be enhanced by modifying certain substructure elements. Bridge columns can be retrofitted using various techniques including reinforced concrete 
jacketing, steel jacketing, active confinement by prestressing wire, and composite fiber/epoxy jacketing, etc. Techniques to retrofit other bridge members have also been developed (FHWA, 2006), (Wright, et al., 2011) for bent caps and footings. In this chapter conventional and emerging retrofit measures capable of improving the seismic resistance of deficient RC bridges are presented and discussed.

\subsection{RETROFIT MEASURES FOR RC COLUMNS}

Previous research studies have shown that the main factors causing RC bridge column failures are their insufficient flexural and shear capacity added to a lack of concrete confinement and lap splice at expected plastic hinge regions at the base of the columns. (Chai, et al., 1991), (Dyngeland, 1998). In order to overcome these deficiencies many retrofitting systems have been developed and experimentally validated. Most of the retrofit measures for RC columns aim to improve the column ductility, shear strength and provide confinement by "Jacketing" the column through the use of various materials. These methods have now been implemented on a large number of deficient bridges throughout seismic regions and have helped preventing bridge failures in major seismic events. (Kawashima, et al., 2011), (Hoshikuma \& Guangfeng, 2013).

\subsubsection{Concrete Jacketing (Concrete Overlays)}

Concrete jacketing was the first method to be employed as a jacketing retrofit measure in practice since 1980. Its application was mainly for RC buildings in Japan as reported by Hayashi et al (1980) and Sugano (1981). The concrete jacketing retrofit measure consists of encasing the existing reinforced concrete column with a jacket of concrete reinforced with longitudinal steel and drilled and grouted dowels or welded wire fabric. Its 
application follows the same principles as any concrete design. Concrete jackets have been primarily used for retrofitting existing RC columns in buildings. Its use in bridges has been limited mainly because its labor intensive and more efficient retrofit measures has been implemented for those structures. An example of concrete jacketing applied to bridge columns is shown in Figure 3.5.

Multiple experimental investigations and field applications have shown that concrete jackets significantly improve the strength and stiffness of as-built columns (Hayashi, et al., 1980), (Teran \& Ruiz, 1992), (Rodriguez \& Park, 1994), (Bracci, et al., 1995). However, an increase in column flexural strength results in increased shear capacity, and consequently in an increased force transfer to cap beams and footings (FHWA, 2006). Therefore, special attention needs to be placed in those components since undesirable effects can be generated on the overall bridge performance.

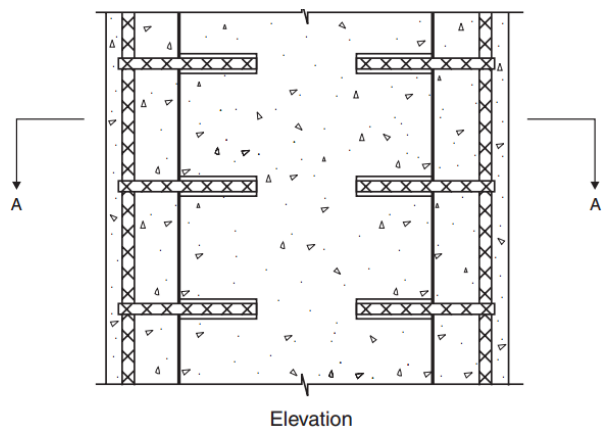

(a)

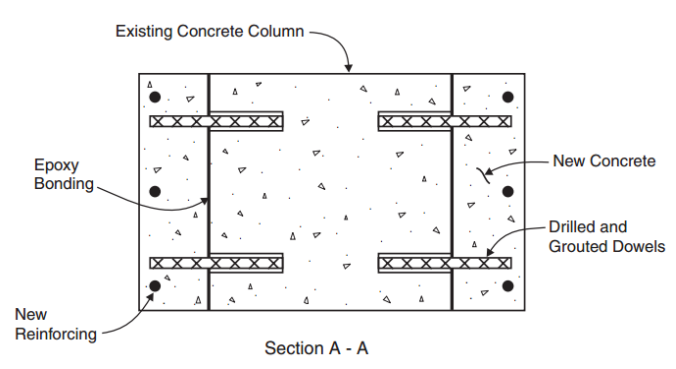

Section A - A

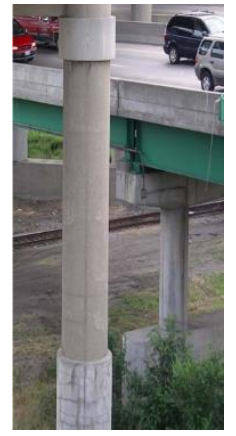

(b)

Figure 3.5 Concrete Jacketing. (a) Concrete overlay (FHWA, 2006), (b) Concrete jacketing of a column in Illinois (Poplar Street Complex)

\subsubsection{Steel Jacketing}

Steel jacketing was originally developed as a retrofit measure for circular columns in the early 90s and since then it has been largely utilized for the retrofitting of deficient columns in California (Caltrans, 1996) and lately throughout the United States. 
Previous research studies (Chai, et al., 1991) have shown that steel jacketing is an effective retrofit technique for seismically-deficient concrete columns. Based on satisfactory laboratory results, steel jackets have been employed to retrofit both circular and rectangular columns as shown in Figure 3.6. For circular columns, the recommended procedure is to cover the entire column or portion of it with two steel plate half-shells slightly oversized for easy installation leaving two vertical seams that are welded in a later stage. The gap between the column and the jacket is filled with grout to provide the required confinement action. An additional gap is required when the full height of the column is steel jacketed in order to minimize flexural strength enhancement, which may cause an undesirable increase in the forces developed in adjacent members. With rectangular columns, the recommended procedure is to use an elliptical shape jacket, which provides continuous confining action similar to that for a circular column. For both cases, the steel jacket acts as a passive confinement, i.e. the steel jacket only provides confinement once the concrete column starts expanding under compressive stresses that induces circumferential stresses in the steel shell. Detailed design guidelines for steel jacketing are found in the Seismic Retrofitting Manual for Highway Structures (FHWA, 2006) and in Chai et al. (1992). Steel jackets are effective in enhancing the confinement of the column, thus increasing the compression strength and strain. Additionally, these jackets can improve column ductility by eliminating the brittle shear and bond mode of failure attributed to a lack of shear reinforcement and lap splices at the base of columns, respectively. The failure mode is consequently shifted to a flexural mode. An undesirable effect of column jacketing is that its application may increase column stiffness. Studies have suggested that this increase could be approximately $10-15 \%$ for the case of partial height jackets (Chai, et al., 1991) and 20-40\% for the case of full height 
jackets (Priestley, et al., 1996). This increase in column stiffness may attract higher forces in the bridge and result in increased forces into bent caps and footings.

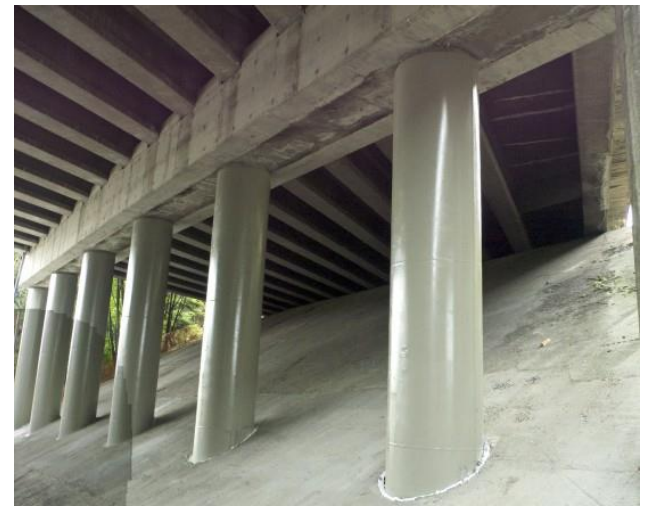

(a)

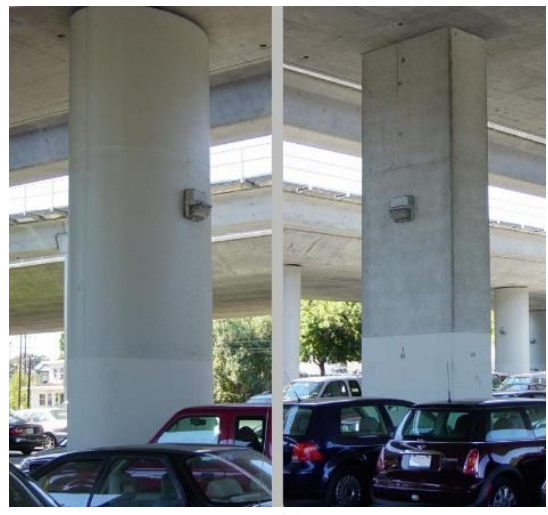

(b)

Figure 3.6 Steel Jacketing for: (a) circular bridge columns in Seattle (photo: Lucia Bill), (b) rectangular bridge column in San Francisco. Left: retrofitted column. Right: As-built column (photo, Leonard G.)

\subsubsection{Composite Jacketing}

Retrofit measures using composite materials has considerable grown since the past decade. The preferred composite material for retrofit application is Fiber Reinforced Polymers (FRPs), which consists of high strength glass, carbon, or aramid fibers bound in an epoxy resin matrix. Recent developments in the manufacturing of fiber reinforced polymer (FRP) composite materials have made these materials available for a wide range of applications, including seismic retrofit of reinforced concrete columns. Compared to steel and concrete jacketing, FRP wrapping has several advantages, including very low weight-tostrength ratios, high elastic moduli, resistance to corrosion, and ease of application. FRP can improve column ductility without considerable stiffness amplification while maintaining the bridge dynamic properties (Haroun \& Elsanadedy, 2005). As mentioned before, carbon fiber reinforced polymer (CFRP), glass fiber reinforced polymer (GFRP) and aramid fiber reinforced polymer (AFRP) are the most commonly used FRP composite materials. CFRP 
has a higher modulus of elasticity and has the highest tensile strength. The Washington State Department of Transportation recommends using CFRP to retrofit bridges because it is less affected by moisture than GFRP (WSDOT, 2006).

Several analytical and experimental studies involving retrofitting of columns using FRPs can be found in the literature. To name some, Seible, et al (1995) describes the jacket design aspects, jacket installation, full-scale field tests and behavior performance of carbon jacket retrofits. Xiao, et al (1999) reported on testing of three large-scale bridge column tests, in which two were retrofitted using a prefabricated composite wrapping system. The results of both studies showed that composite jacketing was a viable retrofit measure for shear enhancement and ductility improvement. Finally, Teng, et al. (2001) provides an excellent overview of the use of advanced composites in the seismic retrofit of concrete structures and its use in Civil Engineering.
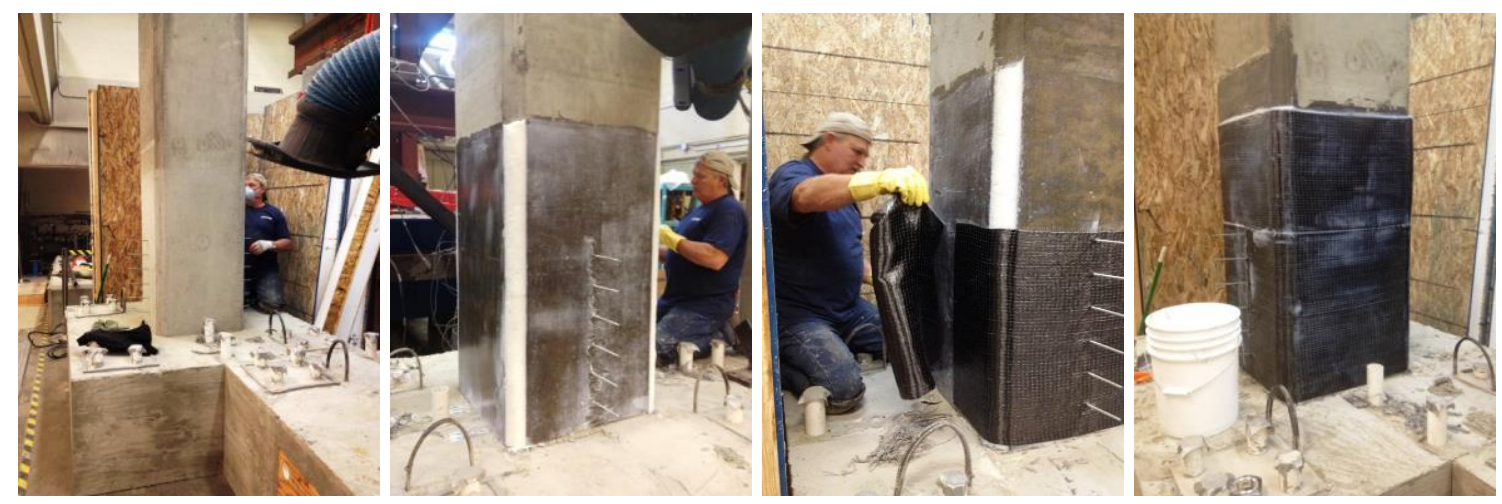

Figure 3.7 Installation of composite jacketing on a seismically deficient RC column 

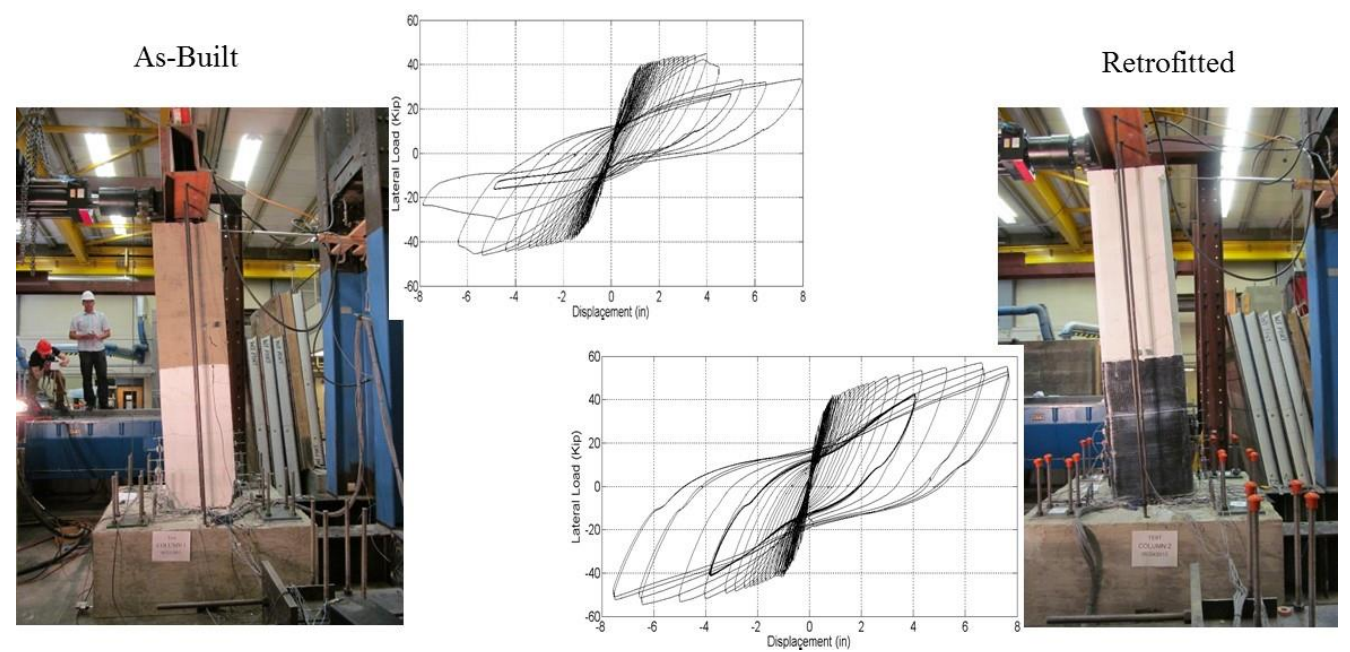

Figure 3.8 Results of an experimental study using CFRP wrapping on a seismically deficient RC column (Mehary, et al., 2014)

Retrofitting of bridge columns using FRP materials can be effectively used for circular columns since circular jackets provide a continuous confinement pressure. On the contrary, for rectangular columns the FRP jacket provides less confinement pressure since the confinement effectiveness is concentrated in the corners. However, recent studies at Portland State University (Mehary, et al., 2014) showed that substandard square reinforced concrete columns can be effectively retrofitted using CFRP. The objective of that study was to mitigate the damage effect that an increasing number of inelastic cycles imposed by subduction zone mega earthquakes has in RC bridge columns. The CFRP composite material called Tyfo®SCH-41 was used for retrofitting the square RC column. Three layers of this composite material was utilized to wrap the base of the column up to 36 inches high in an effort to improve the confinement of the column and prevent lap splice failure at the plastic hinge region as shown in Figure 3.7. The column corners were chamfered prior the installation of the FRP wrap in order to avoid stress concentrations and improve the effect of the FRP confinement. The results of the experimental study demonstrated that the CFRP retrofit measure considerably enhanced the displacement ductility of the column without 
significantly increasing column stiffness and strength as depicted in Figure 3.8. This result is relevant in the evaluation of other bridge components since an increase in column strength would result in an increase in force transfer to cap beams and footings. The retrofit also helped inhibiting bond failures in lap splices.

\subsubsection{External Prestressing Steel}

Different that the retrofitting systems presented before, which rely in passive confinement, the external prestressing steel acts by providing external confining forces into the column. In this retrofit measure, external prestressing steel wires under tension are wrapped around a column to achieve confinement as shown in Figure 3.9. This retrofit method has successfully increased the flexural ductility of circular columns with lap splices at the critical section, but its effect on shear strength has not yet been quantified (FHWA, 2006). An advantage of this practice is that it does not affect the flexural strength and stiffness of the columns. However, the high cost of designing a machine that is big enough to produce the required tension to wrap prestressing wire around the columns makes it uneconomical.

Saatcioglu \& Yalcin (2003) applied external prestressing to columns using individual hoops that consist of prestressing strands and specially designed anchors. They tested the columns under constant axial compression and incrementally increasing lateral deformation reversals. Their results showed that this retrofit methodology can mitigate shear failure, increase flexural capacity and inelastic column deformability.

Recent development in material sciences has increased the spectrum of materials that can be used for retrofitting RC columns. For example, shape memory alloys (SMA) have 
been recently studied to be applied as an active confinement retrofit technique for RC bridge columns (Andrawes, et al., 2010).

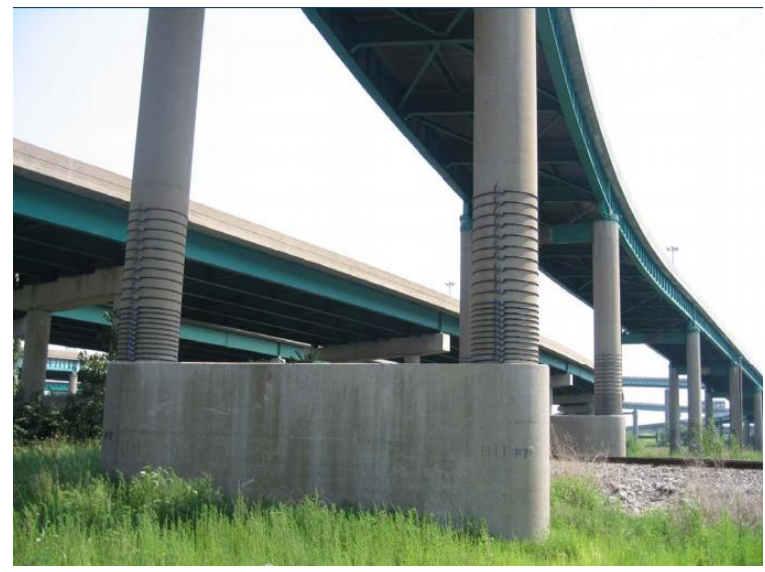

Figure 3.9 External prestressing steel retrofitting of columns in Illinois.

\subsection{RETROFIT MEASURES FOR RC CAP BEAMS}

Cap beams of multi-column RC bridge bents provide the load path from the superstructure to the columns. Cap beams are subjected to shear and flexure when the bridge is seismically excited in the transverse direction. Therefore, cap beams of bents constructed prior 1970 can also result considerably damaged after a major seismic event because of substandard seismic detailing such as premature termination of negative moment and low positive reinforcing steel ratios at the interface with the column face. These details can lead to early formation of plastic hinges in cap beams at the column face and rapid deterioration of beam-column joints. In order to overcome this deficiencies, similar retrofit techniques used in columns can be applied to cap beams. Concrete jacketing, steel jacketing, FRP wrapping and external prestressing retrofit techniques have been used to retrofit cap beams in seismically active regions as shown in Figure 3.10. 


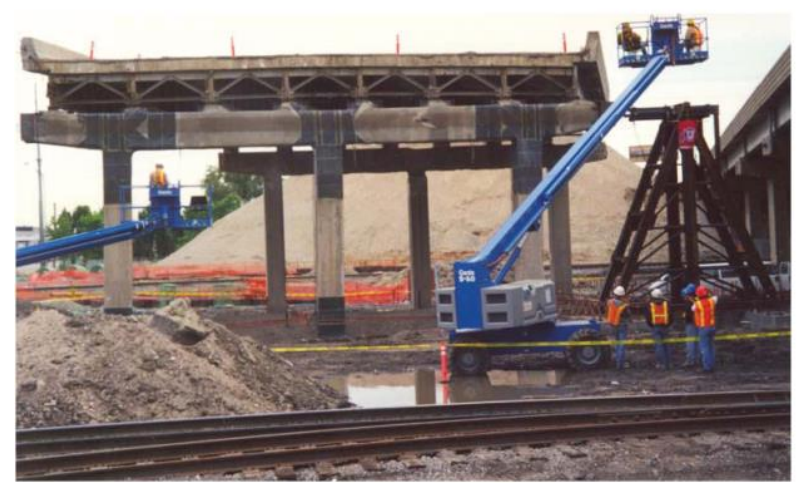

(a)

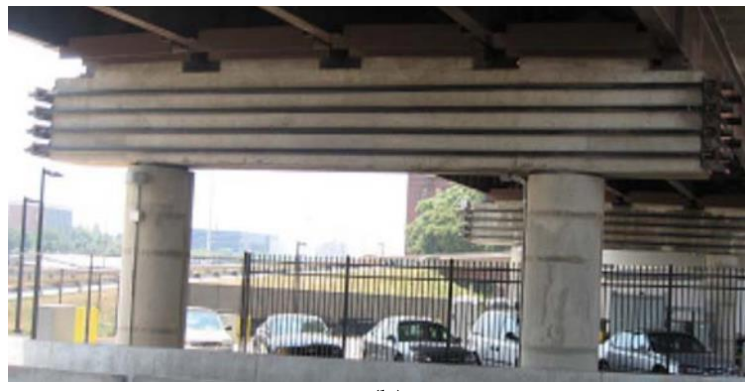

(b)

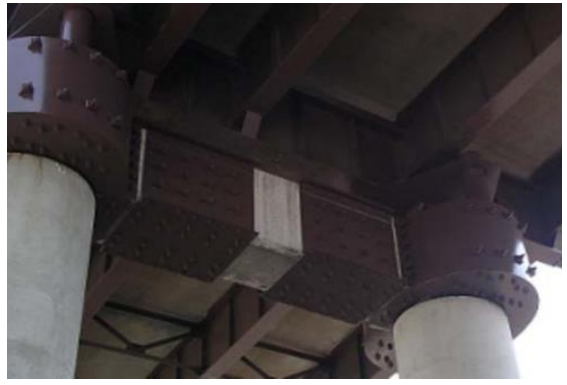

(c)

Figure 3.10 Retrofit measures for cap beams and beam-column joints. (a) FRP wrapping (Pantelides \& Gergely, 2008). (b) Prestressing rods. (c) Steel jackets (Wright, et al., 2011).

\subsection{RETROFIT MEASURES FOR RC BRIDGE BENTS}

Although the retrofit measures discussed in the previous section are also applicable for RC bridge bents, this section presents retrofit alternatives that aim to improve the overall behavior of RC bents more than just improving a specific component.

\subsubsection{Infill Walls}

Infill walls are walls constructed between the columns, cap beam, and footing of a multi-column bent. Adding infill shear walls or braced systems in RC structures has been commonly used as strengthening method in order to increase the shear capacity in the transverse direction, limit the transverse displacements and avoid the formation of plastic hinges in columns and cap beam. Figure 3.11 compares the effect of infill shear walls, also 
referred to as post-cast wall, with different methods used for retrofitting RC structures. As shown in the figure, steel braced frames and infill walls are the most efficient retrofit measures in terms of enhancing transverse shear capacity.

Infill walls have been commonly applied for retrofitting multi-column RC bridge bents due to their lower cost and familiar implementation in the field. The common implementation of infill walls is depicted in Figure 3.12 (FHWA, 2006). The critical aspect of using infill walls is to create an adequate force transfer between the wall, the existing columns and cap beam. In order to obtain an adequate interaction between the surfaces, some extent of the existing cover needs to be chipped off and dowels need to be drilled and grouted before pouring.

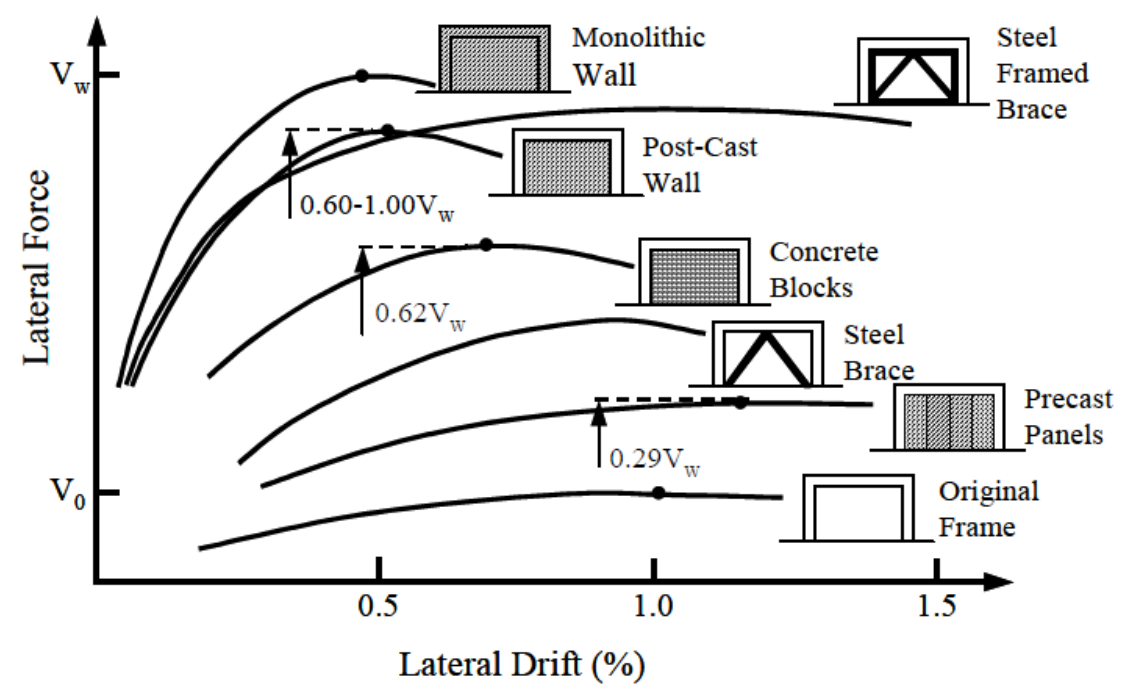

Figure 3.11 Effect of different retrofit measure for RC frames. (Sugano, 1981)

Pulido et al. (2004) performed shake table testing of a $1 / 4$ scale as-built bent representing typical bridge bents constructed before the 1970s and an identical specimen retrofitted with an infill wall. The results of this study showed that the use of infill walls can 
enhance the seismic performance of the as-built bent by increasing both the strength of the bent and its ductility.

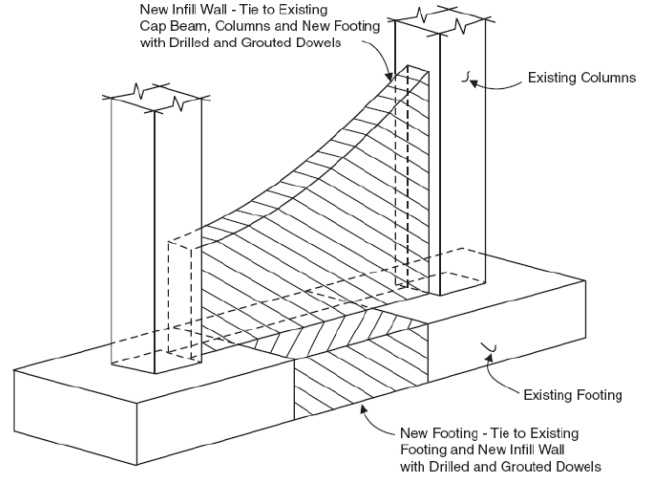

(a)

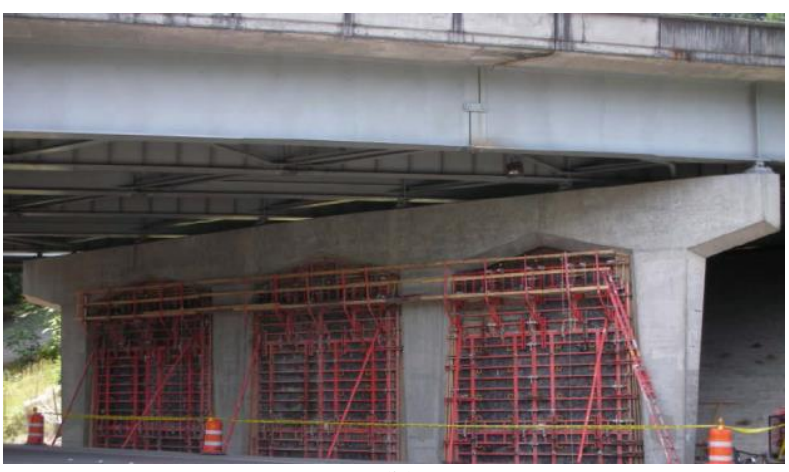

(b)

Figure 3.12 Infill wall. (a) Schematic, (b) Installation on West Lake Sammish Parkway (photo: L.M. Marsh)

\subsubsection{Link Beams}

Another measure used for retrofitting the overall bent behavior is the use of link beams between columns as shown in Figure 3.13. This retrofit technique is usually utilized when the induced forces by a seismic event significantly exceed the component capacity and is generally recommended for the retrofit of tall multi-column bents (Priestley, et al., 1996).

Link beams can be located high in the columns or above the footing. When they are located high in the columns they are an effective technique to reduce the internal forces in the cap beam. If the link beam is located at midheight, the stiffness of the bent will be doubled and then the displacements will be reduced by half. Finally, if the link beam is located just above the footings, then the forces transmitted to the footings will be reduced. Special attention needs to be placed in the design of the link beam to ensure that plastic hinges form in the column below or above the link beam. This is achieved following capacity design principles. 
The use of link beams as a retrofit technique has proved effective to prevent damage after earthquake events (Priestley, et al., 1994).

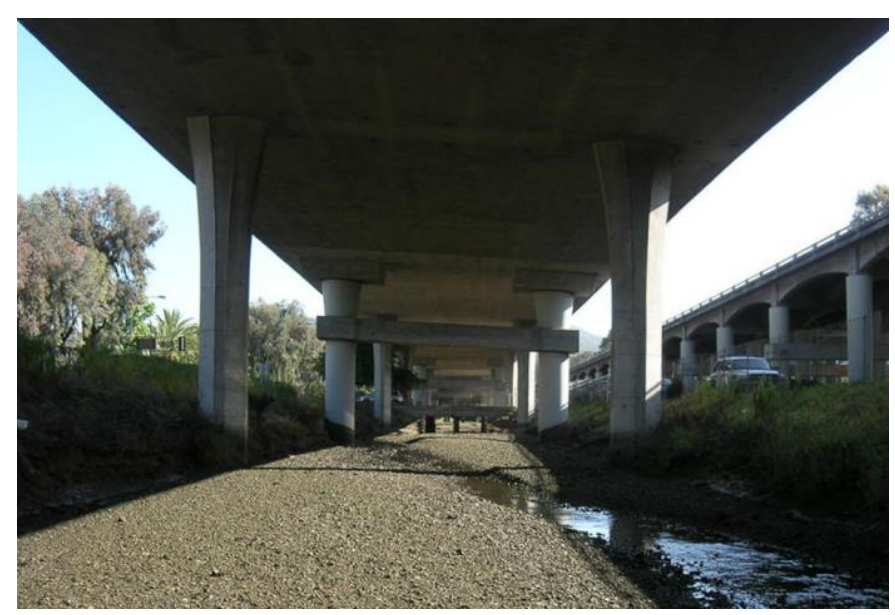

Figure 3.13 Application of link beams in San Rafael Viaduct, California.

\subsubsection{Isolation Devices}

In the case of bearing-supported superstructures, seismic isolation is an effective retrofit technique to reduce the demands that seismic events place on bridges. The isolation of drop-bent bridges is achieved by incorporating seismic isolation bearings or also called simply isolators between the superstructure and the substructure. Isolators are mechanical devices with low horizontal stiffness and are used in bridges for the following reasons: (1) to reduce spectral accelerations in stiff bridges (Figure 3.14a), (2) to reduce strength and displacement demands by increasing energy dissipation (Figure 3.14b), (3) to avoid damage and brittle failure in substandard components such as columns, bents and footings.

Nowadays, several types of isolators are available in the market and have been successfully implemented to protect structures under seismic events (Skinner, et al., 1993), (Constantinou, et al., 2011) (Marsh, et al., 2014). Several seismic isolators have been used in bridges. Some of them are: (1) Laminated bearings or low-damping isolators (LDRB), (2) 
Lead-rubber bearings (LRB) as shown in Figure 3.15, (3) Friction pendulum bearings (FPB),

(4) Flat sliding bearings (FSB) or Eradiquake (EQS), (5) Steel yielding devices (SY), (6)

Combinations of elastomeric bearings with viscous dampers, etc.

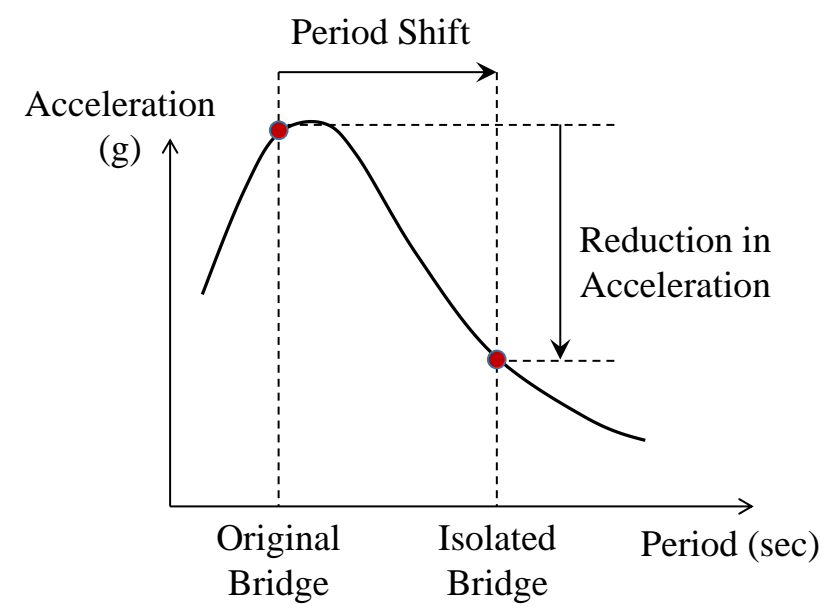

(a)

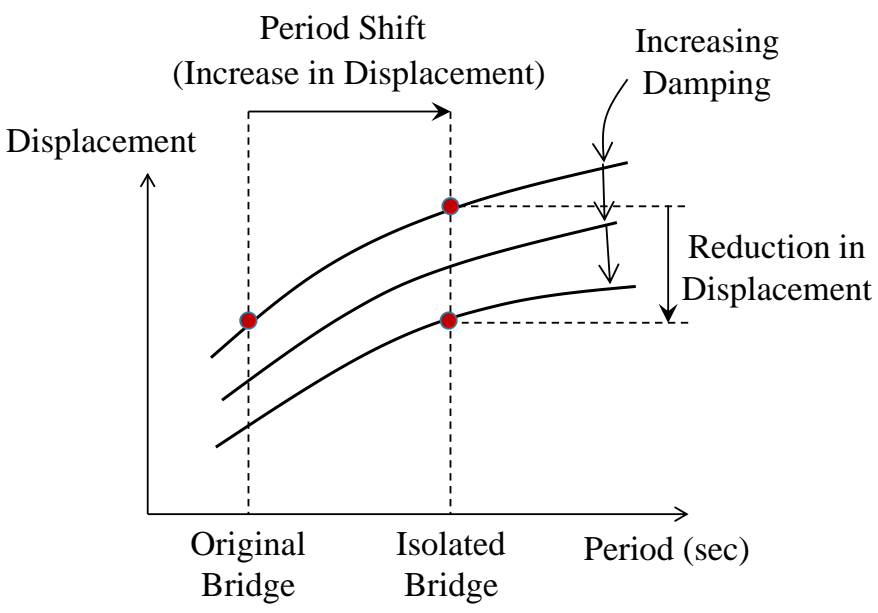

(b)

Figure 3.14 Effect of seismic isolation.

(a) Reduction in spectral acceleration, (b) Effect of increased damping 


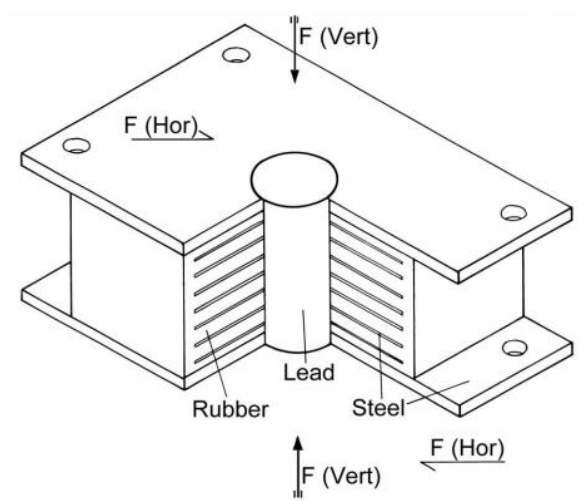

Figure 3.15 Lead rubber bearing (LRB) (Robinson, 1982)

\subsubsection{Energy Dissipation Devices}

Although energy dissipation devices are also used as seismic isolators, this section is focused on energy dissipation devices used on braces. In this case energy dissipation devices are primarily used to increase structural stiffness and reduce displacements.

Several analytical and experimental studies have been conducted on the use of steel bracing as a seismic retrofit technique for RC frame buildings (Badoux \& Jirsa, 1990), (Yamamoto \& Umemura, 1992). Current research into seismic retrofitting RC frames with bracing mostly involves adding supplemental damping devices in an effort to minimize the increase in strength that the retrofit measure would impart to the rest of the components of the structure (McDaniel, 2006). Among the damping devices that have been proposed for seismic retrofitting of structures can be found friction dampers, fluid viscous dampers, viscoelastic dampers, hysteretic dampers and shape memory alloy dampers.

Friction dampers dissipate energy by the friction exerted between the contact surfaces of two elements sliding relatively one another. Generally, friction devices present rectangular hysteretic behavior and are independent to loading velocity. Among friction dampers can be found: (1) Pall friction dampers (Figure 16a) (Pall \& Marsh, 1982), (2) 
Sumitomo damper (Aiken, et al., 1992), (3) Slotted-bolted connections (Grigorian, et al., 1993), (4) Friction damper device (FDD) (Figure 3.16b) (Mualla \& Belev, 2002).
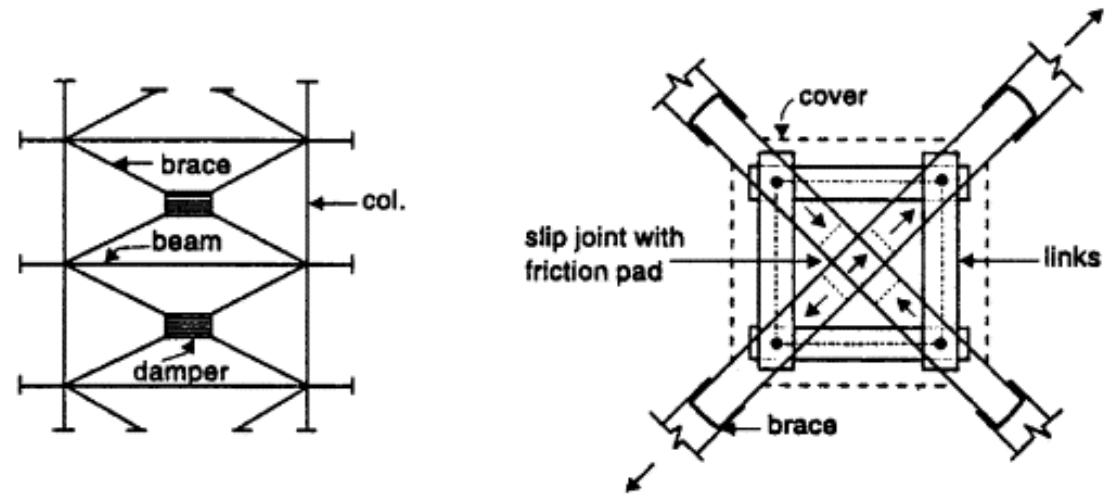

(a)
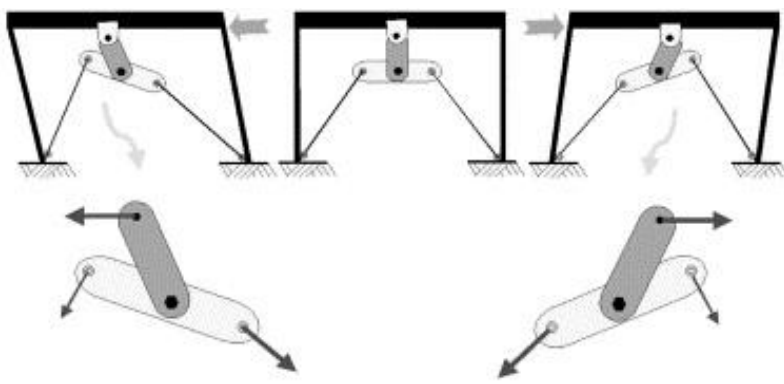

(b)

Figure 3.16 Friction dampers. (a) Pall damper (Pall \& Marsh, 1982), (b) FDD (Mualla \& Belev, 2002)

Fluid viscous dampers dissipate energy through a viscous fluid flowing through orifices inside a cylinder. The damping force exerted by the damper is proportional to the damping ratio, which depends on the pressure difference across the piston head, and the velocity of the piston (Constantinou \& Symans, 1993). Typical details of a fluid viscous damper is shown in Figure 3.17 


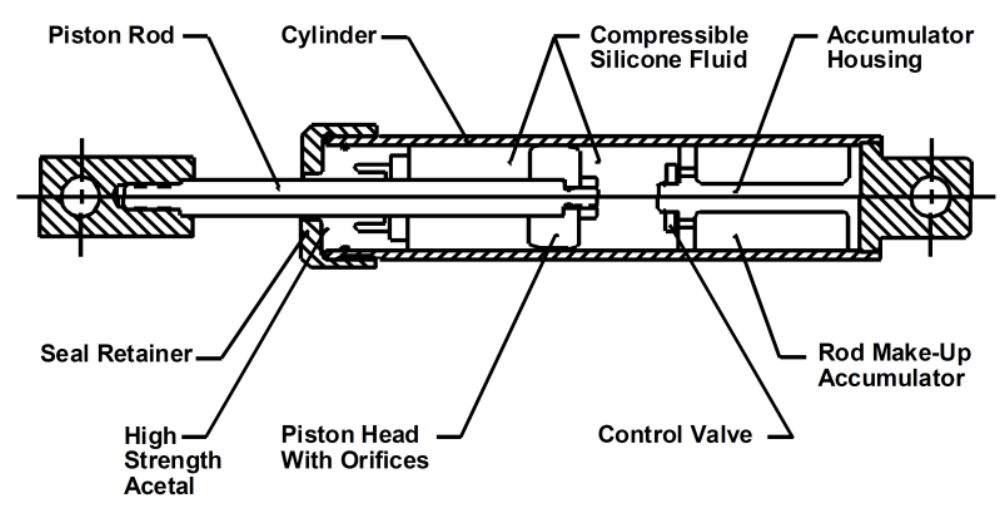

Figure 3.17 Fluid viscous damper. (Constantinou \& Symans, 1993)

Hysteretic dampers, also referred to as metallic dampers, dissipate energy through the inherent inelastic behavior of metals. Therefore, the behavior of the dampers is characterized by the hysteretic behavior of the metal. Many potential hysteretic dampers such as buckling-restrained braces (BRBs), eccentrically braced frames (EBFs), U-shaped dampers and energy dissipating braces (EDBs) can be used for retrofitting RC bridge multicolumn bents as shown in Figure 3.18.

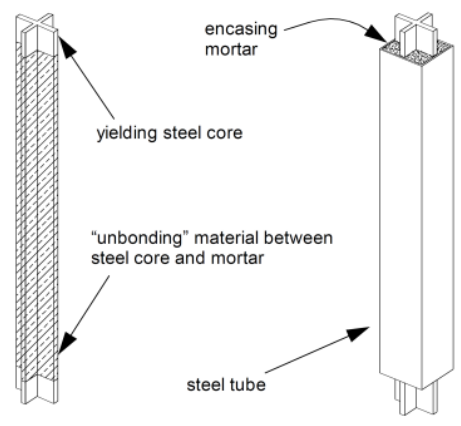

(a)

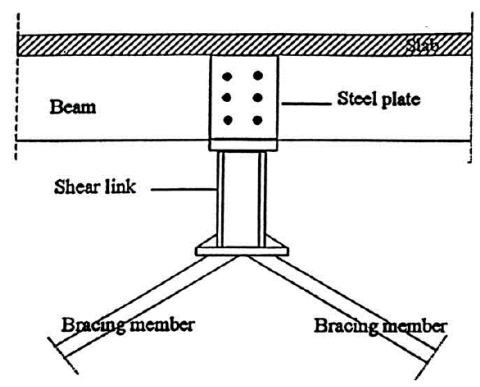

(b)

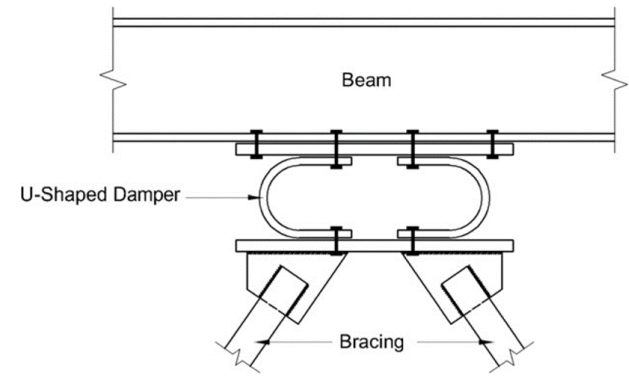

(c) 

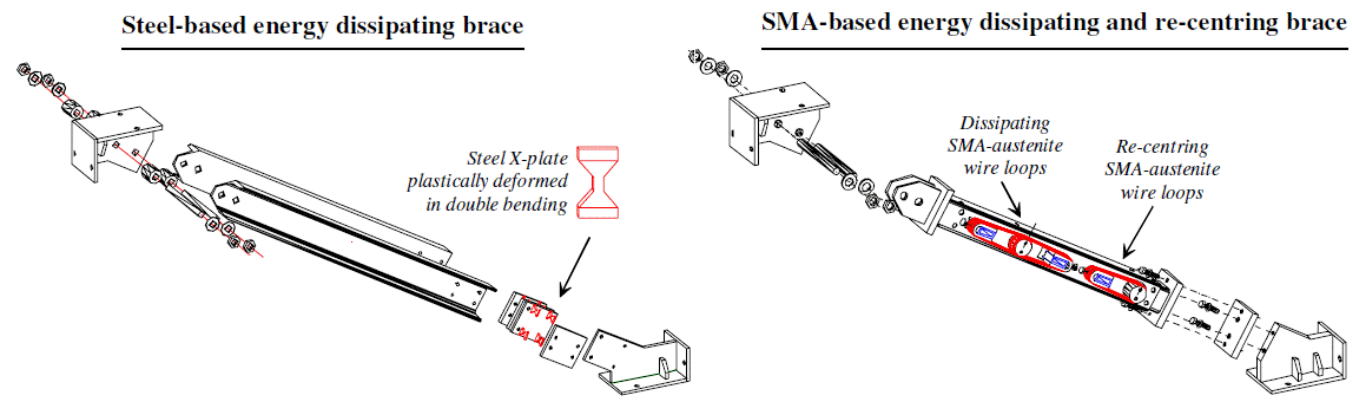

(d)

Figure 3.18 Hysteretic dampers (metallic devices): (a) BRBs (Clark, et al., 1999), (b) EBFs (Mazzolani, et al., 2009), (c) U-Shaped dampers (Bagheru, et al., 2015), (d) EDBs (Dolce, et al., 2005)

Among the hysteretic devices found in the market, buckling-restrained braces (BRBs) have gained notoriety in the field of seismic retrofitting structures for its stable hysteretic behavior and ease of implementation. Buckling-restrained braces, which are the subject of this study, were introduced in the US in the late 1990's. The main characteristic of a BRB is its ability to have a stable hysteretic response through yielding both in tension and compression due to the prevention of global buckling as shown in Figure 3.19 (Clark, et al., 1999). This inherent property of providing higher hysteretic energy dissipation makes BRBs a good candidate in seismic retrofit applications, where the main structure can be designed to respond elastically and the braces become disposable elements capable of absorbing and dissipating the earthquake-induced energy.

Despite the increasing use of this system for buildings, its use has not been materialized on bridge structures. Few BRB applications in bridges can be found, e.g. feasibility of using BRBs for the Vincent Thomas Bridge in Long Beach, California (Lanning, et al., 2011) and seismic retrofit of California's Auburn-Foresthill Road Bridge (Reno \& Pohll, 2010). 


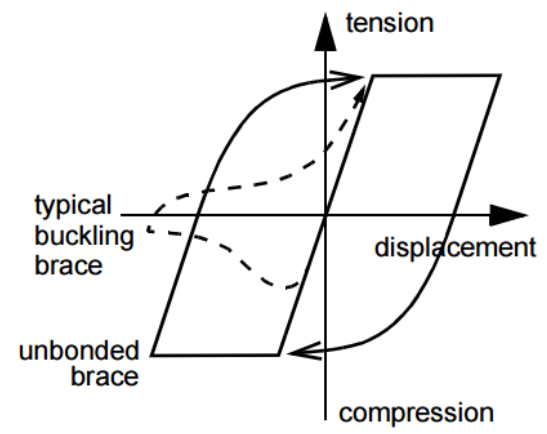

Axial force-displacement behavior

Figure 3.19 Hysteretic behavior of a BRB. (Clark, et al., 1999)

The concept of using BRBs as a seismic retrofit of RC multi-column bents was conceptually proposed by El-Bahey (2011). However, its implementation and experimental validation has not been carried out. The following sections of this study present further development and experimental evaluations of using Buckling-Restrained Braces as a hysteretic device for retrofitting seismically deficient RC bridge bents.

\subsection{SUMMARY}

Typical RC bridge bents built prior 1970 in the Pacific Northwest of the United States are highly vulnerable to seismic events since they were not designed with a seismic criteria in mind, as a consequence, numerous bridges have inadequate reinforcing steel detailing, inadequate transverse reinforcement in columns, cap beams and joints, insufficient confinement, and lap splices within expected plastic hinge zones. In order to overcome these deficiencies, retrofit measures for RC components aim to improve the component ductility, shear strength and provide confinement by "Jacketing" the component through the use of various materials such steel and fiber-reinforced polymers. The method of jacketing the RC component has now been implemented on a large number of deficient bridges throughout seismic regions and have helped preventing bridge failures during major seismic events 
(Kawashima, et al., 2011). Other techniques to retrofit RC bridge bents such as infill walls, link beams, seismic isolators and energy dissipation devices were also presented. In the following chapters buckling-restrained braces as seismic retrofitting technique is further investigated. 


\section{CHAPTER 4}

\section{SEISMIC RETROFIT AND REPAIR OF MULTI-COLUMN RC BRIDGE BENTS UTILIZING BUCKLING-RESTRAINED BRACES}

\subsection{GENERAL}

Current seismic retrofitting manuals and guidelines for existing highway structures in the United States are based on a performance-based seismic design (PBSD) methodology, which uses a multiple-level approach to performance criteria with two seismic hazard levels (FHWA, 2006). In this methodology, damage states can be presented in terms of physical damage and strain limits or curvature limits. Therefore, engineers designing retrofit measures for improving the seismic resistance of existing bridge substructures need to ensure that the structure remains operational under a moderate earthquake and that life safety is preserved after a large earthquake. Seismic retrofit measures including steel jacketing and fiber composite wrapping have been typically utilized to improve the ductility and shear resistance of reinforced concrete substructures as described in the previous chapter. However, these measures neither prevent damaging of columns nor excessive displacements, which in some cases could result in not meeting the intended performance level. In order to overcome this problem, this chapter discusses the option of using sacrificial elements also referred to as structural fuses as a retrofit and repair measure for reinforced concrete bridges and presents the implementation on representative seismically deficient, also referred to as substandard, $\mathrm{RC}$ bridge bents found in the state of Oregon. In this study, the term retrofit refers to 
enhancing or upgrading an undamaged existing bent. Meanwhile, the term repair is used to denote the rehabilitation of a damaged RC bent.

\subsection{Actual Seismic Design ANd Retrofit Philosophy}

Bridges in the United States and in other parts of the world are seismically designed based on the American Association of State Highway and Transportation Officials (AASHTO) seismic specifications. After the devastating earthquakes in San Francisco, CA in 1971 and Loma Prieta, CA in 1989, AASHTO adopted a forced-based design methodology, which was based largely on design criteria developed by the California Department of Transportation (Caltrans). Over the years, it became evident that this methodology did not provide a rational assessment of displacement demand/capacity needed for bridge structures. Consequently, AASTHO modified this methodology for a displacement-based criteria (AASHTO, 2009). This new methodology uses a design earthquake with 7 percent probability of exceedance in 75 years (Approx. 1000-year return period) and four seismic design categories (SDC). Under the AASHTO code requirements bridges are primarily designed to meet a standard performance level based on a life-safety approach, which means the bridge has a very low probability of collapse when subjected to earthquakes that are most likely to occur over the life of the structure.

Nowadays, the seismic design philosophy of bridges is trending to a performancebased seismic design, in which different performance levels need to be satisfied under representative seismic hazards (NCHRP, 2013). For seismic retrofit of bridges the Federal Highway Administration (FHWA) released a performance-based guideline in 2006 (FHWA, 2006), which uses a multi-level performance criteria. In this methodology, damage states can be presented in terms of physical damage and strain limits or curvature limits. Following 
FHWA steps, a few Departments of Transportation in the United States have adopted the performance-based seismic design criteria in their manuals and regulations (NCHRP, 2013). This is the case of the Oregon Department of Transportation (ODOT), which for both new and existing bridges has adopted a two-level performance criteria (ODOT, 2015). The twolevel performance criteria adopted by ODOT comprises a "Life Safety" criteria under a 1000 -year event (7\% probability of exceedance in 75 years) and an "Operational" criteria under a 500 -year event (14\% probability of exceedance in 75 years). The inclusion of this additional lower level performance criteria aims to ensure the serviceability of the bridge. Figure 1 illustrates a four level performance criteria adapted from buildings (Moehle \& Deierlein, 2004) to satisfy the dual ODOT requirement.

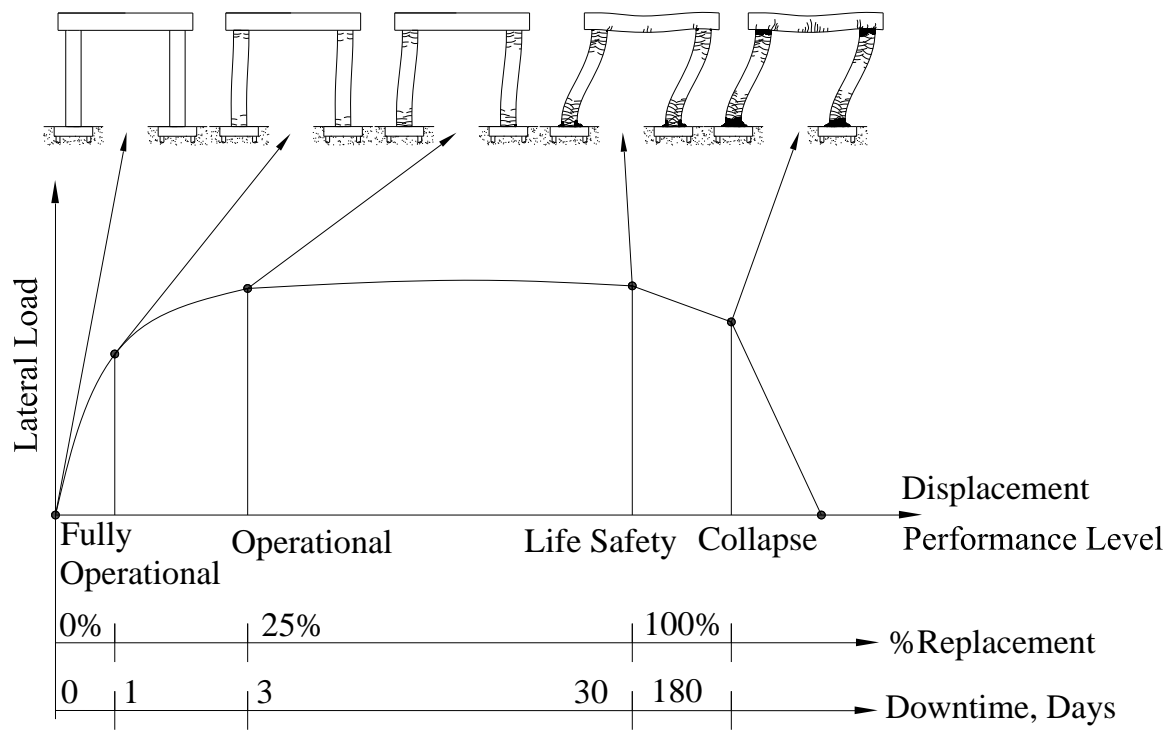

Figure 4.1 Visualization of PBSD (after Moehle and Deierlein 2004), adaptation to ODOT's requirements

\subsubsection{Ground Motion Hazard and Seismic Retrofit Categories}

According the FHWA retrofit design manual (2006), in order to identify evaluation methods and retrofitting measures, the performance-based seismic retrofit category of the 
bridge (SRC) need to be determined. These retrofit categories depend on the bridge's importance, the anticipated service life (ASL), which is one of the factors in deciding the extent of retrofitting, and the seismic hazard. For the representative MPCG Bridge and most of the deficient bridges in Oregon a standard importance category is recommended. The anticipated service life lies in the range 16 to 50 years, which represents an ASL 2 (FHWA, 2006). Next, the performance level for the bridge needs to be determined. The Oregon Bridge Design and Drafting Manual (BDDM) in its 2015 version recommends a dual performance level. The two-level performance criteria for existing bridges recommended by ODOT are "Life Safety" under a 1000-year return period earthquake (7\% probability of exceedance in 75 years) and "Operational" under a 500-year return period event (14 percent probability of exceedance in 75 years). The inclusion of this additional lower level performance criteria ("Operational") is above the standard performance requirements prescribed by AASHTO. Moreover, ODOT raised the lower earthquake ground motion level from a 100-year return period event (50 percent probability of exceedance within 75 years) found in the FHWA retrofit manual to a 500-year event as shown in Table 4.1.

Table 4.1 Minimum performance levels for expected retrofitted bridges in Oregon

\begin{tabular}{c|c}
\hline Ground Motion & $\begin{array}{c}\text { Minimum Performance Level for } \\
\text { Standard Bridges and ASL 2 }\end{array}$ \\
\hline $\begin{array}{c}\text { Lower Level Ground Motion } \\
\text { 100-year (FHWA) } \\
\text { 500-year (ODOT) }\end{array}$ & PL2: Operational \\
\hline $\begin{array}{c}\text { Upper Level Ground Motion } \\
\text { 1000-year (FHWA-ODOT) }\end{array}$ & PL1: Life Safety \\
\hline
\end{tabular}

Depending on the scaled response spectral accelerations for short-period $(0.2 \mathrm{sec})$, $\mathrm{S}_{\mathrm{DS}}$, and long period $(1.0 \mathrm{sec}), \mathrm{S}_{\mathrm{D} 1}$, the FHWA recommends four levels of seismic hazard 
level as indicated in Table 4.2. In the case that two different hazard levels are indicated for a location, the higher level needs to be used.

Table 4.2 Seismic hazard levels

\begin{tabular}{c|c|c}
\hline Hazard Level & Using $\mathrm{S}_{\mathrm{D} 1}$ & Using $\mathrm{S}_{\mathrm{DS}}$ \\
\hline $\mathrm{I}$ & $\mathrm{S}_{\mathrm{D} 1} \leq 0.15$ & $\mathrm{~S}_{\mathrm{DS}} \leq 0.15$ \\
\hline $\mathrm{II}$ & $0.15 \leq \mathrm{S}_{\mathrm{D} 1} \leq 0.25$ & $0.15 \leq \mathrm{S}_{\mathrm{DS}} \leq 0.35$ \\
\hline $\mathrm{III}$ & $0.25 \leq \mathrm{S}_{\mathrm{D} 1} \leq 0.40$ & $0.35 \leq \mathrm{S}_{\mathrm{DS}} \leq 0.60$ \\
\hline $\mathrm{IV}$ & $0.40<\mathrm{S}_{\mathrm{D} 1}$ & $0.60<\mathrm{S}_{\mathrm{DS}}$ \\
\hline
\end{tabular}

Based on the minimum performance level of a potential retrofitted bridge (Table 4.1) and the corresponding seismic hazard level (Table 4.2) the FHWA recommends that the structure should be categorized in one of the four performance-based seismic retrofit categories (SRC), which go from A through D, in increasing order of demand as shown in . Bridges in the seismic retrofit category A do not need to be evaluated or retrofitted. On the contrary, bridges in SRC D require the most rigorous assessment and retrofitting measures. Table 4.3 shows the seismic retrofit categories adapted from the FHWA (2006) to comply with ODOT performance levels for bridges built prior to 1970 in Oregon.

Table 4.3 Seismic retrofit categories, adapted for bridges in Oregon

\begin{tabular}{c|c|c}
\hline Hazard Level & $\begin{array}{c}\text { Operational } \\
\text { (500-year event) }\end{array}$ & $\begin{array}{c}\text { Life Safety } \\
\text { (1000-year event) }\end{array}$ \\
\hline I & B & A \\
\hline II & B & B \\
\hline III & C & B \\
\hline IV & D & C \\
\hline
\end{tabular}

Moreover, the FHWA recommends minimum requirements to evaluate bridges based on their seismic retrofit category and the level of earthquake (500-year or 1000-year 
event). Those evaluation methods are indicated in Table 4.4. Description of each method can be found in the FHWA retrofitting manual (2006).

Table 4.4 Evaluation methods for existing structures

\begin{tabular}{|c|c|c|}
\hline SRC & $\begin{array}{c}\text { Operational } \\
(500 \text {-year event) }\end{array}$ & $\begin{array}{c}\text { Life Safety } \\
\text { (1000-year event) }\end{array}$ \\
\hline A & Not required & Not required \\
\hline $\mathrm{B}$ & Minimum Capacity Checks & Minimum Capacity Checks \\
\hline $\mathrm{C}$ & $\begin{array}{l}\text { Component Capacity/Demand } \\
\text { method using elastic dynamic } \\
\text { analysis }\end{array}$ & $\begin{array}{l}\text { Component Capacity/Demand } \\
\text { method using elastic analysis } \\
\square \text { Nonlinear static procedure } \\
\text { (Pushover) }\end{array}$ \\
\hline $\mathrm{D}$ & $\begin{array}{l}\text { Component Capacity/Demand } \\
\text { method using elastic dynamic } \\
\text { analysis }\end{array}$ & $\begin{array}{l}\square \text { Component Capacity/Demand } \\
\text { method using elastic analysis } \\
\square \text { Nonlinear static procedure } \\
\text { (Pushover) } \\
\square \text { Nonlinear time history analysis }\end{array}$ \\
\hline
\end{tabular}

The seismic hazard level used in this study does not correspond to any specific location, instead the response spectrums used in the study aim to be representative of a vast number of bridges in Oregon. In order to achieve that, the $84^{\text {th }}$ percentile (mean +1 standard deviation) of the acceleration values obtained from the 2002 USGS ground motion maps for the bridges built prior 1970 were assumed as the representative response spectral accelerations. The design response spectrums were developed using the two-point method (AASHTO, 2009) and are depicted in Figure 4.2. The maximum considered earthquake (MCE), which is not used in bridge design since it is used in building codes to define an event having a $2 \%$ probability of exceedance within 50 years, is also included as a point of comparison. 


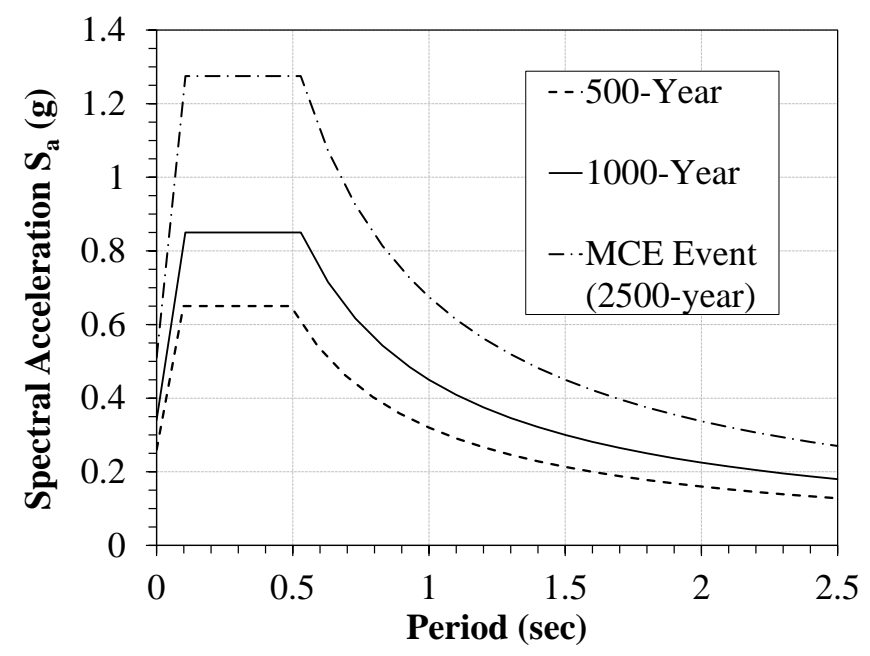

Figure 4.2 Representative response spectrums

The seismic hazard posed by the Cascadia subduction zone (CSZ) in the Pacific Northwest of the United States had been largely underestimated due to the believe that the Cascadia Subduction zone was a quiet fault. However, with the studies carried out by Atwater (2005) and Goldfinger (2003) we now know that a megathrust subduction earthquake is likely to strike the coasts of the Pacific Northwest. In order to overcome this problem and have representative seismic demands, deterministic seismic hazard maps to represent the 500-year event (Operational design level) were developed using the full CSZ rupture model $(M=9.0)$, logic tree and attenuation equations presented in the 2014 update of the United States National Seismic Hazard Maps (Petersen, et al., 2014). The maps generated for the state of Oregon are shown in Figure 4.3 for peak ground acceleration (PGA), 0.2 sec and $1.0 \mathrm{sec}$. The maps present acceleration values for a B/C NEHRP soil category (average shear-wave velocity for the upper $100 \mathrm{ft}$ or $30 \mathrm{~m}$ of the soil profile, $\mathrm{V}_{\mathrm{s} 30}=2500 \mathrm{ft} / \mathrm{s}$ or 760 m/s) (BSSC, 2003), (AASHTO, 2009). Comparison of the spectral accelerations shown in Figure 4.2 and Figure 4.3 lead to the conclusion that the spectral acceleration demands used 
in this study for the 500-year event corresponds to acceleration demands in the Western (coastal) area of Oregon.

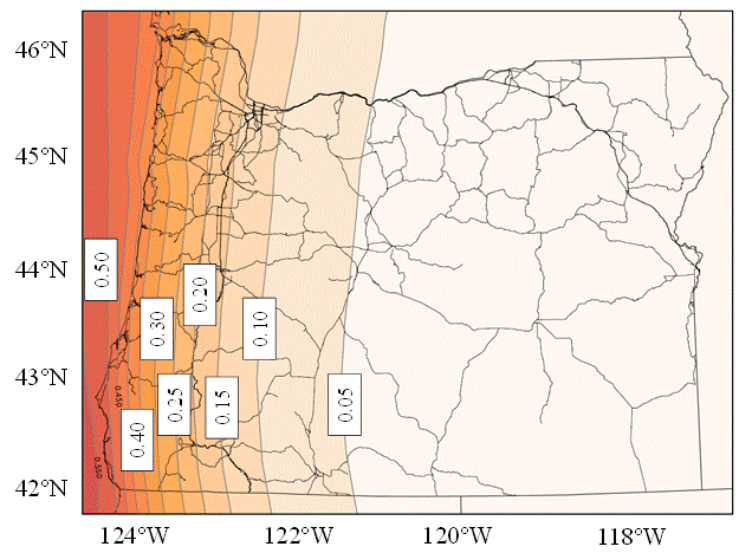

(a)

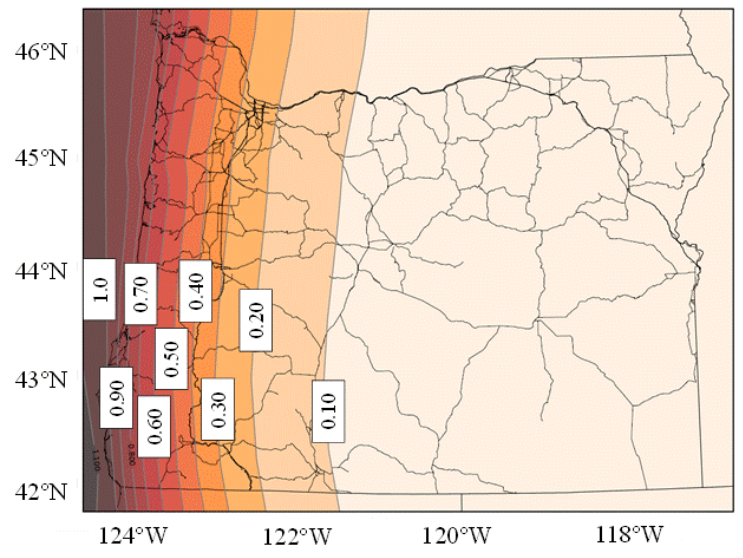

(b)

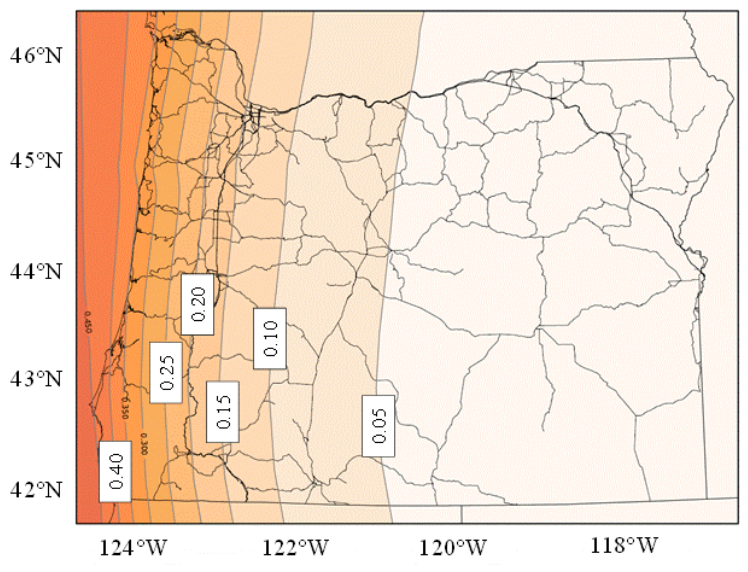

(c)

Figure 4.3 CSZ 2014 full rupture acceleration values for (a) PGA, (b) $0.2 \mathrm{sec}$, (c) $1.0 \mathrm{sec}$ Moreover, Figure 4.4 shows a comparison between the representative response spectrum used in this study for the 500-year event and deterministic response spectrums calculated for three locations in the State of Oregon and for different soil classifications. Average shear-wave velocities of $5000 \mathrm{ft} / \mathrm{s}(1500 \mathrm{~m} / \mathrm{s}), 2500 \mathrm{ft} / \mathrm{s}(760 \mathrm{~m} / \mathrm{s}), 1850 \mathrm{ft} / \mathrm{s}(560$ $\mathrm{m} / \mathrm{s}), 900 \mathrm{ft} / \mathrm{s}(270 \mathrm{~m} / \mathrm{s})$ and $600 \mathrm{ft} / \mathrm{s}(180 \mathrm{~m} / \mathrm{s})$ where assumed for soils $\mathrm{A}, \mathrm{B}, \mathrm{C}, \mathrm{D}$ and $\mathrm{E}$, respectively. From the figure is observed that the spectral accelerations for the 500 -year event used in this study are higher than the deterministic acceleration demands in populated 
areas like Portland (Figure 4.4b), and are similar to the demands in coastal cities like Astoria and Newport (Figure 4.4c and Figure 4.4d) for a B/C NEHRP soil class.

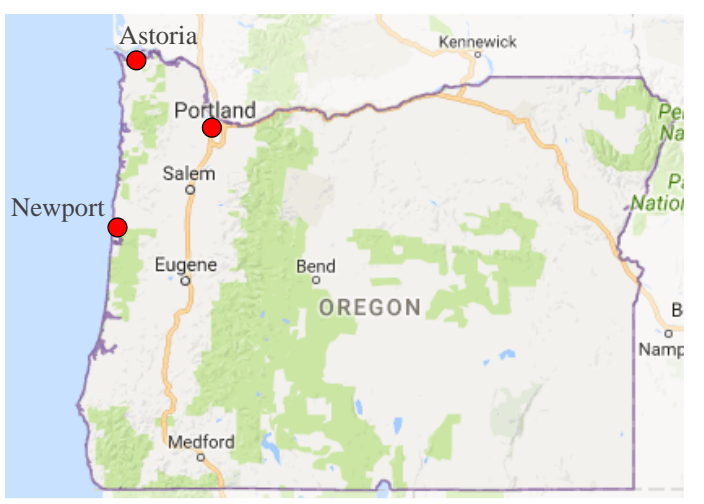

(a)

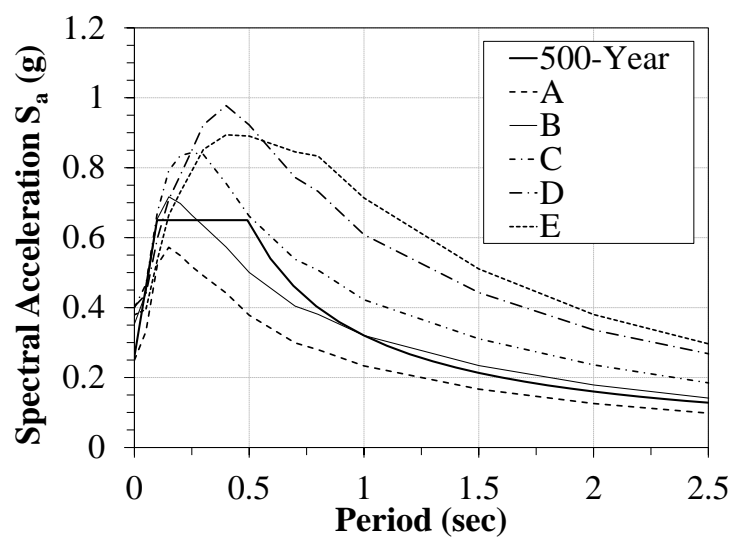

(c)

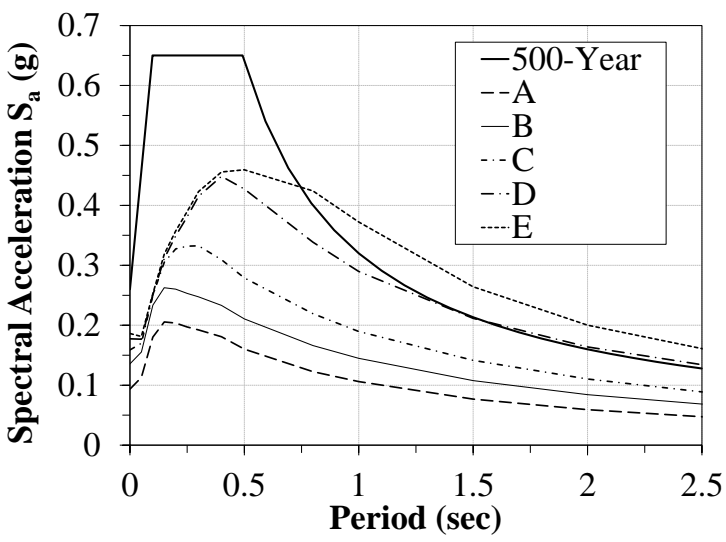

(b)

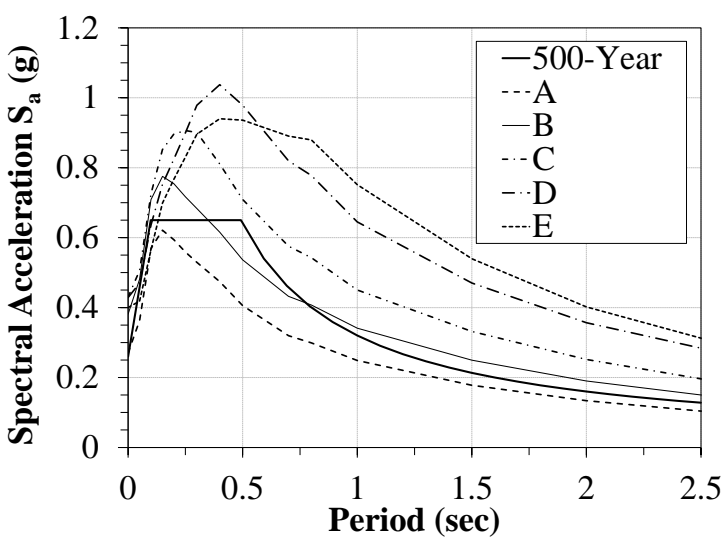

(d)

Figure 4.4 Response spectrum used in this study vs deterministic response spectrum for the 500 -year event. (a) Map of Oregon (Google) (b) Portland, (c) Astoria, (d) Newport.

Once the spectral accelerations are obtained the seismic hazard level and the seismic retrofit category (SRC) can be determined. Considering the response spectrum showed in Figure 4.2, standard bridges built prior 1970 can be categorized in the seismic hazard level IV. Consequently, the SRC for those bridges is as follows:

SRC D for the 500-year event (Operational performance level) SRC C for the 1000-year event (Life Safety performance level) 
The methods of evaluation for the two SRC vary from a minimum analysis of component capacity/demand method to more refined analysis such nonlinear static procedures, as indicated in Table 4.4.

\subsection{Structural Fuse Retrofit Strategy}

The recent occurrence of highly devastating earthquakes near instrumented regions, e.g. 2010 Maule, Chile and 2011 Tohoku, Japan, has again shown the vulnerability of RC bridges subjected to subduction zone mega earthquakes. Various research efforts on structural damage control concepts had taken place in order to develop more effective and reliable retrofit strategies for existing structures subjected to strong ground motions. One alternative that has attracted the focus of current investigations is the use of structural fuses. This structural fuse retrofit concept consists of integrating replaceable components in the main structural system in such a way to restrict the damage undergoing for the primary structure after a damaging earthquake (Connor, et al., 1997) In the case of hysteretic dampers (or metallic dampers), these are designed as replaceable elements in order to take the earthquake-induced energy and dissipate it through nonlinear hysteretic behavior, meanwhile, the remaining structure is expected to behave elastically.

The retrofit strategy adopted in this study is focused in the transverse direction and is based on employing two independent structural systems. The reinforced concrete multicolumn bent, which is the primary system and supports the vertical loading; and a replaceable system that adds lateral stiffness and functions as the seismic energy dissipation device. For the retrofitted bridge bent system, many potential metallic dampers such as buckling-restrained braces (BRBs), eccentrically braced frames (EBFs), U-shaped dampers and energy dissipating braces (EDBs) can be used as a fuse element as shown in Fig. 3.18. 
This structural concept has potential advantages over conventional retrofit systems.

Some of them are:

$\square$ Since the gravity resisting system is designed to remain elastic after a major earthquake event, higher performance levels can be achieved for the 500-year and 1000-year events.

Energy dissipation through stable nonlinear hysteretic behavior.

$\square$ The stiffness of the system is increased providing a drift-controlled system

Ideally, the sacrificial elements can be easily replaced, minimizing the repair time and allowing uninterrupted service in the bridge.

By forcing the inelastic demand to the fuses, the behavior of the system becomes more predictable.

This retrofitting technique is not exempt of disadvantages and challenges. For example, special assessment of element's capacities needs to be performed since the internal forces in the system may change due to the added strength and stiffness of the replaceable element, and consequently affect other bridge components such as footings. And, the connection design between steel and concrete components also needs special attention. Moreover, since the retrofit strategy mainly affects the response in the transverse direction, in the longitudinal direction, depending on the type of abutment, the bridge can engage the abutments that significantly influence the deformation demand. This is the case for bridges with integral and semi-integral abutments where the passive resistance of the soil against the backwall increases the seismic force transferred to the abutment backfill, and decreases the seismic demands on the bridge bents. 


\subsection{DESIGN AND IMPLEMENTATION OF STRUCTURAL FUSES}

\subsubsection{General Design and Implementation}

As in any design, in order to retrofit deficient RC multi-columns bridge bents by using structural fuses is necessary to iterate until all the limit states of each component are verified and a proper design is achieved. For this purpose a retrofit procedure needs to follow at least 4 main steps as shown in Figure 4.5 and described as follows:

Step 1: Assessment of the as-built RC bent. The capacity of the bare (as-built) bent and the demand obtained from two seismic hazards (500-year and 1000-year events) need to be assessed.

Step 2: Sacrificial element needs to be design following the structural fuse concept. Location, configuration, stiffness and dimensions of the fuse needs to be determined.

$\square$ Step 3: Connection needs to be designed to sustain the load transferred from the fuse element. The connection between sacrificial elements to gusset plate and gusset plate to concrete components need to be performed.

$\square$ Step 4: Column, cap beam and footing capacity need to be checked to ensure that the primary system remains elastic or with minor damage after the dual seismic demand (500-year and 1000-year events).

Step 5 (Optional): Repeat steps 2 to 4 if needed. 


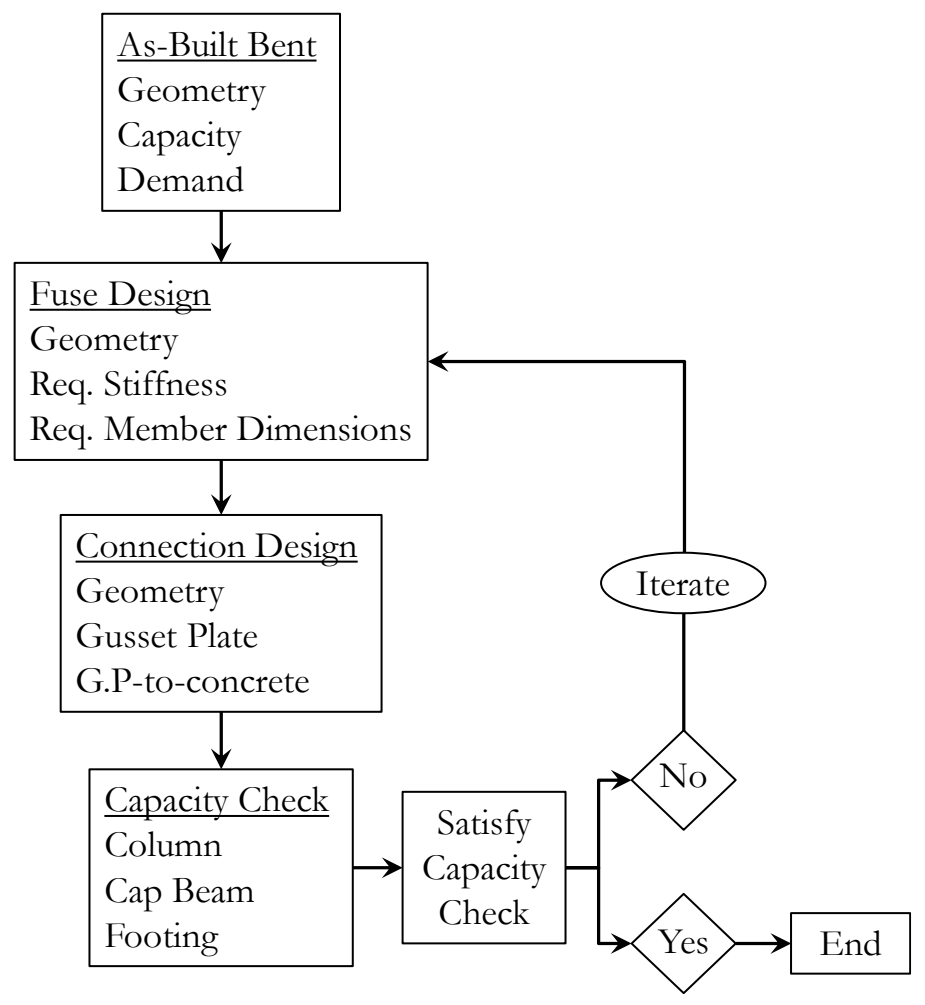

Figure 4.5 Structural fuse design implementation.

\subsubsection{Step1: Preliminary Assessment of the As-built RC bent}

The as-built RC multi-column bridge bent capacity and demand from two seismic hazards (500-year and 1000-year events) need to be assessed. A nonlinear static analysis, or also referred to as pushover analysis, is required to determine the capacity curve of the RC bent as shown in Figure 4.5.

The pushover analysis required the use of material properties from actual testing, such as reinforcing steel tensile tests or mill certificates and compression tests on concrete cylinders taken from representative areas of the bridge. In cases when testing of samples is not feasible expected material properties can be used. Expected material properties can be determined by applying factors to the specified properties. Factors of 1.3-1.5 and 1.1 are recommended in the literature for the compressive strength of concrete and the yield stress 
of steel, respectively (Priestley, et al., 1996), (AASHTO, 2009). It is worth mentioning that a general pushover analysis is suitable in this case since the first mode usually controls the behavior of RC bridge bents in the transverse direction.

The demand is obtained from subjecting the as-built bent to two seismic hazards, which are a 500-year event (14 percent probability of exceedance in 75 years) and a 1000year event ( 7 percent probability of exceedance in 75 years). A first estimation of the displacement demand in the RC bent can be calculated using Eq. (4.1), which is based on an elastic analysis of an equivalent single degree of freedom system. The use of this analysis requires the determination of the response spectrum for each seismic hazard and applying a displacement magnification factor $\left(\mathrm{R}_{\mathrm{d}}\right)$ for short-period structures as per AASHTO (2009).

$$
\begin{gathered}
\delta_{(500,1000)}=R_{d} \frac{S_{a,(500,1000)} T_{e}^{2} g}{4 \pi^{2}}=R_{d} S_{a} g \frac{m}{K_{S}} \\
R_{d}=\left\{\begin{array}{c}
\left(1-\frac{1}{\mu_{D}}\right) \frac{1.25 T_{s}}{T_{e}}+\frac{1}{\mu_{D}} \geq 1.0 \text { for } \frac{1.25 T_{s}}{T_{e}}>1.0 \\
1.0 \text { for } \frac{1.25 T_{s}}{T_{e}} \leq 1.0
\end{array}\right.
\end{gathered}
$$

Where, $T_{e}$ is the fundamental period of the SDOF system; $S_{a}$ is the spectral acceleration given by the respective response spectrum; $g$ is the standard gravity constant; $m$ is the inertial mass of the system, $K_{s}$ is the stiffness of the system, $T_{s}$ is the period at the end of constant design spectral acceleration plateau and $\mu_{\mathrm{D}}$ is the maximum local member displacement ductility demand. AASHTO states that $\mu_{\mathrm{D}}$ may be taken as 6 for multi-column bents. 


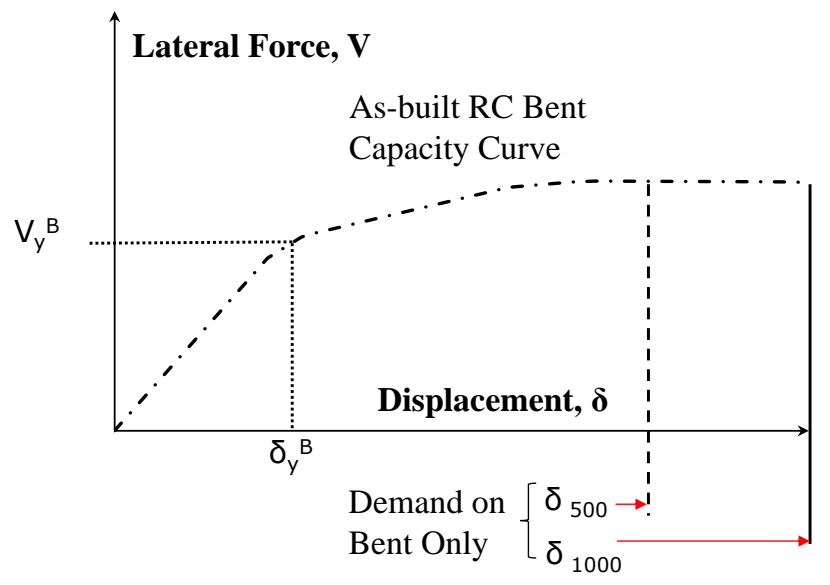

Figure 4.6 Assessment of as-built RC bent.

\subsubsection{Step 2: Structural Fuse Design}

The structural fuse concept design procedure presented in this study is similar to the one proposed by Vargas and Bruneau (2009) for buildings and El-Bahey and Bruneau (2011) for bridges but with some modifications. The design concept, as already stated, requires that the primary structural system remains essentially elastic after a major earthquake. In order to fulfill this design concept and satisfy the performance criteria for existing bridges described in the FHWA (2006) guideline, the fuse component needs to be designed in such way to reduce the displacement demands on the as-built bent, which ideally would respond elastically under the 500-year and 1000-year event. However, due to the increased forces in the system, in some cases the demands in the concrete elements exceed their capacity making the design and implementation of the fuse infeasible. For that reason, minor inelastic excursions are allowed to occur under the 1000-year event as shown in Figure 4.7. A maximum displacement of 140 percent the yield displacement $\left(1.4 \delta_{y}\right)$ is recommended, which is equivalent to a displacement ductility of $\mu=1.4$ for the as-built bent. Priestley et al. (1996) stated that the displacement ductility at which the operational performance level 
occurs depends on many factors such as section properties, dimensions and level of axial load, and suggested an average ductility $(\mu)$ of 2 to limit the serviceability or operational performance level. Kowalsky (2000) calculated displacement ductility values at different design limit states. The displacement ductility values for the serviceability limit states ranged from 1.1 to 3 . Therefore, the use of a displacement ductility of 1.4 is recommended in an effort to limit damage in the concrete components, still provide for a system ductile response and achieved an operational performance level.

For the response of the structural fuse is assumed a bi-linear response with hardening as depicted in Figure 4.7. Important parameters that define the response of the structural fuse, which is depicted as fuse contribution in Figure 4.7, are the yield displacement of the fuse $\left(\delta_{\mathrm{y}}^{\text {Fuse }}\right)$, the lateral force corresponding at the yield displacement $\left(\mathrm{V}_{\mathrm{y}}^{\text {Fuse }}\right)$, the initial stiffness $\left(\mathrm{k}_{\mathrm{i}}^{\text {Fuse }}\right)$ and any over-strength factor affecting the hardening of the fuse. The strength hardening effect is important since it increases the demands in the concrete elements.

The effect of retrofitting the RC bent by incorporating BRBs can be observed in the increment on lateral strength capacity and the reduction on displacement demand for both seismic hazards as shown in Figure 4.7. The increased strength is reflected in the curve referred to as "Retrofitted Bent", in which at the yield displacement of the fuse the strength is $V_{y 1}{ }^{R}$ and at the yield displacement of the as-built bent is $V_{y 2}{ }^{R}$.

The first step to design the structural fuses is to set the following parameters:

Material properties $\left(\mathrm{f}_{\mathrm{y}}, \mathrm{E}_{\mathrm{s}}\right)$ that are limited to commercial products

Configuration of the fuse system $\left(\mathrm{L}_{\mathrm{b}}, \theta\right)$ that is limited by the existing bridge dimensions. 
The following step is to set the displacement demand equal to or less than the yield displacement of the bent $\left(\delta_{\mathrm{y}}{ }^{\mathrm{B}}\right)$, which was previously obtained in the assessment of the asbuilt RC bent, for the 500-year event and equal to or less than 1.4 times the yield displacement of the as-built bent for the 1000-year event as shown in Eq. (4.2) and (4.3), respectively.

$$
\begin{aligned}
& \delta^{R}{ }_{(500)}=R_{d-500} S_{a-500} g \frac{m}{K_{S}} \leq \delta_{y}{ }^{B} \\
& \delta^{R_{(1000)}}=R_{d-1000} S_{a-1000} g \frac{m}{K_{S}} \leq 1.4 \delta_{y}{ }^{B}
\end{aligned}
$$

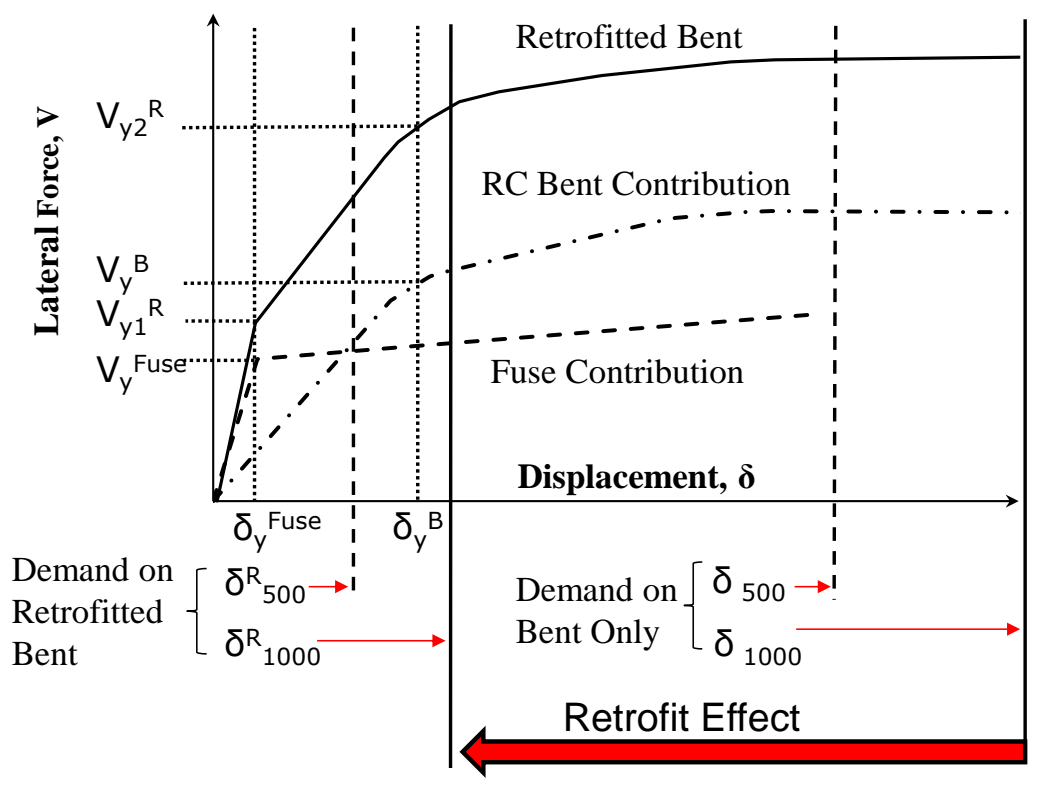

Figure 4.7 Idealized structural fuse retrofit design

The system composed by the $\mathrm{RC}$ bent and the structural fuse can be analyzed as a system in parallel. In such configuration, the displacement in global coordinates $(\delta)$ is the same for both the RC bent and the structural fuse. The strength (V) and stiffness $(\mathrm{k})$ of each element is added to obtain the system strength and stiffness as follows: 


$$
\begin{aligned}
& V^{R}=V^{B}+V^{\text {Fuse }} \\
& K_{s}=k_{\text {eff }}{ }^{B}+k_{i}^{\text {Fuse }}
\end{aligned}
$$

The effective initial stiffness of the as-built $\mathrm{RC}$ bent $\left(\mathrm{k}_{\mathrm{eff}}{ }^{\mathrm{B}}\right)$ can be directly determined from the pushover curve or by using an elastic analysis assuming crack sections as shown in Eq. (4.6).

$$
\begin{gathered}
k_{e f f}{ }^{B}=\frac{24 E_{c c} I_{c, \text { crack }}}{H^{3}} \cdot \frac{12 \rho+1}{12 \rho+4} \\
\rho=\frac{E_{c b} I_{b}}{L} \cdot \frac{H}{2 E_{c c} I_{c}}
\end{gathered}
$$

Where, $E_{c c}$ and $E_{c b}$ are the modulus of elasticity of concrete in the column and beam respectively; $I_{c, \text { crack }}$ is the cracked inertia of the column; $I_{b}$ and $I_{c}$ are the inertia of the beam and column respectively; $\mathrm{H}$ is the height of the bent; $\mathrm{L}$ is the span of the bent and $\varrho$ is a modification factor that take into account that the cap beam is not infinitely rigid.

Since the demand depends on the fundamental period of the system, the structural fuse design, as discussed in this paper, is reduced to iterate until the stiffness of the fuse system $\left(k_{i}^{\text {Fuse }}\right)$ is determined as shown in Eq.

$$
k_{i}^{\text {Fuse }} \geq \max \left(\frac{R_{d-500} S_{a-500} m g}{\delta_{y}{ }^{B}}, \frac{R_{d-1000} S_{a-1000} m g}{1.4 \delta_{y}{ }^{B}}\right)-k_{e f f}{ }^{\text {в }}
$$

In summary, the aim of the structural fuse design is to reduce the displacement demands of the as-built bent for the 500-year event $\left(\delta_{500}\right)$ and 1000 -year event $\left(\delta_{1000}\right)$ to displacement demands below the yield displacement of the as-built bent $\left(\delta_{\mathrm{y}}{ }^{\mathrm{B}}\right)$ for the case of the retrofitted bent under the 500-year event $\left(\delta^{\mathrm{R}}{ }_{500}\right)$ and 1.4 times the yield displacement of the as-built frame for the case of the retrofitted bent under the 1000 -year event $\left(\delta^{\mathrm{R}}{ }_{1000}\right)$. 


\subsubsection{Step 3: Connection Design}

Any steel brace-to-gusset plate connection shall be designed to resist the demands based on adjusted strengths in an effort to satisfy capacity design principles as per AISC (2010) and shown in Eq. (4.8).

$$
P_{a d}=\Omega R_{y} P_{y}
$$

Where, $\mathrm{P}_{\text {ad }}$ is the adjusted strength; $\Omega$ is an over-strength factor; $\mathrm{R}_{\mathrm{y}}$ is the ratio of the expected yield stress to the specified minimum yield stress (usually taken as 1.1), and $\mathrm{P}_{\mathrm{y}}$ is the yield strength of the connecting member. Over-strength factors shall be obtained from qualification tests or provided by the manufacturer.

The limit states that need to be checked for the design of the gusset plate according to AASHTO (2009) and AISC steel manual (2011) are: tensile yielding of Whitmore section, tensile rupture of Whitmore section, block shear in tension, buckling in compression, yield moment strength, plastic moment strength, weld/bolts for gusset to brace connection, and buckling of unsupported edge. For bolted connections is recommended the use of slip critical connections.

Anchor rods are recommended for connecting the gusset plate to concrete. The design forces are obtained from the adjusted strength and the corresponding gusset plate free body diagram. According ACI318 Appendix D (2011), tensile and shear strength must be considered in the connection design. For tensile strength, five possible failure modes can appear in this load direction, namely, steel failure of anchor in tension, concrete breakout, pullout, concrete side-face blowout, and concrete splitting. For shear strength, three failure modes are considered, namely, steel failure in shear, concrete breakout and concrete pryout. The failure modes in tension and shear mainly depend on steel grades, bolt spacing, concrete 
cover and bolt embedment. Additionally, for anchors that are subjected to both shear and tension a shear-tension interaction shall be satisfied.

\subsubsection{Step 4: Capacity Check}

Due to the incorporation of structural fuses, the internal forces in the RC bent components (columns, cap beam and footings) may increase considerably. This is particularly important when an oversized metallic damper is used. For that reason, a capacity check of the RC elements is crucial for the success of the structural fuse design concept. The capacity of concrete elements can be computed using the requirements of AASHTO (2009) and needs to be compared to the internal forces generated using adjusted (or factored) loads. If strength requirements of any reinforced concrete element are not satisfied, a new structural fuse design iteration is required as shown in Figure 4.5. Moreover, at the ultimate damage state the formation of plastic hinges has to be verified in order to avoid any undesirable mechanism of collapse.

\subsection{BRBs as Structural Fuse For Multi-Column RC Bridge Bents}

BRBs were introduced over two decades ago, and many experimental tests and postearthquake reconnaissance in multi-story frame buildings have shown that these structures may be efficiently retrofitted using BRBs (Tremblay, et al., 2006), (Cameron, et al., 2004). However, their potential implementation in bridges has been limited to research in steel girder diaphragms and steel bridges (Carden, et al., 2006). Recently, El-Bahey \& Bruneau (2011) proposed the used of BRBs as a structural fuse in the seismic retrofitting of RC bents. El-Bahey's studies were based on the previous work carried out by Vargas \& Bruneau (2006) for buildings. The main characteristic of a BRB is a stable hysteretic behavior through 
yielding in both tension and compression by restraining the core from global buckling (Clark, et al., 1999). This inherent property of stable hysteretic response, which may be translated to higher hysteretic energy dissipation, makes BRBs a good candidate in seismic retrofit applications. As with any metallic dissipation device, BRB behavior depends on its geometry and mechanical characteristics, and its effectiveness depends on its energy dissipation capacity. BRBs are typically composed of a steel core and an encasing system that is unbounded from the steel core as illustrated in Figure 4.8, which also depicts typical characteristic segments. The main function of the steel core is to transfer the axial force, while the encasing system prevents buckling of the core under compression. Additionally, a layer of unbounded material is used in the interface between the steel core and the encasing system to eliminate the shear transfer during elongation and contraction of the steel core. BRBs are a versatile system allowing different configurations and material composition (Miller, et al., 2012), (Dusicka \& Tinker, 2013).

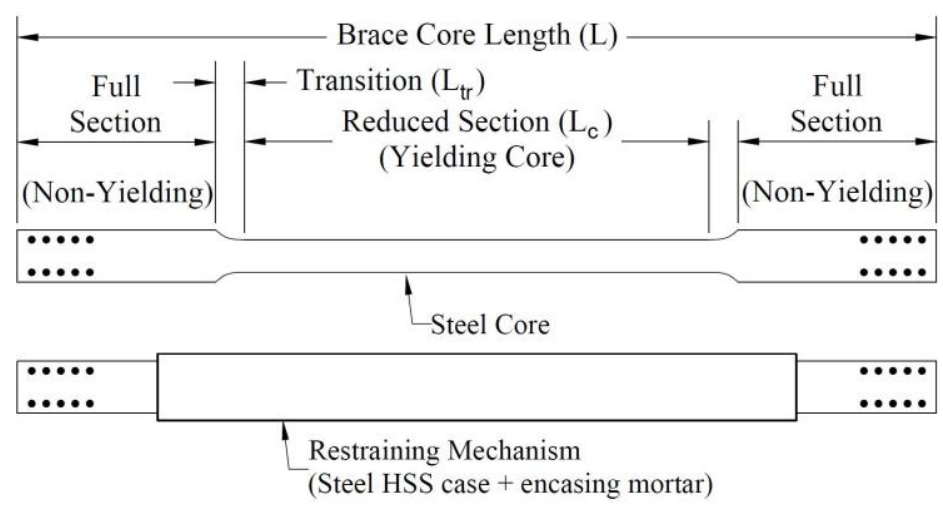

Figure 4.8 Configuration of a typical buckling-restrained brace.

Despite the increasing use of this system for buildings, its use has not been materialized in bridge structures. Few BRB applications in bridges can be found, e.g. feasibility of using BRBs for the Vincent Thomas Bridge in Long Beach, California (Lanning, et al., 2011) and seismic retrofit of California's Auburn-Foresthill Road Bridge 
(Reno \& Pohll, 2010). However, none has been implemented for seismic retrofitting of common reinforced concrete multi-column RC bridge bents. The objective of this research is to advance the seismic retrofit of reinforced concrete bridges subjected to moderate and large earthquakes by using buckling-restrained braces as a retrofit measure. For the retrofitted bridge bent system, the BRB is designated as the "fuse or replaceable" element and the $\mathrm{RC}$ bent is designated as the primary system as shown in Figure 4.9. The proposed configuration consists of a single diagonal brace (Figure 4.9(b)) but its use is not limited to that configuration as shown in Figure 4.9(c). This approach has the potential to improve the overall seismic behavior and the expected performance levels especially under performance driven design criteria.

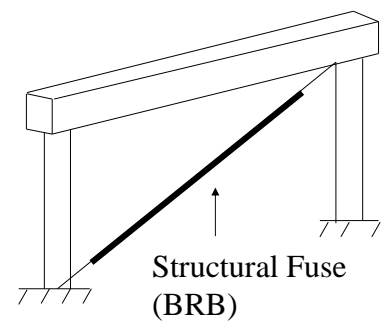

(a)

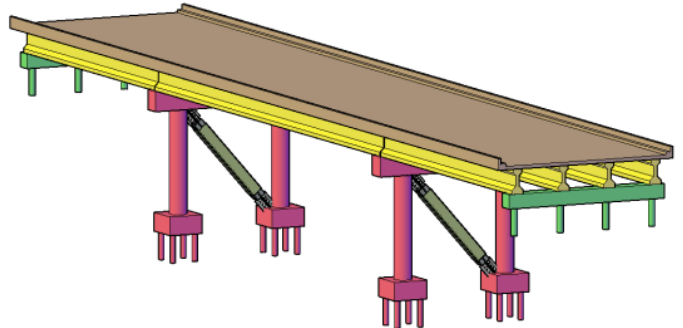

(b)

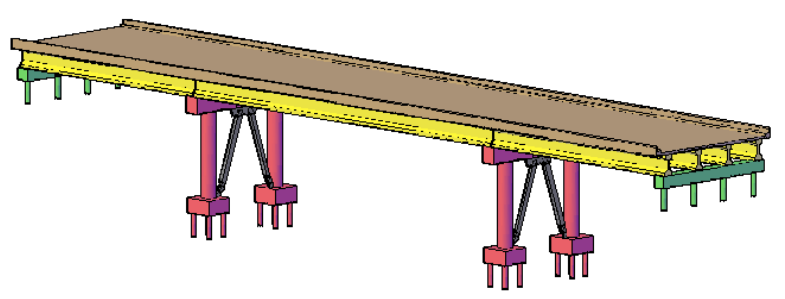

(c)

Figure 4.9 BRB retrofit configuration. (a) Structural fuse schematic. (b) Diagonal configuration. (c) Chevron configuration

\subsubsection{Implementation of the Structural Fuse Concept Using BRBs}

The inclusion of a BRB aims to achieve an operational performance level for both seismic hazard events as depicted in Figure 4.10. 


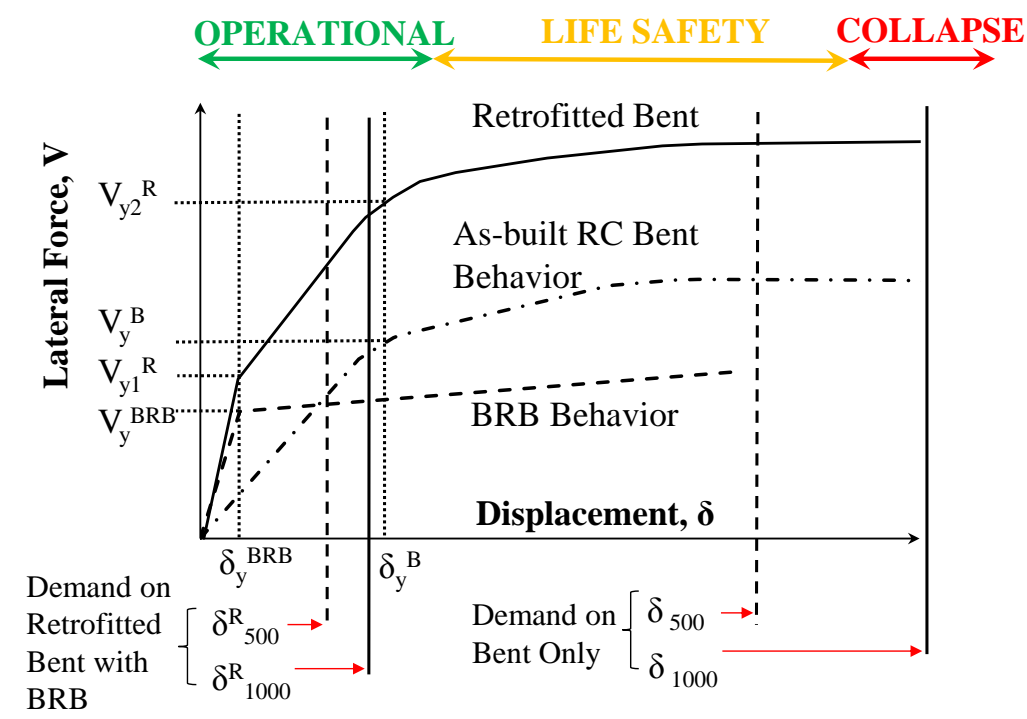

Figure 4.10 Toward operational performance using BRBs

In order to represent the behavior of buckling-restrained braces, a bi-linear response with hardening is utilized as illustrated in Figure 4.11. The BRB parameters are the steel core area $\left(A_{s c}\right)$, the steel core yield strength $\left(f_{y}\right)$, the initial stiffness $\left(k_{i}^{B R B}\right)$ that as a first approximation can be assumed as the stiffness provided by the reduced section, the overstrength factors in tension $(\omega)$ and compression $(\beta \omega)$, steel core elongation at initial yield $\left(\Delta_{y}\right)$, the elongation at the design story drift $\left(\Delta_{\mathrm{BM}}\right)$, which should not be less than $1 \%$ the story drift and as first approximation can be assumed as $7.5 \Delta_{y}$, the axial yield strength of steel core $\left(\mathrm{P}_{\mathrm{ysc}}\right)$ and the adjusted brace strength $\left(\mathrm{P}_{\mathrm{ad}}\right)$. 


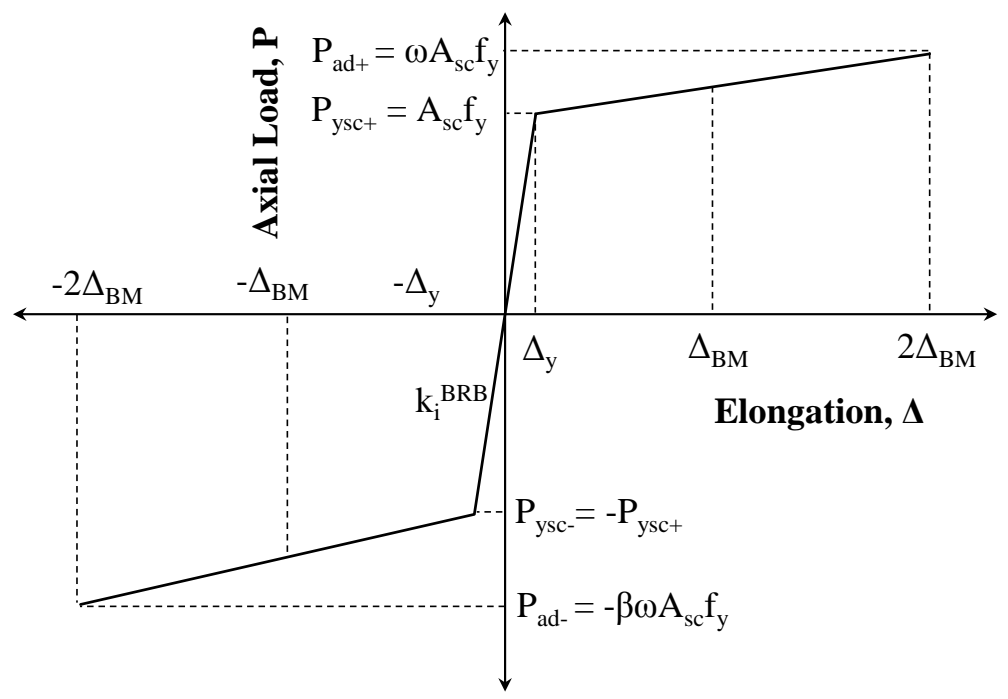

Figure 4.11 Idealized response of BRBs

Additional transformation for the BRB parameters is needed depending on the BRB configuration. The proposed configuration consists of a single diagonal brace. Thus, the aforementioned BRB parameters that are in a local coordinate system can be transformed to a global coordinate system as shown in Figure 4.12.

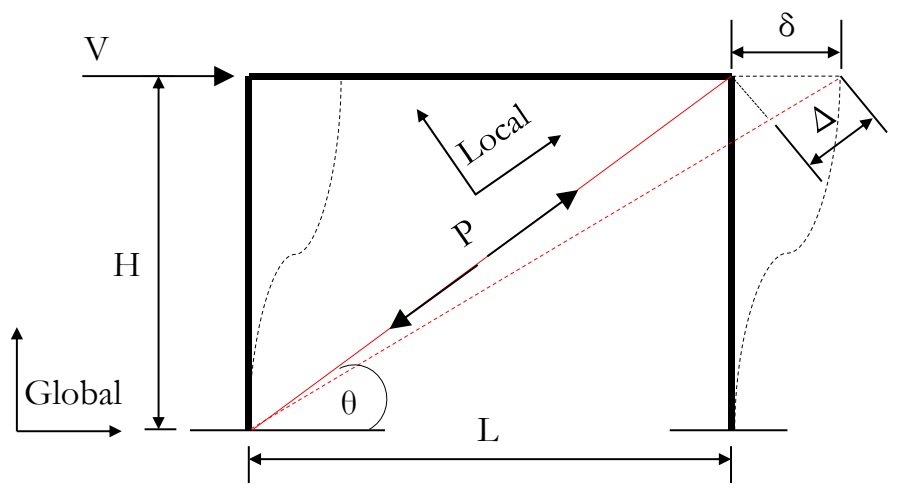

Figure 4.12 BRB parameters transformation

The relation between the elongation of the $\operatorname{BRB}(\Delta)$ and the lateral displacement of the RC bent ( $\delta$ ) is given by Eq. (4.9).

$$
\delta=\frac{\Delta}{\cos (\theta)}
$$


At yield, the stress-strain relationship for the BRB is given by Eq.(4.10) . Where, $f_{y}$ is the yield stress, $\mathrm{E}_{\mathrm{s}}$ is the modulus of elasticity of steel and $\varepsilon_{\mathrm{y}}$ is the strain at yield.

Furthermore, the strain at yield is assumed as the strain produced at the reduced section $\left(\mathrm{L}_{c}\right)$, i.e. the deformations from the transition sections $\left(\mathrm{L}_{\mathrm{tr}}\right)$ and full sections $\left(\mathrm{L}_{\mathrm{f}}\right)$ are neglected.

$$
f_{y}=E_{s} \cdot \varepsilon_{y} \rightarrow \Delta_{y}{ }^{B R B}=\frac{f_{y} L_{c}}{E_{s}}
$$

Therefore, the BRB yield displacement $\left(\delta_{\mathrm{y}}{ }^{\mathrm{BRB}}\right)$ is obtained substituting Eq. (4.10) into Eq. (4.9), which yields Eq. (4.11).

$$
\delta_{y}{ }^{B R B}=\frac{f_{y} L_{c}}{E_{s} \cos (\theta)}
$$

Finally, the lateral strength and stiffness contribution to be used in the structural fuse design, as presented in Section 4.4.3, is determined by using equations (4.12) and (4.13).

$$
\begin{gathered}
V_{y}{ }^{B R B}=A_{s c} f_{y} \cos (\theta) \\
K_{i}{ }^{B R B}=k_{i}{ }^{B R B} \cdot \cos ^{2}(\theta)=\frac{E_{s} A_{s c} \cdot \cos ^{2}(\theta)}{L_{c}}
\end{gathered}
$$

Since the demand depends on the fundamental period of the system, the BRB design is reduced to iterate until the BRB stiffness is determined using Eq. (4.14). Consequently, the steel core area and the reduced length section $\left(\mathrm{L}_{c}\right)$ of the BRB can be obtained using Eq.

$$
\begin{gathered}
K_{i}^{B R B}=\frac{E_{s} A_{s c} \cdot \cos ^{2}(\theta)}{L_{c}} \geq \max \left(\frac{R_{d-500} S_{a-500} m g}{\delta_{y}^{B}}, \frac{R_{d-1000} S_{a-1000} m g}{1.4 \delta_{y}^{B}}\right)-k_{e f f}^{B} \\
\frac{A_{s c}}{L_{c}} \geq\left\{\max \left(\frac{R_{d-500} S_{a-500} m g}{\delta_{y}^{B}}, \frac{R_{d-1000} S_{a-1000} m g}{1.4 \delta_{y}^{B}}\right)-k_{e f f}^{B}\right\} \frac{1}{E_{s} \cos ^{2}(\theta)}
\end{gathered}
$$


The final implementation of BRBs is recommended to be in coordination with a BRB manufacturer. This is because some of the parameters needed for the BRB design depend on the design of the brace itself and may differ from manufacturer to manufacturer. Also, it is worth mentioning that in many practical cases the design would result in short reduced sections $\left(\mathrm{L}_{c}\right)$, making not accurate the assumption that the initial BRB stiffness $\left(\mathrm{k}_{\mathrm{i}}^{\mathrm{BRB}}\right)$ is solely given by the reduced section. Thus, an effective stiffness for the BRB ( $\mathrm{k}_{\mathrm{eff}}^{\mathrm{BRB}}{ }^{\mathrm{B}}$ that considers the contribution of the transition sections and full sections can be used instead as follows:

$$
k_{e f f}^{B R B}=\frac{1}{\frac{1}{k_{i}^{B R B}}+\frac{2}{k_{t r}}+\frac{2}{k_{f}}}
$$

Where, $\mathrm{k}_{\mathrm{tr}}$ is the stiffness of the transition section and $\mathrm{k}_{\mathrm{f}}$ is the stiffness of the full section of the steel core. This effective stiffness is usually provided by the BRB manufacturer.

The BRB-to-gusset connection shall be designed to resist the demands based on the adjusted brace strength as per AISC (2010).

$$
P_{a d}=\beta \omega \cdot R_{y} \cdot P_{y s c}
$$

Where, $P_{a d}$ is the adjusted brace strength; $\beta \omega$ is the over-strength factor in compression; $\mathrm{R}_{\mathrm{y}}$ is the ratio of the expected yield stress to the specified minimum yield stress (usually taken as 1.1) and $\mathrm{P}_{\mathrm{ysc}}$ is the axial yield strength of steel core. Over-strength factors vary from 1.2 to 2.2 and shall be obtained from a qualification test or provided by the manufacturer. However, an initial assumption of 1.45 for $\beta \omega$ is suggested in the preliminary design. 


\subsection{IMPLEMENTATION ON SUBSTANDARD RC BRIDGES}

Previous studies (Nako, et al., 2009) have stated that many bridges owned by the state of Oregon are highly vulnerable to damage after an earthquake. The main reason being the majority of state owned bridges were designed and built in a period of time (1950-1975) when seismic loading was not thoroughly considered as indicated in Table 4.5.

Table 4.5 Seismic design code considerations, seismic hazard adopted by ODOT, and number of bridges built over time, adapted from Nako et al. (2009)

\begin{tabular}{|c|c|c|c|}
\hline Year & AASHTO Design Code & $\begin{array}{c}\text { Ground Motion } \\
\text { Hazard } \\
\text { Adopted by ODOT }\end{array}$ & $\begin{array}{l}\text { No. of } \\
\text { Bridges } \\
\text { Built }\end{array}$ \\
\hline Prior 1958 & $\begin{array}{l}\text { Seismic loading was not typically } \\
\text { considered }\end{array}$ & $\mathrm{N} / \mathrm{A}$ & 451 \\
\hline $1958-1974$ & $\begin{array}{l}\text { Bridges were designed for seismic } \\
\text { force from } 2 \%-6 \% \text { of structure weight }\end{array}$ & $\mathrm{N} / \mathrm{A}$ & 1294 \\
\hline 1971 & \multicolumn{3}{|c|}{ San Fernando, CA Earthquake } \\
\hline 1975-1990 & $\begin{array}{l}\text { Bridges were designed for seismic } \\
\text { force from } 2 \%-6 \% \text { of structure weight } \\
\text { based on AASHTO Interim } \\
\text { Specifications }\end{array}$ & $\begin{array}{l}\text { Seismic Hazard Maps } \\
\text { appeared in AASHTO }\end{array}$ & 522 \\
\hline 1989 & \multicolumn{3}{|c|}{ Loma Prieta, CA Earthquake } \\
\hline 1990 & $\begin{array}{l}\text { Adopt } 1983 \text { AASHTO Seismic } \\
\text { Design Guide }\end{array}$ & $\begin{array}{l}1990 \text { Interim ODOT } \\
\text { Seismic Specifications } \\
\text { Hazard Map }\end{array}$ & \multirow{2}{*}{409} \\
\hline 2004-2008 & $\begin{array}{l}\text { Include liquefaction effects into } \\
\text { design }\end{array}$ & $\begin{array}{l}2002 \text { USGS hazard } \\
\text { maps, 1000-year base } \\
\text { design event }\end{array}$ & \\
\hline 2009 & - & $\begin{array}{l}\text { Design check for } \\
\text { serviceability level, 500- } \\
\text { year event }\end{array}$ & - \\
\hline
\end{tabular}

According to the National Bridge Inventory (NBI), Stringer/Multi-beam or Girder Bridges are the dominant type of bridge in the state of Oregon. For that reason, this section is focused on multi-span reinforced concrete and prestressed concrete continuous stringer/girder bridges that were built before 1970 since those bridges were designed primarily for gravity loads without much consideration to lateral forces from seismic loading. 
As a result, columns lack sufficient transverse reinforcement to provide satisfactory performance in a major seismic event. Typically, No. 3 or No. 4 hoops at 12 inches on center were provided in columns regardless of the column cross-sectional dimensions. The stirrups were anchored by $90^{\circ}$ hooks with short extensions and intermediate ties were seldom used. Minimal restraint provided by the hoops can cause the longitudinal reinforcement to buckle once the concrete cover spalls. Furthermore, bridges built prior to 1970 have undesirable short to medium lap splices at the base of RC column. This lap splice detailing can potentially cause reduced column ductility and result in rapid loss of flexural strength.

\subsubsection{Multi-Column Prestressed Concrete Stringer/Girder (MPCG) Bridge Bent}

Prestressed concrete stringer/girder bridges, such as the one schematically depicted in Figure 4.13, are the second most common type of bridge in Oregon (Mehary \& Dusicka, 2015). These bridges consist of a superstructure that is not monolithically casted with the substructure. In these bridges the superstructure comprises a deck slab, parapets and girders. The substructure consists of bents (cap beam + columns), abutments and foundations.

The characteristics of the representative MPCG bridge used in this study were inferred from the 2010 ODOT inventory for prestressed concrete stringer/girder bridges built prior 1970 as follows:

- Selected from 364 Prestressed Concrete Stringer/Girder bridges built prior 1970

- Number of spans equal to 3, since $180 / 364$ bridges has that number of spans

- Number of lanes equal to 2 , since $132 / 364$ bridges has that number of lanes

- 2 round $3 \mathrm{ft}$ diameter columns per bent

- Longitudinal reinforcement varies: No. 10 or No. 11 
- Transverse reinforcement varies: No. 3 or No. 4 spaced 12 inches center-to-center

- Integral or semi-integral abutments

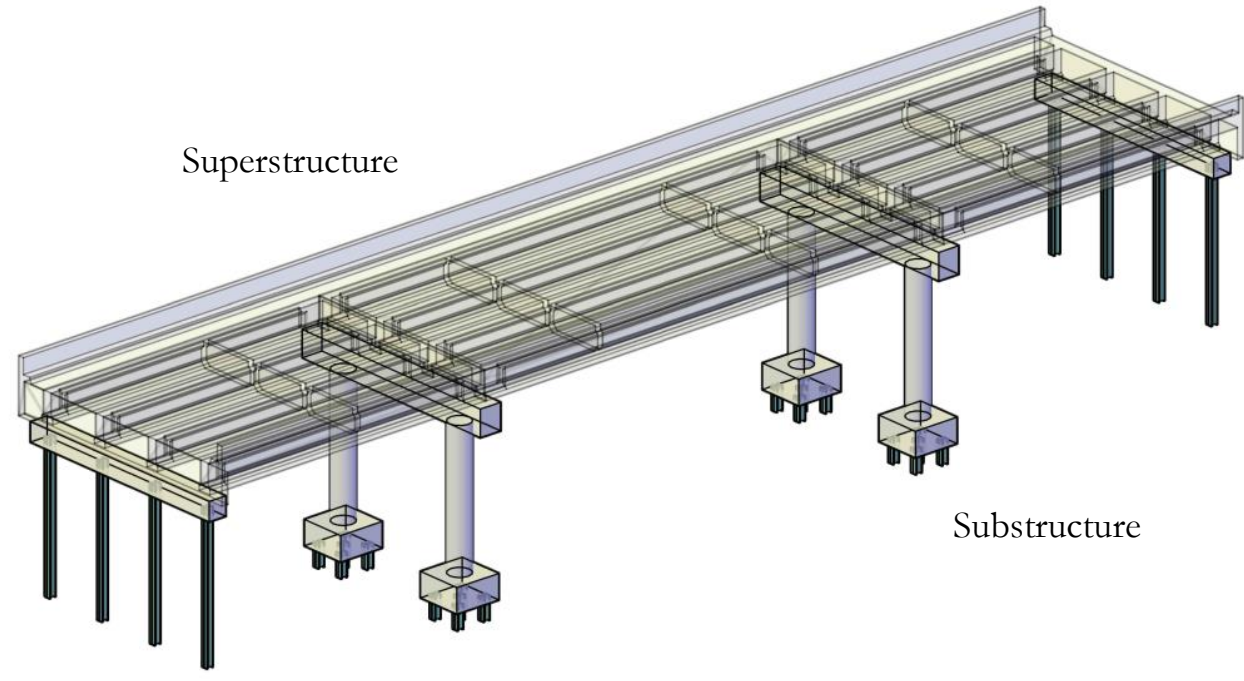

(a)

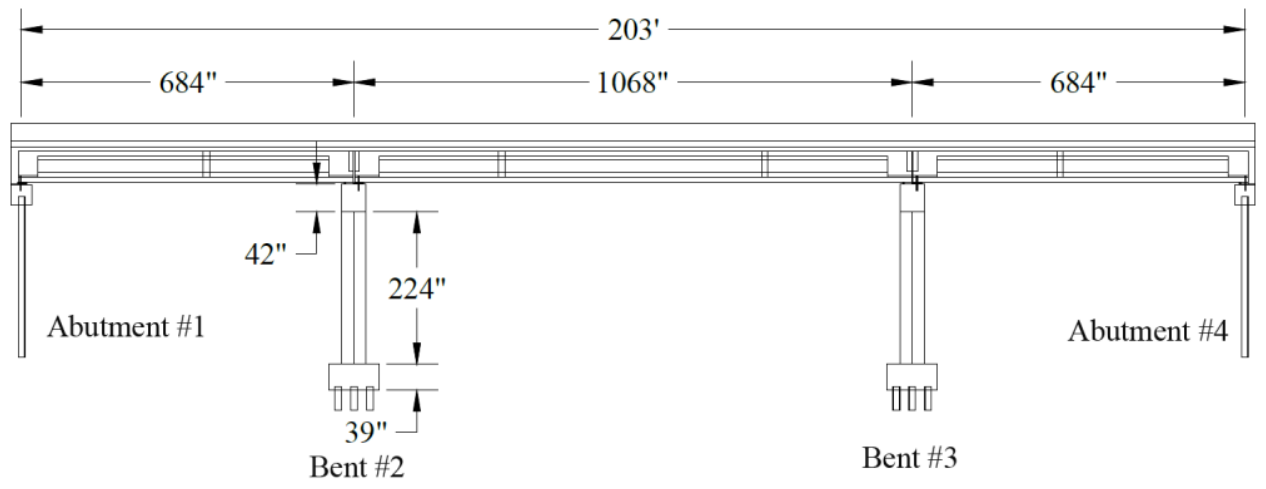

(b)

Figure 4.13 Typical MPCG bridge. (a) 3D schematic, (b) Elevation

The representative multi-column prestressed concrete stringer/girder bridge bent, also referred to as MPCG bridge bent, corresponds to an existing RC multi-column bridge bent constructed in the 1950 to mid-1970 in the state of Oregon. As many of the bridge structures built at that time in the Pacific Northwest, the bridge substructure was designed and built with minimum seismic considerations. This resulted in inadequate transverse 
reinforcement, no seismic detailing, and lap-splices of length from 20 to 40 times the diameter of the longitudinal reinforcing steel $\left(\mathrm{d}_{\mathrm{b}}\right)$ in expected plastic hinge zones.

The prototype bridge bent consists of two circular columns per bent, a rectangular cap beam and rectangular pile cap footings. The column longitudinal reinforcement ratio is $\varrho_{\mathrm{L}}=1.2 \%$, which is just above the minimum required by AASHTO (2009). The provided column shear reinforcement and confinement does not meet code requirements since \#4 circular hoops spaced at 12 in were provided. The cap beam has premature termination of top reinforcement and low quantity of bottom steel reinforcement at the ends of the span, which might result in the formation of premature hinges in the cap beam at the column face. Moreover, lap splices of $39 \mathrm{~d}_{\mathrm{b}}$ can be found at the base of columns and no seismic detailing was specified. The specified material properties for the representative bridge were $3.3 \mathrm{ksi}$ as compressive strength of concrete at 28 days and Grade 40 steel. Typical details for the representative two column RC bridge bent are illustrated in Figure 4.14.
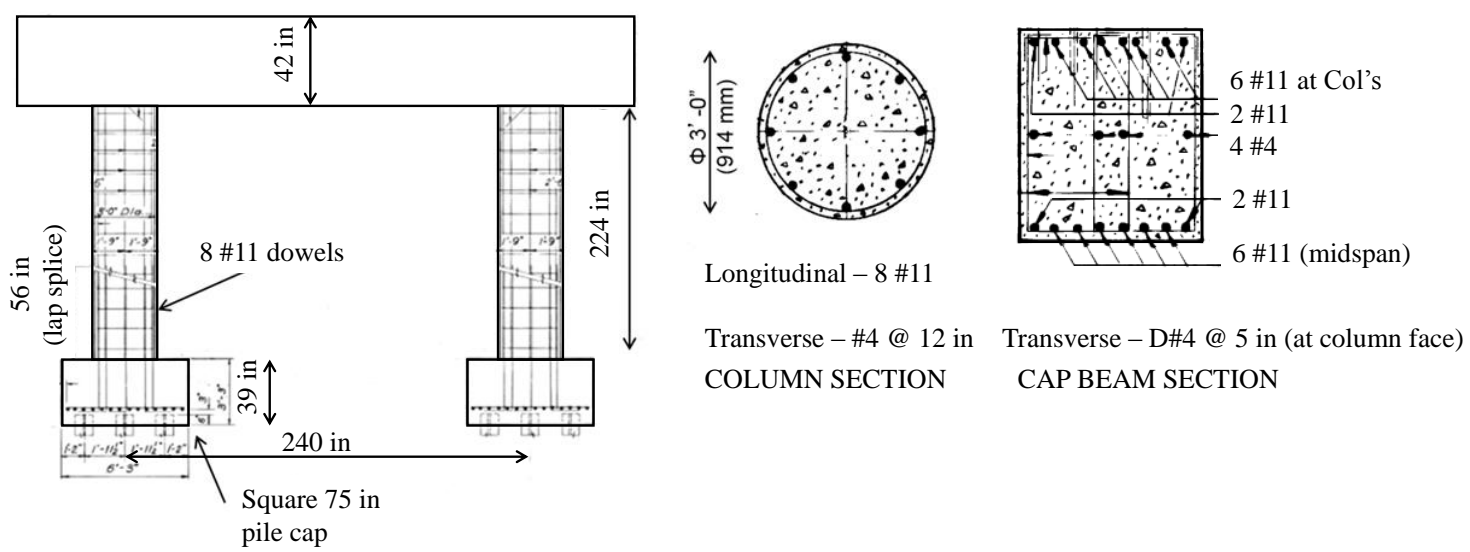

Figure 4.14 Typical RC bent used in MPCG bridges

As described in Section 4.2.1, the methods to assess the behavior of existing bridges vary from a minimum component capacity/demand analysis to more refined analysis such nonlinear procedures (Pushover analysis and Nonlinear Time History analysis). In the 
assessment of the MPCG bridge bent two methods were used, namely, the displacement demand/capacity method and the pushover method.

Displacement demands on both directions were based on multi-mode spectral elastic analysis, combination of orthogonal displacement demands and were magnified through the use of the displacement magnification factor for short period structures, $R_{d}$. SAP2000 models, as the one shown in Figure 4.15, were generated for the MPCG bridge in order to determine the demands in the transverse and longitudinal direction. Link elements were used to model the connection between girders and cap beam. Additionally, the moment transfer between girders and cap beam was released from the model to capture that no moment was transferred from the superstructure to the substructure.

In this preliminary assessment, the foundations were assumed rigid (fixed-base columns) following the Modeling Method I recommended for SDC C bridges by AASHTO (2009). Typical MPCG bridges found in Oregon present semi-integral abutments. Those abutments were modeled through the use of linear springs with an initial longitudinal stiffness equal to $20 \mathrm{kip} / \mathrm{in} / \mathrm{ft}$ that was adjusted proportional to the diaphragm height following ODOT recommendation (ODOT, 2015) and Eq. (4.18), which is based on empirical relationships developed from full-scale tests conducted at UC Davis (Maroney, et al., 1994).

$$
K_{a b u t}=k_{i} \cdot w \cdot \frac{H}{5.5}(\mathrm{kip} / \mathrm{in})
$$

Where, $\mathrm{k}_{i}$ is the initial stiffness, $\mathrm{w}$ is the width of the backwall in feet, and $\mathrm{H}$ is the height of the backwall in feet. ODOT recommends the use of $\mathrm{k}_{i}=20 \mathrm{kips} / \mathrm{in} / \mathrm{ft}$ for the initial stiffness. 
In addition to the stiffness provided by the backfill soil when the abutment backwall is pressed into the soil, abutment piles were also considered. A value of 40 kips/in per pile was used to represent the stiffness of the piles. 4 piles were assumed for the representative MPCG bridge as shown in Figure 4.13. In the transverse direction the stiffness was conservatively estimated, ignoring any wingwalls, as 40 kips/in per pile.

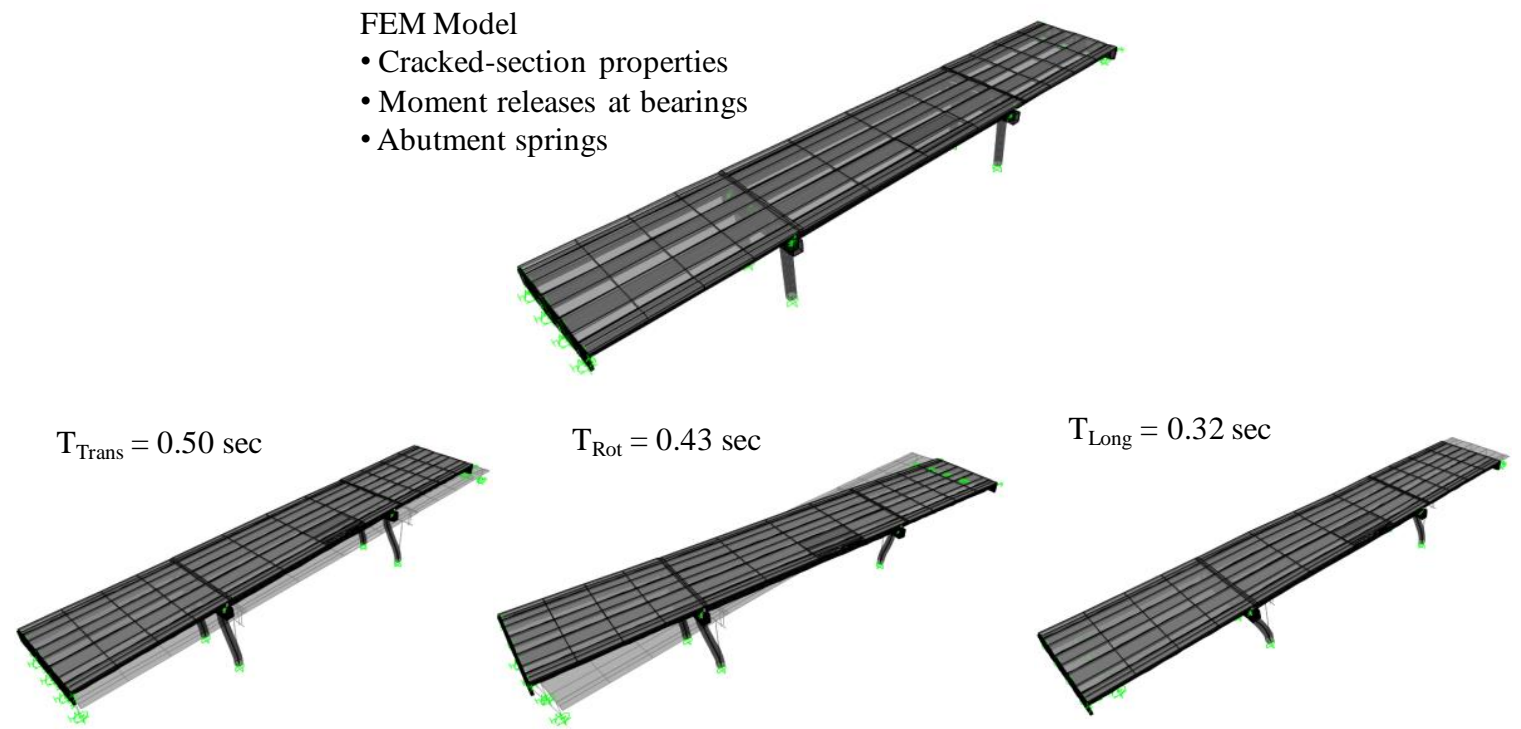

Figure 4.15 SAP2000 model of the MPCG Bridge

Table 4.6 Displacement demand for the MPCG Bridge

\begin{tabular}{c|c|c}
\hline $\begin{array}{c}\text { Seismic } \\
\text { Hazard }\end{array}$ & $\begin{array}{c}\text { Transverse } \\
\text { (in) }\end{array}$ & $\begin{array}{c}\text { Longitudinal } \\
\text { (in) }\end{array}$ \\
\hline 500-year & 2.2 & 1.8 \\
\hline 1000-year & 3.4 & 2.5 \\
\hline
\end{tabular}

In order to determine the capacity of the MPCG bridge. The method described in AASHTO (2009) was utilized. A preliminary displacement capacity of the bridge was obtained from the approximation given by Eq. (4.19) for the seismic design category (SDC) C since typical bridges under the acceleration demands used in this study can be categorized 
in that SDC. It is worth noting that the SDC is for new bridges and is different that the SRC described in Section 4.2.1.

$$
\begin{gathered}
\Delta_{C}{ }^{L}=0.12 H_{o}(-2.32 \ln (x)-1.22) \geq 0.12 H_{o} \\
x=\frac{\Lambda B_{o}}{H_{o}}
\end{gathered}
$$

Where, $\mathrm{H}_{\mathrm{o}}$ is the column clear height in feet, $\mathrm{B}_{\mathrm{o}}$ is the column diameter and $\Lambda$ is a factor that accounts for the column end restraint condition, 1 for fixed-free and 2 for fixed top and bottom. This equation, however, was developed to represent the displacement capacity of well-detailed columns designed in accordance with the AASHTO design specifications (AASHTO, 2009). In order to overcome this problem, Imbsen (2006) recommended the use of equations (4.20) and (4.21) for lap-splice and poor confinement columns, respectively.

$$
\begin{aligned}
& {\Delta_{C}}^{L}=4.0 \cdot(0.0013)^{x} \\
& \Delta_{C}{ }^{L}=3.9 \cdot(0.0070)^{x}
\end{aligned}
$$

In order to find the capacity from more refined analysis. A nonlinear static procedure, also referred to as pushover analysis, was performed using the expected material properties and the modeling guidelines contained in AASHTO (2009). Expected material properties can be determined by applying factors to the specified properties. Factors of 1.31.5 and 1.1 are recommended in the literature for the compressive strength of concrete and the yield stress of steel, respectively. Expected compressive strength of 4290 psi and yield stress of $44 \mathrm{ksi}$ were used to model the concrete and steel, respectively. The pushover analysis was carried out using the software SAP2000, in which potential plastic hinges were assigned at the top and bottom of columns in the transverse direction and only at the 
bottom of the columns in the longitudinal direction. A schematic of the potential plastic hinges formed in the bent when loaded in the transverse direction is shown in Figure 4.16.

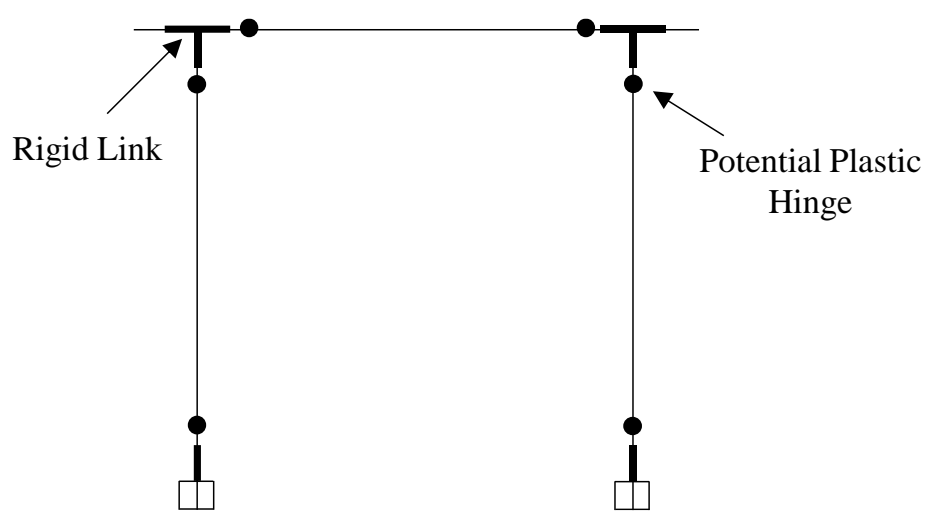

Figure 4.16 Modeling approach in the transverse direction

Plastic hinges were also defined in the cap beam, specifically in the interface between cap beam and column, in order to capture the low positive moment capacity caused by a greatly reduced area of longitudinal positive (bottom) steel reinforcement as compared to the negative (top) steel reinforcement. Lap splice behavior was also considered at the base of columns through the use of the method suggested by Priestley et al. (1996). This method indicates that columns with lap splices may not develop its full nominal flexural capacity $\left(\mathrm{M}_{\mathrm{n}}\right)$ because splice failure mechanism inhibit yielding of the longitudinal reinforcement. Instead a reduced moment capacity $\left(M_{s}\right)$ is achieved as shown in line 1 of Figure 4.17. In cases when the longitudinal reinforcement reaches yielding (line 2 in Figure 4.17), the lap splice becomes prone to slipping when the concrete compressive strain reaches 0.002 . Thus, degradation of lap splice strength with increasing ductility results in a loss of moment capacity down to a residual value $\left(\mathrm{M}_{\mathrm{r}}\right)$ based on axial force alone. The expected behavior of an unconfined section without lap splices is also depicted in Figure 4.17 as a reference. The typical MPCG bent used in this study presents a lap splice length of 56 inches $\left(\sim 40 \mathrm{~d}_{b}\right)$ and an expected $\mathrm{f}_{\mathrm{c}}$ ( $\mathrm{f}_{\text {ce }}$ equal to $4.3 \mathrm{ksi}$. Results of using Eq. (2.8) lead to the conclusion that the column will be 
able to develop its full nominal flexural strength. Consequently, the behavior of the plastic hinge at the base of the MPCG column can be modeled with line 2 as shown in Figure 4.17.

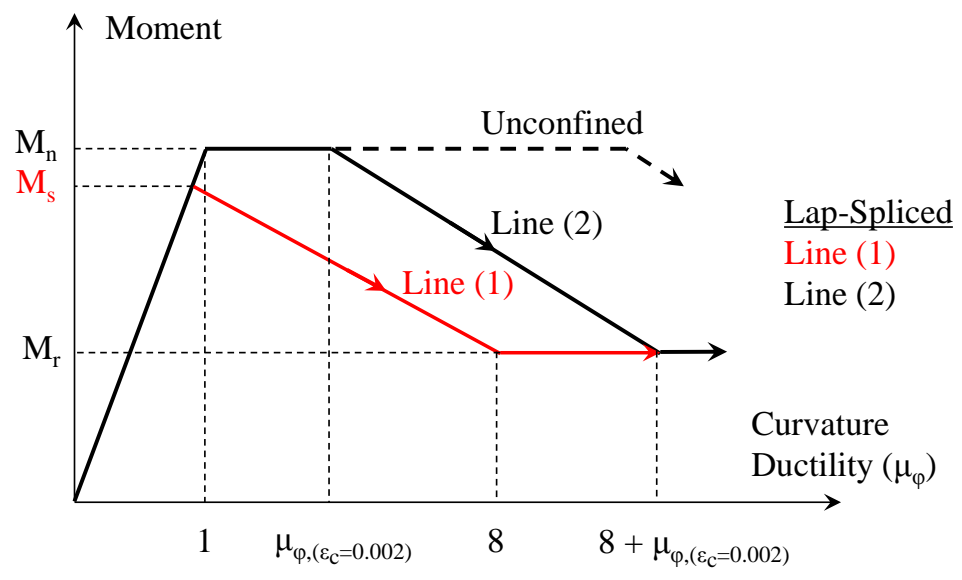

Figure 4.17 Response of lap-spliced columns. Adapted from Priestley et al. (1996)

The pushover analysis indicated that the first plastic hinge in the transverse direction is generated in the cap beam followed by hinge formations at the base of the columns as shown in Figure 4.18.

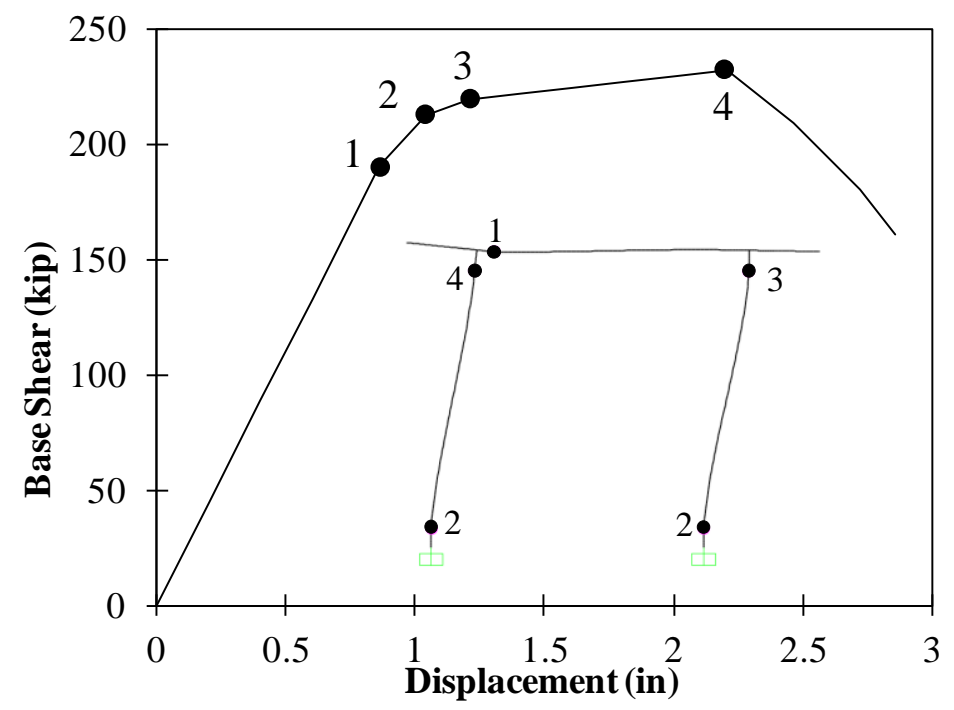

Figure 4.18 Pushover analysis of the MPCG bent in the transverse direction

The bridge displacement capacity calculated using the aforementioned equations and pushover analysis are shown in Table 4.7. Comparing the demand (Table 4.6) to the capacity 
(Table 4.7) demonstrates that the capacity of RC bents built prior 1970 is limited and may not satisfy the performance levels required by ODOT (2015), especially in the transverse direction.

Table 4.7 Displacement capacities for the MPCG bridge bent

\begin{tabular}{c|c|c|c|c}
\hline Direction & $\begin{array}{c}\text { SDC C } \\
\text { Eq. } \\
(4.19)\end{array}$ & $\begin{array}{c}\text { Lap-Splice } \\
\text { Eq. (4.20) }\end{array}$ & $\begin{array}{c}\text { Poor } \\
\text { Confinement } \\
\text { Eq. (4.21) }\end{array}$ & $\begin{array}{c}\text { Pushover } \\
\text { SAP2000 }\end{array}$ \\
\hline Transverse & 3.2 in & 1.0 in & 1.6 in & 2.5 in \\
\hline Longitudinal & 6.8 in & 2.8 in & 3.5 in & 3.8 in \\
\hline
\end{tabular}

\subsubsection{Multi-Column Continuous Concrete Stringer/Girder (MCCG) Bridge Bent}

Continuous concrete stringer/girder bridges are the most common type of bridge in Oregon (Mehary \& Dusicka, 2015). These bridges consist of a superstructure that is monolithically casted with the substructure as shown in Figure 4.19.

The characteristics of the representative MCCG Bridge used in this study were inferred from the 2010 ODOT inventory for continuous concrete stringer/girder bridges built prior 1970 as follows:

- Selected from 523 Continuous Concrete Stringer/Girder bridges built prior 1970.

- Number of spans equal to 3, since 290/523 bridges has that number of spans.

- Number of lanes equal to 2 , since $233 / 523$ bridges has that number of lanes.

- 2 square $2 \mathrm{ft}$ columns per bent

- Longitudinal reinforcement varies: No. 10 or No. 11

- Transverse reinforcement varies: No. 3 or No. 4 spaced 12 inches center-to-center

- Integral or semi-integral abutments 


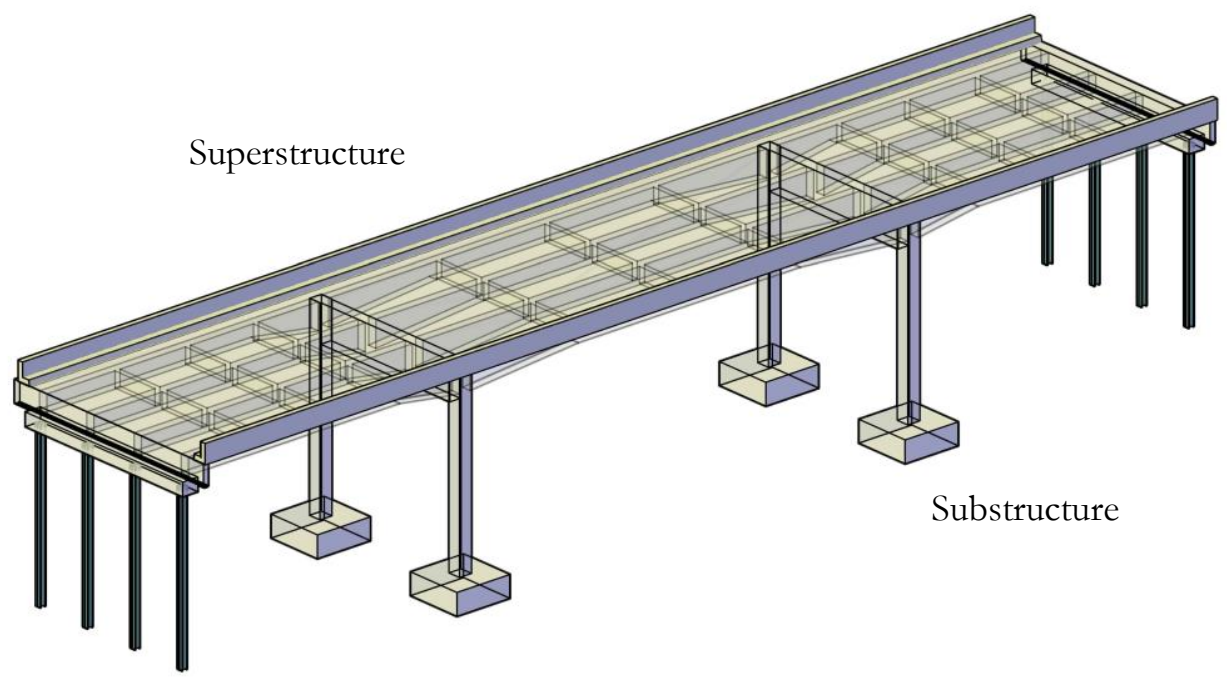

(a)

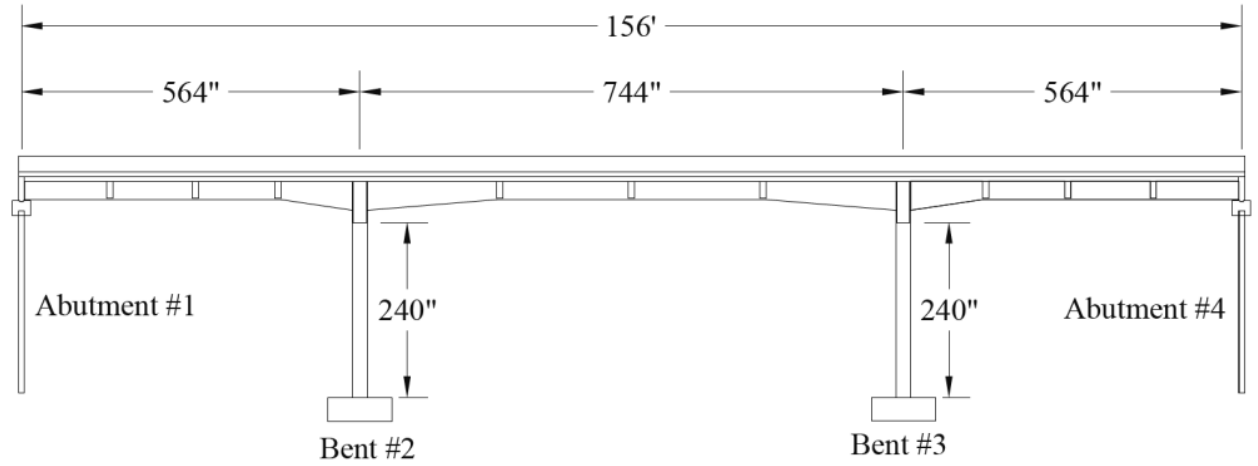

(b)

Figure 4.19 Typical MCCG bridge. (a) 3D schematic, (b) Elevation

The representative MCCG Bridge bent consists of two square columns per bent and a rectangular cap beam. Specified materials comprise $3.3 \mathrm{ksi}$ and $40 \mathrm{ksi}$ for the compressive strength of concrete and yield stress of steel, respectively. The column longitudinal reinforcing steel consists of 4 \#10 rebars. Typically, \#3 or \#4 hoops at 12 inches on center were provided in columns regardless of the column cross-sectional dimensions. As a consequence, the provided column shear reinforcement and confinement does not meet current code requirements. The stirrups were anchored by $90^{\circ}$ hooks with short extensions and intermediate ties were seldom used. Minimal restraint provided by the hoops can cause 
the longitudinal reinforcement to buckle once the concrete cover spalls. Furthermore, bridges built prior to 1970 have undesirable lap splices at the base of the columns. The lap splice detailing combined with the poor transverse reinforcing may cause reduced column strength and ductility, and result in rapid deterioration of flexural strength. Typical details for the representative multi-span continuous stringer/girder RC bridge bent are illustrated in Figure 4.20.

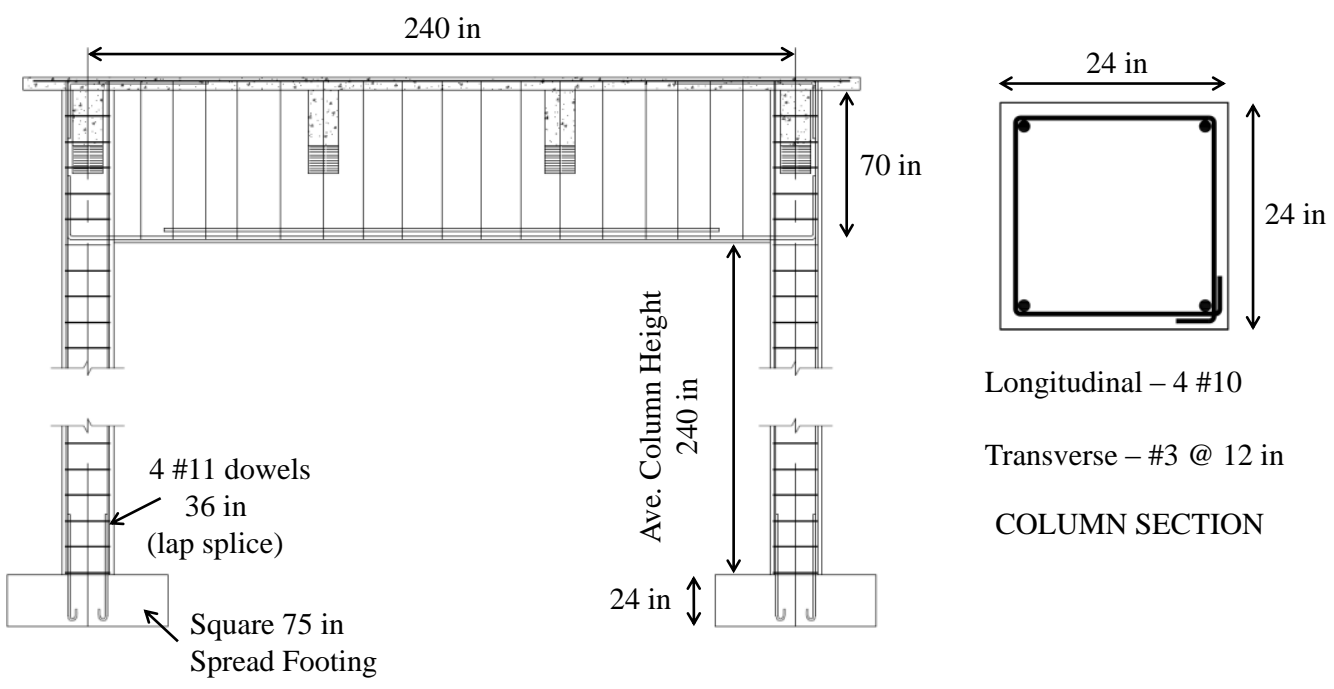

Figure 4.20 Typical RC bent used in MCCG bridges

The SAP2000 model of the representative MCCG bridge is shown in Figure 4.21. Similar methodology and modeling assumptions described for the MPCG bridge were used to determine the displacement demand (Table 4.8) and capacity (Table 4.9) of the MCCG bridge. The main difference in this case was the use of plastic hinges at the top and bottom of the columns on both directions (longitudinal and transverse) for the pushover analysis.

Based on the results shown in Table 4.9, the displacement capacity of the bent obtained using pushover analysis is more than the capacity calculated for a well-detailed column using Eq. (4.19) for SDC C. This result is most likely caused by the low ratio of longitudinal reinforcement and the low axial demand $\left(\sim 10 \% \mathrm{f}_{\mathrm{c}} \mathrm{A}_{\mathrm{g}}\right)$ imposed in the columns as 
was observed during the full-scale experiments presented in Chapter 2, Section 2.11. In this case, the RC bent may not need to be retrofitted despite its substandard detailing.

FEM Model

- Cracked-section properties

- Abutment springs
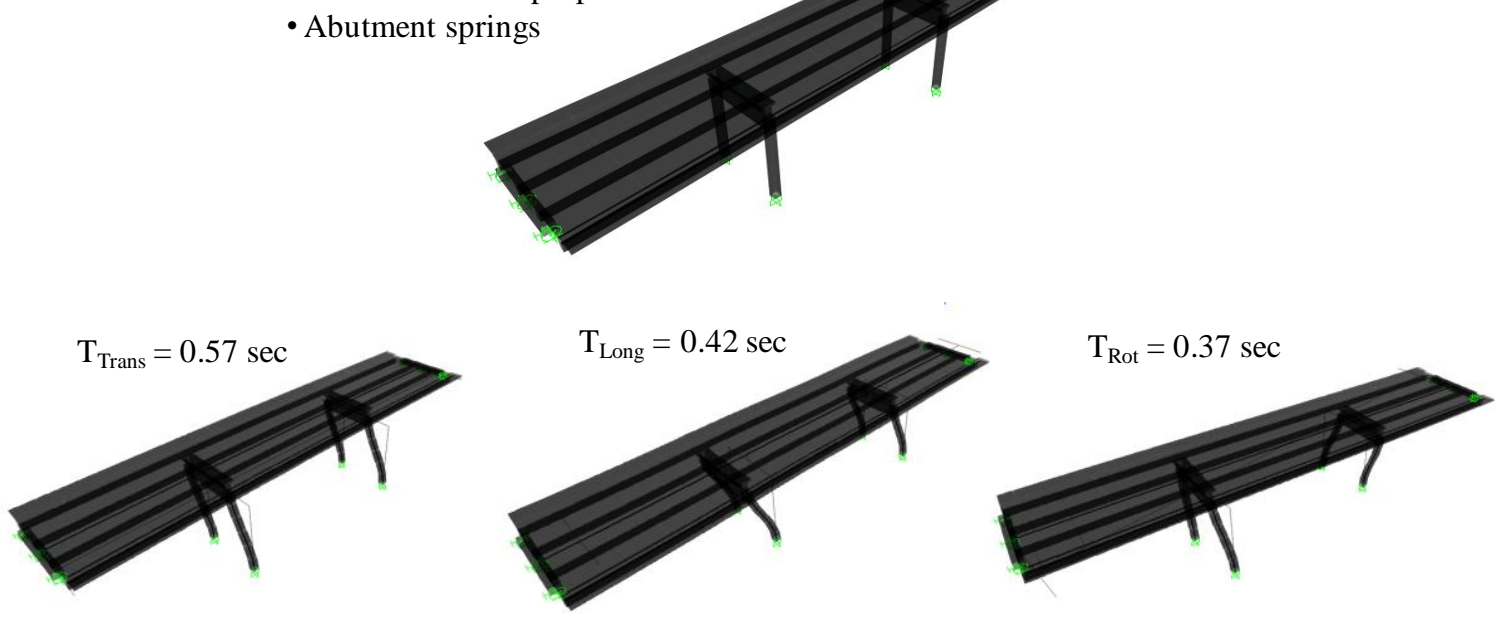

Figure 4.21 SAP2000 model of the MCCG Bridge

Table 4.8 Displacement demand for the MCCG bridge bent

\begin{tabular}{c|c|c}
\hline $\begin{array}{c}\text { Seismic } \\
\text { Hazard }\end{array}$ & $\begin{array}{c}\text { Transverse } \\
\text { (in) }\end{array}$ & $\begin{array}{c}\text { Longitudinal } \\
\text { (in) }\end{array}$ \\
\hline 500-year & 3.2 & 2.2 \\
\hline 1000-year & 4.8 & 2.8 \\
\hline
\end{tabular}

Table 4.9 Displacement capacities for the MCCG bridge bent

\begin{tabular}{c|c|c|c|c}
\hline Direction & $\begin{array}{c}\text { SDC C } \\
\text { Eq. } \\
(4.19)\end{array}$ & $\begin{array}{c}\text { Lap-Splice } \\
\text { Eq. (4.20) }\end{array}$ & $\begin{array}{c}\text { Poor } \\
\text { Confinement } \\
\text { Eq. (4.21) }\end{array}$ & $\begin{array}{c}\text { Pushover } \\
\text { SAP2000 }\end{array}$ \\
\hline Transverse & 5.4 in & 1.8 in & 2.5 in & 5.8 \\
\hline Longitudinal & 5.4 in & 1.8 in & 2.5 in & 6.2 \\
\hline
\end{tabular}

\subsubsection{BRB Design}

In order to retrofit the substandard bridge bents shown in previous sections through the inclusion of buckling-restrained braces, the following considerations were made: 
The BRB length and angle are limited by the dimensions of the bridge bent under consideration. For the MPCG bent a span length $\mathrm{L}=240$ in and height $\mathrm{H}=224$ in were considered, while $\mathrm{L}=240$ in and $\mathrm{H}=240$ in were considered for the MCCG bent. Brace angles $(\theta)$ of 48 and 45 degrees were considered appropriate for the MPCG and MCCG bridge bents, respectively.

Inertial masses of $1.81 \mathrm{kip}-\mathrm{s}^{2} /$ in and $1.24 \mathrm{kip}-\mathrm{s}^{2} /$ in were obtained from the superstructure dead load for the MPCG bent and MCCG bent, respectively.

$\square$ Response spectrums with maximum spectrum acceleration $\left(S_{\mathrm{a}}\right)$ of $0.65 \mathrm{~g}$ with $\mathrm{T}_{\mathrm{s}}$ equal to $0.49 \mathrm{sec}$ for the 500 -year event and $0.85 \mathrm{~g}$ with $\mathrm{T}_{\mathrm{s}}$ equal to $0.53 \mathrm{sec}$ for the 1000 year event were considered as shown in Figure 4.2.

$\square$ An expected compressive strength of concrete equal to $4.3 \mathrm{ksi}$ and an expected yield stress of $44 \mathrm{ksi}$ for the steel reinforcement were considered. For the brace, a yield stress of $42 \mathrm{ksi}$ was considered appropriate for this application.

$\square$ The obtained as-built bent parameters from the pushover analysis were: $\delta_{\mathrm{y}}{ }^{\mathrm{B}}=0.98$ in and $\mathrm{k}_{\mathrm{i}}{ }^{\mathrm{B}}=252 \mathrm{kip} / \mathrm{in}$ for the MPCG bridge bent, and $\delta_{\mathrm{y}}{ }^{\mathrm{B}}=1.25$ in and $\mathrm{k}_{\mathrm{i}}{ }^{\mathrm{B}}=65$ kip/in for the MCCG bridge bent.

Following the procedure described in Section 4.5.1, the minimum lateral stiffness of the BRB $\left(\mathrm{K}_{\mathrm{i}}^{\mathrm{BRB}}\right)$ was computed as $686 \mathrm{kip} /$ in and $345 \mathrm{kip} /$ in for the MPCG and MCCG bent, respectively. Thus, potential BRB designs would require a steel core area of 3.4 in $^{2}$ and $1.8 \mathrm{in}^{2}$, and a reduced section length $\left(\mathrm{L}_{\mathrm{c}}\right)$ of 60 in and 70 in for the MPCG and MCCG bridge bent, respectively. The total system demands were then calculated as: $\delta^{\mathrm{R}}{ }_{500}=0.96$ in and 1.21 in, and $\delta^{\mathrm{R}}{ }_{1000}=1.35$ in and 1.69 in for the MPCG and MCCG bent, respectively. Other BRB designs are also acceptable if the strength of the concrete elements is sufficient. 
In this case, the steel core area was reduced in order to satisfy capacity checks, which resulted in minor inelastic excursions for the 1000-year event. Thus, following the structural fuse concept the target performance levels were achieved, resulting in an "Operational" performance level for the bridge for both seismic hazards, 500-year event as well as 1000year event.

\subsection{Post-Earthquake Damage Repair of RC Bents using BRBs}

Bridges are an important component of a highway network. Interruption and even restriction in traffic caused by excessive damage in a bridge can have severe consequences in the transportations services, the economic activities around the damaged area, and also in the prompt response of emergency vehicles to provide first aid and evacuation services in a timely manner. Moreover, recent earthquakes have demonstrated that damage in bridges is not only caused by the earthquake mainshock but also can be caused by aftershocks, which are a series of subsequent seismic events.

Several investigations on damaged bridges following an earthquake have been conducted in an effort to develop repair techniques capable of minimizing the impact that the disruption of traffic may cause, provide enough capacity to sustain aftershock events, and enhance resiliency of the transportation network. In this study, the term repair is used to denote the rehabilitation of a damaged RC bent. On the contrary, the term retrofit, used in previous sections, refers to enhancing or upgrading an undamaged existing bent.

Post-earthquake reconnaissance of damaged structures often reports visual damage that varies from minor cracks to considerable damage such as complete crushing of concrete, longitudinal rebar buckling and bar fracture. In general, seismic repairs in concrete elements depends on the severity of the reported damage. For example, in the case of RC 
components such as columns and cap beams that bar fracture has not occurred, repair techniques usually involve epoxy injection into concrete cracks, remove loose concrete and patch spalled zones, and provide passive or active confinement through encasing the concrete with FRP wrapping, RC jackets, steel jackets, and prestressing strands (Chai, et al., 1991), (Saadatmanesh, et al., 1997), (Chang, et al., 2004) (Vosooghi \& Saiid Saiidi, 2013) (Fakharifar, et al., 2016). Once the longitudinal bar has buckled or fractured, repair techniques usually include the replacement of the fractured bars through the use of mechanical splices, reinstallation of transverse reinforcement, and enhancing the concrete confinement through the same methods previously mentioned (Lehman, et al., 2001), (Cheng, et al., 2003), (Shin \& Andrawes, 2011), (He, et al., 2013).

Here, buckling-restrained braces are analyzed as a repair measure for earthquakedamaged RC bents. The objective of implementing BRBs in damaged $\mathrm{RC}$ bents is the same as the one described in the retrofitting case, which was limiting the damage in the main components of the gravity resisting system (cap beam and columns) and concentrating the damage and energy dissipation in the replaceable component (BRB). With that aim, the general repair design follows a similar procedure to the one shown in Figure 4.5 with modifications to steps 1 and 2 as follows:

Step 1: Change assessment of the as-built bent for the assessment of the damaged structure in an effort of determining the level of damage and also the residual strength and stiffness of the structure.

FEMA306 (1998) and FEMA307 (1998) suggested the use of a modified plastic hinge model to account for the reduced strength, reduced stiffness and the residual deformation of the damaged component as shown in Figure 4.22. Where, $\mathrm{K}$ and $\mathrm{Q}_{\mathrm{CE}}$ are the 
stiffness and strength of the undamaged component, respectively. K' and Q' ${ }_{\mathrm{CE}}$ are the stiffness and strength of the damaged component, respectively. $\lambda_{\mathrm{K}}$ is a stiffness modification factor that accounts for change in effective initial stiffness resulting from damage, and $\lambda_{\mathrm{Q}}$ is a modification factor that accounts for change in expected strength resulting from damage.

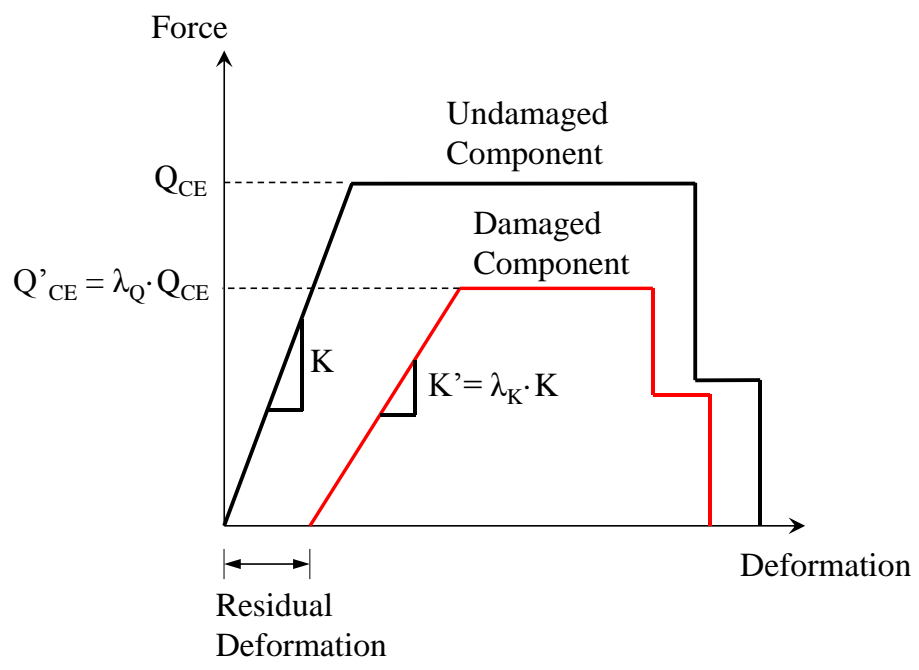

Figure 4.22 Modeling of damaged component. Adapted from FEMA306

According to FEMA306 the modification factors can be stablished from experimental results of critical components or using the tables prescribed in the report as indicated in Table 4.10.

Table 4.10 Modification factors for damaged RC components

\begin{tabular}{c|c|c|c|c}
\hline $\begin{array}{c}\text { Mode of } \\
\text { Failure }\end{array}$ & \multicolumn{4}{|c}{ Ductile Flexural } \\
\hline $\begin{array}{c}\text { Damage } \\
\text { Severity }\end{array}$ & $\begin{array}{c}\text { Insignificant } \\
\text { (minor cracks) }\end{array}$ & $\begin{array}{c}\text { Slight } \\
\text { (crack width }<1 / 4 \\
\text { in }\end{array}$ & $\begin{array}{c}\text { Moderate } \\
\text { (spalling) }\end{array}$ & Heavy \\
\hline$\lambda_{\mathrm{K}}$ & 0.8 & 0.6 & 0.5 & $\begin{array}{c}\text { Not } \\
\text { used }\end{array}$ \\
\hline$\lambda_{\mathrm{Q}}$ & 1.0 & 1.0 & 0.8 & $\begin{array}{c}\text { Not } \\
\text { used }\end{array}$ \\
\hline
\end{tabular}


Ludovico et al (2013)studied the modification factors for damaged substandard RC

columns. In Ludovico's study, experimental-based formulations were introduced based on 23 experiments conducted on non-conforming (or substandard) columns with deformed bars and 13 with smooth bars. They proposed equations (4.22) and (4.23) for the stiffness and strength factors, respectively, to be used on the theoretical assessment of the residual capacity using pushover analyses.

$$
\begin{gathered}
\lambda_{K}=1-\left[1.01-0.96 \cdot\left(\theta / \theta_{y}\right)^{-1.1}\right] \\
\lambda_{Q}=1-0.05 \cdot\left(\theta / \theta_{y}-3.9\right) \text { for } 3.9<\theta / \theta_{y} \leq \theta_{u} / \theta_{y}
\end{gathered}
$$

Where $\theta$ is the peak rotation (or drift) of the component, $\theta_{\mathrm{y}}$ is the yield rotation (or drift) and $\theta_{\mathrm{u}}$ is the ultimate rotation (or drift).

Preliminary assessment of the residual strength and stiffness of the damaged structure can be computed using the information presented in Table 4.10 or through equations (4.22) and (4.23).

Step 2: Since the damaged RC bent has a reduced stiffness, satisfying the condition of limiting the maximum displacement of the repaired bent below the yield displacement of the undamaged RC bent for the 1000-year event is not feasible. Thus, larger inelastic excursions are permitted in an effort to still provide for a ductile response without considerably increasing the damage as shown in Figure 4.23 and expressed in Eq. (4.24). In this case, a factor of 2.5 applied to the yield displacement of the undamaged bent is recommended. Then the BRB design is reduced to iterate until the BRB stiffness is determined using Eq. (4.25).

$$
\delta_{1000}^{\mathrm{Re}}=R_{d-1000} S_{a-1000} g \frac{m}{K_{i}{ }^{B R B}+\lambda_{K} k_{e f f}^{B}} \leq 2.5 \cdot \delta_{y}{ }^{B}
$$




$$
K_{i}^{B R B} \geq \frac{R_{d-1000} S_{a-1000} m g}{2.5 \cdot \delta_{y}^{B}}-\lambda_{K} k_{e f f}^{B}
$$

The implementation of this repair technique is not restricted to pre-1970 bridges, i.e. it can be applied to any earthquake-damaged RC bent. Depending on the type of bridge and the abutment condition, additional repair techniques may be required in the longitudinal direction.

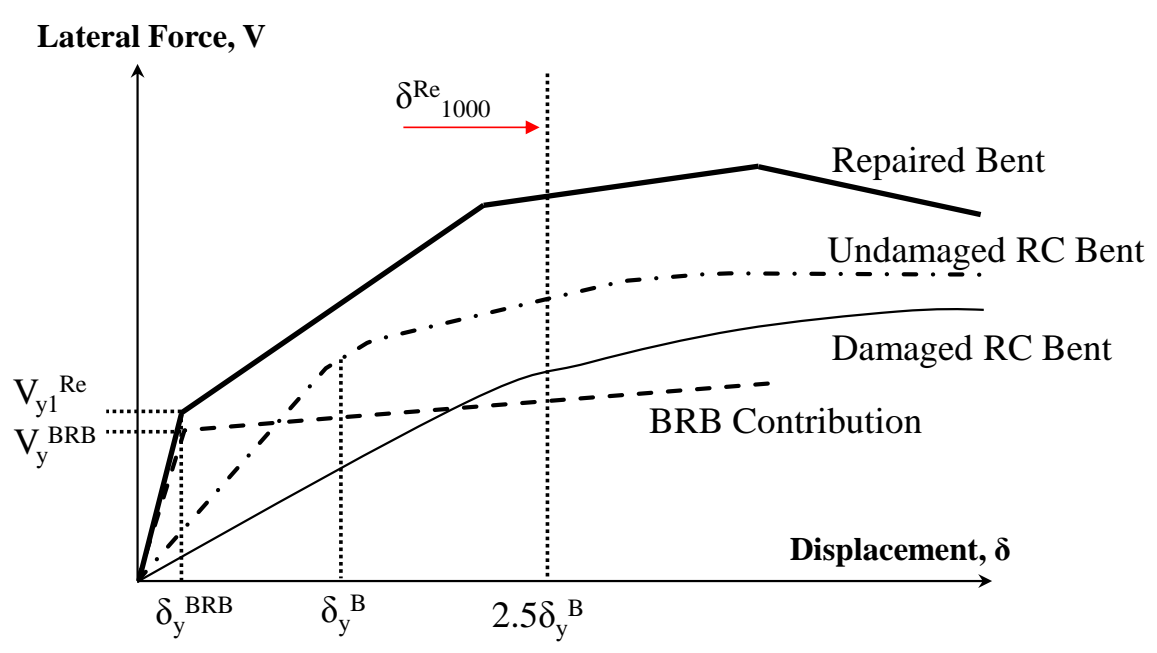

Figure 4.23 BRBs as seismic repair for damaged RC bents

\subsection{SUMMARY}

The design implementation of buckling-restrained braces using a structural fuse concept as a transverse direction retrofit measure for reinforced concrete multi-column bridge bents was presented. The structural fuse design concept aims to concentrate all the induced-energy during an earthquake event in disposable elements, while the reinforced concrete bent behavior is essentially in the elastic range. Based on this retrofit strategy, case studies of two representative bridge bents found in Oregon were discussed. Results showed that by implementing buckling-restrained braces, the retrofitted and repaired bents were 
significantly stiffer than the as-built and earthquake-damaged bent, respectively.

Consequently, displacement demands decreased and expected or further damage in columns and cap beam can be avoided, which would allow the structure to achieve operational performance levels of response.

Even though, the proposed design implementation was developed for a diagonal configuration in mind, its application can be extended to other cases. 


\section{CHAPTER 5}

\section{EXPERIMENTAL EVALUATION}

\subsection{GENERAL}

Over the years, earthquakes have exposed the vulnerability of reinforced concrete structures under seismic loads. The recent occurrence of highly devastating subduction zone mega earthquakes near instrumented regions has demonstrated the catastrophic impact of such natural force upon reinforced concrete structures. Typical reinforced concrete bridge bents constructed in the 1950 to mid-1970 in the Pacific Northwest were designed and built with minimum seismic considerations. This resulted in inadequate detailing within plastic hinge zones, leaving numerous RC bridge bents highly susceptible to damage following an earthquake. In order to overcome this deficiency, buckling-restrained braces have been proposed for retrofitting and repairing multi-column reinforced concrete bridge bents.

This chapter focuses in the experimental results of seismic performances of representative half-scale bridge bents in a retrofitted and repaired state using bucklingrestrained braces in a diagonal configuration. As-built, retrofitted and repaired states were tested under subduction loading protocols in an effort to reflect the displacement demands in $\mathrm{RC}$ bridge bents subjected to subduction zone earthquakes. The braces were designed utilizing the structural fuse concept presented in Chapter 4. In this concept, the main structural system is integrated with sacrificial (or fuse) components in order to restrict damage to the primary structure imposed by a damaging earthquake. The fuse elements, which are the buckling-restrained braces, were designed to take the earthquake-induced 
energy and dissipate it through nonlinear hysteretic behavior; meanwhile, the remaining structure is expected to behave elastically or with minor inelastic excursions. The motivation of this retrofit strategy for bridges is to minimize extensive damage in bents, and potentially satisfy serviceability criteria such that the bridges remain usable following a major earthquake event.

Two BRBs, namely, BRB1 and BRB2, were designed and experimentally evaluated with the aim of assessing the influence of BRB stiffness on the overall performance of the retrofitted bent. In the case, of the repaired bent only one BRB, namely, BRB3, was studied. Moreover, the use of a novel gusset plate detailing is proposed to connect the BRB to the concrete elements.

In addition to the RC bent tests, subassemblage tests of three buckling-restrained braces were conducted in an effort to study the response of these elements and allow potential nonlinear characterization for the analysis of the retrofitted and repaired systems.

\subsection{DESIGN OF SPECIMENS}

The experimental program consisted of two stages comprising five tests of largescale models of two nominally identical RC bridge bents. The first stage evaluated the retrofit option and consisted of three tests. Meanwhile, the repaired bent was evaluated in the second stage and consisted of two tests.

In the first stage, the first two experiments consisted on studying different BRB designs in an effort to assess the influence of BRB stiffness on the overall structural performance. In the third test, the bent was evaluated in the as-built non-retrofitted condition but in a damaged state. In the second stage, the first experiment consisted of 
testing the as-built bent in an undamaged state. In the second test, the cyclic performance of the repaired bent was evaluated.

The performance of these specimens was intended to reveal vulnerabilities in existing deficient RC bents and more importantly assess the behavior of their retrofitted and repaired condition.

\subsubsection{As-Built RC Bent}

In order to represent a typical seismically deficient $\mathrm{RC}$ bridge bent, a large-scale bent of a representative bridge was designed, hereinafter referred to as "As-built". The representative RC bent corresponded to a commonly found multi-column bridge bent constructed in the 1950 to 1970 in the State of Oregon, USA, as shown in Figure 4.15. As many of the bridge structures built at that time in the Pacific Northwest, the bridge bent was designed and built with minimum seismic considerations. This resulted in inadequate transverse reinforcement and confinement, no seismic detailing, and presence of lap-splices within plastic hinge zones. Similitude laws were used in order to design the large-scale RC bent. Table 5.1 illustrates the scaling process and includes relevant dimensions and reinforcement details for the test specimen.

Two bent specimens (models), namely, As-built Bent 1 and As-built Bent 2, were constructed and consisted of two 18 in $(457 \mathrm{~mm})$ diameter circular columns per bent and a rectangular 18 in $(457 \mathrm{~mm})$ x 21 in $(533 \mathrm{~mm})$ cap beam as illustrated in Figure 2.2. A column longitudinal reinforcement ratio of $\varrho_{\mathrm{L}}=1.2 \%$, which is just above the minimum required by AASHTO $\left(\varrho_{\mathrm{L}}>1 \%\right)\left(\right.$ AASHTO, 2009), and a transverse reinforcement ratio of $\varrho_{\mathrm{T}}=0.2 \%$, which does not meet current requirements $\left(\varrho_{\mathrm{T}}>0.5 \%\right)$, represent the same reinforcement ratios found in the representative bridge bent. The longitudinal reinforcement in the 
columns consisted of $10 \# 5$ ( $\phi 16 \mathrm{~mm})$ bars equally spaced. The transverse hoop reinforcement was deformed wire D5 $(\phi 6.4 \mathrm{~mm})$ spaced at 6 in $(152.4 \mathrm{~mm})$ center to center. Lap splices were located at the base of the test specimens through the incorporation of 10 \#5 (\$16mm) dowels. The lap splice length was 25 in $(635 \mathrm{~mm})$, which corresponds to 40 times the diameter of the longitudinal steel reinforcement $\left(40 \mathrm{~d}_{\mathrm{b}}\right)$ and replicated the detailing in the representative bridge. The reinforcing steel used to construct the test specimens consisted of Grade 40, $f_{y}=40 \mathrm{ksi}(275.8 \mathrm{MPa}), f_{u}=60 \mathrm{ksi}(413.7 \mathrm{MPa})$, deformed bar conforming to the American Society of Testing and Materials (ASTM) designation A615. The transverse steel consisted of deformed wire D5 conforming the ASTM A496.

Table 5.1 Dimension of representative bridge and test specimen.

\begin{tabular}{l|c|c|c}
\hline \multicolumn{1}{c|}{ Parameter } & Prototype & Specimen & $\begin{array}{c}\text { Scale } \\
\text { Factor }\end{array}$ \\
\hline Column Diameter (in) & 36 & 18 & 0.5 \\
\hline Column Height (in) & 224 & 112 & 0.5 \\
\hline Beam Depth (in) & 42 & 21 & 0.5 \\
\hline Beam Width (in) & 36 & 18 & 0.5 \\
\hline Beam Length (in) & 240 & 120 & 0.5 \\
\hline QLong $(\%)$ & 1.2 & 1.2 & 1 \\
\hline Hoop Spacing (in) & 12 & 6 & 0.5 \\
\hline QTrans $(\%)$ & 0.2 & 0.2 & 1 \\
\hline $\begin{array}{l}\text { Lap splice }\left(\mathrm{d}_{\mathrm{b}}\right) \\
\mathrm{d}_{\mathrm{r}} \text { diameter of long. } \\
\text { reinforcement }\end{array}$ & 40 & 40 & 1 \\
\hline Concrete cover columns (in) & 2 & 1 & 0.5 \\
\hline Concrete cover cap beam (in) & 1.5 & 0.75 & 0.5 \\
\hline Axial Load (\% $\left.\mathrm{A}_{\mathrm{g}} \mathrm{f}_{\mathrm{c}}\right)$ & 10 & 10 & 1 \\
\hline Yield stress, $\mathrm{f}_{\mathrm{y}}(\mathrm{ksi})$ & 40 & 40 & 1 \\
\hline Compressive strength, $\mathrm{f}_{\mathrm{c}}(\mathrm{psi})$ & 3300 & 3300 & 1 \\
\hline
\end{tabular}




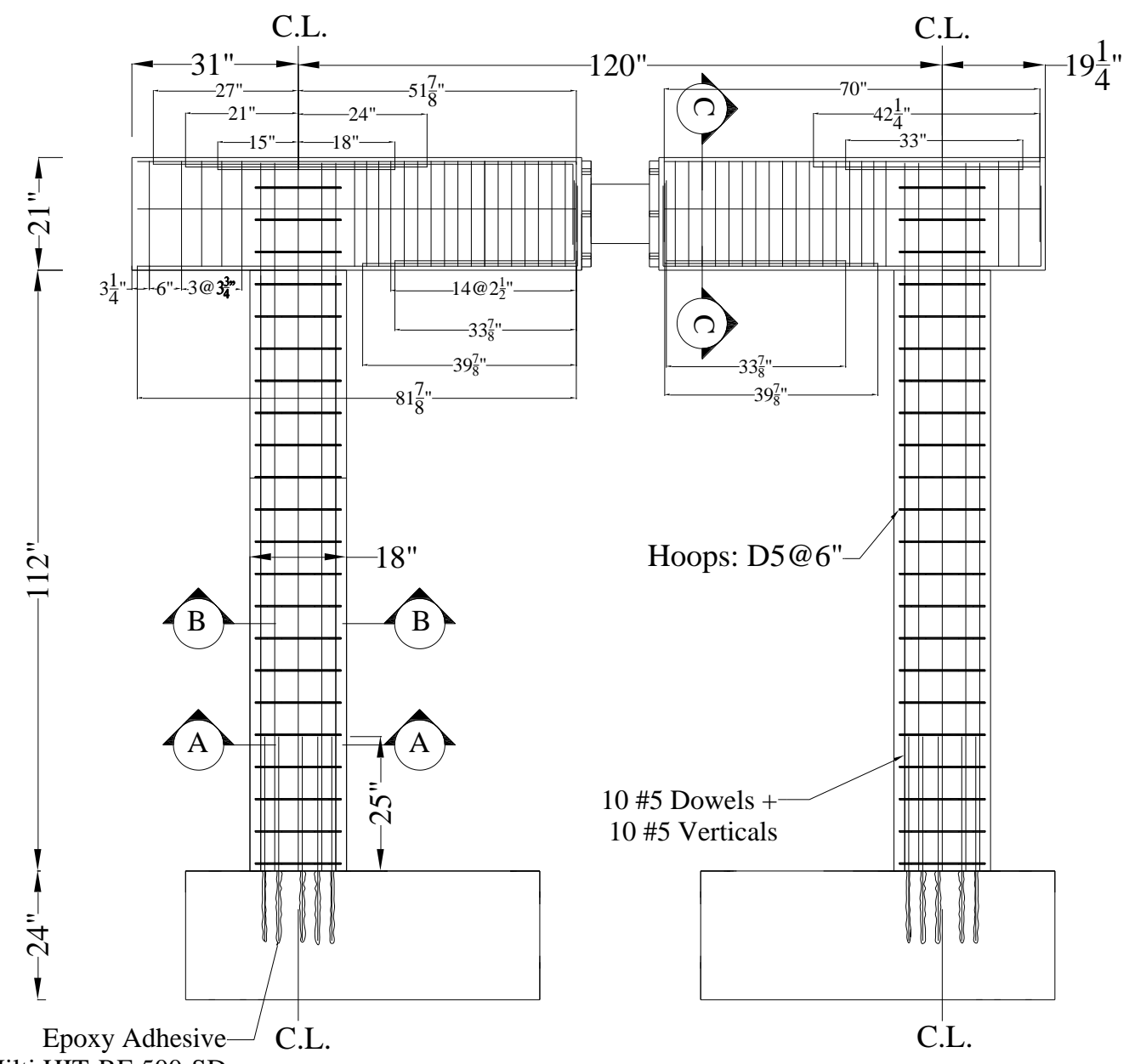
Hilti HIT-RE 500-SD Embedment Depth = 13"

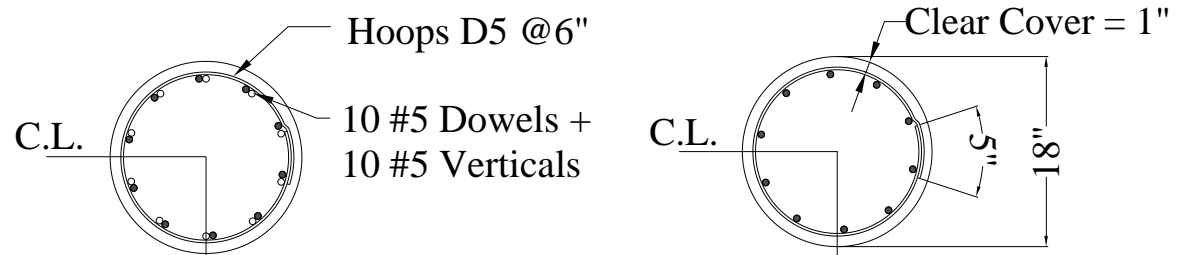

C.L.

\section{Section A - A}

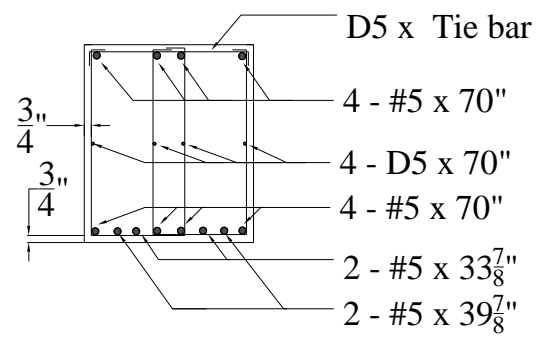

Section C - C
C.L.

Section B - B

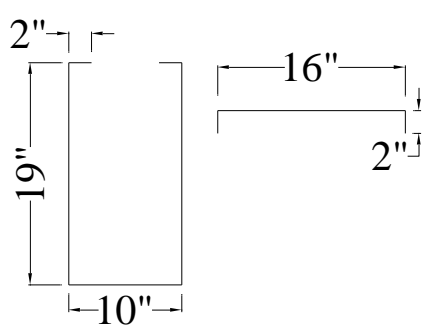

D5 - Double Stirrups + Tie

Figure 5.1 Geometry and reinforcement of RC Bridge bent specimens 
Normal weight concrete was used to construct the test specimens with a target 28day strength $\left(f^{\prime}\right)$ of $3.3 \mathrm{ksi}(22.8 \mathrm{MPa})$. The concrete cover was 1 inch $(25.4 \mathrm{~mm})$ for columns and $3 / 4 "$ " $(19 \mathrm{~mm})$ for the cap beam. Standard compression testing of 6 -inch by 12 -inch concrete cylinders was performed at 7-day, 28 days and at the day of test completion. The average of the concrete cylinder tests are shown in Table 5.2. The yield $\left(f_{y-m e}\right)$ and tensile $\left(f_{u-m e}\right)$ stress, and the elongation of the reinforcing steel obtained from tensile tests are shown in Table 5.3. It is worth noting that the measured values of $f_{c-m e}$ are close to the assessment strengths, $f_{c a}^{\prime}=1.5 f_{c}^{\prime}=34.1 \mathrm{MPa}$ (4.95 ksi), suggested by Priestley for existing bridges (1996).

Table 5.2 Compressive strength of concrete cylinders for RC bents

\begin{tabular}{c|c|c|c|c}
\hline \multirow{2}{*}{ Stage } & Concrete Pour & \multicolumn{3}{|c|}{ Compressive Strength $\left(f^{\prime}\right)$, psi } \\
\cline { 2 - 4 } & & Columns & $\begin{array}{c}\text { Cap } \\
\text { Beam }\end{array}$ & Footing \\
\hline \multirow{2}{*}{$\begin{array}{c}\text { Common for } \\
\text { both stages }\end{array}$} & 7 day & 3961 & 3774 & \\
\cline { 2 - 4 } & 28 day & 4326 & 4168 & \\
\hline $\begin{array}{c}\text { Stage 1, } \\
\text { day of testing } \\
\text { (Retrofit) }\end{array}$ & Bent/BRB1 & 4833 & 4739 & \multirow{2}{*}{4922} \\
\cline { 2 - 4 } & Bent/BRB2 & 4971 & 4776 & \\
\cline { 2 - 4 } $\begin{array}{c}\text { Stage 2, } \\
\text { day of testing } \\
\text { (Repair) }\end{array}$ & As-built 1 & 4998 & 4810 & \\
\cline { 2 - 4 } & Bent/BRB3 & 4906 & 4766 & \\
\hline
\end{tabular}

Table 5.3 Reinforcing steel properties

\begin{tabular}{c|c|c}
\hline Parameter & $\begin{array}{c}\text { Rebar \#5 } \\
(\phi 16 \mathrm{~mm})\end{array}$ & $\begin{array}{c}\text { Wire D5 } \\
(\phi 6.4 \mathrm{~mm})\end{array}$ \\
\hline $\mathrm{f}_{\mathrm{y}_{-} \mathrm{me}, \mathrm{ksi}(\mathrm{MPa})}$ & $50(344.7)$ & - \\
\hline $\mathrm{f}_{\mathrm{y}_{-} \mathrm{me}, \mathrm{ksi}(\mathrm{MPa}}$ & $75.8(522.6)$ & $537.8(78)$ \\
\hline Elongation, $\%$ & 17.5 & 6 \\
\hline
\end{tabular}




\subsubsection{Buckling-Restrained Braces for Retrofitted Bent}

The design of BRBs followed the procedure described in Chapter 4, which uses a structural fuse concept as basis for design. The structural fuse concept states that the Asbuilt bent would respond elastically or with minor damage under major earthquake events. In order to fulfill this design concept and satisfy performance criteria for existing bridges (FHWA, 2006), (ODOT, 2015), the BRB was designed to reduce the displacement demands on the As-built bent under the 500-year and 1000-year earthquake events. This reduction in displacement demand is a direct effect of the retrofit measure as illustrated in Figure 4.7. In this figure, the BRB and the As-built RC bent responses are idealized through loaddisplacement curves. Relevant load-displacement parameters are the yield displacement $\left(\delta_{\mathrm{y}}\right)$ and yield force $\left(V_{y}\right)$ denoted by the superscripts BRB, B and $R$ to designate the brace, Asbuilt and retrofitted responses, respectively. The total capacity of the retrofitted bent can be obtained by adding the contribution of the As-built bent and the BRB since both structural systems act in parallel. The design of a BRB is then reduced to iterate until the BRB stiffness, and consequently the BRB steel core area $\left(\mathrm{A}_{\mathrm{sc}}\right)$ and the length of the reduced section $\left(\mathrm{L}_{\mathrm{c}}\right)$ are determined.

Two BRB designs were considered in the first stage of this study in an effort to assess the influence of BRB stiffness on the overall bent performance. The first BRB design, hereinafter referred to as Bent/BRB1, was specifically designed following the structural fuse concept. The second model, hereinafter referred to as Bent/BRB2, was designed to reflect a common industry practice where the length of the BRB reduced section is equivalent to two thirds of the total BRB length. This resulted in a more flexible brace as compared to Bent/BRB1. For the brace cores, a yield stress of $44.2 \mathrm{ksi}$ (305 MPa) given by the BRB 
manufacturer (Star Seismic), a brace angle $(\theta)$ of 48.7 degrees and a BRB length of 130.625 in (3318 mm) were considered for this application. The Bent/BRB1 was designed for a response spectrum with maximum spectrum acceleration $\left(S_{a}\right)$ of $0.65 \mathrm{~g}$ with a period at the end of constant design spectral acceleration plateau $\left(T_{s}\right)$ equal to $0.49 \mathrm{sec}$ for the 500 -year event, and $0.85 \mathrm{~g}$ with $T_{s}$ equal to $0.53 \mathrm{sec}$ for the 1000 -year event as shown in Figure 4.2. These spectrum accelerations were chosen in an effort to represent acceleration demands of a vast number of vulnerable bridges in the State of Oregon. The area of the BRB steel core within the reduced section and the length of the reduced section $\left(L_{c}\right)$ for both models are included in Table 2.1. While both braces have the same nominal strength, the brace in Bent/BRB1 has the reduced section length approximately 1/3 of the brace in Bent/BRB2. This resulted in approximately three times the overall brace stiffness. The specified material properties for the restraining mechanism were steel A500-Gr B, $f_{y}=46 \mathrm{ksi}(317 \mathrm{MPa})$ and $f_{u}$ $=58 \mathrm{ksi}(400 \mathrm{MPa})$, and $f_{c}^{\prime}=5 \mathrm{ksi}(34.5 \mathrm{MPa})$ for the steel HSS and encasing mortar, respectively. An example of the BRBs layout used in this study is shown in Figure 5.2.

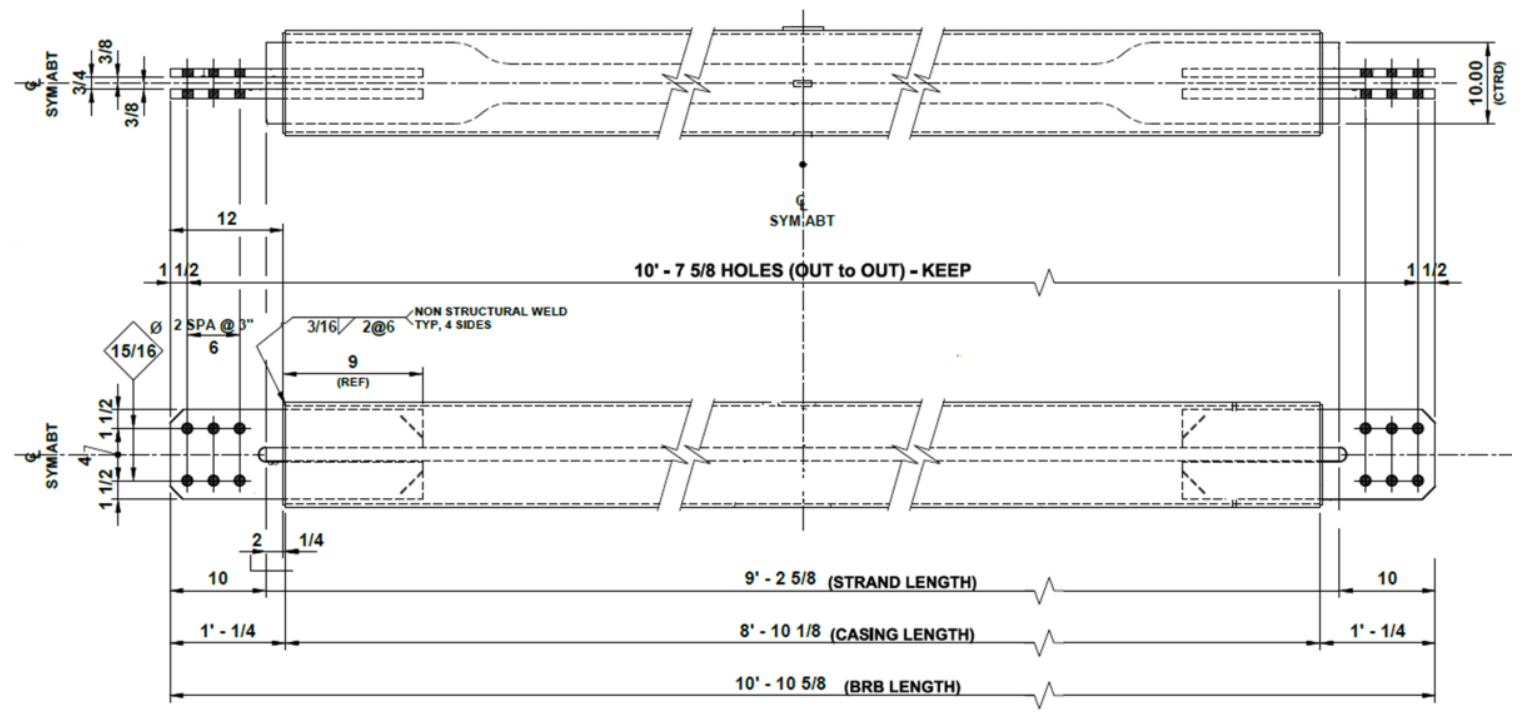

Figure 5.2 Example of BRB layout 
Table 2.1 also shows the displacement demands in the retrofitted bent. The analytical response for each retrofitted system is depicted in Figure 5.3. This figure shows that the Bent/BRB1 can achieve better performance levels than those required by ODOT (2015), resulting in an "Operational" performance level for both seismic hazards (500-year as well as 1000-year events). Further, the Bent/BRB2 still satisfies ODOT requirements, which are an “Operational" performance level under the 500-year event and a "Life Safety" performance level under the 1000-year event.

Table 5.4 Results of BRB designs for Bent/BRB1 and Bent/BRB2

\begin{tabular}{c|c|c|c|c}
\hline \multirow{2}{*}{ Specimen } & \multirow{2}{*}{$\begin{array}{c}\text { Steel Core } \\
\text { Area }\left(\text { in }^{2}\right)\end{array}$} & \multirow{2}{*}{$\begin{array}{c}\text { Reduced Section } \\
\text { Length (in) }\end{array}$} & \multicolumn{2}{|c}{$\begin{array}{c}\text { Displacement Demand } \\
\text { (in) }\end{array}$} \\
\cline { 4 - 5 } & & 30 & $\delta^{\mathrm{R}}{ }_{500}$ & $\delta^{\mathrm{R}}{ }_{1000}$ \\
\hline Bent/BRB1 & 1.2 & 88.6 & 0.5 & 0.7 \\
\hline Bent/BRB2 & 1.2 & 0.8 & 1.2 \\
\hline
\end{tabular}

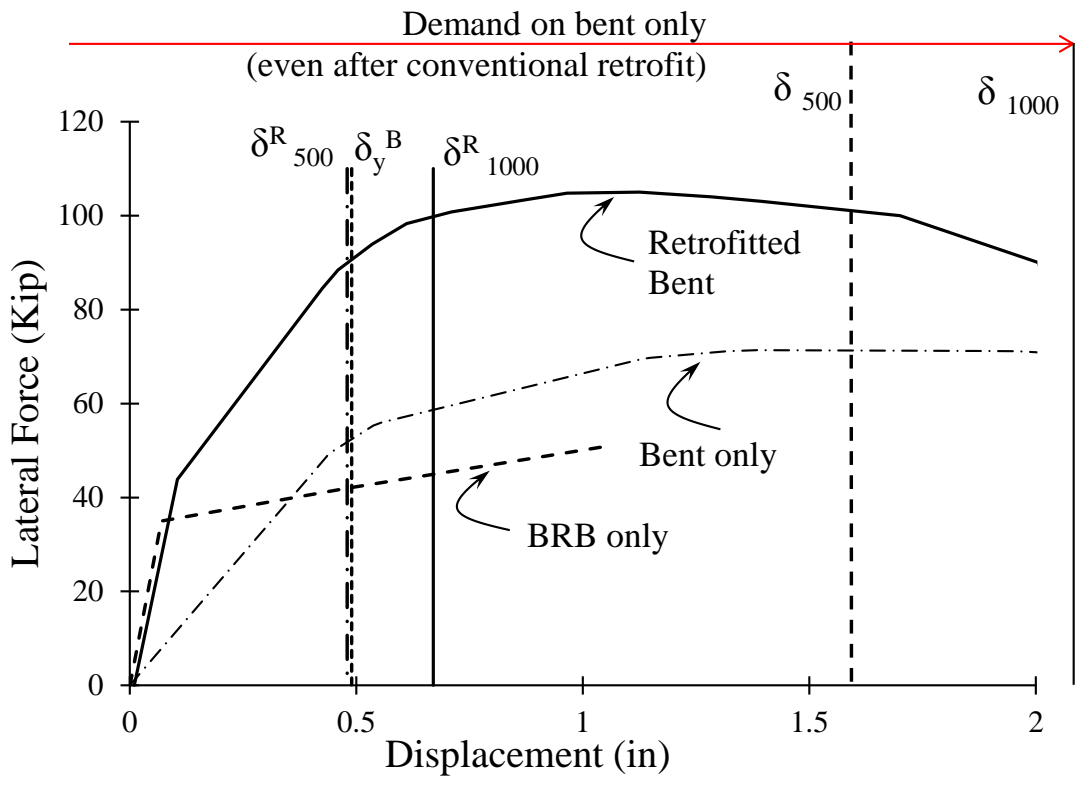

(a) 


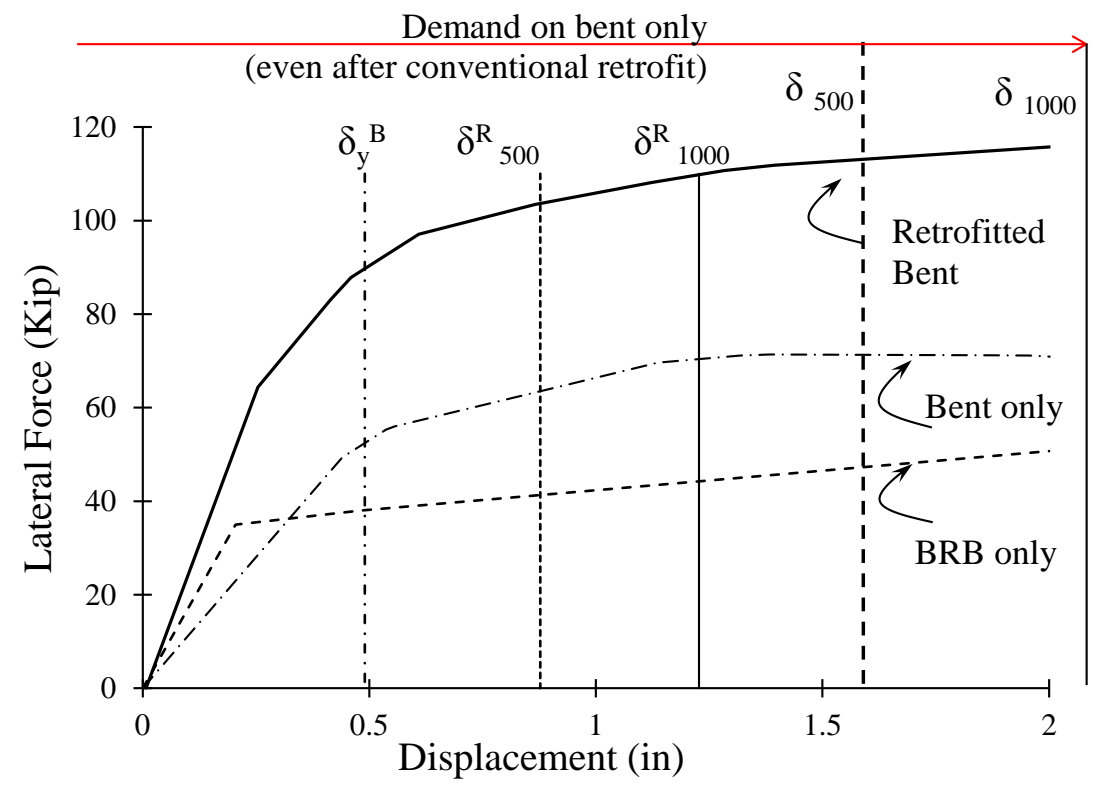

(b)

Figure 5.3 Expected load vs displacement behavior of retrofitted bent specimens.

(a) Bent/BRB1; (b) Bent/BRB2

\subsubsection{Buckling-Restrained Brace for Repaired Bent}

One BRB design (BRB3) was considered for repairing a previously damaged bent

(As-Built 2). The design was performed following the structural fuse concept stated on

Chapter 4. To account for the reduced stiffness and strength of the damaged bent a stiffness factor $\left(\lambda_{\mathrm{k}}\right)$ equal to 0.5 and a strength factor $\left(\lambda_{\mathrm{k}}\right)$ equal to 0.8 were applied to the stiffness and strength of the undamaged bent. These factors were assumed in an effort to represent the stiffness when the concrete has already spalled. For the brace, a yield stress of $43.4 \mathrm{ksi}$ (299 $\mathrm{MPa}$ ) given by the BRB manufacturer (CoreBrace), a brace angle ( $\theta)$ of 48.7 degrees and a BRB length of 130.625 in $(3318 \mathrm{~mm})$ were considered for this application. The Bent/BRB3 was designed for the response spectrums shown in Figure 4.2. The area of the BRB steel core within the reduced section and the length of the reduced section $\left(L_{c}\right)$ for the BRB used in the repaired bent is indicated in Table 5.5. The expected response for the repaired system 
is depicted in Figure 5.4. This figure shows that the Bent/BRB2 may achieve operational performance level for both seismic hazard events (500-year and 1000-year events).

Table 5.5 Results of BRB design for repaired bent

\begin{tabular}{c|c|c|c|c}
\hline \multirow{2}{*}{ Specimen } & \multirow{2}{*}{$\begin{array}{c}\text { Steel Core } \\
\text { Area }\left(\text { in }^{2}\right)\end{array}$} & \multirow{2}{*}{$\begin{array}{c}\text { Reduced Section } \\
\text { Length (in) }\end{array}$} & \multicolumn{2}{|c}{$\begin{array}{c}\text { Displacement Demand } \\
\text { (in) }\end{array}$} \\
\cline { 4 - 5 } & & & $\delta^{\mathrm{Re}}{ }_{500}$ & $\delta^{\mathrm{Re}}{ }_{1000}$ \\
\hline Bent/BRB3 & 1.2 & 68.9 & 0.9 & 1.2 \\
\hline
\end{tabular}

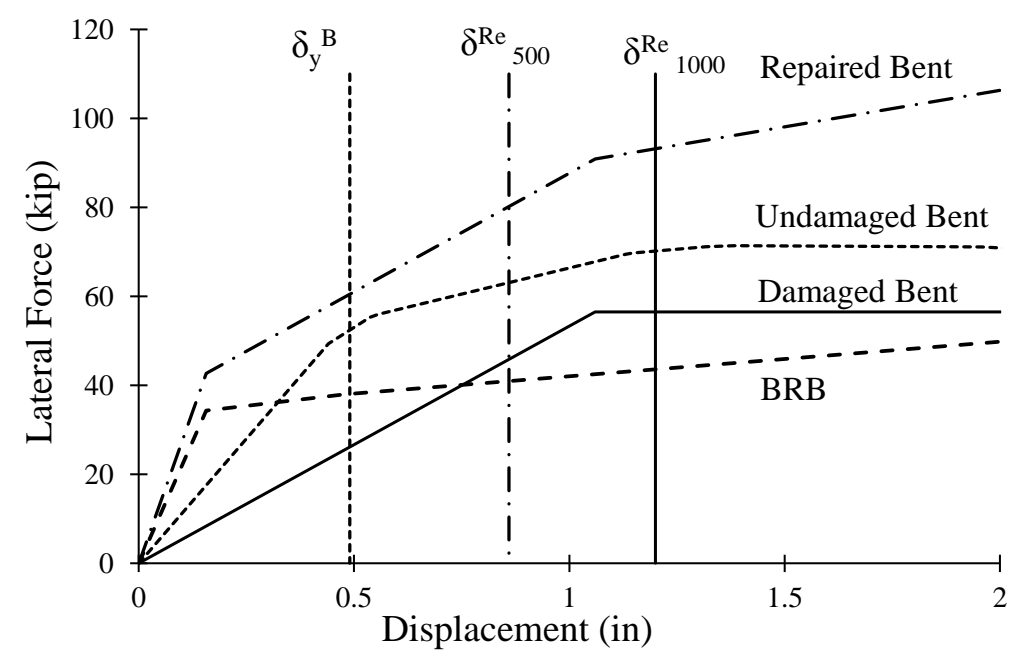

Figure 5.4 Expected load vs displacement behavior of the repaired bent specimen

\subsubsection{Brace-to-Bent Connection}

A novel gusset plate to RC bent connection was developed in the experimental program. In this connection, the gusset plates were directly connected to horizontal RC elements without interfering with the columns. A gap of 1 in $(25 \mathrm{~mm})$ was intentionally designed to allow column movement relative to the gusset plates as shown in Figure 5.5. This connection was utilized since previous experimental tests in steel frames have shown that high stresses caused by opening and closing the angle between the column and the horizontal element in column-footing and column-beam interfaces can lead to extensive damage and deterioration of the connection itself and the surrounding area (Mahin, et al., 
2004). This approach also avoided potential shortening of the effective height of the columns.
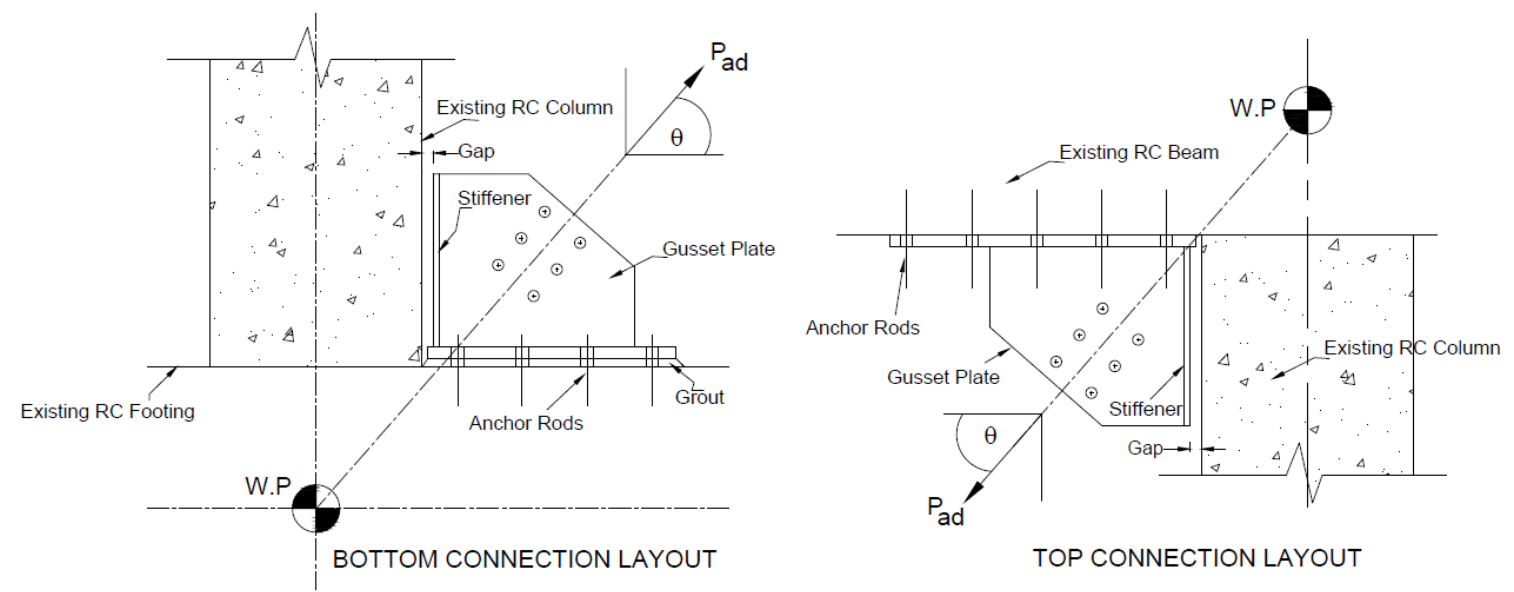

Figure 5.5 Novel brace-to-bent connection

Any BRB to gusset plate connection need to be designed to resist the demands based on an adjusted brace strength as per AISC 341-10 (2010). In this study the adjusted brace strength in compression was computed as $\beta \omega R_{y} P_{y s c}=76.9$ kips $(342 \mathrm{kN})$, where $\beta \omega$ is an over-strength factor in compression; $R_{y}$ is the ratio of the expected yield stress to the specified minimum yield stress, and $P_{y s c}$ is the axial yield strength of the steel core. A factor of 1.45 for $\beta \omega$ was used as suggested by the manufacturer, and $R_{y}$ was computed as 1 since the value of $P_{y s c}$ was established using the yield stress directly measured from a steel coupon. The limit states that were checked for the design of the gusset plates according to AASHTO (2009) and AISC, Section J (2010) are: tensile yielding of Whitmore section, tensile rupture of Whitmore section, block shear in tension, buckling in compression, yield moment strength, plastic moment strength, weld/bolts for gusset-to-brace connection, slip critical connection, and buckling of unsupported edges. Six 7/8 in $(\phi 22 \mathrm{~mm})$ high strength Group A (ASTM A325) bolts were used in the BRB-to-gusset connection. For the bolted connection the shear and the slip-critical capacity were compared to the adjusted demand as follows: 
The reduced shear strength $\left(\phi \mathrm{R}_{\mathrm{nv}}\right)$ of six $7 / 8$ in Group A bolts is given by Eq. (2.1) (ANSI/AISC 360-10, 2010).

$$
\phi R_{n v}=N_{\text {bolts }} \cdot 0.75 \cdot F_{n v} \cdot A_{b} \cdot n_{s}=292 k i p s>77 k i p s \quad O K
$$

Where $\mathrm{N}_{\text {bolts }}$ is the number of bolts used in the connection; $\mathrm{F}_{\mathrm{nv}}$ is the nominal shear stress that for Group A bolts when the threads are not excluded from shear planes is equal to $54 \mathrm{ksi} ; \mathrm{A}_{\mathrm{b}}$ is the nominal area of bolt; and $\mathrm{n}_{\mathrm{s}}$ is the number of shear planes that in this case was equal to 2 . The results show that the shear capacity of the bolts vastly exceeds the demand on them.

The slip-critical strength for the same group of bolts is given by Eq. (5.2) (ANSI/AISC 360-10, 2010).

$$
\phi R_{n-s l i p}=N_{b o l t s} \cdot \mu \cdot D_{u} \cdot h_{f} \cdot T_{b} \cdot n_{s}=159 \text { kips }>77 \text { kips } \quad O K
$$

Where $\mu$ is the mean slip coefficient that for Class A surfaces is equal to $0.30 ; D_{u}$ is a multiplier (1.13) that accounts for the difference between the mean installed bolt pretension and the specified minimum bolt pretension; $h_{\mathrm{f}}$ is a factor that reflects the use of fillers that for two or more fillers is equal to 0.85 and the other cases equal to 1.0; and $\mathrm{Tb}$ is the minimum fastener pretension that for $7 / 8$ in bolts is equal to 39 kips. The demand-tocapacity ratio for the slip-critical connection was 0.49 .

In the gusset plates, steel ASTM A572 Gr50 with a thickness of 5/8 inches was considered appropriate for this application. The demand-to-capacity ratios of the limit states required by AASHTO and AISC are indicated in Table 5.6, which shows that a conservative design was done in order to prevent any failure in the connection. Moreover, in order to prevent buckling, the maximum length of any free edge $\left(\mathrm{L}_{\mathrm{fg}}\right)$ was computed using Eq. (5.3) . 
Where, $\mathrm{E}$ is the modulus of elasticity of steel (29000 ksi), $\mathrm{f}_{\mathrm{y}}$ is the yield stress $(50 \mathrm{ksi})$, and $t$ is the plate thickness.

$$
L_{f g}(\text { in })=0.75 \cdot \sqrt{\frac{E}{f_{y}}} \cdot t=11 \text { in }
$$

Table 5.6 Capacity-to-demand ratios for the gusset plate

\begin{tabular}{c|c|c|c}
\hline Limit State & Capacity (kips) & Demand (kips) & Ratio \\
\hline Bolt Bearing & 442 & 77 & 0.17 \\
\hline Block shear & 244 & 77 & 0.32 \\
\hline Whitmore section & 272 & 77 & 0.28 \\
\hline Buckling & 251 & 77 & 0.31 \\
\hline
\end{tabular}

Post-installed adhesive anchors were designed for connecting the gusset plate to the concrete elements. The design forces were obtained from the adjusted brace strength and the corresponding gusset plate free body diagram as shown in Figure 5.6. Tensile and shear strength were considered in the connection design according ACI318-11, Appendix D (2011). The shear-tension interaction for the post-installed adhesive anchors using the trilinear interaction approach was 1 . The anchor rod design was also checked using the free software Profis developed by Hilti as shown in Figure 5.7.

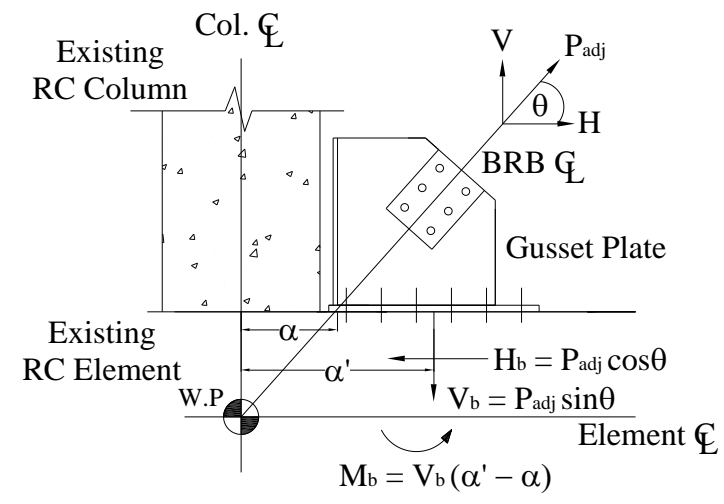

Figure 5.6 Free body diagram used in the design of the gusset plate-to-bent connection 


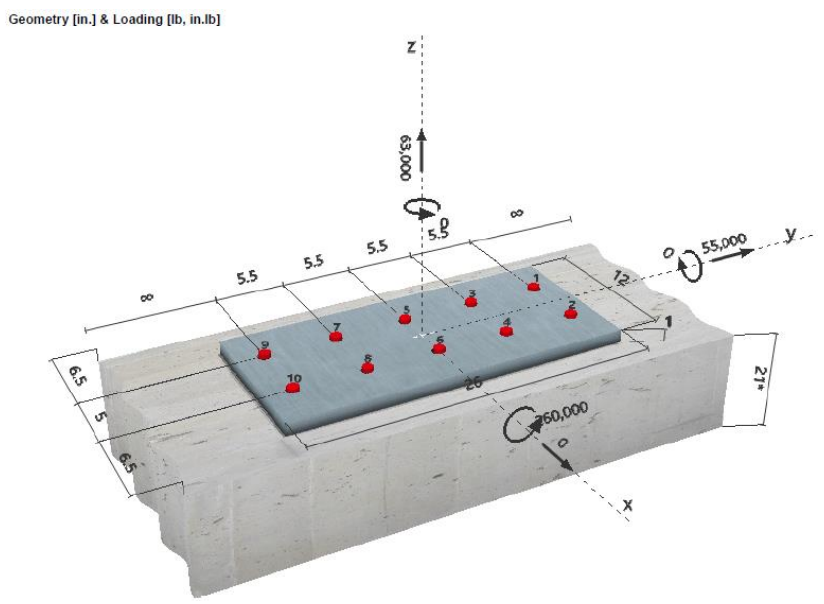

Figure 5.7 Anchor rods design example (top connection)

The retrofit design resulted in a unique steel-concrete retrofit connection that did not rely on an additional steel frame. Figure 5.8 and Figure 5.9 show the layout of the base and top connection, respectively. Figure 5.10 shows the connection as implemented in the experimental program.

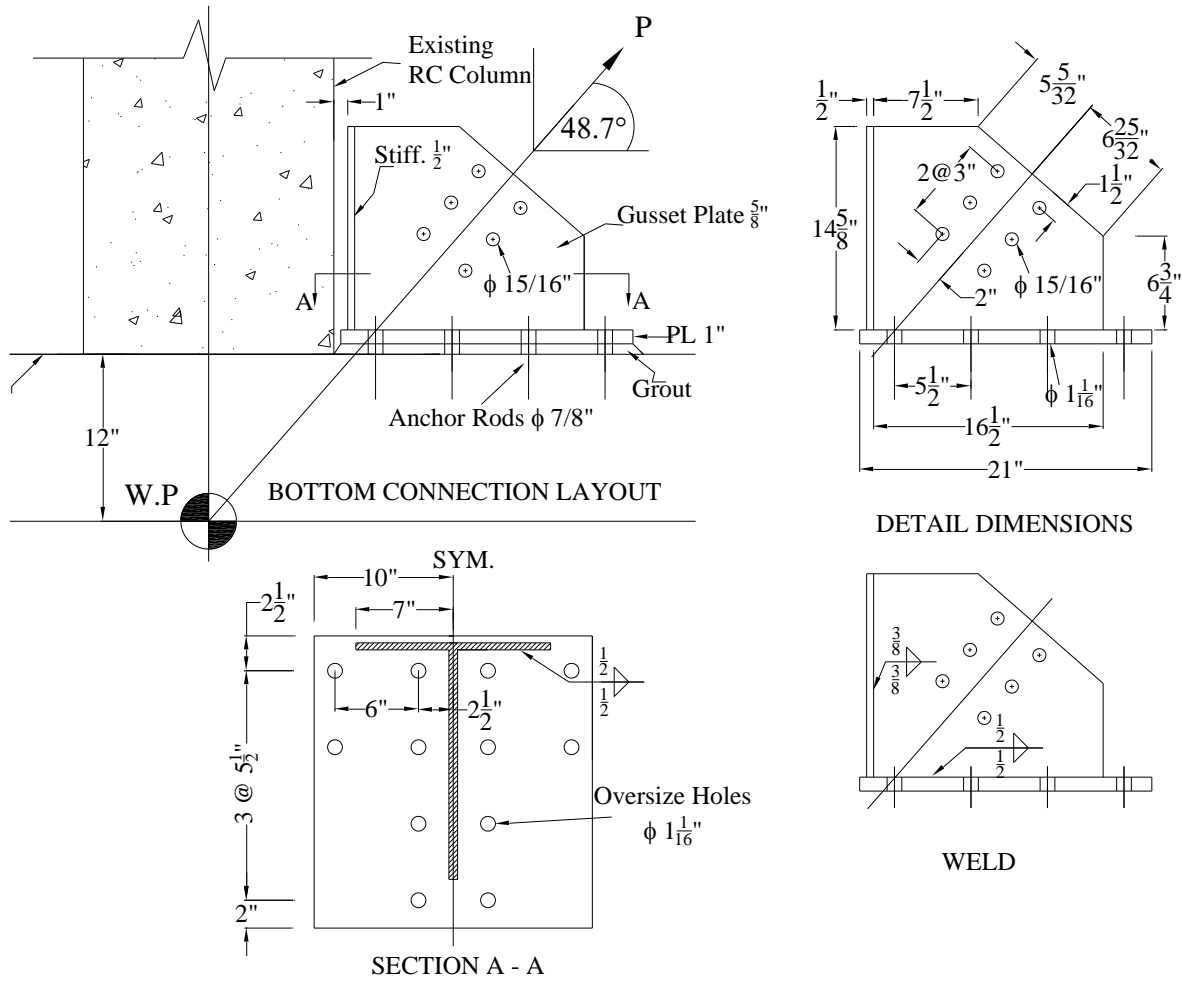

Figure 5.8 Detailing of base connection 


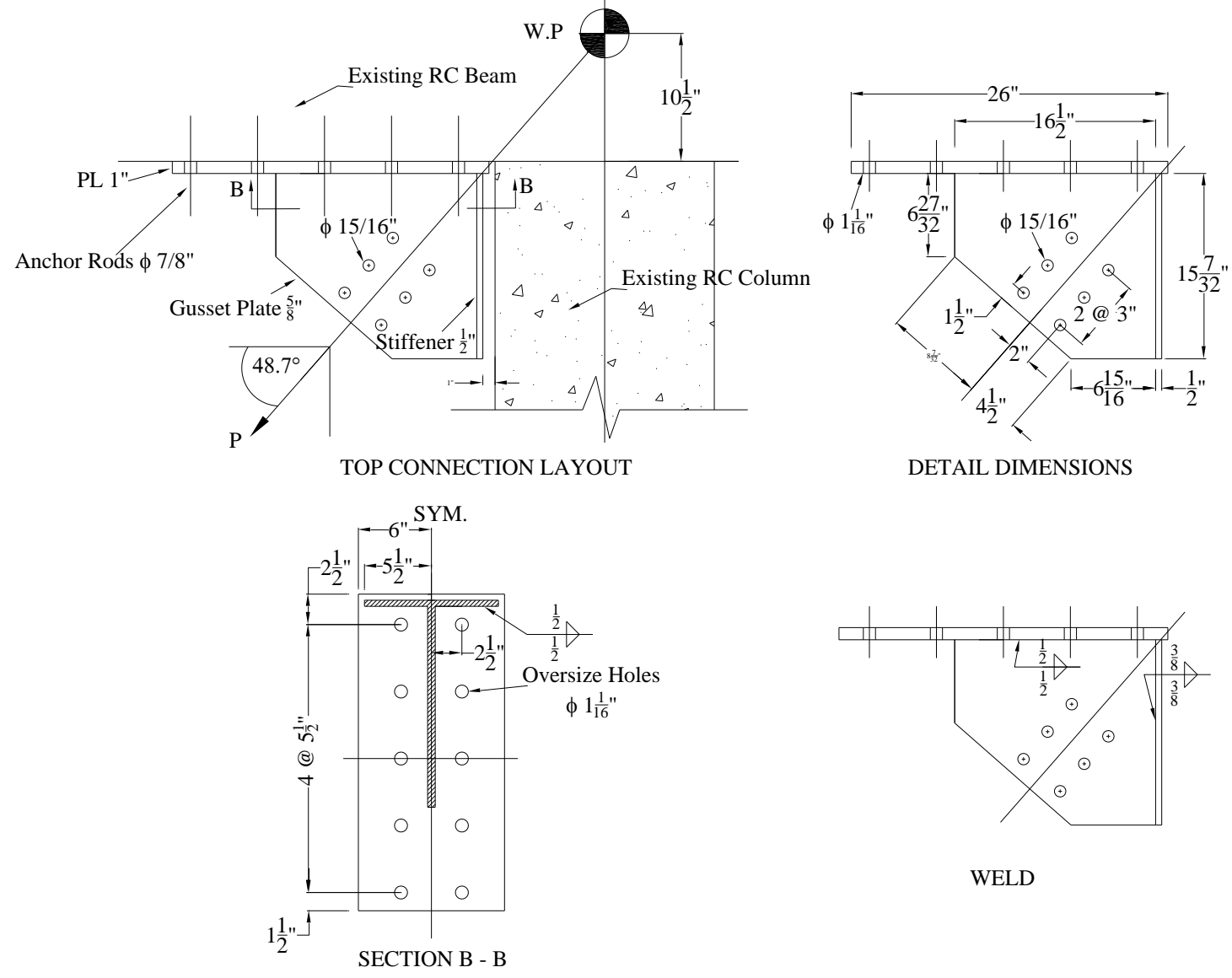

Figure 5.9 Detailing of top connection

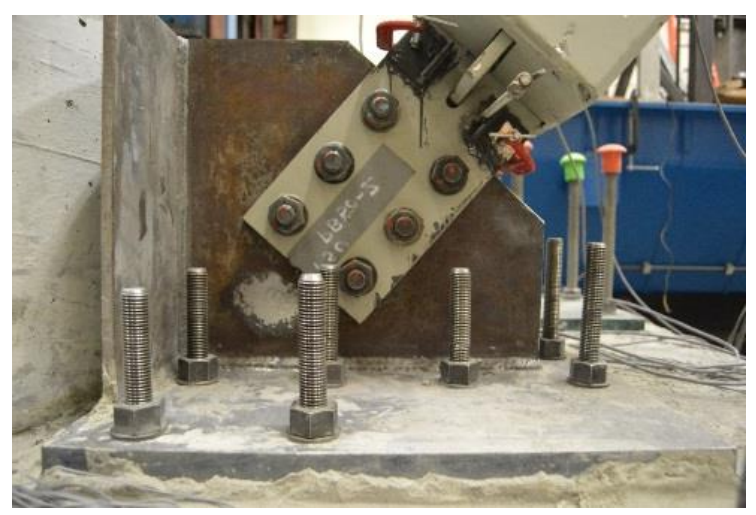

(a)

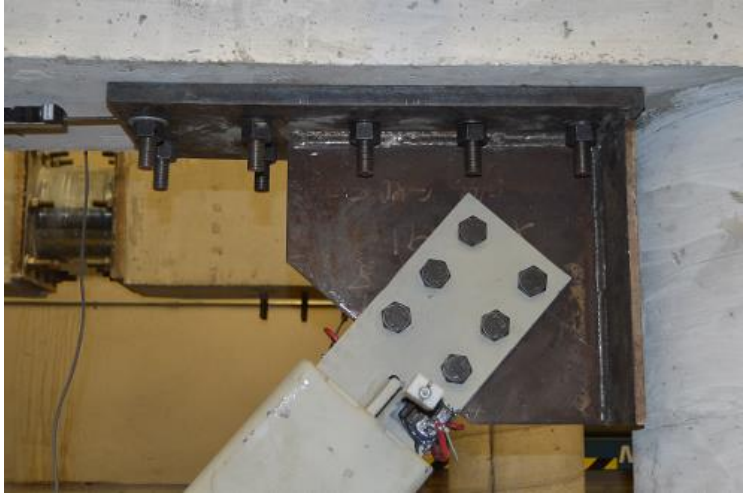

(b)

Figure 5.10 Gusset plate connection. (a) Base; (b) Top 


\subsection{EXPERIMENTAL LAYOUT}

A schematic representation and the actual test setup are shown in Figure 5.11. The cyclic lateral loading was applied through a horizontal hydraulic actuator capable of applying a maximum load of \pm 220 kips $( \pm 979 \mathrm{kN})$ with a maximum stroke of \pm 10 inches. The actuator was controlled through a MTS Flex'Test controller. The actuator was attached to a steel connector beam at the bent cap level. The connector beam was linked to end plates on the other side of the cap beam through the use of high strength rods that were not tensioned, but instead used to apply the reversing load on the opposite side of the bent. The lateral force was applied under displacement control and load cells were used to monitor the applied lateral load during testing. To simulate the superstructure dead load on the columns, a constant load equal to $10 \%$ of the nominal column axial capacity $\left(0.10 f^{\prime}{ }^{\prime} A_{g}=84\right.$ kips, 373.5 $\mathrm{kN}$ ) was applied through two high-strength rods and hydraulic rams. The application of this axial load was performed after the BRB and all the instrumentation were installed. The gravity load was continuously monitored throughout the testing using load cells located on top of the rams. A six degree of freedom (6DOF) load cell was integrated at mid-span of the cap beam in order to directly measure the internal forces that were transmitted from one side of the bent to the other, and thereby allow for indirect calculation of the internal loads within the bent, including the axial force in the BRB. In order to prevent out-of-plane displacements of the RC bent a restraining system was implemented through two HSS steel sections pin connected on one side to the cap beam and on the other to a horizontal steel beam as shown in Figure 5.12. The RC footing was secured to the laboratory floor with post-tensioning rods. Test setup details are depicted in Figure 5.13. 

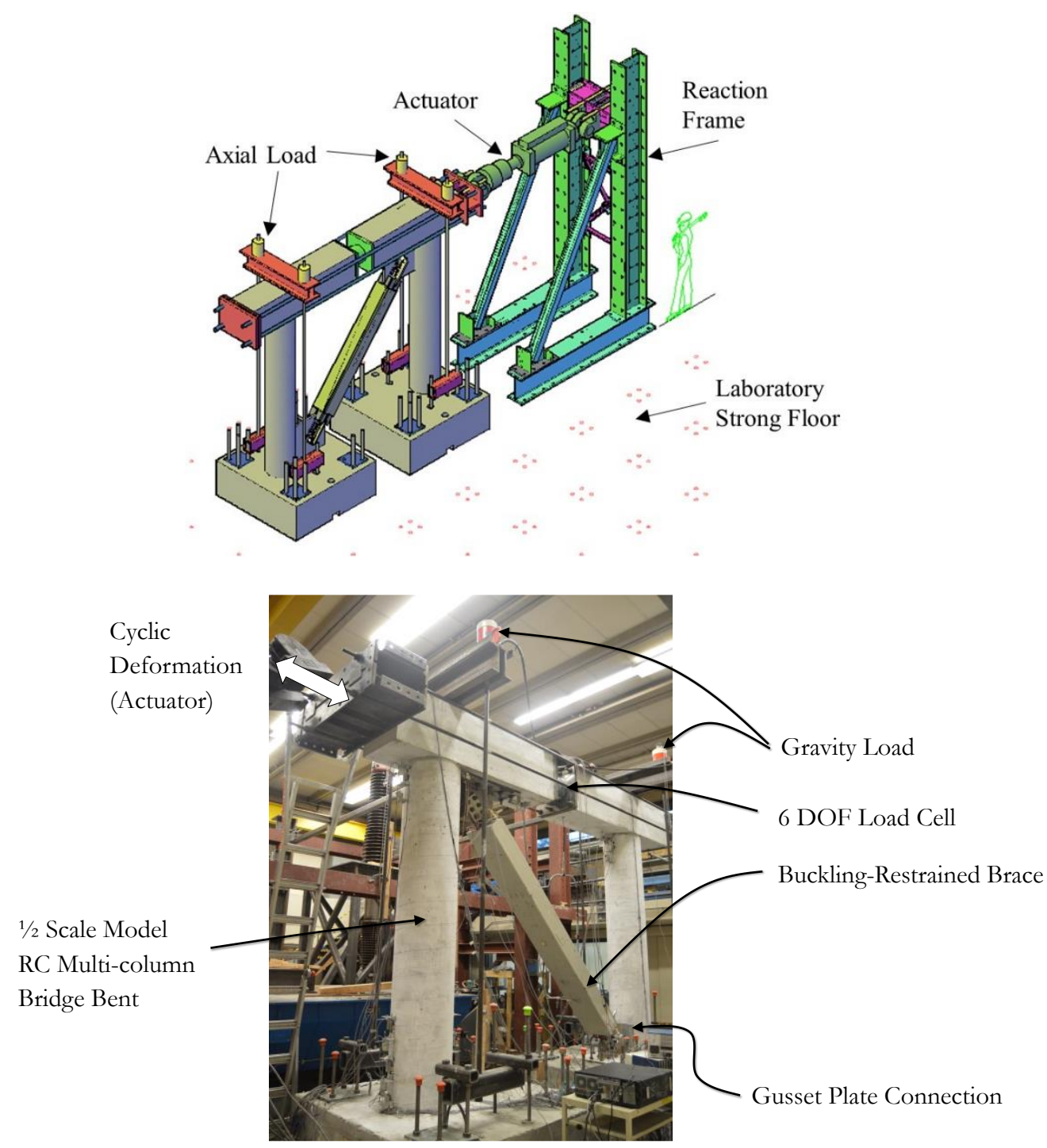

Figure 5.11 Schematic and actual of test setup configuration
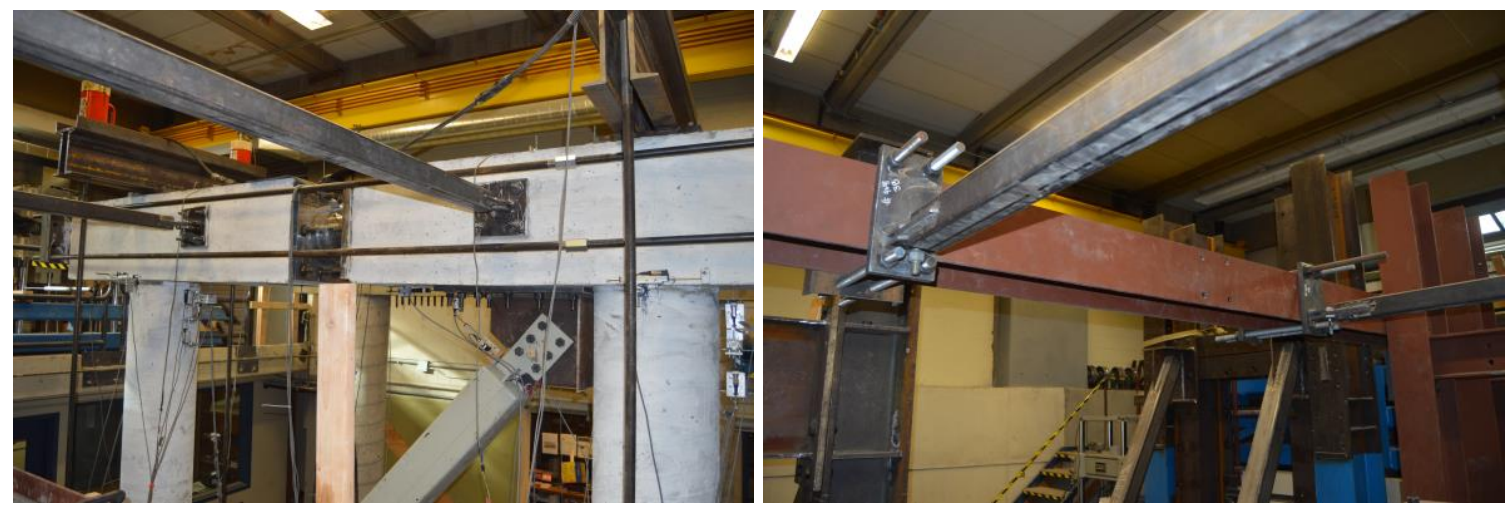

Figure 5.12 Out-of-Plane restraining system 


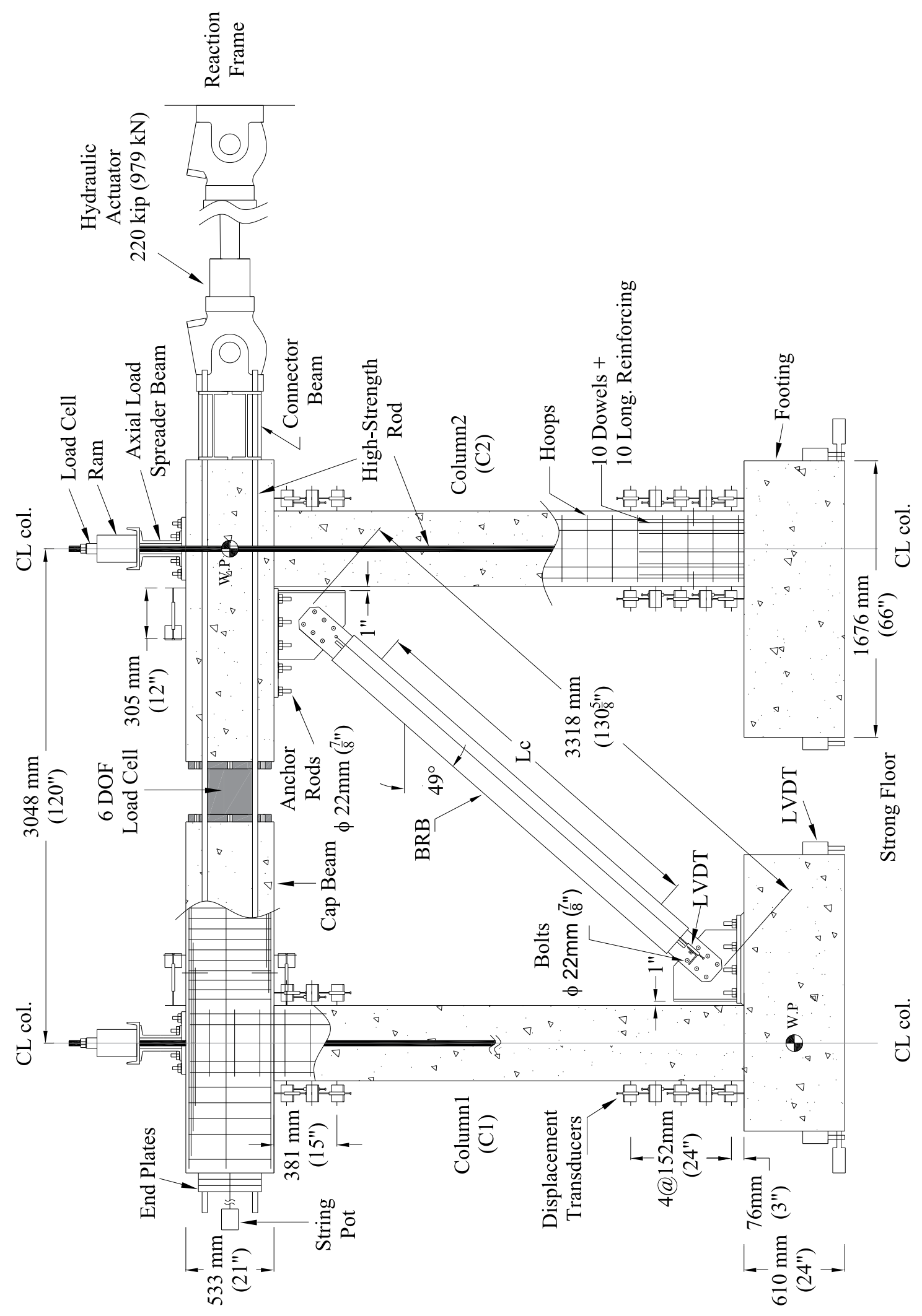

Figure 5.13 Test setup and instrumentation on RC bent 


\subsubsection{Instrumentation}

In order to analyze the response of the specimens, the bents were fully instrumented via linear variable displacement transducers (LVDTs), string pots, strain gauges, and load cells.

LVDTs were utilized to measure relative displacements and rotations, namely, top displacement of bent through the use of a string pot located at mid-height of the cap beam (3112 mm, $122.5 \mathrm{in}$ ), curvature of columns and beams through the use of displacement transducers located at the base and top of columns, and top and bottom of beam, rotation and slippage of foundations through the use of three LVDTs on each footing, and elongation of the BRB through the use of four displacement transducers located on opposite sides of the BRB as shown in Figure 5.13. Additional LVDTs (not shown in Figure 5.13 for clarity) were also installed in the gusset plate to measure, namely, the slippage between brace and gusset plate, rotation of the gusset plate, and slip between the gusset plates and the RC elements as depicted in Figure 5.14.

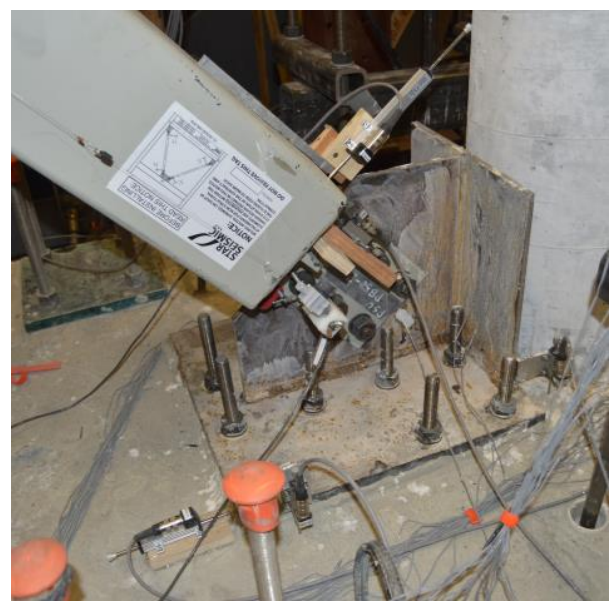

Figure 5.14 Additional instrumentation on brace-to-bent connection

Strain gauges were used to measure strains at specific locations within the specimens as shown in Figure 5.15. A total of 90 strain gauges were installed on each bent: 8 strain 
gauges in the dowels embedded in the footing, 24 in the column dowels, also referred to as starter bars, 48 in the longitudinal reinforcement of the columns, 4 in the transverse reinforcement of the columns, 4 in the cap beam, and 2 in the gusset plates. Strain gauges in the $\mathrm{RC}$ bent were placed at expected plastic hinge zones of columns and cap beam.

Installation of strain gauges was performed prior to the placement of the concrete as shown in Figure 5.16.

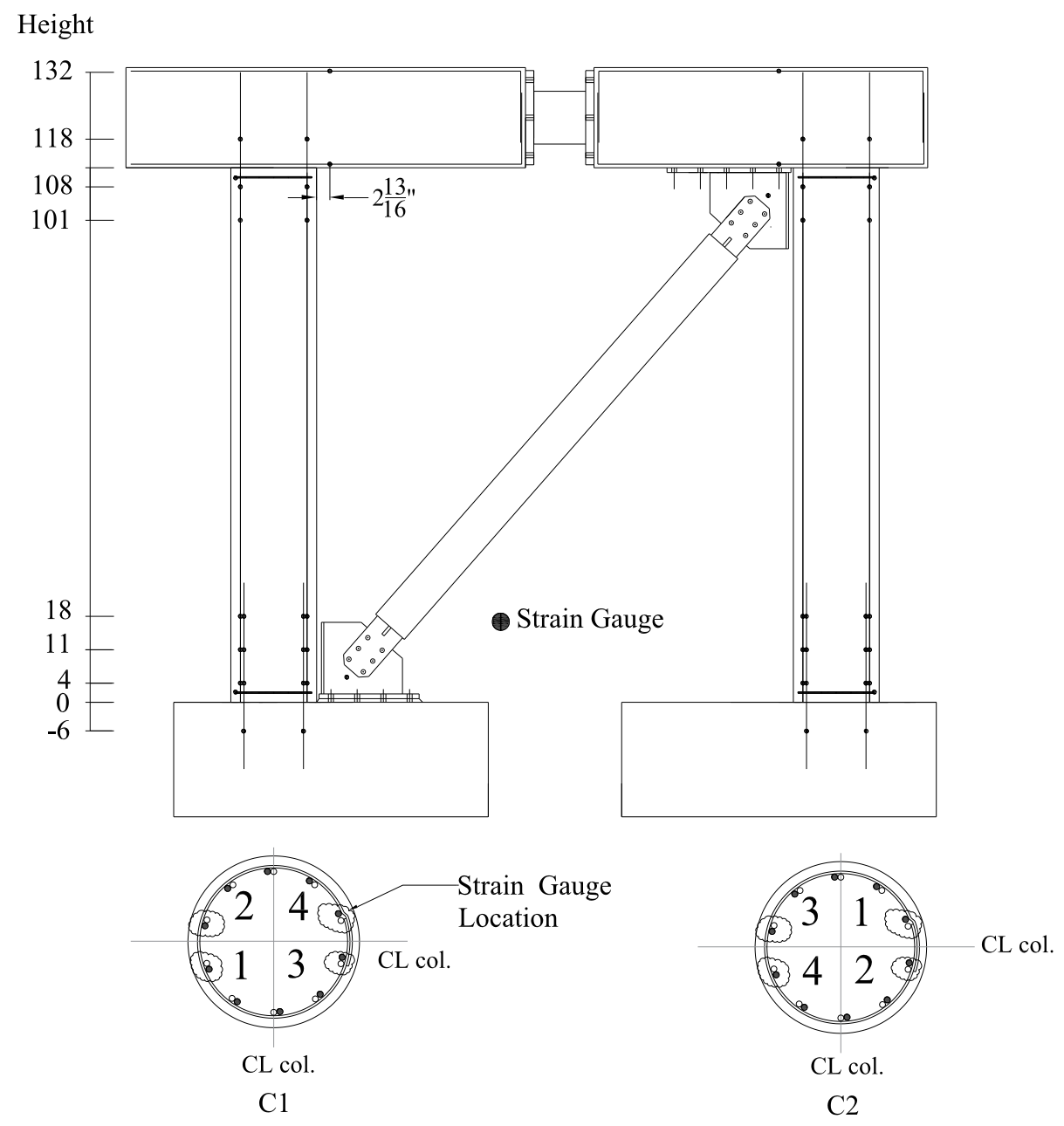

Figure 5.15 Location of strain gauges 


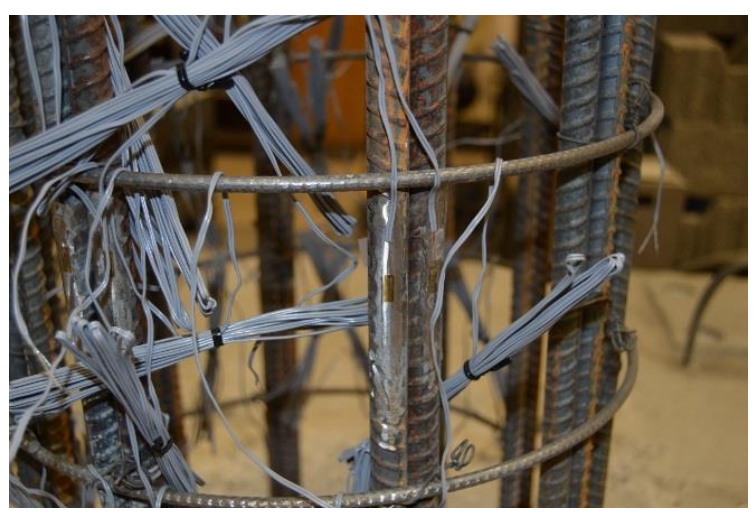

(a)

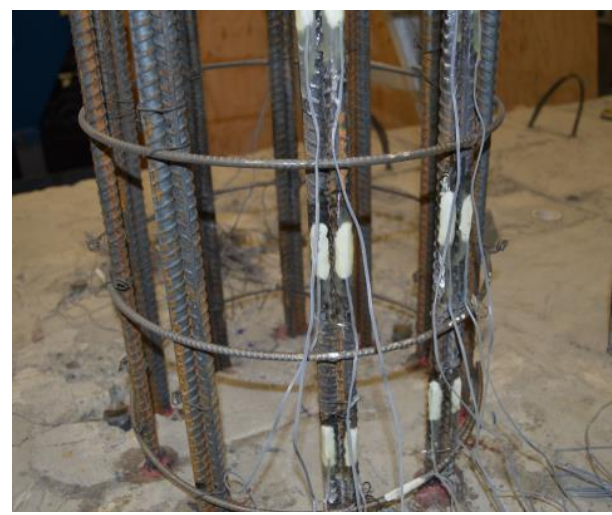

(b)

Figure 5.16 Installation of strain gauges. (a) Strain gauges on longitudinal reinforcement and dowel, (b) Strain gauges with protective coating.

Load cells were used to measure the load transferred to the specimen. The load cells used in this study comprise a load cell located in the actuator to monitor the applied lateral load during testing; four load cells with a capacity of 100 kips in compression to monitor the application of axial load as shown in Figure 5.17; and a six degree of freedom (6DOF) load cell integrated at mid-span of the cap beam in order to directly measure the internal forces that were transmitted from one side of the bent to the other. The 6DOF load cell had an axial capacity of 500 kips, shear capacity of 350 kips, and a moment capacity of 1500 kip-in. Figure 5.18 shows the load cell used in this study.

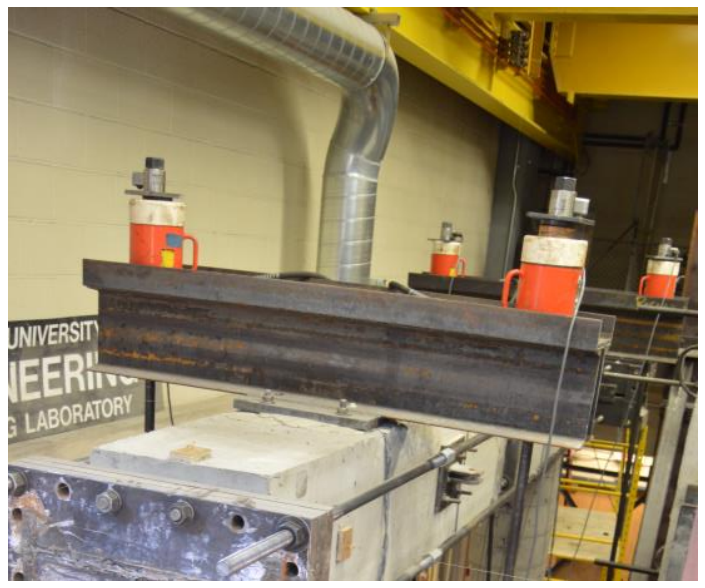

Figure 5.17 Load cells to monitor axial load 

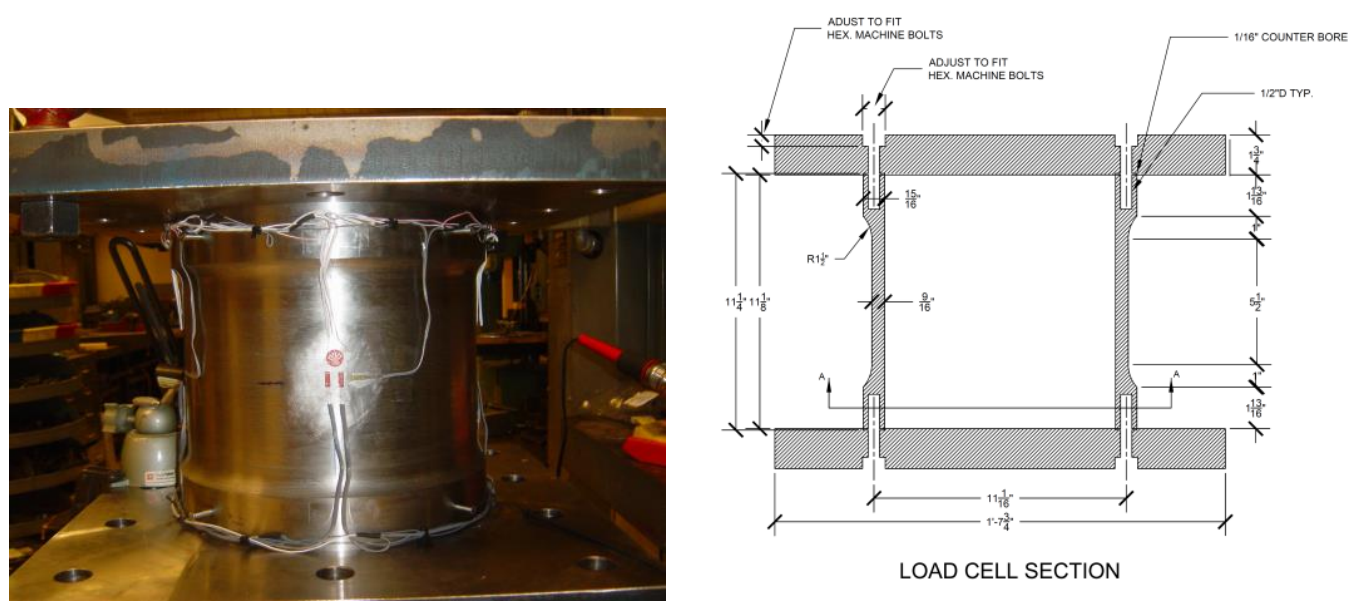

Figure 5.18 6-DOF load cell used in the experimental program

All test data from LVDTS, load cells and strain gauges was collected using two

National Instrument Data Acquisition Systems (DAQs) connected to a terminal computer.

Data were displayed and recorded using the software LabView from National Instruments.

\subsubsection{Construction Sequence}

The sequence of construction for the RC bents is depicted in Figure 5.19. Existing rectangular footings were used in the experimental program. For that reason, in order to install No. 5 dowels into the footings, holes were drilled and epoxy adhesive, Hilti RE-500 SD, was used as shown in Figure 5.19(a). Dowels were embedded 13 inches into the footing in an effort to develop the maximum tensile strength of the bars. After the dowels were installed, the longitudinal reinforcing steel and the circular hoops were tied as shown in Figure 5.19(b). Once the steel cages for the columns were completed and strain gauges installed, 18 in diameter Sonotubes were utilized as formwork. Sonotubes were braced together in order to restrict any movement during the concrete pour as shown in Figure 5.19(c). Four columns were pour using the same day and batch of concrete. Once the columns were built, they were put in position, steel cages for the cap beams were assembled, 
cap beam formwork was built, and concrete was poured as shown in Figure 5.19(d), (e), (f) and (g), respectively.

Gusset plates were installed using post-installed anchors as shown in Figure 5.20. The braces were installed using an overhead crane and a system of pulleys as shown in Figure 5.21. The braces were connected to the gusset plates through eight high strength bolts. The turn-of-the nut method was followed to tight the bolts and allowed for a slipcritical connection.

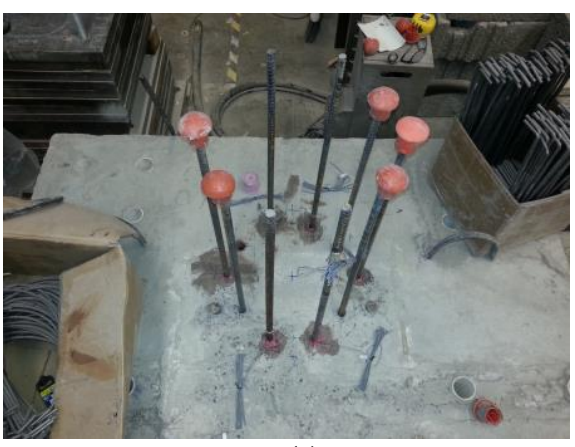

(a)

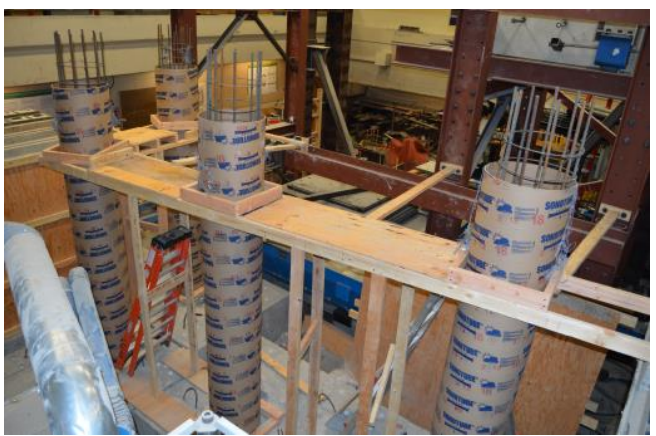

(c)

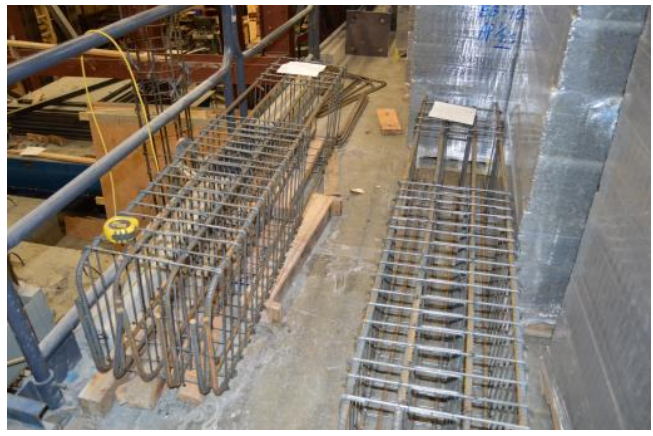

(e)

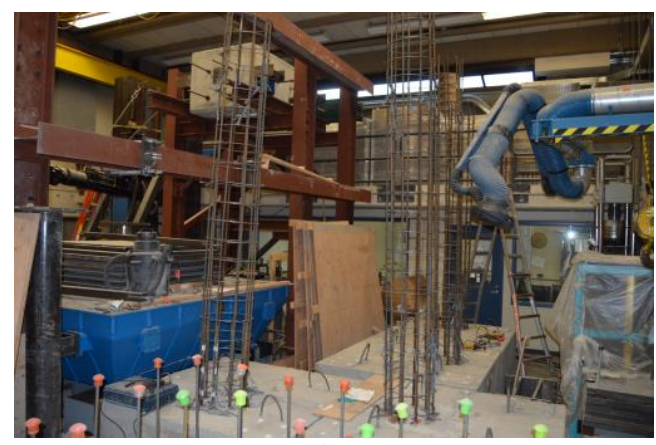

(b)

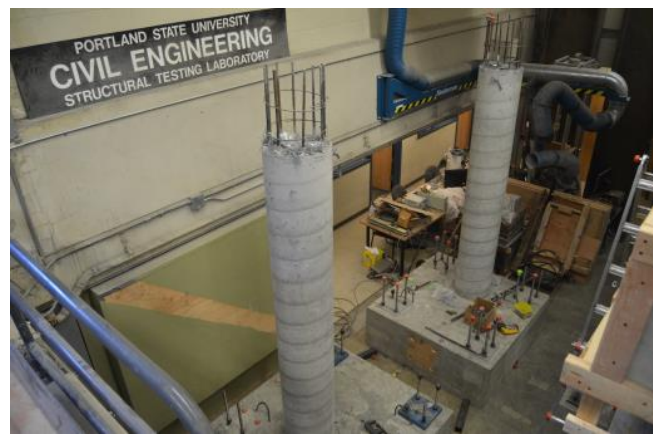

(d)

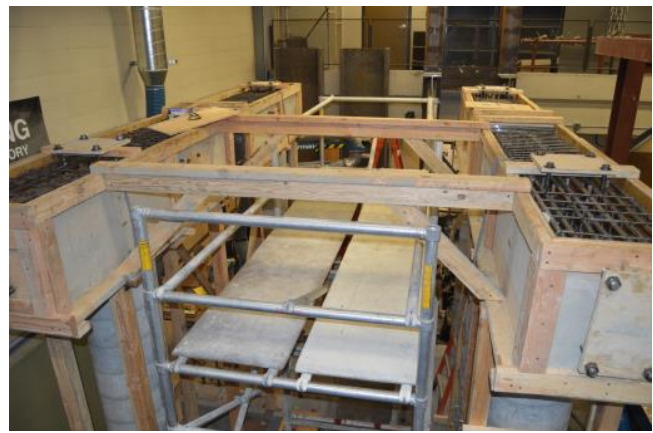

(f) 


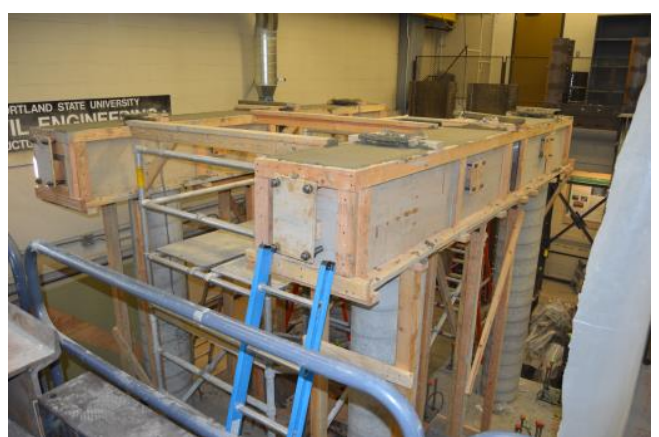

(g)

Figure 5.19 Construction sequence of RC bents

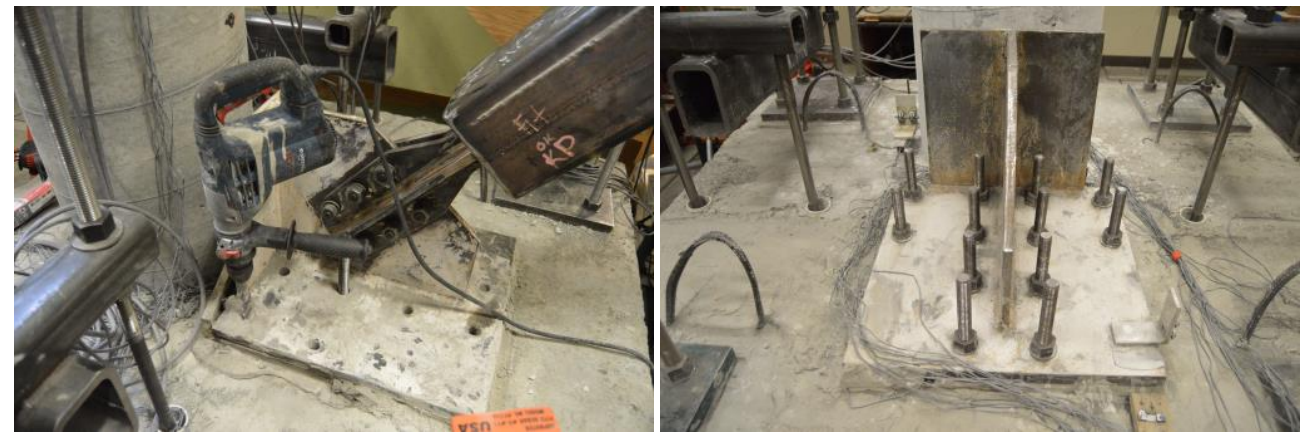

Figure 5.20 Post-installed anchors for gusset plates

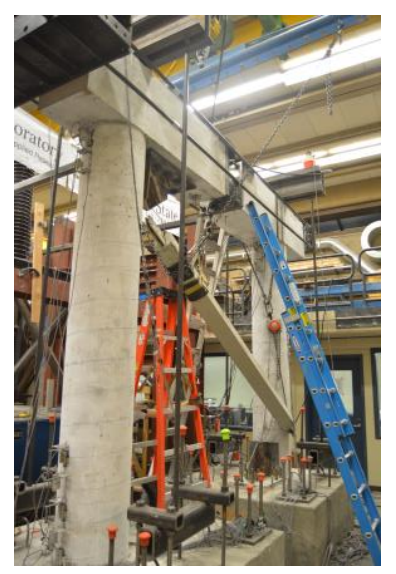

Figure 5.21 BRB installation

\subsection{CyClic LOAdING History}

Loading of the test specimens was quasi-statically applied using cyclic loading aiming to reflect subduction zone earthquake demands as discussed in Chapter 2. The deformation 
cycles were determined through analyses of structural response to subduction earthquake records. For the retrofitted specimens the loading protocol corresponded to target displacement ductility $(\mu) 8$ and fundamental period of $0.2 \mathrm{sec}$. This period was found to be representative of the retrofitted condition because the inclusion of the BRB increases the overall transverse system stiffness, which in turns shortens the fundamental period of the structure. The loading protocol was expected to subject the Bent/BRB systems to cumulative inelastic deformation of 437 times the yield deformation $\left(\delta_{y}\right)$. This cumulative inelastic deformation was calculated following the section K3 of the Seismic Provisions of AISC 341-10 (2010). This value of cumulative inelastic deformation is greater than twice of that required in cyclic test for qualification of $\mathrm{BRBs}$, which requires a cumulative inelastic deformation of at least $200 \delta_{\mathrm{y}}$ (AISC 341-10, 2010). For the As-built specimen the loading protocol was adjusted to correspond to structures with fundamental period of $0.5 \mathrm{sec}$, which is more representative of highway bridges with unbraced multi-column bents. For the repaired bent was assumed that the stiffness of the damaged structure would be restored. Therefore, the loading history for structures with fundamental period of $0.5 \mathrm{sec}$ was also used for the repaired bent.

The resulting horizontal displacements were applied based on a pattern of progressively increasing cycles, referenced to the horizontal displacement to cause yield as shown in Figure 5.22. The system displacement ductility was defined as $\mu=\delta / \delta_{y}$, where $\delta$ is the top lateral displacement at a specific cycle and $\delta_{\mathrm{y}}$ is the yield displacement of the system. The nominal yield displacement was initially calculated from material properties for all the specimens and the corrected on each test. Failure was considered at a point when lateral load degraded by $20 \%$ relative to the peak recorded load. 


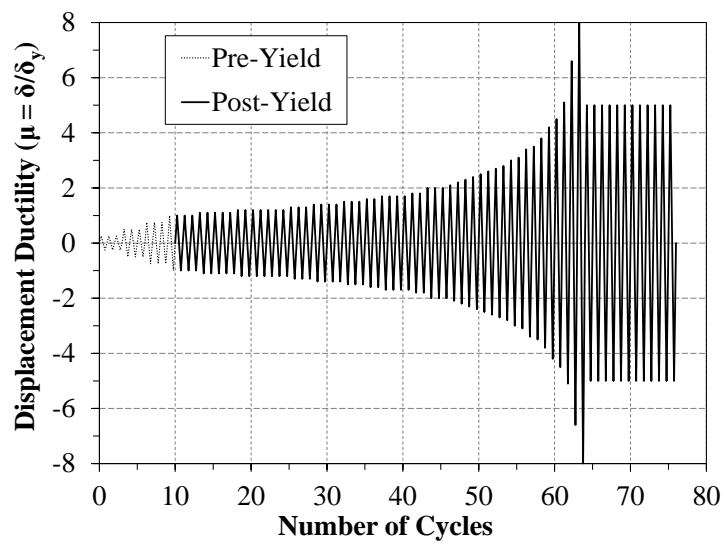

(a)

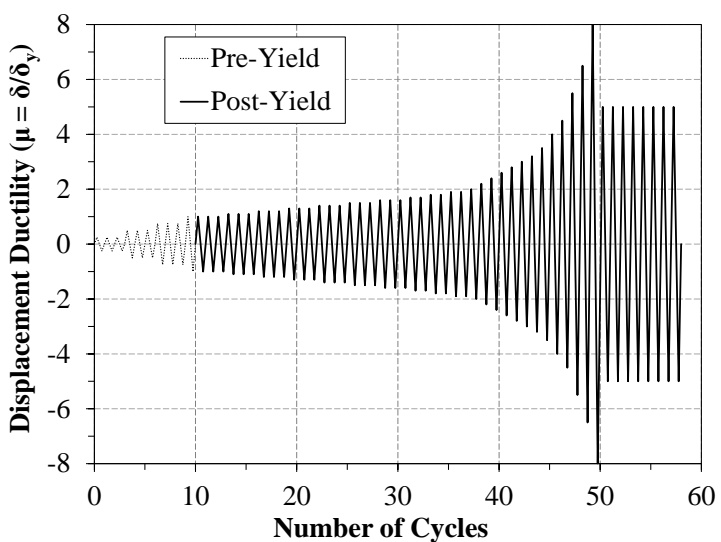

(b)

Figure 5.22 Cyclic loading histories. (a) Retrofitted bents, (b) As-built and repaired bents.

\subsection{First StAgE - EXPERIMENTAL EVALUATION OF RETROFITTED BENT}

The first stage of the experimental program consisted of evaluating the feasibility of using $\mathrm{BRBs}$ as a retrofit measure for $\mathrm{RC}$ bents. The test sequence comprised testing a $\mathrm{RC}$ bent retrofitted with a BRB specifically designed using the structural fuse concept, referred to as Bent/BRB 1, and a second BRB, referred to as Bent/BRB 2, designed to reflect typical design dimensions for BRBs. Finally, a third test was conducted in the as-built nonretrofitted condition, referred to as As-built Bent 1.

\subsubsection{Cyclic Behavior and Damage Progression}

The experimental outcomes are discussed with reference to global and local behavior.

\subsubsection{Retrofitted Bent/BRB 1}

The buckling-restrained brace used in Bent/BRB1 was designed following the structural fuse concept, which resulted on limiting the displacement of the bent to 0.7 in under the 1000-year event in order to prevent significant yielding of the cap beam and columns. This design resulted in a considerable stiff BRB as shown in Figure 5.23. 
The recorded lateral load-deformation behavior for this specimen shown in Figure 5.24 indicates ductile behavior and high energy dissipation via non-degrading hysteretic cycles. Bent/BRB1 exhibited ductile behavior up to ductility 8 , which was the maximum ductility considered in this case. The nominal yield displacement of the brace was computed as 0.07 in $(1.8 \mathrm{~mm})$. However, this yield displacement was corrected during the test to represent the first significant change in slope of the load-deformation curve in an effort to adjust for the actual response of the test specimen. The experimental yield displacement depicted in Figure 5.24 was 0.085 in $(2.2 \mathrm{~mm})$, which is a $22 \%$ increase with respect to the nominal value.

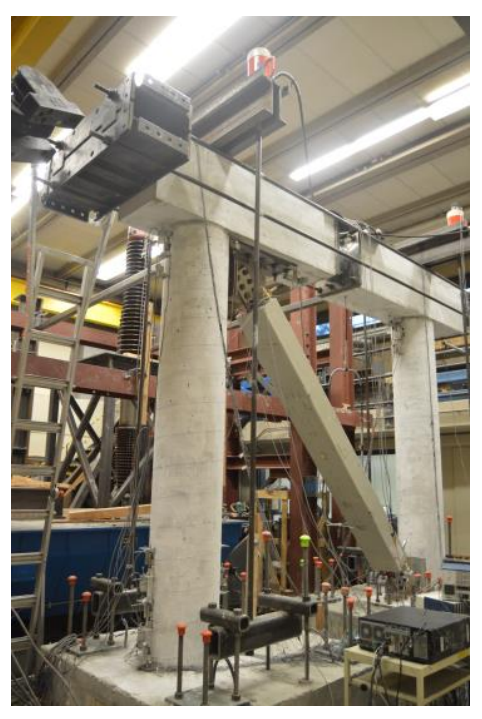

Figure 5.23 Bent/BRB 1

The first horizontal crack of width less than $0.05 \mathrm{~mm}$ was observed following a cycle excursion to a displacement of 0.25 in $(6.35 \mathrm{~mm})$, which corresponded to a lateral load of 79 kips $(351 \mathrm{kN})$. The influence of cracking can also be observed in Figure 5.24 through the change in slope of the load-deformation curve. The peak lateral load was 113 kips (503 kN) and occurred at a lateral displacement of 0.67 in $(17 \mathrm{~mm})$. First yield of the columns occurred at a system ductility of 6.5 , which is equivalent to a displacement of 0.56 in $(14.2$ 
$\mathrm{mm})$. This value was inferred by comparing the nominal curvature at first yield to curvature values calculated from the displacement transducers located at the base and top of columns and also using strain gauge measurements. Despite of the first yield of the bent, the specimen did not exhibit a decrease in lateral strength.

At the end of testing, only horizontal hairline cracks of maximum width $0.05 \mathrm{~mm}$ were observed at the base and top of columns as shown in Figure 5.25. This result demonstrated that a well-designed BRB limits the displacement of the RC bent, prevents significant yielding of the columns or damage in the beams and still provides a ductile response. Inspection of the gusset plates, which was conducted following the removal of the $\mathrm{BRB}$, did not reveal any damage, showing the potential replaceability of the BRB after a major seismic event.

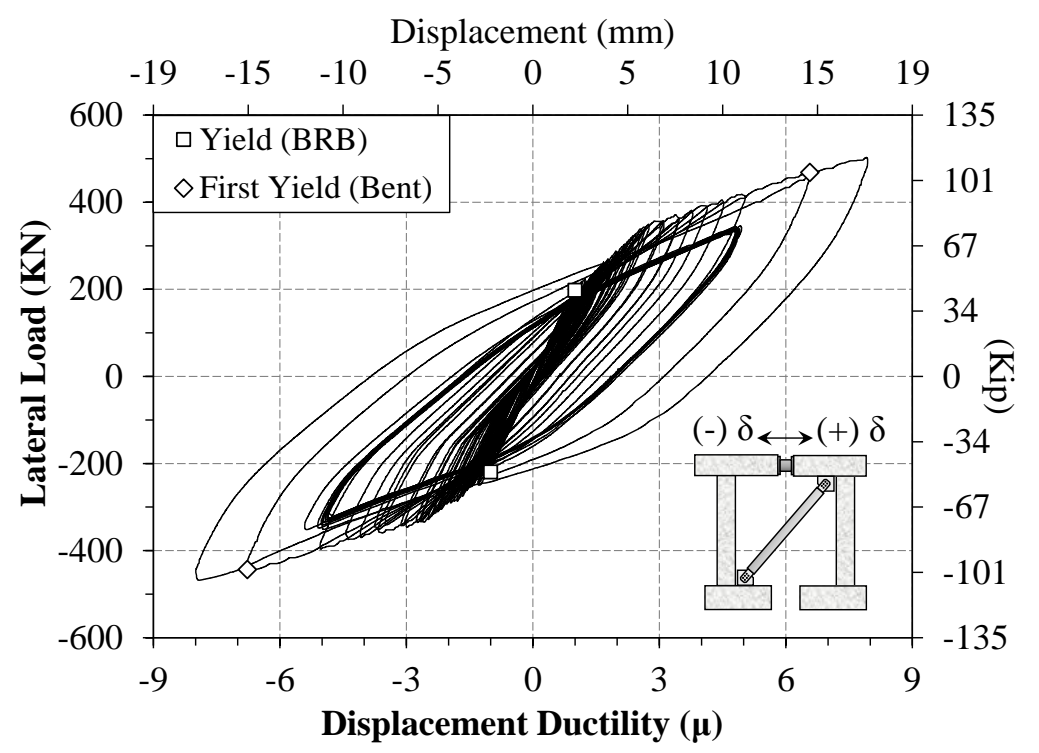

Figure 5.24 Hysteretic response of Bent/BRB 1 

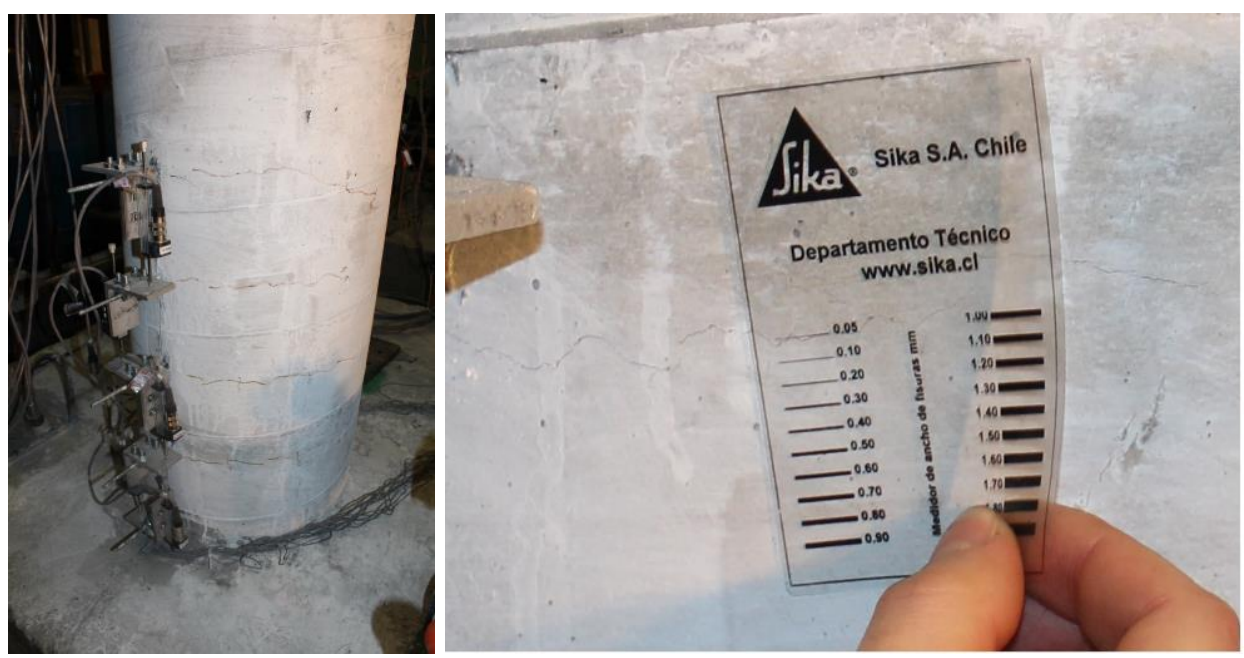

Figure 5.25 Damage in Bent/BRB 1

\subsubsection{Retrofitted Bent/BRB 2}

The brace for Bent/BRB2 was designed following a typical approach of BRB design, which considers a reduced section $\left(L_{c}\right)$ equal to two thirds of the brace length. Since the BRB was intentionally not designed for targeting a specific performance level, the brace was more flexible than that used in Bent/BRB1 as shown in Figure 5.26.

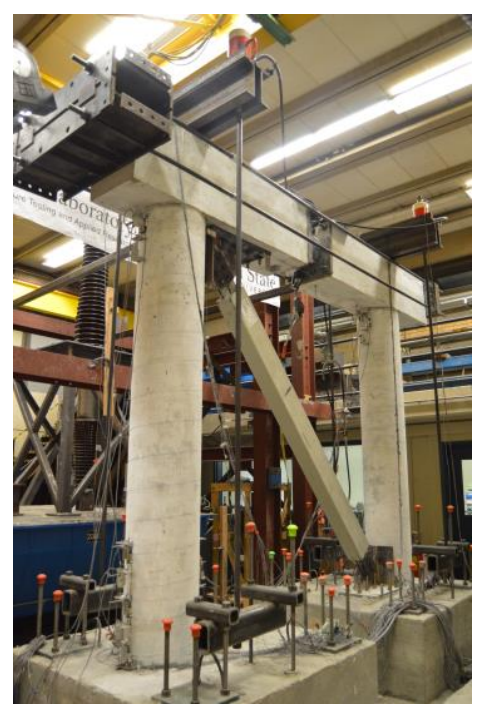

Figure 5.26 Bent/BRB 2 
The lateral load vs. deformation hysteresis curve shown in Figure 5.27 indicates ductile behavior and high energy dissipation up to a ductility value of 4.2 , which is equivalent to a displacement of 1.22 in $(33 \mathrm{~mm})$. The nominal yield displacement of the BRB was calculated as 0.20 in $(5.2 \mathrm{~mm})$ and the experimental one was 0.29 in $(7.3 \mathrm{~mm})$, which is a $40 \%$ increase with respect to the nominal value. First yield of the column occurred at a ductility of 1.9 or a displacement of 0.56 in $(14.2 \mathrm{~mm})$. This value was indirectly obtained using curvature values calculated from the displacement transducers located at the base and top of the columns and compared to the strain gauge measurements. The effective yield of the RC bent occurred at a ductility of 2.5 or a displacement of 0.73 in $(18.5 \mathrm{~mm})$. The effective yield was assumed at the occurrence of change in slope of the load-deformation curve as depicted in Figure 5.27. This effective yield displacement is important to be computed since it is required in bridge codes, such as AASHTO (2009), to compute the displacement ductility of the RC bent and to check ductility requirements for seismic design category (SDC) D. The effective yield is commonly larger than the first yield in flexure because of the additional displacement caused by reinforcing bar slip and shear.

As the progressively increasing displacements were applied, the lateral load increased up to 104 kips $(463 \mathrm{kN})$ for the brace compression direction. From that displacement forward, the specimen exhibited a significant decrease in lateral strength during the compression half-cycles. As was found later, buckling of the steel core likely started developing at this point giving place to fracture of the steel core once the BRB was tensioned after a cycle in compression as shown in Figure 5.27. Once the BRB failed, most of the load capacity was resisted by the RC bent, with a small contribution from the BRB. 
The peak lateral load for this retrofitted bent was 114.9 kips $(511 \mathrm{kN})$ and occurred at a ductility of 4 or lateral displacement of 1.18 in $(30 \mathrm{~mm})$.

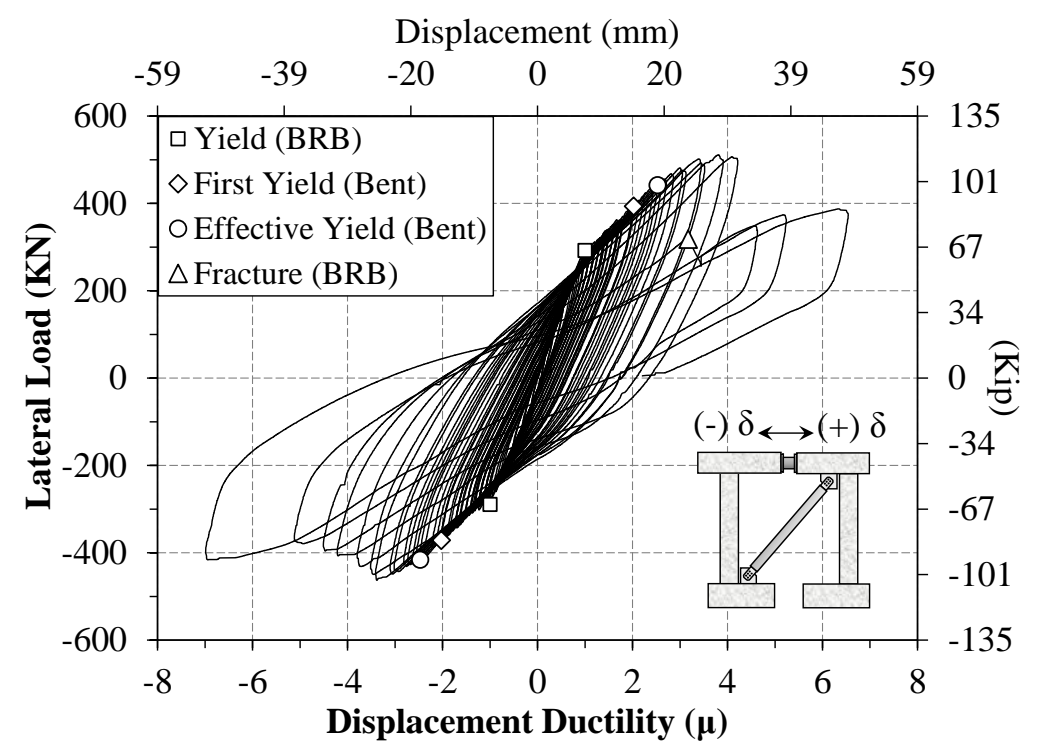

Figure 5.27 Hysteretic response of Bent/BRB 2

Regarding damage progression, horizontal cracks formed throughout the expected

plastic hinge zones of the columns as shown in Figure 5.28 and progressed in length and width. At the end of testing, crack width measurements registered maximum horizontal crack width of $0.4 \mathrm{~mm}$ and vertical cracks width less than $0.6 \mathrm{~mm}$. The horizontal cracks were located at the ends of the columns and the vertical cracks were measured in the cap beam in areas of negative moment. Minimal spalling of concrete at the base of the columns was also observed as illustrated in Figure 5.28. The crack widths along with observed spalling of concrete indicated that both columns and beams reached significant inelasticity during this test. These results showed that a BRB designed without explicitly considering the fuse concept can still provide moderate ductility and energy dissipation without significant damage on the RC bent. However, this BRB did not prevent yielding of columns and cap beam, the latter not being desirable and not being permissible without additional retrofit for 
an earthquake-resisting system according to AASHTO (2009). Further inspections after removing the $\mathrm{BRB}$ from the $\mathrm{RC}$ bent showed that the gusset plates did not exhibit any damage, thereby allowing an expedited replacement of the brace after a damaging seismic event. However, due to the damage in the cap beam, other repairs may be needed before the BRB can be replaced.

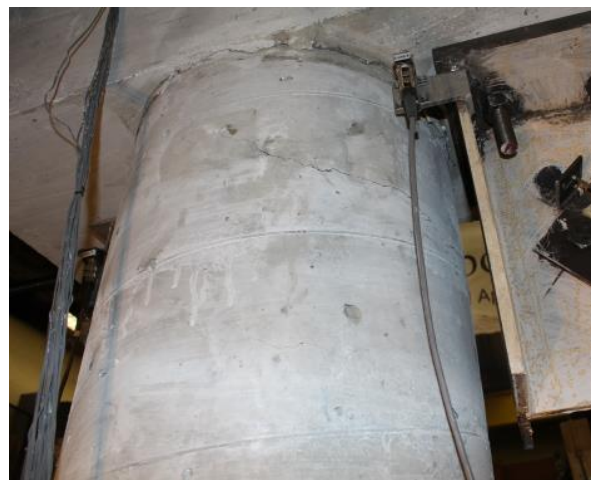

(a)

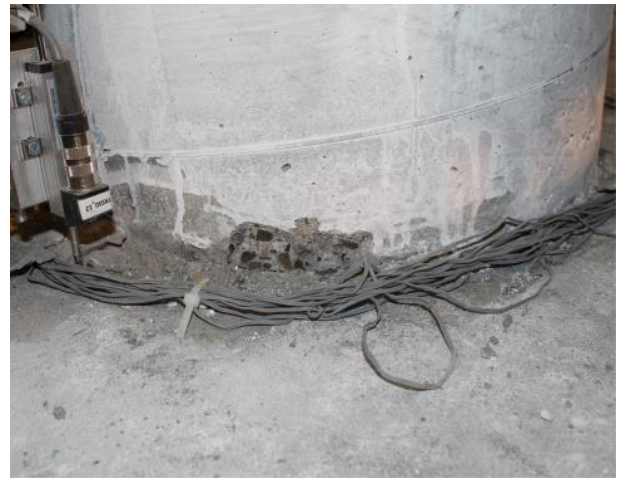

(b)

Figure 5.28 Damage in Bent/BRB 2. (a) Horizontal cracks, (b) Spalling of concrete

\subsubsection{As-built Bent 1}

The recorded lateral load-deformation behavior for the As-built Bent 1 is shown in Figure 5.29. The peak lateral load was 70 kips $(311 \mathrm{kN})$ and occurred at a lateral displacement of approximately $2.45 \mathrm{in}(62 \mathrm{~mm})$. The yield displacement values are depicted in Figure 5.29.

The initial damage consisted of horizontal cracks that were propagated throughout the height of the expected plastic hinge zones to give place to spalling of concrete in early stages of the cyclic sequence. Vertical cracks in the bent beam increased in width up to 0.8 $\mathrm{mm}$. Through visual inspection, buckling of the first rebar was observed in the column at a ductility of 4.8 or an equivalent displacement of 3.51 in $(89 \mathrm{~mm})$ as depicted in Figure 5.29. 


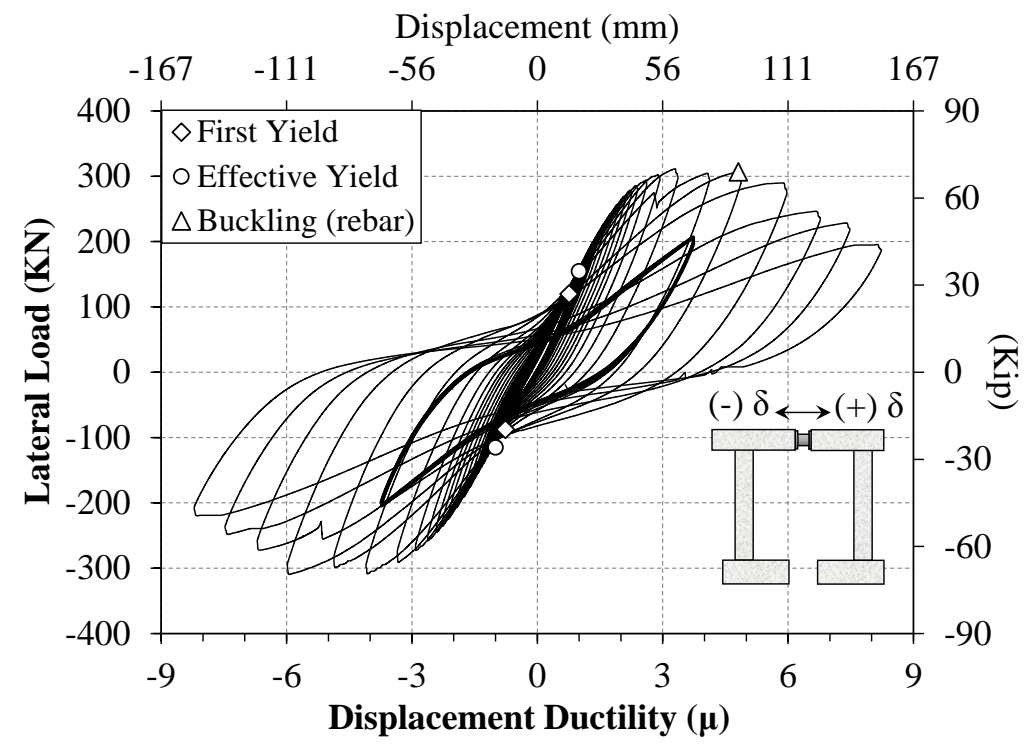

Figure 5.29 Hysteretic response of As-built Bent 1

The ultimate mode of failure was crushing of concrete and extensive buckling of the longitudinal reinforcing steel. Crushing of the concrete at the base and top of the columns began due to flexural deformations, exposing the column reinforcement. Once the concrete cover was lost, the longitudinal bars and dowels in those regions began to buckle and finally fracture as shown in Figure 5.30. No diagonal shear cracks were observed on the specimens. The As-built RC bent was able to attain a ductility of 6.7 or an equivalent displacement of 4.92 in $(125 \mathrm{~mm})$ before the load at reversal dropped below $80 \%$ of the peak load. Despite the design deficiencies of the As-built RC bent, namely, lap splices at the base of columns, low ratio of transverse reinforcement and no seismic detailing, this bent exhibited a moderately ductile behavior and energy dissipation. 


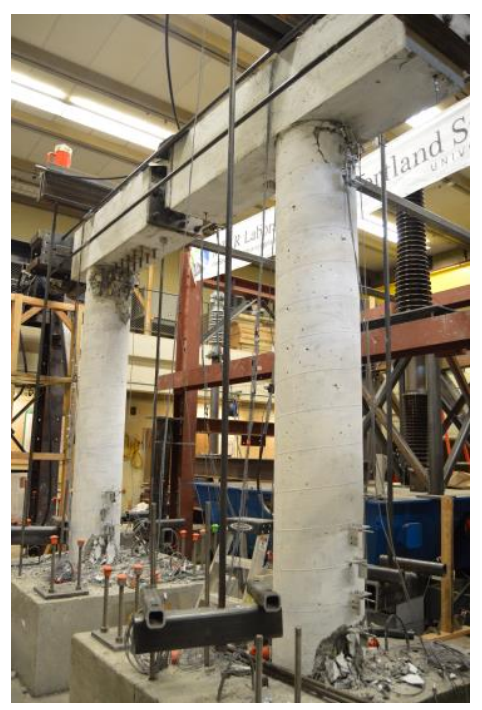

(a)

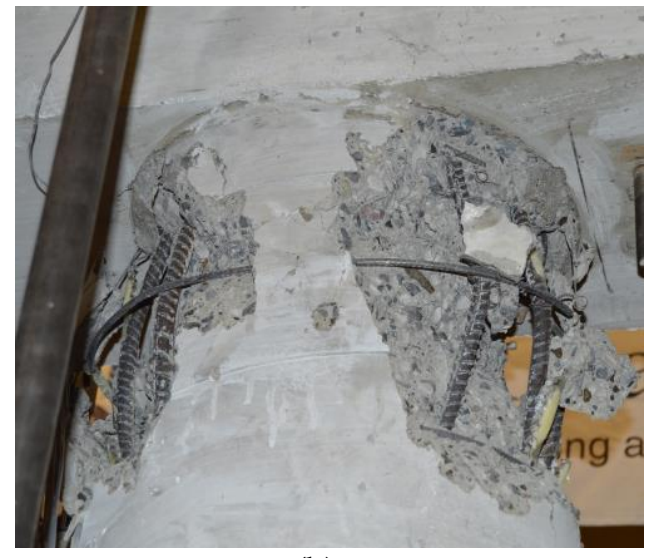

(b)

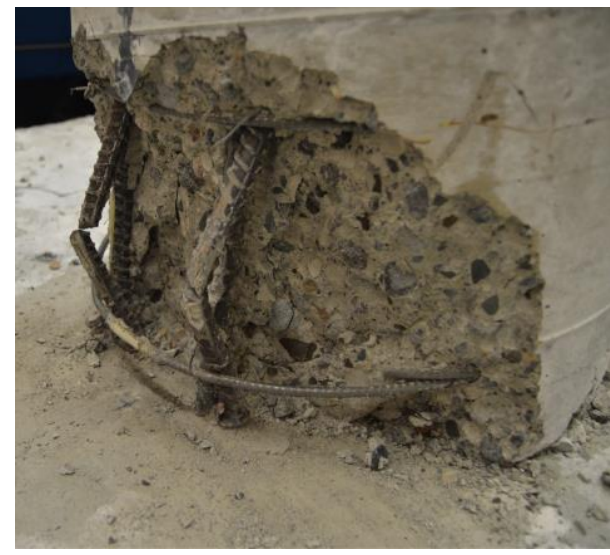

(c)

Figure 5.30 Damage in As-built Bent 1; (a) Overall damage, (b) Buckling of steel, (c) Rupture of steel

\subsubsection{Buckling-Restrained Brace (BRB) Component Response}

The elongation of the BRB was measured via four LVDTs. Two of them measured the relative displacement between the steel core and the steel casing at the top and bottom connections, referred to as Top and Bottom elongations. The other two, referred to as West and East, were located on the sides of the brace to measure the total elongation. The brace elongation for the Bent/BRB 1 and Bent/BRB 2 are shown in Figure 5.31 and Figure 5.32, respectively. 


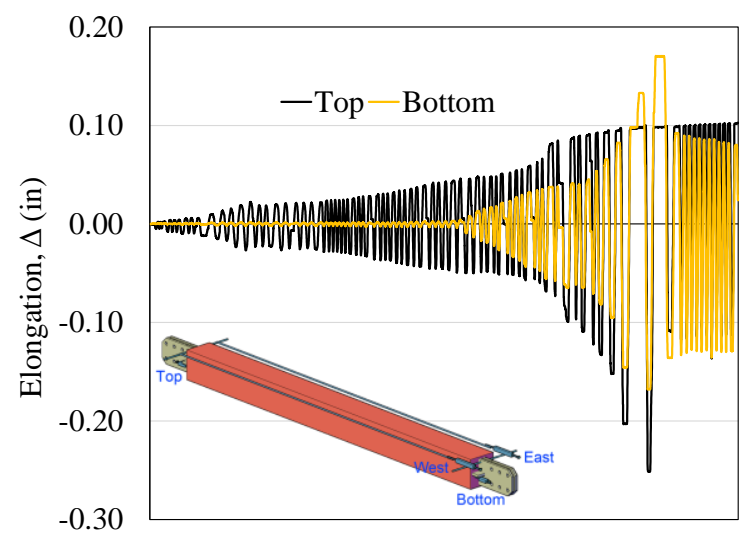

(a)

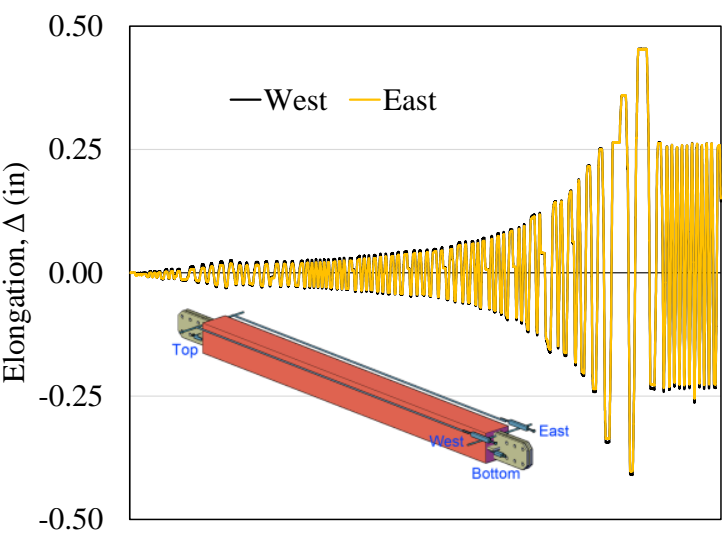

(b)

Figure 5.31 BRB elongation in Bent/BRB 1. (a) Top and Bottom, (b) West and East
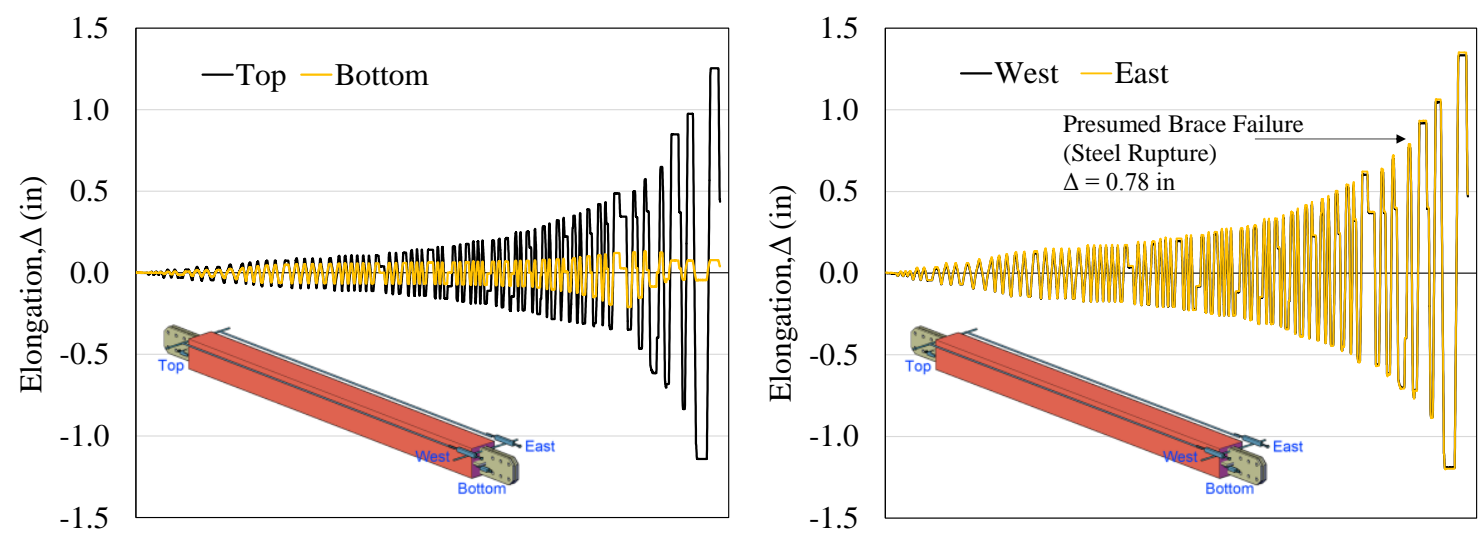

Figure 5.32 BRB elongation in Bent/BRB 2. (a) Top and Bottom, (b) West and East

Comparing the top elongation to the bottom elongation, it can be observed that inelastic deformation demands were mostly concentrated at the top of the BRB, i.e. at the connection with the cap beam. This behavior was critical for the Bent/BRB 2, in which the top elongation was up to 20 times greater than the bottom elongation. As a result, the brace failed at the top part at an elongation of 0.78 inches. The elongation at rupture was lower than the expected elongation since according the BRB design the brace should have attained a maximum elongation of 1.78 inches. The unsatisfactory behavior of the BRB in the Bent/BRB 2 system was attributed to the poor confinement of the brace within the transition section $\left(\mathrm{L}_{\mathrm{tr}}\right)$. 
The BRB axial response was indirectly obtained using the measurements from the 6DOF load cell, which was located at mid-span of the beam, the load cells located on top of the rams, and the corresponding free body diagrams. In order to resolve the brace axial force, shear and moment in the BRB were assumed to be negligible. BRB response envelope during the Bent/BRB1 and Bent/BRB2 test is illustrated in Figure 5.33(a) and Figure 5.33(b), respectively.

The numerical response was computed from using material properties and an overstrength factor $(\beta \omega)$ of 1.45 for compression and 1.3 for tension $(\omega)$ at 15 times the yield elongation, a convenient bi-linear approximation suitable for design as shown in Figure 4.11. Numerical yield elongations of 0.046 in $(1.2 \mathrm{~mm})$ and 0.14 in $(3.4 \mathrm{~mm})$ were used for Bent/BRB1 and Bent/BRB2, respectively. Actual yield elongations were computed as 0.056 in $(1.4 \mathrm{~mm})$ for Bent/BRB1 and 0.19 in $(4.9 \mathrm{~mm})$ for Bent/BRB2. The actual yield elongations were calculated by converting the yield displacement on the retrofitted system to elongation in the BRB. This was done by multiplying the yield displacement by cosine of $\theta$. Comparison of the inferred envelopes and the numerically obtained bi-linear responses in Figure 5.33 shows that the numerical approximations fit well with the experimental curves in the elastic region. Once the BRB yielded, the experimental values exceeded the numerical. The greater experimentally obtained values of axial load in the post-yield range are likely due to the assumptions made in indirectly obtaining the axial load in the first place. 


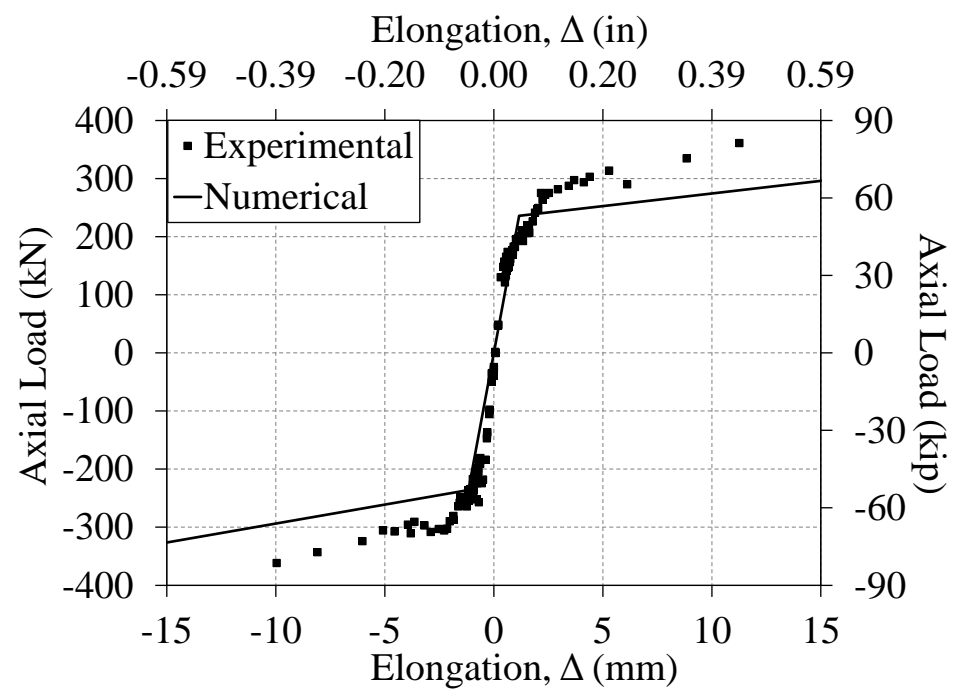

(a)

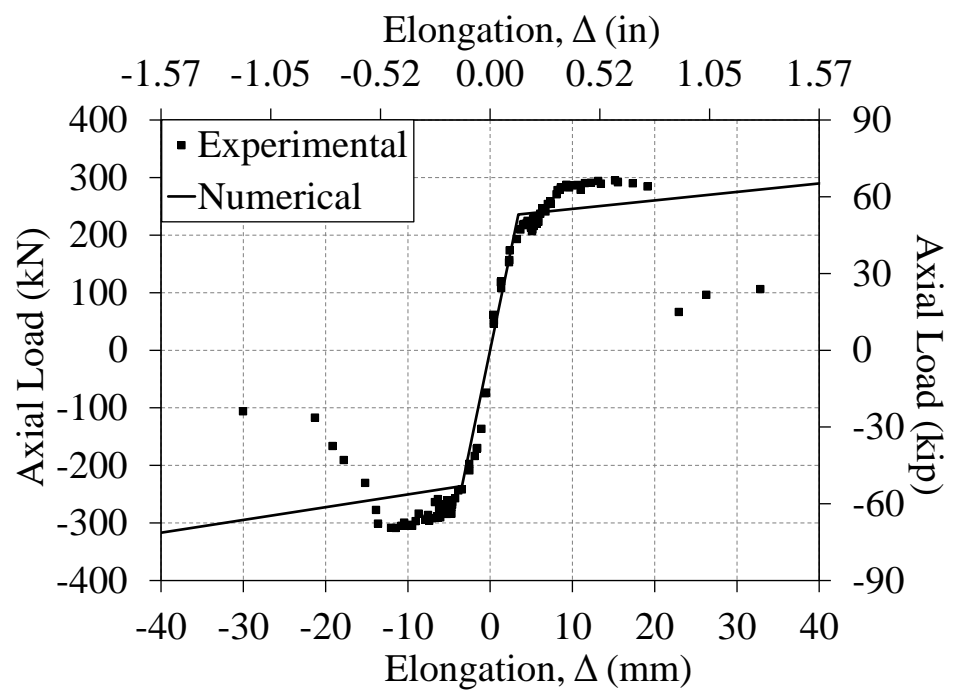

(b)

Figure 5.33 Buckling-restrained brace response. (a) Bent/BRB 1; (b) Bent/BRB 2

The axial strains in the steel core can be estimated by assuming that all the elongation/contraction is concentrated within the reduced section (yielding core) and the deformations in the elastic components are negligible. The estimated maximum axial strains of the steel core at the end of the tests were computed dividing the maximum elongation by the reduced section length, and were approximately $1.5 \%$ for both BRBs. This value of 
estimated axial strain is less than the maximum axial strain $(2 \%)$ recommended for the design of BRBs (AISC 341-10, 2010), (Lopez \& Sabelli, 2004).

Following the tests, the braces were deconstructed in order to observe any internal damage. Figure 5.34(a) shows the deformations of the steel core of Bent/BRB1, in which minor damage in the intersection between the transition section $\left(L_{t r}\right)$ and the reduced section $\left(L_{c}\right)$ can be seen. This damage was attributed to high stress concentrations from changing the cross sectional shape of the steel core. This minor damage in the steel core showed that even though the Bent/BRB1 attained a highly ductile behavior, the brace still had remaining deformation capacity. This capacity is vital in cases when the seismic event exceeds the demands considered in the design process or for resisting strong aftershocks.

The damage presented in the steel core of Bent/BRB2 is shown in Figure 5.34(b). The fracture of the steel core occurred in the transition section between the non-yielding and the reduced section of the core. This mode of failure was attributed to poor confinement in the transition section $\left(L_{t r}\right)$ within the brace and to the uneven deformation demands measured for the top and bottom of the brace as stated previously. The fracture of the steel core occurred at an elongation of 0.78 in $(19.8 \mathrm{~mm})$, which represents an estimated axial strain in the BRB core of $0.88 \%$. The fracture of the steel core was unexpected since this brace followed the typical design dimensions for BRBs.

The contribution of the BRB at the peak load of the retrofitted system was $47 \%$ and $40 \%$ for Bent/BRB1 and Bent/BRB2, respectively. This result showed that almost half of the system maximum lateral load was resisted by the BRB. 

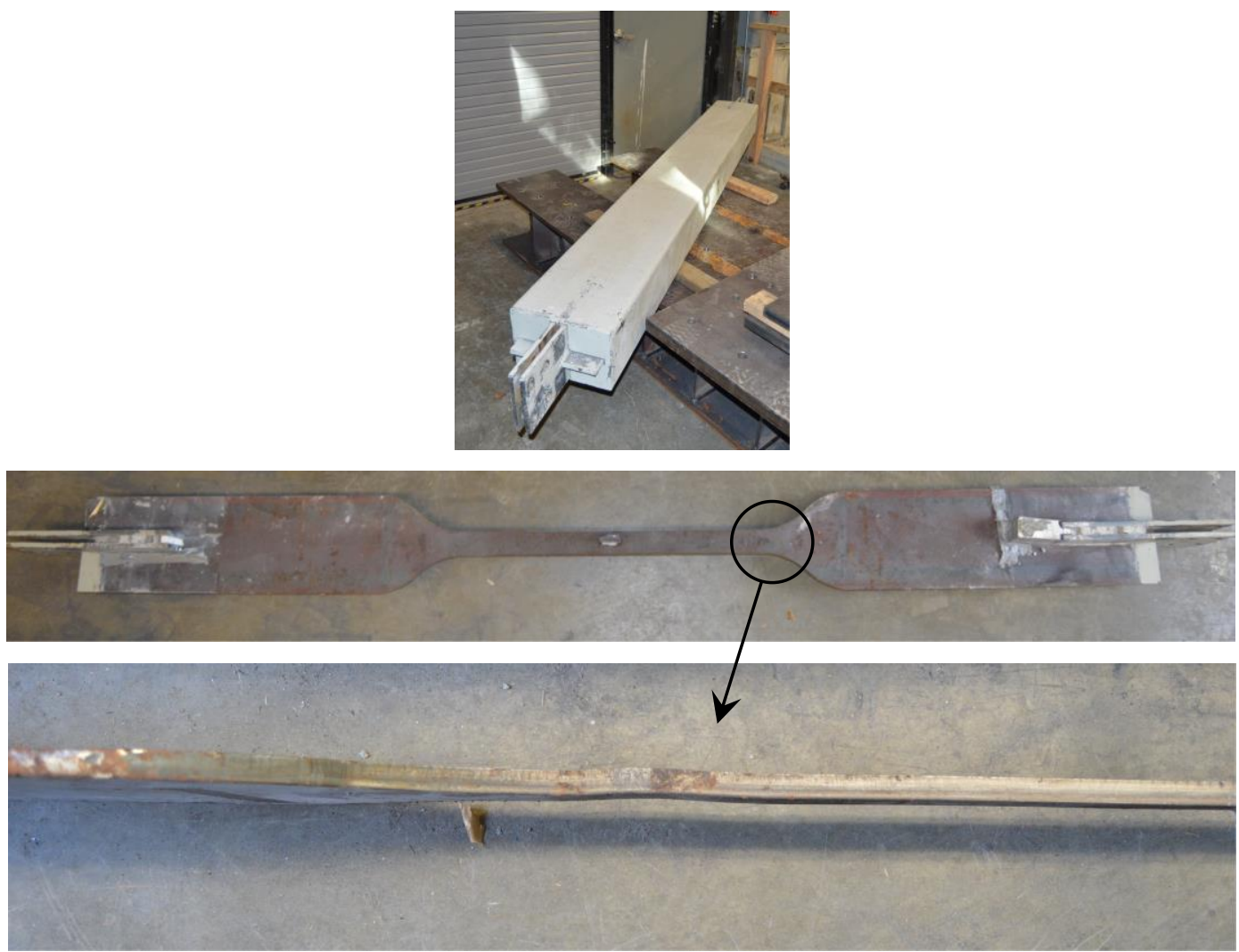

(a)
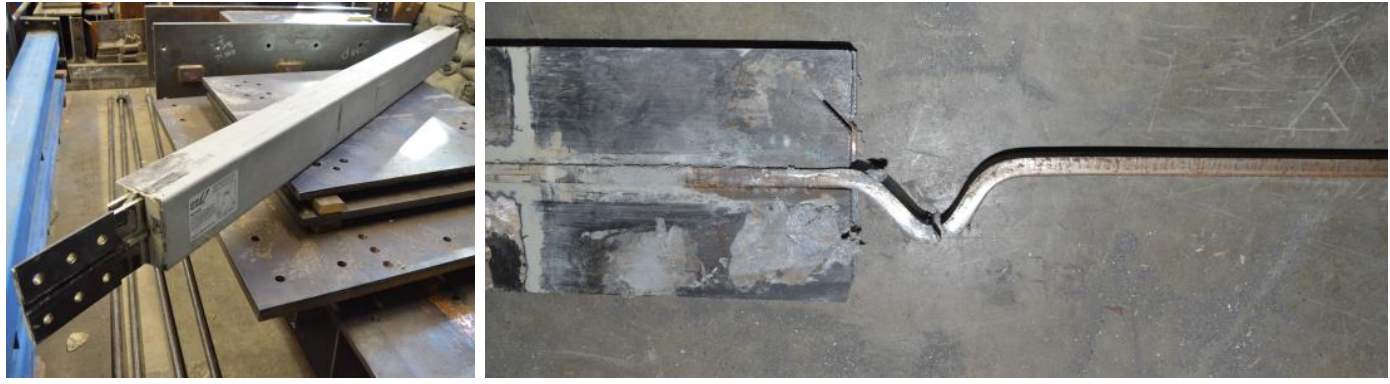

(b)

Figure 5.34 Damage in BRBs. (a) BRB 1; (b) BRB 2

\subsubsection{Columns Component Response}

The internal axial loads in column 1 (C1) and column 2 (C2) were calculated by adding the load cell measurements of dead load located on top of each column, the shear measurement of the 6DOF load cell located at the mid-span of the cap beam, and decomposing the axial load contribution from the BRB in the retrofitted case. The internal 
axial loads in C1 and C2 for Bent/BRB1, Bent/BRB2 and As-built bent are shown in Figure 5.35(a), (b) and (c), respectively.
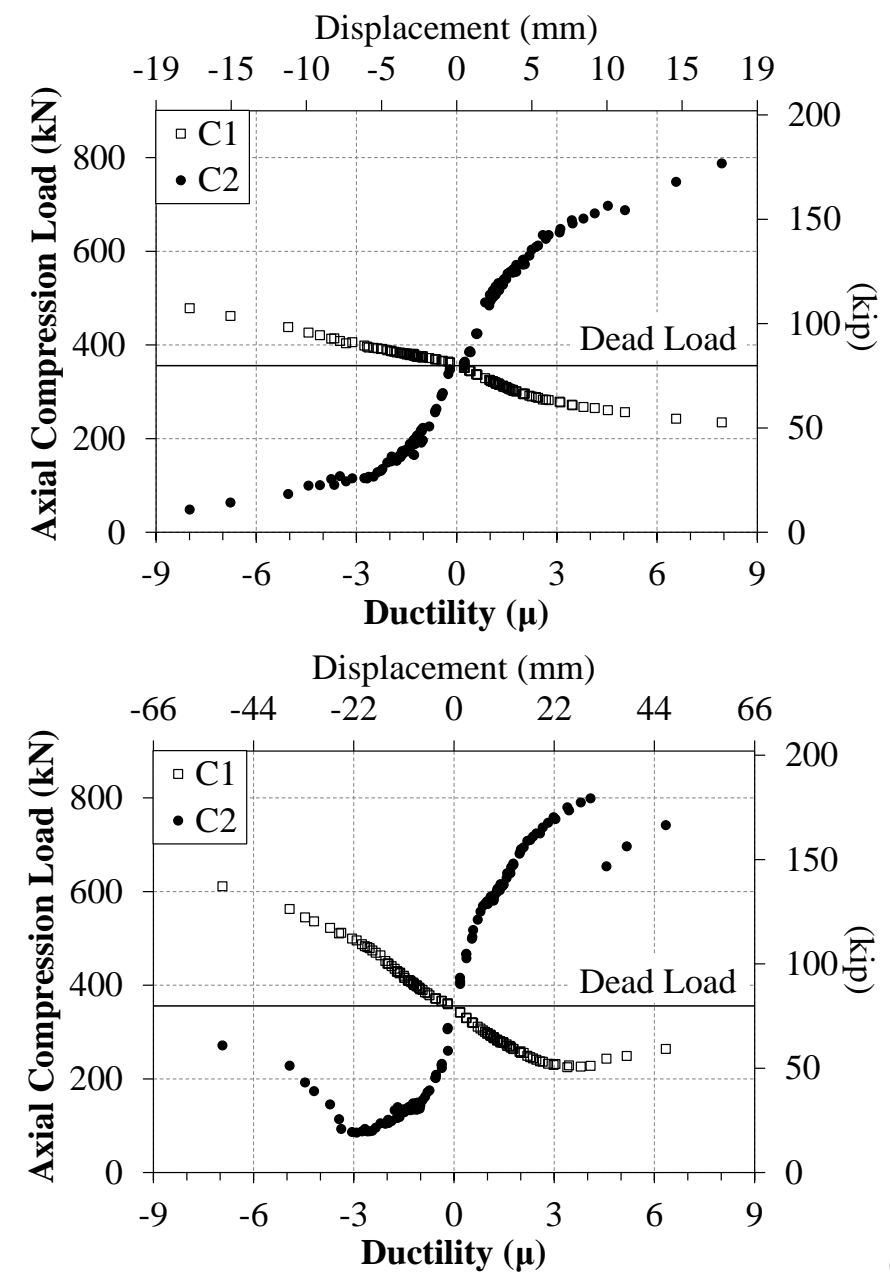

(a)

Displacement (mm)

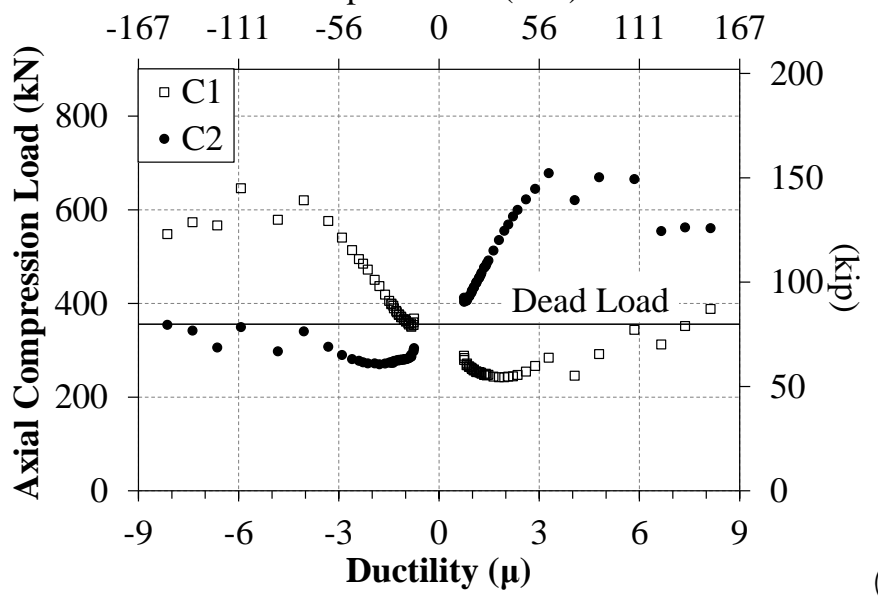

b)

(c)

Figure 5.35 Internal column axial load. (a) Bent/BRB 1; (b) Bent/BRB 2; (c) As-built Bent 1. 189 
From these figures, two effects of the BRB in the column axial load can be observed. First, the influence of the $\mathrm{BRB}$ on $\mathrm{C} 1$ is negligible as compared to the As-built condition. This behavior is due to the bottom connection itself. Since the brace is connected using a gusset plate that is only connected to the footing all the axial load is transferred directly to the foundation. This has positive implications on the axial load in the columns since the forces are similar to the As-built condition. However, its implications in the foundations need to be considered as part of the retrofit. On the contrary, the axial load in C2 is directly affected by the inclusion of the BRB since the axial load in the BRB is decomposed into an additional axial load in that column. This can be seen in an increase in peak compression load in positive displacement in about twice the axial load for the Bent/BRB1 and 120\% for the Bent/BRB2 as compared to the As-built case. In negative displacement direction, the opposite occurs since the change of loading direction reduces the compression in $\mathrm{C} 2$ to approximately $80 \%$ for the Bent/BRB1 and $70 \%$ for the Bent/BRB2 with respect to the Asbuilt value at the same displacement. The effect of axial loading in columns due to the inclusion of BRBs is of crucial importance in the retrofitting of existing structures since the change in axial load needs to be accounted for in the design and will affect the subsequent assessment and retrofit of the various components.

\subsubsection{Plastic Hinge Damage}

Plastic hinge damage of the As-Built bent is shown in Figure 5.36 and Figure 5.37 for columns and cap beam, respectively. As described in the response of the As-built bent, the first longitudinal bar buckled at a ductility of 4.8 after 37 cycles following the first yield displacement. At progressing cycle increments of displacement ductility more longitudinal reinforcing bars buckled at the column top and dowels buckled at the column base. The first 
dowel in column 2 fractured during the cycle at ductility 6.6. After the first dowel fractured, crushing of concrete extended into the core during the remaining cycles as shown in Figure 5.36(a), (b), (c) and (d) for the base of column 1, top of column 1, base of column 2, and top of column 2, respectively. This type of failure has been typically observed in experimental studies (McLean, et al., 1998) and post-reconnaissance damage of bridges after major earthquakes (Kawashima, et al., 2011).

In order to compare the plastic hinge length $\left(L_{p}\right)$ with the extent of damage in the plastic hinge region, an approximate plastic hinge length (Priestley, et al., 1996) was calculated as:

$$
\begin{array}{ll}
L_{p}=0.08 \frac{H}{2}+0.022 f_{y-m e} d_{b} \geq 0.044 f_{y-m e} d_{b} & (M P a, m m) \\
L_{p}=0.08 \frac{H}{2}+0.15 f_{y-m e} d_{b} \geq 0.3 f_{y-m e} d_{b} & (k s i, i n)
\end{array}
$$

Where, $H$ is the column height, $f_{y-m e}$ is the measured yield stress of the reinforcing steel, and $d_{b}$ is the diameter of the longitudinal reinforcing steel. The point of moment contra-flexure was assumed to occur at mid-height of the column for purposes of calculating the plastic hinge length. The plastic hinge length using Eq. (5.4) was 9.4 in (239 mm). Although, that equation provides a fictitious length for analytical purposes, the analytical plastic hinge is in agreement with the observed extent of damage at the end of testing, in which plastic hinge lengths of 9 in $(229 \mathrm{~mm})$ to 10 in $(254 \mathrm{~mm})$ were observed as shown in Figure 5.36. The top of column 2 exhibited more localized damage as shown in Figure 5.36(d). This damage was likely the consequence of greater axial forces in column 2 as shown in Figure 5.35(c). 


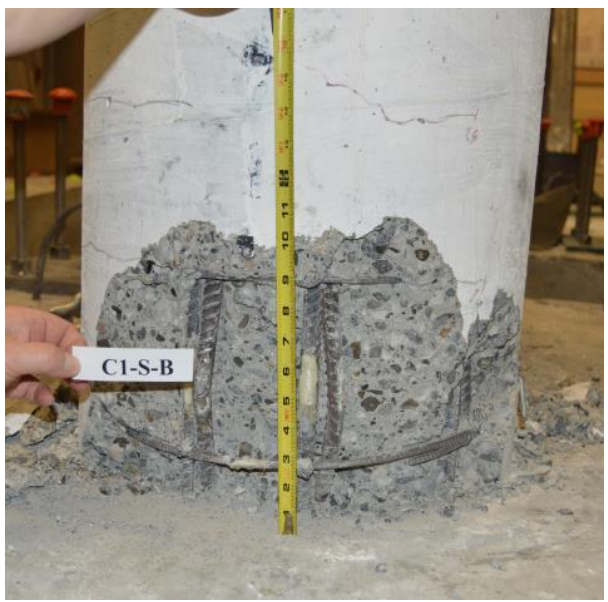

(a)

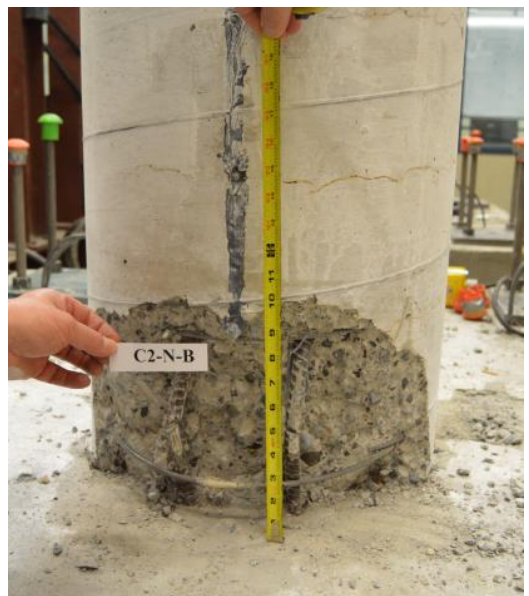

(c)

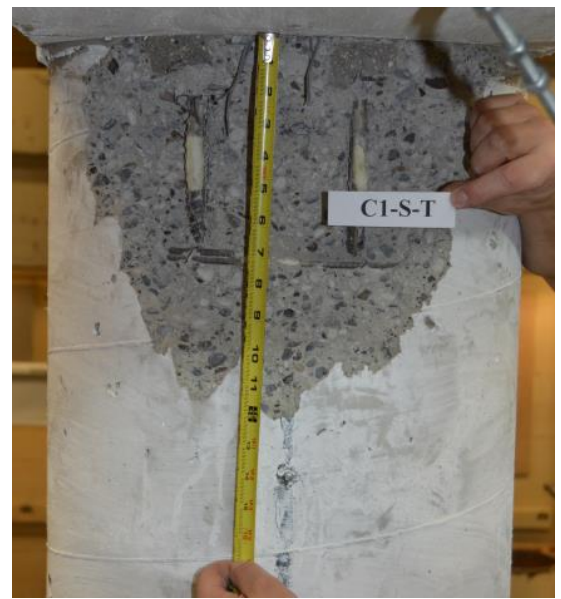

(b)

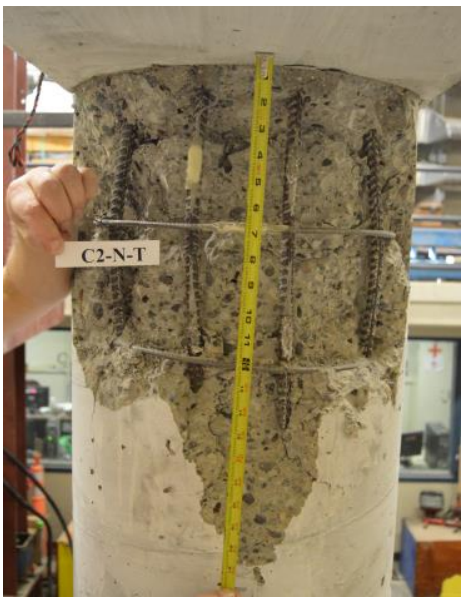

(d)

Figure 5.36 Plastic hinge damage in columns. (a) C1-Base; (b) C1-Top; (c) C2-Base; (d) C2-Top

Figure 5.37 depicts significant damage in the cap beam indicating that a plastic hinge also formed in the cap beam; furthermore, this figure also shows diagonal cracks in the cap beam-column joint that indicate some level of flexural-shear damage caused by high shear stresses acting at the joint boundary and the poor detailing of the joint. This result is of significance since both retrofitted bents limited the formation of plastic hinges in not only the columns but also in the cap beam, potentially reducing the need for retrofit of these components when the BRBs are implemented. 


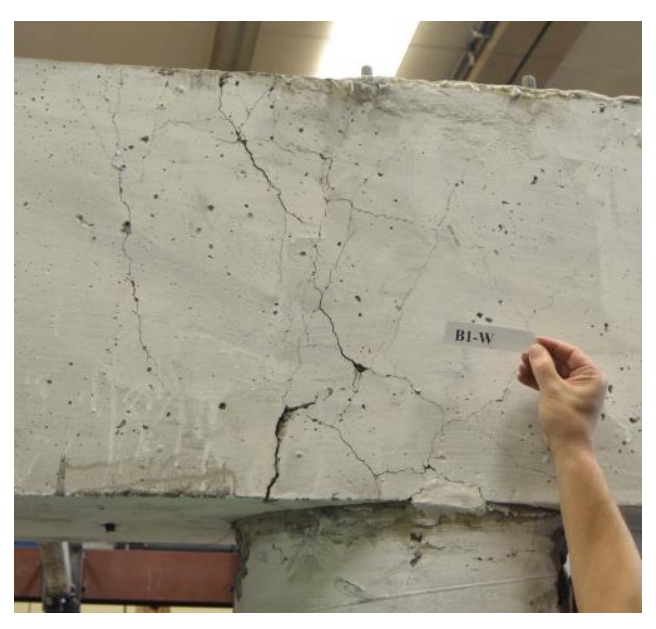

Figure 5.37 Plastic hinge damage in cap beam

\subsubsection{Bent and Components Ductility}

Displacement ductility $(\mu)$ was defined as the ratio of the top lateral displacement at a specific cycle to the top displacement at a reference yield displacement. The reference yield can be calculated as the nominal yield or measured from the experiments.

\subsubsection{Nominal Bent Yield Displacement}

Nominal yield displacements for Bent/BRB1 and Bent/BRB2 were computed converting the nominal yield elongation of the BRB to top lateral displacement. This calculation was done dividing the elongation $\left(\Delta_{\mathrm{y}}\right)$ by $\operatorname{cosine}(\theta)$ as shown in Figure 4.12. For the RC bent the nominal yield displacement was computed herein using the approximated yield displacement $\left(\delta_{y}\right)$ defined by Priestley (2007), which neglects shear deformations and in which strain penetration is included by increasing the effective height of the column through the use of a fictitious length $L_{s p}$. Thus, the nominal yield displacement for the As-built bent is calculated using equations (5.5) to (5.7).

$$
\phi_{y}=\frac{2.25 \varepsilon_{y}}{D}
$$




$$
\begin{gathered}
\delta_{y}=\frac{\phi_{y}\left(H+2 L_{S P}\right)^{2}}{6} \\
L_{S P}=0.022 f_{y-m e} d_{b} \quad(M P a, m m) \\
L_{S P}=0.15 f_{y-m e} d_{b} \quad(k s i, i n)
\end{gathered}
$$

Where $\varepsilon_{y}$ is the yield strain of the longitudinal reinforcement, $D$ is the column diameter and $\phi_{y}$ is the effective yield curvature. Summary of the nominal yield displacements are provided in Table 5.7 for all the bents. As expected, the nominal yield displacement for the Bent/BRB2 is almost three times greater than the value for the Bent/BRB1 since the BRB used in Bent/BRB2 was more flexible.

\subsubsection{Measured Curvatures and Yield Displacements}

Actual yield displacements for the retrofitted specimens, Bent/BRB1 and Bent/BRB2, were obtained using the experimentally inferred yield elongation of the brace. The corresponding displacement ductilities were computed using the actual yield displacements. Curvature profiles were used to obtain the first yield displacement of the RC bent and are presented in Figure 5.38 for columns and Figure 5.39 for the cap beam at three different displacement ductilities. First yield curvature values of $\phi_{y c}=1.58 \mathrm{e}-4 \mathrm{rad} / \mathrm{in}(0.0062$ $\mathrm{rad} / \mathrm{m})$ for columns and $\phi_{y b}=1.6 \mathrm{e}-4 \mathrm{rad} / \mathrm{in}(0.0063 \mathrm{rad} / \mathrm{m})$ for the cap beam were used. These values were computed using moment-curvature relations and are represented by vertical dashed lines in Figure 5.38 and Figure 5.39 for columns and beam, respectively.

The column curvatures were calculated using the displacements measured by the displacement transducers located at the base and top of the columns. Similarly, the beam curvatures were calculated using the displacement transducers located on top and below the cap beam. Curvatures that in this case represent the average curvature along the vertical 
distance between the transducers were derived from rotation, which was computed by dividing the algebraic summation of the relative displacements between opposite transducers at the same height to the horizontal distance between them. The curvatures for the Bent/BRB1 are considerably lower than for the other two tests due to the smaller imposed displacements. The average curvatures for Bent/BRB1 in columns and cap beam were smaller than the calculated yield curvature. This result shows that a BRB designed specifically for achieving performance targets can indeed limit the displacements and damage of the bent and still provide a ductile response of the structure. An exception can be seen at the columnfooting and column-beam interface, where additional deformations caused by longitudinal reinforcement bond slip resulted in the average column curvatures to be larger than at other locations. For Bent/BRB2 as well as the As-built bent, both column and beam curvatures exceeded the computed yield values throughout the plastic hinge zone, which means that during those tests both columns and beam reached yielding. The beam yielding can be seen in Figure 5.39(b) for the Bent/BRB2 where a change in slope occurred at the exact location of the computed first yield in both directions of loading. Moreover, majority of the curvatures measured in the As-built bent were larger than the nominal first yield.

The displacement ductility for the As-built bent was computed using an effective yield displacement, which was assumed at the occurrence of change in slope of the loaddeformation curve as depicted in Figure 5.27. Values of yield displacement and final displacement ductility are summarized in Table 5.7. Comparison of the RC bent nominal yield displacement and the effective displacement shows that the RC bent has a larger actual yield displacement. This result is due to the fact that shear deformations were neglected in 
the computation of the nominal yield displacement and due to an underestimation of rebar slip effects in the column-footing and column-beam interface.
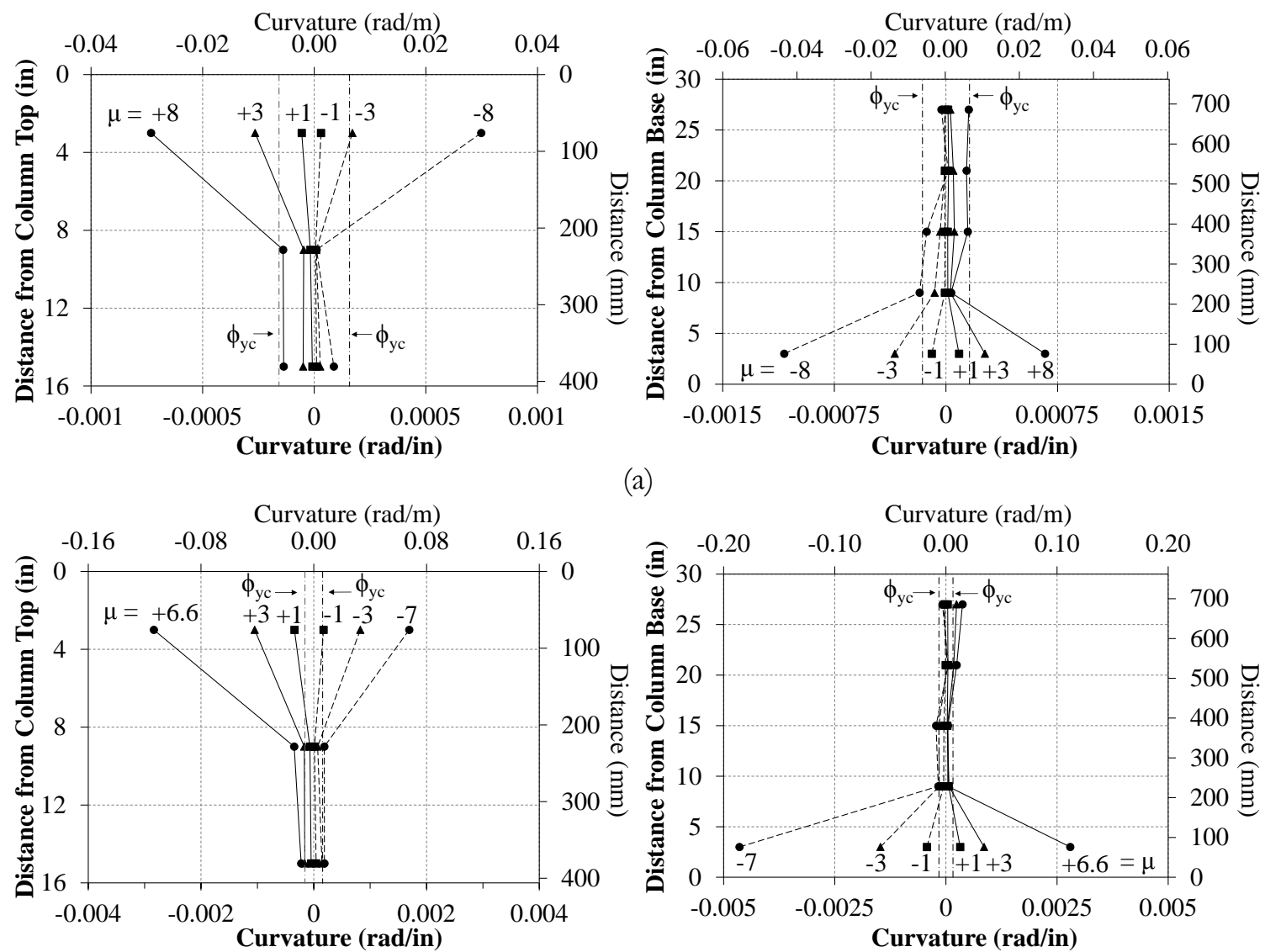

(a)

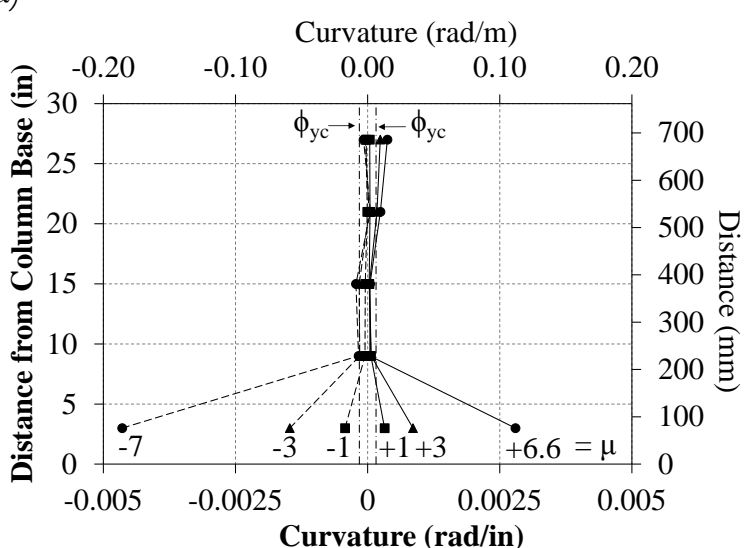

(b)
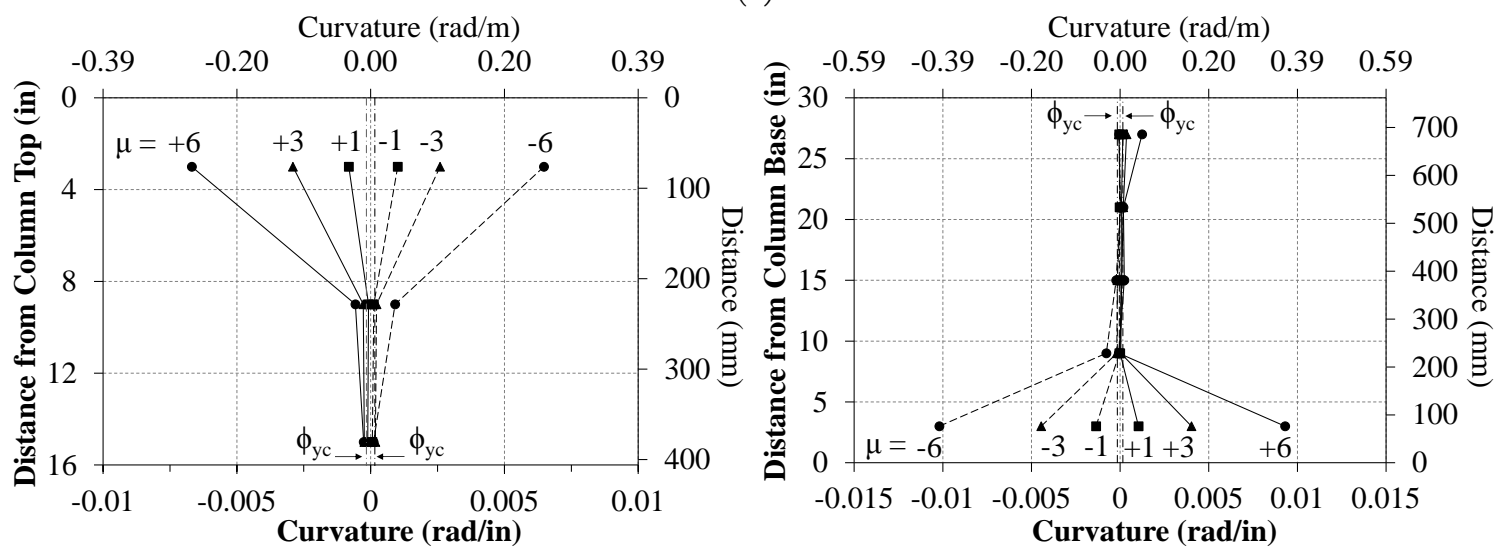

(c)

Figure 5.38 Average curvature profiles in columns. (a) Bent/BRB 1; (b) Bent/BRB2;

(c) As-built Bent 1. 


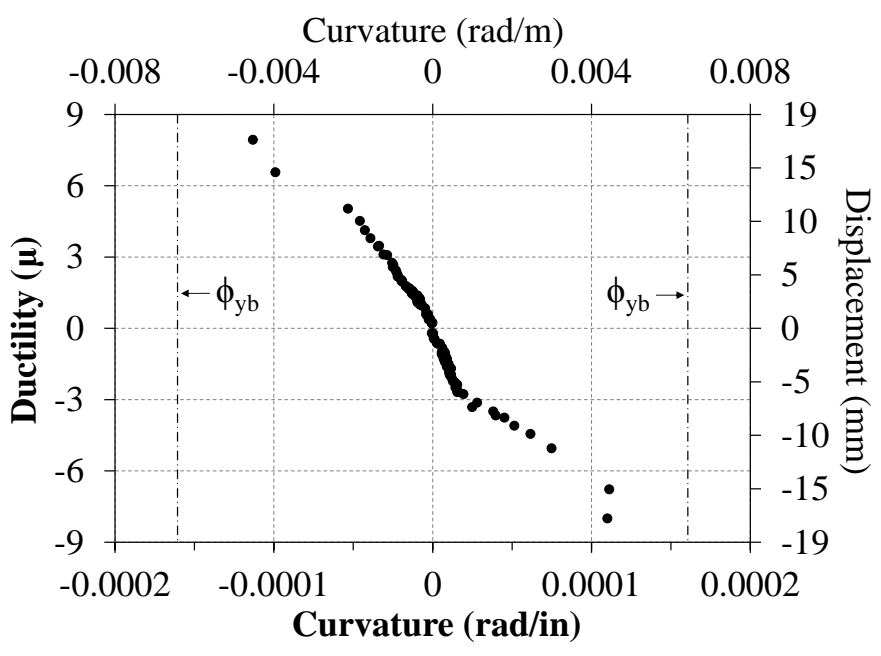

(a)

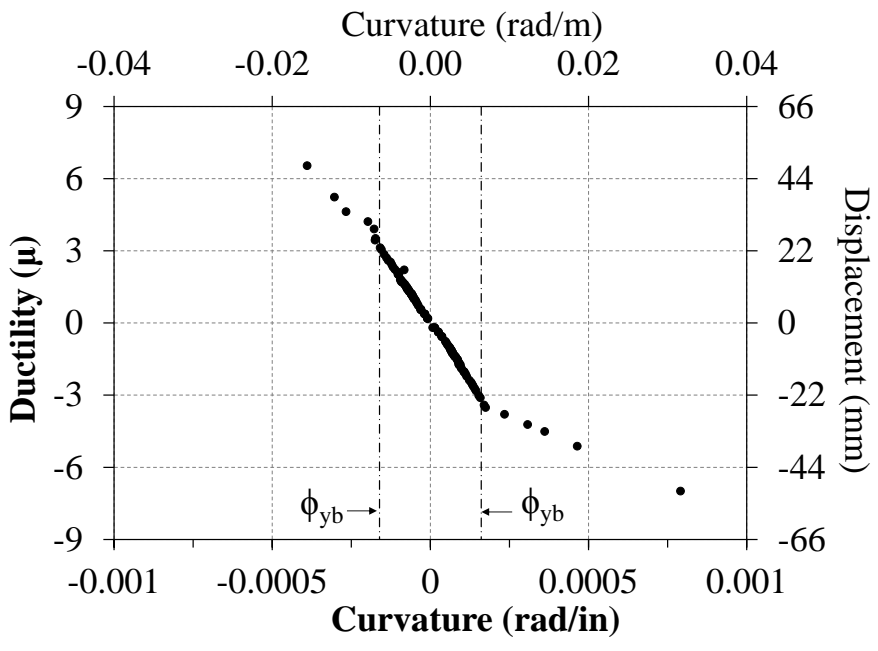

(b)

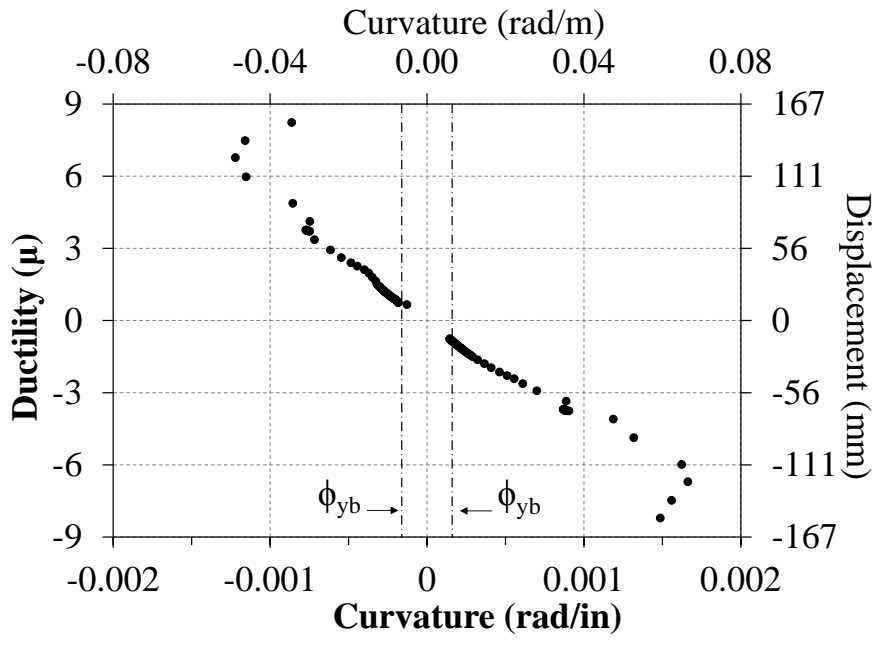

(c)

Figure 5.39 Average curvature profiles in cap beam. (a) Bent/BRB 1; (b) Bent/BRB2; (c) As-built 1. 
Table 5.7 Displacement ductility of retrofitted bents

\begin{tabular}{c|c|c|c|c}
\hline Test & $\begin{array}{c}\text { Nominal } \\
\text { yield } \\
\text { in (mm) }\end{array}$ & $\begin{array}{c}\text { Measured first } \\
\text { yield } \\
\text { mm (in) }\end{array}$ & $\begin{array}{c}\text { Effective yield } \\
\mathrm{mm}(\text { in) }\end{array}$ & $\begin{array}{c}\text { Max. } \\
\text { Displacement } \\
\text { ductility }(\mu)\end{array}$ \\
\hline Bent/BRB 1 & $0.069(1.8)$ & $0.085(2.2)$ & $0.085(2.2)$ & 8.0 \\
\hline Bent/BRB 2 & $0.205(5.2)$ & $0.29(7.4)$ & $0.29(7.4)$ & 7.0 \\
\hline As-built Bent 1 & $0.53(13.4)$ & $0.56(14.2)$ & $0.73(18.5)$ & 6.7 \\
\hline
\end{tabular}

The displacement ductility in the As-built test at the point of failure, which was defined as the point when the strength degraded more than $20 \%$, was $\mu=6.7$. This moderate ductility value was partially caused by the low column axial loads that prevented early rebar buckling and concrete crushing, and due to a relative long lap splice $\left(\sim 40 \mathrm{~d}_{\mathrm{b}}\right)$ at the base of the columns. Despite achieving this moderate levels of ductility, the bent exhibited significantly larger inelastic curvatures than the retrofitted bents at similar levels of ductility.

\subsubsection{Backbone Curve Comparison}

Backbone curves were utilized to visualize and understand the difference in terms of overall load and displacement capacity that the specimens exhibited. Figure 5.40 compares the backbone curves from the three experiments in terms of ductility of the As-built condition, i.e., ductility one corresponds to the effective yield of the As-built RC bent $\left(\delta_{\mathrm{ye}}\right)$. As expected, the retrofitted bents exhibited larger strength and stiffness as compared to the As-built bent. For the retrofitted state, the strength increased by $60 \%$ with respect to the strength in the As-built condition. This behavior was expected because the inclusion of the $\mathrm{BRB}$, which acts in parallel with the RC bent, adds stiffness and strength to the overall system. Bent/BRB1 and Bent/BRB2 presented similar peak strength since both BRB 
specimens had the same material properties and cross sectional area of steel core. The main difference between the retrofitted specimens was the brace stiffness, which for the Bent/BRB1 was almost three times the stiffness of the Bent/BRB2. It is worth noting that the Bent/BRB1, which was designed using a structural fuse concept, did not reach the effective yield of the RC bent for the displacement demand considered. This result confirms that a fuse based design utilizing BRBs can limit the inelastic displacement demands in the columns and beams and still provide a highly ductile behavior and energy dissipation. The fact that the Bent/BRB2 had an abrupt drop in load was attributed to the unexpected BRB fracture caused by poor confinement of the brace between the steel core and the nonyielding length.

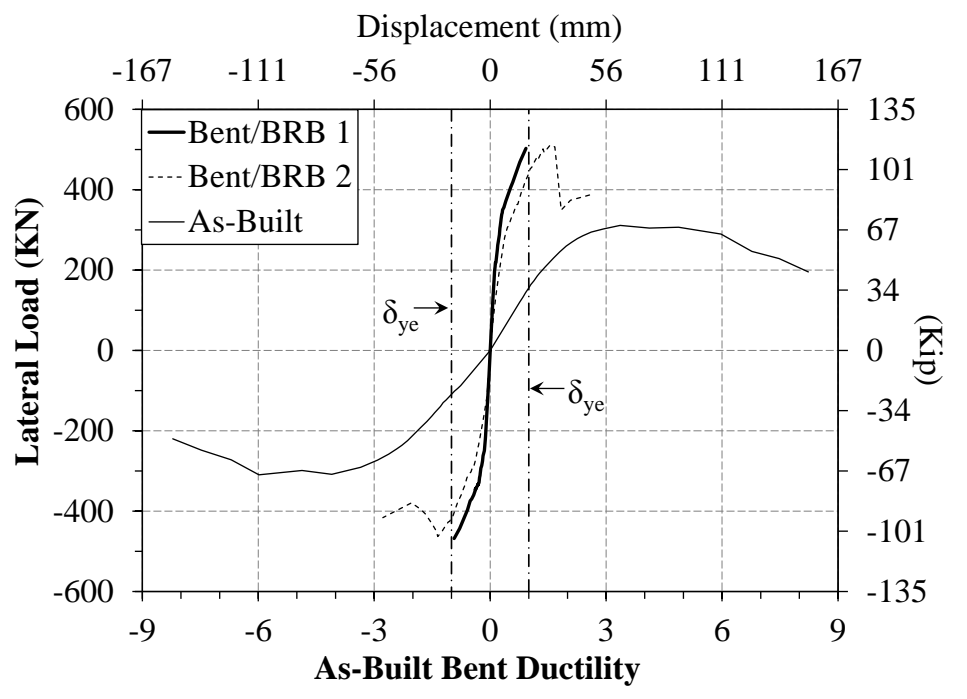

Figure 5.40 Backbone curves comparison (1 ${ }^{\text {st }}$ stage $)$

Despite the seismic detailing deficiencies, the backbone curve for the As-built bent showed moderate displacement capacity. Even though this behavior was unexpected because of the vast number of deficiencies in the as-built bent, similar results of flexural dominated columns can be found in the literature (McLean, et al., 1998), (Eberhard \& Marsh, 1997), 
(ElGawady, et al., 2010). The ductile performance is likely a result of a relatively long lap splice length $\left(40 \mathrm{~d}_{\mathrm{b}}\right)$, and low axial column loads $\left(0.1 \mathrm{f}_{\mathrm{c}} \mathrm{A}_{\mathrm{g}}\right)$, demonstrating a level of conservatism in assessing the response of flexural columns with detailing that does not fully conform to current standards.

\subsubsection{Stiffness Degradation}

The stiffness degradation is an important property in RC bridges subjected to seismic events because of the influence on the effective natural period of the structure. Stiffness degradation of the retrofitted bents can be attributed to BRB nonlinear behavior, concrete nonlinear behavior caused by flexural and shear cracking, slippage of steel reinforcement, etc. In this study, the normalized stiffness, represented as the secant stiffness $\left(k_{\text {sec }}\right)$ divided by the yield stiffness $\left(\mathrm{k}_{\mathrm{y}}\right)$, at different displacement ductilities, was utilized as the stiffness degradation parameter. It is worth noting that the yield displacement used in the calculations corresponds to the yield displacement of the BRB as that was the first sign of inelasticity of the retrofitted bents.

In terms of stiffness degradation both BRB test specimens showed similar stiffness degradation up to a displacement ductility of 3 as shown in Figure 5.41. However, for ductilities above 3, the Bent/BRB1 showed a lower stiffness degradation than the Bent/BRB2. This corresponds to the hysteretic response showed by the two specimens, in which the Bent/BRB1 showed a stable response throughout the test. Bent/BRB2 showed greater rate of stiffness degradation in the negative direction due to strength deterioration in compression at a displacement ductility exceeding 3.2. Moreover, the Bent/BRB2 exhibited an abrupt decay at a displacement ductility of 4.2 due to the fracture of the BRB steel core. 
Gulkan and Sozen (1974) proposed a relation between the displacement ductility ( $\mu$ ) and the secant stiffness $\left(\mathrm{k}_{\mathrm{sec}}\right)$ as shown in Eq. (5.8). That relation does not agree well with the stiffness degradation for the BRB specimens since that stiffness ratio was developed to be representative of deficient RC columns. In order to incorporate the stiffness degradation calculated for the retrofitted specimens the equation proposed by Gulkan and Sozen was modified by incorporating an exponential factor (c). In the original equation this c factor is equal to 1 . However, a $\mathrm{c}$ factor of 0.6 was deemed suitable to fit the experimental data of the specimens retrofitted with BRBs. This result, as expected, implies that the retrofitted bent has a lower rate of stiffness degradation than deficient RC components.

$$
\frac{k_{\mathrm{sec}}}{k_{y}}=\frac{1}{\mu^{c}} \leq 1
$$

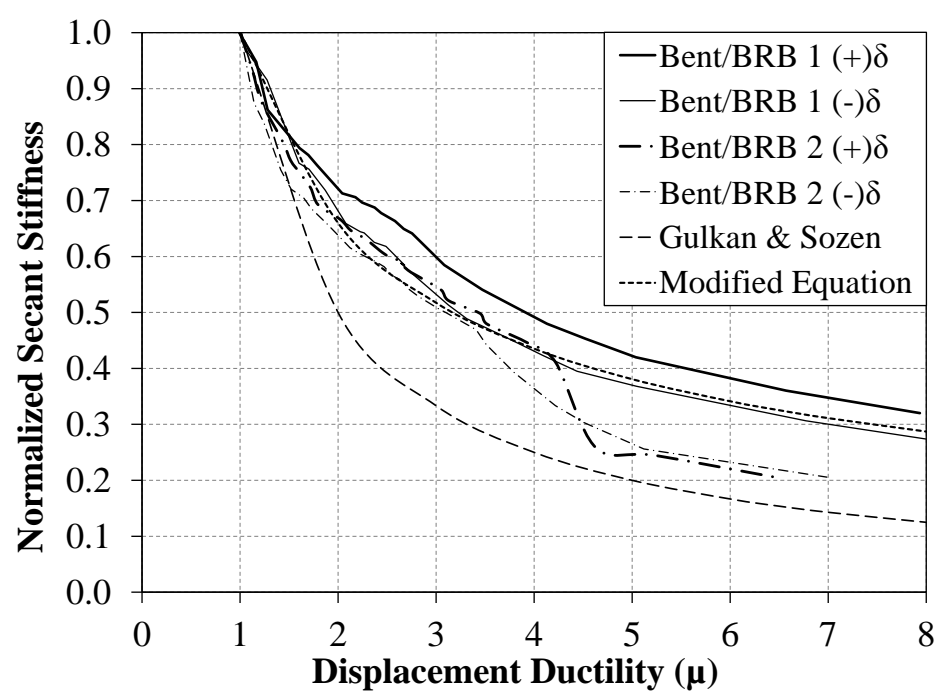

Figure 5.41 Stiffness degradation (1 ${ }^{\text {st }}$ Stage)

\subsubsection{Energy Dissipation and Equivalent Viscous Damping}

The property of dissipating energy through hysteretic behavior is desirable in structures subjected to major seismic events. A central task in any modern seismic analysis 
method as a measurement of energy dissipation is to determine the equivalent viscous damping of a structure. This is especially important in bridges that are designed and retrofitted using displacement based analysis. The total equivalent viscous damping $\left(\xi_{\mathrm{eq}}\right)$ was obtained by adding the initial elastic damping $\left(\xi_{\mathrm{el}}\right)$, which is typically considered as $5 \%$ for RC structures, and the hysteretic damping $\left(\xi_{\text {hys }}\right)$. The hysteretic damping (Priestley, et al., 2007) was calculated using Eq. (5.9).

$$
\xi_{\text {hys }}=\frac{1}{4 \pi} \frac{A_{d}}{A_{\text {strain }}}=\frac{1}{2 \pi} \frac{A_{d}}{F_{\max , \text { cycle }} \cdot \delta_{\text {cycle }}}
$$

Where, $A_{d}$ is the energy dissipated in a full cycle and $A_{\text {strain }}$ is the strain energy (or stored energy) measure at the peak force of each cycle $\left(F_{\max }, y c l\right)$. The equivalent viscous damping $\left(\xi_{\mathrm{eq}}\right)$ was calculated for each specimen and is illustrated in Figure 5.42.

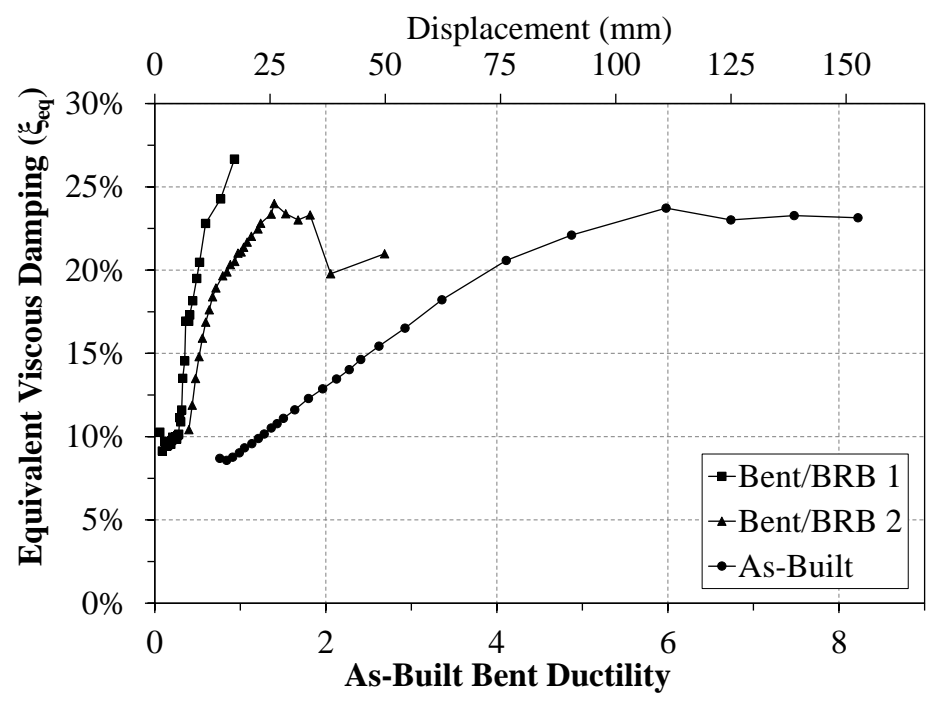

Figure 5.42 Equivalent viscous damping (1 ${ }^{\text {st }}$ stage $)$

The results showed that the Bent/BRB1 has the greatest equivalent viscous damping $\left(\xi_{\mathrm{eq}} \approx 27 \%\right)$ as compared to the other specimens. This result is consistent with the stable hysteretic behavior and wide loops shown by the Bent/BRB1, which is typical of energy 
dissipation systems. The equivalent viscous damping increased with increasing levels of drift up to failure. For the As-built specimen the $\xi_{\text {eq }}$ remained constant with increasing drift due to the gradual degradation of strength. In the Bent/BRB2, the equivalent viscous damping abruptly decreased due to the failure of the steel core signifying the BRB contribution to energy dissipation.

All the specimens achieved a maximum $\xi_{\text {eq }}$ values of approximately $25 \%$ as they each reached their respective ductility levels of approximately 8 . However, the retrofitted bent specimens reached that level of equivalent viscous damping at much smaller displacements than the As-built. And, in the case of the Bent/BRB1, this level was even reached prior to the RC bent started to contribute to the dissipating energy by yielding of the columns. This result shows that the BRB retrofitted bent can reach high levels of energy dissipation without even relying on the energy dissipated by the original structure.

\subsubsection{Comparison to Analytical Displacement Demands}

Figure 5.43 shows a comparison between the experimental results and the analytical displacement demands under the 500-year earthquake event and the 1000-year event. The results further demonstrate that the target performance levels required by ODOT (2015) were achieved, resulting in a fully "Operational” performance level for the Bent/BRB 1 under the serviceability level earthquake (500-year event) as well as for the design level earthquake (1000-year event). Further, for Bent/BRB 2 the Operational performance criteria under the 500-year event and the Life Safety criteria under the 1000 year event were still satisfied. 


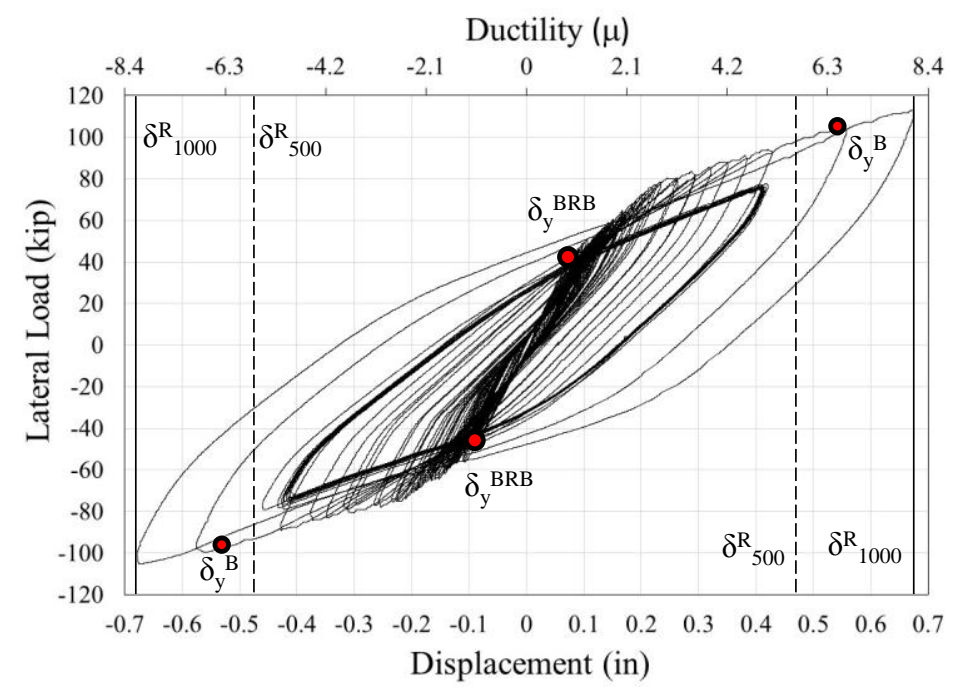

(a)

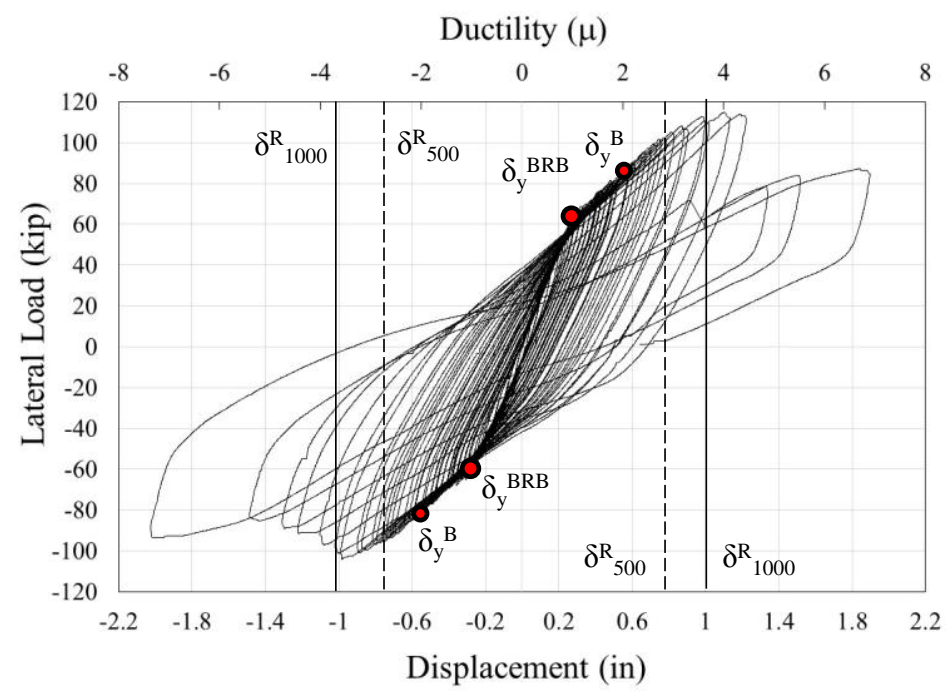

(b)

Figure 5.43 Comparison between experimental results and analytical demand; (a) Bent/BRB 1, (b) Bent/BRB 2

\subsubsection{Performance Assessment Based on Strain Measurements}

In order to assess the performance of test specimens, it is common practice to relate strain data to certain limit states (Kowalsky, 2000). For example, the two-level performance criteria required by ODOT in terms of strains is indicated in Table 5.8. Other researchers have also proposed different strain limits as shown in Table 5.8. 
Table 5.8 Quantitative limit states in terms of strains

\begin{tabular}{|c|c|c|c|c|}
\hline $\begin{array}{c}\text { Performance } \\
\text { Level }\end{array}$ & $\begin{array}{l}\text { Seismic } \\
\text { Hazard }\end{array}$ & $\begin{array}{l}\text { ODOT } \\
(2015)\end{array}$ & $\begin{array}{l}\text { Kowalski } \\
(2000)\end{array}$ & Sheikh \& Légeron (2014) \\
\hline $\begin{array}{c}\text { Fully } \\
\text { Operational }\end{array}$ & - & - & - & $\begin{aligned} & \mathrm{f}_{\mathrm{c}}<\mathrm{f}_{\mathrm{cr}}= 0.4 \sqrt{f^{\prime}{ }_{c}}(\mathrm{MPa}) \\
& \mathrm{f}_{\mathrm{s}}<\mathrm{f}_{\mathrm{y}}\end{aligned}$ \\
\hline $\begin{array}{c}\text { Operational } \\
\text { (Serviceability) } \\
\end{array}$ & $\begin{array}{l}500 \text {-year } \\
(\text { ODOT }) \\
\end{array}$ & $\begin{array}{c}\varepsilon_{\mathrm{cc}}=0.005 \\
\varepsilon_{\mathrm{s}} \leq 2 \varepsilon_{\mathrm{sh}}=0.03\end{array}$ & $\begin{array}{l}\varepsilon_{\mathrm{c}} \leq 0.004 \\
\varepsilon_{\mathrm{s}} \leq 0.015\end{array}$ & $\begin{array}{l}\varepsilon_{\mathrm{c}} \leq 0.004 \\
\varepsilon_{\mathrm{s}} \leq 0.007\end{array}$ \\
\hline $\begin{array}{c}\text { Life Safety } \\
\text { (Damage } \\
\text { Control) }\end{array}$ & $\begin{array}{l}\text { 1000-year } \\
\text { (ODOT) }\end{array}$ & $\begin{array}{c}\varepsilon_{\mathrm{cc}}=0.9 \varepsilon_{\mathrm{cu}} \approx 0.008 \\
\varepsilon_{\mathrm{s}} \leq \varepsilon_{\mathrm{su}}^{\mathrm{R}}=0.09\end{array}$ & $\begin{aligned} \varepsilon_{\mathrm{cu}} & \approx 0.018 \\
\varepsilon_{\mathrm{s}} & \leq 0.06\end{aligned}$ & $\begin{array}{c}\varepsilon_{\mathrm{c}}=\varepsilon_{\mathrm{cc} 50} \\
\text { (initial core crushing) } \\
\varepsilon_{\mathrm{c}}=\varepsilon_{\mathrm{cu}} \\
\text { (fracture of hoops) } \\
\varepsilon_{\mathrm{s}} \leq \varepsilon_{\mathrm{su}}=0.07 \\
\text { (rebar fracture) } \\
\varepsilon_{\mathrm{s}} \leq \varepsilon_{\mathrm{scr}} \\
\text { (onset of buckling) }\end{array}$ \\
\hline
\end{tabular}

Where, $f_{c}$ is the stress in concrete; $f_{c r}$ is the tensile strength of concrete; $f_{c}$ is compressive strength of concrete; $f_{s}$ is the stress in steel; $f_{y}$ is the yield strength of steel; $\varepsilon_{s}$ is the reinforcing steel strain; $\varepsilon_{\mathrm{sh}}$ is the reinforcing steel strain at the onset of strain hardening; $\varepsilon_{\text {su }}^{\mathrm{R}}$ is the reduced ultimate tensile strain in the reinforcing steel; $\varepsilon_{\mathrm{su}}$ is the tensile strain at fracture; $\varepsilon_{\mathrm{scr}}$ is the steel strain at the onset of buckling of longitudinal; $\varepsilon_{\mathrm{c}}$ is the concrete strain in concrete, $\varepsilon_{\mathrm{cc}}$ is the concrete strain in the confined section; $\varepsilon_{\mathrm{cu}}$ is the ultimate concrete strain computed using Mander's model. The values of $\varepsilon_{\text {sh }}$ and $\varepsilon_{\text {su }}^{R}$ depend on the size of the rebar and are given in AASHTO (2009). ODOT's performance levels based on the rebar size used in this study are indicated in Table 5.8. From that table, it can be observed that the difference in concrete strains between the operational and the life safety performance level is only 0.003 . This low margin was caused by the low concrete confinement, which is typical of RC bents built prior to 1970 . Reinforcement strains and concrete strains are presented and discussed in the following sections. Maximum strains at the end of each test are compared to the limit states given in Table 5.8. 


\subsubsection{Steel Reinforcement Strains}

The strains in rebars were measured through 90 strain gauges as shown in Figure 5.15. The results of these measurements are depicted in Figure 5.44 to Figure 5.47 for columns of Bent/BRB1 and Figure 5.48 to Figure 5.51 for columns of Bent/BRB2 at different displacement ductilities. The designation used in the figures is as follows: column number (C1 or C2), the quadrant (1, 2, 3 or 4), and the type of rebar (dowel: D or continuous: C). For example, C1-2-C corresponds to Column1 $-2^{\text {nd }}$ quadrant - Continuous rebar. In the figures, some strain measurements are missing since during the concrete pouring a few strain gauges got damaged. Moreover, positive values of ductility was defined when the BRB was in tension. The results clearly demonstrate that the columns of Bent/BRB1 underwent only minor inelastic excursions. The maximum strains were at the top of column C2 in quadrants 3 and 4 with strain values close to 0.002 . This greater demand in column 2 was presumably caused by the larger axial loads transferred from the BRB to that column as shown in Figure 5.35(a). Comparing the maximum steel strain reached by the columns of Bent/BRB1 to the minimum steel strain limit presented in Table 5.8 for the Operational performance level $\left(\varepsilon_{\mathrm{s}}=0.007\right)$, it is evident that this retrofitted bent remained Operational. In the case of Bent/BRB2 the maximum strain demand was also in column C2 with a value of 0.015 , which is still lower than the steel strain limit required by ODOT for the Operational level but is close to the value suggested by Kowalski (2000) and definitely greater than the one recommended by Sheikh and Légeron (2014). Moreover, Figure 5.52 shows that the cap beam remained elastic for Bent/BRB 1 and minor inelastic strain excursions occurred in Bent/BRB2. 


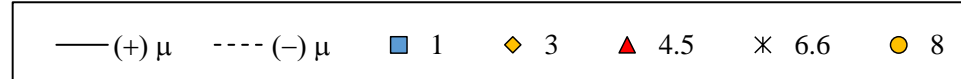
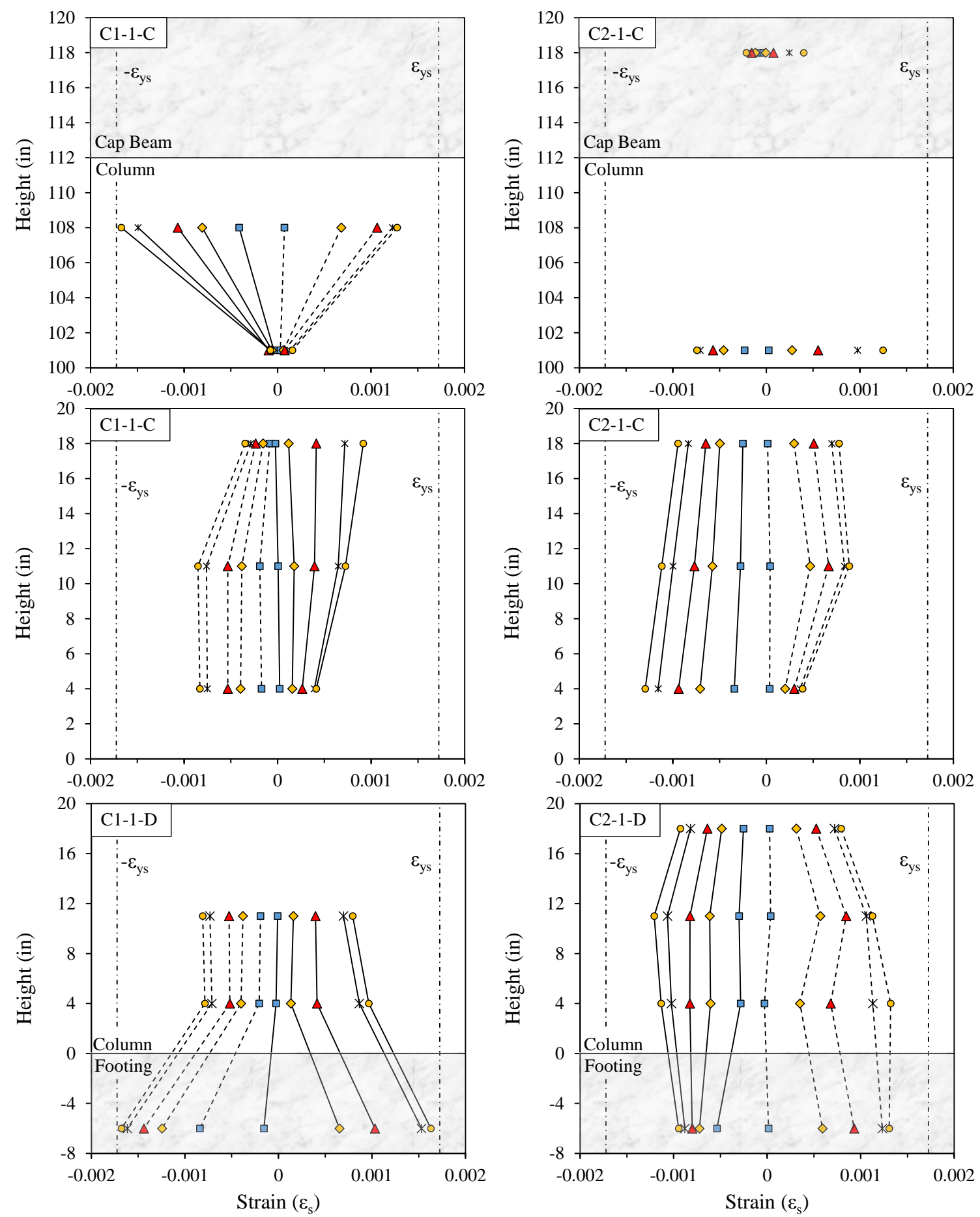

Figure 5.44 Strains in reinforcing steel for Bent/BRB 1 (1 ${ }^{\text {st }}$ quadrant) 


$$
-(+) \mu \quad--(-) \mu \quad \square 1 \quad \diamond 3 \quad \Delta 4.5 \quad * 6.6 \quad 0 \quad 8
$$
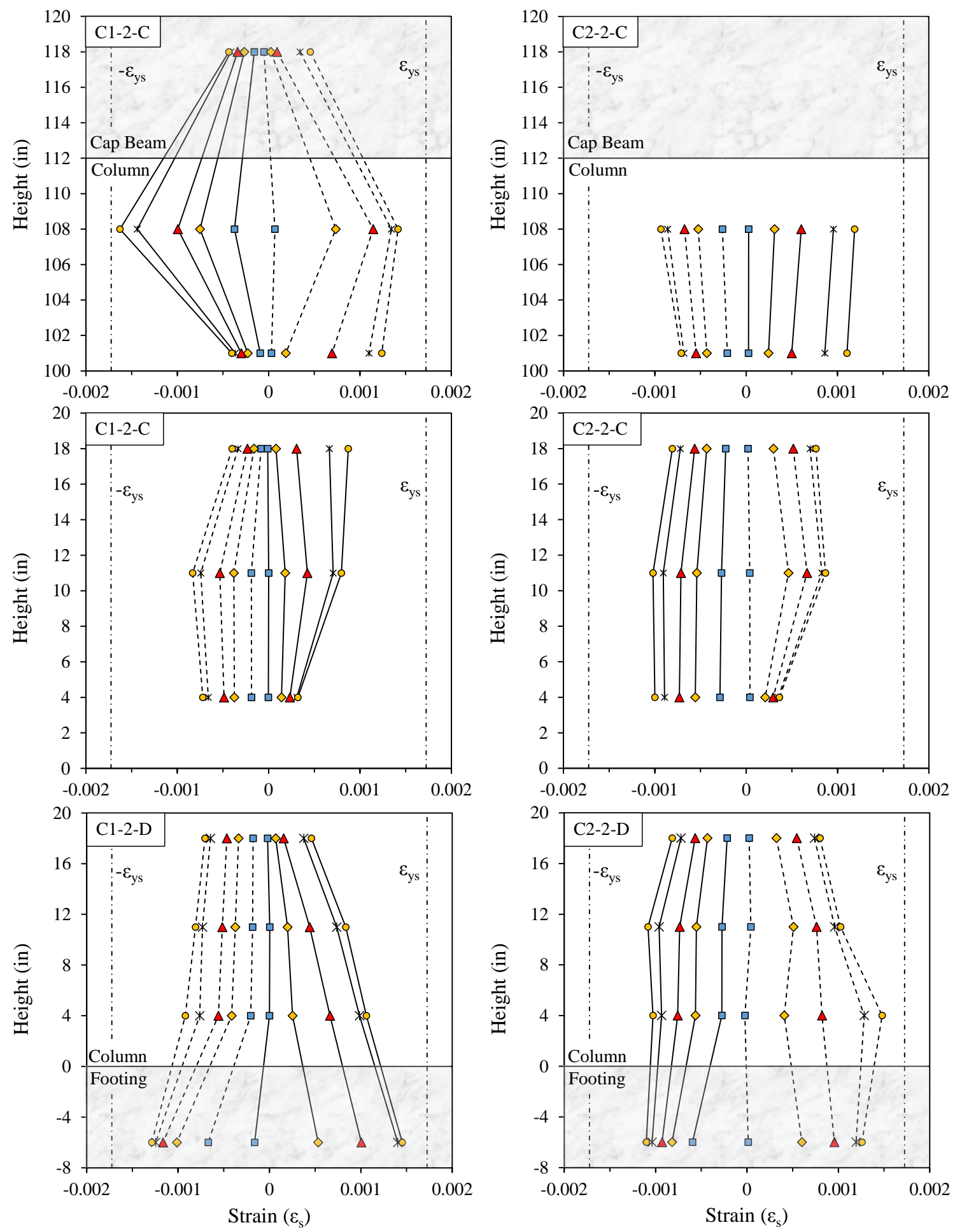

Figure 5.45 Strains in reinforcing steel for Bent/BRB 1 (2 $2^{\text {nd }}$ quadrant) 


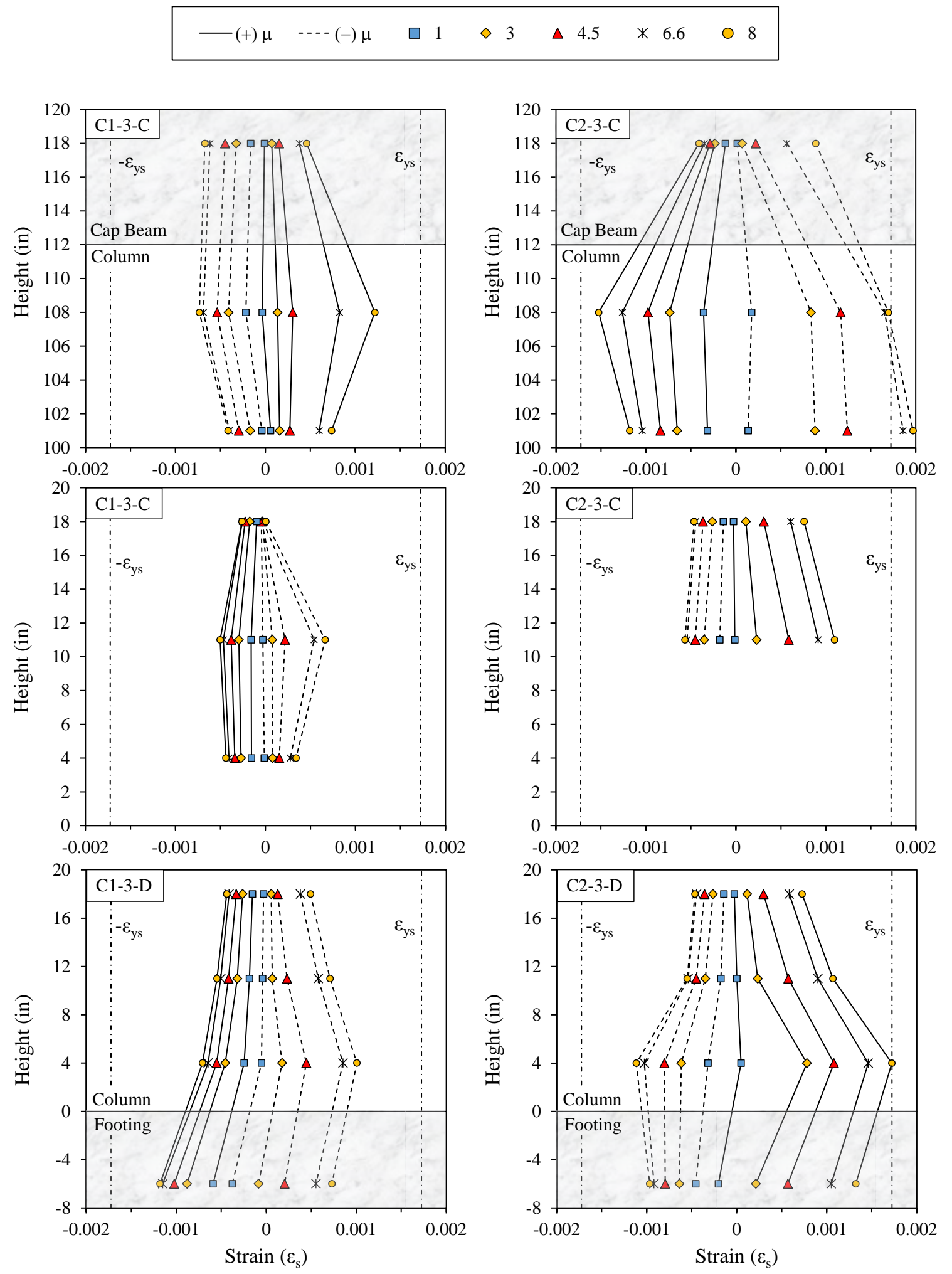

Figure 5.46 Strains in reinforcing steel for Bent/BRB 1 ( $3^{\text {rd }}$ quadrant) 


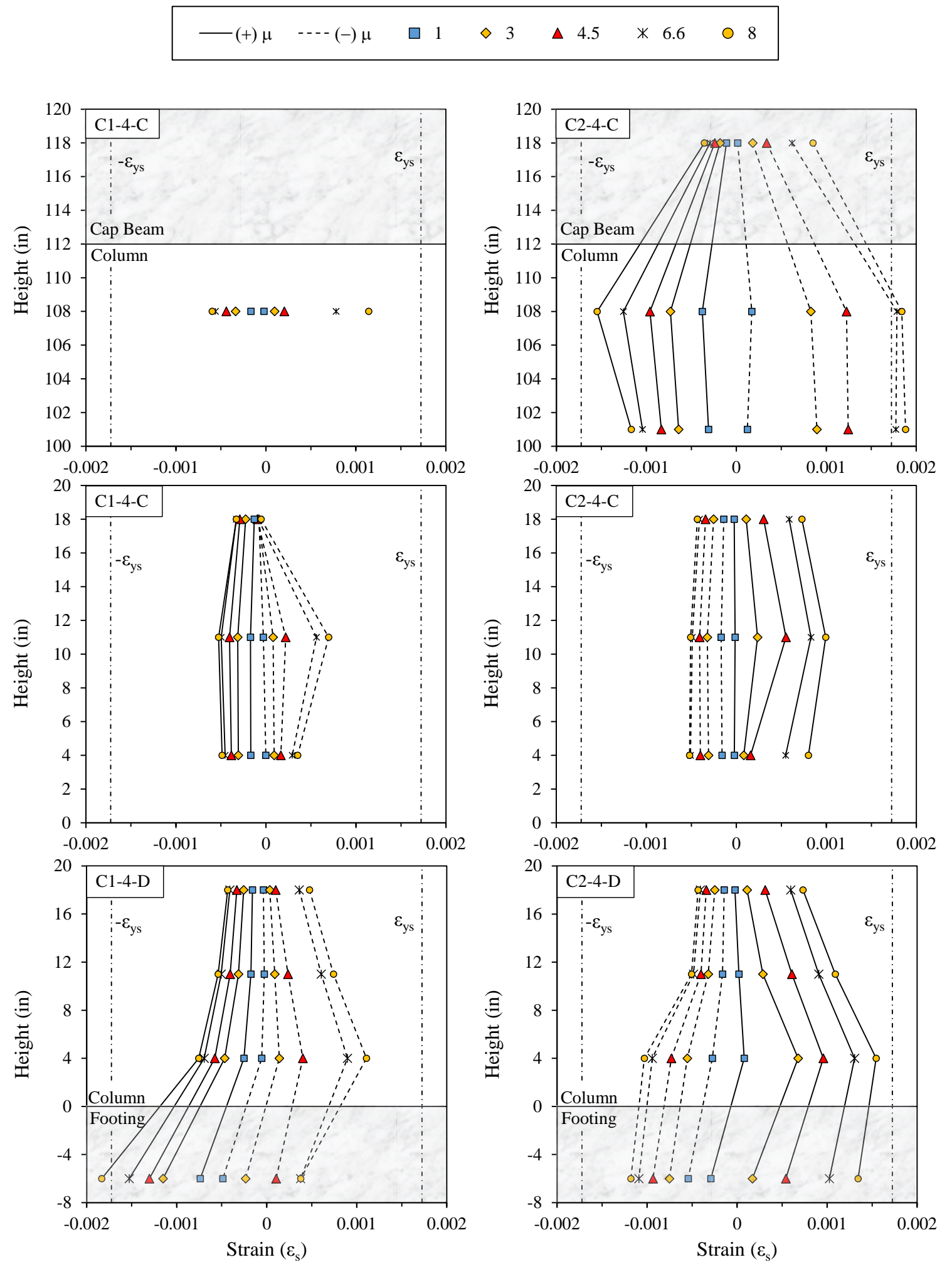

Figure 5.47 Strains in reinforcing steel for Bent/BRB 1 (4th quadrant) 


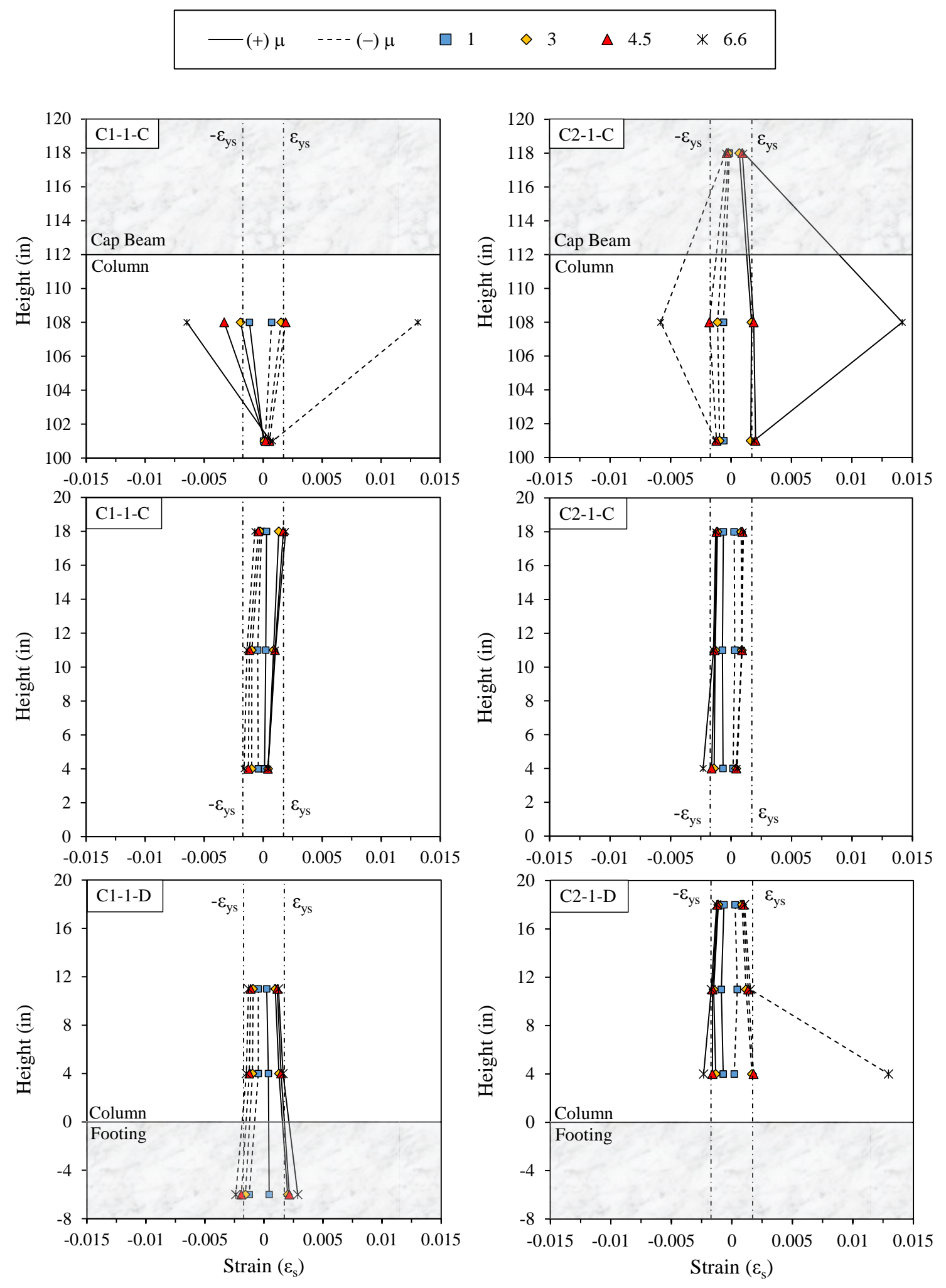

Figure 5.48 Strains in reinforcing steel for Bent/BRB 2 (1 ${ }^{\text {st }}$ quadrant) 


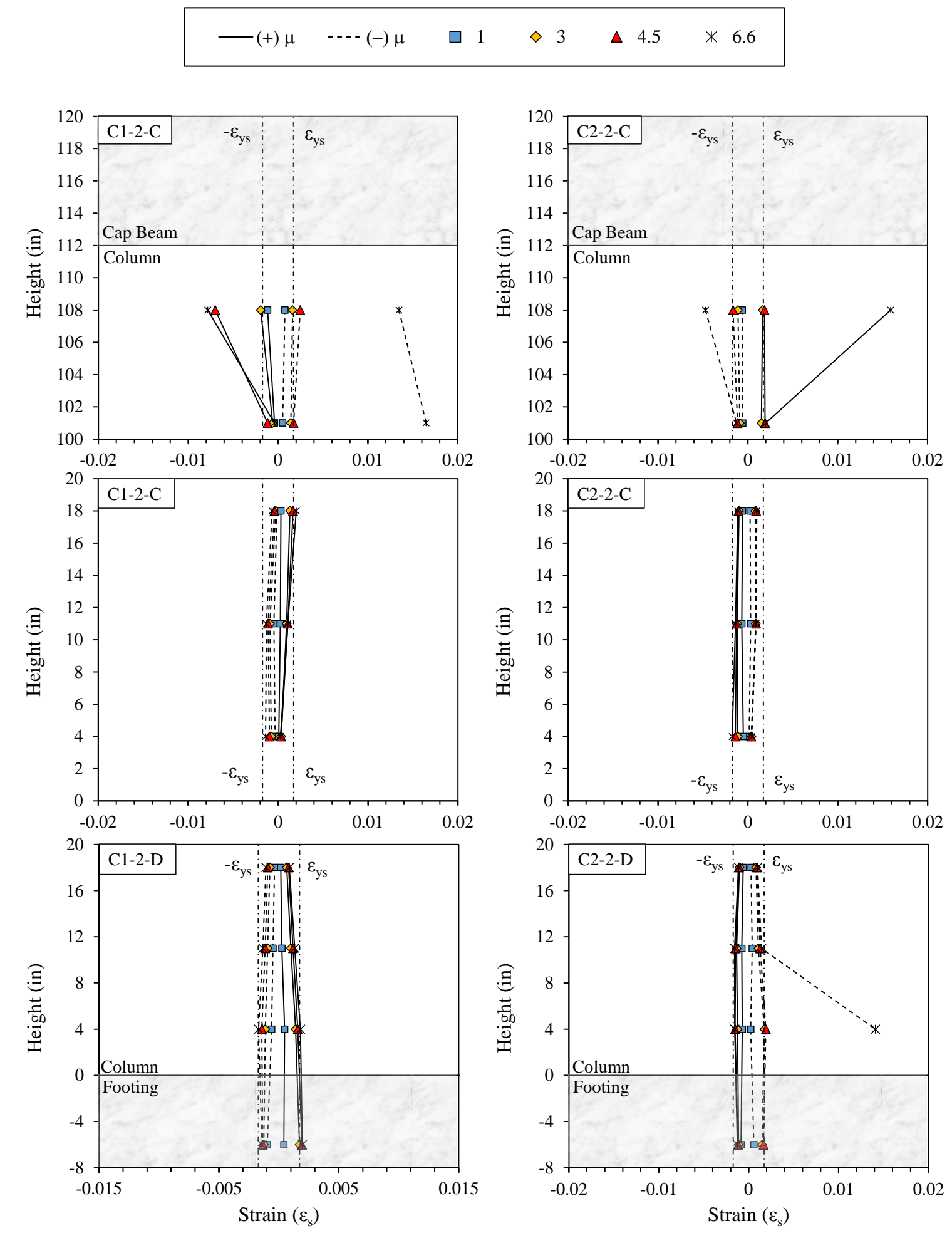

Figure 5.49 Strains in reinforcing steel for Bent/BRB 2 (2 ${ }^{\text {nd }}$ quadrant) 


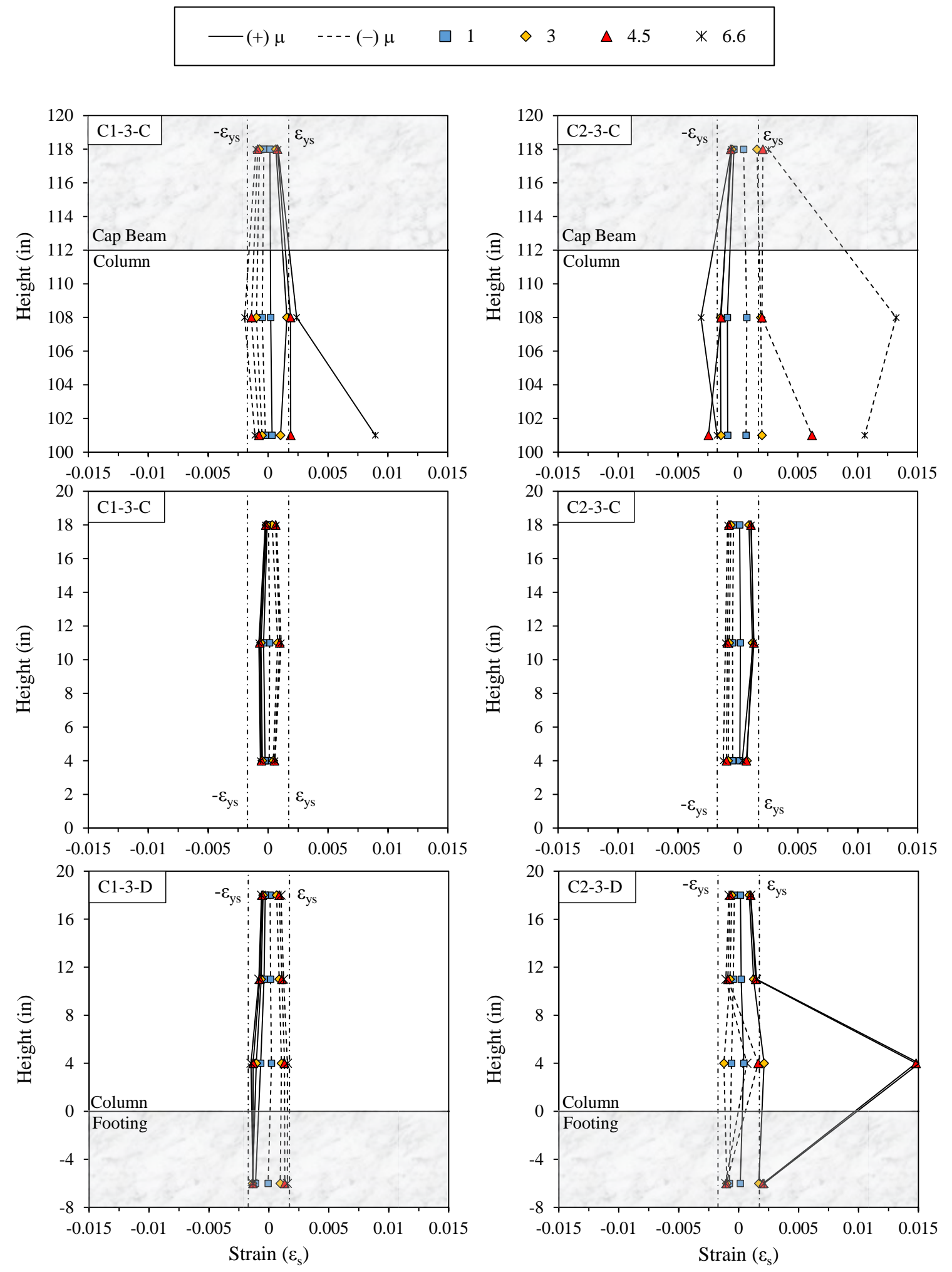

Figure 5.50 Strains in reinforcing steel for Bent/BRB 2 (3rd quadrant) 


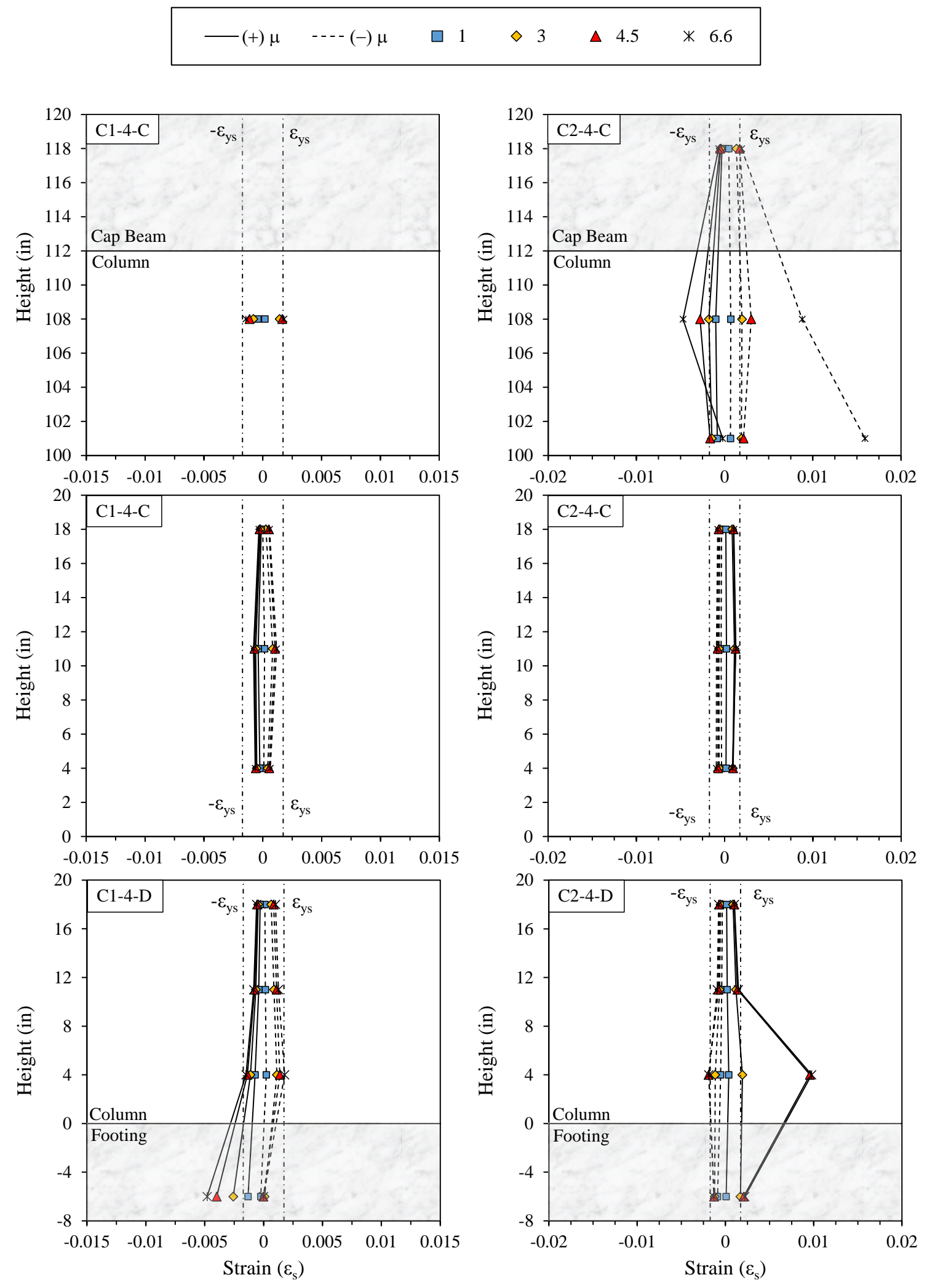

Figure 5.51 Strains in reinforcing steel for Bent/BRB 2 (4th quadrant) 

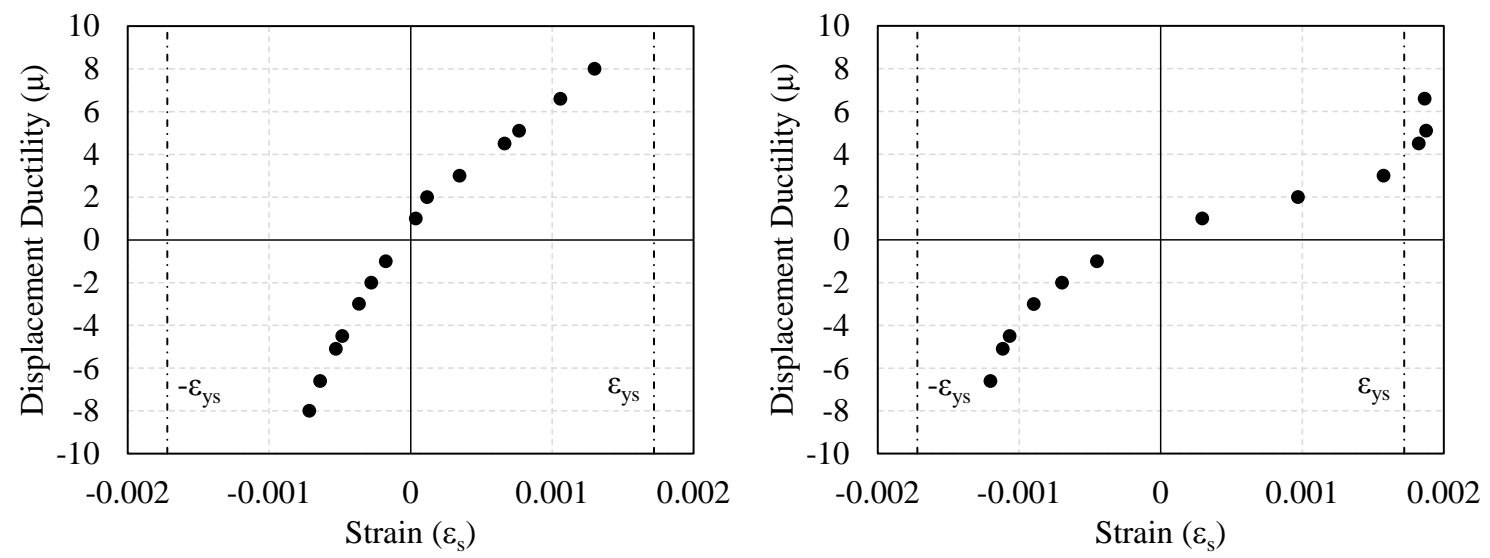

Figure 5.52 Rebar strains in cap beam. (a) Bent/BRB1; (b) Bent/BRB2

\subsubsection{Concrete Strains}

The concrete strains at the base of the columns were obtained using the results from the strains in the reinforcing steel and the linear strain profile for a circular section shown in Figure 5.53. In order to obtain the compression strains in the concrete at the column base, only tensile strains measurements in the dowels were used; meanwhile, both dowels and longitudinal reinforcement strain gauge measurements were used for the compression strains. The use of the tensile strain in the dowels was used to avoid the discrepancy in tensile strains caused by the lap splice. Concrete strains at the top of the columns were computed using tensile and compression strains, which were measured using strain gauges installed on the longitudinal reinforcing steel.

Results of using this methodology are presented in Figure 5.54. Maximum strains for Bent/BRB1 occurred at the top of the columns with values close to $0.002 \mathrm{in} / \mathrm{in}$. This result again confirms that Bent/BRB1 did not exceed the strain limits for the Operational performance level presented in Table 5.8. On the contrary, the concrete strains in Bent/BRB2 exceeded the strain limits for the Operational performance and were close to the strain limits for the Life Safety level. 


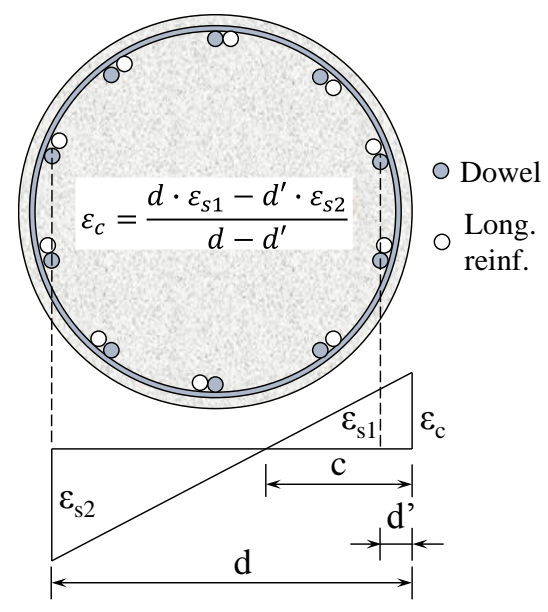

Figure 5.53 Linear strain profile for a circular section

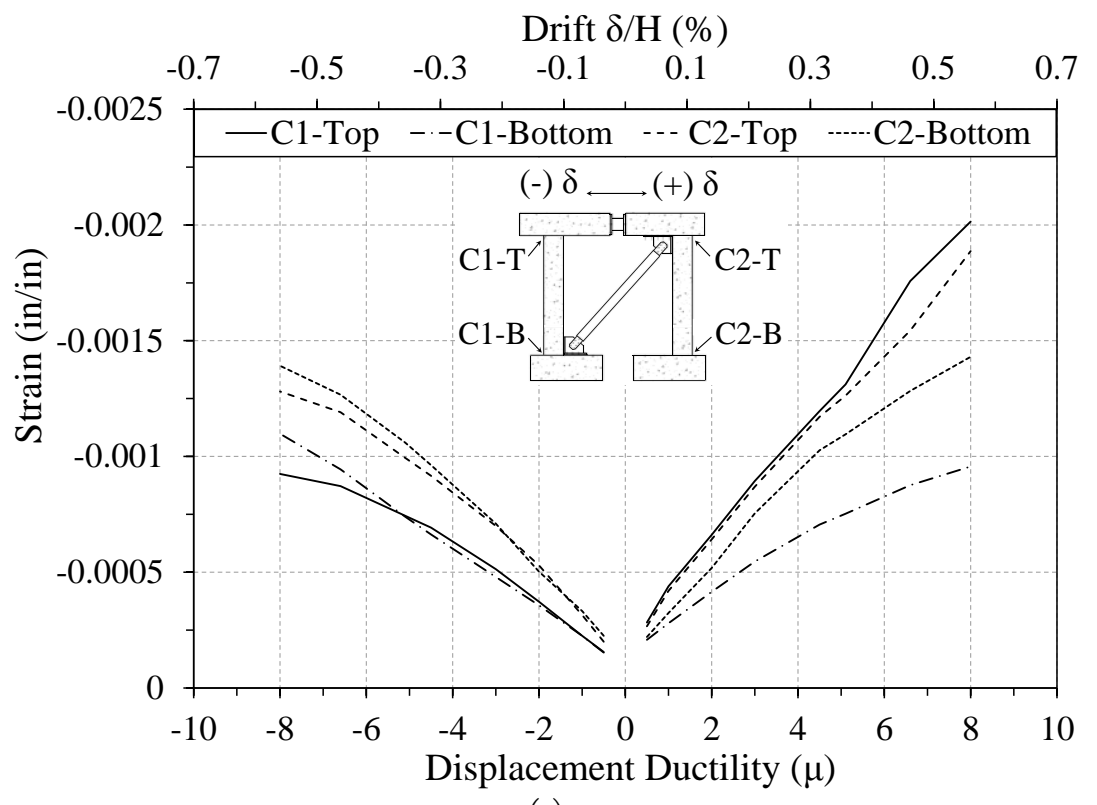

(a) 


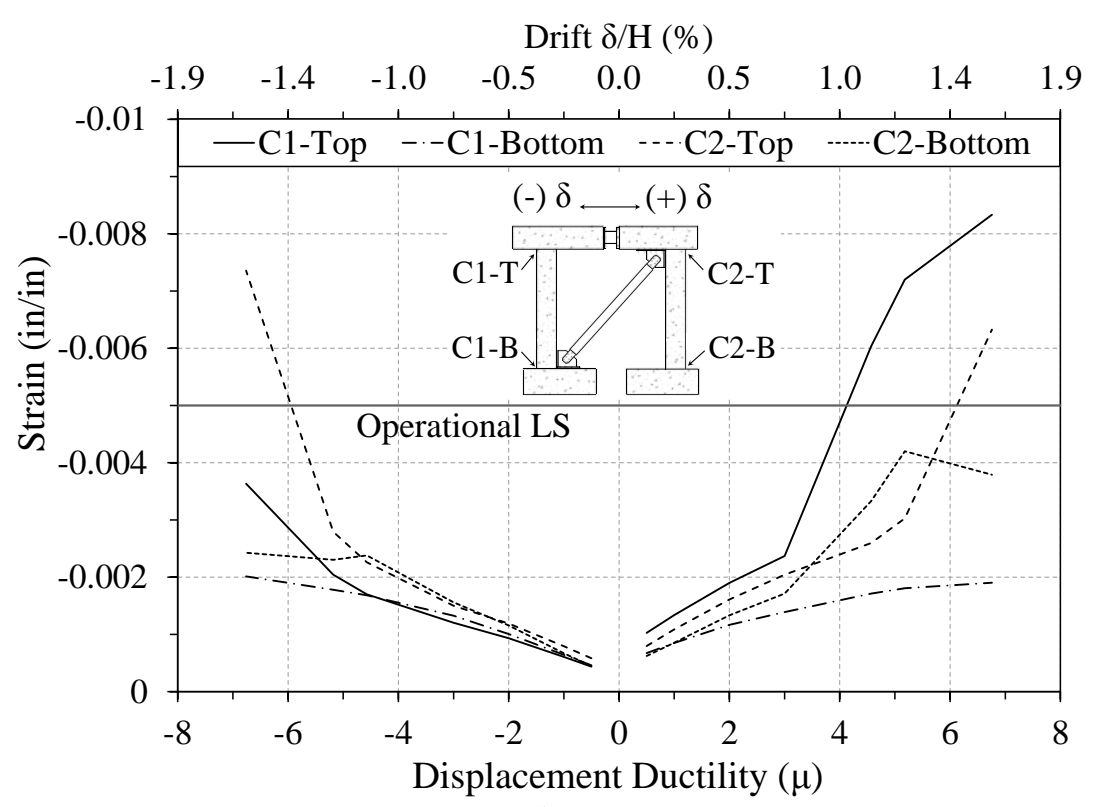

(b)

Figure 5.54 Compression strains in concrete (a) Bent/BRB1; (b) Bent/BRB2

\subsubsection{RC Bent Limit States}

In order to relate the global performance levels, namely, Operational and Life Safety, to component's levels, a five level component performance is shown in Table 5.9. This table is based on the work carried out by Hose \& Seible (1999) and adapted to correspond with ODOT's performance criteria. The table also shows the corresponding performance criteria suggested by Hose \& Seible, in which the Operational level corresponds to first yielding and minor cracking, and Life Safety corresponds to the onset of spalling.

Results of using the five level component performance for the As-built Bent 1 are illustrated in Figure 5.55 and values indicated in Table 5.10. The results show that the Operational performance level is reached at a ductility of 1.7 and the Life Safety performance at a ductility of 4.8. Comparing these values of displacement ductility to the backbone curves depicted in Figure 5.40 further demonstrate that the Bent/BRB 1 remained within the Operational Performance level; while, the Bent/BRB 2 satisfy the Life Safety 
performance. It is worth mentioning that the As-built bent showed moderate displacement ductility capacity, which indicates that retrofitting this bent may not be needed.

Table 5.9 Component Performance Levels adapted from Hose \& Seible

\begin{tabular}{c|c|c|c|c}
\hline Level & $\begin{array}{c}\text { Component } \\
\text { Performance }\end{array}$ & $\begin{array}{c}\text { Repair } \\
\text { Description }\end{array}$ & $\begin{array}{c}\text { Hose \& Seible } \\
\text { Global } \\
\text { Performance }\end{array}$ & $\begin{array}{c}\text { ODOT's } \\
\text { Performance } \\
\text { Levels }\end{array}$ \\
\hline I & Cracking & No Repair & $\begin{array}{c}\text { Fully } \\
\text { Operational }\end{array}$ & - \\
\hline II & First Yield & Possible Repair & Operational & - \\
\hline III & Effective Yield & Possible Repair & - & - \\
\hline IV & Onset of Spalling & Minimum Repair & Life Safety & Operational \\
\hline V & Buckling or Rupture & Replacement & Collapse & Life Safety \\
\hline
\end{tabular}

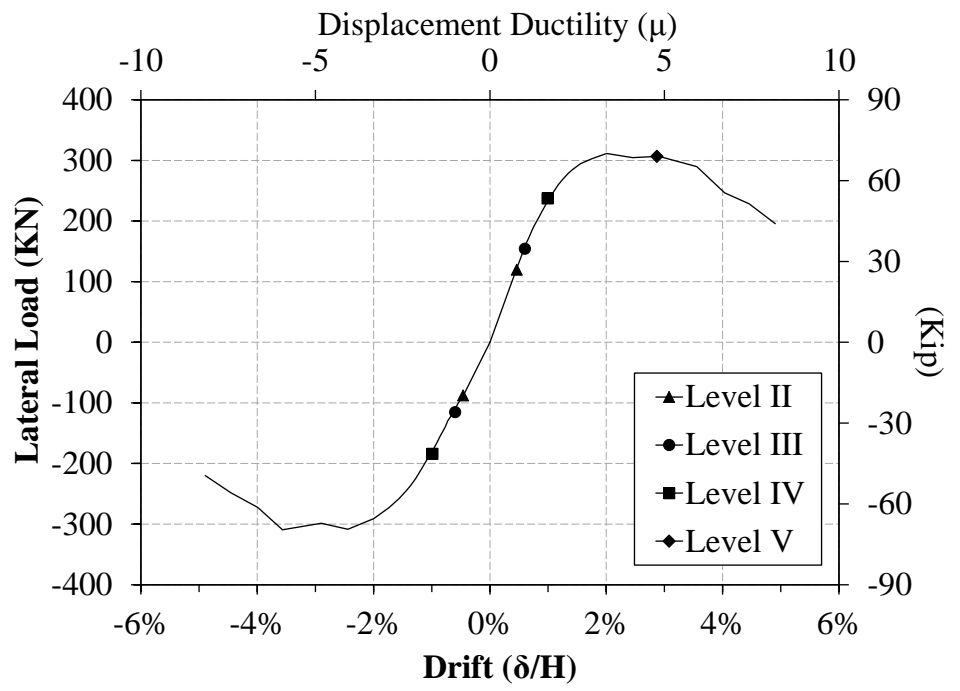

Figure 5.55 Limit States for As-built Bent 1

Table 5.10 As-built Bent 1 Performance Parameters (Limit States)

\begin{tabular}{c|c|c|c|c|c}
\hline Level & Limit State & $\begin{array}{c}\text { Steel } \\
\text { Strains }\left(\varepsilon_{\mathrm{s}}\right)\end{array}$ & $\begin{array}{c}\text { Concrete } \\
\text { Strains }\left(\varepsilon_{\mathrm{c}}\right)\end{array}$ & $\begin{array}{c}\% \\
\text { Drift }\end{array}$ & $\begin{array}{c}\text { Ductility } \\
(\mu)\end{array}$ \\
\hline I & Cracking & 0.0008 & 0.0007 & 0.21 & 0.3 \\
\hline II & First Yield & 0.0017 & 0.0012 & 0.46 & 0.8 \\
\hline III & Effective Yield & 0.0020 & 0.0017 & 0.60 & 1 \\
\hline IV & Onset of Spalling & 0.010 & 0.0042 & 0.99 & 1.7 \\
\hline V & $\begin{array}{c}\text { Buckling or } \\
\text { Rupture }\end{array}$ & 0.048 & 0.0080 & 2.87 & 4.8 \\
\hline
\end{tabular}




\subsection{SECOND StAge - EXPERIMENTAL EVALUATION OF REPAIRED BENT}

The second stage of the experimental program consisted of evaluating the feasibility of using BRBs as repair measure for damaged RC bents. The test sequence comprised testing an undamaged RC bent, hereinafter referred to as As-built Bent 2, until considerable damage was observed. Then, a second test comprised repairing the already damaged bent with BRBs, hereinafter referred to as Bent/BRB 3. Both tests were conducted using a cyclic loading that aims to reflect subduction zone earthquake displacement demands as shown in Figure 5.22(b).

\subsubsection{Cyclic Behavior and Damage Progression}

The experimental outcomes are discussed with reference to global and local behavior.

\subsubsection{As-built Bent 2}

This bent was tested using the proposed protocol discussed in Chapter 2 for structures of ductility 8 and fundamental period of $0.5 \mathrm{sec}$. However, in order to avoid reaching the Collapse performance level (Table 5.9), this bent was tested up to displacement ductility 4.5. This ductility value was selected based on the result obtained for the As-built Bent 1, which suggested that rebar buckling would occur at a displacement ductility of 4.8 as shown in Table 5.10.

The recorded lateral load-deformation behavior for the As-built Bent 2 is shown in Figure 5.56. The peak lateral load was 66 kips $(294 \mathrm{kN})$ and occurred at a lateral displacement of approximately 3.22 in $(82 \mathrm{~mm})$. The first yield occurred at a displacement of 0.56 in $(14 \mathrm{~mm})$ and the effective yield was computed as 0.73 in $(19 \mathrm{~mm})$. These yield 
displacements match the values obtained for the retrofitted bents (Bent/BRB $1 \& 2)$ and also the As-built Bent 1.

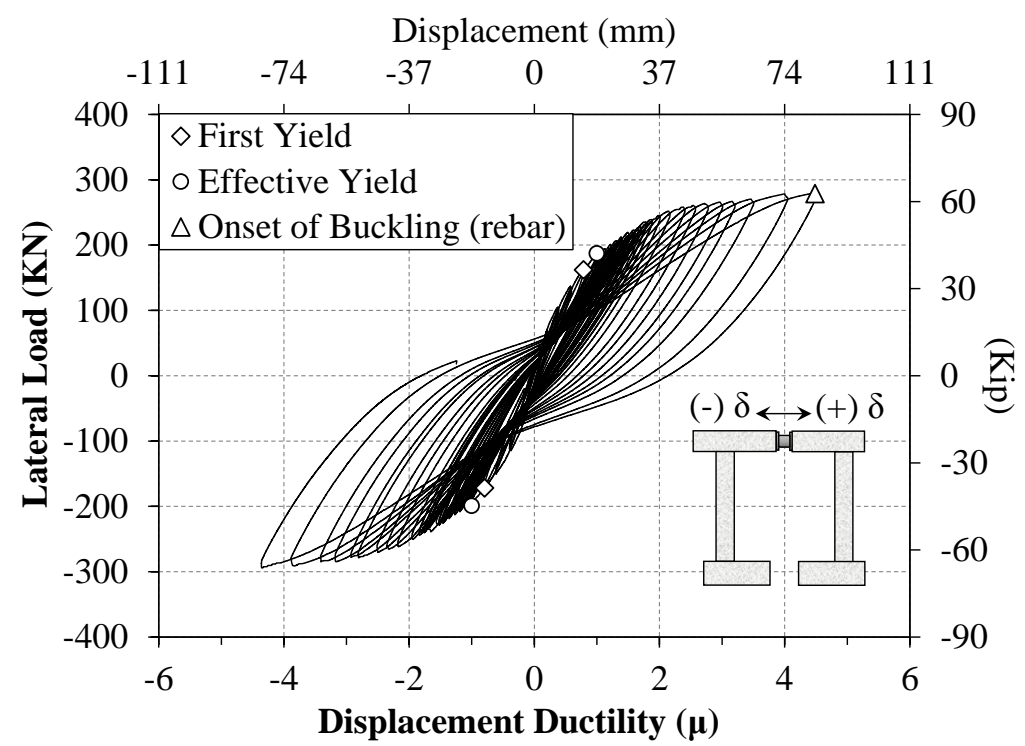

Figure 5.56 Hysteretic response of As-built Bent 2

The initial damage consisted of horizontal cracks that were propagated throughout the height of the expected plastic hinge zones of columns. Contrary to the case of the retrofitted bents, in the As-built Bent 2 was observed early formation of vertical cracks in the cap beam at the face of column C1 (see Figure 5.13 for column designations). Vertical cracks in the bent beam increased in width up to $0.6 \mathrm{~mm}$ as shown in Figure 5.57 (a). Significant spalling of concrete at expected plastic hinge locations was observed during the last cycles as shown in Figure 5.57(b). Also at the end of testing some vertical cracks were observed at the column base, which can be attributed to the lap splice at that location. Onset of dowel buckling was observed at the base of column C2 during the last cycle at a displacement ductility of 4.5 as depicted in Figure 5.57(c). Similar to the results obtained for the As-built Bent 1, the As-built Bent 2 exhibited moderate ductile response and stable behavior. In this case failure of the specimen was not reached. 


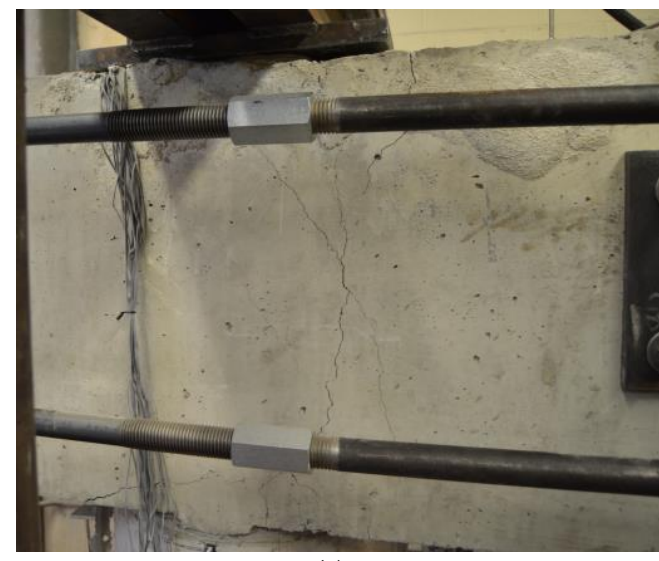

(a)

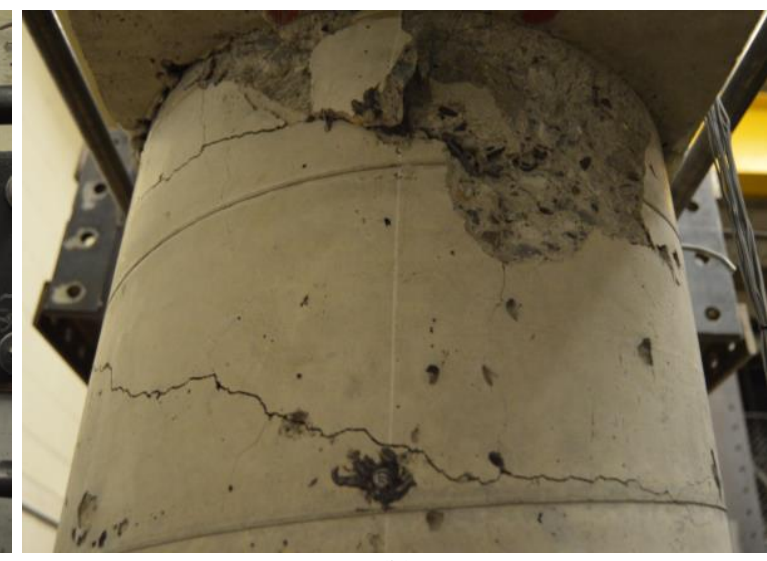

(b)

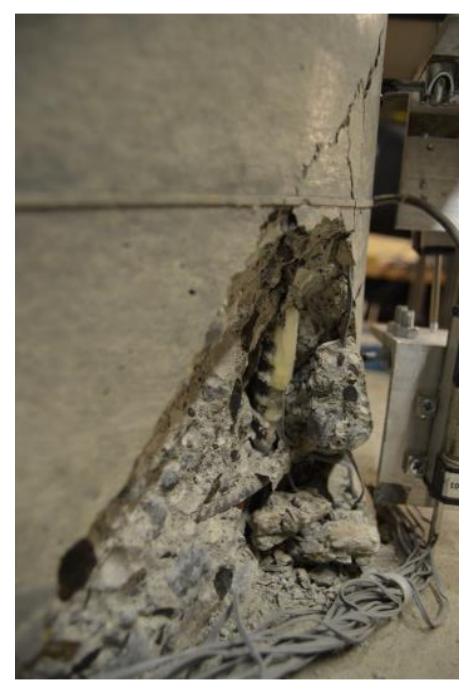

(c)

Figure 5.57 Damage in As-built Bent 2; (a) Damage in cap beam, (c) Significant spalling in columns, (c) Onset of rebar buckling

\subsubsection{Repaired Bent/BRB 3}

The buckling-restrained brace used in Bent/BRB3 was designed following the structural fuse concept applied to a damaged bent as described in Chapter 4 and exemplified in Section 5.2.3, which resulted on limiting the displacement of the bent to 1.2 in under the 1000 -year event in order to prevent significant damage in cap beam and columns. From the results of the As-built Bent 2, it was concluded that repairing the cap beam was also needed. With that aim, the use of a post-tensioning load that increases compressive axial stresses in the beam and in consequence increases the capacity of both cap-beam and column-beam 
joint was deemed suitable for this application. Thus, a post-tensioning force was applied using the 4 horizontal high-strength rods attached to the connector beam on one side and to end plates on the other as shown in Figure 5.13. The force applied to the cap beam was calculated with the aim of restricting further damage in the cap beam. Analysis performed in SAP2000 resulted in a post-tension force of 70 kips. This design resulted in a Bent/BRB system as shown in Figure 5.58.

It is worth mentioning that columns were not further repaired and that for this phase of the experimental program a different BRB manufacturer provided the BRB.

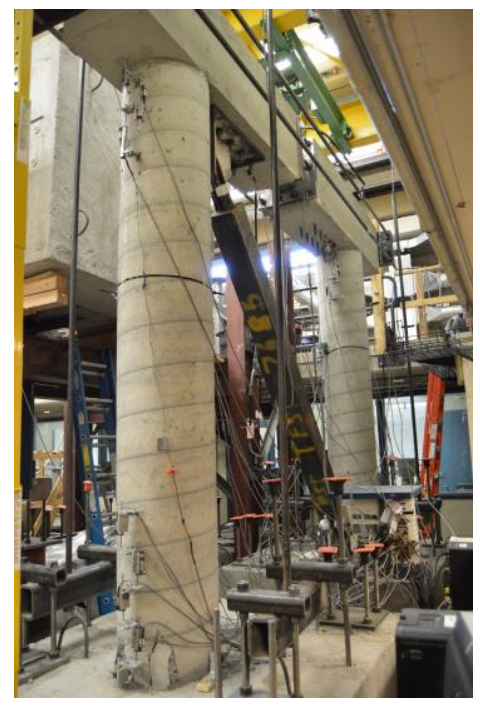

Figure 5.58 Bent/BRB 3

Despite of preliminary calculations that indicated the maximum demand for this bent was 1.2 inches $(30 \mathrm{~mm})$ under the 1000-year event, testing was initially performed until completion of the loading protocol targeting ductility 8 . However, during the test was observed that the hysteretic behavior of the bent did not show any sign of strength deterioration and the damage in the RC components was still acceptable. Thus, cycles of increasing amplitude were performed after the proposed loading history. The test was ended after 5 more cycles of increased displacement amplitude because the hysteretic response of 
the Bent/BRB3 did not show noticeable strength deterioration and more importantly because the large amplitude displacements in conjunction with excessive out-of-plane displacements jeopardized the safety of the equipment in the lab.

The lateral load vs. deformation hysteresis curve shown in Figure 5.59 indicates highly ductile behavior and high energy dissipation up to a displacement ductility of 17.7 , which is equivalent to a displacement of 4.53 in $(115 \mathrm{~mm})$. The nominal lateral displacement at yield for the BRB was calculated as 0.16 in $(4.1 \mathrm{~mm})$ and the experimental one was 0.26 in $(6.6 \mathrm{~mm})$, which is a $62 \%$ increase with respect to the nominal value. Displacement ductility was calculated using the yield displacement of the BRB. The effective yield of the RC bent is also depicted in Figure 5.59 as a point of comparison with the results of the As-built Bent 2 .

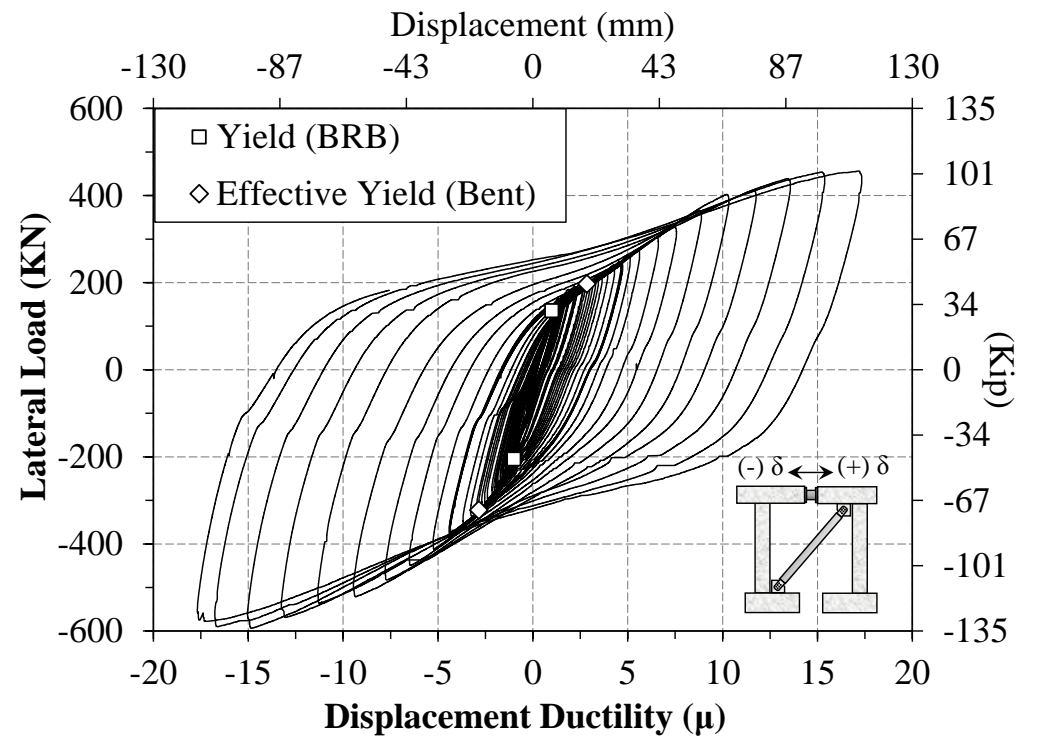

Figure 5.59 Hysteretic response of Bent/BRB 3

As the progressively increasing displacements were applied, the lateral load increased up to 134 kips $(596 \mathrm{kN})$ for the brace compression direction and $103 \mathrm{kips}(458 \mathrm{kN}$ ) for the brace tension direction. This difference in strength capacity was expected since Figure 5.59 clearly shows that the response was dominated by the stable hysteretic behavior of the BRB, 
which is also known for presenting more strength capacity in compression due to the additional friction forces exerted in the interface between the steel core and the concrete confining it.

Regarding damage progression, at the beginning of the test the columns and cap beam presented the same damage shown at the end of the As-built Bent 2 test (Figure 5.57). No further damage was registered in RC components until a displacement ductility of 8 , which is equivalent to 2.1 in $(53 \mathrm{~mm})$. After that point, crushing of the concrete at the base and top of the columns was noticeable, exposing the column reinforcement. Once the concrete cover was lost, the longitudinal bars and dowels in those regions began to buckle as shown in Figure 5.60(a) and (b). Despite of the columns sustaining significant damage that can be categorized in the Collapse limit state, the system showed only minor strength deterioration, in particular in the last cycle when the BRB was in compression. Moreover, at the end of the test no further damage was observed in the cap beam as shown in Figure 5.60(c), which demonstrates that post-tensioning the cap beam was an effective method to restrict the damage in that component.

Inspection of the gusset plates, which was conducted following the removal of the $\mathrm{BRB}$, did not reveal any damage. It is worth mentioning that the same gusset plates were used for all the tests, showing the excellent behavior of those components. 


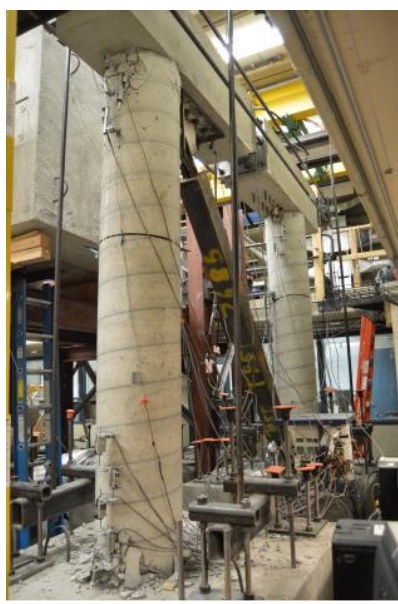

(a)

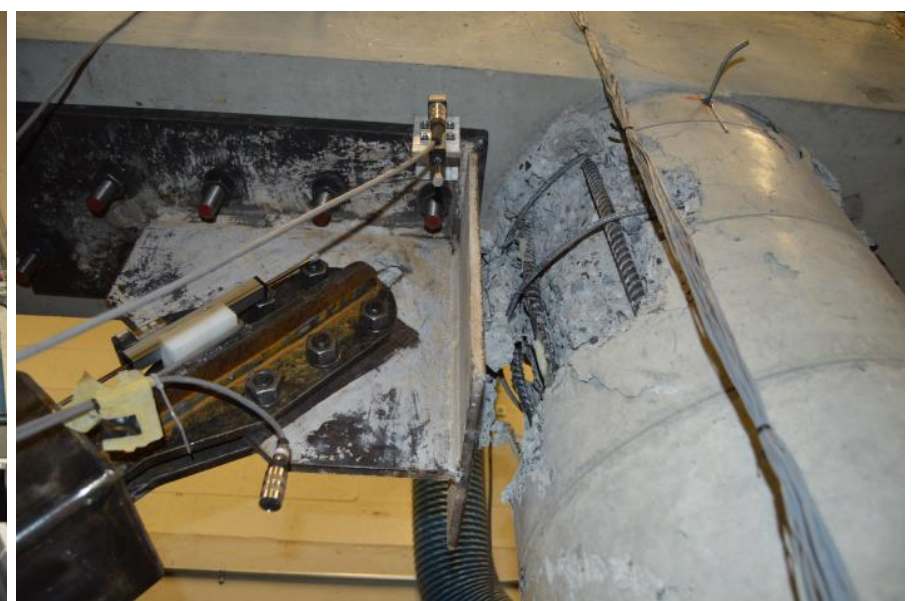

(b)

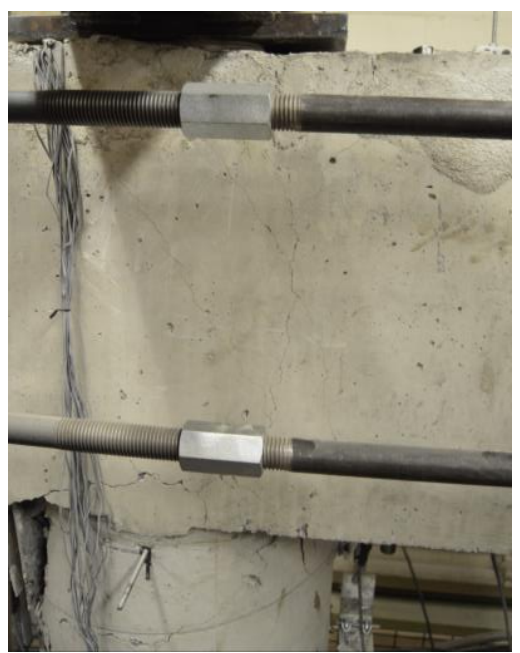

(c)

Figure 5.60 Damage in Bent/BRB3; (a) Overall damage, (b) Buckling of steel, (c) Damage in cap beam

\subsubsection{Yield Displacement and Ductility}

Actual yield displacement for the repaired specimen, Bent/BRB3, was obtained using the experimentally inferred yield elongation of the brace. The corresponding displacement ductilities were computed using the actual yield displacements. The displacement ductility for the As-built Bent 2 was computed using an effective yield displacement that was calculated to be the same that the one for the As-built Bent 1. Values of yield displacement and final displacement ductility for each specimen are summarized in Table 5.11. It is worth 
mentioning that neither the As-built Bent 2 nor the Bent/BRB3 reached failure, which was defined as the point when the strength degraded more than $20 \%$.

Table 5.11 Displacement ductility of repaired bent

\begin{tabular}{c|c|c|c|c}
\hline Test & $\begin{array}{c}\text { Nominal } \\
\text { yield } \\
\text { in (mm) }\end{array}$ & $\begin{array}{c}\text { Measured first } \\
\text { yield } \\
\text { mm (in) }\end{array}$ & $\begin{array}{c}\text { Effective yield } \\
\mathrm{mm} \text { (in) }\end{array}$ & $\begin{array}{c}\text { Displacement } \\
\text { ductility }(\mu) \text { at } \\
\text { the end of the } \\
\text { test }\end{array}$ \\
\hline As-built Bent 2 & $0.53(13.4)$ & $0.56(14.2)$ & $0.73(18.5)$ & 4.5 \\
\hline Bent/BRB 3 & $0.16(4.1)$ & $0.26(6.6)$ & $0.26(7.4)$ & 17.7 \\
\hline
\end{tabular}

\subsubsection{Buckling-Restrained Brace (BRB) Component Response}

Similar to the retrofitted bent, the elongation of BRB3 was measured via four LVDTs. Two of them measured the relative displacement between the steel core and the steel casing at the top and bottom connections. The other two were located on the sides of the brace to measure the total elongation. The brace elongation for the Bent/BRB 3 is shown in Figure 5.61. Results show that inelastic deformation demands were evenly distributed at the top and bottom of the BRB except the last two cycles. This result may explain why the BRB presented a stable response and did not fail prematurely like the BRB in Bent/BRB2.

The BRB axial response was obtained following the same procedure that for the retrofitted bents as discussed in Section 5.5.1.4 with the difference that the post-tensioning force was added in the free body diagram of the system. BRB response envelope during the test of Bent/BRB3 is illustrated in Figure 5.62. The numerical response was computed from using material properties and over-strength factors. The same over-strength factors used for the retrofitted bents were used in this case. Numerical and actual yield elongation were calculated as 0.1 in $(2.5 \mathrm{~mm})$ and 0.17 in $(4.3 \mathrm{~mm})$, respectively. Comparison of the inferred 
envelope and the numerically obtained bi-linear response in Figure 5.62 shows that the numerical approximation fits well with the experimental curves in the elastic range.

However, in the inelastic range the preliminary over-strength factors underestimated the BRB strength. This result was presumably caused by using a BRB provided by a different manufacturer, which proved to be effective in achieving greater elongation capacity but also detrimental in the excessive over-strength factors $(\sim 2)$ that the BRB attained.

The estimated maximum axial strain of the steel core at the end of the test was computed dividing the maximum elongation by the reduced section length, and was approximately $4 \%$.

The contribution of the BRB at the peak load of the repaired system was $73 \%$, which showed that most than half of the system maximum lateral load was resisted by the BRB.

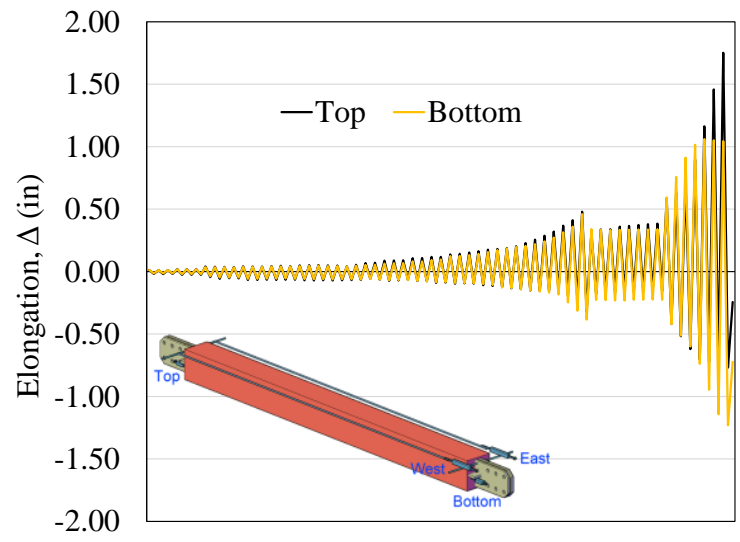

(a)

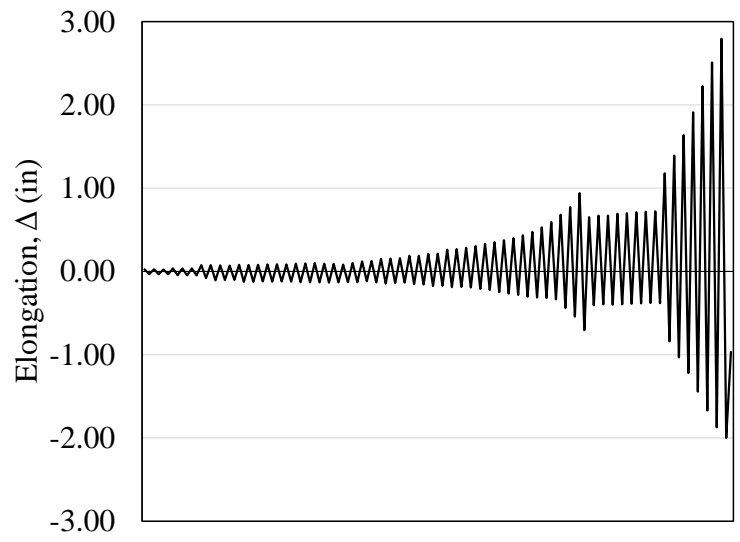

(b)

Figure 5.61 BRB deformation in Bent/BRB 3. (a) Top and Bottom, (b) Total deformation 


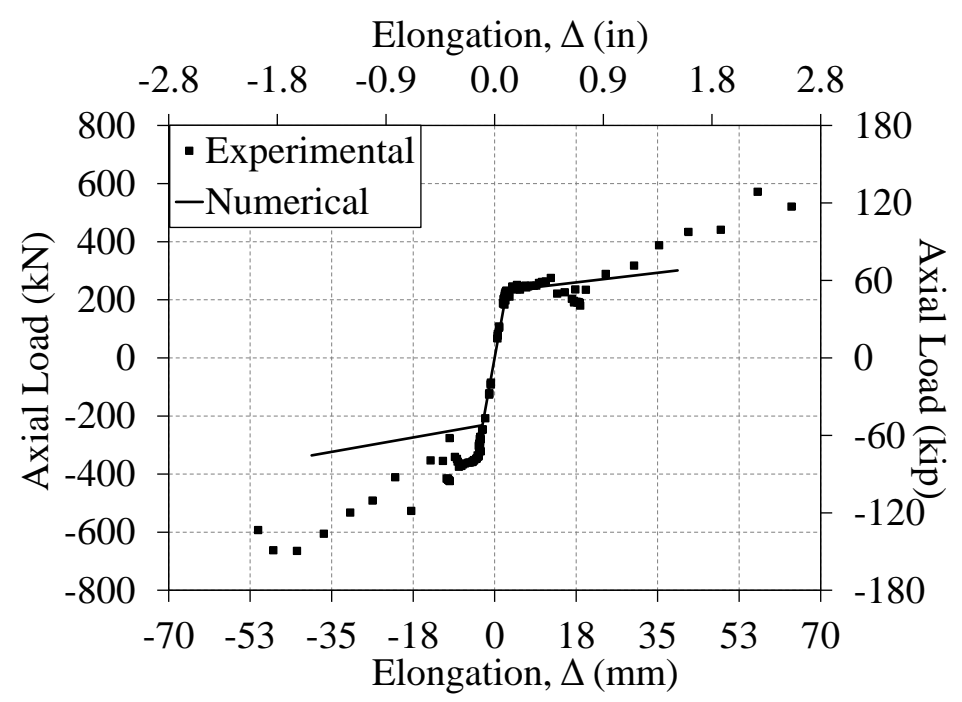

Figure 5.62 Buckling-restrained brace response for Bent/BRB 3

\subsubsection{Columns Component Response}

The internal axial loads in column 1 (C1) and column 2 (C2) were calculated in a similar way to that of the retrofitted bents. The internal axial loads in C1 and C2 for the specimens are shown in Figure 5.35. The results showed the influence of the BRB on C1 is negligible as compared to the As-built condition. On the contrary, the axial load in $\mathrm{C} 2$ is directly affected by the inclusion of the BRB since the axial load in the BRB is decomposed into an additional axial load in that column. The effect is such that at some point C2 is completely under tension loads as shown in Figure 5.35(b). These results are a direct consequence of the brace-to-bent connection, which connected the brace to the footing on one side and to the cap beam on the other. Any significant variation in the internal axial load of columns needs to be accounted for in the design and will affect the subsequent assessment and retrofit of other components. 


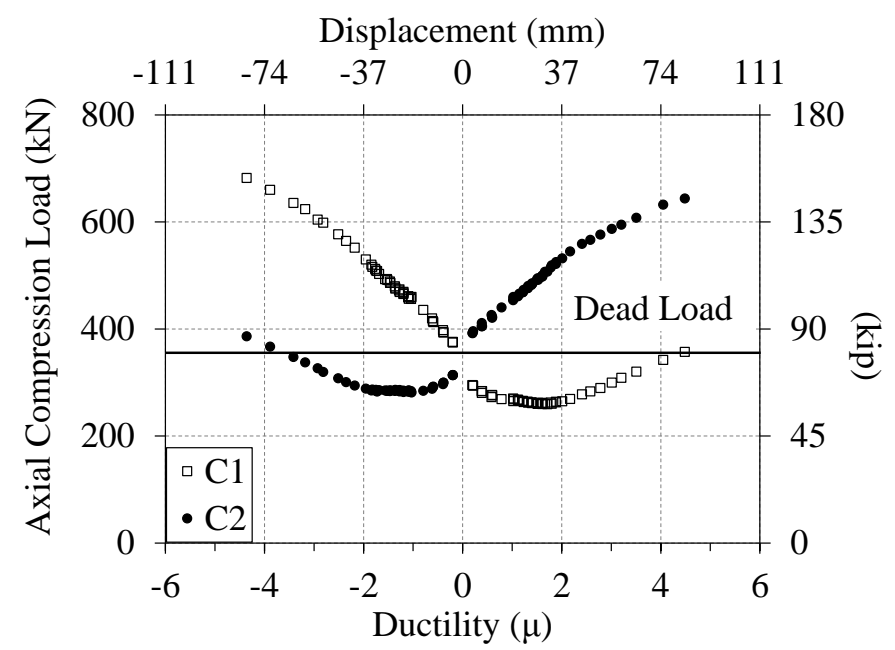

(a)

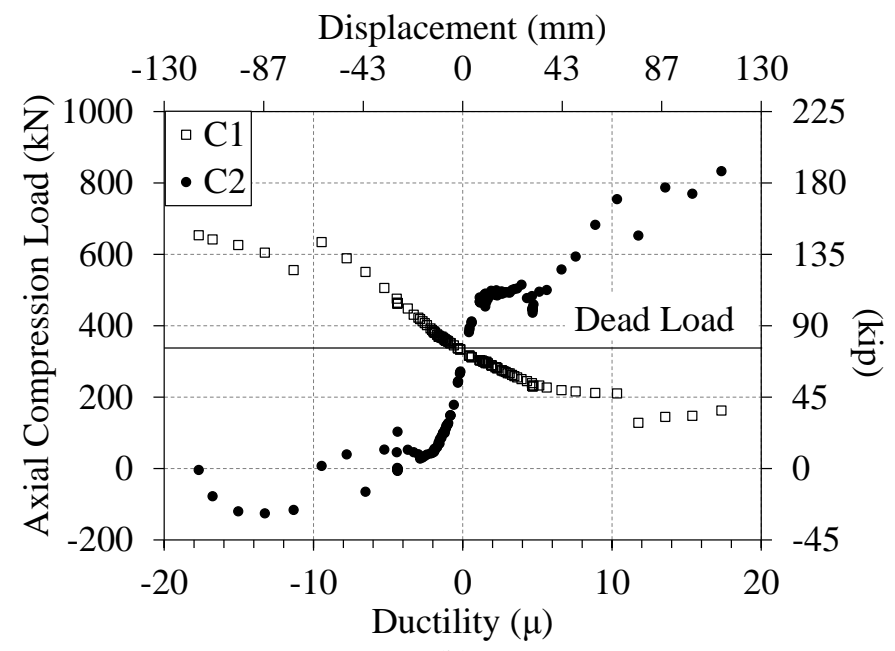

(b)

Figure 5.63 Internal column axial load. (a) As-built Bent 2; (b) Bent/BRB 3

\subsubsection{Backbone Curve Comparison}

Backbone curves were utilized to visualize and understand the difference in terms of initial stiffness, overall load and displacement capacity that the specimens exhibited. Figure 5.64 compares the backbone curves from the two experiments in terms of ductility of the As-built bent condition, i.e., ductility one corresponds to the effective yield of the As-built Bent 2. For the repaired state, results showed that the strength increased by $63 \%$ in the positive direction and $102 \%$ in the negative direction with respect to the strength of the 
undamaged As-built condition. While, the yield stiffness also increased by $158 \%$ in the repaired condition. As expected, the repaired bent exhibited larger strength and stiffness as compared to the As-built bent, demonstrating that repairing damaged RC bents with BRBs is a feasible option to restore the stiffness and strength of the system.

Figure 5.65 shows the comparison of backbone curves for all the $\mathrm{RC}$ bent conditions tested in this study. This figure clearly demonstrates the effect in stiffness and strength of retrofitting and repairing RC bridge bents with BRBs.

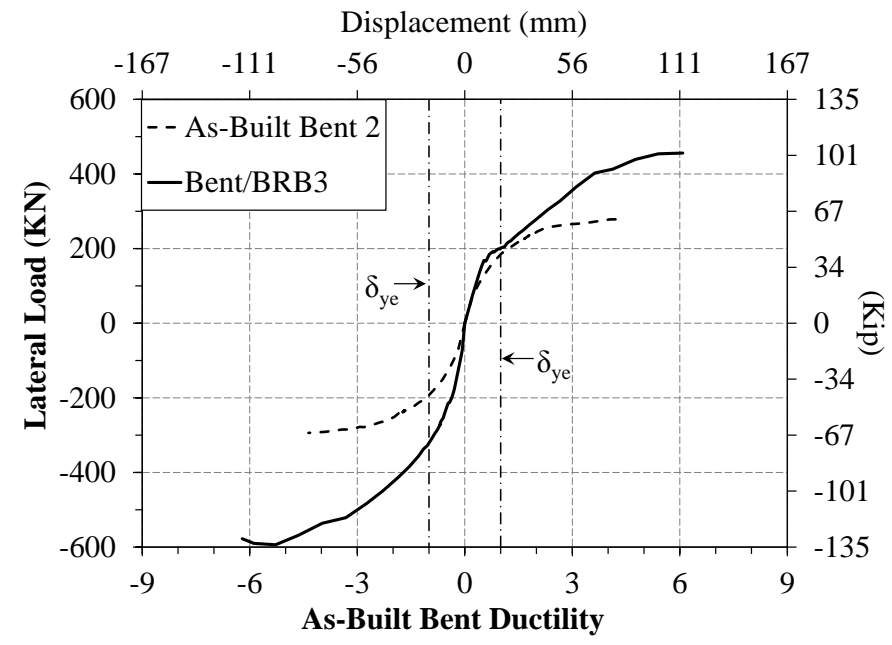

Figure 5.64 Backbone curves comparison (2 ${ }^{\text {nd }}$ stage)

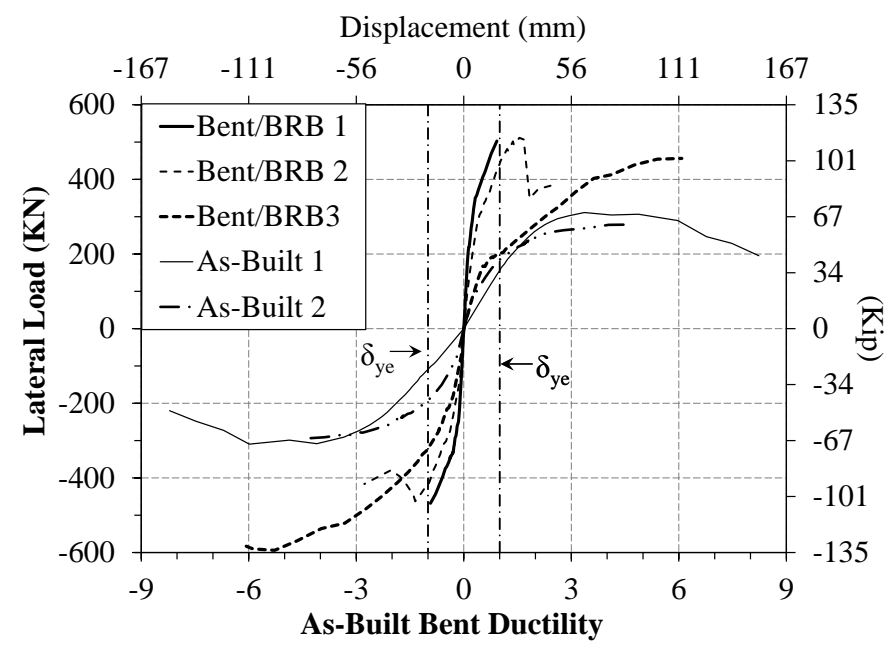

Figure 5.65 Backbone curves comparison for all specimens 


\subsubsection{Stiffness Degradation}

The normalized stiffness, represented as the secant stiffness $\left(k_{\mathrm{sec}}\right)$ divided by the yield stiffness $\left(\mathrm{k}_{\mathrm{y}}\right)$, at different displacement ductilities, was utilized as the stiffness degradation parameter. It is worth noting that the yield displacement used in the calculations corresponds to the effective yield displacement calculated for the As-built Bent 2 and the yield displacement of the BRB for the Bent/BRB 3 as those were the first sign of inelasticity of the as-built bent and repaired bent, respectively.

In terms of stiffness degradation (shown in Figure 5.66), the results for the As-built bent agree with the modified equation proposed for the retrofitted bents, Eq. (5.8) with c $=0.6$, up to ductility 2.5. After that point the As-built bent showed greater rate of stiffness degradation. Despite of being built with substandard detailing, the as-built bent did not agree with the equation, Eq. (5.8) for c=1 (Gulkan \& Sozen, 1974), which was intended for deficient RC columns. The BRB test specimen, on the other hand, showed a lower stiffness degradation than the As-built Bent 2, in particular in the positive direction. This lower stiffness degradation of Bent/BRB3 was caused by the stable and highly ductile hysteretic response showed throughout the test by the repaired bent. The results also showed that the modified equation provides a lower bound for the stiffness deterioration of the repaired bent. Thus, the modified equation is recommended for assessing both retrofitted and repaired bents with BRBs. 


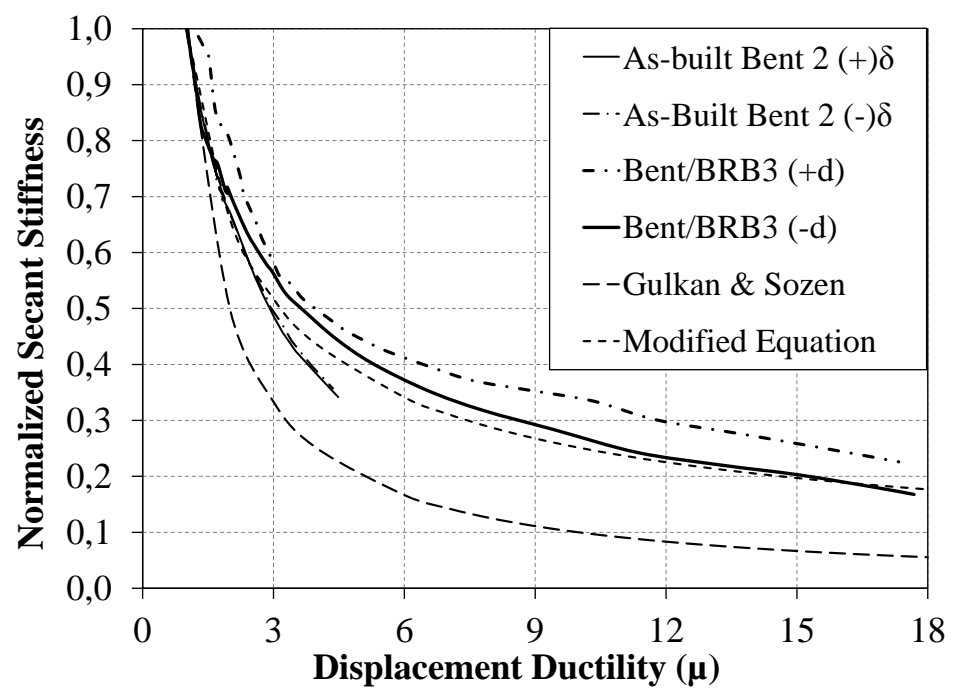

Figure 5.66 Stiffness degradation (2nd stage)

\subsubsection{Equivalent Viscous Damping}

The total equivalent viscous damping $\left(\xi_{\mathrm{eq}}\right)$ was obtained for the As-built bent 2 and the Bent/BRB3 as described in Section 5.5.5. Figure 5.67 shows that the Bent/BRB3 has greater equivalent viscous damping $\left(\xi_{\mathrm{eq}} \approx 39 \%\right)$ as compared to the as-built condition. This result is consistent with the stable hysteretic behavior and wide loops shown by the Bent/BRB3, which is typical of BRB systems, and demonstrates that repairing damaged RC bents with BRBs is a good option to reach high levels of energy dissipation. 


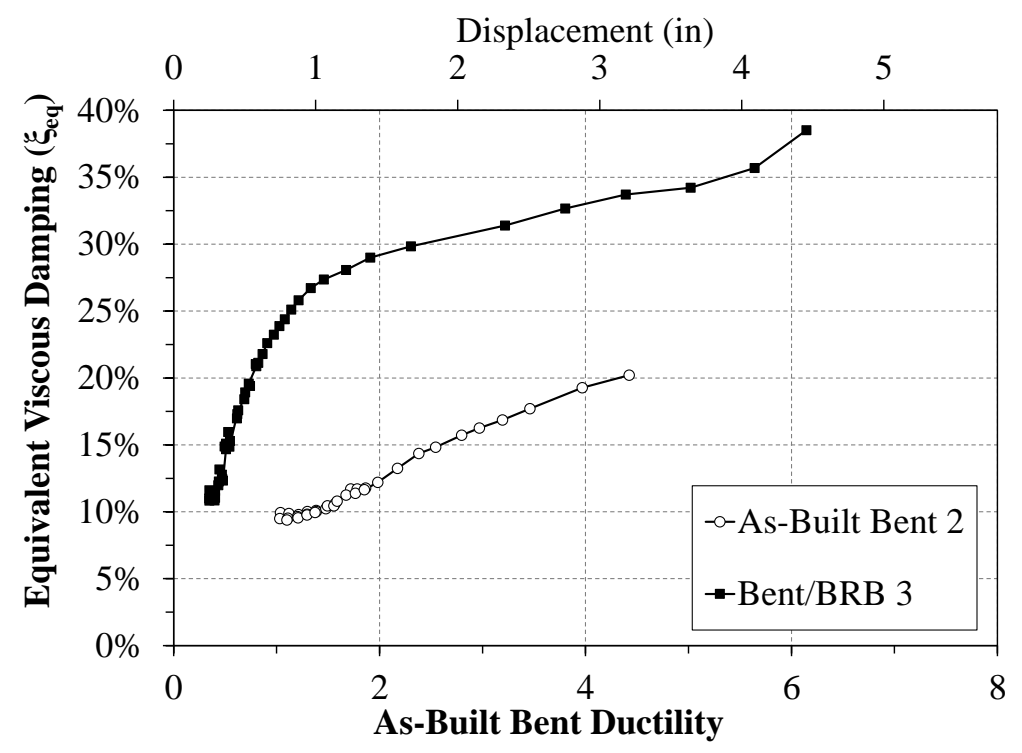

Figure 5.67 Equivalent viscous damping (2nd stage)

\subsection{Component Evaluation OF BRBs}

Subassemblage tests of three buckling-restrained braces were conducted in an effort to evaluate and characterize the hysteretic response of BRBs with the same dimensions and properties to the ones described in previous sections, i.e. BRB 1 and BRB2 for the retrofitted bent, and BRB 3 for the repaired bent. The specimens were designated according to their counterpart in the retrofitted and repaired condition by adding a letter "Q" at the end of their designation.

\subsubsection{Test Setup}

A subassemblage test setup, which comprised a steel beam connected to the strong floor through sixteen high strength rods, a steel column pinned at its base, a hydraulic actuator with a capacity of 220 kips, two gusset plates and a BRB specimen, was used as shown in Figure 5.68. In addition, a lateral support using a pin-pin steel brace was installed 
on the upper part of the column to prevent out-of-plane displacements as shown in Figure

5.69.

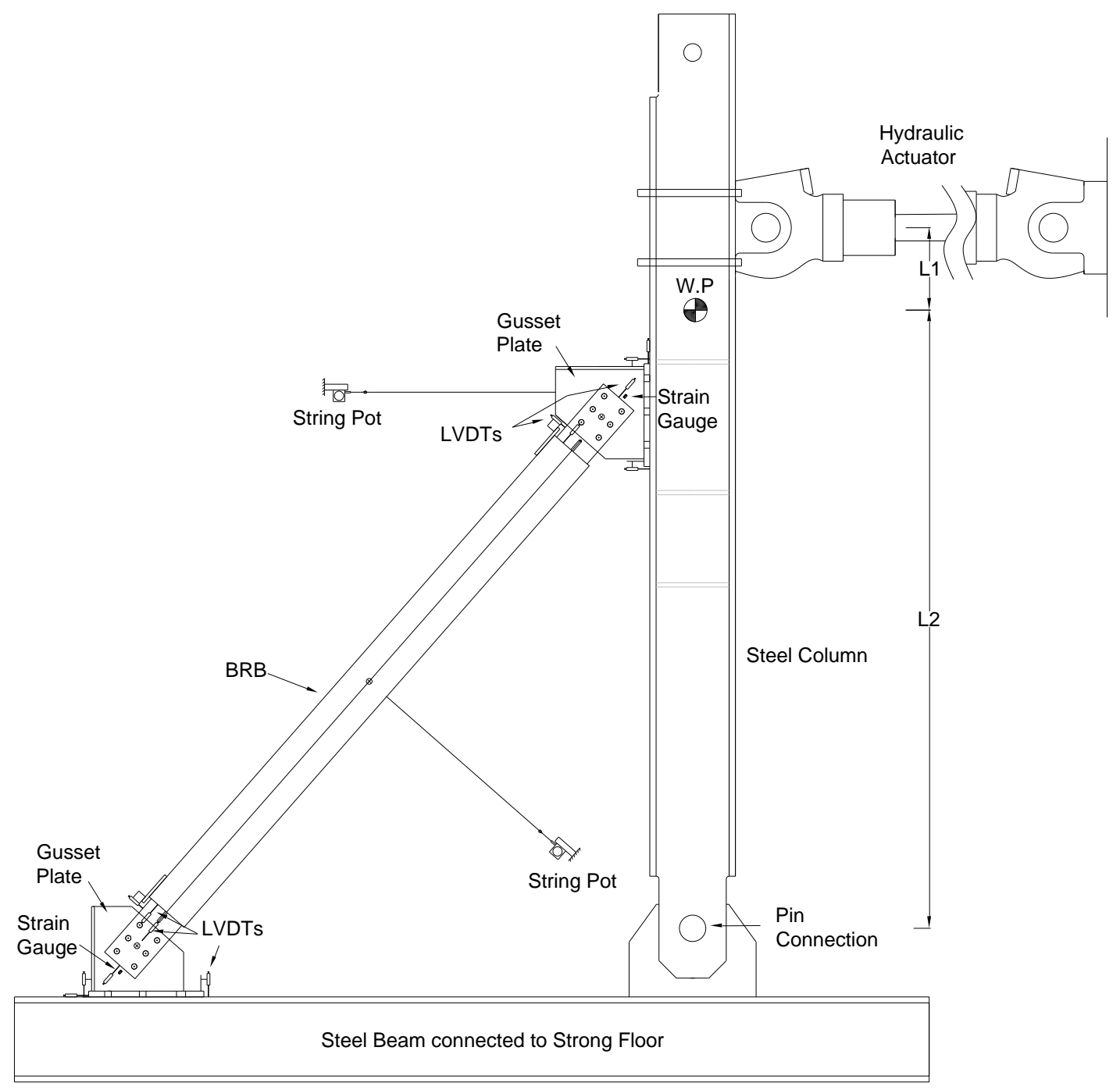

Figure 5.68 Test setup for qualification of BRBs 


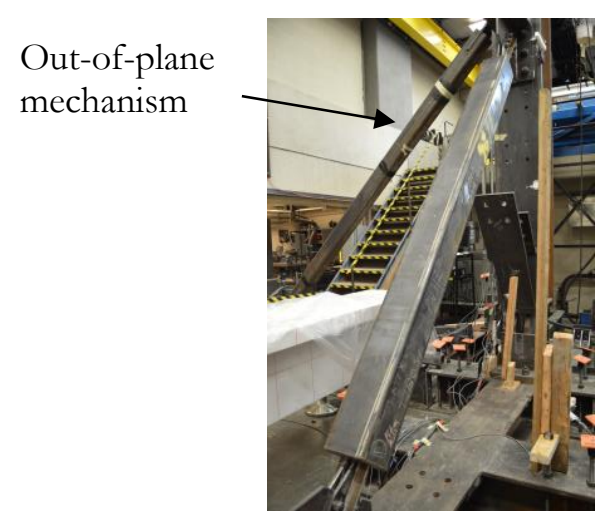

Figure 5.69 Out-of-plane mechanism in subassemblage tests

\subsubsection{Gusset plates}

For the subassemblage tests, gusset plates were designed with the aim of replicating the gusset plates used in the retrofitted and repaired cases. Steel ASTM A572 Gr50 with a thickness of 5/8 inches was used. Details of the gusset plates used in the tests are shown in Figure 5.70 and Figure 5.71 for the top and bottom gusset plate, respectively.
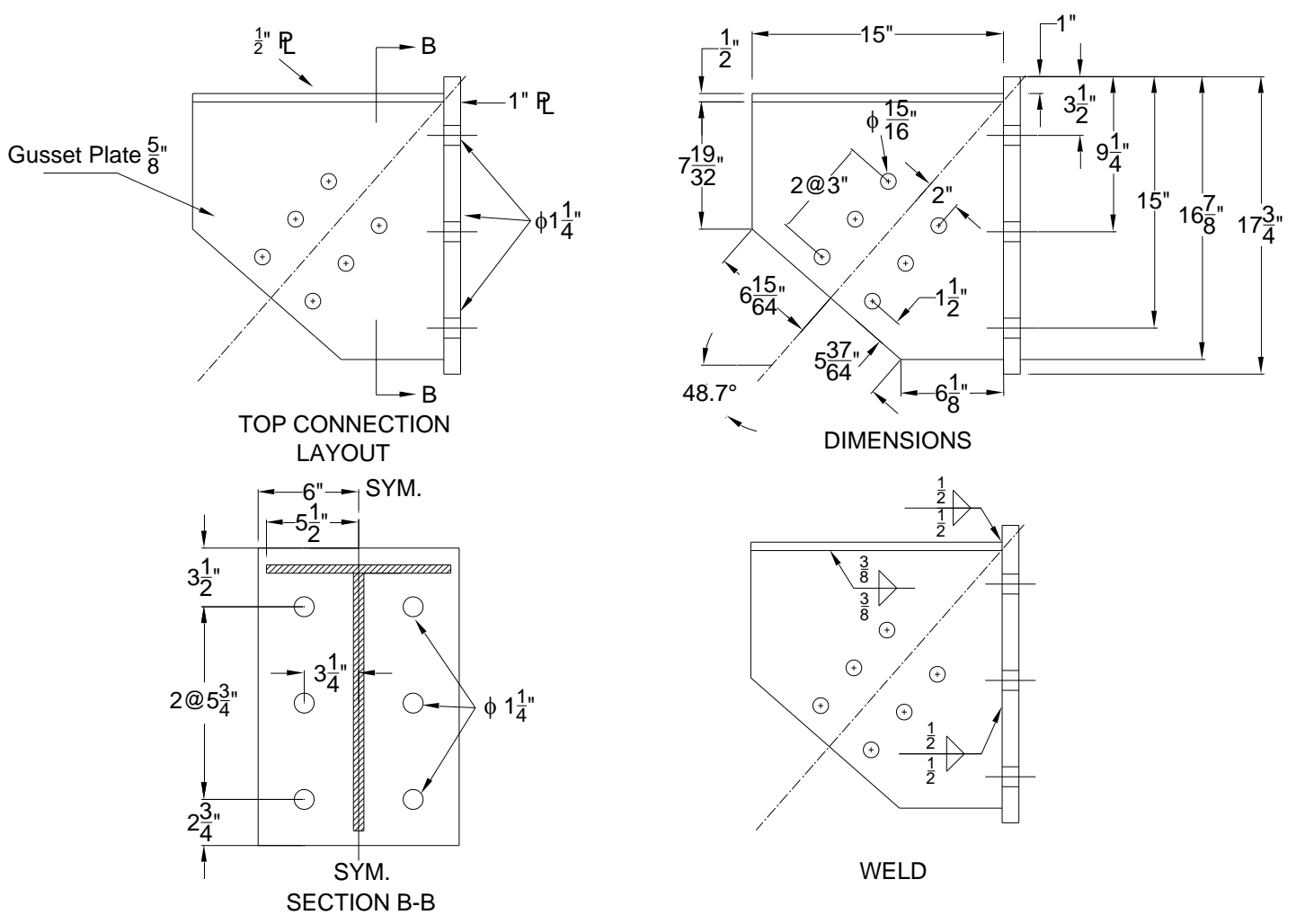

Figure 5.70 Top gusset plate for qualification of BRBs 


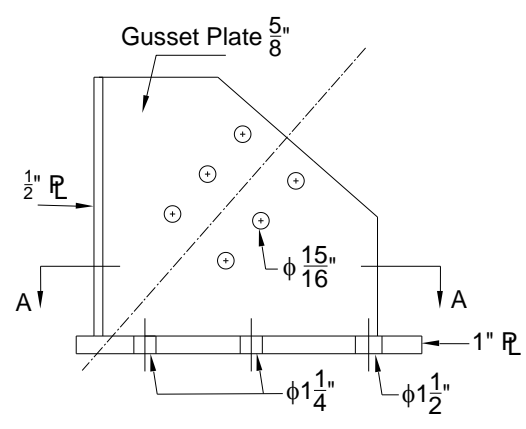

BOTTOM CONNECTION

LAYOUT

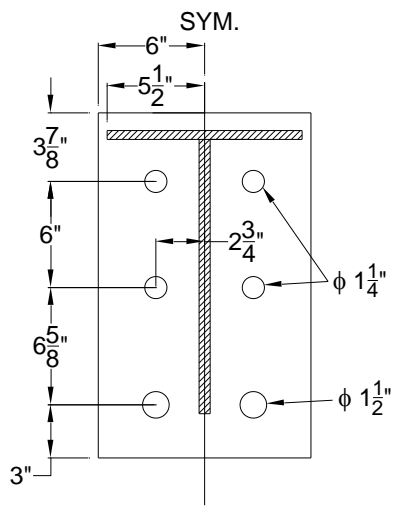

SYM.

SECTION A - A

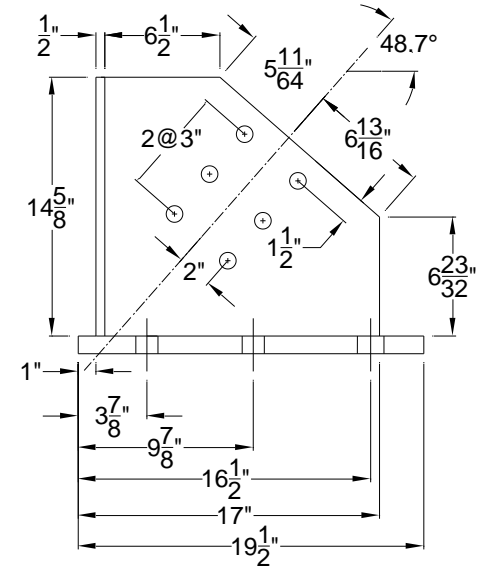

DIMENSIONS

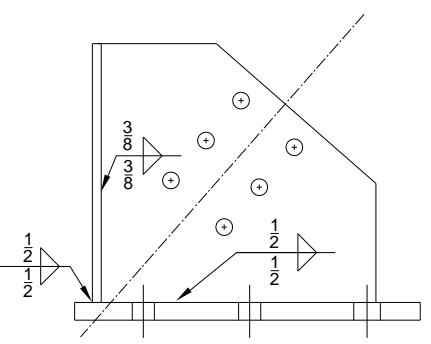

WELD

Figure 5.71 Bottom gusset plate for qualification of BRBs

\subsubsection{Instrumentation}

The general instrumentation layout is depicted in Figure 5.68. Four LVDTs measured the axial deformation of the BRBs. Two of them measured the relative displacement between the steel core and the steel casing at the top and bottom connections. The other two were located on the sides of the brace to measure the total elongation as shown in Figure 5.72(a). LVDTs were also utilized to measure slippage in the BRB-gusset plate connection, out-of-plane displacement of the gussets, uplift of gussets, and slippage between gusset plate and steel beam at the base and between gusset plate and steel column at the top as shown in Figure 5.72(a) and (b). String pots were used to measure the lateral displacement at the top gusset connection, and to monitor any significant out-of-plane 
displacement of the BRB in an effort to assess potential global buckling failure as shown in Figure 5.72(c). Strain gauges were installed on the gussets in order to assess the behavior of those components.

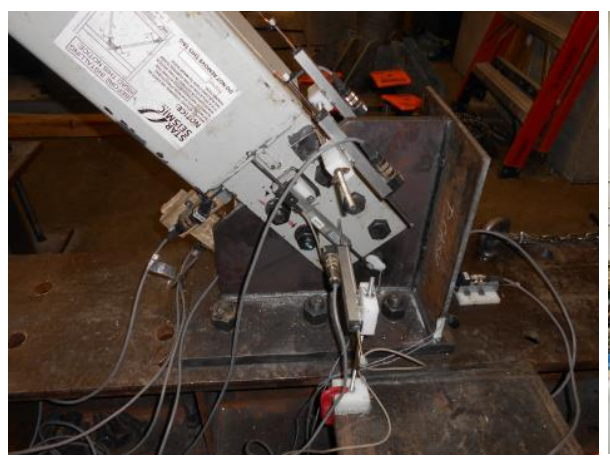

(a)

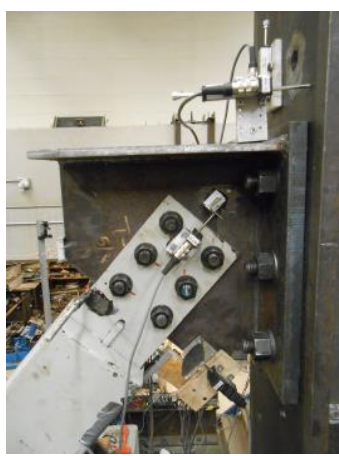

(b)

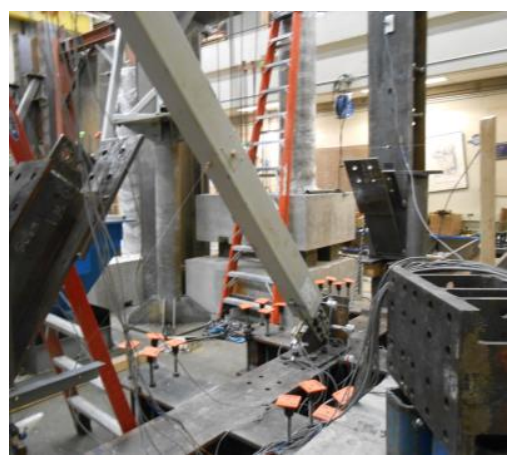

(c)

Figure 5.72 Instrumentation on subassemblage tests (a) Base, (b) Top, (c) String pots

\subsubsection{Loading Protocol}

The loading protocol used to evaluate the response of the specimens was based on the qualification loading protocol of AISC (2010) with some modifications as shown in Figure 5.73. The loading sequence suggested in the AISC Section K3.4c is as follows:

(1) 2 cycles of loading at the deformation corresponding to $\Delta_{\text {by }}$

(2) 2 cycles of loading at the deformation corresponding to $0.5 \Delta_{\mathrm{bm}}$

(3) 2 cycles of loading at the deformation corresponding to $\Delta_{\mathrm{bm}}$

(4) 2 cycles of loading at the deformation corresponding to $1.5 \Delta_{\mathrm{bm}}$

(5) 2 cycles of loading at the deformation corresponding to $2 \Delta_{\mathrm{bm}}$

(6) Additional complete cycles of loading at the deformation corresponding to $\Delta_{\mathrm{bm}}$ as required for the brace test specimen to achieve a cumulative inelastic axial deformation of at least 200 times the yield deformation (not required for the subassemblage test specimen). 
Where, $\Delta_{\text {by }}$ is the deformation at first significant yield and $\Delta_{\text {bm }}$ is the deformation corresponding to the design story drift. $\Delta_{\text {by }}$ was calculated using the nominal properties of the brace. The design story drift $\left(\Delta_{\mathrm{bm}}\right)$ was selected using the minimum of $1 \%$ the story height or $7.5 \Delta_{\mathrm{by}}$. As a result, the value of $\Delta_{\mathrm{bm}}$ for BRB1-Q and BRB3-Q was controlled by $7.5 \Delta_{\text {by }}$ and for BRB2-Q using the $1 \%$ story height. The values of $\Delta_{\text {by }}$ and $\Delta_{\text {bm }}$ for each test are summarized in Table 5.12.

Table 5.12 Values of $\Delta_{\text {by }}$ and $\Delta_{\text {bm }}$

\begin{tabular}{c|c|c}
\hline Test & $\Delta_{\text {by }}$ (in) & $\Delta_{\text {bm }}$ (in) \\
\hline BRB1-Q & 0.05 & 0.34 \\
\hline BRB2-Q & 0.14 & 0.89 \\
\hline BRB3-Q & 0.10 & 0.77 \\
\hline
\end{tabular}

In this study, the sequence (6) was replaced by additional two cycles at $2.5 \Delta_{\mathrm{bm}}$ and 3.0 $\Delta_{\mathrm{bm}}$ in an effort to achieve a cumulative inelastic axial deformation of at least 350 times the yield deformation as required by the OSHPD (Office of Statewide Health Planning and Development). In cases when the brace did not fail, additional cycles of loading at a deformation corresponding to $2.0 \Delta_{\mathrm{bm}}$ were performed.

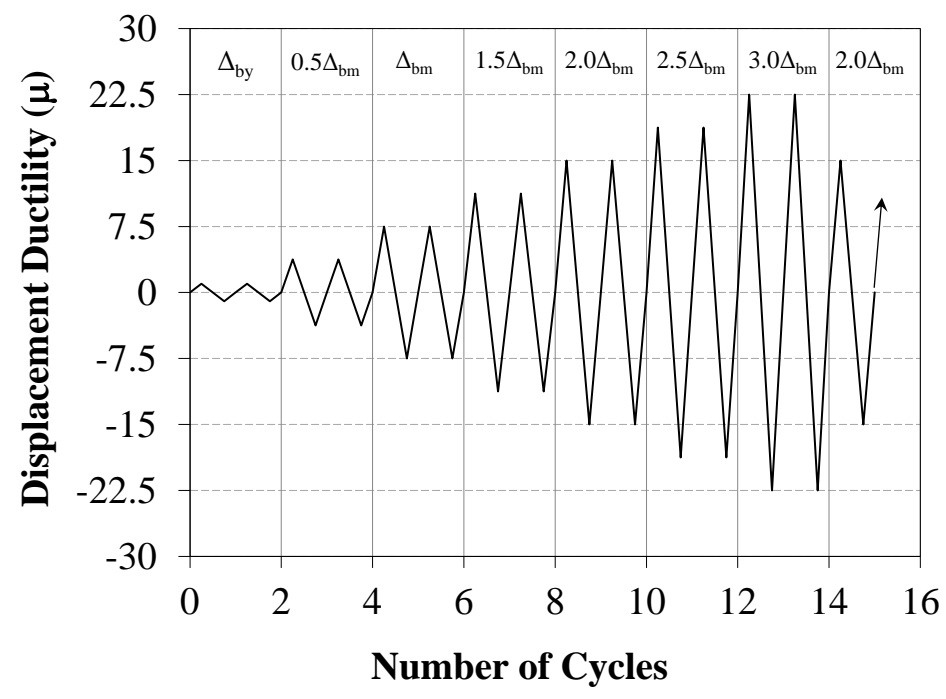

Figure 5.73 Loading protocol for evaluation of BRBs 238 


\subsubsection{Cyclic Behavior and Damage}

\subsubsection{Data Reduction}

In the following sections, the brace axial deformation $(\Delta)$ that is reported corresponds to the average of the LVDTs installed on each side of the BRB. Since the actuator was not located at the same height that the top brace connection, the axial force in the brace was calculated using Eq. (5.10).

$$
\text { Axial Force }=\frac{\text { Actuator } \cdot\left(L_{1}+L_{2}\right)}{L_{2} \cdot \cos (\theta)}
$$

Where, Actuator is the measured actuator load, $\mathrm{L}_{1}$ is the distance from the actuator to the brace work point (WP), and $\mathrm{L}_{2}$ is the distance from the WP to the pin at the base of the column. Values of 28.5 in and 106.6 in were used for $L_{1}$ and $L_{2}$, respectively.

\subsubsection{BRB1-Q}

This BRB was designed using the structural fuse concept in order to limit the displacement demand in a RC bent as described in Section 5.2.2. As a result, a stiff BRB with a reduced section length equal to 30 in and a steel core area equal to $1.2 \mathrm{in}^{2}$ was specified. The subassemblage test setup for this brace is shown in Figure 5.74.

The recorded axial load-deformation behavior for this specimen shown in Figure 5.75 indicates ductile behavior and high energy dissipation via stable hysteretic cycles. The nominal yield deformation of the brace was computed as 0.05 in $(1.3 \mathrm{~mm})$. Although, the loading protocol was defined up to $3.0 \Delta_{\mathrm{bm}}, \mathrm{BRB} 1-\mathrm{Q}$ was tested up to an axial deformation of 1.21 in $(30.7 \mathrm{~mm})$, which is equivalent to approximately $3.5 \Delta_{\mathrm{bm}}$ or ductility 24 and axial strain of 4\%. Peak loads of 85 kips and 95 kips were recorded for the brace in tension and compression, respectively. These loads occurred at 1 in of axial deformation. Figure 5.75 
demonstrates that this brace exceeds the axial deformation of $2.0 \Delta_{\mathrm{bm}}$ required by code for qualification purposes. Thus, this brace satisfied the requirements of qualification.
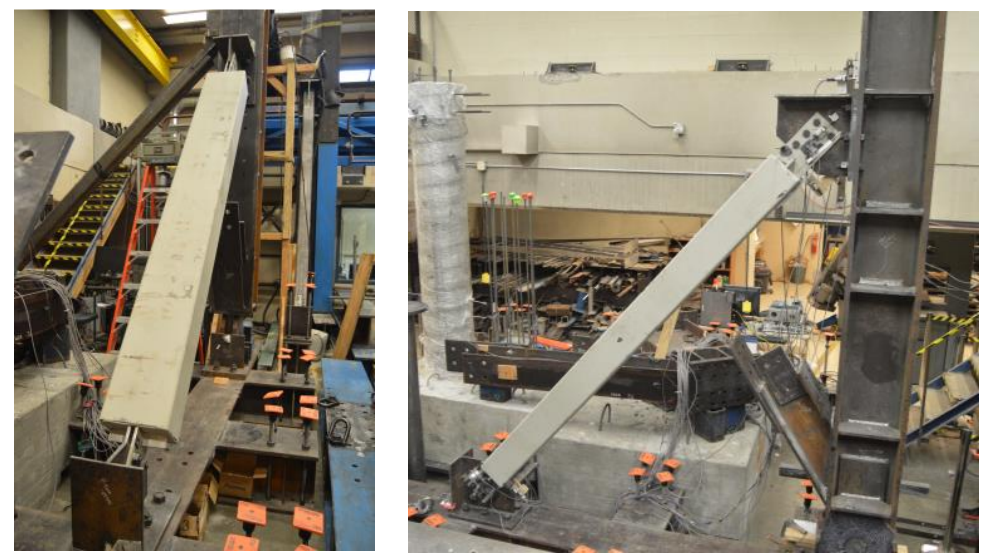

Figure 5.74 Subassemblage test of BRB1-Q

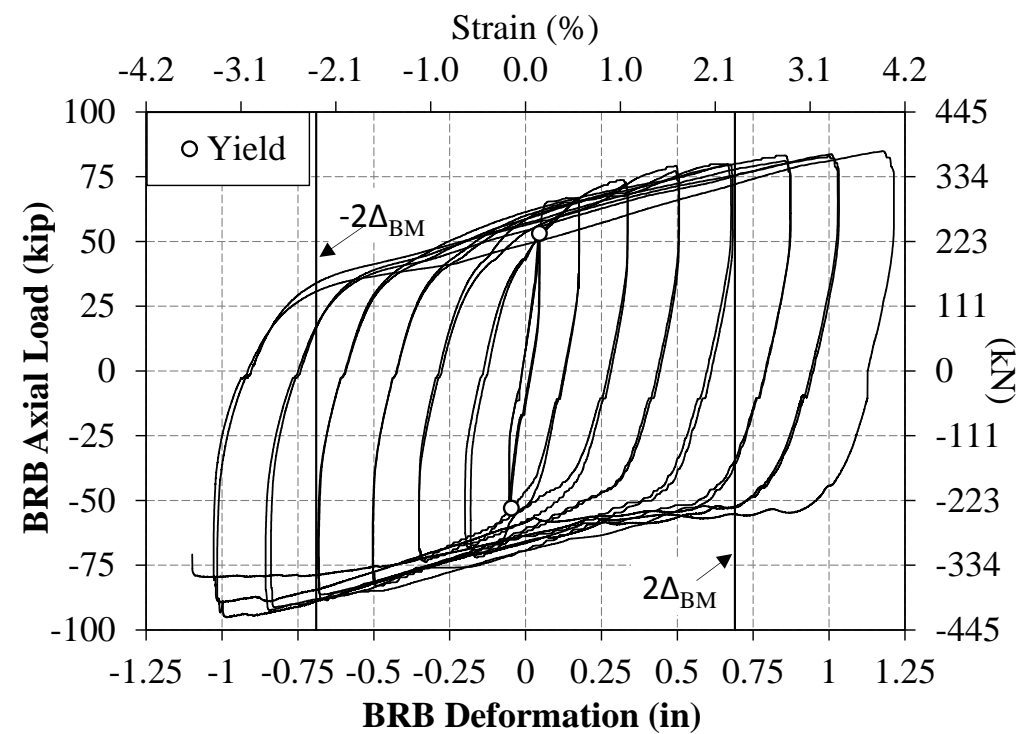

Figure 5.75 Hysteretic response of BRB1-Q

Comparison of top and bottom axial deformations, which were measured through two LVDTs installed on the top and bottom part of the brace, is depicted in Figure 5.76. This figure indicates a nearly symmetric distribution of axial deformation. However, the figure also show that during the cycle of yield amplitude $\left(\Delta_{\text {by }}\right)$ and during the subsequent cycles, axial deformations tended to move out from the initial 0 value. This result indicates 
that the encasing system moved relatively to the steel core to a different position than the one the casing was originally. In other words, this behavior can be described as a residual displacement of the encasing system.

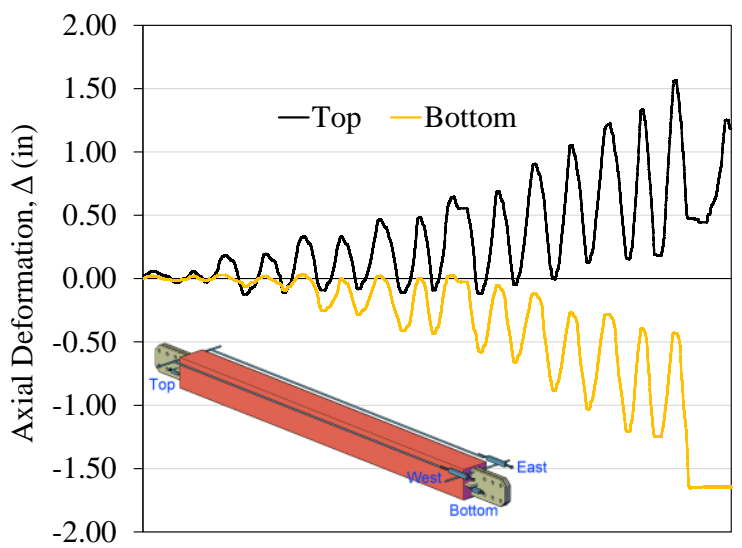

Figure 5.76 Distribution of axial deformation on BRB1-Q

At the end of the test, localized damage was observed in the steel casing as shown in Figure 5.77(a). This damage was caused by the internal buckling of the steel core in its week axis which in turn damaged the concrete surrounding it and bend the steel casing. The damage sequence was clear once the brace was deconstructed in order to observe the internal damage as shown in Figure 5.77(b) and (c).

Further inspections after removing the BRB from the test setup showed that the gusset plates did not exhibit any damage. This result was also reflected in the strain gauge measurements that indicated that the gussets were not even close to yielding. The maximum strain recorded in the gusset plates was $0.000735 \mathrm{in} / \mathrm{in}$, which is lower than the strain at yield (0.00172 in/in). 


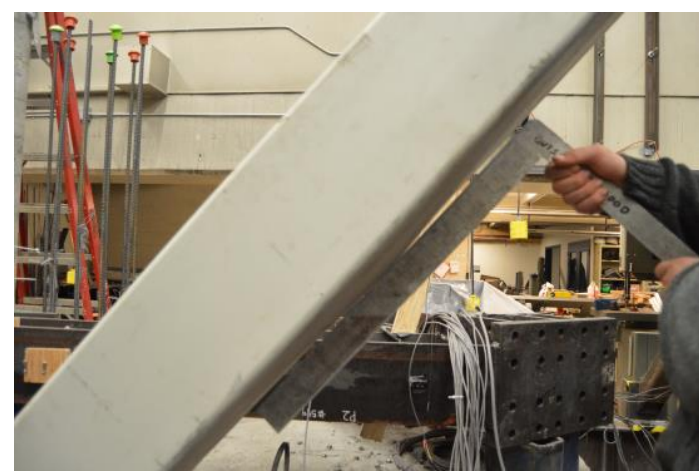

(a)

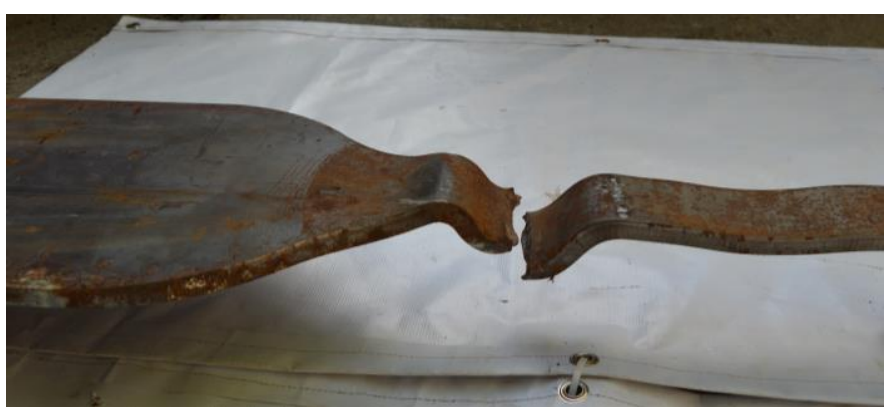

(c)

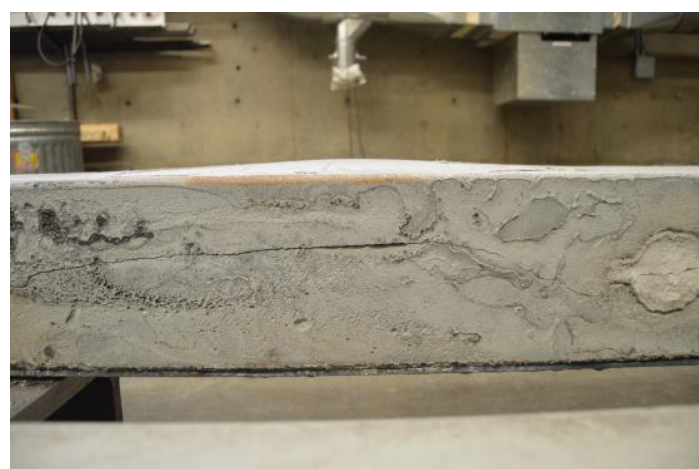

(b)

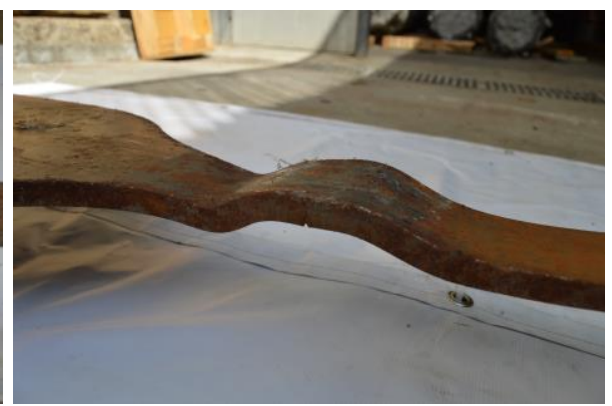

Figure 5.77 Damage in BRB1-Q. (a) Damage in steel casing, (b) Damage in concrete, (c) Failure mode of the steel core

\subsubsection{BRB2-Q}

BRB2-Q was designed following a typical approach of BRB design, which considers a reduced section $\left(L_{c}\right)$ equal to two thirds of the brace length $(88.6$ in). As a result, a more flexible brace as compared to BRB1-Q was utilized as shown in Figure 5.78. It is worth mentioning that this brace had the same area of steel core and material properties than BRB1-Q. Thus, the same yield strength was computed for both braces. 

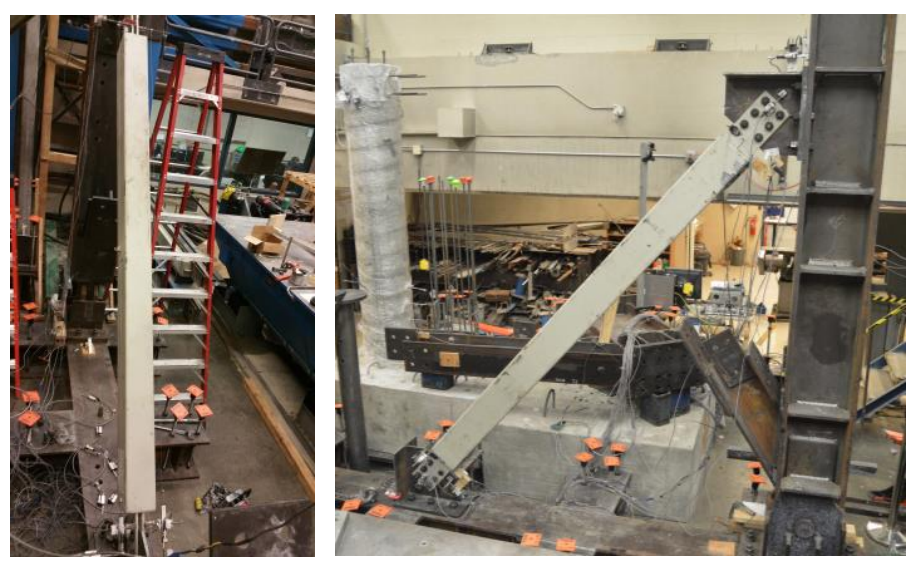

Figure 5.78 Subassemblage test of BRB2-Q

The hysteretic response of BRB2-Q shown in Figure 5.79 indicates that this brace did not reach the axial deformation $\left(2 \Delta_{\mathrm{bm}}\right)$ required for qualification purposes. Peak loads of 70 kips and 87 kips were recorded for the brace in tension and compression, respectively. The nominal yield deformation of the brace was computed as 0.14 in $(3.6 \mathrm{~mm})$ and the failure of the brace was observed during the last compression half-cycle at an axial deformation of 1.32 in, which is equivalent to a ductility of 9.4.

The response of this brace was similar to the response inferred for Bent/BRB2, which also presented lower axial deformation than expected and similar axial strength. It is worth mentioning that the brace in Bent/BRB2 failed at an axial deformation of 0.78 in, while BRB2-Q failed after an axial deformation of 1.32 in. Further comparisons of top and bottom axial deformations of BRB2-Q shown in Figure 5.82 indicate a more symmetric distribution of axial deformations as compared to the results recorded for Bent/BRB2. In the latter, the inelastic deformation demands were mostly concentrated at the top of the $\mathrm{BRB}$. The difference in the distribution of axial deformations and also in axial deformation capacity between BRB2-Q and Bent/BRB2 was presumably caused by the different loading protocol used in Bent/BRB2, in which a loading history with more inelastic cycles of low 
amplitude was utilized to reflect inelastic demands from subduction zone ground motions. As a consequence, the brace in Bent/BRB2 was subjected to many more inelastic axial strains than BRB2-Q.

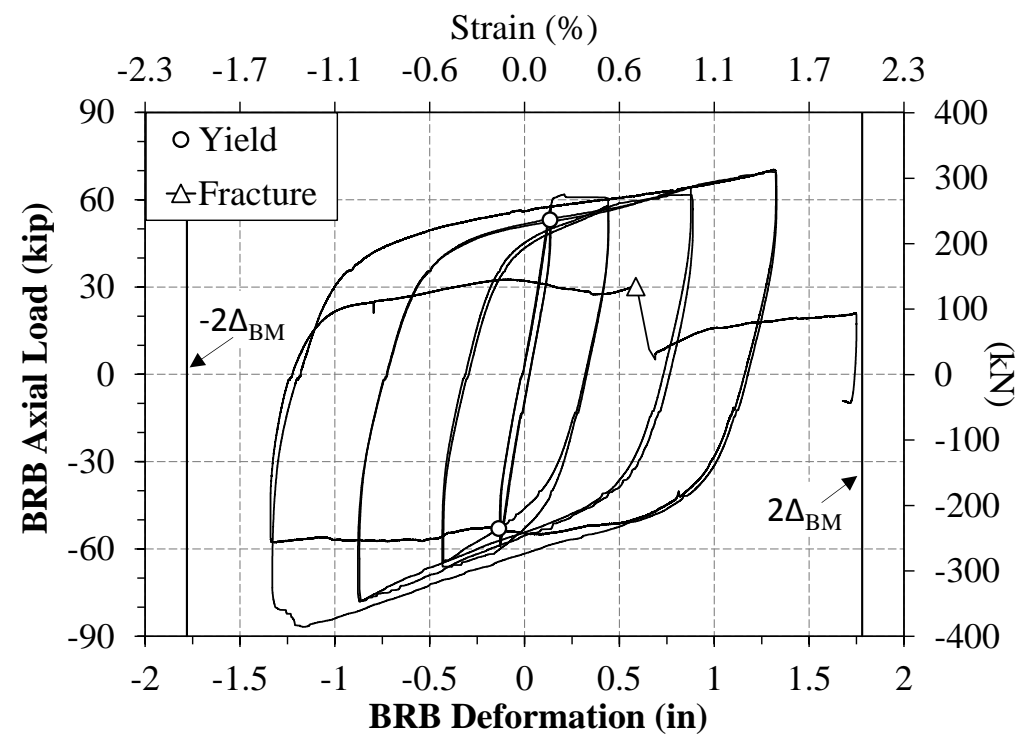

Figure 5.79 Hysteretic response of BRB2-Q

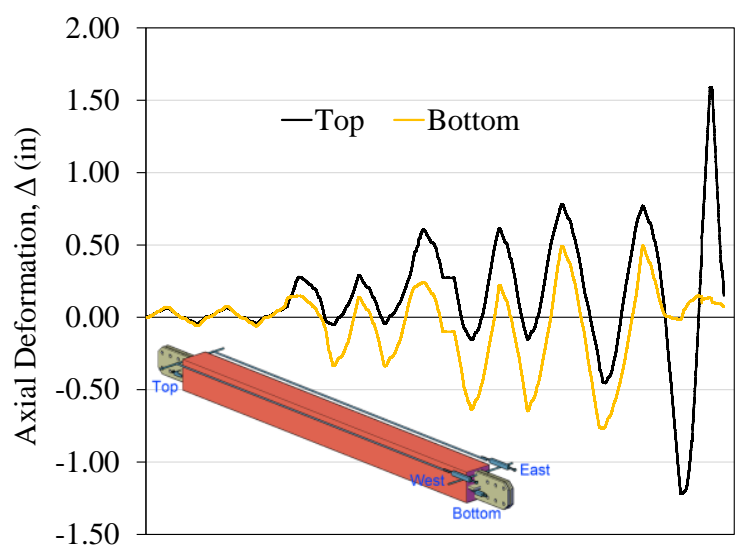

Figure 5.80 Distribution of axial deformation on BRB2-Q

No visual damage was observed throughout the test. However, buckling of the steel core likely started developing during the last compression half-cycle through significant decrease in axial strength to give place to fracture of the steel core during the tension halfcycle as shown in Figure 5.79. The damage presented in the steel core of BRB2-Q is shown 
in Figure 5.81. This damage agrees with the recorded distribution of axial deformations shown in Figure 5.80, in which it can be observed that during the last cycles the axial deformation was concentrated at the top part of the BRB leading to buckling of the steel core in the compression half cycle and then to rupture during the tension half cycle as shown in Figure 5.81(a). Minimal internal buckling but more evenly distributed was observed in the bottom part of the brace as shown in Figure 5.81(b). Similarly to Bent/BRB2, the fracture of the steel core occurred in the transition section between the non-yielding and the reduced section of the core. This mode of failure was attributed to poor confinement in the transition section $\left(L_{t r}\right)$ within the brace.

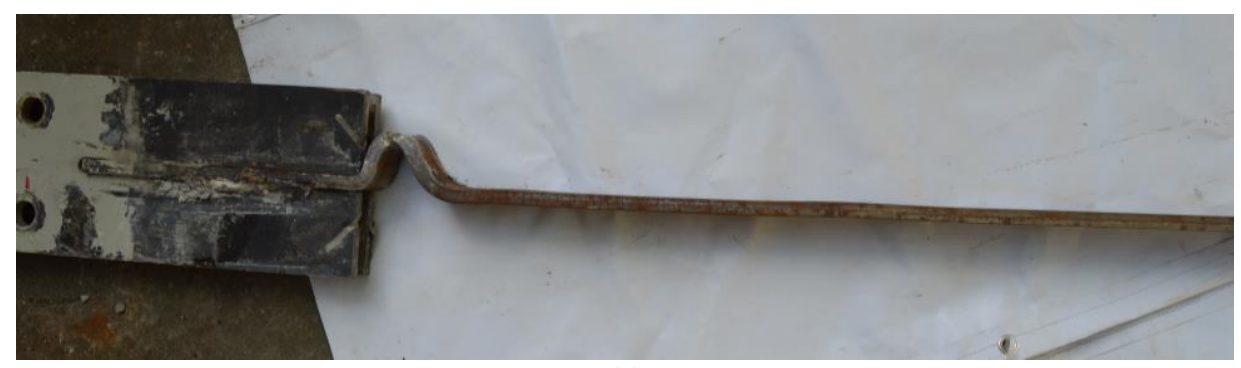

(a)

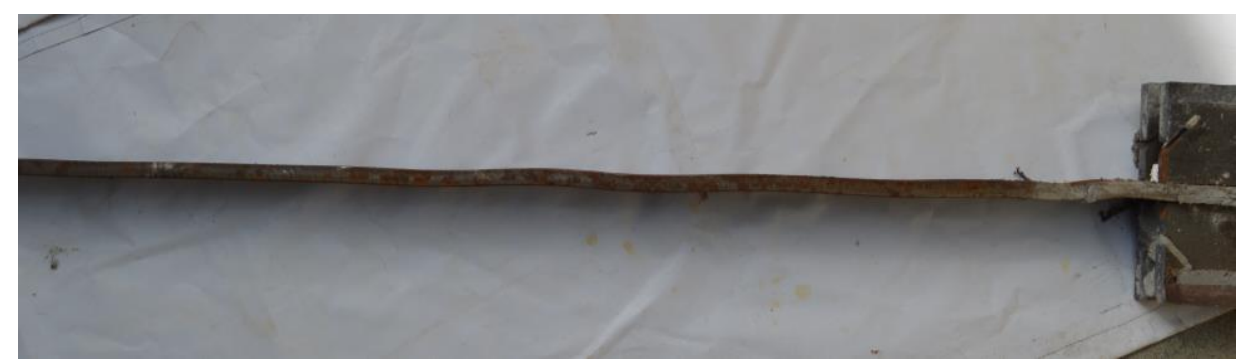

(b)

Figure 5.81 Damage in BRB2-Q. (a) Top, (b) Bottom

\subsubsection{BRB3-Q}

BRB3-Q was designed to satisfy the repair procedure stated in Chapter 4 and exemplified in Section 5.2.3. The same steel core area (1.2 $\left.\mathrm{in}^{2}\right)$ than BRB1-Q and BRB2-Q was specified for this brace. However, a reduced section length of 68.9 in was used for this 
brace, resulting in a brace more flexible than BRB1-Q but stiffer than BRB2-Q as shown in Figure 5.82.
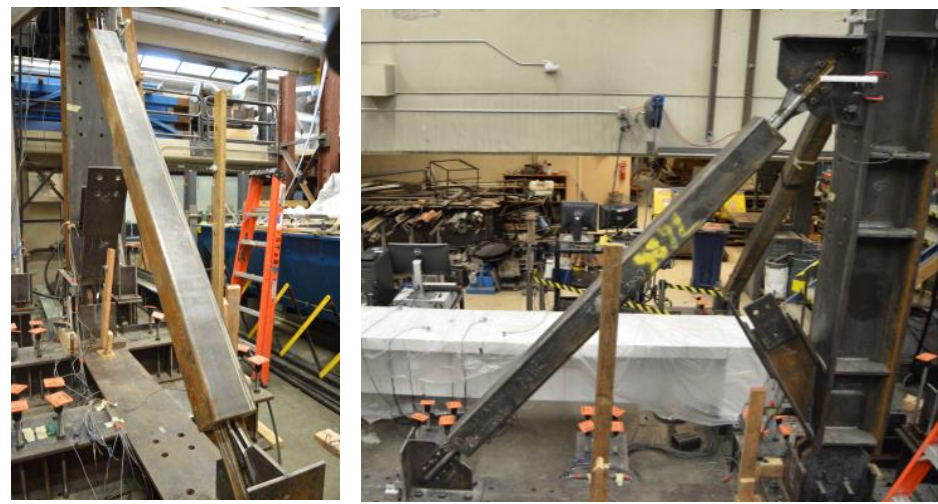

Figure 5.82 Subassemblage test of BRB3-Q

It is worth mentioning that a different BRB manufacturer provided this BRB. As a consequence, the hysteretic response for this brace shown in Figure 5.83 indicates greater strain hardening as compared to BRB1-Q and BRB2-Q. The response of this brace showed a ductile and stable hysteretic behavior but highly asymmetric in tension and compression strength. Peak loads of 80 kips and 124 kips were recorded in tension and compression, respectively. In terms of axial deformation, the brace exceeded the axial deformation required by the AISC seismic provisions (2010). The nominal yield deformation of the brace was computed as 0.10 in $(2.5 \mathrm{~mm})$ and the maximum deformation of the brace was 1.96 in (49.8 mm), which is equivalent to a ductility of 19.6. Larger deformations were not possible during the test due to a limitation in the maximum stroke of the LVDTs. For that reason, after reaching the maximum stroke of the LVDTs ( 2 in) cycles at an amplitude of $2 \Delta_{\mathrm{bm}}$ (ductility of 15, axial deformation of 1.55 in) were performed until failure of the specimen was reached. Failure of the specimen was observed after 5 cycles at that amplitude. 


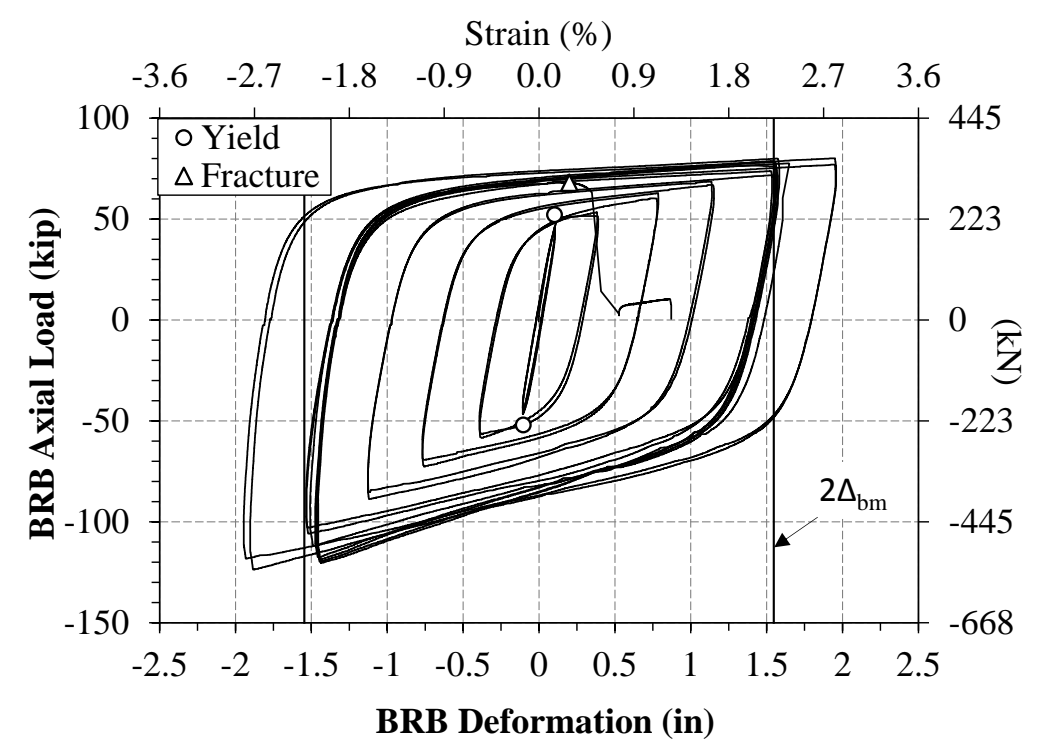

Figure 5.83 Hysteretic response of BRB3-Q

Comparing the top and bottom axial deformations of BRB3-Q shown in Figure 5.84 indicates a symmetric distribution of axial deformations. Similar to BRB1-Q axial deformations tended to move out from the initial 0 value, effect that was attributed to the encasing system moving from its initial position.

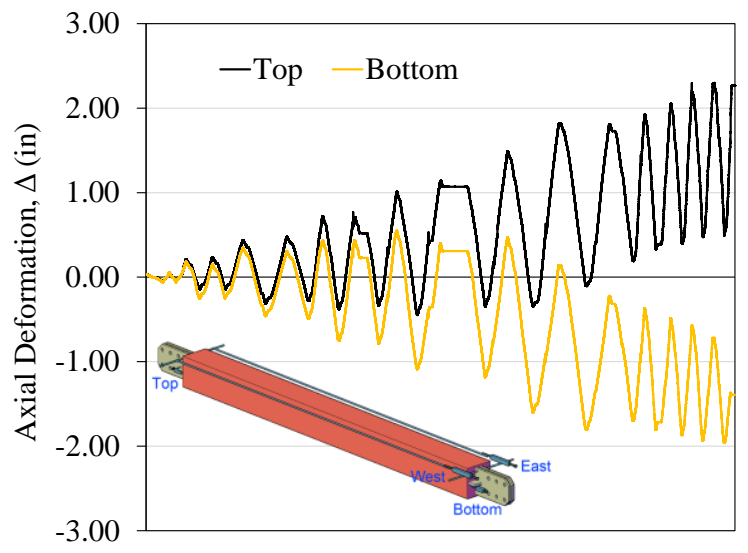

Figure 5.84 Distribution of axial deformation on BRB3-Q

No visual damage was observed during the test. However, the significant decrease in axial strength shown in Figure 5.83 and a deformation larger than the measured one at the end of the test as shown in Figure 5.85 indicates that fracture of the steel core had occurred. 


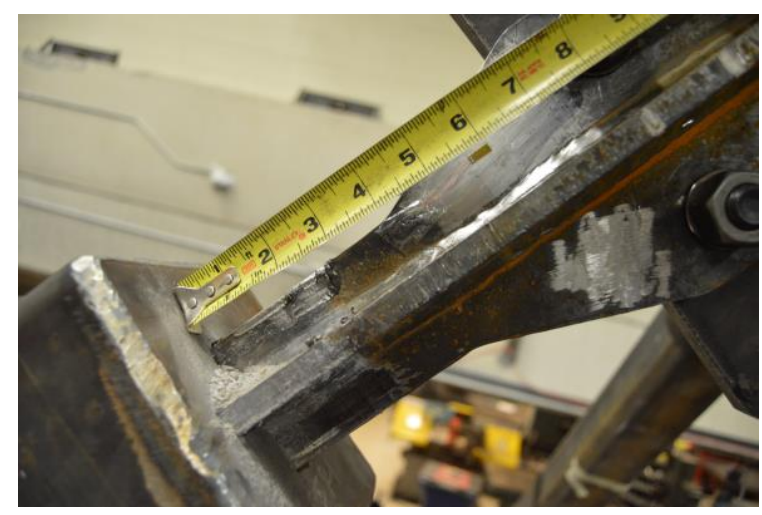

Figure 5.85 Damage in BRB3-Q

Visual inspections after removing the BRB from the test setup showed that the gusset plates did not exhibit any damage. This result was corroborated with strain gauge measurements that indicated that the gussets remained elastic with a maximum strain of $0.00134 \mathrm{in} / \mathrm{in}$, which is lower than the strain at yield (0.00172 in/in) for ASTM A572 Gr50 plates. It is worth mentioning that the same gusset plates were used for the three BRBs, showing the excellent behavior and reusability of those components.

\subsubsection{Over-strength Factors}

The tension over-strength factor, $\omega$, was computed using Eq. (5.11) and the compression adjustment factor, $\beta$, was computed using Eq. (5.12).

$$
\begin{gathered}
\omega=\frac{T_{\max }}{P_{y}}=\frac{T_{\max }}{f_{y} \cdot A_{s c}} \\
\beta=\frac{P_{\max }}{T_{\max }}
\end{gathered}
$$

Where, $P_{y}$ is the nominal axial force at yield, $f_{y}$ is the nominal yield stress, $A_{s c}$ is the area of the steel core, $\mathrm{T}_{\max }$ is the maximum tension load at each cycle, and $\mathrm{P}_{\max }$ is the maximum compressive load. AISC (2010) recommends that the $\beta$ factor should not exceed 
1.3. Strength adjustment factors at different displacement ductilities are shown in Figure 5.86 and Figure 5.87 for tension and compression, respectively. Results showed that the BRBs presented similar over-strength factors in tension at the same ductility, e.g. at a ductility of 7.5 the tension adjustment factor is close to 1.2 for all the specimens. On the contrary, the compression factor was significantly different for all the specimens. BRB1-Q had the lowest compression adjustment factor with values close to 1.1 for all the considered ductilities. BRB3-Q had the highest compression adjustment factor at $2 \Delta_{\mathrm{bm}}$ with a value of 1.57 . This compression adjustment factor exceeds the limit recommended by the AISC seismic provisions. These results agree with the $\mathrm{BRB}$ response inferred during the retrofitted and repaired bent tests.

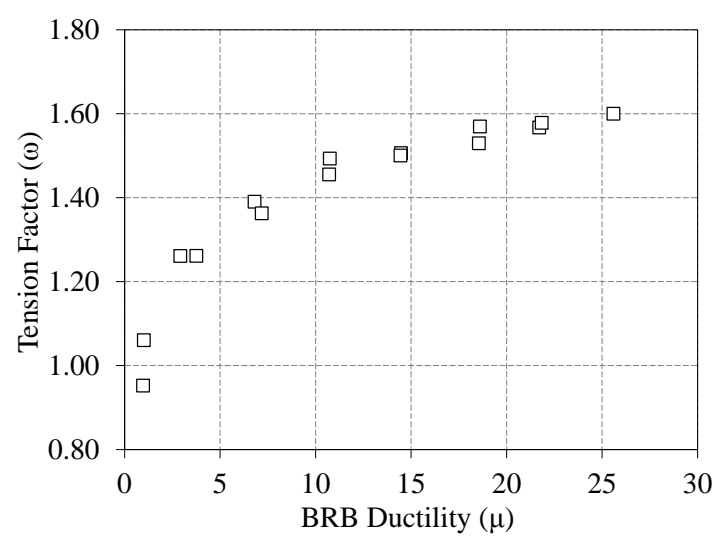

(a)

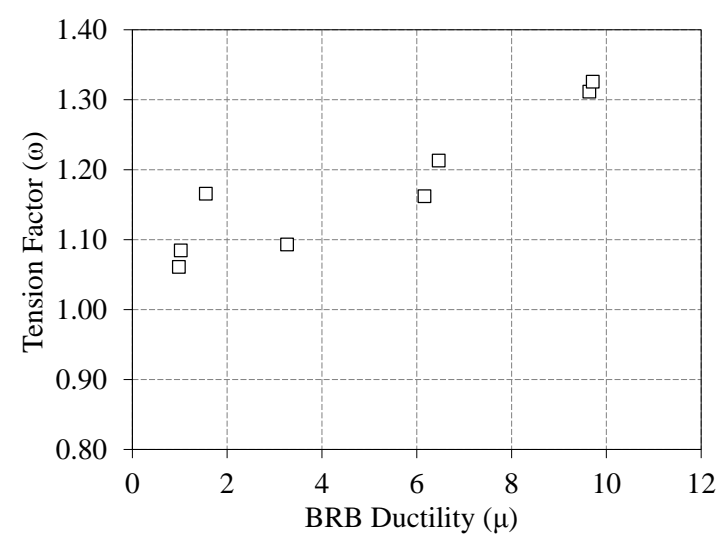

(b)

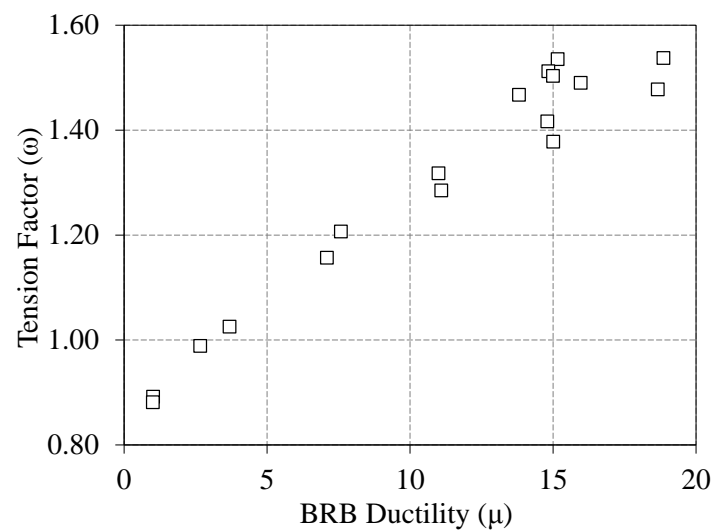

(c)

Figure 5.86 Tension adjustment factor. (a) BRB1-Q, (b) BRB2-Q, (c) BRB3-Q 


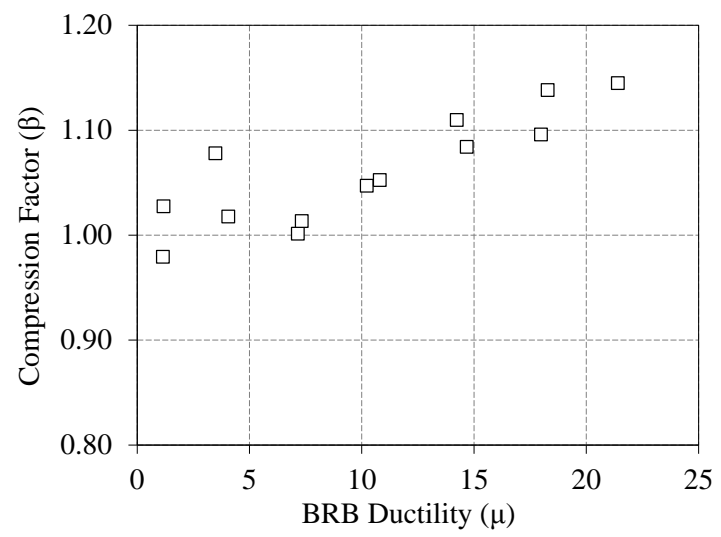

(a)

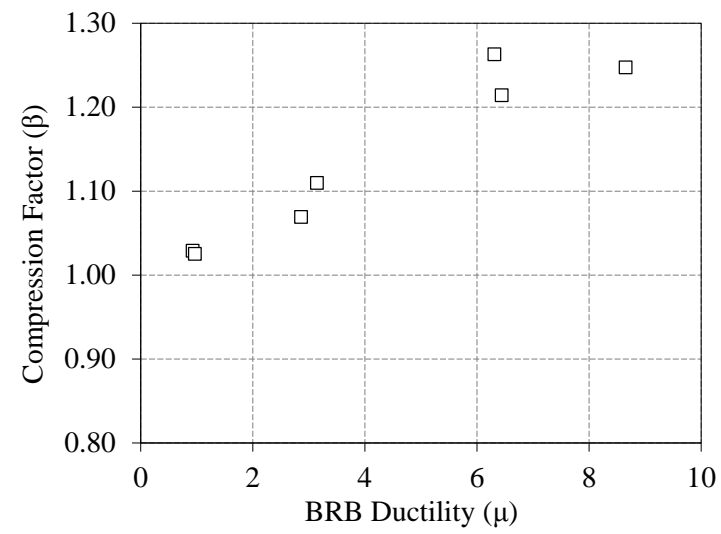

(b)

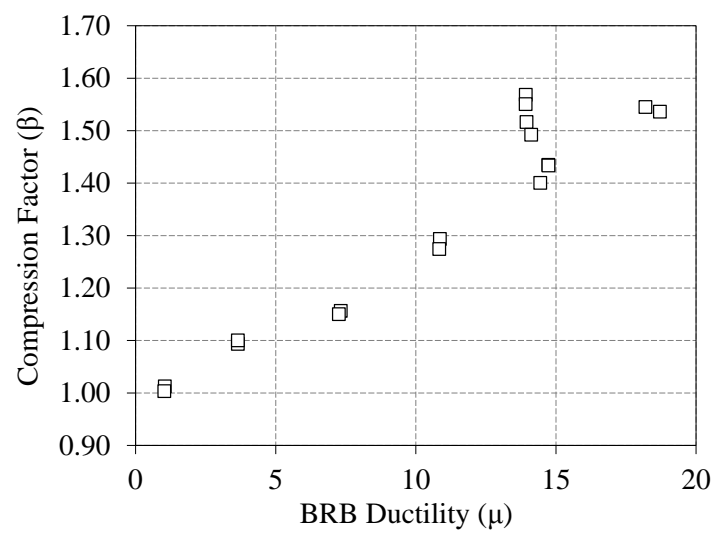

(c)

Figure 5.87 Compression adjustment factor. (a) BRB1-Q, (b) BRB2-Q, (c) BRB3-Q

\subsubsection{Cumulative Inelastic Deformation and Energy Dissipation}

Cumulative inelastic deformations were calculated following the section $\mathrm{K} 3$ of the Seismic Provisions of AISC 341-10 (2010). According to the AISC seismic provision (2010), a BRB specimen needs to achieve a cumulative inelastic axial deformation of at least 200 times the yield deformation. However, this level of cumulative inelastic deformation is not required for subassemblage tests. The cumulative inelastic axial deformation for each specimen at failure is indicated in Table 5.13.

The results show that the cumulative inelastic deformation for BRB1-Q and BRB3$\mathrm{Q}$ is greater than three times of that required for qualification of $\mathrm{BRBs}$, which requires a 
cumulative inelastic deformation of at least $200 \Delta_{\text {by }}$ (AISC 341-10, 2010). On the contrary, the BRB2-Q attained lower cumulative deformation than that required by AISC 341-10. This result is consistent with the poor performance of a similar brace tested in the retrofitted case (Bent/BRB2).

Table 5.13 Cumulative inelastic axial deformations

\begin{tabular}{c|c}
\hline Specimen & $\begin{array}{c}\text { Cumulative Inelastic Axial } \\
\text { Deformation }\left(\mathrm{x} \Delta_{\text {by }}\right)\end{array}$ \\
\hline BRB1-Q & 683 \\
\hline BRB2-Q & 159 \\
\hline BRB3-Q & 690 \\
\hline
\end{tabular}

The energy dissipation of the specimens, which was calculated using the area enclosed by the hysteretic curves, is depicted in Figure 5.88. Although, BRB2-Q was expected to reach larger axial deformations than the other two BRBs, the results showed that BRB2-Q dissipated less energy. This lower energy dissipation capacity was caused by the early failure of the steel core that this BRB presented.

Another important parameter that demonstrates the energy dissipation capacity that a structural component can achieve is the hysteretic damping. The hysteretic damping for each specimen was calculated according to Section 5.5.5 and results are shown in Figure 5.89. All the braces reached a minimum of $40 \%$ hysteretic damping before failing. This result demonstrates the excellent energy dissipation capacity that BRBs can attain. 


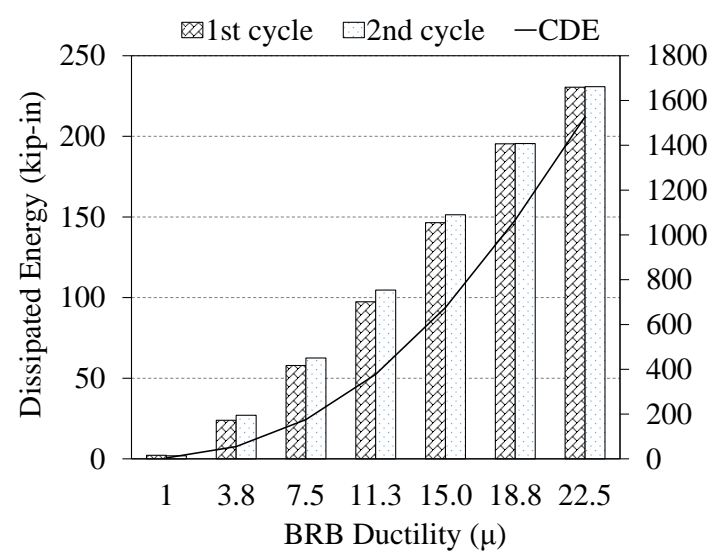

(a)

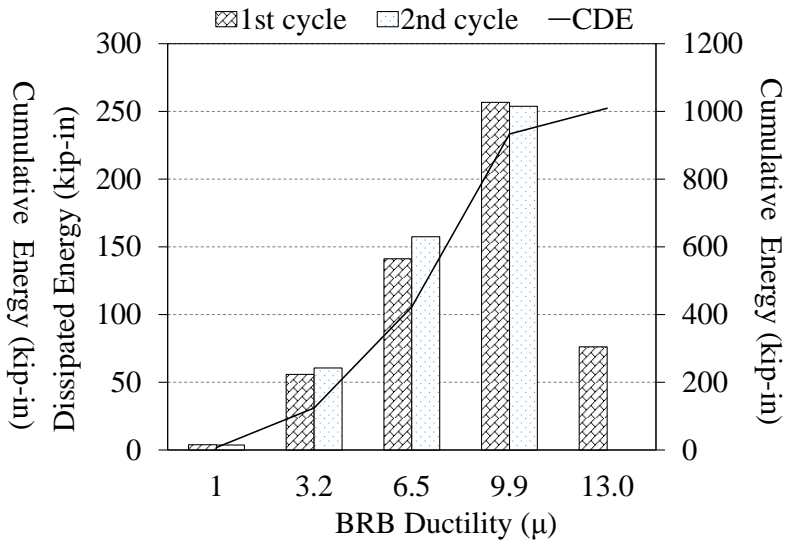

(b)

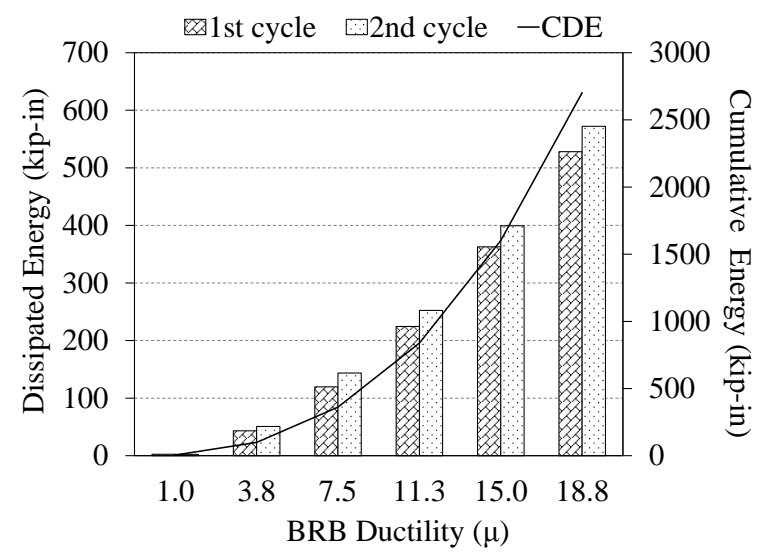

(c)

Figure 5.88 Dissipated energy. (a) BRB1-Q, (b) BRB2-Q, (c) BRB3-Q

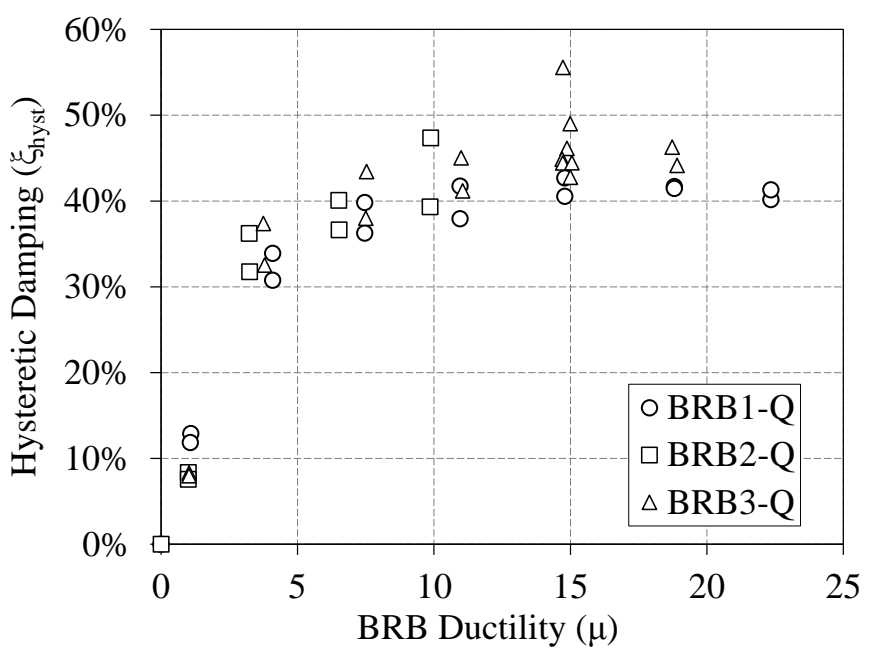

Figure 5.89 Hysteretic damping of BRBs 


\subsection{SUMMARY AND CONCLUSIONS}

Experimental evaluation of a large-scale reinforced concrete bridge bent designed to mimic a representative RC bent constructed in the 1950 to 1970 in the Pacific Northwest was presented in an effort to assess the viability and evaluate the cyclic behavior of seismic retrofitting and repairing with BRBs. Retrofitted, repaired and as-built cases were tested using cyclic loading protocols representative of the displacement demands in $\mathrm{RC}$ bridge bents subjected to subduction zone earthquakes. Two BRB retrofits were considered; Bent/BRB1 that was specifically designed using the fuse concept and Bent/BRB2 that utilized BRB of typical design proportions. A repaired bent (Bent/BRB3) was also tested. Based on the experiments, the following observations are made from this study:

The results of these large-scale experiments successfully demonstrated the effectiveness of utilizing BRBs for achieving high displacement ductility and energy dissipations in retrofitted and repaired cases, while also controlling excessive damage in columns and cap beam. The lack of damage and inelastic behavior of the RC components in Bent/BRB1 implies that no additional retrofit is needed of the RC column and the RC bent beam, especially when specific performance levels are targeted in the design using the fuse concept.

Repairing RC bents with BRBs proved to be an effective measure to restore the stiffness and strength of a damaged bent and provide high levels of energy dissipation.

The potential for improving the overall bent seismic behavior and the achieved performance levels with BRBs offers bridge design professionals a viable method to comply with serviceability criteria used in the retrofit of existing bridge bents. 
The incorporation of the BRB led to increasing forces in components of the $\mathrm{RC}$ bent. The experimental results showed an increase in compressive forces as compared to the As-Built specimen when the loading direction subjected the column to more compression. Furthermore, the results also showed a decrease in compressive forces when the loading direction was inverted. While this is not expected to significantly affect the column capacity for bridges, which often experience low axial demand/capacity ratio, additional care is recommended in the assessment of the footings due to the increase loading conditions.

No damage was observed in the gusset connection regions of the brace throughout the loading history, leaving the potential for rapid replaceability of the sacrificial BRB element after a major seismic event, and allowing for minimal interruption of service for the bridge.

A modified equation to calculate the stiffness degradation rate for BRB retrofitted bents was proposed by adding an exponential factor to the equation used for RC bents in order to account for the reduced rate in stiffness degradation.

The results also indicated that despite the detailing deficiencies of the multi-column RC bridge bent, the cyclic response of the as-built cases exhibited moderately ductile performance. The As-built Bent 1 specimen reached a displacement ductility of 6.7, which is likely the result of a relatively long lap splice length, and low axial column loads in the flexural dominated columns. The fact that the representative bent performed better than would be expected for bridges with similar detailing deficiencies can have potential positive impact on future retrofit plans for bridge owners. 
$\square \quad$ Qualification tests of BRBs agreed with the BRB responses and failures observed during the retrofitted and repaired bent tests and demonstrated the high level of energy dissipation that BRBs can attain. 


\section{CHAPTER 6}

\section{NONLINEAR CHARACTERIZATION BASED ON EXPERIMENTAL RESULTS}

\subsection{GENERAL}

Refined modeling techniques calibrated with experimental results are essential to achieve reliable displacement demands in structures subjected to strong ground motions. For that reason, this chapter focuses in the nonlinear characterization and modeling of bucklingrestrained braces and RC bridge bents in their as-built, retrofitted and repaired conditions. Refined numerical models are presented and validated in an effort to aid researchers and designers in performing nonlinear analyses. The parameters used in numerical modeling are based on the experimental results described in Chapter 5. Two software are utilized to perform such modeling, namely, SAP2000 and OpenSees. Nonlinear static analyses were performed using SAP2000 (Computers and Structures Inc., 2011) in an effort to compare the experimental results with a widely used commercial software. The Open System for Earthquake Engineering Simulation (OpenSees) software was also utilized for modeling and analyzing the nonlinear response of the tested specimens. OpenSees was utilized for the numerical simulations due to its widely use in earthquake engineering research and its wide range of material models, elements, and solution algorithms to model and analyze the nonlinear response of systems.

In addition to that, nonlinear modeling of two representative $\mathrm{RC}$ bridges is presented to reflect the use of refined nonlinear modeling techniques based on experimental results. Also, the performance assessment of a representative reinforced concrete bridge bent 
retrofitted using BRBs is investigated by performing nonlinear time history analyses. Special attention is put on assessing the structures subjected to subduction zone ground motions.

\subsection{Numerical Model OF BUCKLING-Restrained Braces}

In this section, numerical models of BRBs are presented in an effort to characterize the response of these components recorded from the subassemblage tests presented in Chapter 5. Further comparisons between experimental and numerical results are also presented.

\subsubsection{SAP2000 Model}

Numerical models representing the subassemblage test of each BRB were performed in SAP2000. The nonlinear model used in this study is shown in Figure 1.1. In this model, an elastic column pinned at its base was used in conjunction with rigid links and a single element for the brace. The use of a single element for the brace does not reflect that the nonlinear behavior is concentrated in a yielding zone. As a result, the stiffness in the model needs to be modified to reflect the influence on brace stiffness caused by a reduced section within the brace. In this study, the stiffness of the brace was modified by changing the modulus of elasticity applied to the element. Thus, an equivalent modulus of elasticity $\left(E_{\text {eq }}\right)$ was calculated using Eq. (6.1) (Zsarnoczay, 2013).

$$
E_{\text {eq }}=E_{\mathrm{s}} \frac{L_{\text {model }}}{L_{\mathrm{c}}+2 L_{\mathrm{tr}} \frac{b_{\mathrm{c}}}{\sqrt{b_{\mathrm{el}} \cdot b_{\mathrm{c}}}}+2 L_{\mathrm{el}} \frac{b_{\mathrm{c}}}{b_{\mathrm{el}}}}
$$

Where, $\mathrm{E}_{\mathrm{s}}$ is the steel modulus of elasticity $(29,000 \mathrm{ksi}) ; \mathrm{L}_{\text {model }}$ is the BRB length used in the model; $\mathrm{L}_{\mathrm{c}}$ is the length of the reduced section (yielding zone); $\mathrm{L}_{\mathrm{tr}}$ is the length of the 
transition section; $\mathrm{L}_{\mathrm{el}}$ is the length of the elastic section (full zone); $\mathrm{b}_{\mathrm{c}}$ is the width of the reduced section; and $b_{\text {el }}$ is the width of the full section.

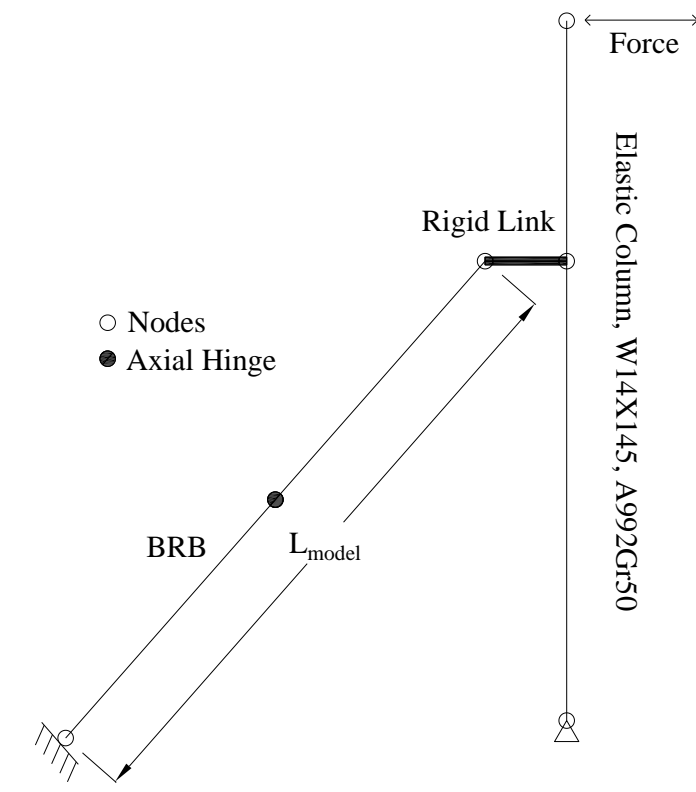

Figure 6.1 SAP2000 model of subassemblage tests

The BRB inelastic response was modeled using an axial hinge that was controlled by deformation. The behavior of the axial hinge was modeled as elastoplastic with hardening as depicted in Figure 4.11. The preliminary parameters used for modeling the three BRBs presented in Chapter 5, namely, BRB1-Q, BRB2-Q and BRB3-Q are indicated in Table 6.1. The values to define the maximum strength of the BRBs $\left(\mathrm{P}_{\mathrm{ad}}\right)$ were initially based on overstrength factors of 1.3 in tension $(\omega)$ and 1.45 in compression $(\beta \omega)$ as discussed in Chapter 4. Moreover, a maximum axial deformation equal to $15 \Delta_{\text {by }}$ was assumed.

Results of using those parameters are shown in Figure 6.2. Results showed that the numerical models underestimated the strength of the BRBs, especially in compression. This result was caused by two reasons. Firstly, the experimental axial strength at yield was greater than the nominal value for BRB1-Q and BRB2-Q. Secondly, the experimental over-strength 
factors in tension and compression (shown in Figure 5.86 and Figure 5.87, respectively) were also greater than the assumed factors.

Table 6.1 Initial parameters for nonlinear modeling of BRBs

\begin{tabular}{c|c|c|c|c|c|c}
\hline Specimen & $\mathrm{f}_{\mathrm{y}}, \mathrm{ksi}$ & $\mathrm{A}_{\mathrm{sc}}$, in $^{2}$ & $\mathrm{P}_{\mathrm{ysc}}$, kips & $\mathrm{P}_{\text {ad }}$, kips & $\mathrm{P}_{\text {ad- }}$, kips & $\Delta_{\text {by }}$, in \\
\hline BRB1-Q & 44.2 & 1.2 & 53.0 & 68.9 & 76.9 & 0.046 \\
\hline BRB2-Q & 44.2 & 1.2 & 53.0 & 68.9 & 76.9 & 0.135 \\
\hline BRB3-Q & 43.4 & 1.2 & 52.1 & 67.7 & 75.5 & 0.103 \\
\hline
\end{tabular}

The model also underestimated the maximum axial deformation of BRB1-Q and BRB3-Q since those braces attained axial deformations equal to 22.5 and 18.8 times the yield deformation $\left(\Delta_{\text {by }}\right)$, which were greater than the assumed value of $15 \Delta_{\text {by. }}$ On the contrary, the axial deformation of BRB2-Q was overestimated by the model since this brace failed unexpectedly as described in Chapter 5.

In order to improve the numerical results, axial strength at yield, over-strengthfactors using the updated axial strength, and maximum axial deformations were modified based on the experimental results. Updated parameters are indicated in Table 6.2 and numerical results using those parameters are shown in Figure 6.3.

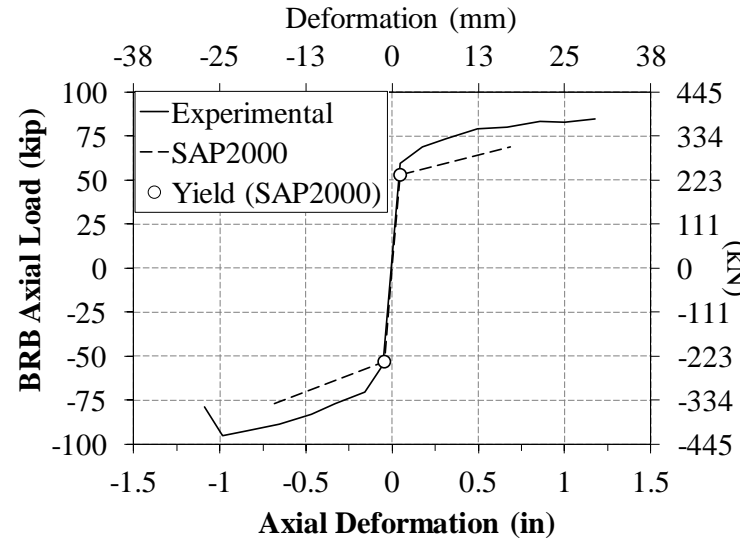

(a)

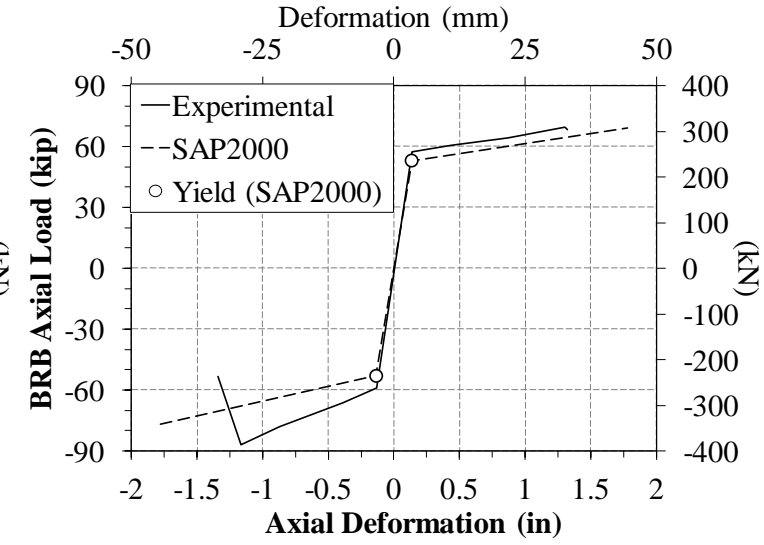

(b) 


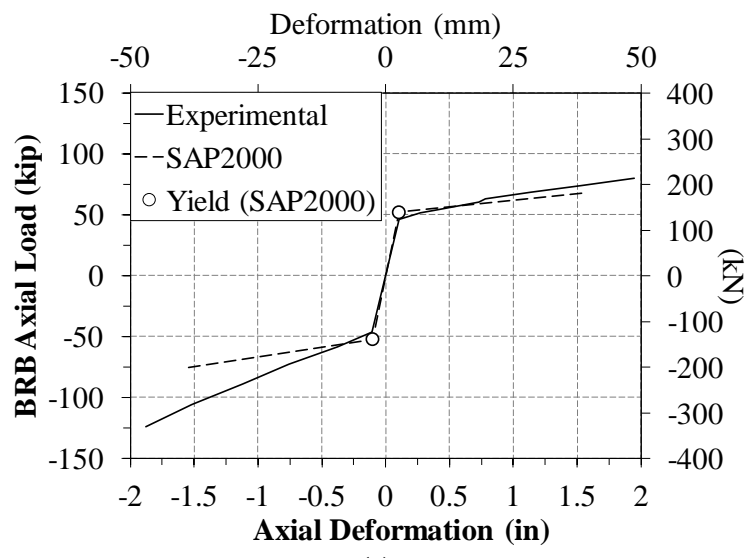

(c)

Figure 6.2 Preliminary SAP2000 results. (a) BRB1-Q, (b) BRB2-Q, (c) BRB3-Q

Table 6.2 Improved parameters for nonlinear modeling of BRBs

\begin{tabular}{c|c|c|c|c}
\hline Specimen & $\begin{array}{c}\mathrm{P}_{\text {ysc }}, \\
\text { kips }\end{array}$ & $\begin{array}{c}\omega \\
\text { (tension) }\end{array}$ & $\begin{array}{c}\beta \cdot \omega \\
\text { (compression) }\end{array}$ & $\begin{array}{c}\Delta_{\mathrm{u}}(\mathrm{x} \\
\Delta_{\text {by }}\end{array}$ \\
\hline BRB1-Q & 64 & 1.33 & 1.46 & 22.5 \\
\hline BRB2-Q & 58 & 1.22 & 1.49 & 10 \\
\hline BRB3-Q & 52 & 1.54 & 2.39 & 18.8 \\
\hline
\end{tabular}

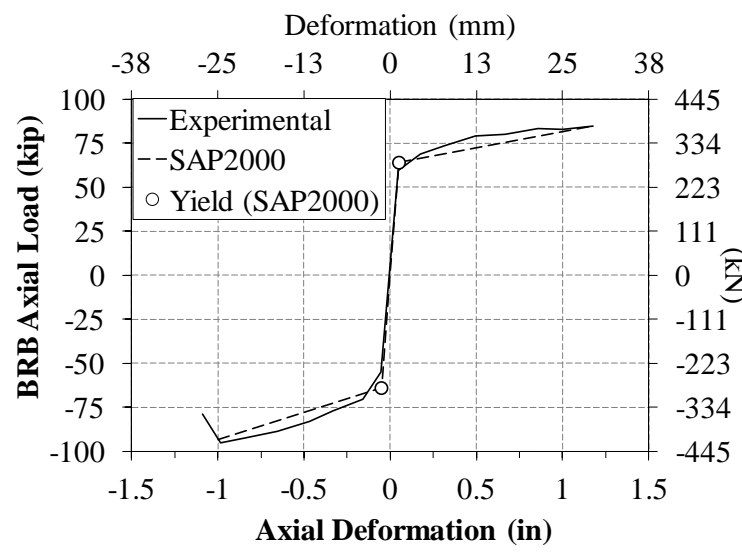

(a)

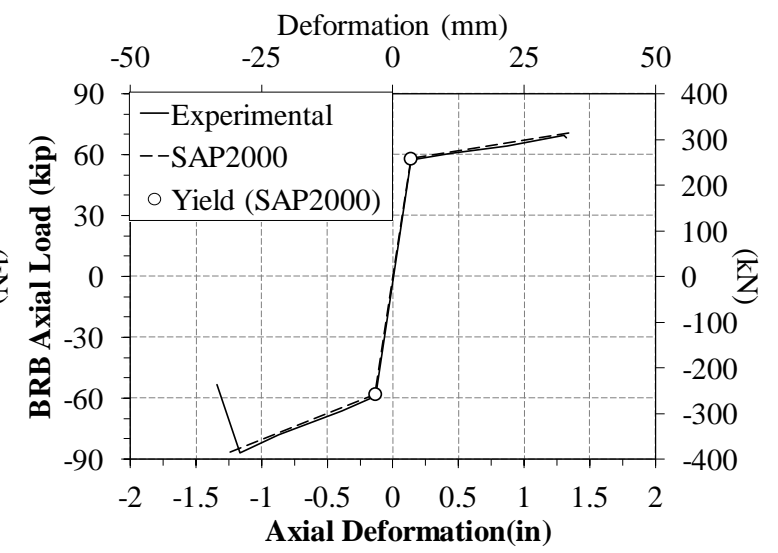

(b) 


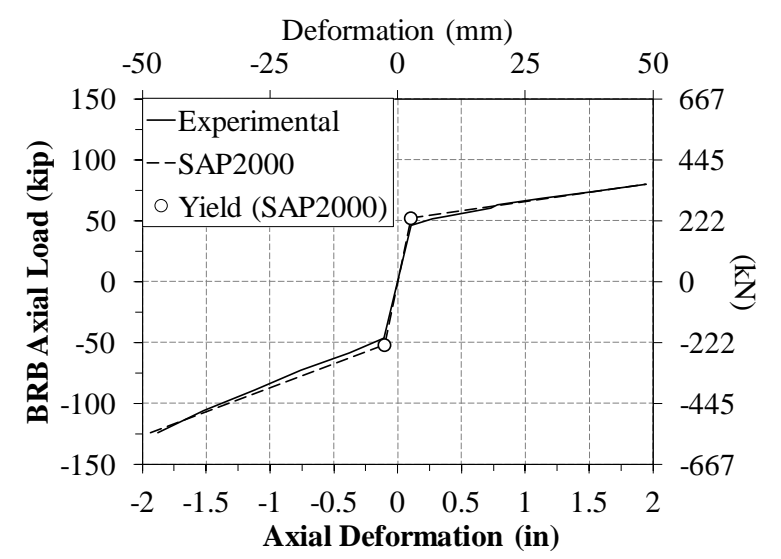

(c)

Figure 6.3 SAP2000 models based on experimental results. (a) BRB1-Q, (b) BRB2-Q, (c) BRB3-Q

Numerical results demonstrated the variability that important parameters such as axial strength at yield, over-strength factors and ultimate deformation can have with respect to experimental results. In particular, the compression adjustment factor was greatly underestimated.

\subsubsection{OpenSees Model}

Numerical models were also performed in OpenSees (2013) due to its vast library of uniaxial materials and nonlinear analysis capabilities. A similar nonlinear model to the one used in SAP2000 (Figure 1.1) was utilized to perform cyclic pushovers based on the loading history applied to each BRB. The main difference with respect to the SAP2000 model was the use of a truss element to define each brace. The stiffness of the BRB was also modified through the use of an equivalent elastic stiffness as described in Section 6.2.1.

In order to characterize the nonlinear response of the BRB two uniaxial materials were used, namely, Steel02 and Steel04. The Steel02 material, which is based on the GiuffréMenegotto-Pinto model with isotropic hardening, was used since this material has been widely used by researchers to model the inelastic response of BRBs (Korzekwa \& Tremblay, 
2009), (Ariyaratana \& Fahnestock, 2011). In order to define the Steel02 material, a strain hardening ratio of 0.03 was used in all the models. The rest of the parameters are indicated in Table 6.3.

Table 6.3 Steel02 parameters

\begin{tabular}{c|c|c|c|c|c|c|c}
\hline \multirow{2}{*}{ Specimen } & \multicolumn{7}{|c}{ Parameters } \\
\cline { 2 - 8 } & R0 & CR1 & CR2 & a1 & a2 & a3 & a4 \\
\hline $\begin{array}{c}\text { BRB1-Q } \\
\text { BRB2-Q }\end{array}$ & 20 & 0.925 & 0.15 & 0.05 & 0.9 & 0.0 & 1.0 \\
\hline BRB3-Q & 20 & 0.925 & 0.15 & 0.08 & 0.9 & 0.03 & 0.9 \\
\hline
\end{tabular}

Where, R0, CR1 and CR2 are parameters to control the transition from elastic to plastic branches; and a1 to a4 are parameters to control the isotropic hardening in compression (a1 and a2) and in tension (a3 and a4).

The cyclic behavior of buckling-restrained braces was also modeled using the Steel04 and the fatigue material as proposed by Zsarnóczay (2013). According to Zsarnóczay, the steel04 material better represents the Bauschinger effect, limits the maximum stress that can be developed in the material, and by adding the fatigue material is able to limit the otherwise infinite deformation capacity that the Stee 02 material presents. The fatigue material, which accounts for the effect of low cycle fatigue using Miner's rule, was defined based on the recommendation of Zsarnóczay (2013) in an effort to adjust the inelastic strains that occur in the yielding zone (reduced section) and to be consistent with the use of a single element to model the brace. Then, the value of strain $\left(\boldsymbol{\varepsilon}_{\mathrm{o}}\right)$ at which one cycle will cause failure was calculated using Eq. (6.2). In addition to that, the slope of Coffin-Manson curve in log-log space (m) was set to 0.4 as suggested by Zsarnóczay (2013). 


$$
\varepsilon_{o}=0.14 \cdot \frac{L_{c}}{L_{\text {model }}}
$$

Where, $\mathrm{L}_{\mathrm{c}}$ is the reduced section length and $\mathrm{L}_{\text {model }}$ is the length of the BRB used in the model. It is worth mentioning that for BRB2-Q, the maximum strain $\left(\boldsymbol{\varepsilon}_{\mathrm{o}}\right)$ was reduced to reflect the lower deformation capacity attained by that brace. The values of strain amplitude at which one complete cycle is sufficient to cause failure of the brace were set equal to 0.035 for BRB1-Q and BRB2-Q and equal to 0.079 for BRB3-Q.

Figure 6.4 shows the results of using Steel 02 and Steel04 plus fatigue. The numerical simulations using the Steel02 material showed good agreement with the experimental results. However, as identified by others (Zsarnoczay, 2013) this material results in infinite strength and deformation, which can lead to erroneous assessment of strength and deformation capacities in nonlinear time history analysis when not combined with other materials such as the fatigue material. On the contrary, the numerical simulations that were performed using the Steel04 plus the fatigue material agree well with experimental results in terms of maximum strength and ultimate deformation. Moreover, a benefit of incorporating the fatigue material into the model is that BRB failure was artificially captured in the model. Thus, the model using Steel04 plus the fatigue material is recommended to simulate the nonlinear behavior of BRBs. 


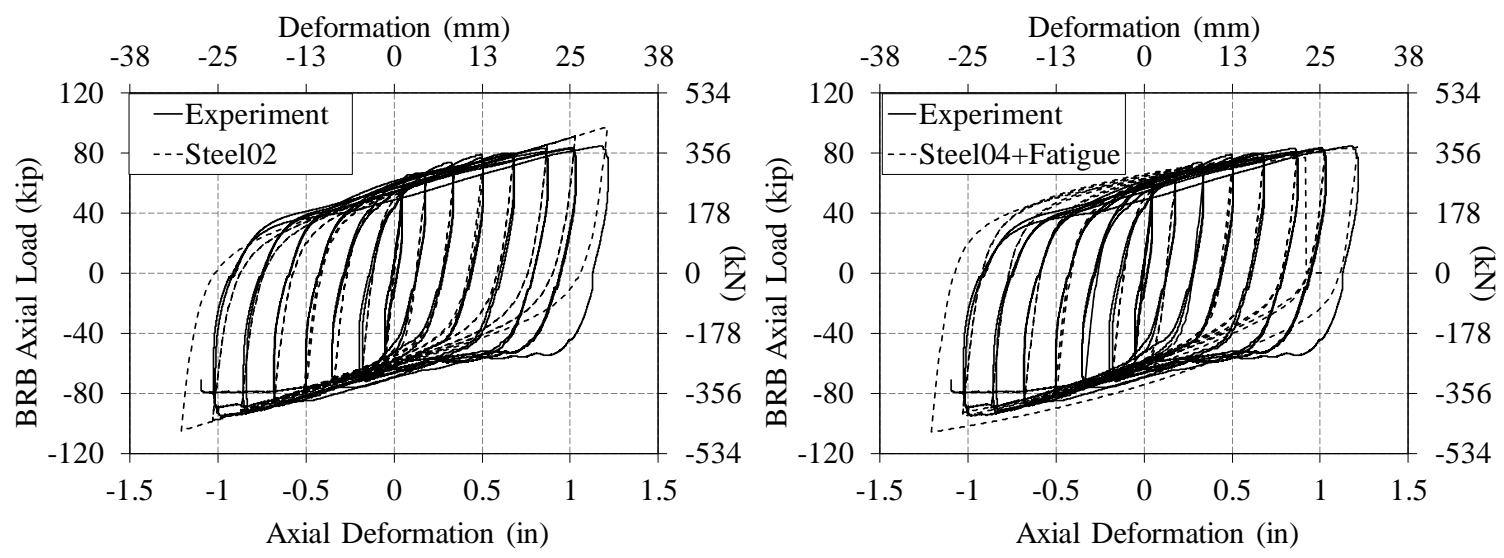

(a)

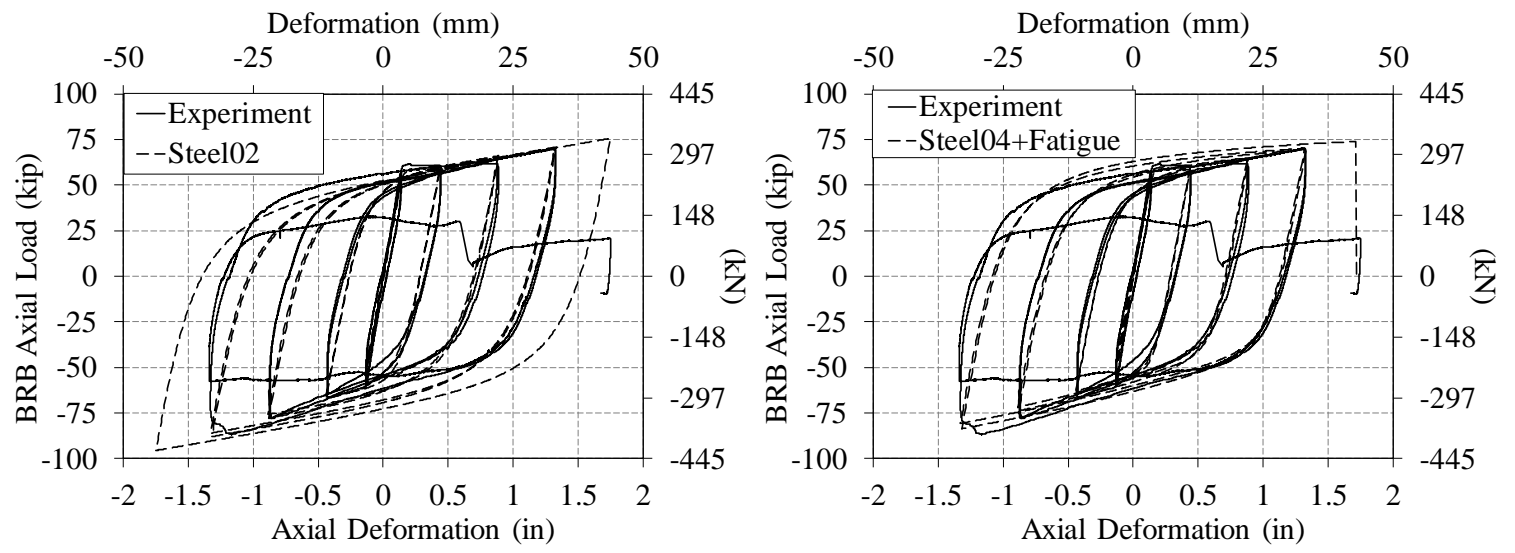

(b)

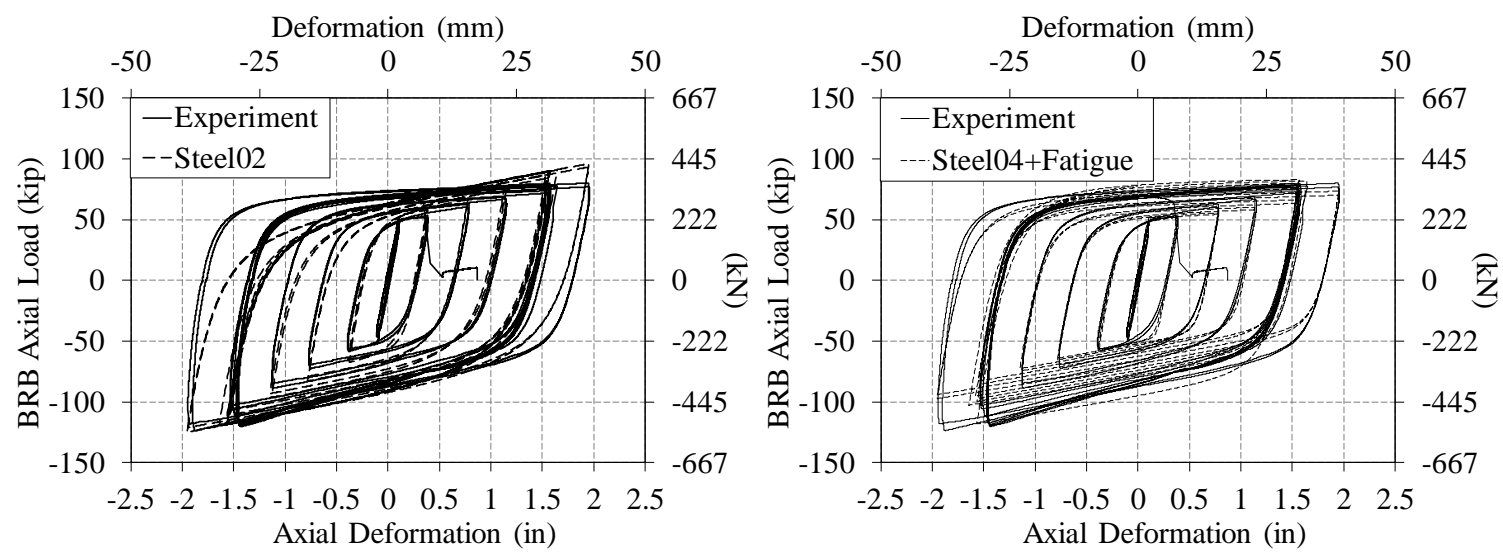

(c)

Figure 6.4 OpenSees simulations based on experimental results.

(a) BRB1-Q, (b) BRB2-Q, (c) BRB3-Q 


\subsection{Numerical MOdEL OF LARGE-SCALE BRIDGE BENTS}

In this section, numerical models are evaluated to characterize the response of the large-scale RC bridge bents tested in an as-built, retrofitted and repaired states as described in Chapter 5. The large-scale bridge bent specimens consisted of two 18 in (457 mm) diameter circular columns per bent and a rectangular 18 in $(457 \mathrm{~mm})$ x 21 in $(533 \mathrm{~mm})$ cap beam. For the retrofitted and repaired conditions a BRB in a diagonal configuration was added.

\subsubsection{Sap2000 Model}

The model used for the nonlinear static analyses is depicted in Figure 6.5. In this model, cap beam and columns were modeled using elastic frame elements with property modifiers obtained from moment-curvature analysis in an effort to reflect cracked sections. Additional property modifiers of 0.8 and 0.5 were applied for the numerical models of Asbuilt bent 1 and Bent/BRB3, respectively, since those tests started from an already damaged state.

The inelastic behavior of columns and cap beam was modeled assuming Mander concrete parametric stress-strain curve for the unconfined and confined concrete (Mander, et al., 1988) and the Park parametric stress-strain curve for reinforcing steel. The hinges in columns and cap beam were defined using an idealized moment-curvature behavior as described in Caltrans(2013) and were lumped at expected plastic hinge locations as shown in Figure 6.5. Eq. (5.4) was used to define the plastic hinge length as required by AASHTO (2009). In addition to the cap beam and column elements, rigid links were utilized to represent the beam-column joint and gusset plates. The BRB response was modeled using an axial hinge that was controlled by deformation as described in a previous section. The 
parameters used for modeling the three BRBs evaluated in the retrofitted and repaired bents are indicated in Table 6.2. The parameters for the adjusted strength in tension $\left(\mathrm{P}_{\text {adt }}\right)$ and compression $\left(\mathrm{P}_{\mathrm{ad}}-\right)$ were modified based on the results obtained from the experimental evaluation of BRBs, namely, BRB1-Q, BRB2-Q and BRB3-Q. The stiffness in the model was also modified as described in Section 6.2.1.

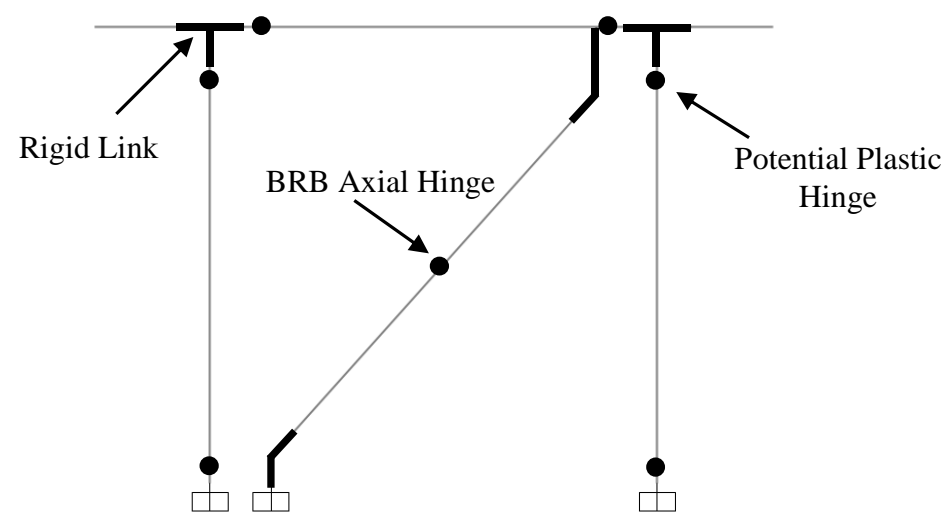

Figure 6.5 Model schematic for nonlinear static analysis in SAP2000

Comparisons between experimental and numerical results are shown in Figure 6.6(a), (b), (c), (d) and (e) for Bent/BRB1, Bent/BRB2, As-Built 1, As-Built 2 and Bent/BRB3, respectively. Results for Bent/BRB1, which was tested up to the expected displacement demand under the 1000 -year event ( 0.7 in) showed close agreement between the experimental and numerical load-displacement envelope curves, especially for the initial stiffness and yield displacement. Figure 6.6(b) compares the experimental result for the Bent/BRB2 with those from the pushover analysis using SAP2000. In this case, the numerical results agree well with the experimental results until failure of the BRB, after that point the model is incapable of capturing the unexpected failure for this BRB.

Numerical simulations for As-built bent 1, As-built bent 2 and Bent/BR3 matched the experimental results in terms of strength and displacement capacity. Thus, the 
experimental response envelopes for the retrofitted and repaired conditions can be satisfactorily characterized using a relatively simple nonlinear model.

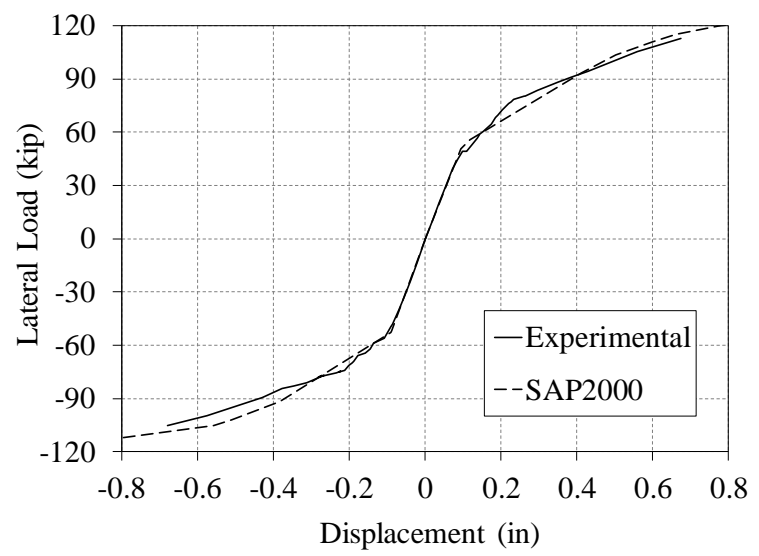

(a)

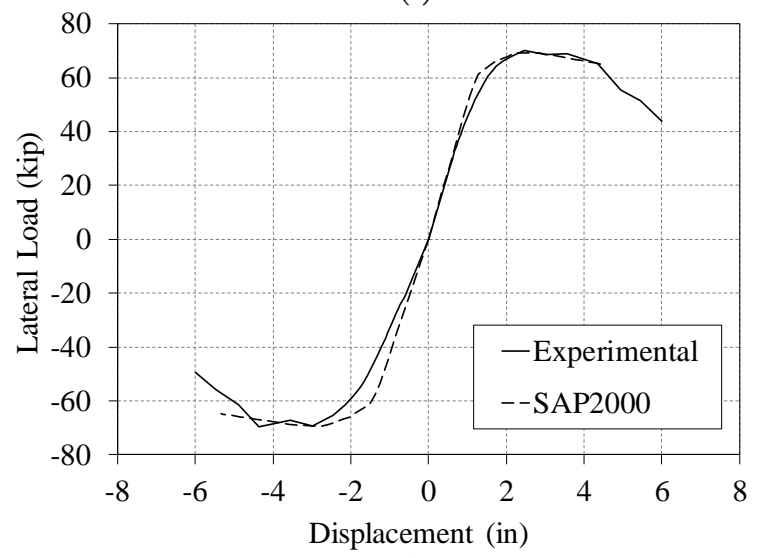

(c)

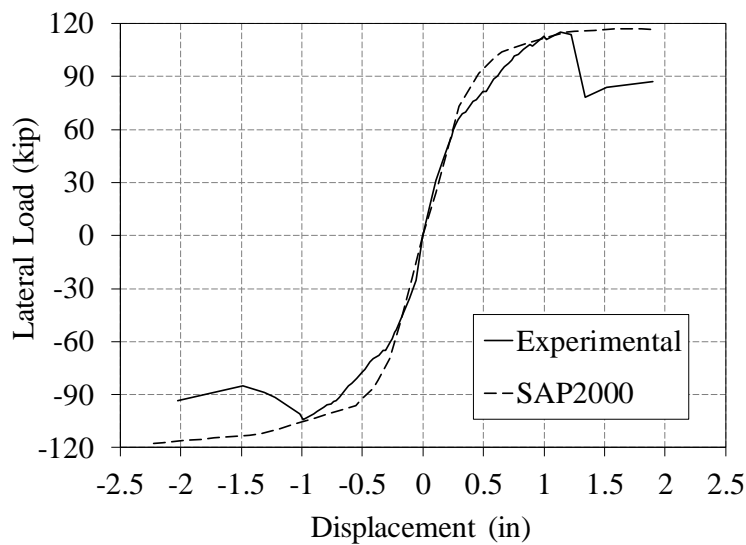

(b)

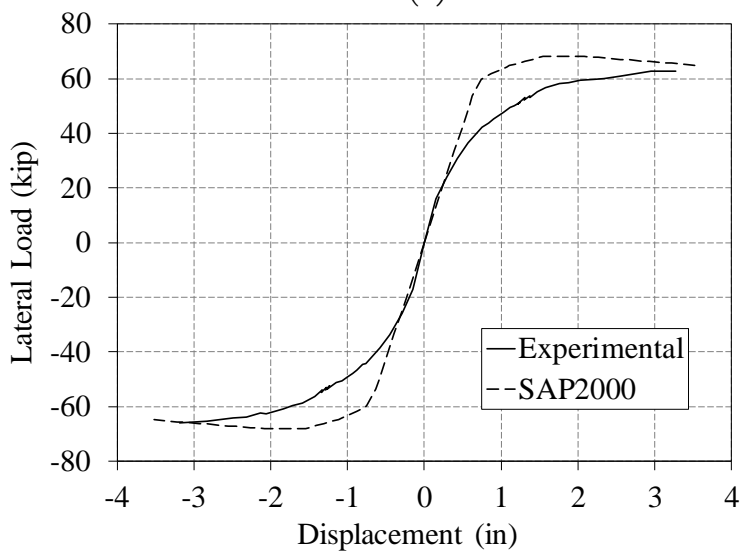

(d)

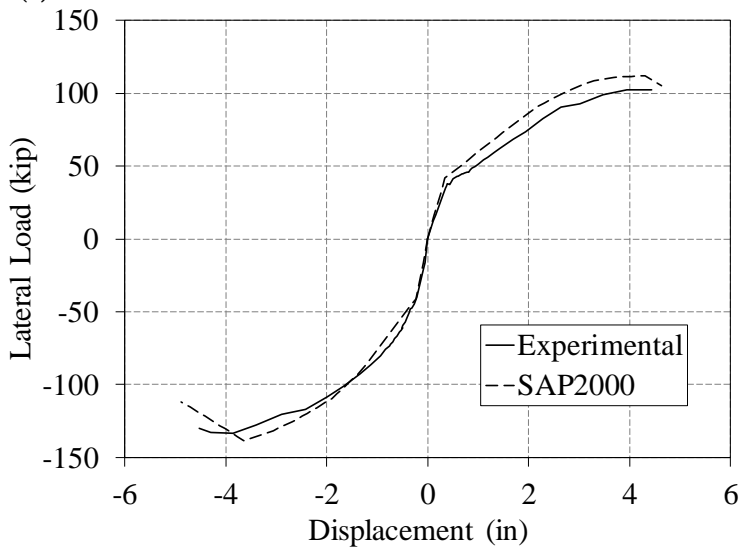

(e)

Figure 6.6 SAP2000 models based on experimental results. (a) Bent/BRB1, (b) Bent/BRB2, (c) As-built bent 1, (d) As-built bent 2, (e) Bent/BRB3 


\subsubsection{OpenSees Model}

The large-scale bridge bents used in the experimental program were modeled using a distributed-plasticity method similar to the model described in Chapter 2. In this method, the nonlinear behavior of structural components such as columns and beams was distributed along the length of the member through the use of fiber-based elements and integration points. OpenSees (2013) was utilized for the numerical simulations.

A schematic of the model is depicted in Figure 6.7, where force-based beam-column elements with six integration points between nodes were used to represent the columns and the cap beam. The Concrete02 with linear tension softening uniaxial material was used in this study to model both confined and unconfined concrete. The longitudinal reinforcing steel was modeled using the Hysteretic uniaxial material since it is able to capture the pinching behavior observed in columns with lap splices and is also capable of simulating strength and stiffness degradation. Fiber-based models can accurately capture the flexural behavior of a component. However, deformations due to shear and bond slip are not considered unless additional elements or stiffness modifiers are introduced into the model. In this study, shear deformations were introduced in the model through the use of the section aggregator command, in which a constant shear modulus equal to $0.2 E_{c}$ was used following the recommendations of Elwood and Eberhard (2009). Bond slip deformations at the base and top of column and at the ends of the cap beam were modeled through the use of a zero-length section following the model proposed by Ghannoum (2007).

As described in the previous section, the cyclic behavior of buckling-restrained braces was best modeled using the Steel04 and the fatigue uniaxial materials as proposed by Zsarnóczay (2013). A benefit of incorporating the fatigue material into the model was that 
BRB failure was artificially captured in the model. The BRB in the model of the large-scale bridge bent had an effective length of $130.625 \mathrm{in}(3318 \mathrm{~mm})$ and two rigid links that represent the end connections (gusset plates). In order to reflect that the gusset plates were not connected to the columns, the rigid links were directly connected to the work points in the cap beam and footing as shown in Figure 6.7(b).

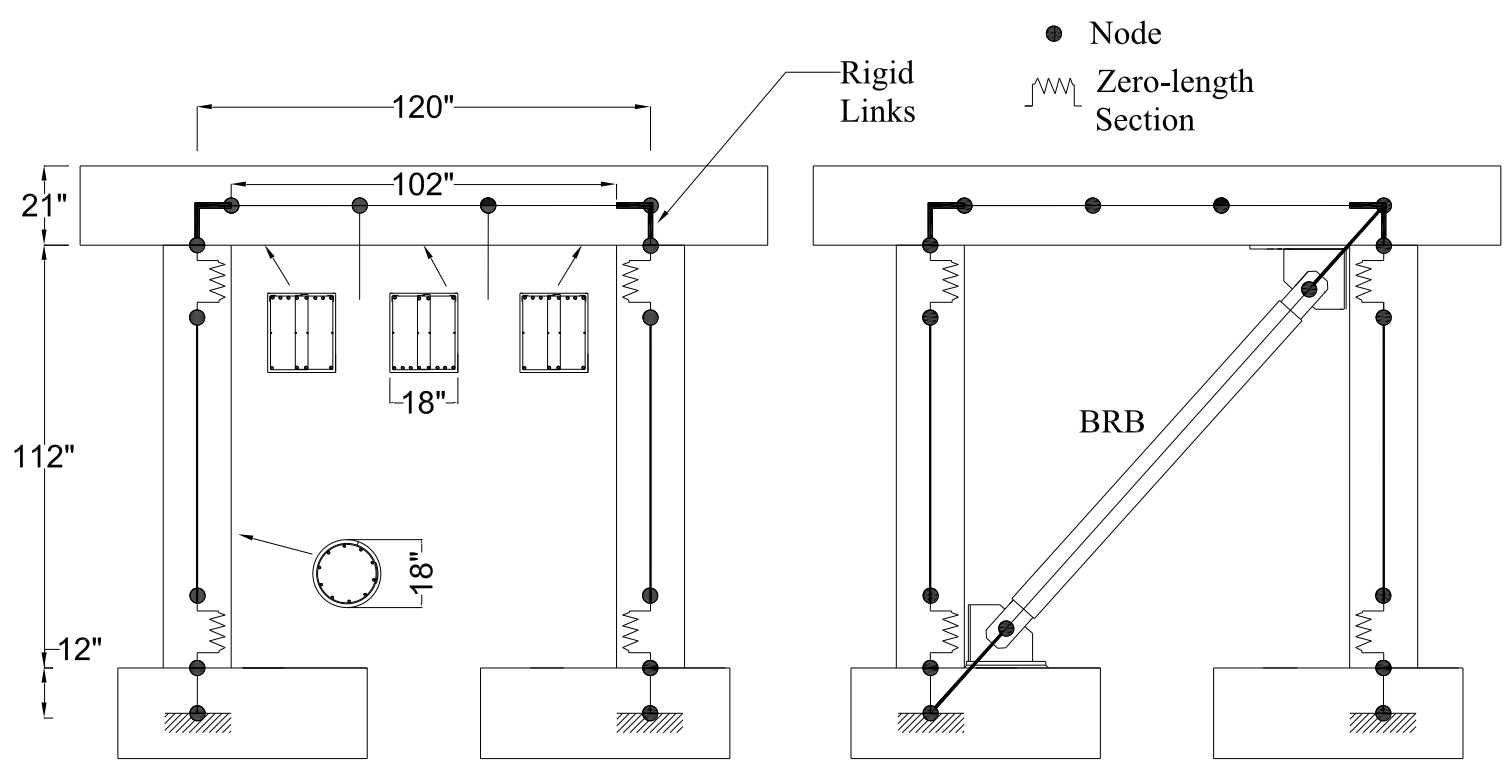

Figure 6.7 Numerical model of tested RC bent. (a) As-built, (b) Retrofitted and repaired bents

Results of the OpenSees simulations are shown in Figure 6.8. The results demonstrate that the numerical models were able to characterize the behavior of the tested bents. Initial stiffness, strength and deformation capacities, pinching effect, and strength and stiffness degradation were closely represented by the models. Figure 6.8(e) shows that the numerical model for the Bent/BRB3 overestimated the strength capacity of the bent when the $\mathrm{BRB}$ was in tension. This effect was presumably caused by the complexity of modeling the RC bent from an originally damaged state. 


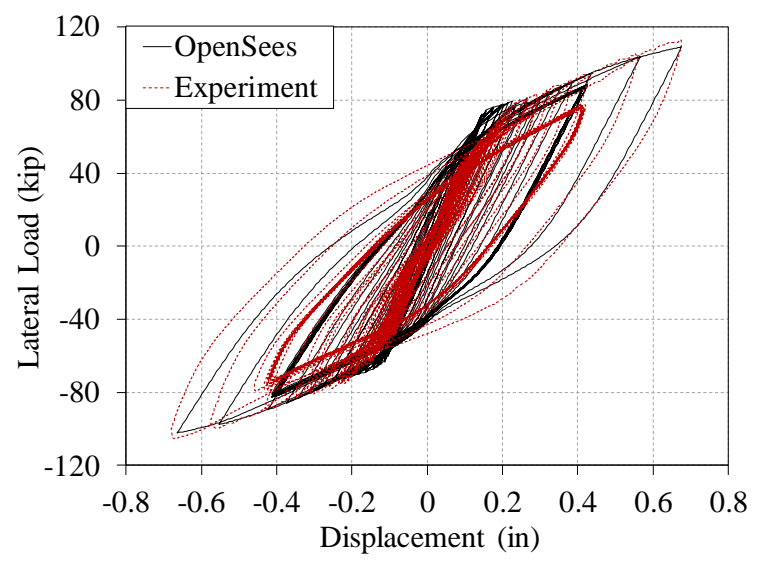

(a)

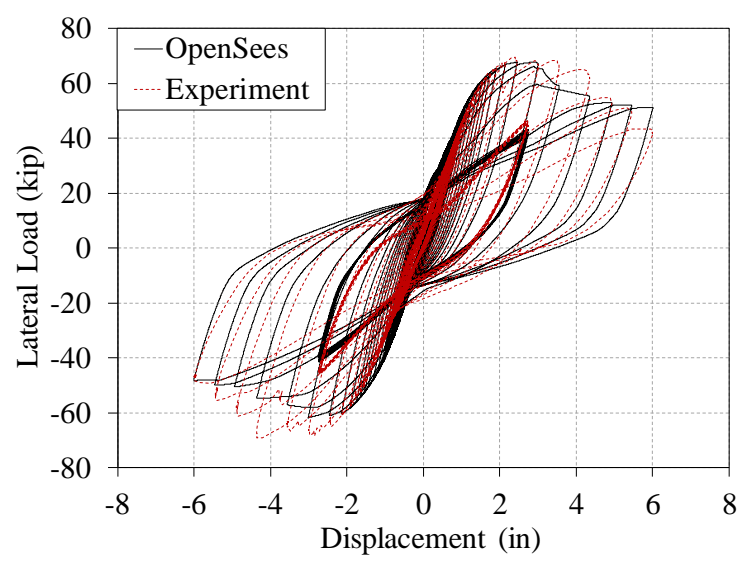

(c)

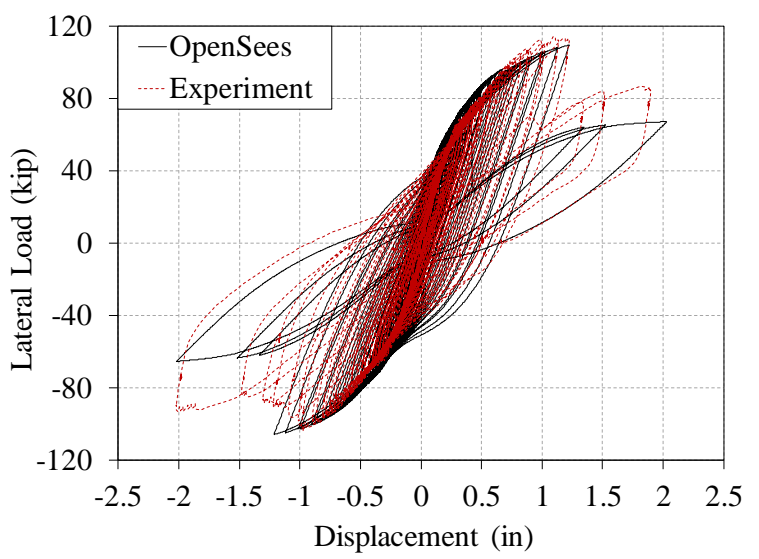

(b)

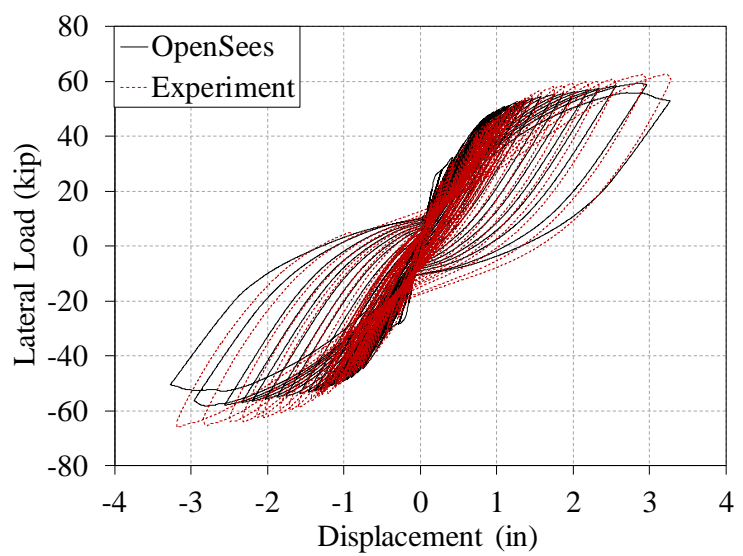

(d)

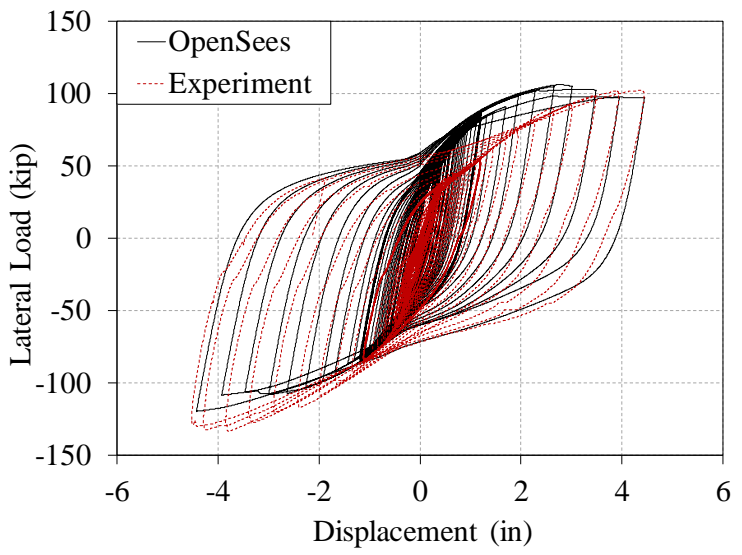

(e)

Figure 6.8 OpenSees simulations based on experimental results.

(a) Bent/BRB1, (b) Bent/BRB2, (c) As-built 1, (d) As-built 2, (e) Bent/BRB3 


\subsection{Modeling of Multi-Column Prestressed ConCrete Stringer/Girder BRIDGE}

This section presents details for modeling the components of a typical multi-column prestressed concrete stringer/girder (MPCG) bridge using numerical models calibrated based on the experimental results.

\subsubsection{Overview of As-built Numerical Model}

The numerical model for a MPCG bridge is shown in Figure 6.9. In this model, specific modeling approaches, which are described in following sections, were utilized to characterize the response of different bridge components such as abutments, bearings, foundation, etc., as shown in Figure 6.9(a). The dimensions for the bridge are depicted in Figure 6.9(b). These dimensions were utilized to represent typical dimensions observed in MPCG bridges of Oregon.

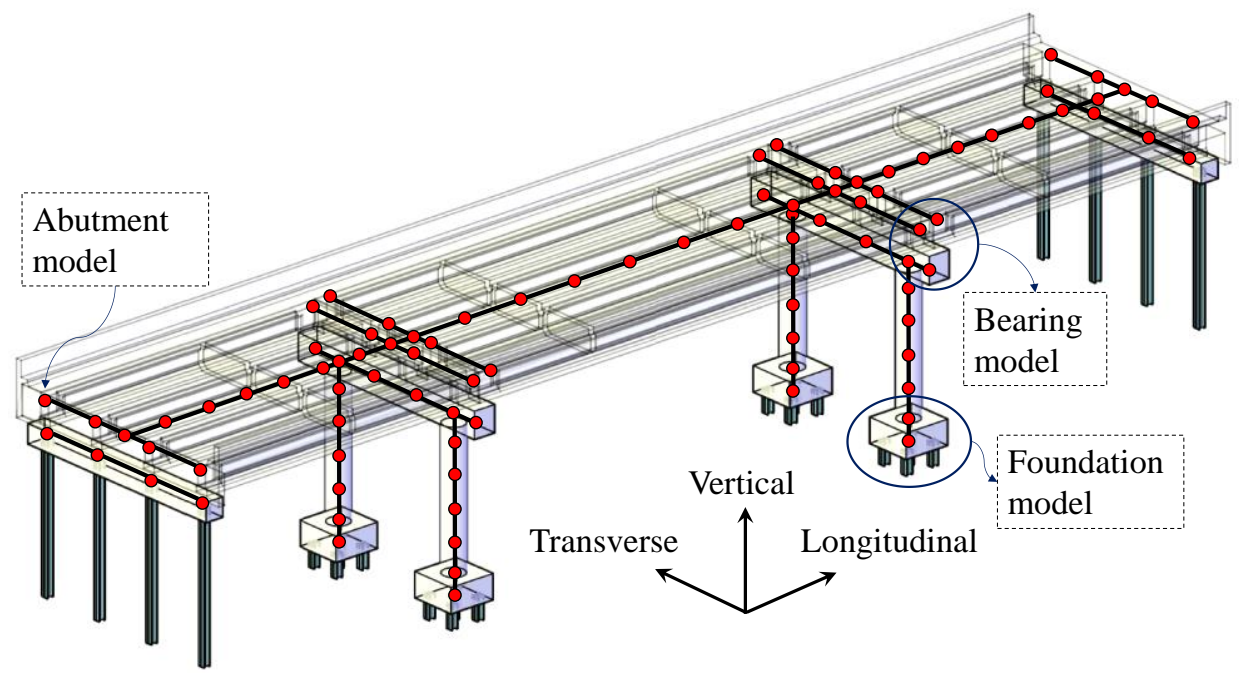

(a) 


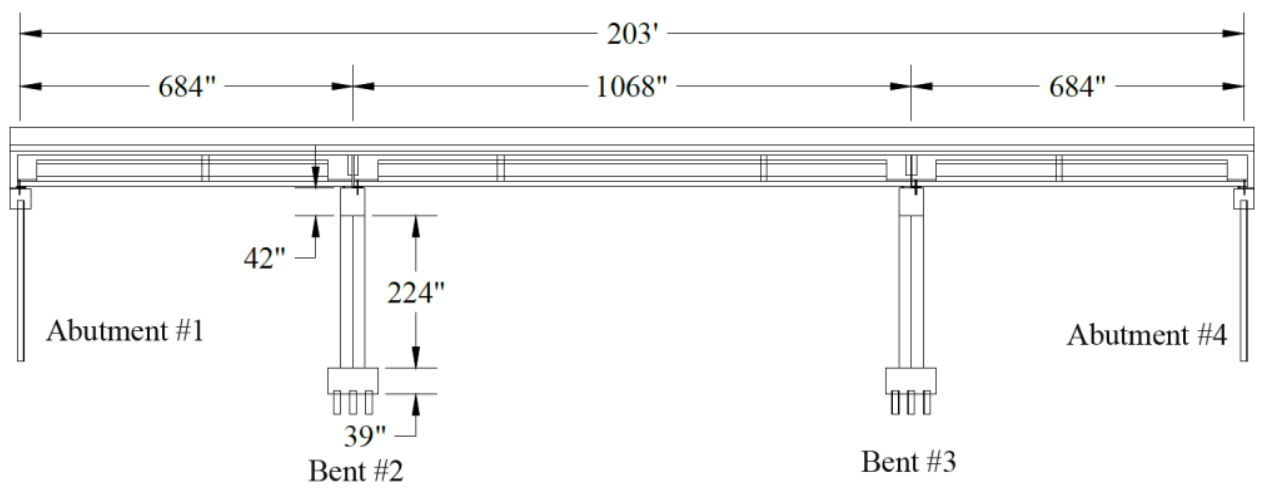

(b)

Figure 6.9 General layout of a MPCG bridge (a) 3D Model (b) Elevation

\subsubsection{Superstructure}

\subsubsection{General Description}

The superstructure in multi-column prestressed concrete girder bridges comprises all the bridge components that are located on top of the cap beam, such as girders, diaphragms and slab. The girders typically found in Oregon bridges consists of Type III or Type IV prestressed concrete girders. The slab or deck consists of a thin layer of concrete that is made continuous by casting it in place on top of the girders. The girders present bend bars extending from its top surface and shear keys in order to create a composite action between the girders and the deck. The deck thickness usually ranges from 5" to 8”. In this study, Type IV girders and a deck thickness of 7" were assumed. Additional transverse beams usually referred to as diaphragms are located at the end and middle of the spans in order to increase the stiffness of the superstructure and to make it continuous. Figure 6.10 shows a 3D schematic of a typical superstructure for MPCG bridges. 


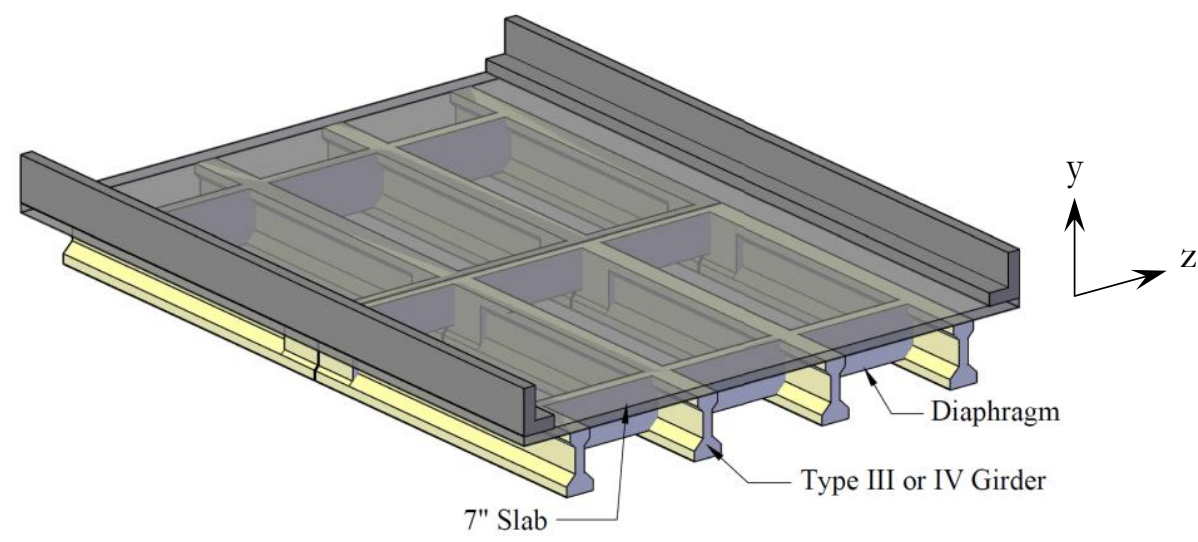

Figure 6.10 Typical superstructure used in MPCG bridges

\subsubsection{Superstructure Model}

The superstructure was modeled using a spine-line model. In the model, elastic beam column elements with the properties calculated from the composite action between girders and deck were used. This modeling assumption was made because the superstructure is expected to remain in the elastic range of response during a seismic event. Specified compressive concrete strength, $f_{c}^{\prime}$, were used in order to calculate the modulus of elasticity and ultimately the composite properties. Values of 3,300 psi and 6,000 psi were used for the slab and girders, respectively. The elastic element was defined using the values given in Table 2.1. Since a spine model was used to model the superstructure, seven intermediate nodes, i.e. 8 elements, were utilized to lump the masses based on tributary volume of the system. This discretization approximates the translational distributed mass of the bridge. Additional rotational mass was added in the model in order to characterize the torsional properties of the superstructure. The rotational mass, $M_{\text {rot }}$, was assigned according to Eq. (6.3) (Aviram, et al., 2008).

$$
M_{r o t}=\frac{M d_{w}{ }^{2}}{12}=\frac{(m / L) \cdot L_{t r i b} \cdot d_{w}{ }^{2}}{12}
$$


Where, $\mathrm{M}$ is the mass of the superstructure segment that is tributary to the node, $m / L$ is the mass per unit length, $L_{\text {trib }}$ is the tributary length of any specific node, and $d_{w}$ is the superstructure width. The elastic elements were placed 44 inches above the cap beam to reflect the centroid of the superstructure.

Table 6.4 Superstructure modeling parameters for MPCG Bridge

\begin{tabular}{l|c}
\hline \multicolumn{1}{c|}{ Description } & Value \\
\hline Cross-sectional area & $6883 \mathrm{in}^{2}$ \\
\hline Modulus of elasticity $(\mathrm{E})$ & $3274 \mathrm{ksi}$ \\
\hline Shear modulus $(\mathrm{G})$ & $1364 \mathrm{ksi}$ \\
\hline Second moment of area about the local z axis $\left(\mathrm{I}_{\mathrm{z}}\right)$ & $3.646 \times 10^{6} \mathrm{in}^{4}$ \\
\hline Second moment of area about the local y axis $\left(\mathrm{I}_{\mathrm{y}}\right)$ & $1.158 \times 10^{8} \mathrm{in}^{4}$ \\
\hline Torsional moment of inertia $(\mathrm{J})$ & $2.985 \times 10^{5} \mathrm{in}^{4}$ \\
\hline $\begin{array}{l}\text { Weight of superstructure }+ \text { overlay and barriers } \\
\text { (kip/in) }\end{array}$ & 0.77 \\
\hline Superstructure width (in) & 360 \\
\hline Centroid from bottom of girder & $44 \mathrm{in}$ \\
\hline
\end{tabular}

\subsubsection{RC Bent}

\subsubsection{General Description}

The representative reinforced concrete bent corresponds to an existing RC multicolumn bridge bent constructed in the 1950 to mid-1970 in the state of Oregon. As many of the bridge structures built at that time in the Pacific Northwest, the bridge substructure was designed and built with minimum seismic considerations. This resulted in inadequate transverse reinforcement, no seismic detailing, and lap-splices of length from 20 to 40 times the diameter of the longitudinal reinforcing steel $\left(\mathrm{d}_{\mathrm{b}}\right)$ in expected plastic hinge zones.

The prototype bridge bent consists of two circular columns per bent, a rectangular cap beam and rectangular pile cap footings. The column longitudinal reinforcement ratio is 
$\varrho_{\mathrm{L}}=1.2 \%$, which is just above the minimum required by AASHTO (2009). The provided column shear reinforcement and confinement does not meet code requirements since \#4 circular hoops spaced at 12 in were provided. The cap beam has premature termination of top reinforcement and low quantity of bottom steel reinforcement at the ends of the span, which might result in the formation of premature hinges in the cap beam at the column face. Moreover, lap splices of $40 \mathrm{~d}_{\mathrm{b}}$ can be found at the base of columns and no seismic detailing was specified. The specified material properties for the representative bridge were $3.3 \mathrm{ksi}$ as compressive strength of concrete at 28 days and Grade 40 steel. Typical details for the representative two column RC bridge bent are illustrated in Figure 4.14.

\subsubsection{Bent Model}

The model to characterize the response of the representative bent was based on the model presented in Section 6.3.2, which was calibrated with the experimental tests of largescale RC bent specimens. As a consequence, the numerical model for the MPCG bridge bent consisted of force-based fiber beam-column elements in conjunction with rigid links and zero length sections as shown in Figure 6.11. The discretization of the fibers comprised the use of 16 core radial subdivisions, 2 cover radial subdivision, and 36 core and cover transverse subdivisions for the column, and 28 by 22 subdivisions for the bent cap. The expected hysteretic behavior of the bent numerical model under the subduction loading protocol described in Chapter 2 is shown in Figure 6.12. A comparison with a pushover performed in SAP2000 is shown in the same figure. That comparison shows a good agreement in the initial force-displacement behavior captured for both software, and indicates that the response obtained using SAP2000 represents an approximate envelope of the hysteretic behavior obtained using OpenSees. 


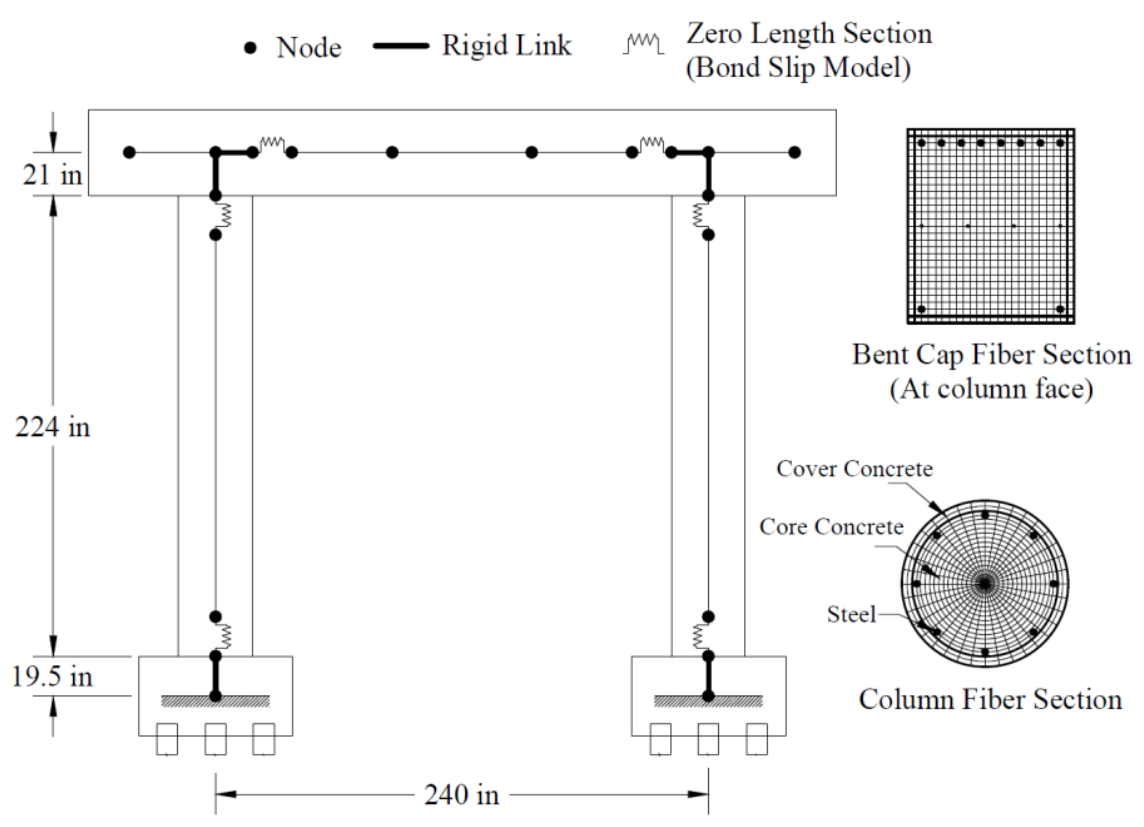

Figure 6.11 Numerical model for MPCG bridge bents

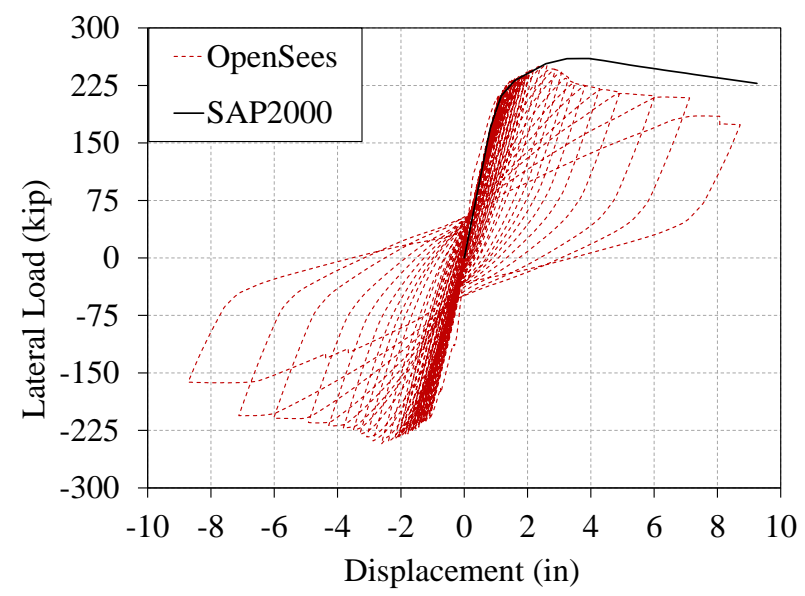

Figure 6.12 Expected force-displacement behavior

For the nonlinear time history analyses, 5\% Rayleigh damping was used and additional rotational mass was added in the model in order to characterize the torsional properties of the columns. The rotational mass, $M_{\text {rot-col }}$, was assigned according to Eq. (6.4) (Aviram, et al., 2008).

$$
M_{r o t-c o l}=\frac{M D_{c o l}^{2}}{8}=\frac{(m / L) \cdot L_{t r i b} \cdot D_{c o l}^{2}}{8}
$$


Where, $\mathrm{M}$ is the mass of the column segment that is tributary to the node, $m / L$ is the mass per unit length, $L_{\text {trib }}$ is the tributary length of any specific node, and $D_{c o l}$ is the column diameter.

\subsubsection{Foundations}

\subsubsection{General Description}

Although the foundation type in bridge varies from pile foundation systems to spread footings. Typical MPCG bridges present pile foundations, which consist of a pile cap and a group of piles as illustrated in Figure 6.13(a). The piles commonly found in Oregon's MPCG bridges comprise steel H-piles, which are driven deep into the ground with the primary purpose of resisting vertical loads. However, they also provide a mechanism to resist horizontal loads. The vertical load resisting mechanism of the pile foundation can be created from the friction between the pile and the surrounding soil or from end bearing capacity. Hpiles are usually considered for a design load between 80 kips and 500 kips, and they are commonly used as end bearing piles. In this study, five piles 10 BP42 (area $\left.=12.4 \mathrm{in}^{2}\right)$ ASTM A-36 were utilized in the pile foundation system. The distance between piles, the dimensions of the square pile cap, and the steel reinforcement of the pile cap are shown in Figure 6.13(b). The poor reinforcement provided to the footing added to the low embedment length of the piles, which varies from 6" to 12", into the pile cap make the foundation of pre-1970 bridges vulnerable to damage after a seismic event. 


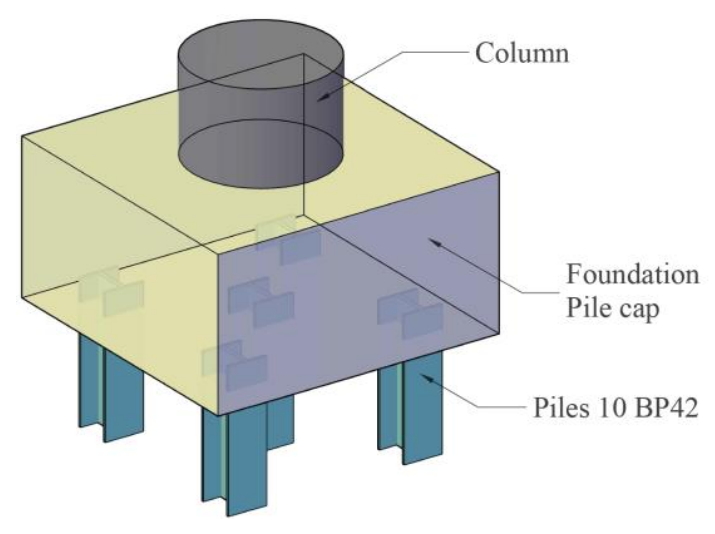

(a)

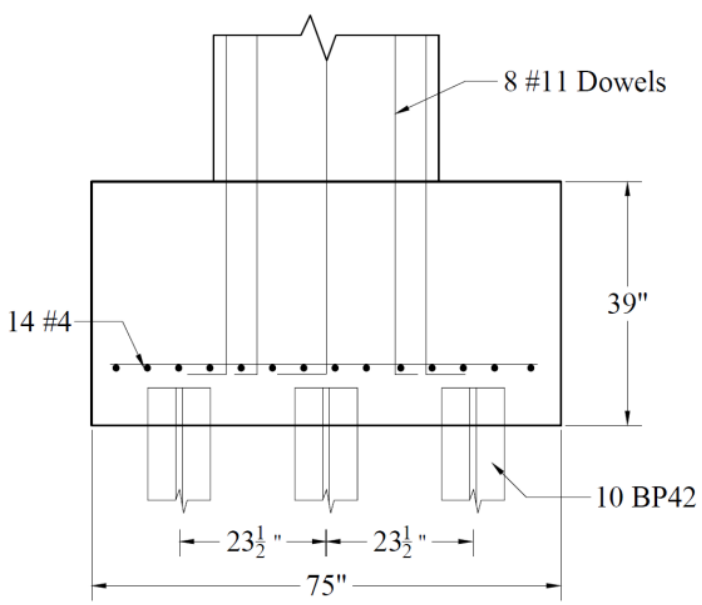

(b)

Figure 6.13 Typical foundations used in MPCG bridges (a) 3D schematic (b) Elevation

\subsubsection{Foundation Model}

Two models were utilized to represent the foundation behavior. The first model comprised the use of fixed foundations to represent foundations in competent soil or foundations that have been previously retrofitted. The second numerical model, also known as substructure method (Wolf, 1985), was created using linear and non-linear springs that takes into account the contribution of the pile group and the pile cap, and tries to realistically capture the foundation flexibility. Both fixed and spring models are depicted in Figure 6.14.

A linear spring was used to represent the vertical stiffness of the pile group. The vertical stiffness of each pile is difficult to estimate since a length of pile needs to be assumed. In this study, an axial stiffness of $1030 \mathrm{kip} /$ in was computed for a single pile following the recommendation of ODOT (2015). On the other hand, non-linear translational and rotational springs were used to represent the horizontal and rotational stiffness of the footing.

Translational stiffness was computed including the total lateral pile stiffness plus the stiffness provided by the passive soil stiffness acting on the face of the pile cap. Single pile 
stiffness equal to $34 \mathrm{kip} /$ in and $25 \mathrm{kip} /$ in were used for the strong and weak orientation of the pile as per ODOT (2015).

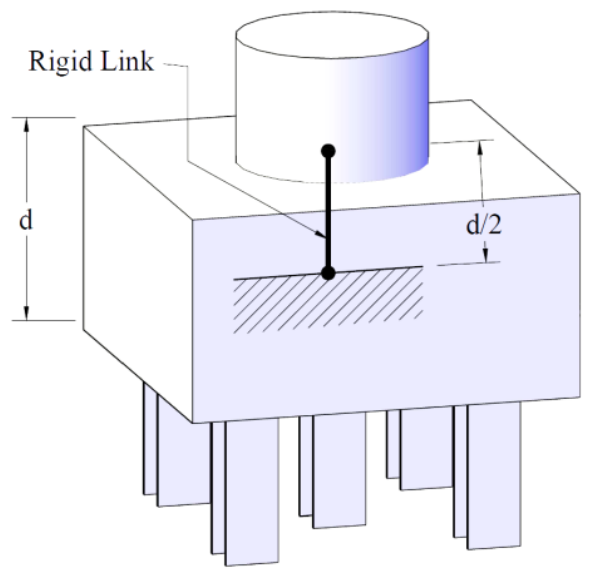

(a)

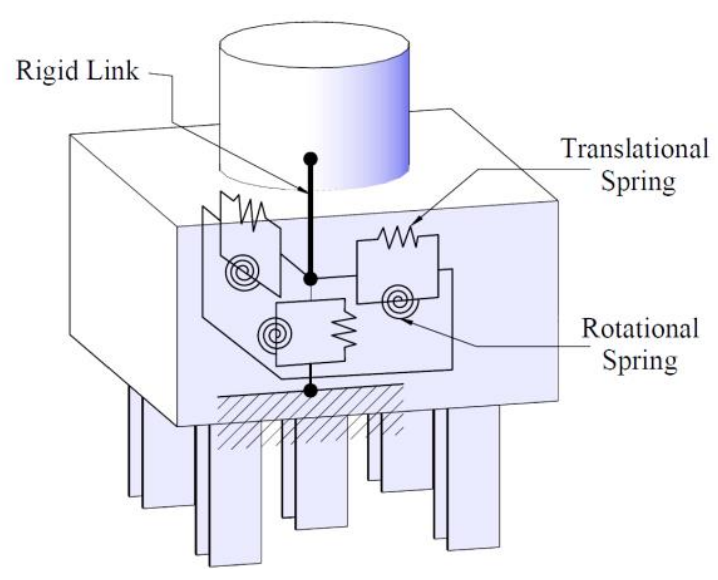

(b)

Figure 6.14 Numerical models for pile foundations used in this study.

(a) Fixed model, (b) Flexible model

The pile cap horizontal stiffness was calculated using Eq. (6.5), which is usually used for spread footings, with the exception that the stiffness contribution along the base of the pile cap was neglected.

$$
k_{\text {pile-cap }}=\alpha \beta \cdot \frac{8 G R}{2-v}
$$

Where, $a$ and $\beta$ are correction factor obtained from graphs (ODOT, 2015), $G$ is the shear modulus, $R$ is the equivalent footing radius, and $v$ is the Poisson's ratio. Values for $a$, $\beta$, $\mathrm{R}, \mathrm{G}$, and $v$ of $1.02,1.5-1.0,42.3 \mathrm{in}, 1100 \mathrm{ksf}$ (7.6 ksi), and 0.35 were used, respectively, which gives a pile cap stiffness of $795 \mathrm{kip} / \mathrm{in}$. Therefore, total horizontal stiffness of 965 kip/in and $920 \mathrm{kip} /$ in for longitudinal and transverse directions, respectively, were used per footing. The nominal translational capacity of the pile-supported cap footing was calculated using the nominal passive resistance of the pile cap, neglecting the resistance from the base, plus the resistance of the piles. Nominal pile translational capacities per pile of 29 kips and 
17 kips were used for strong and weak axis, respectively. Only passive resistance developed on the front face of the footing was considered and was computed as 35 kips. As a result, a total resistance equal to $120 \mathrm{kip} /$ footing was utilized.

Rotational stiffness was calculated using Eq. (6.6) (Priestley, et al., 1996).

$$
k_{\text {rot }}=\sum_{i=1}^{n} K_{a x i a l, i} \cdot x_{i}^{2}
$$

Where, $K_{a x i a l, i}$ is the axial stiffness of the $\mathrm{i}^{\text {th }}$ pile and $\mathrm{x}_{\mathrm{i}}$ is the distance from the centroid of the pile group to the $i^{\text {th }}$ pile. As a result, a rotational spring constant of $2.275 \times 10^{6}$ kip-in/rad for each footing and a moment capacity of 11,750 kip-in was used. The springs in the OpenSees model were incorporated using zero length elements.

\subsubsection{Elastomeric Bearing Pads}

\subsubsection{General Description}

Elastomeric bearing pads were commonly used in prestressed concreted bridges built pre-1970 in the State of Oregon. The typical composition of these elastomeric bearings comprises a rubber or neoprene pad and steel dowels that are embedded into the pier cap and into the underside of the prestressed concrete girder as shown in Figure 6.15. These type of bearings transfer the horizontal forces from the prestressed concrete girder to the cap beam by friction in the elastomeric pad and by a beam type action of the steel dowel, which provides a restraining mechanism for the lateral and vertical movement. Previous studies and reconnaissance efforts have shown that elastomeric bearings have performed relatively well during seismic events. However, cases of bearing failure known as "walk-out", which occurs when the bearing is not properly secured, have been reported by Imbsen \& Nutt(1981). 
Typical elastomeric bearing pad sizes found in MPCG bridges varies from 1'-10” to 2'-2" long, 6" to 8" wide and 3/4" to 1" thick as shown in Figure 6.16. The diameter of the steel dowel also varies from 1" to 1-1/2". In addition to that, the steel dowels are inserted into a hole that is $1 / 4$ " larger than the steel dowel.

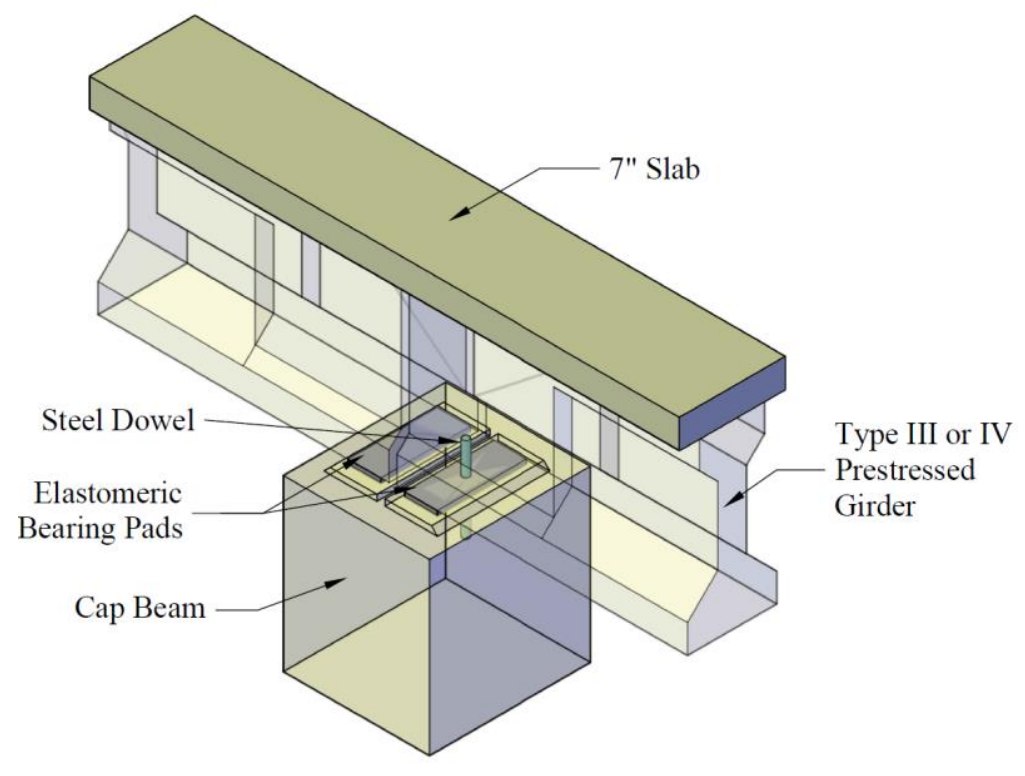

Figure 6.15 Elastomeric bearing pad configuration used in MPCG bridges

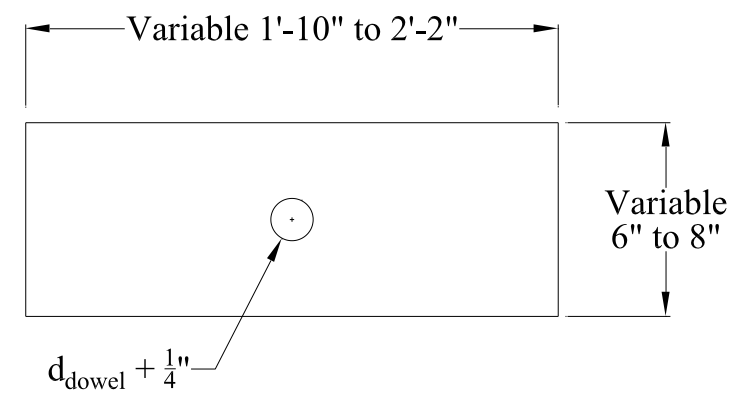

Figure 6.16 Typical elastomeric pad sizes

\subsubsection{Elastomeric Bearing Model}

Both the elastomeric pad and the steel dowel contributes to the behavior of the bearing under lateral loading. Therefore, each component needs to be characterized in order to model the response of the bearing. The elastomeric pads, as previously mentioned, 
transfer the loads by friction and their behavior is controlled by sliding. The sliding behavior is highly dependent on the initial stiffness and the coefficient of friction between the elastomeric pad and the surrounding concrete. Once this coefficient of friction is exceeded the stiffness of the bearing pad tends to zero (Schrage, 1981). The initial stiffness, $\mathrm{k}_{\mathrm{i}-\mathrm{pad}}$, is calculated using Eq. (6.7) (Choi, 2002)

$$
k_{i-p a d}=\frac{G A}{h}
$$

Where, $\mathrm{G}$ is the shear modulus, $\mathrm{A}$ is the area of the elastomeric bearing, and $\mathrm{h}$ is the thickness. Values of area and thickness can be easily obtained from drawings of typical bridges found in Oregon. However, the shear modulus, G, was assumed from previous studies. Following the recommendation of Choi (2002) and Nielson (2005), values for the shear modulus G varies from 96 psi to 300 psi and is highly dependent on the hardness of the elastomeric pad. This study is focused on bridges built prior 1970. Consequently, a high level of hardness is expected in the elastomeric pads. For modeling purposes, values of 250 psi, $168 \mathrm{in}^{2}$, and 0.75 in were assumed for the shear modulus, area, and thickness of the elastomeric pads, respectively.

The coefficient of friction depends on the normal stress on the bearing, $\sigma_{\mathrm{n}}$ (in psi), and based on experiments carried out by Schrage (1981) is calculated using Eq. (6.8). In this study, a normal stress, $\sigma_{\mathrm{n}}$, equal to $200 \mathrm{psi}$ was used. As a result, a coefficient of friction of 0.34 and an initial stiffness of $56 \mathrm{kip} /$ in were used.

$$
\mu=0.05+\frac{58.015}{\sigma_{n}}
$$

The maximum frictional force, $\mathrm{F}_{\mathrm{pad}}$, developed in the elastomeric pad is obtained by multiplying the coefficient of friction, $\mu$, between the elastomeric pad and the surrounding 
concrete, and the normal force, $\mathrm{N}$, applied on the bearing. As previously mentioned, once this force is exceeded the stiffness drops to zero, as a consequence, the behavior of the elastomeric pad can be characterized with an elastic perfectly-plastic response. In order to achieve this behavior, the elastomeric pad was modeled in OpenSees using the steel01 uniaxial material with an initial stiffness of $56 \mathrm{kip} /$ in, a strain hardening ratio equal to 0 , and a force at which the material reaches the plastic state given by $F_{\text {pad }}=\mu \mathrm{N}$ as shown in Figure 6.17.

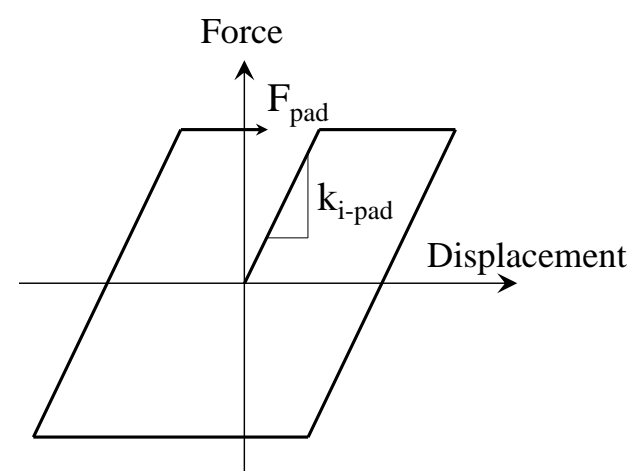

Figure 6.17 Hysteretic behavior of elastomeric pad

The steel dowels, which restrict the movement between the cap beam and the girders, vary in diameter from 1" to 1-1/2". An elastic behavior of the steel dowel is expected under normal load conditions. However, under seismic events, incursions in the nonlinear range are expected (Nielson, 2005). The estimated strength of the dowel was calculated using Eq. (6.9), which assumes that pure shear controls the capacity of the dowel.

$$
F_{\text {dowel }}=0.6 \cdot f_{s} \cdot A_{b}
$$

Where, $\mathrm{f}_{\mathrm{s}}$ is the stress in the steel and $\mathrm{A}_{\mathrm{b}}$ is the cross-sectional area of the dowel. Values of $36 \mathrm{ksi}, 60 \mathrm{ksi}$, and $1.77 \mathrm{in}^{2}$ were used as yield stress, ultimate stress, and area of the steel dowel, respectively. As a result, the estimated yield and ultimate strength was 38.2 kips and 63.7 kips, respectively. Values of $0.1 \mathrm{~d}_{\mathrm{b}}$ and $0.4 \mathrm{~d}_{\mathrm{b}}$ were used to estimate the yield and 
ultimate displacements based on the diameter of the steel dowel, $\mathrm{d}_{\mathrm{b}}$. In order to model the behavior of the steel dowel in OpenSees, the Hysteretic uniaxial material was utilized as depicted in Figure 6.18. A gap of 1/8" was included in the behavior to represent the influence of the oversized hole. The pinching parameters, pinchx and pinchy were set equal to 1.0 and 0.0 , respectively, in order to represent the desired response. Additionally, the parameters damage1, damage2 and beta were set equal to 0.

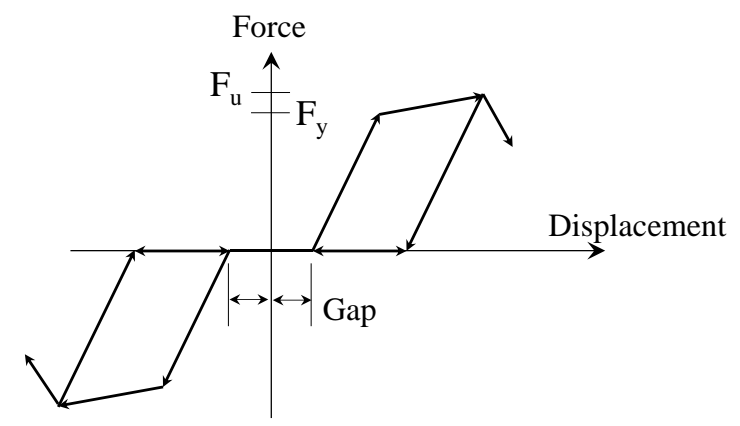

Figure 6.18 Hysteretic behavior of a steel dowel

The final implementation of the bearing response in OpenSees was accomplished by adding both the steel01 material and the hysteretic material with gaps into a parallel uniaxial material, which in the model was represented by a zero length element. The elastomeric bearing behavior is shown in Figure 6.19.

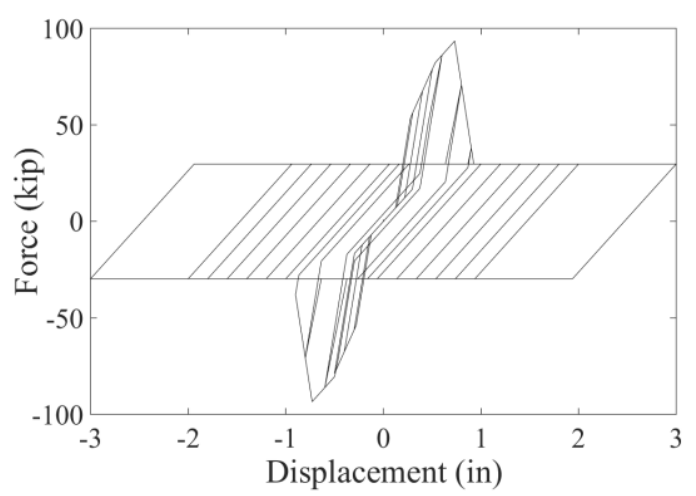

Figure 6.19 Hysteretic behavior of elastomeric bearings 
The implementation of the bearings in the overall bridge model was accomplished by using rigid links and zero length elements as shown in Figure 6.20. It is worth noting that at some supports the steel dowel was not utilized in order to reflect a free end. In those cases, the bearing behavior was only characterized with the elastic perfectly plastic response of the elastomeric pad by using the steel01 material as shown in Figure 6.17.

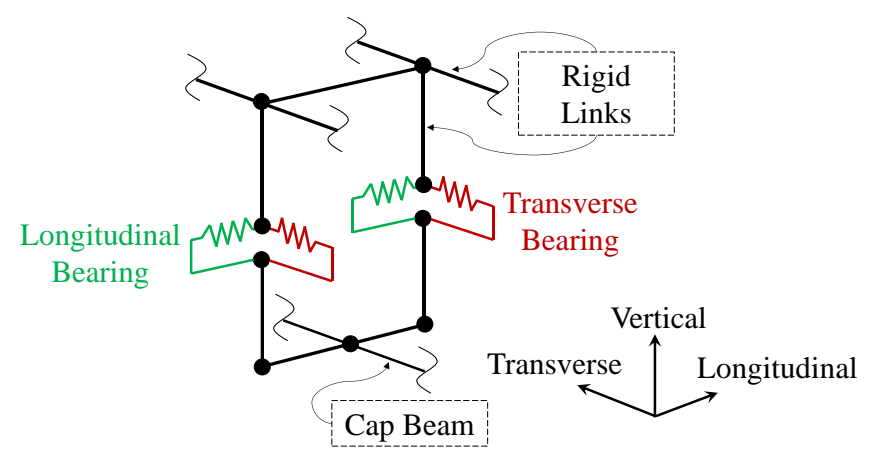

Figure 6.20 Numerical model for bearings

\subsubsection{Abutments}

\subsubsection{General Description}

Diaphragm abutments on steel piles as shown in Figure 6.21(a) were the focus of this study since they are commonly found in pre-1970 Oregon bridges. These abutments provide vertical support to the bridge superstructure and connect the bridge road with the roadway approaches. This type of abutment is typically cast monolithically with the deck/slab creating an integral or semi-integral abutment capable of engaging the soil passive resistance when the diaphragm is pressed into the backfill soil, which in turns provides passive longitudinal resistance. In addition to this passive resistance, these type of abutments also rely in the response provided by piles driven deep into the soil. Pile resistance contribution is particularly important when the abutment is pulled away from the backfill. In the transverse 
direction, the passive pressure of the backfill soil is usually low enough to neglect it depending on the existence of a large wing wall (Priestley, et al., 1996).

Figure 6.21(b) shows typical dimensions and configuration of a semi-integral diaphragm abutment found in MPCG bridges. A moment release is placed in the connection between the diaphragm and the pile cap in order to alleviate the thermal and live load demands transferred from the system to the pile cap. This connection is possible by inserting an expanded polystyrene in the interface between superstructure and pile cap, and a bearing support. Steel H-pile 10BP42 were found to be representative of the piles used in Oregon.

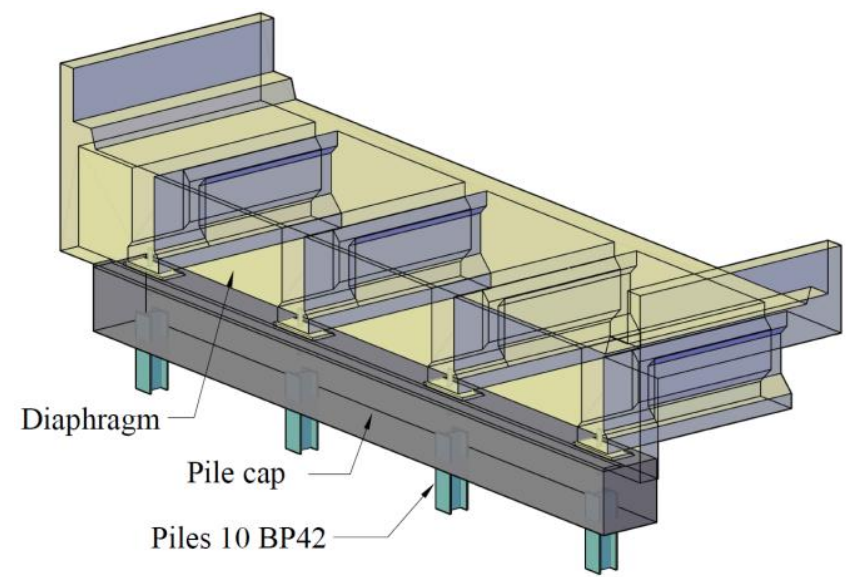

(a)

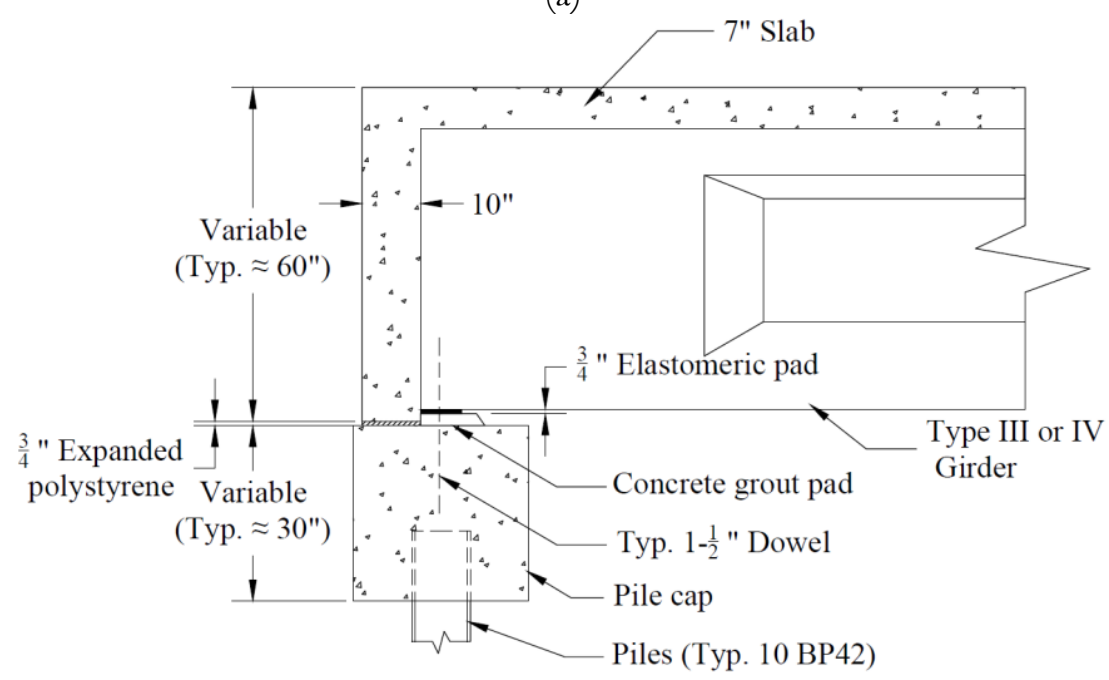

(b)

Figure 6.21 Typical abutments used in MPCG bridges (a) 3D schematic (b) Elevation 


\subsubsection{Abutment Model}

Abutments are an important component of highway bridges and can significantly affect the response of the system by providing longitudinal and transverse stiffness. Therefore, modeling the contribution of abutments is of vital importance to assess the overall bridge seismic response. Previous studies have found that abutment behavior, soilstructure interaction and embankment flexibility significantly influence the response of short and medium span bridges with a relatively stiff superstructure (Wilson \& Tan, 1990), (Goel \& Chopra, 1997), (Aviram, et al., 2008); (Shamsabadi, et al., 2007); (Shamsabadi, et al., 2010). Moreover, Kotsoglou \& Pantazopoulou (2009) determined that embankment flexibility and soil mobilization of the embankment under large seismic demands can considerably increase the displacement demands in bridge bents of RC bridges with integral abutments supported on flexible pile foundations. Bridge-embankment interaction is a problem of considerable complexity and focus of many ongoing research studies. In this study, a spring model is used following the recommendation of Aviram et al. (2008). The spring model used in this study was based on the model developed by Mackie and Stojadinovic (2006) with the modeling characterization and parameters suggested by Choi (2002) and Shamsabadi et al. (2010) for pile response and backfill soil response, respectively. In this model, the longitudinal response of the abutment considers the contribution of elastomeric bearing pads, backfill passive pressure and abutment piles. In the transverse direction, the response is based on the behavior of elastomeric bearing pads and abutment piles. The contribution of wingwalls in the transverse response was neglected since Caltrans (2013) pointed out that the effect of wingwalls decreases as the width of the abutment increases. The response of each 
component is captured by using nonlinear springs, which are described in subsequent paragraphs.

The piles, as stated previously, were modeled using the trilinear representation proposed by Choi (2002). The original model assumed that piles become plastic at a deformation of 1 in and that first yielding occurs at 0.3 in. However, that assumption generated a contradiction between the effective stiffness and the ultimate strength. In order to overcome that problem, the deformation at which the piles become plastic was computed dividing the ultimate strength of the pile by the pile effective stiffness. The pile effective stiffness and strength were the same as the ones used in the foundation model. The trilinear model is depicted in Figure 6.22. The Hysteretic material assigned to a zero length element was utilized to model the pile response in OpenSees.

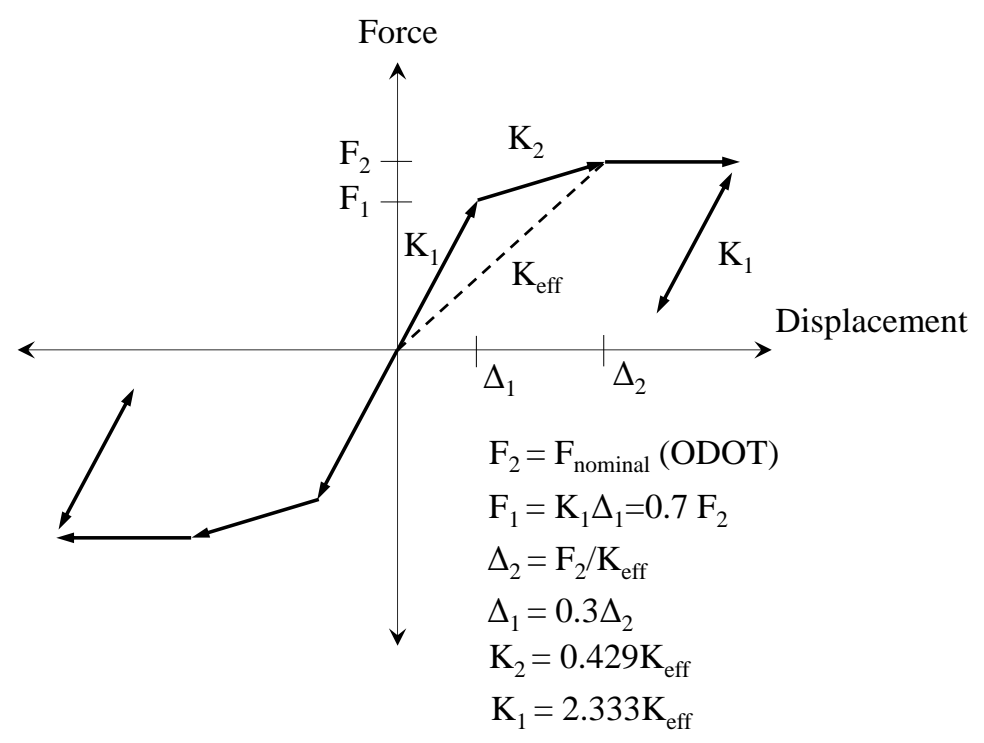

Figure 6.22 Tri-linear response for piles (Choi, 2002)

In order to capture the backwall-soil interaction, the hyperbolic soil model proposed by Shamsabadi et al. (2007) and (2010) was used. This model was calibrated with full-scale tests performed at the University of California, Los Angeles (UCLA), for seat type 
abutments with granular backfill, and at the University of California, Davis (UCD), for integral abutments with cohesive backfill. Both tests comprised abutments 66" tall, which is similar to the backwall height found in typical MPCG bridges as shown in Figure 6.21.

Figure 6.23 shows the abutment force-displacement backbone, where $\mathrm{K}$ is the average soil stiffness, $F_{\text {ult }}$ is the maximum abutment force, $\mathrm{y}_{\max }$ is the maximum displacement, and $\mathrm{y}_{\text {ave }}$ is the displacement corresponding to half the maximum force. The hyperbolic response can be obtained using Eq. (6.10) and (6.11) for abutments 66 in tall and different backfill soils. The ultimate force, $\mathrm{F}_{\text {ult, }}$ was computed assuming that the maximum displacement of the backwall is 0.05 the abutment height $(\mathrm{H})$ for granular backfills and $0.1 \mathrm{H}$ for cohesive backfills. Using those values for $y_{\max }$ and Eq. (6.10) and (6.11) for granular soil and cohesive soil, respectively, the ultimate force was computed as 30 kips per foot of wall for both backfill soils. The average soil stiffness, $\mathrm{K}$, can be assumed as $50 \mathrm{kip} / \mathrm{in} / \mathrm{ft}$ and 25 kip/in/ft for granular and cohesive backfills, respectively (Shamsabadi, et al., 2007).

$$
\begin{aligned}
& F(y)=\frac{90.84 \cdot y}{1+2.7 \cdot y} \quad(\text { Granular } \quad \text { soil }) \\
& F(y)=\frac{45.42 \cdot y}{1+1.35 \cdot y} \quad \text { (Cohesive soil) }
\end{aligned}
$$

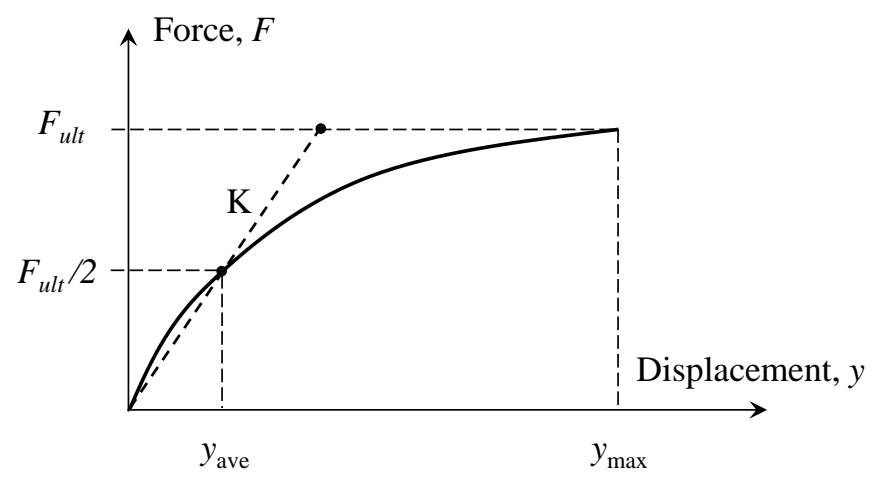

Figure 6.23 Hyperbolic response for abutment backwall (Shamsabadi, et al., 2007) 
The Hyperbolic Gap Material assigned to a zero length element was utilized in OpenSees to capture the abutment response. Cohesive backfill was assumed in this study. Values of $25 \mathrm{kip} / \mathrm{in} / \mathrm{ft}$ and $30 \mathrm{kips}$ per foot of abutment width were utilized to represent the average stiffness and the ultimate (maximum) passive resistance, respectively. The gap assumed in the model was set equal to zero in order to reflect the diaphragm abutment. Figure 6.24 shows the numerical model used in OpenSees. A $5 \mathrm{kip}-\mathrm{s}^{2} /$ in concentrated mass at the abutments was used in order to capture the additional inertial force that is generated at the abutment-embankment interaction during an earthquake (Mackie \& Stojadinovic, 2006).

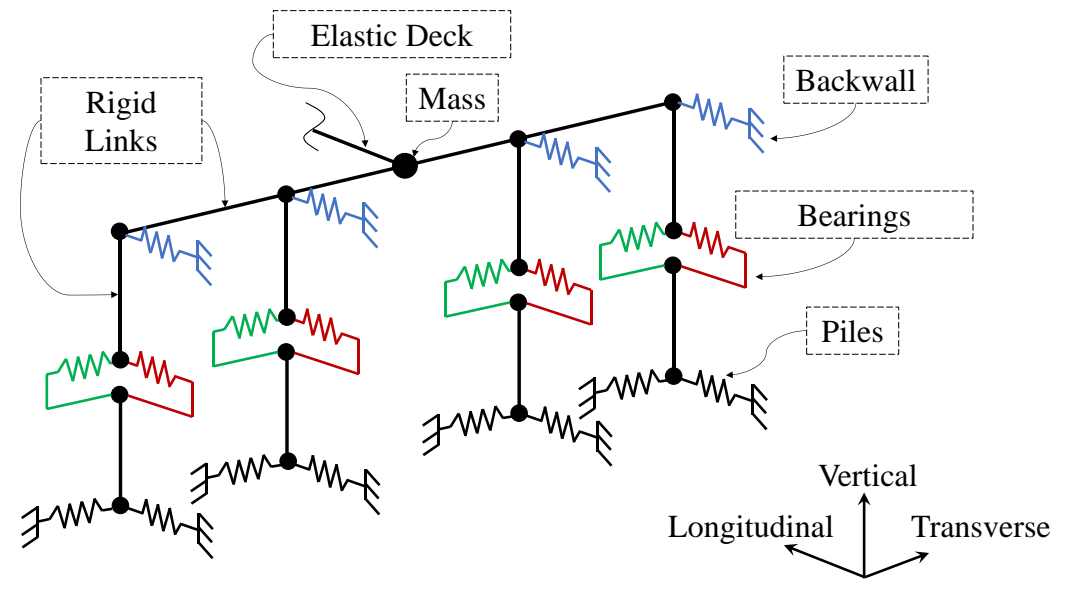

Figure 6.24 Numerical model for abutments

\subsubsection{Nonlinear Time History Analysis (NLTHA)}

In order to study the nonlinear response of the representative MPCG bridge, nonlinear time history analyses of a three dimensional bridge model were performed in OpenSees (2013) using the aforementioned component modeling for superstructure, bearings, RC bent, footings and abutments. The ground motions used in the NLTHA were selected from the Maule, Chile M8.8 earthquake in an effort to simulate a potential Cascadia Subduction scenario and the 1000-year event described in Chapter 4. With that aim, ground motions recorded at the Curico station were used in the transverse and longitudinal direction 
of the bridge as shown in Figure 6.25(a) and (b), respectively. The 5\% damped response spectrum of the ground motions is depicted in Figure 6.25(c) and is compared to the 1000year event.

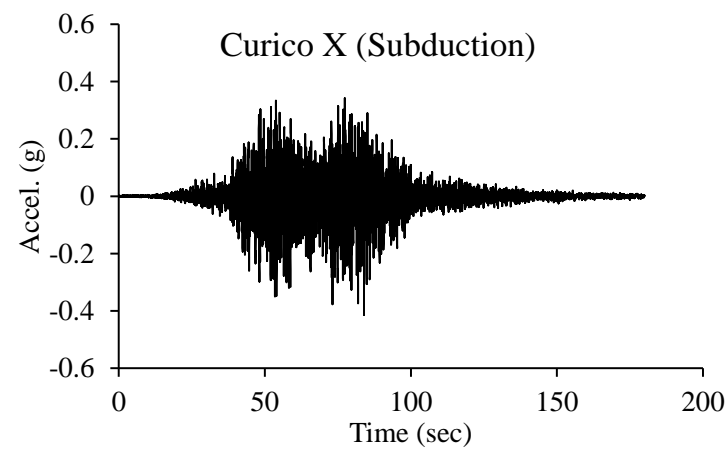

(a)

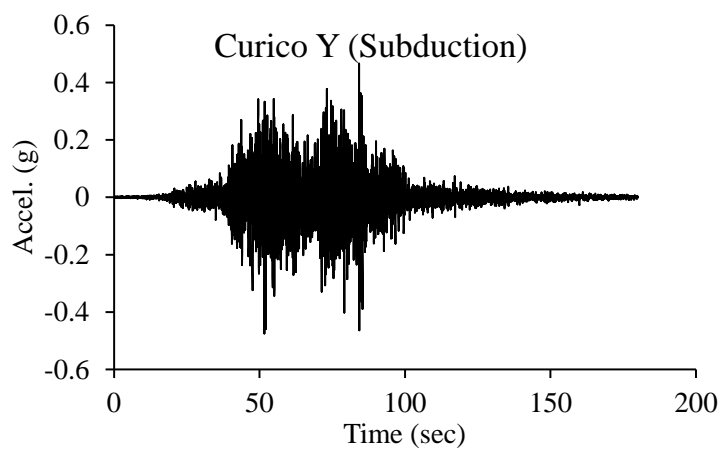

(b)

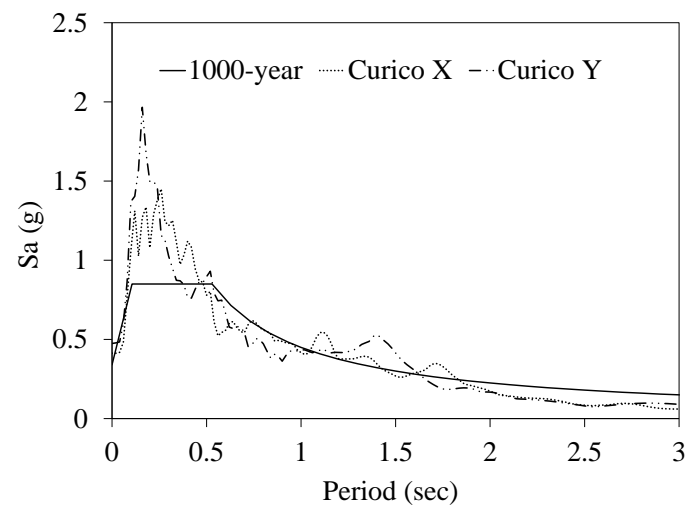

(c)

Figure 6.25 Ground motions used in NLTHA of 3D bridge models.

(a) Transverse direction, (b) Longitudinal direction, (c) Response Spectrum

Results obtained of performing nonlinear time history analysis in the representative MPCG bridge model with fixed supports and no additional mass from the embankments are shown in Figure 6.26(a) and (b) for bents in the transverse and longitudinal direction, respectively. The response of the abutments that is shown in Figure 6.26(c) and (d) corresponds to the response of the backfill and a single pile, respectively. The results indicate that the bridge has displacement demands of 2.9 inches in the transverse direction and 1.8 inches in the longitudinal direction. 
Figure 6.26(c) shows that the abutment response in the transverse direction is controlled by the hysteretic response of the piles. Significant inelastic excursions are expected in the piles. In the longitudinal direction, the backfill, as expected, acts when it is compressed and limits the maximum displacement in the longitudinal direction.

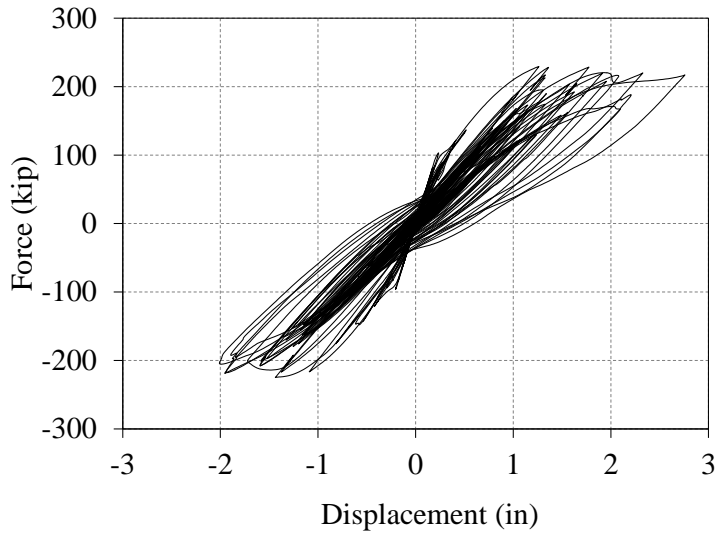

(a)

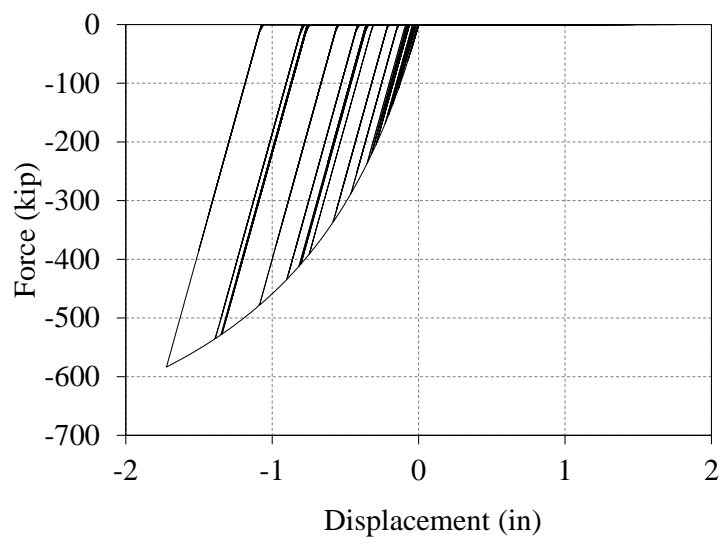

(c)

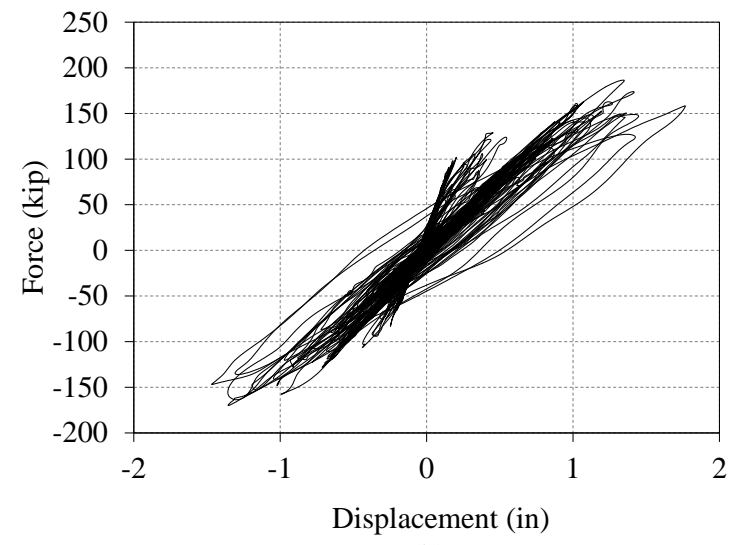

(b)

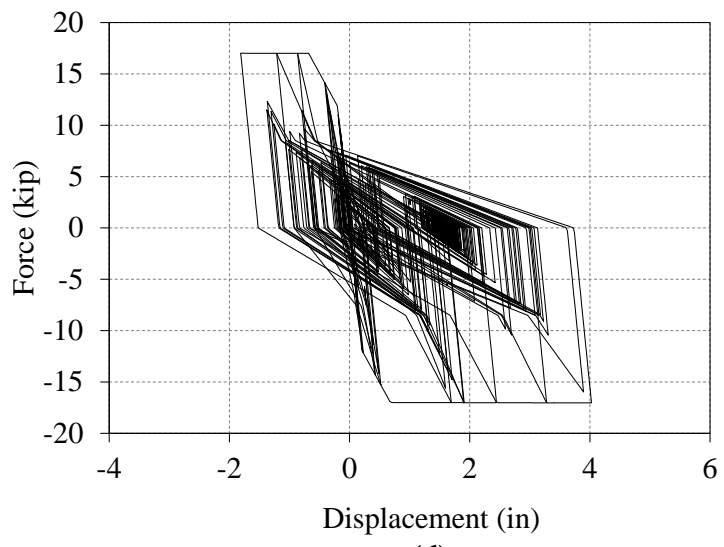

(d)

Figure 6.26 Results of NLTHA for MCCG bridge with fixed supports.

(a) Bent-Transverse, (b) Bent-Longitudinal, (c) Abutment-Backfill, (d) Abutment-Pile

Results of considering an additional concentrated mass of $5 \mathrm{kip}-\mathrm{s}^{2} /$ in at the

abutments (Mackie \& Stojadinovic, 2006) are depicted in Figure 6.27. In this case, the bridge has displacement demands of 3.3 in and 2.5 in in the transverse and longitudinal direction, respectively. In the transverse direction, strength and stiffness degradation was observed at a displacement ductility demand of 2.8. This result implies that adding the concentrated mass 
at the abutments increases the displacement demands in both directions. In particular, the displacement demand in the RC bents increased $14 \%$ and $39 \%$ with respect to the case without additional mass in the transverse and longitudinal direction, respectively.

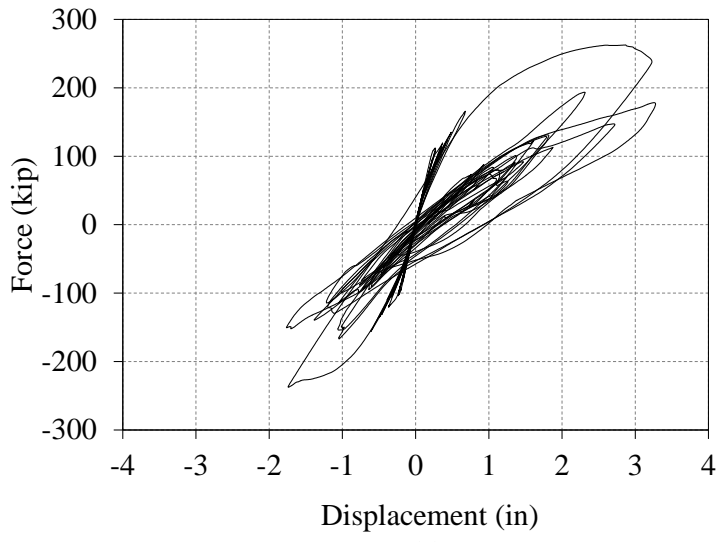

(a)

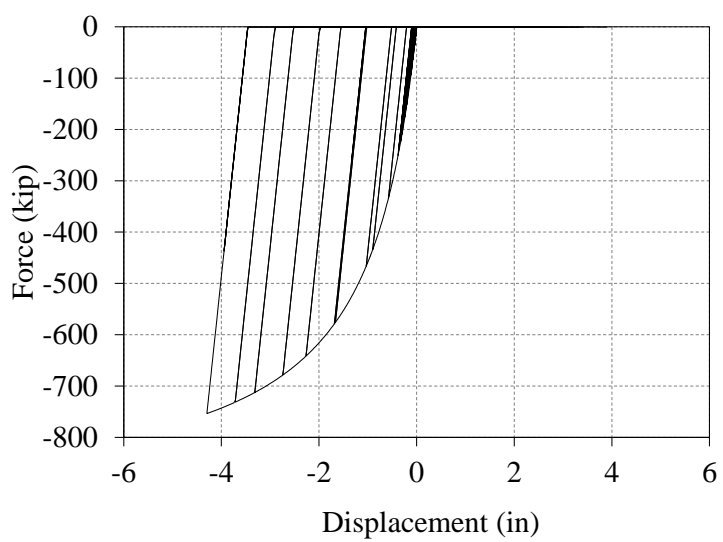

(c)

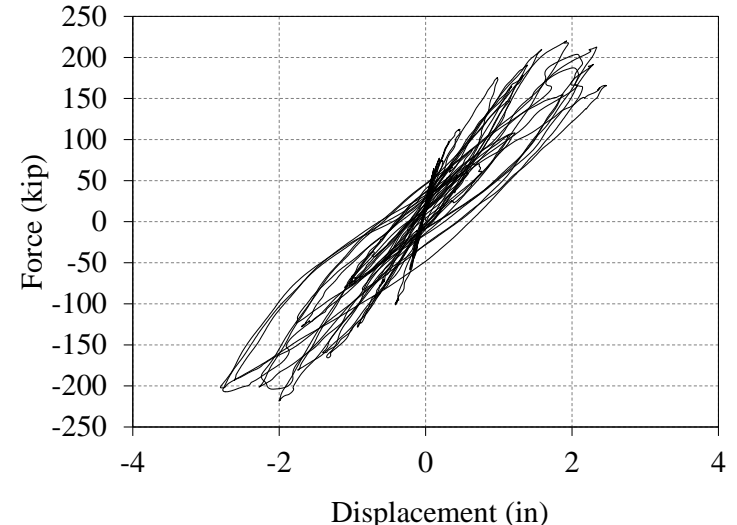

(b)

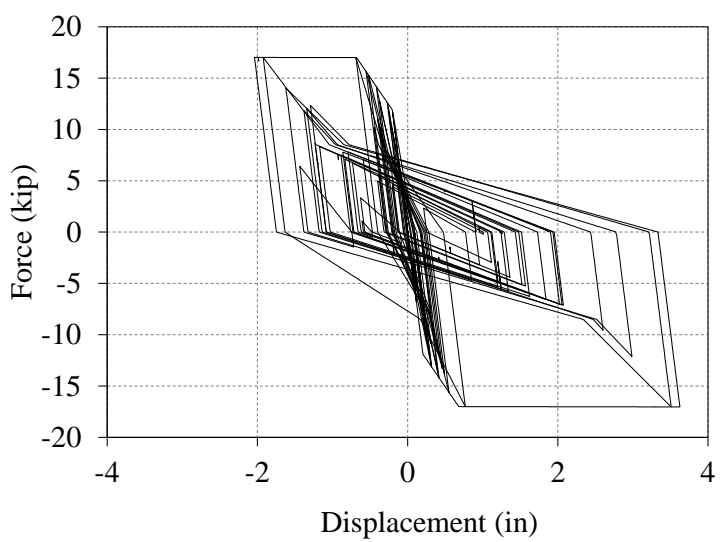

(d)

Figure 6.27 Results of NLTHA for MCCG bridge with additional mass at abutments.

(a) Bent-Transverse, (b) Bent-Longitudinal, (c) Abutment-Transverse, (d) Abutment-Longitudinal

Results considering foundation flexibility (spring-model) are depicted in Figure 6.28.

In this case, displacement demands of 4.0 inches in the transverse direction and 1.7 inches in the longitudinal direction were recorded. These results indicate an increase on displacement demand of $48 \%$ in the transverse direction and a reduction of $6 \%$ in the longitudinal direction with respect to the fixed condition. 
Thus, retrofitting this RC bent with BRBs may reduce the displacement demands in the transverse direction on both RC bents and abutments.

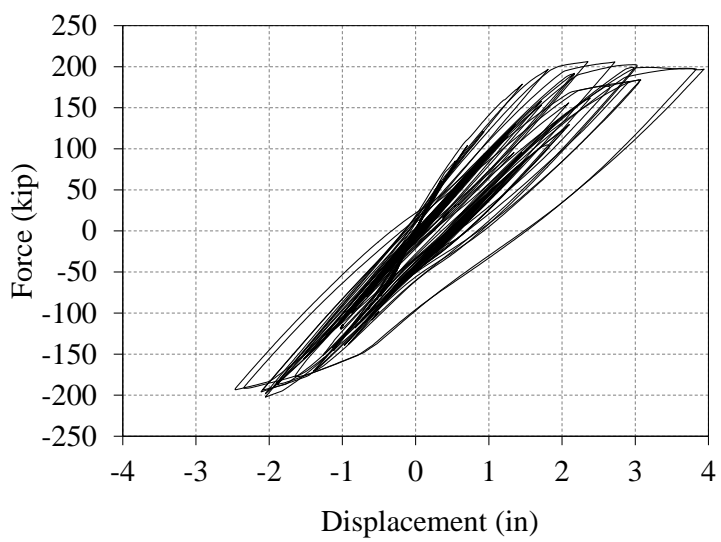

(a)

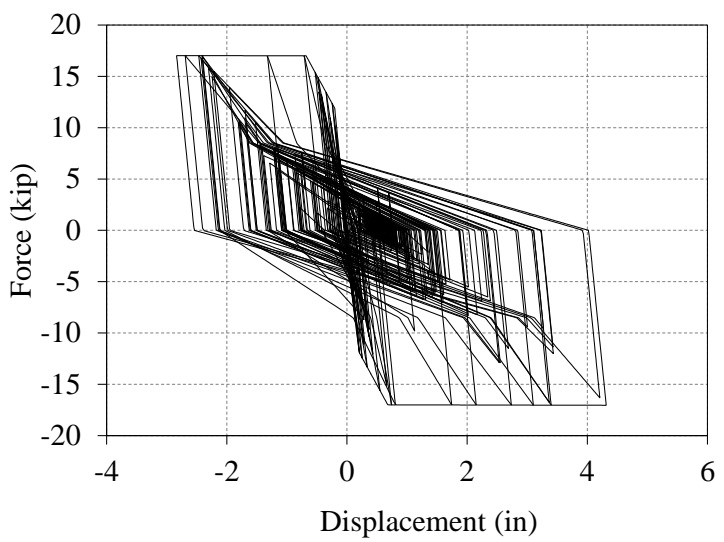

(c)

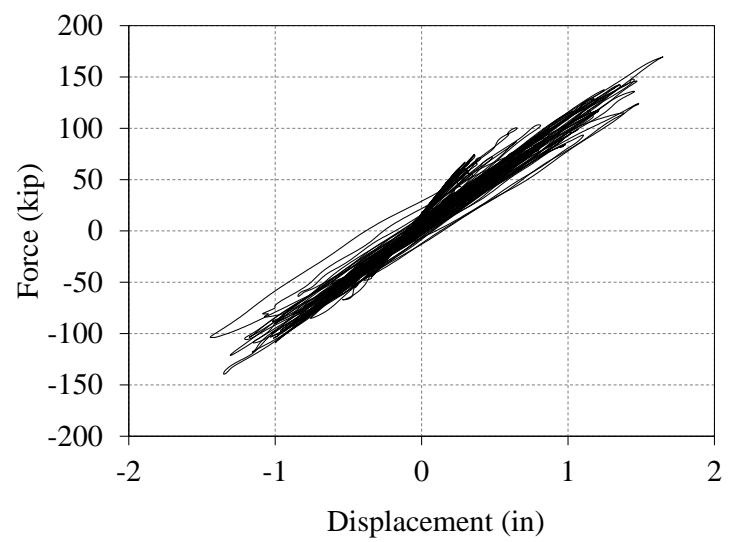

(b)

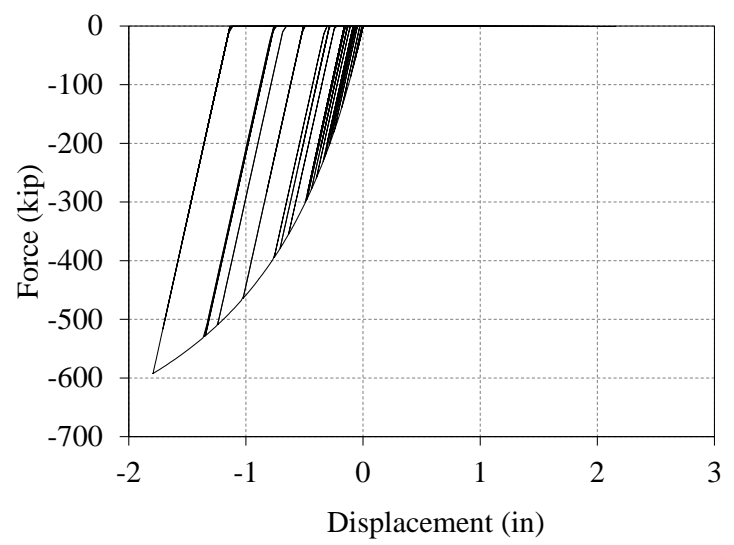

(d)

Figure 6.28 Results of NLTHA for MCCG bridge with spring foundations.

(a) Bent-Transverse, (b) Bent-Longitudinal, (c) Abutment-Transverse, (d) Abutment-Longitudinal

\subsection{Modeling of Multi-Column Concrete Continuous Stringer/Girder \\ BRIDGE}

This section presents details for modeling the components of a typical multi-column concrete continuous stringer/girder (MCCG) bridge based on experimental results presented in previous chapters. 


\subsubsection{Overview of As-built Numerical Model}

The numerical model for the MCCG bridge is shown in Figure 6.29. In this model, specific modeling approaches, which are described in following sections, were utilized to characterize the response of different bridge components such as abutments and foundations, as shown in Figure 6.29(a). The dimensions for the bridge are depicted in Figure 6.29(b). These dimensions were utilized to represent typical dimensions observed in MCCG bridges of Oregon.

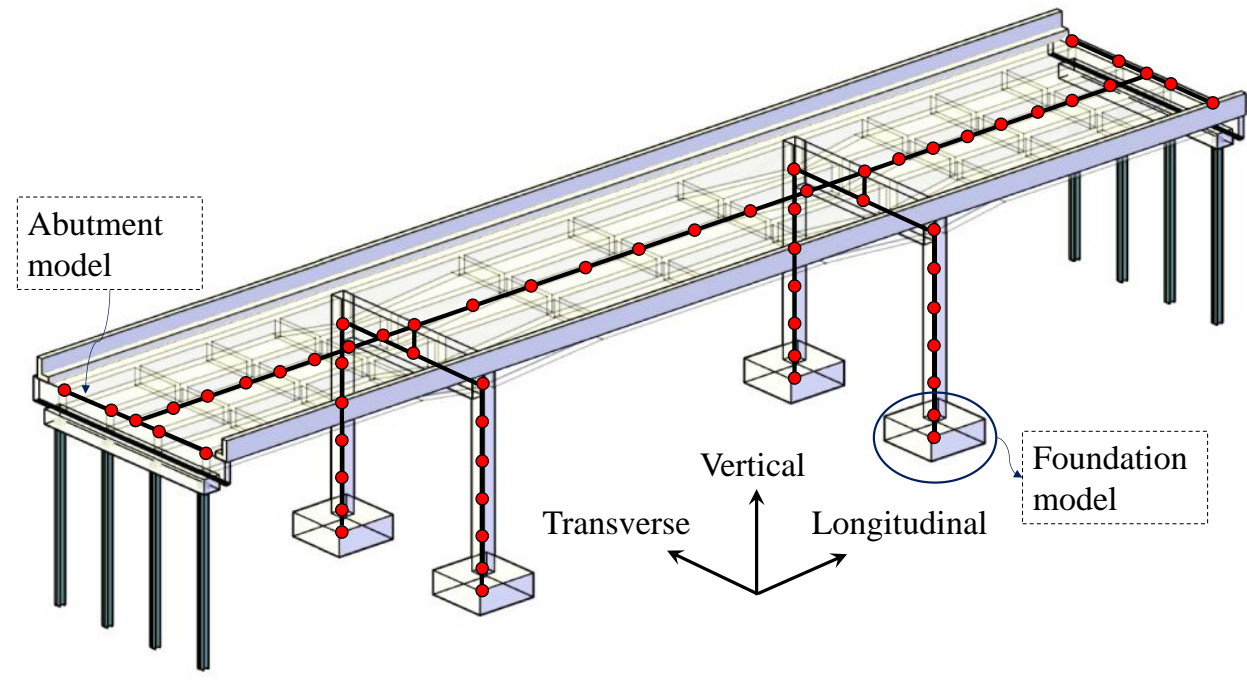

(a)

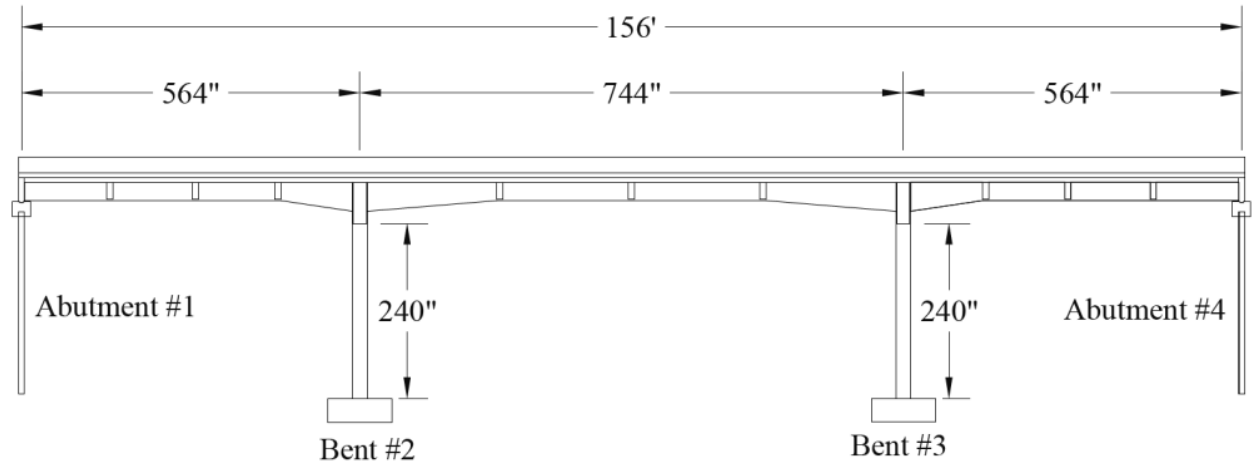

(b)

Figure 6.29 General layout of a MCCG bridge (a) 3D Model (b) Elevation 


\subsubsection{Superstructure}

\subsubsection{General Description}

The superstructure of MCCG bridges consists of continuous girders/beams, a slab/deck, a wearing surface, parapets, and diaphragms or transverse beams as depicted in Figure 6.30. The dimensions of these elements vary from bridge-to-bridge. The slab thickness usually ranges from 5" to 8". In this study, a slab thickness of 7" was assumed. Continuous girders with a cross section of 16.5 'x 30 ” were assumed. Additional transverse beams usually referred as diaphragms are located at the end and middle of the spans in order to increase the stiffness of the superstructure and avoid excessive deflections caused by the live loads.

\subsubsection{Superstructure Model}

The superstructure was characterized using a simple spine model since modeling the superstructure with three-dimensional finite elements (solids or plates) is not typically necessary for the seismic analysis of a medium span bridge with a rigid superstructure. In the spine model the members of the superstructure are assumed to create a composite action and are consolidated into one element, hereinafter referred to as deck. The location of the deck in the model was calculated considering all the components of the superstructure in order to simulate the center of mass (C.M.) of the system. Translational and rotational mass were assigned to each node based on tributary volume associated with the node.

Under seismic loadings the deck is assumed to behave essentially elastic. Therefore, the deck was modeled in OpenSees (2013) using the Elastic Beam Column element. In order to represent the stiffness and mass distribution of the superstructure, the deck was defined using the values given in Table 6.5. 


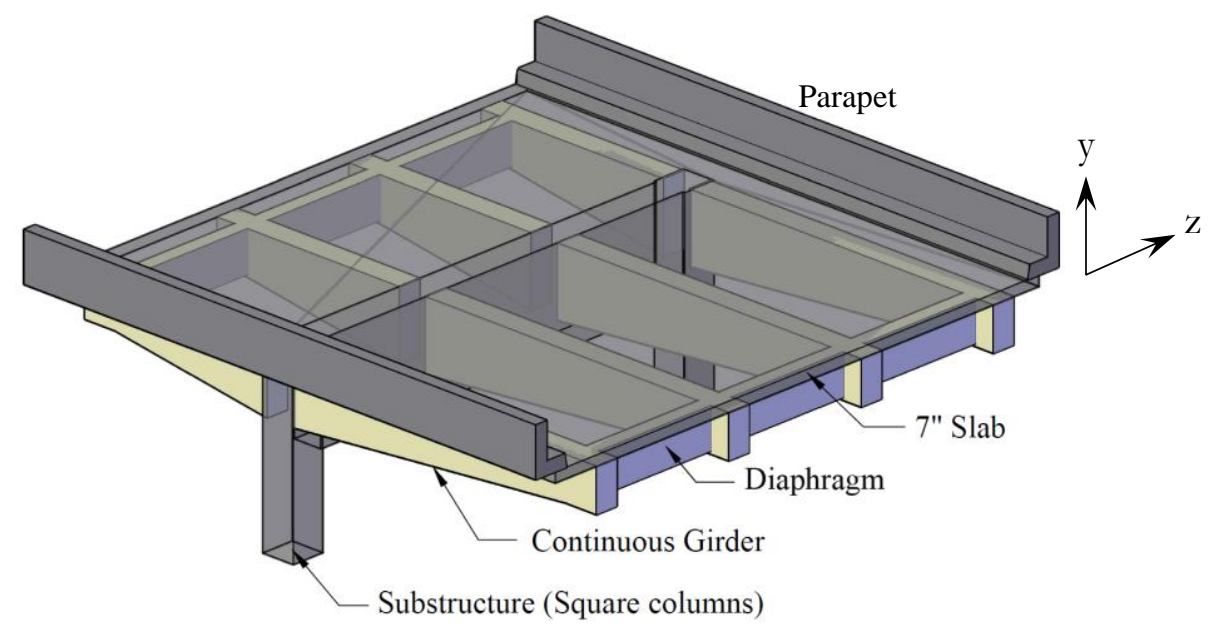

Figure 6.30 General layout (a) 3D Model (b) Elevation

Table 6.5 Superstructure modeling parameters for MCCG Bridge

\begin{tabular}{l|c}
\hline \multicolumn{1}{c|}{ Description } & Value \\
\hline Cross-sectional area & $5256 \mathrm{in}^{2}$ \\
\hline Modulus of elasticity $(\mathrm{E})$ & $3274 \mathrm{ksi}$ \\
\hline Shear modulus $(\mathrm{G})$ & $1364 \mathrm{ksi}$ \\
\hline Second moment of area about the local $\mathrm{z}$ axis $\left(\mathrm{I}_{\mathrm{z}}\right)$ & $6.897 \times 10^{5} \mathrm{in}^{4}$ \\
\hline Second moment of area about the local y axis $\left(\mathrm{I}_{\mathrm{y}}\right)$ & $8.506 \times 10^{7} \mathrm{in}^{4}$ \\
\hline Torsional moment of inertia $(\mathrm{J})$ & $2.224 \times 10^{5} \mathrm{in}^{4}$ \\
\hline $\begin{array}{l}\text { Weight of superstructure }+ \text { overlay and barriers } \\
\text { (kip/in) }\end{array}$ & 0.74 \\
\hline Centroid from bottom of girder & $65 \mathrm{in}$ \\
\hline
\end{tabular}

\subsubsection{Bent}

\subsubsection{General Description}

The representative MCCG Bridge bent consists of two square columns per bent and a rectangular cap beam. Specified materials comprise $3.3 \mathrm{ksi}$ and $40 \mathrm{ksi}$ for the compressive strength of concrete and yield stress of steel, respectively. The column longitudinal reinforcing steel consists of 4 \#10 rebars. Typically, \#3 or \#4 hoops at 12 inches on center were provided in columns regardless of the column cross-sectional dimensions. As a 
consequence, the provided column shear reinforcement and confinement does not meet current code requirements. The stirrups were anchored by $90^{\circ}$ hooks with short extensions and intermediate ties were seldom used. Minimal restraint provided by the hoops can cause the longitudinal reinforcement to buckle once the concrete cover spalls. Furthermore, bridges built prior to 1970 have undesirable lap splices at the base of the columns. The lap splice detailing combined with the poor transverse reinforcing may cause reduced column strength and ductility, and result in rapid deterioration of flexural strength. Typical details for the representative multi-span continuous stringer/girder $\mathrm{RC}$ bridge bent are illustrated in Figure 4.20.

\subsubsection{Bent Model}

The model to characterize the response of the representative MCCG bent was based on the distributed-plasticity model presented in Chapter 2, which was calibrated with experimental tests of full-scale RC square columns. The numerical model for the MCCG bridge columns consisted of force-based fiber beam-column elements, rigid links and zero length sections as shown in Figure 6.31. The discretization of the column fibers comprised the use of 24 cover subdivisions in the local-y direction, 24 cover subdivisions in the local-z direction, and 20 core subdivisions. On the other hand, an elastic beam element was used to represent the behavior of the bent cap since in MCCG bridges the cap beam acts monolithically with the superstructure where yielding is not expected. Material properties for of $4.95 \mathrm{ksi}$ and $48 \mathrm{ksi}$ were used to represent the compressive strength of concrete and the yield stress of steel, respectively. 


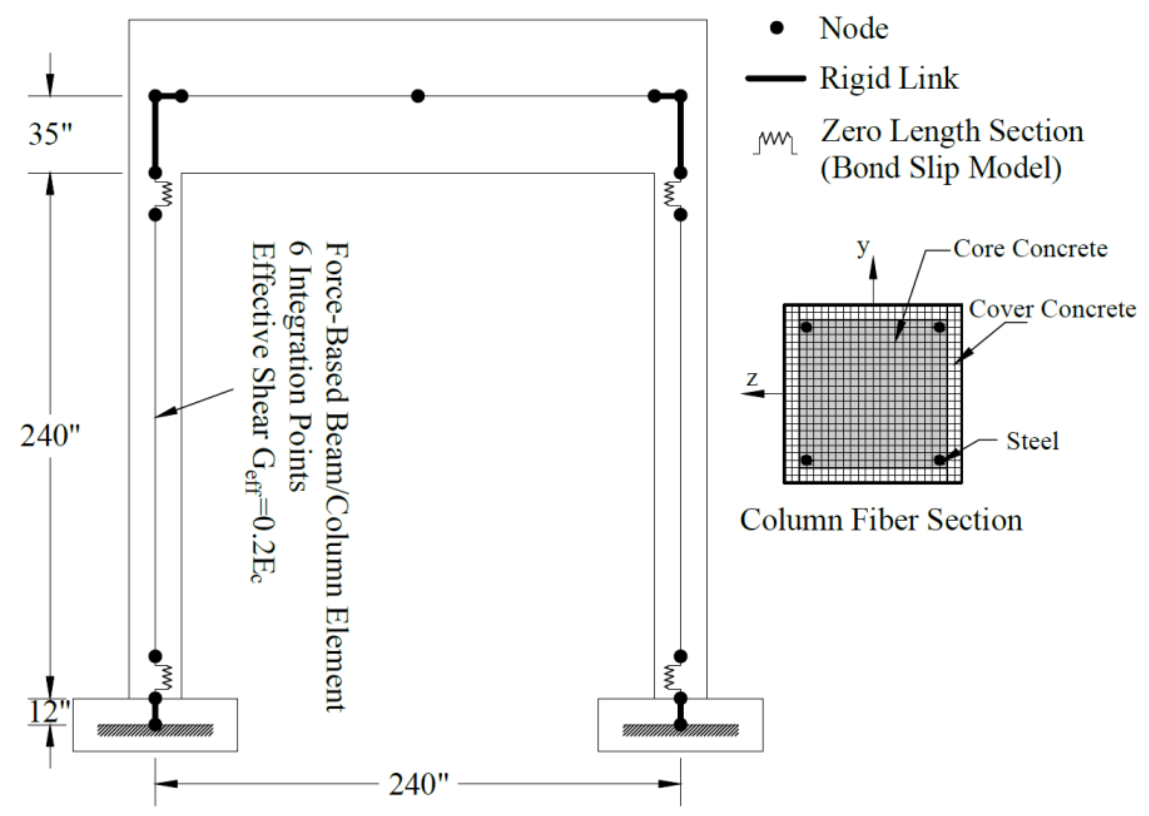

Figure 6.31 Numerical model for MCCG bridge bent

The expected hysteretic behavior of the bent under the subduction loading protocol described in Chapter 2 is shown in Figure 6.32. A comparison with a pushover performed in SAP2000 shows a good agreement in the initial stiffness of the bent. However, there is a difference in the maximum strength captured for each model. This difference is caused by the assumptions made in first place. The OpenSees model was based on experimental tests of full-scale square columns, which showed that the subduction protocol causes an early bond degradation at the base of the columns that in turns inhibits the full development of the force transferred through the lap splice. On the other hand, the SAP2000 model was performed according conventional static non-linear analysis. 


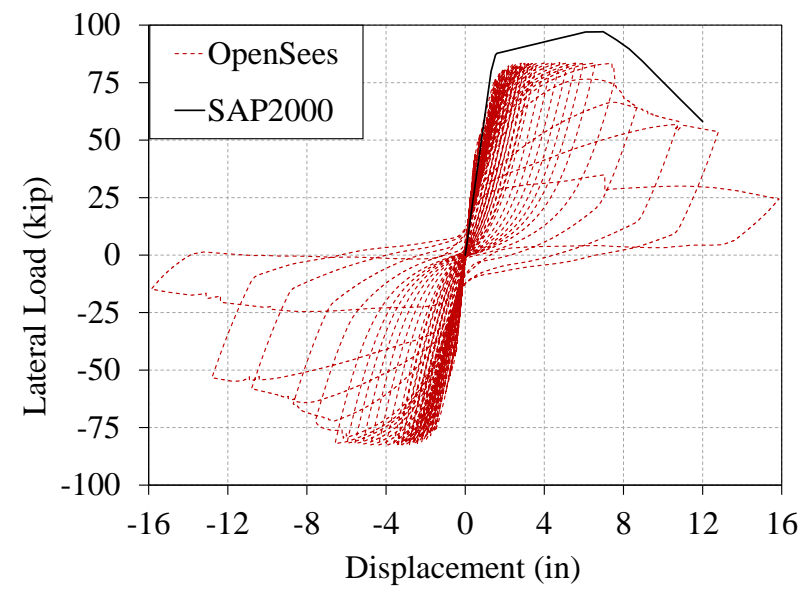

Figure 6.32 Expected force-displacement behavior

\subsubsection{Foundation}

\subsubsection{General Description}

MCCG bridges built in Oregon prior to 1970 present spread foundations or pile foundations depending on site conditions. In this study, square spread footings as depicted in Figure 6.33(a) were utilized to represent the dimensions and properties of typical foundations found in three span multi-column continuous concrete girder bridges. These footings consist of 90 in square footing with a depth of 24 in as shown in Figure 6.33(b).

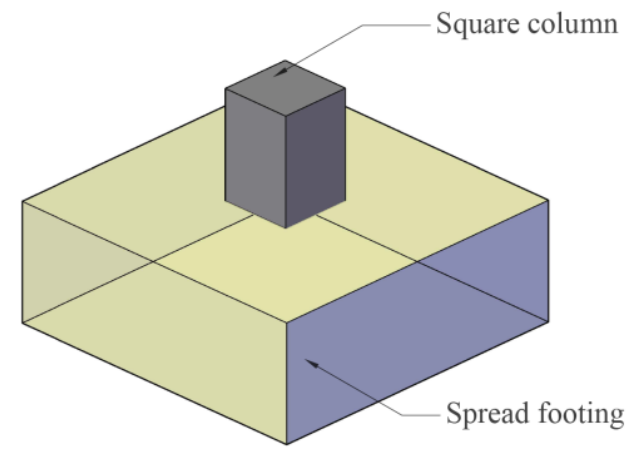

(a)

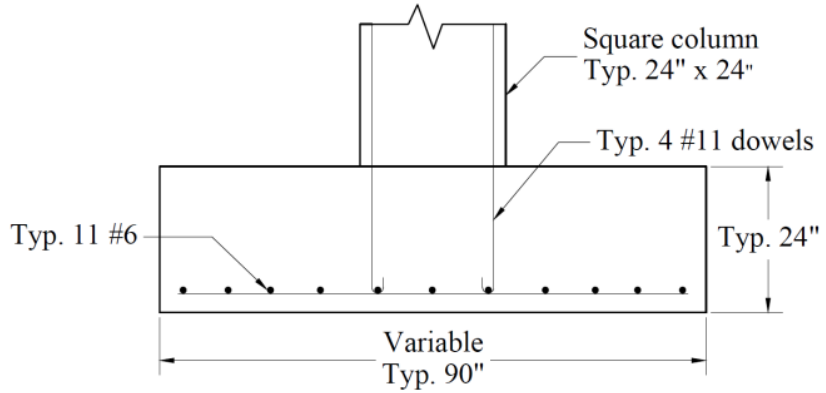

(b)

Figure 6.33 Typical foundations used in MCCG bridges (a) 3D schematic (b) Elevation 


\subsubsection{Foundation Model}

The MCCG foundation model considered one model with fixed foundations and one model with translational and rotational springs similarly to the model for the MPCG pile footing (Figure 6.14). The first model tries to represent footings built into a rock mass with a modulus of elasticity greater than $14,000 \mathrm{ksf}$. The translational and rotational stiffness of the second model were computed using Eq. (6.12) to (6.15) and typical values recommended by ODOT (2015) for dense granular fill. Result of using these equations are indicated in Table 6.6.

$$
\begin{aligned}
& k_{\text {vertical }}=\alpha \beta \cdot \frac{4 G R}{1-v} \\
& k_{\text {horizontal }}=\alpha \beta \cdot \frac{8 G R}{2-v} \\
& k_{\text {torsional }}=\alpha \beta \cdot \frac{16 G R^{3}}{3} \\
& k_{\text {rotational }}=\alpha \beta \cdot \frac{8 G R^{3}}{3 \cdot(1-v)}
\end{aligned}
$$

Where, $\alpha$ and $\beta$ are correction factors obtained from graphs, $G$ is the shear modulus, and $v$ is the Poisson's ratio. Values for $\alpha, \beta, G$, and $v$ of 1.02, 1.6, $1100 \mathrm{ksf}$ (7.6 ksi), and 0.35 were used, respectively. The value of $\mathrm{R}$, which is the equivalent footing radius, varies depending on the considered direction. For a 90 in square footing, the equivalent radius was computed using Eq. (6.16) and (6.17). Where, $\mathrm{L}$ is half the footing width.

$$
\begin{aligned}
& R_{\text {translatinal }}=\sqrt{\frac{4 L^{2}}{\pi}}=50.8 \mathrm{in} \\
& R_{\text {rotational- torsional }}=\left[\frac{16 L^{4}}{3 \pi}\right]^{\frac{1}{4}}=51.4 \mathrm{in}
\end{aligned}
$$


Table 6.6 Stiffness of spread footing

\begin{tabular}{c|c}
\hline Stiffness & Value \\
\hline Vertical & $3877 \mathrm{kip} /$ in \\
\hline Horizontal & $3055 \mathrm{kip} /$ in \\
\hline Rotational & $8.983 \times 10^{6} \mathrm{kip}-\mathrm{in} / \mathrm{rad}$ \\
\hline Torsional & $6.910 \times 10^{6} \mathrm{kip}-\mathrm{in} / \mathrm{rad}$ \\
\hline
\end{tabular}

\subsubsection{Abutments}

\subsubsection{General Description}

Pre-1970 MCCG bridges built in Oregon generally present integral or semi-integral abutments on piles. In this study, the semi-integral abutment configuration depicted in Figure 6.34(a) was utilized to represent the typical abutment found in three span multicolumn continuous concrete girder bridges. Figure 6.34(b) shows the abutment details, where a moment release can be observed between the diaphragm and the pile cap. The connection consists of placing a steel dowel, a mastic, and a shear key. This connection is made with the aim of alleviating the thermal and live load demands that are transferred to pile cap and piles.

\subsubsection{Abutment Model}

A spring model was used to represent the behavior of MCCG abutments. The spring abutment model for MCCG bridges is similar to the one presented for MPCG bridges in Figure 6.24, excepting that the bearing pad was not considered in this case. In addition to that, the hyperbolic force-displacement that captures the abutment response in the longitudinal direction was adjusted to reflect the difference in heights between the UCD fullscale tests and the effective abutment height of the representative MCCG bridge. This adjustment was accomplished by assuming an effective abutment height equal to 39 in and 
using the height-adjustment factors developed by Shamsabadi et al. (2010). Consequently, a maximum abutment backwall force of $18.2 \mathrm{kips} / \mathrm{ft}$ was used.

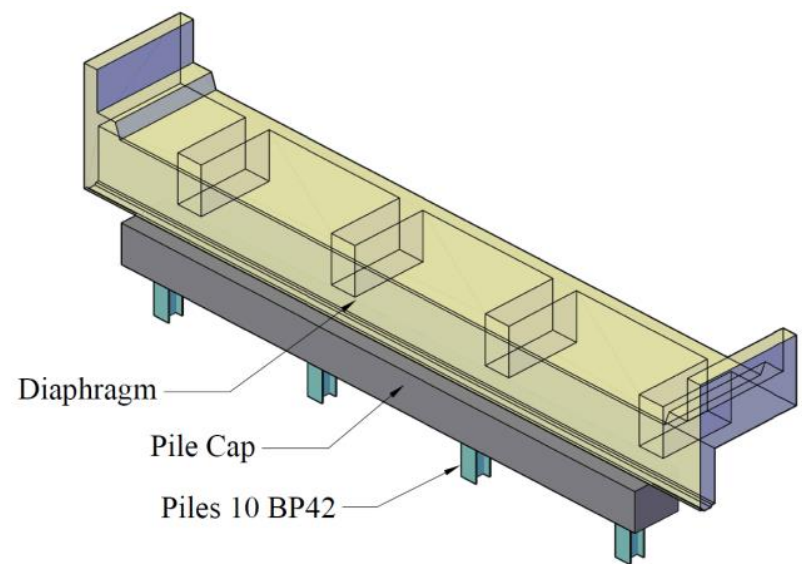

(a)

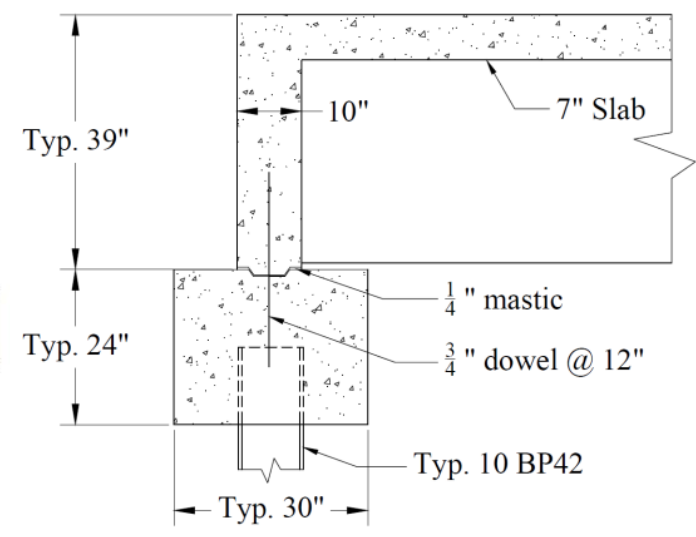

(b)

Figure 6.34 Typical abutments used in MCCG bridges (a) 3D schematic (b) Elevation

\subsubsection{Nonlinear Time History Results}

In order to study the nonlinear response of MCCG Bridge, a nonlinear time history analysis of a three dimensional bridge model was performed in OpenSees (2013) using the aforementioned component modeling for superstructure, RC bent, footings and abutments. The ground motions used in the NLTHA were identical to the one used for the MPCG bridge.

Results obtained of performing nonlinear time history analysis in the representative MCCG bridge model with fixed supports and no additional mass from the embankments are shown in Figure 6.35(a), (b), (c) and (d) for bents and abutments in the transverse and longitudinal direction, respectively. The results indicate that the bridge has displacement demands of 3.6 inches in the transverse direction and 1.9 inches in the longitudinal direction. Figure 6.36(c) shows that the abutment response in the transverse direction is controlled, as expected, by the hysteretic response of the piles. Moreover, significant inelastic excursions 
are expected in the abutments especially in the transverse direction. Thus, retrofitting this $\mathrm{RC}$ bent with $\mathrm{BRBs}$ may reduce the displacement demands in the transverse direction on both RC bents and abutments.

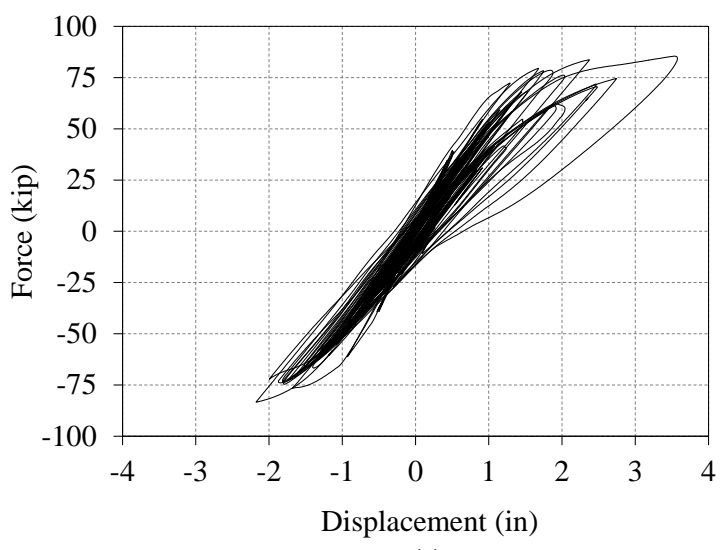

(a)

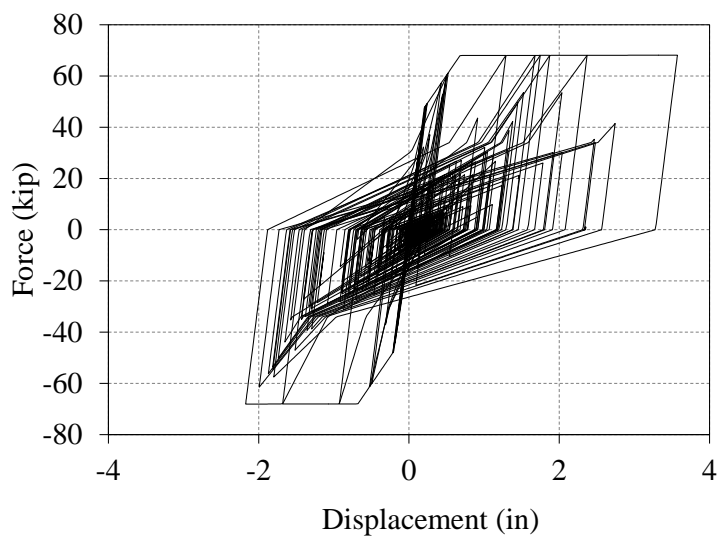

(c)

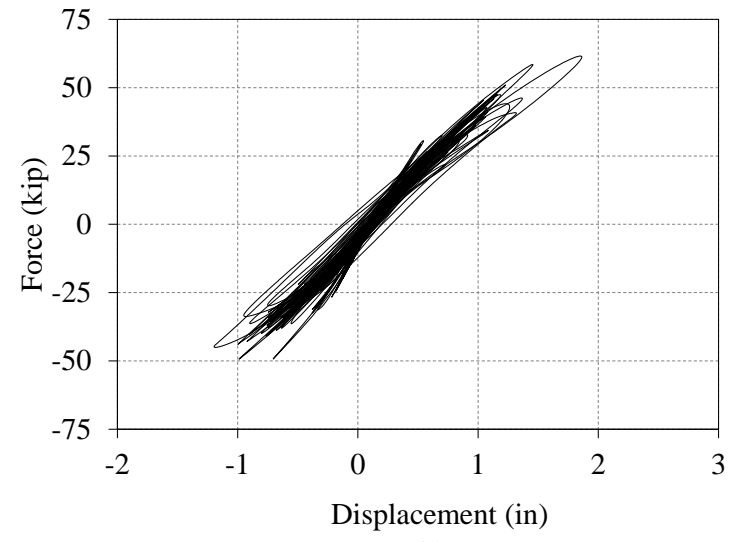

(b)

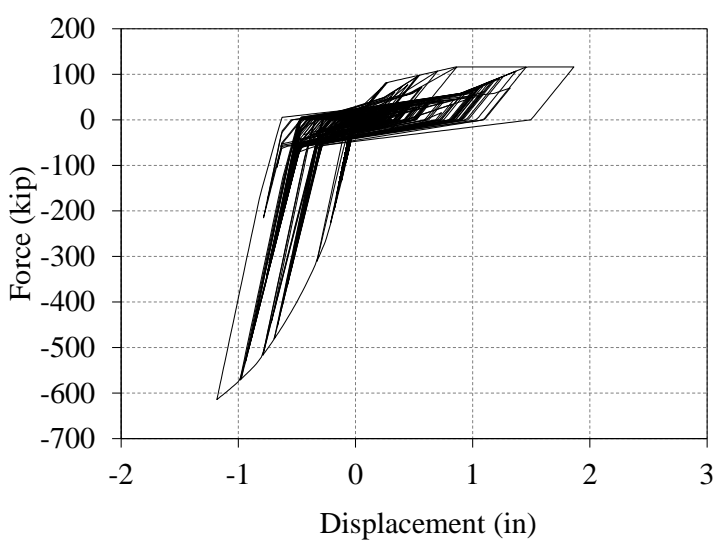

(d)

Figure 6.35 Results of NLTHA for MCCG bridge with fixed supports.

(a) Bent-Transverse, (b) Bent-Longitudinal, (c) Abutment-Transverse, (d) Abutment-Longitudinal

Results of considering an additional concentrated mass of $5 \mathrm{kip}-\mathrm{s}^{2} /$ in at the abutments (Mackie \& Stojadinovic, 2006) are depicted in Figure 6.36. In this case, the bridge has displacement demands of 4 inches on both directions, showing slightly more strength and stiffness degradation in the transverse direction. This result implies that adding this concentrated mass at the abutments significantly affects the displacement demands in the longitudinal direction, which increased more than $100 \%$ with respect to the case without 
additional mass. Significant inelastic excursions are expected in the abutment on both directions.

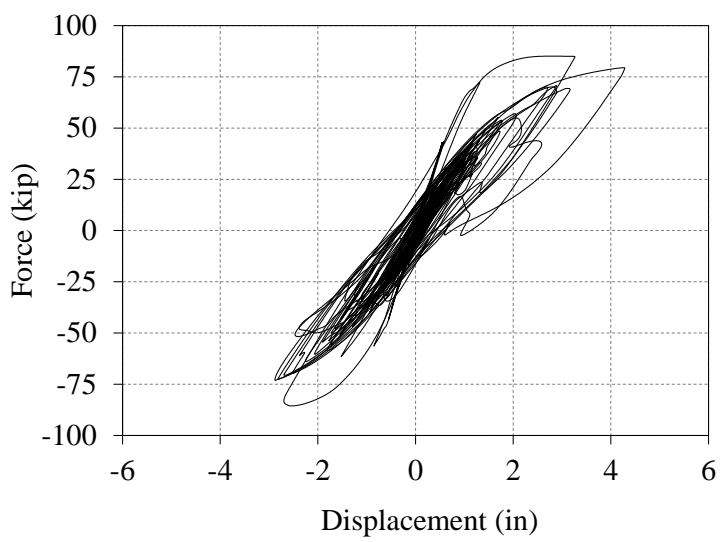

(a)

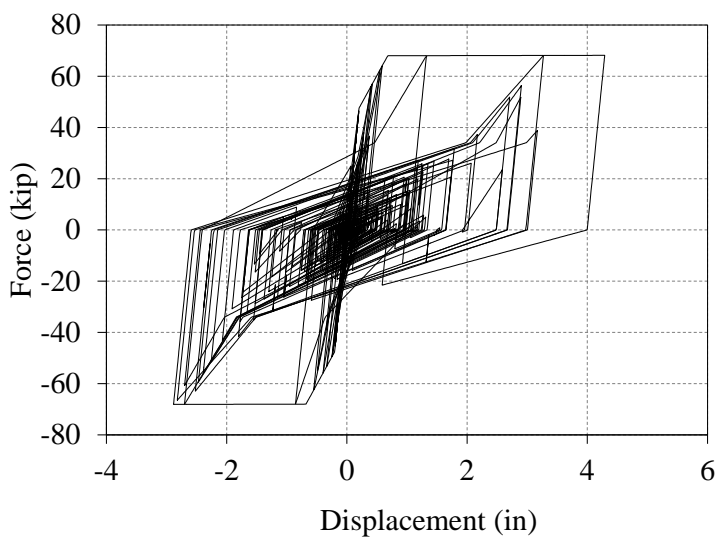

(c)

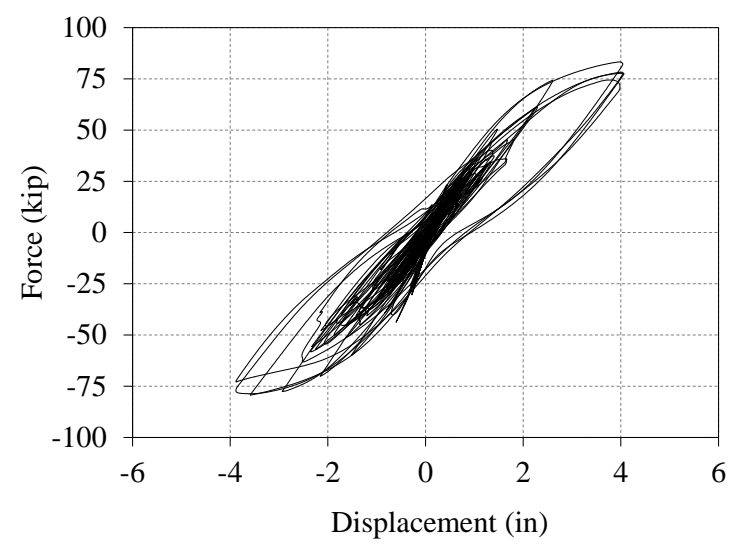

(b)

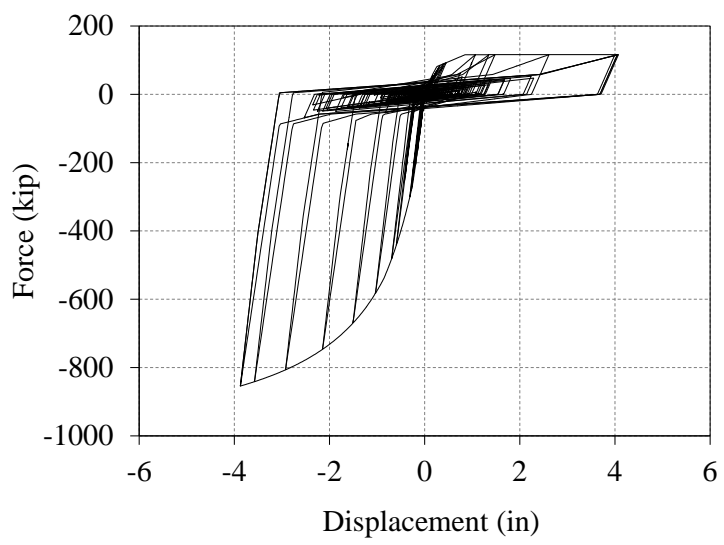

(d)

Figure 6.36 Results of NLTHA for MCCG bridge with additional mass at abutments.

(a) Bent-Transverse, (b) Bent-Longitudinal, (c) Abutment-Transverse, (d) Abutment-Longitudinal

Results considering flexibility on the foundations (spring-model) are depicted in

Figure 6.37. These results show a slightly increase on displacement demands on both transverse and longitudinal demands of bents and abutments. In this case, displacement demands of 3.7 inches in the transverse direction and 1.9 inches in the longitudinal direction were recorded. This negligible variation with respect to the results of the fixed support was presumably caused by assuming granular dense soil for calculating the linear stiffness of the footings, which resulted in very stiff foundations. 


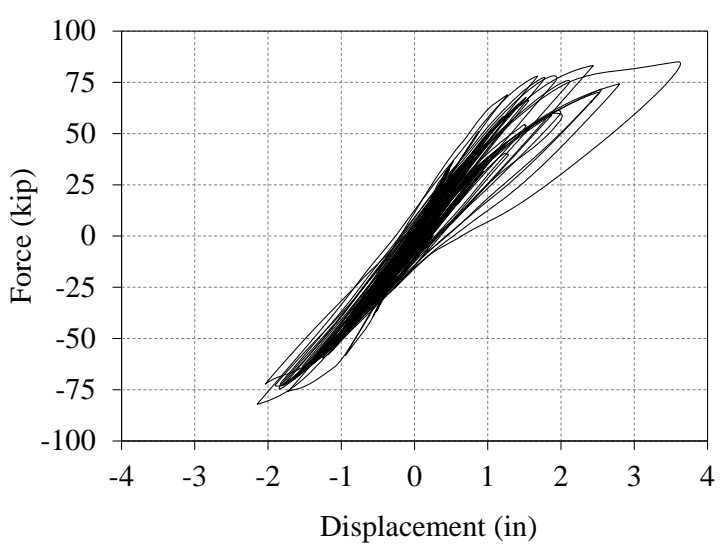

(a)

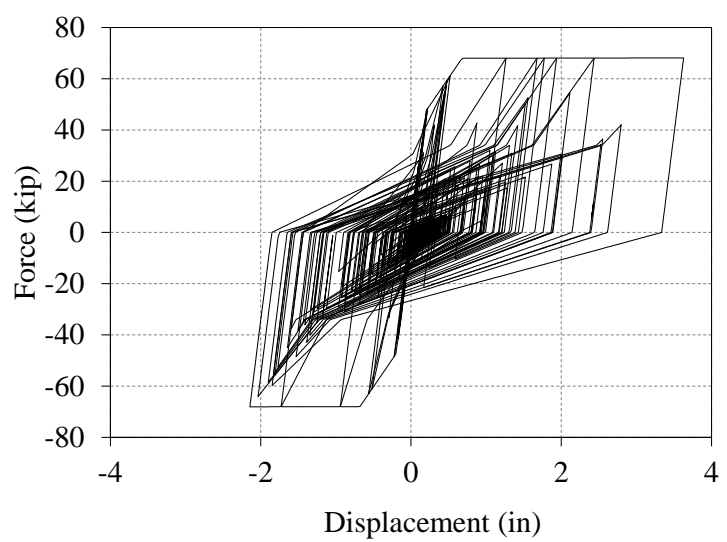

(c)

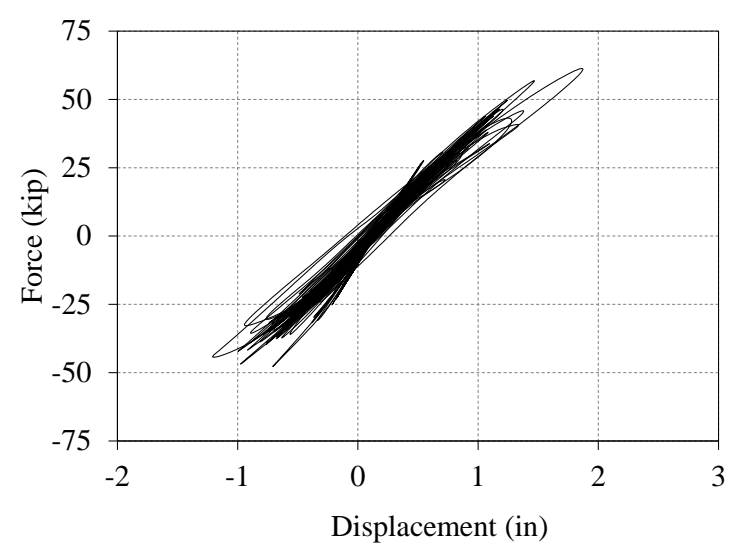

(b)

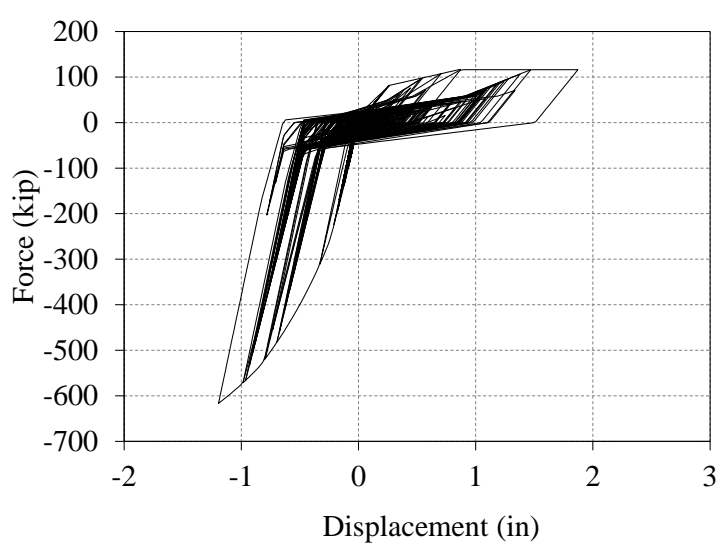

(d)

Figure 6.37 Results of NLTHA for MCCG bridge with spring foundations.

(a) Bent-Transverse, (b) Bent-Longitudinal, (c) Abutment-Transverse, (d) Abutment-Longitudinal

\subsection{Case Study OF RETRofitted Bent}

Nonlinear response analysis has been widely used to assess the performance of structures by the earthquake engineering community. In this section, the performance assessment of a representative reinforced concrete bridge bent retrofitted using BRBs is investigated performing nonlinear time history analysis. The refined numerical model presented in Section 6.3.2 was used to perform such nonlinear analyses. Special attention is put on assessing the bridge bent subjected to subduction zone ground motions. 
The representative reinforced concrete bent corresponds to an existing RC multicolumn bridge bent constructed in the 1950 to mid-1970 in the state of Oregon. As many of the bridge structures built at that time in the Pacific Northwest, the bridge substructure was designed and built with minimum seismic considerations. The prototype bridge bent model consists of two circular columns per bent, a rectangular cap beam and rectangular pile cap footings as shown in Figure 6.38. Force-based beam-column elements with six integration points between nodes were used to represent the columns and the cap beam. The BRB in the model of the prototype bridge bent had an effective length of 261 in and two rigid links that represent the end connections (gusset plates). Fixed based supports were assumed at the base of the columns. Fundamental periods of $0.5 \mathrm{sec}$ and $0.26 \mathrm{sec}$ were computed for the As-built and the retrofitted bent, respectively.

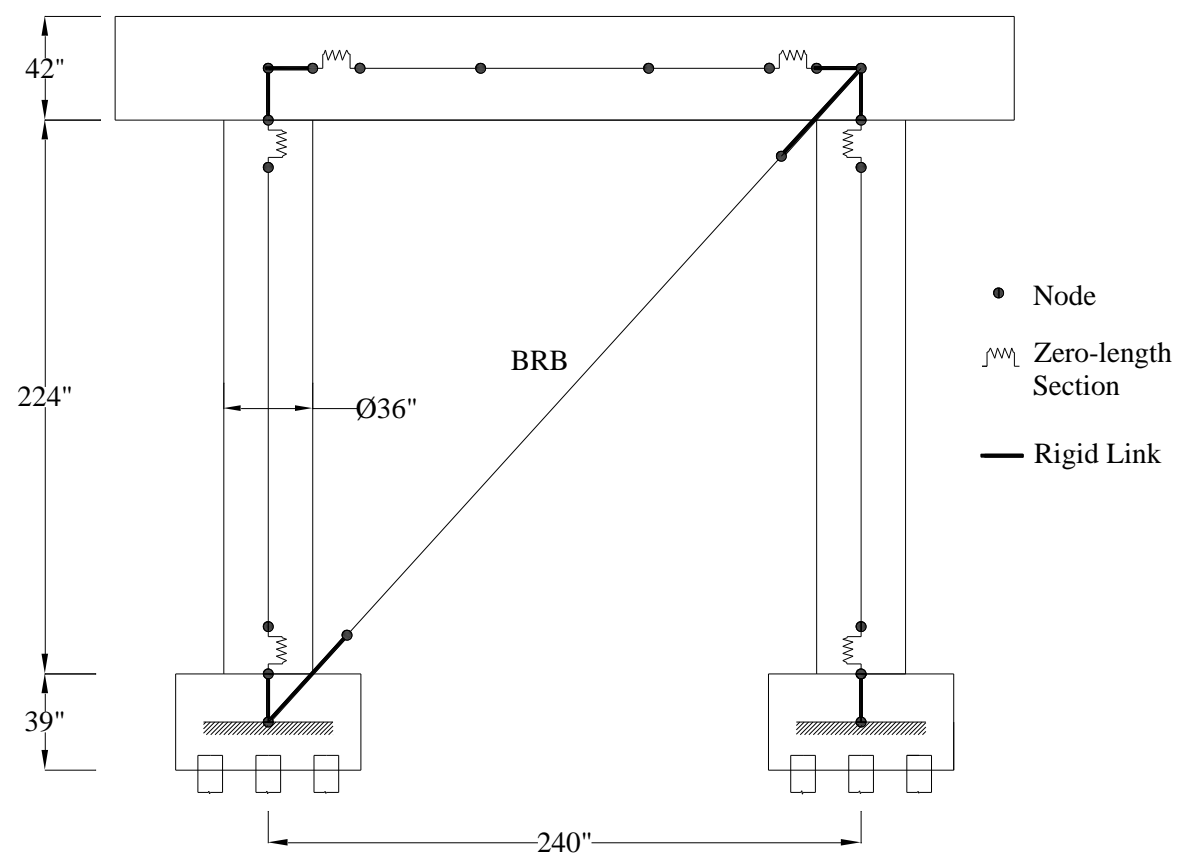

Figure 6.38 Numerical model for case study 
Six unscaled ground motions were used to evaluate the performance of the typical $\mathrm{RC}$ bridge bent in an effort to represent the 1000-year event described in Chapter 4 . The ground motions were selected from crustal and subduction sources. Ground motions recorded at the Capitola station during the 1989 Loma Prieta earthquake were selected to represent the crustal source. Four subduction ground motions were selected from the 2010 Maule, Chile and 2011 Tohoku, Japan earthquakes as presented in Table 6.7. Ground motions with different NEHRP site classes were used with the aim of representing different soil conditions.

Table 6.7 also shows the duration of the ground motion records. Even though, there is not a wide consensus in the duration definition of a ground motion, in this study two duration metrics were used, namely, bracketed duration and significant duration. Bracketed duration was taken as the time interval from the first to the last occurrence of an acceleration of $0.05 \mathrm{~g}$. Significant duration was taken as the time interval from $5 \%$ to $95 \%$ of the total energy. From Table 6.7 it can be observed that subduction ground motions have longer duration than crustal ground motions. This longer duration implies more inelastic cycles imposed in the structure as described in Chapter 2 and may lead to more damage and risk of collapse (Chandramohan, et al., 2015), (Raghunandan \& Liel, 2013).

Figure 6.39 shows the $5 \%$ damped response spectrum of the six ground motions used in this study. Moreover, three response spectrum representing the 500 -year event (operational criteria), the 1000-year event (life safety criteria), and the maximum considered earthquake (MCE) are presented in Figure 6.39. The 1000-year event was based on the $84^{\text {th }}$ percentile of the response spectrum of numerous bridges in the state of Oregon in an effort of representing the seismic demand of a vast number of bridges. 
Table 6.7 Ground motion records used in the case study

\begin{tabular}{|c|c|c|c|c|c|c|}
\hline Earthquake & Station & $\begin{array}{l}\text { Magnitude } \\
\qquad\left(\mathrm{M}_{\mathrm{w}}\right)\end{array}$ & Component & $\begin{array}{l}\text { PGA } \\
(\mathrm{g})\end{array}$ & $\begin{array}{c}\text { Bracketed } \\
\text { Duration } \\
\text { (sec) } \\
\end{array}$ & $\begin{array}{c}\text { Significant } \\
\text { Duration } \\
(\mathrm{sec}) \\
\end{array}$ \\
\hline \multirow{2}{*}{$\begin{array}{l}\text { Loma Prieta } \\
\text { (Crustal) }\end{array}$} & \multirow{2}{*}{ Capitola } & \multirow{2}{*}{6.9} & $00(\mathrm{X})$ & 0.53 & 25 & 12 \\
\hline & & & $90(Y)$ & 0.44 & 20 & 13 \\
\hline \multirow{2}{*}{$\begin{array}{l}\text { Maule, Chile } \\
\text { (Subduction) }\end{array}$} & \multirow{2}{*}{ Curico } & \multirow{2}{*}{8.8} & $\mathrm{EW}(\mathrm{X})$ & 0.41 & 91 & 52 \\
\hline & & & NS (Y) & 0.47 & 91 & 50 \\
\hline \multirow{2}{*}{$\begin{array}{c}\text { Tohoku, } \\
\text { Japan } \\
\text { (Subduction) }\end{array}$} & \multirow{2}{*}{ Kakuda } & \multirow{2}{*}{9.0} & $\mathrm{EW}(\mathrm{X})$ & 0.36 & 140 & 130 \\
\hline & & & NS $(Y)$ & 0.32 & 208 & 227 \\
\hline
\end{tabular}

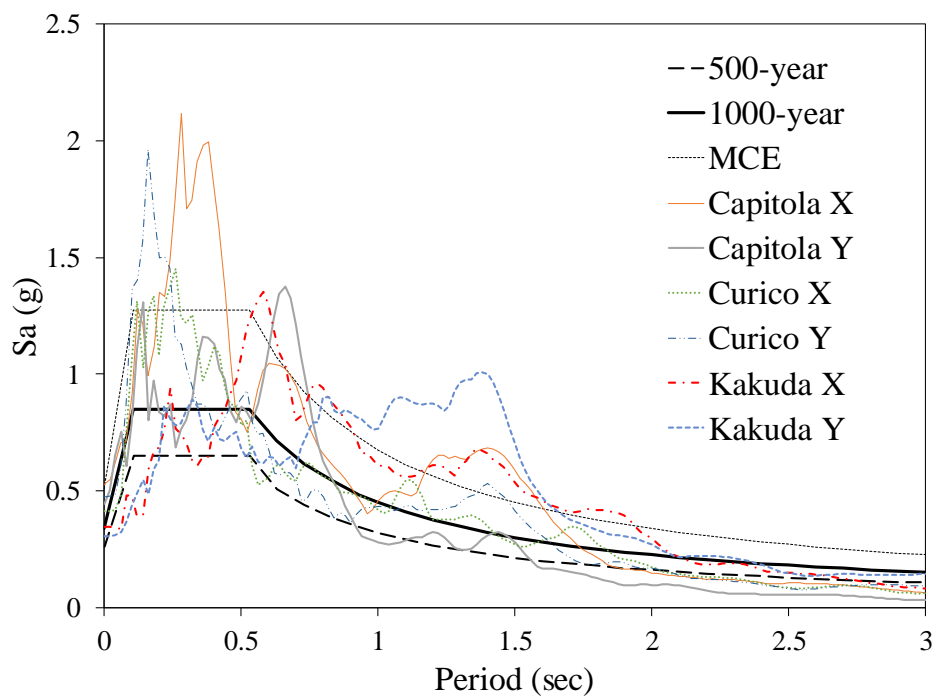

Figure 6.39 Ground motion records and response spectrum for 5\% damping

In order to perform the nonlinear time history analysis, a 5\% tangent-stiffness elastic damping was assigned to the RC elements in order to represent damping in the initial stages of response. No Rayleigh damping was used for the BRB, instead energy dissipation through hysteretic damping was assumed to be well captured by the numerical model.

The results of the analysis (Figure 6.40) show that the As-built bent exhibits strength deterioration as well as hysteretic pinching, which is expected in inadequately detailed reinforced concrete elements. The retrofit hysteresis on the other hand exhibit hysteretic 
behavior with much reduced strength degradation or pinching. The subduction ground motions, namely, Curico and Kakuda, subjected both the As-built bent and the retrofitted bent to many more inelastic cycles of small amplitude as compared to the crustal ground motion. Consequently, the As-built bent subjected to the CapitolaX ground motion did not show clear signs of deterioration from one cycle to the next, which is known as cyclic deterioration, but instead it showed in-cycle deterioration. On the other hand, the Kakuda ground motions subjected the As-built bent to many inelastic cycles causing an increase in cyclic deterioration. Further, based on the numerical results the KakudaY ground motion would cause severe damage and even collapse of the bent. This result can be a consequence of the long duration and distinctive site condition (NEHRP class E) of the Kakuda ground motions. Comparing the behavior of the retrofitted bent and the as-built bent, it can be observed that for the selected ground motions, the retrofitting technique was able to effectively reduce the drift demands and provide a ductile response. Ductility demand ratios over 8 were calculated for the retrofitted bent in all the cases.

Results at component level are shown in Figure 6.41. These results show that the BRBs performed, as expected, with stable hysteretic behavior and wide loops that reflect the good energy dissipation capacity of those components. Further, Figure 6.41 also shows that BRBs subjected to subduction ground motions underwent significantly more inelastic excursions of low amplitude. 

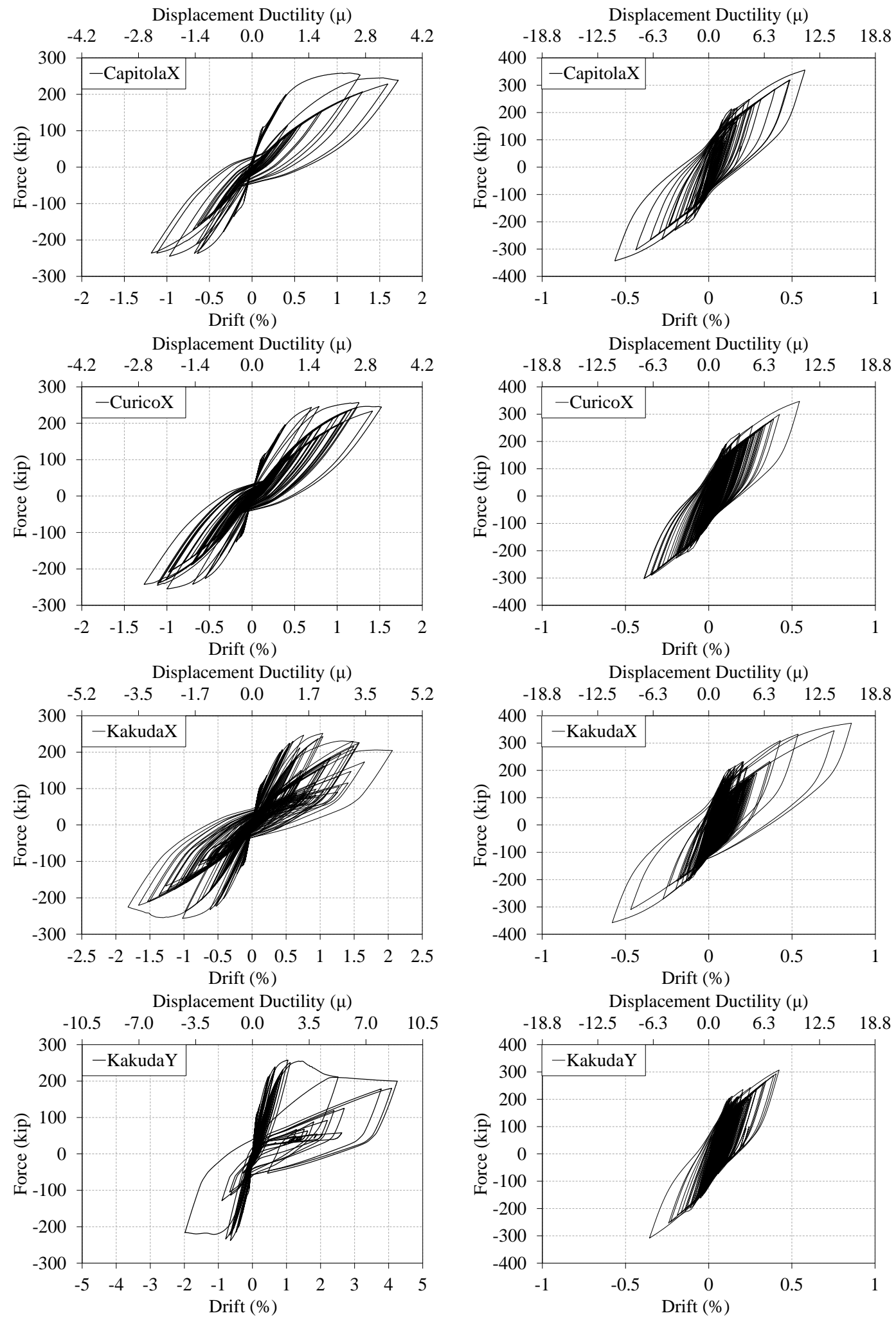

(a)

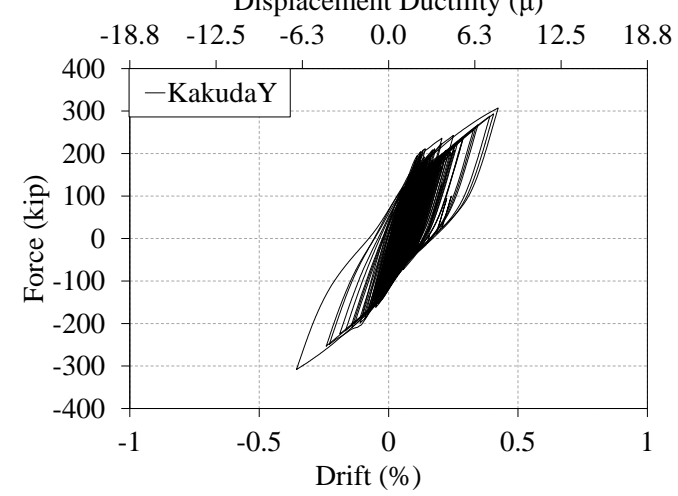

(b)

Figure 6.40 Results of nonlinear analysis. (a) As-built bent, (b) Retrofitted bent 

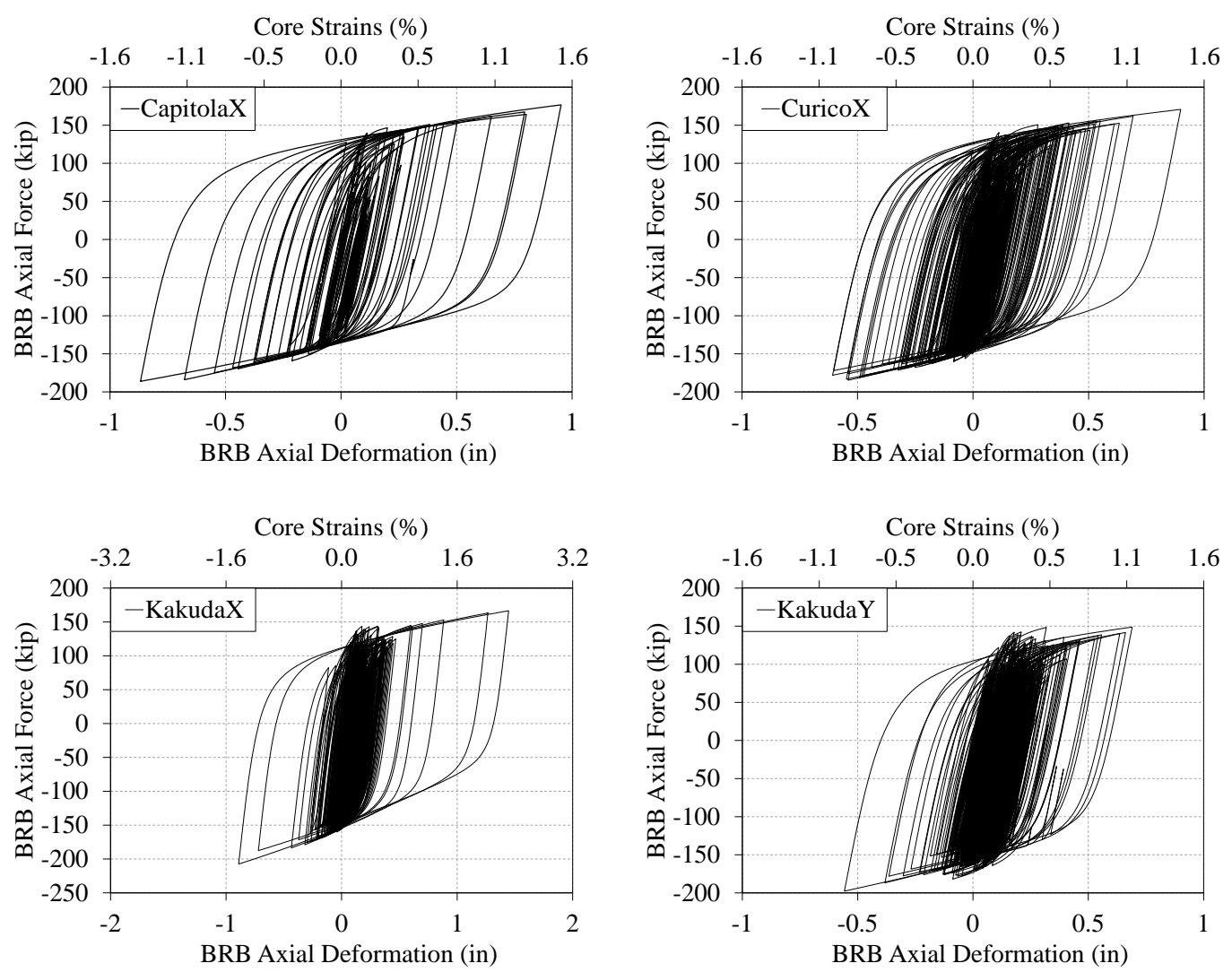

Figure 6.41 Response of BRBs in the nonlinear analysis

Maximum drift comparisons are summarized in Figure 6.42, comparing the

performance between the As-built and retrofitted bent responses. Retrofitting the As-built bent with buckling-restrained braces was an effective measure to reduce the drift demands more than $1 \%$, consequently limiting the damage of the As-built bent components. A comparison with the two-level performance criteria required by the state of Oregon, which is based on strain limitations for concrete and steel, is also depicted in Figure 6.42. This figure demonstrates that an operational performance level of response can be achieved for ground motions targeting the 1000-year event, and even for the MCE event if we take into account that for the retrofitted bent, which has a fundamental period of $0.26 \mathrm{sec}$, the demands generated from the Capitola and Curico ground motions would be close to the demands calculated from the MCE response spectrum shown in Figure 6.39. 


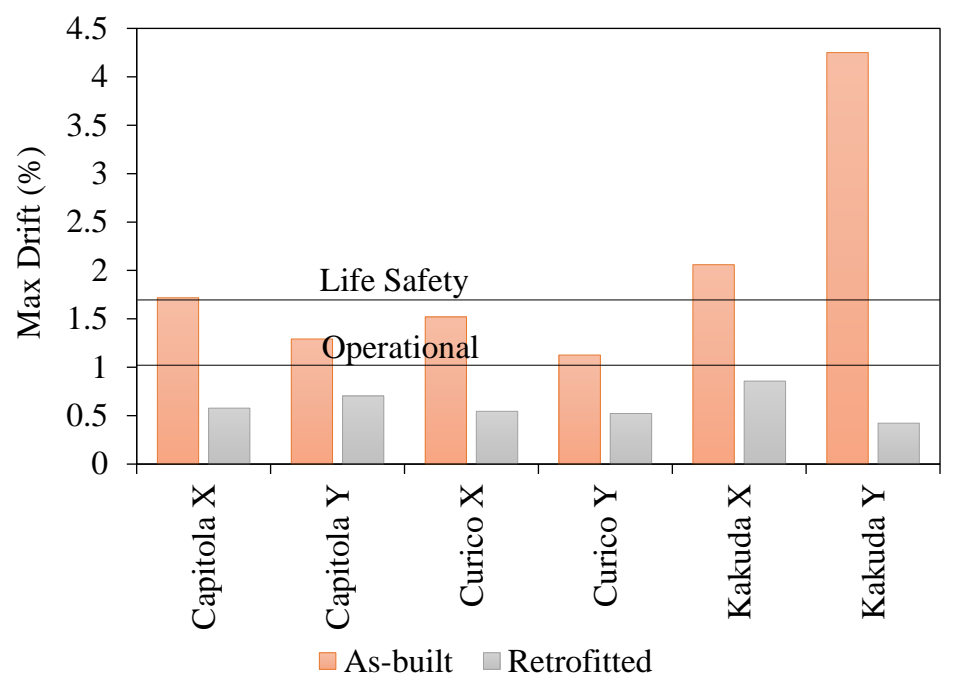

Figure 6.42 Toward Operational performance level

Interestingly, the retrofit technique was more effective for the case where the Asbuilt bent had the greatest demand, i.e. for the KakudaY ground motion. This is most likely caused by the shift in fundamental period and consequently the reduction in the demands for shorter period structures in that record as shown in Figure 6.39, and can imply that when soil effects are expected to amplify the demands for medium and long period structures, retrofitting them with a stiff and ductile hysteretic damper can be an effective retrofit option.

\subsection{SUMMARY}

Numerical models were developed in SAP2000 and OpenSees in order to characterize the nonlinear response of buckling-restrained braces and RC bridge bents in their as-built, retrofitted and repaired conditions. In addition to that, nonlinear modeling of two representative $\mathrm{RC}$ bridges was presented to reflect the use of refined nonlinear modeling techniques in the assessment of bridges. Finally, a case study of a representative RC bridge bent retrofitted with BRBs was investigated based on the suggested nonlinear models. 
Numerical results showed the importance of modeling the rate of strength and stiffness deterioration in RC bridges. This is of paramount importance in regions susceptible to be struck by subduction zone mega earthquakes since the faster the rate of deterioration the more significant the expected effect of number of inelastic cycles on column behavior.

The numerical results also showed that the models could provide a reasonable approximation of the nonlinear behavior of the BRBs and bents, including stiffness and strength degradation. This demonstrates the potential of these models to be used in the numerical assessment of substandard reinforced concrete bridges.

The numerical case study showed that by implementing buckling-restrained braces, the retrofitted bent was significantly stiffer than the as-built bent and yet provided for ductile response without significant damage to the concrete elements. Thus, adding BRBs in RC bridge bent can be a suitable retrofit measure for successfully achieving performance based dual-level design approaches. 


\section{CHAPTER 7}

\section{SEISMIC FRAGILITY ASSESSMENT}

\subsection{GENERAL}

The economic impact that a severe earthquake has on a highway network has led governmental entities and research groups to develop reliable methods for the damage assessment of components and highway structures in general. One of these methodologies is to base the damage assessment in fragility curves. Bridge damage fragility curves describe the conditional probability of exceeding a level of direct or indirect bridge damage for a given level of seismic hazard. Nowadays, fragility curves have emerged as an important decision tool to prioritize bridge retrofitting and estimate potential losses during and after a major earthquake.

In this chapter a brief state of the art in bridge fragility curve development is presented, limitations of each fragility development method are discussed, and fragility curves for a representative bridge bent in its as built and retrofitted conditions are developed by using analytical methods. In order to develop those fragility curves actual subduction zone strong motion records in conjunction with nonlinear analyses performed in SAP2000 and OpenSees were used. 


\subsection{SEISMIC FRAGILITY}

Seismic fragility analysis was born as a consequence of lifeline earthquake engineering. Current highway structure design methodologies are trending to performancebased design, in which fragility curves play an important role of describing the performance of a structure or component at different levels of a selected seismic intensity measure (Mackie and Stojadinovic 2005). The fragility of a structure or component can be expressed as a conditional probability that a defined limit state (LS) is exceeded for a given level of ground motion intensity (IM), as follows:

$$
\text { Fragility }=P[L S \mid I M]
$$

\subsection{FragiLity DEVELOPMENT METHODS}

The development of fragility cures has been widely discussed in the literature (Shinozuka, Feng and Lee, et al. 2000), (Stojadinović and Mackie 2007), (Vosooghi and Saiid Saiidi 2012) and several methods have been applied for its development. Fragility development methods can be categorized in empirical methods, experimental methods, expert opinions, and analytical methods. A detailed literature review of fragility curves development methods can be found in Padgett (2007), and in Billah and Alam (2014).

\subsubsection{Empirical Methods}

Empirical fragility curves are developed by utilizing observed damage data during past earthquakes. These damage data was collected primarily during the 1971 San Fernando earthquake, 1989 Loma Prieta earthquake, and the 1994 Northridge earthquake, which showed the high vulnerability of deficient bridges subjected to strong ground motions. 
Empirical fragility curve development is relatively straightforward since earthquake damage reports are used to establish the relationship between the ground motion intensity and the damage state of each bridge.

Several reasearchers have conducted studies to develop bridge fragility curves by statistically analyzing empirical damage data from damage reports (Basoz and Kiremidjian 1998), (Yamazaki, Ohnishi and Tayama 1999), (Shinozuka, Feng and Lee, et al. 2000).

Despite empirical methods may represent a more realistic approach due to the use of actual damage reports, they lack generality and have a large degree of uncertainty, which is primarily due to potential discrepancies in damage observation between inspection teams.

\subsubsection{Expert Opinion}

In this method the opinion of experts is collected and analyzed to estimate structural damages. The expert opinion is usually considered through surveys, which are then analyzed and represented in a damage probability matrix describing damage state for different levels of ground motion intensity. Finally, the probability matrix generated from the survey results is used for developing the fragility curves (ATC 1991), (Grossi 2000).

The expert opinion is the only source for the development of fragility curves. As a result, this method depends on the experience of the engineer and the number of expert's opinion gathered, which can lead to largely bias fragility curves and question its reliability.

\subsubsection{Experimental Methodology}

In this method the results from large-scale or full-scale experiments are needed for the development of fragility curves. Vosooghi and Saiidi (2012) developed experimental fragility curves for reinforced concrete bridge columns based on data from 32 bridge column models tested on shake tables. However, a lack of data at the same damage state is evident 
due to the limited amount of large scale testing. This lack of data highly limits the application of the experimental fragility curves.

\subsubsection{Analytical Methods}

\subsubsection{Elastic Spectral Analysis}

In this method the capacity/demand ratio of different components are determined to evaluate their potential seismic damage. In order to develop fragility curves using this method, damage states are defined and correlated to the capacity/demand ratio of the component via statistical analysis. The capacity/demand ratios are obtained using elastic spectral analysis (Hwang, Jernigan and Lin 1999) (Hwang, Jernigan and Lin 2000) (Hwang, Liu and Chiu 2001).

Despite the easy implementation of this method, its limitation is apparent at not be able to account for nonlinear behavior.

\subsubsection{Nonlinear Static Analysis (NSA)}

Nonlinear static analysis (NSA), also called Pushover analysis, is used in conjuntion with probabilistic analysis to determine fragility curves (Mander and Basöz 1999), (Shinozuka, Feng and Kim, et al. 2000), (Liao and Loh 2004).

In this method, uncertainty in capacity and demand is considered by plotting lognormal distributions over the capacity and demand curves. For a particular intensity measure (IM) the probability of failure can be estimated by using the intersection of capacity and demand distribution as shown in Figure 7.1. Finally, the fragility curves can be generated by increasing the level of intensity measure and measuring the response at various damage states (DS). 
Shinozuka et al. (2000) examined fragility curves of a bridge by time history analysis and the capacity spectrum method which is one of the nonlinear static procedures developed for buildings. Their comparison of fragility curves generated by the nonlinear static procedure with those by time-history analysis indicated that there was good agreement for the state of minor damage, but not as good for the state of major damage where nonlinear effects played an important role. However, they also concluded that even for the state of major state the agreement between the fragility curves based on NSA and nonlinear time history analysis was adequate considering the large number of assumptions that are performed for obtaining the fragility curves.

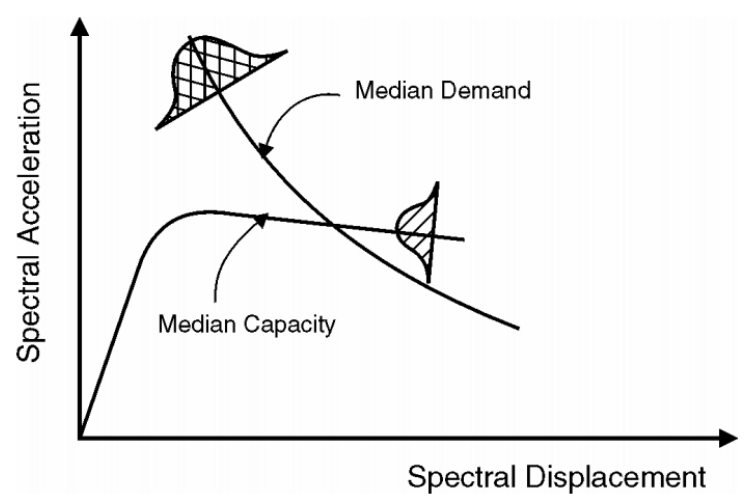

Figure 7.1 Capacity vs demand spectra showing uncertainty in structural behavior and ground motion response (FHWA 2006)

This method overcomes the deficiencies of performing an elastic spectral analysis by considering the nonlinearity of materials. However, in this method not all nonlinear effects are considered, which limits its application.

\subsubsection{Nonlinear Time History Analysis (NLTHA)}

Nonlinear time histories are used to construct analytical fragility curves. In this method ground motion time histories are selected to represent the seismic hazard at a specific area of interest. Nonlinear time histories analysis are conducted to obtain the 
response of the structure in study. Damage states and intensity measures are defined to quantify the damage undergone by the structure. Probabilistic analysis of the median and log-standard deviation parameters are then estimated by maximum likelihood procedure and the fragility curves are generated. (Shinozuka, Feng and Kim, et al. 2000), (Hwang, Liu and Chiu 2001), (Shinozuka, et al., 2001), (DesRoches, Nielson and Choi 2003), (Pan 2007), (Nielson and DesRoches 2007), (Simon, Bracci and Gardoni 2010).

Despite of being the most computationally demanding and time consuming, this method is the most reliable one for generating fragility curves.

\subsubsection{Fragility Curves for Retrofitted Bridges}

Currently, limited bridge fragility has been developed for retrofitted bridges since most of fragility curve development has been focused on the as-built condition of bridges. Some of the researches focused on fragility curves of retrofitted $\mathrm{RC}$ bridges are summarized below.

Shinozuka et al (2002) developed fragility curves for two retrofitted bridges by means of steel jacketing of columns. In order to develop the fragility curves, they used SAP2000 for modeling the bridges and sixty ground motion recordings representative of Los Angeles.

Padgett (2005), (Padgett, 2007) reviewed bridge seismic retrofit practice in the Central and Southeastern United States. Padgett used 48 strong ground motions as a seismic hazard and the Open System for Earthquake Engineering Simulation (OpenSees) computational analysis program to model the bridges. Non-linear time history analysis were conducted to capture parameters of interest for each component. The fragility was modeled by a lognormal cumulative distribution function where the structural demand and capacity were assumed to be lognormal distributed. 
Padgett and DesRoches (2007), (2008), (2009) presented an analytical methodology

for developing fragility curves for retrofitted bridge systems for the Central and Southeastern U.S. Since there were no records of strong motions in that area, they used two suites of synthetic ground motions for the study.

Billah et al. (2012), (2014) developed fragility curves for retrofitted multicolumn bridge bents subjected to near-fault and far field ground motions using a probabilistic seismic demand model and incremental dynamic analyses (IDA). The retrofit measures considered in that study were concrete jacketing, steel jacketing, carbon fiber reinforced polymer (CFRP) jacketing, and cementitious composite jacket. The results, as anticipated, showed that the bent retrofitted with concrete jacketing was more vulnerable to seismic ground motions. On the contrary, the bent retrofitted with CFRP and cementitious composite showed less vulnerability under both far-field and near-fault earthquakes.

\subsection{Fragility CurVES For REPRESENTATIVE BRIDGE BENT}

In this study, nonlinear analysis is used to evaluate the seismic fragility of the representative bridge bent built prior 1970 in the State of Oregon, as described in Chapter 4, and its retrofitted state with buckling-restrained braces. Two-dimensional (2D) finiteelement model scheme modeled in SAP2000 and OpenSees, which were described in Chapter 6, were used in the analytical modeling because of the good agreement between experimental and analytical results for the retrofitted and as-built condition. Moreover, for simplicity, the bridge bent is assumed to be supported on rigid foundations.

Two methodologies for fragility curve development are used in this study. Firstly, fragility curves were developed using SAP2000 and the method proposed by Shinozuka et al. (2000), which is based on a Capacity Spectrum method using nonlinear static analysis. 
Secondly, incremental dynamic analysis (IDA) (Luco \& Cornell, 1998), (Vamvatsikos \& Cornell, 2002) were used to develop fragility curves. In this method, a series of nonlinear time history analysis are performed at increasing levels of ground motions in an effort to capture the entire range of nonlinear response of a structure, from elastic behavior until failure, under earthquake actions.

\subsubsection{Strong Ground Motions}

A total of 30 ground motions, of which 10 are from the Tohoku earthquake M9.0, 10 from the Maule, Chile earthquake M8.8, and 10 from the Valparaiso, Chile earthquake M7.8 were utilized to evaluate the likelihood of exceeding the seismic capacity of the bridge bent in an effort to represent a potential Cascadia earthquake scenario. The seismic actions were represented by means of 5\% damped elastic response spectra as shown in Figure 7.2. The records selected for performance assessment of RC bridge bents are listed in

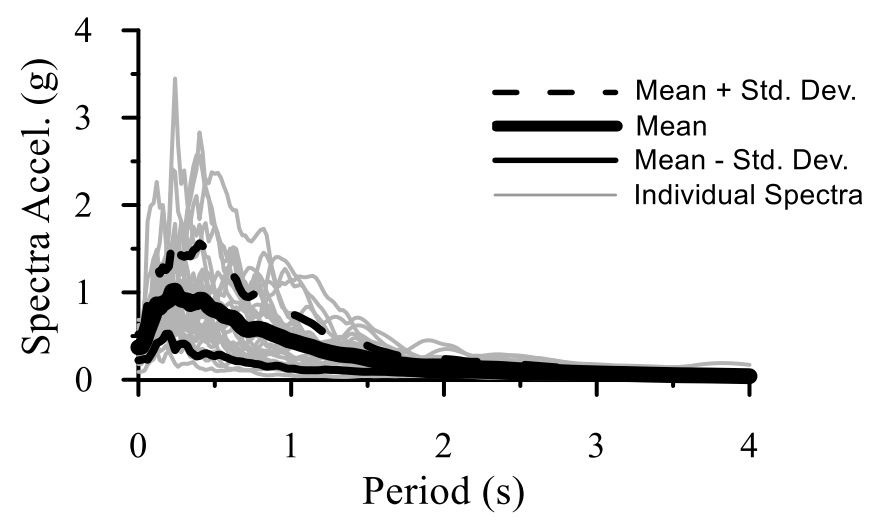

Figure 7.2 Response spectra for the 30 time histories used in this study (5\%damping)

\subsubsection{Damage States}

Damage states (DS) for a bridge component should provide a useful qualitative and quantitative representation for that component. These DS are often based on visual representations or strain and stress levels obtained from experimental studies. In this work, 
the engineering demand parameter (EDP) used for measuring the damage state of the bridge bent was displacement ductility, $\mu$. The EDP values are based on available literature (Hwang, et al., 2001) and the experimental results presented in Chapter 5. The values for the EDP are shown in Table 7.2. The displacement ductility for the damage state of collapse was adapted to the maximum ductility of the representative bridge bent.

Table 7.1 Selected earthquake ground motion records

\begin{tabular}{c|c|c|c}
\hline Earthquake & Station & PGA $_{\mathrm{x}}$ & PGA $_{\mathrm{y}}$ \\
\hline \multirow{4}{*}{$\begin{array}{c}\text { Valparaiso } \\
\text { Chile }\end{array}$} & Rapel & 0.09 & 0.22 \\
\cline { 2 - 4 } & Pichilemu & 0.18 & 0.26 \\
\cline { 2 - 4 } & San Felipe & 0.31 & 0.43 \\
\cline { 2 - 4 } & San Fernando & 0.34 & 0.29 \\
\cline { 2 - 4 } & Viña del Mar & 0.24 & 0.36 \\
\hline \multirow{4}{*}{$\begin{array}{c}\text { Maule } \\
\text { Chile }\end{array}$} & Constitucion & 0.54 & 0.35 \\
\cline { 2 - 4 } & Curico & 0.41 & 0.47 \\
\cline { 2 - 4 } & Stgo. Centro & 0.21 & 0.31 \\
\cline { 2 - 4 } & Viña, Centro & 0.33 & 0.22 \\
\cline { 2 - 4 } & Viña, El Salto & 0.34 & 0.35 \\
\hline \multirow{4}{*}{$\begin{array}{c}\text { Tohoku } \\
\text { Japan }\end{array}$} & Iitate & 0.51 & 0.58 \\
\cline { 2 - 4 } & Sukagawa & 0.50 & 0.69 \\
\cline { 2 - 4 } & Takahagi & 0.60 & 0.53 \\
\cline { 2 - 4 } & Nakoso & 0.27 & 0.39 \\
\cline { 2 - 4 } & Mizusawa & 0.37 & 0.21 \\
\hline
\end{tabular}

Table 7.2 Damage states for fragility curve development

\begin{tabular}{c|c|c|c|c}
\hline \multirow{2}{*}{ EDP } & \multicolumn{4}{|c}{ Damage States } \\
\cline { 2 - 5 } & Slight & Moderate & Extensive & Collapse \\
\hline Displacement ductility $(\mu)$ & $\mu>1$ & $\mu>1.4$ & $\mu>2$ & $\mu>4.5$ \\
\hline
\end{tabular}




\subsubsection{Fragility Curves}

\subsubsection{Nonlinear static analysis (Pushover)}

Nonlinear static analyses were performed in SAP2000 by using characteristic material properties for reinforcing steel and concrete, and the model described in Chapter 6.

For a given damage state, the fragility curve defines the conditional probability that the damage state be equaled or exceeded as a function of an intensity measure (IM). Following the work carried out by Shinozuka et al. (2000), the intensity measure considered in this study was the peak ground acceleration (PGA). The strong ground motion time histories were scaled to specific PGAs. The PGA ranged from $0.05 \mathrm{~g}$ to $0.8 \mathrm{~g}$ in increments of 0.1g. (e.g. $0.05,0.1,0.2 \ldots 0.8 \mathrm{~g})$. For each group of PGA the mean and the mean $\pm 1 \sigma$ (standard deviation) was calculated. This process generated three elastic acceleration response spectra, and consequently three spectral displacements were determined by intersecting the capacity spectrum with the demand spectrum. The displacement demands determined from this process are depicted in Figure 7.3 and Figure 7.4 for the as-built RC bridge bent and retrofitted bent, respectively. Since the distribution of spectral displacement was not symmetric, different standard deviations $(\sigma+$ and $\sigma-)$ were determined.

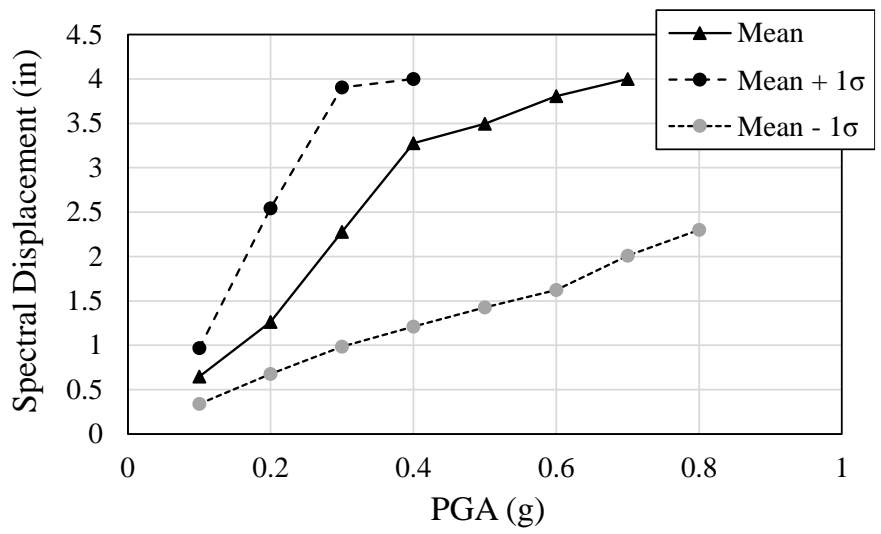

Figure 7.3 Performance displacement of as-built RC bridge bent 


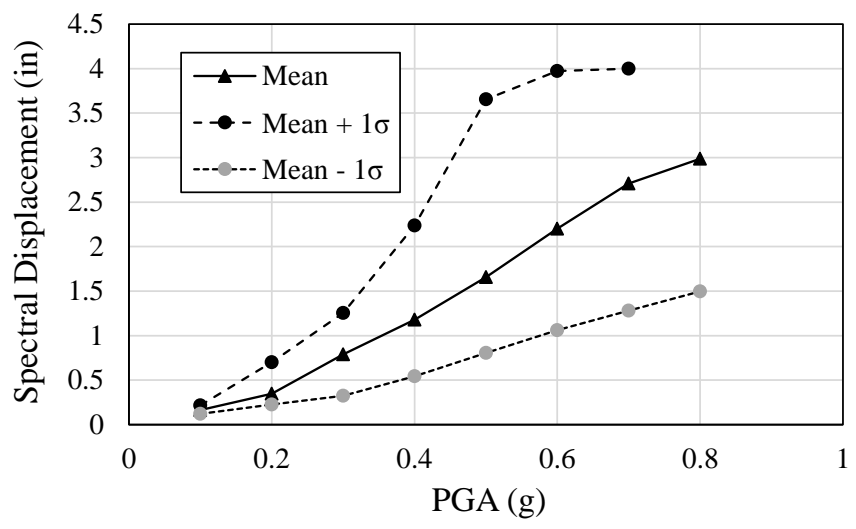

Figure 7.4 Performance displacement of retrofitted RC bridge bent

In this study the displacement ductility was determined by dividing the spectral displacement by the equivalent spectral displacement at yield. Thus, the displacement ductility has mean $\overline{\mu_{d}}$ and standard deviation, $\sigma$, redefined as $\sqrt{\sigma^{+} \cdot \sigma^{-}}$.

Eq. (7.2) defines the fragility curve.

$$
P\left[\mu_{d} \geq \mu \mid P G A\right]=1-\Phi\left[\frac{1}{\xi} \ln \left(\frac{\mu}{c}\right)\right]
$$

Where, $\Phi$ is the standardized normal distribution function, $\mu_{\mathrm{d}}$ is the displacement demand ductility, $\mu$ is the displacement demand capacity shown in Table $7.2, \xi$ and c are the standard deviations and the mean values of the corresponding normal distribution. As proposed by Shinozuka et al. (2000), the standard deviation $\xi$ and the mean $c$ at each PGA can be obtained from Eq. (7.3) and (7.4), respectively.

$$
\begin{gathered}
\overline{\mu_{d}}=c \cdot \exp \left[\frac{\xi^{2}}{2}\right] \\
\sigma^{2}=\left\{\overline{\mu_{d}}\right\}^{2}\left[\exp \left(\{\xi\}^{2}\right)-1\right]
\end{gathered}
$$

The probability of exceedance for the selected damage states is depicted in Figure 7.5 and Figure 7.6 for the as-built RC bent and its retrofitted condition, respectively. It is worth noting, that the displacement ductility for the retrofitted case was performed with respect to 
the yield displacement of the as-built bent instead of the yield displacement of the BRB.

Fragility curves for the selected damage states are summarized in Figure 7.7 for the as-built and retrofitted cases. These figures showed that the representative as-built $\mathrm{RC}$ bridge bent has a high probability of reaching a moderate level of damage for ground motions with PGA over $0.3 \mathrm{~g}$, and extensive damage for PGAs over $0.4 \mathrm{~g}$. For the retrofitted bent, high probability of damage for moderate and extensive damage states are reached for PGAs over $0.7 \mathrm{~g}$. Moreover, the collapse damage state, as desired in the retrofitted case, has a low probability for even PGAs over $0.7 \mathrm{~g}$.

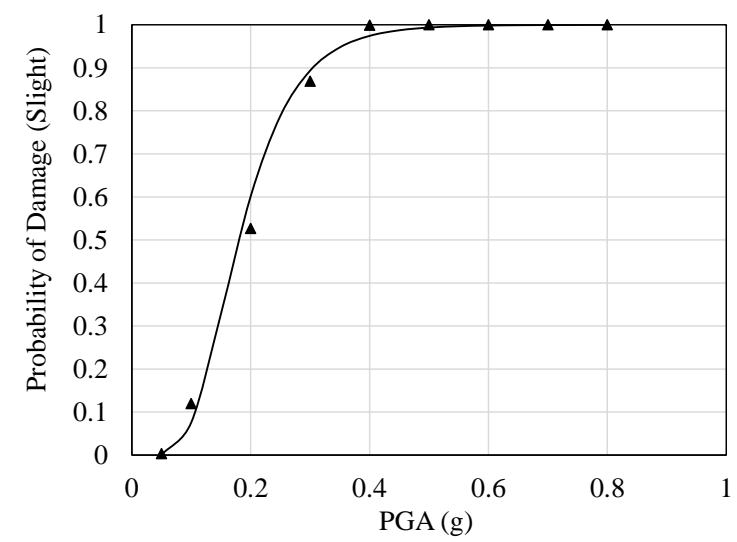

(a)

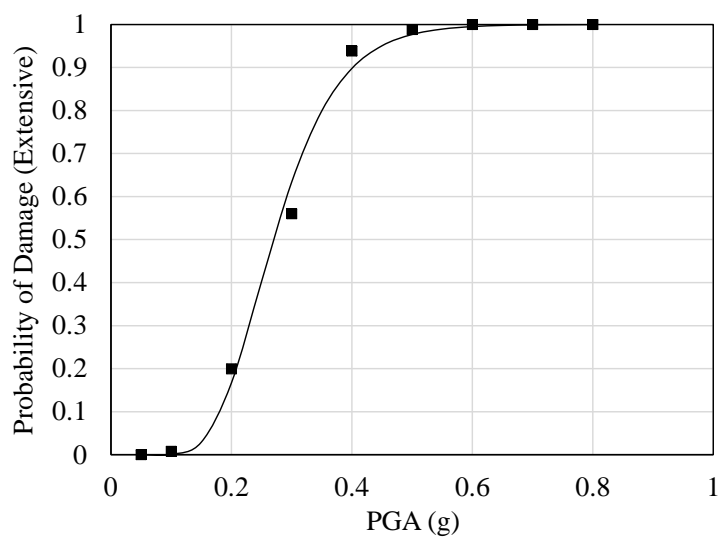

(c)

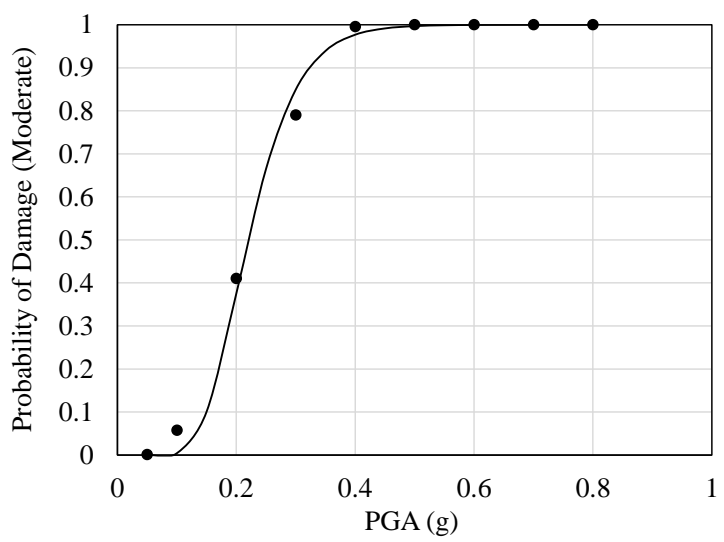

(b)

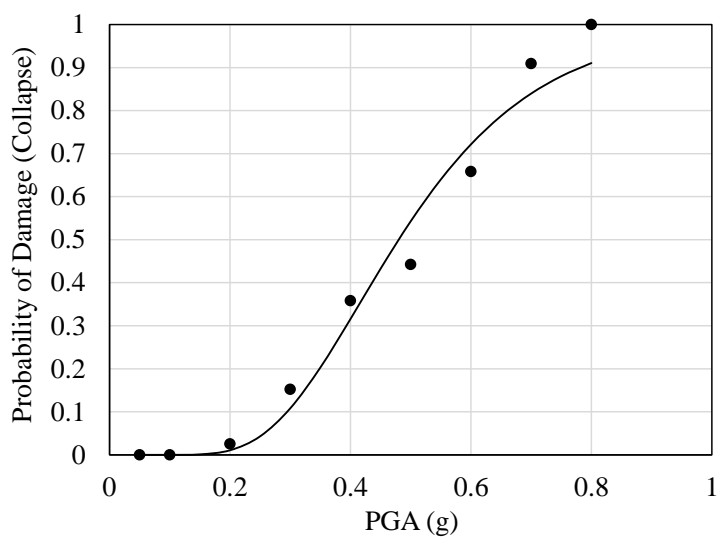

(d)

Figure 7.5 Fragility curves for representative as-built RC bridge bent for damage state: (a) Slight, (b) Moderate, (c) Extensive, (d) Collapse. 


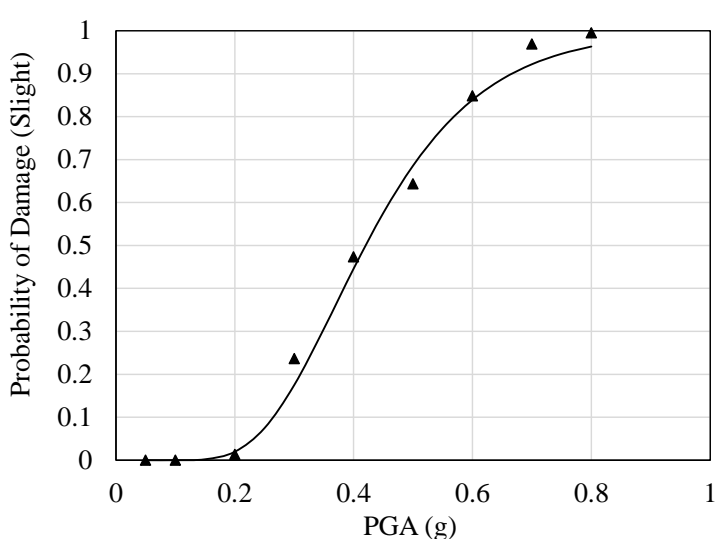

(a)

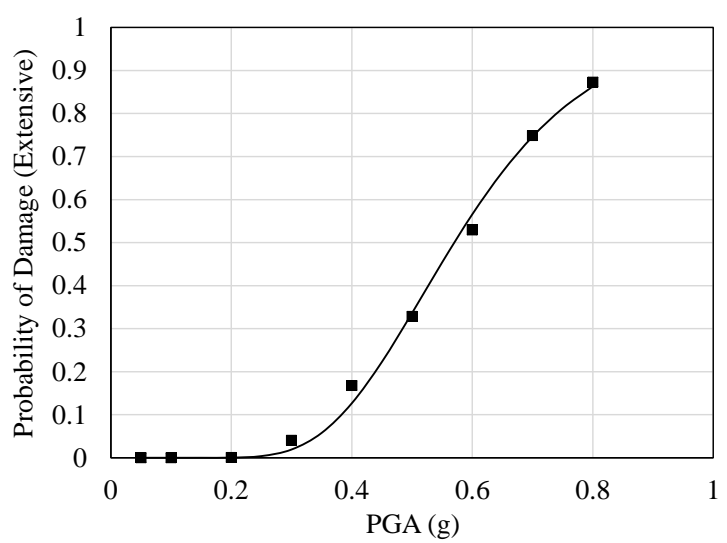

(c)

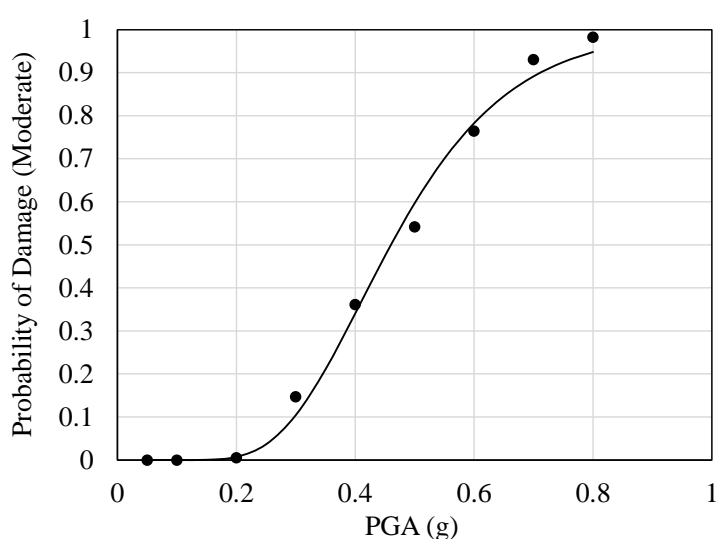

(b)

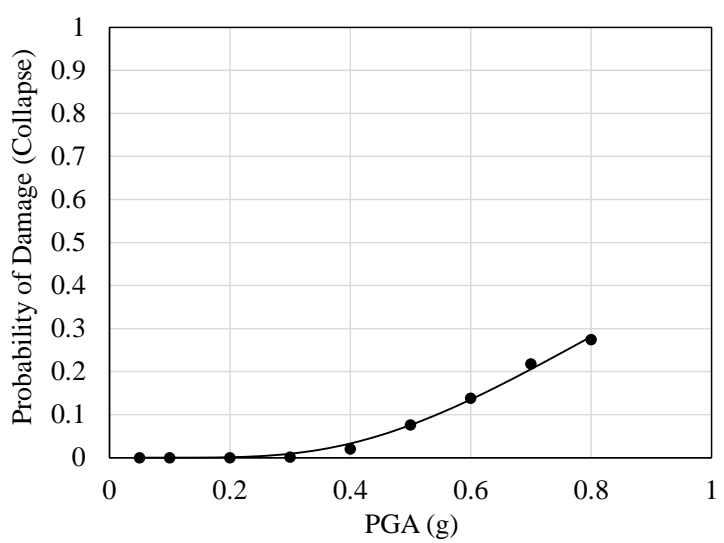

(d)

Figure 7.6 Fragility curves for retrofitted RC bridge bent for damage state: (a) Slight, (b) Moderate, (c) Extensive, (d) Collapse.

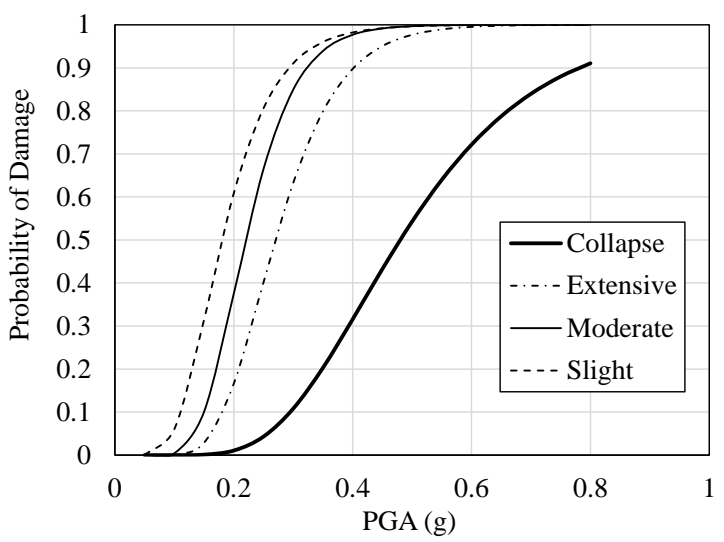

(a)

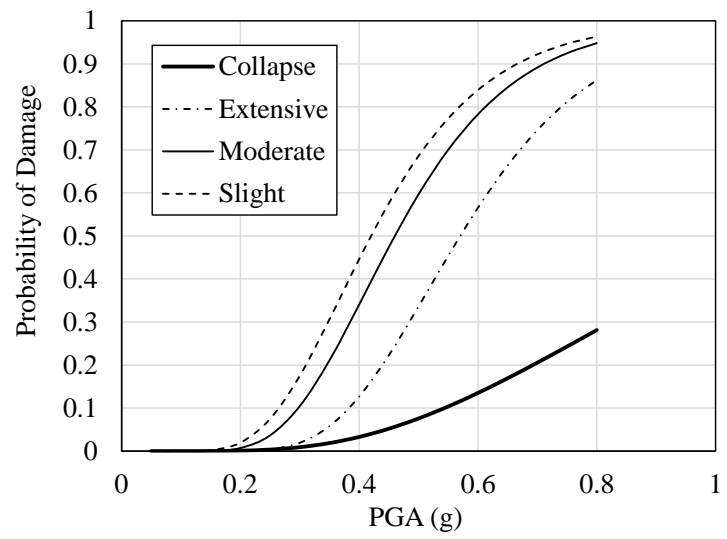

(b)

Figure 7.7 Fragility curves. (a) As-built bent, (b) Retrofitted bent 
Median values and dispersion for each damage state are shown in Table 7.3 and

Table 7.4 for the as-built and retrofitted bent, respectively. Comparison of the median values for the as-built state to the retrofitted state is shown in Figure 7.8. As can be observed in the figure, the retrofitted state presents a reduced probability of damage for all the damage states as compared to the as-built condition. Moreover, the median PGA value, which represents a probability of exceedance of $50 \%$, for the retrofitted bent is more than twice that the one for the as-built bent. This result implies, as expected, the retrofitted bridge bent is less vulnerable to seismic actions than the as-built bent.

Table 7.3 Fragility curve values for representative RC bridge bent

\begin{tabular}{c|c|c|c|c}
\hline \multirow{2}{*}{ Parameter } & \multicolumn{4}{|c}{ Damage States } \\
\cline { 2 - 5 } & Slight & Moderate & Extensive & Collapse \\
\hline Median & 0.18 & 0.22 & 0.27 & 0.48 \\
\hline Dispersion & 0.38 & 0.30 & 0.31 & 0.38 \\
\hline
\end{tabular}

Table 7.4 Fragility curve values for retrofitted RC bridge bent

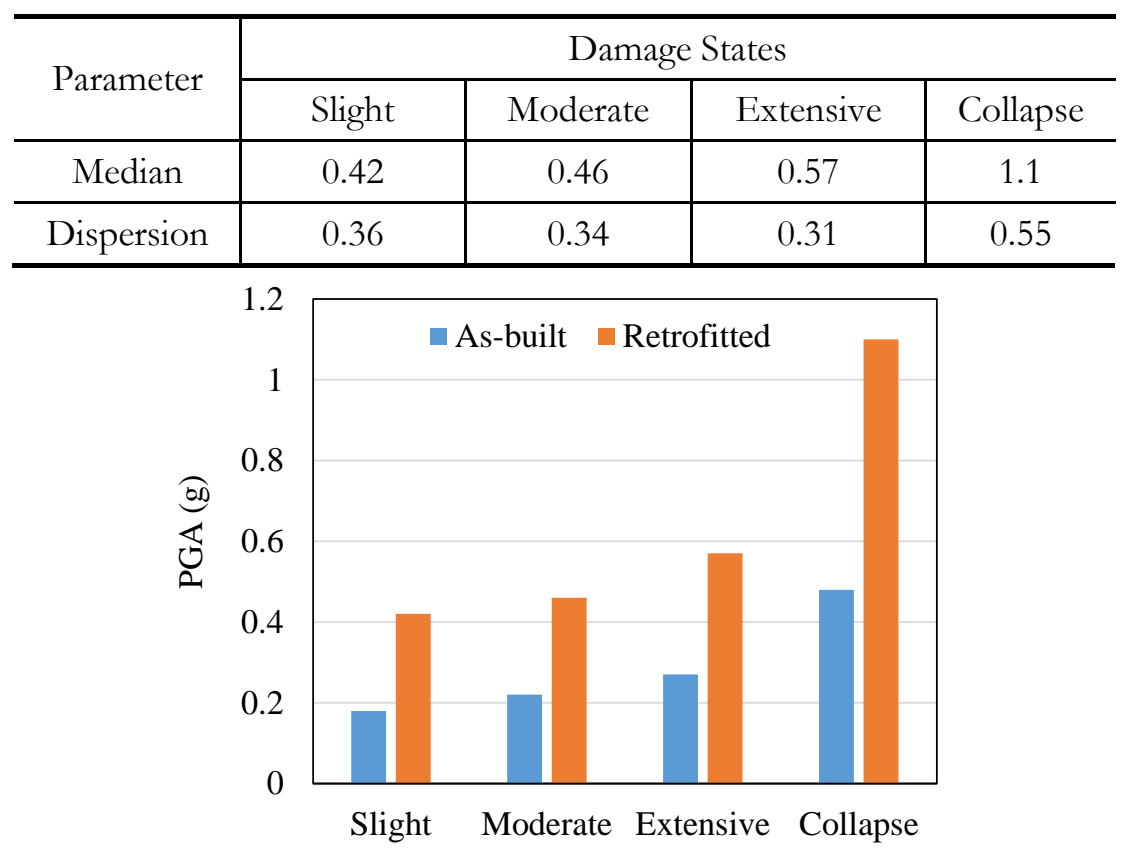

Figure 7.8 Comparison of median values of PGA for representative RC bridge bent 


\subsubsection{Nonlinear Time History Analysis}

The objective of this section is to perform fragility based seismic performance of a representative RC bridge bents in its as-built and retrofitted state by performing incremental dynamic analysis. Figure 7.9 illustrates the methodology adopted in this study. In this methodology, the nonlinear characterization using OpenSees was utilized to perform NLTHA at increasing levels of ground motion intensity. Peak ground acceleration was used as the intensity measure (IM) as suggested by Padgett et al. (2008). In order to be consistent with the fragility curves developed using nonlinear static analysis in SAP2000, the engineering demand parameter (EDP) used for measuring the damage state of the bridge bent was displacement ductility, $\mu$. Then, using the IDA method the fragility curves can be obtained using the damage probability at a given IM level. This damage probability is calculated as the ratio of the number of damage cases " $n_{i}$ " for the damage state " $i$ " over the number of total simulation cases $\mathrm{N}$ as shown in Eq. (7.5) (Zhang \& Huo, 2009).

$$
P\left[D I \geq L S_{i} \mid I M\right]=\frac{n_{i}}{N} \quad(i=1-4)
$$

In this study, a log-normal cumulative distribution function using Eq. (7.6) was fitted to the data in order to generate the IDA fragility curves using the maximum likelihood method as suggested by Baker (2015).

$$
P\left[D I \geq L S_{i} \mid I M\right]=\int_{0}^{I M} \frac{1}{i m \sqrt{2 \pi} \xi_{I M}} \exp \left[-\frac{\left(\ln (i m)-\lambda_{I M}\right)^{2}}{2 \xi_{I M}^{2}}\right] d(i m)
$$

Where, $\lambda$ and $\xi$ are the standard deviation and mean value of IM to reach the specified damage state. 
Incremental Dynamic Analysis (IDA)

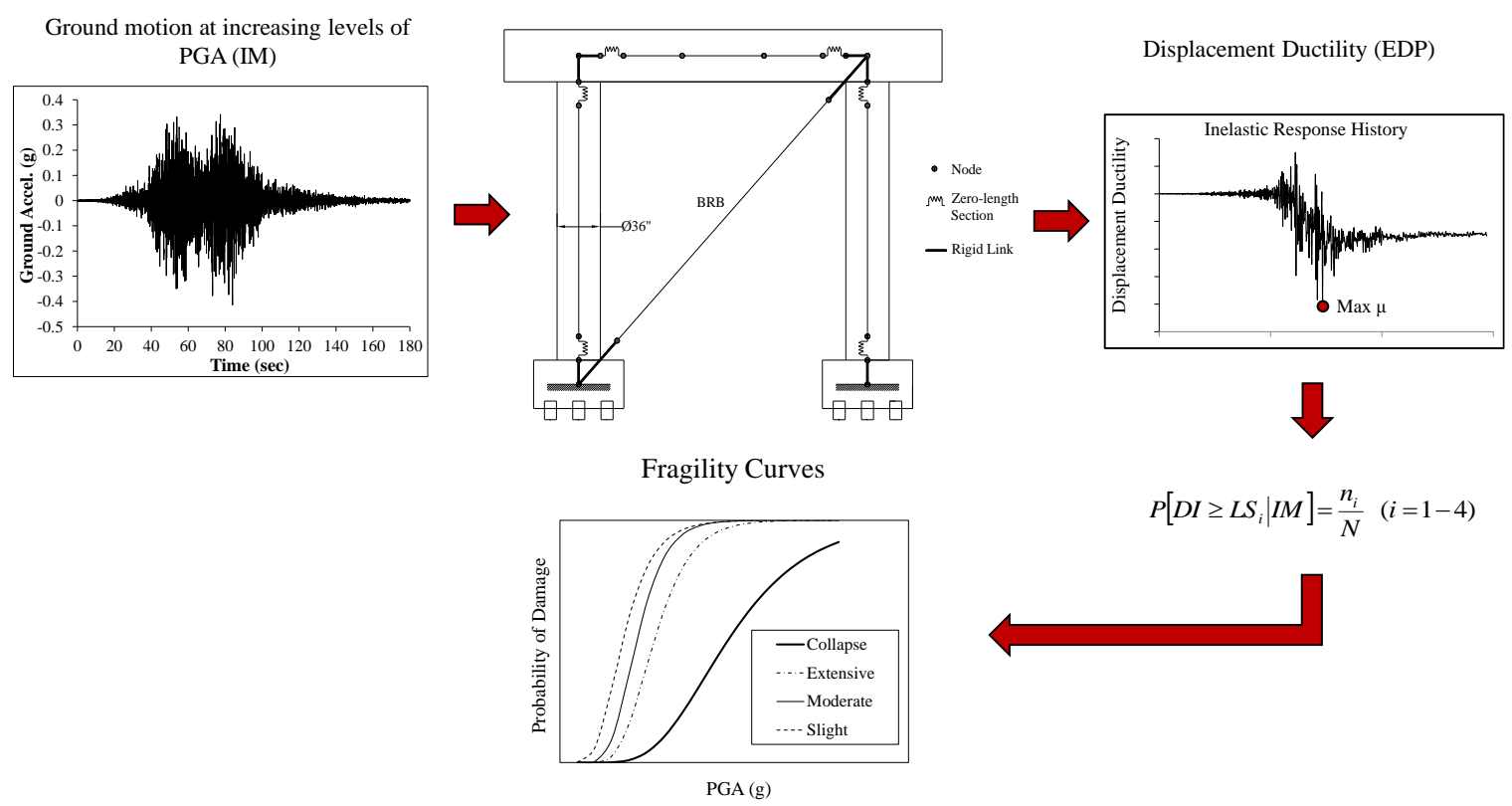

Figure 7.9 Methodology for seismic risk assessment of RC bridge bents

Fragility curves for the selected damage states obtained using NLTHA are shown in

Figure 7.10 for the as-built and retrofitted cases. These figures showed that the as-built RC bridge bent has a high probability ( $>80 \%$ ) of reaching a moderate level of damage for ground motions with PGA over $0.3 \mathrm{~g}$, and extensive damage for PGAs over $0.4 \mathrm{~g}$. For the retrofitted bent, high probability of damage for moderate and extensive damage states are reached for PGAs over $0.7 \mathrm{~g}$. Moreover, for the retrofitted bent collapse damage state has a low probability for even PGAs over $0.7 \mathrm{~g}$. Median values and dispersion for each damage state are shown in Table 7.5 and Table 7.6 for the as-built and retrofitted bent, respectively. Median values using NLTHA agree with the results obtained using NSA. Thus, results show that the retrofitted bridge bent is less vulnerable to seismic actions than the as-built bent. 


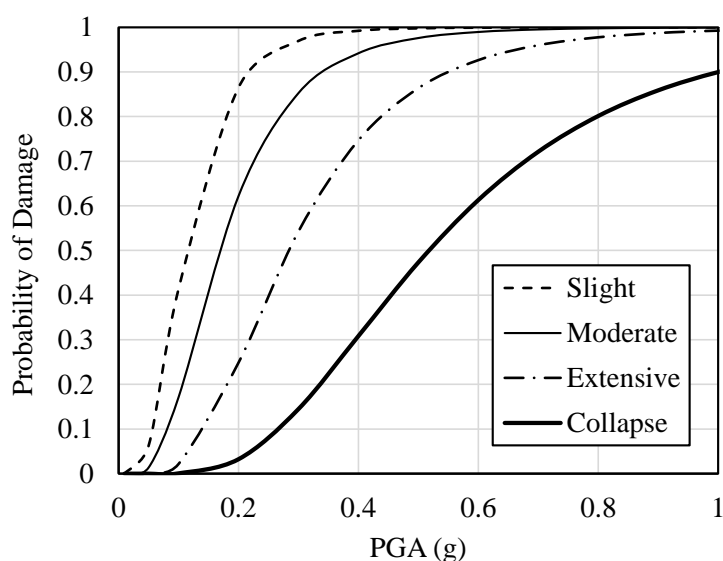

(a)

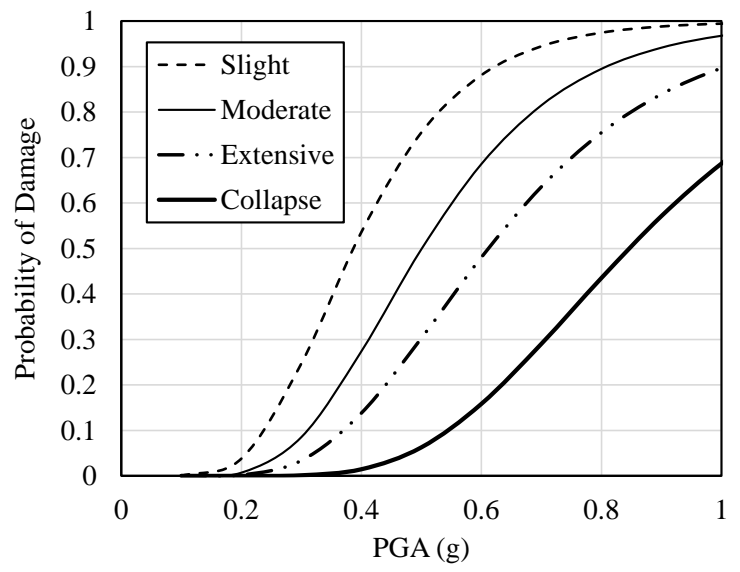

(b)

Figure 7.10 Fragility curves. (a) As-built bent, (b) Retrofitted bent

Table 7.5 Fragility curve values for representative RC bridge bent

\begin{tabular}{c|c|c|c|c}
\hline \multirow{2}{*}{ Parameter } & \multicolumn{4}{|c}{ Damage States } \\
\cline { 2 - 5 } & Slight & Moderate & Extensive & Collapse \\
\hline Median & 0.11 & 0.17 & 0.28 & 0.52 \\
\hline Dispersion & 0.52 & 0.55 & 0.52 & 0.51 \\
\hline
\end{tabular}

Table 7.6 Fragility curve values for retrofitted RC bridge bent

\begin{tabular}{c|c|c|c|c}
\hline \multirow{2}{*}{ Parameter } & \multicolumn{4}{|c}{ Damage States } \\
\cline { 2 - 5 } & Slight & Moderate & Extensive & Collapse \\
\hline Median & 0.39 & 0.50 & 0.61 & 0.85 \\
\hline Dispersion & 0.37 & 0.37 & 0.39 & 0.34 \\
\hline
\end{tabular}

\subsection{SUMMARY}

Fragility curves for a seismically deficient RC bridge bent and the bent retrofitted with buckling-restrained braces were developed. The methodology used for the development of the fragility curves involved the use of 30 subduction zone ground motions and the use of nonlinear analytical models. The analytical methods used in the study were nonlinear static analysis (pushover) performed in SAP2000 following the study carried out by Shinozuka et 
al. (2000), and NLTHA performed in OpenSees using incremental dynamic analysis. The NSA method was selected due to its simplicity and the good agreement between the experimental results shown in a previous chapter and the analytical results computed by means of pushover analysis. While, IDA analyses were performed to better capture the nonlinearity of the materials, and strength and stiffness deterioration due to the greater number of inelastic cycles that the structure would undergo when subjected to subduction zone ground motions. Through the process, the impact of the retrofit measure and the vulnerability of the as built bridge bent was evaluated.

The numerical results showed that the representative as-built bridge bent is more susceptible to subduction zone ground motions as compared to the response of the RC bent retrofitted with buckling-restrained braces. As a result, the analysis of the fragility curves revealed the effectiveness of the proposed retrofit measure in mitigating probable damage undergo by the structure during a major seismic event. The fragility curves and the retrofit measure as presented in this study aim to improve the criteria for retrofitting prioritization, estimation of potential losses and help with the decision of selecting a suitable retrofit measure in areas prone to subduction zone mega earthquakes. 


\section{CHAPTER 8}

\section{CONCLUSIONS AND FUTURE WORK}

\subsection{CONCLUSIONS}

Quasi-static loading protocols were developed to represent the increase of cumulative inelastic demands in reinforced concrete bridge columns. The inelastic time history results from a degrading numerical model in conjunction with the simplified rainflow counting procedure were used for the development of the protocols. The proposed loading protocols include a larger number of small amplitude inelastic cycles as compared to conventional protocols, revealing that conventional loading protocols commonly used in experimental testing tend to impose unrepresentative drift demands through imposing numerous large inelastic reversals on the component. Despite the higher number of large inelastic cycles, the overall normalized cumulative plastic displacement demands were similar when compared to the proposed protocols.

A representative pre-1970 lightly reinforced and lap-spliced bridge column was studied to observe the effect of the proposed protocols on the behavior of reinforced concrete bridge columns. Experimental results of deficient square RC columns showed that

the proposed subduction protocols influenced the response of reinforced concrete columns due to an increase in the overall number of inelastic cycles. This influence in response was observed in a reduced displacement capacity and strength resistance of the column subjected to one of the subduction protocols as compared to a conventional protocol. The experimental results of this study indicated that square columns present in bridges built 
before 1970 in the Pacific Northwest have unexpected deformation capacity. This moderately ductile performance was predominantly observed in columns with low axial load level $\left(0.07 \mathrm{f}_{\mathrm{c}} \mathrm{A}_{\mathrm{g}}\right)$ and low longitudinal steel reinforcement ratio $(\varrho=1.2 \%)$. In addition to the experimental results, a numerical case study showed the importance of modeling the rate of strength and stiffness deterioration in $\mathrm{RC}$ bridges. This is of paramount importance in regions susceptible to be struck by subduction zone mega earthquakes since the faster the rate of deterioration the more significant the expected effect of number of inelastic cycles on column behavior. Both lumped and distributed-plasticity numerical models were able to reasonably reproduce the initial stiffness, and strength as well as the stiffness degradation of the specimens. This demonstrates the potential of these models to be used in the numerical assessment of reinforced concrete bridges. Severe degradation parameters were needed to appropriately capture the damage on substandard columns. Since pre-1970 columns were built without seismic detailing, the behavior of these columns is expected to be represented by $\lambda$ values near 2 in the lumped-plasticity model. In the distributed-plasticity model, values of 0.006 and 0.002 for the damage 1 and damage 2 , respectively, were suggested. These higher damage parameters should be incorporated in areas where subduction earthquakes are expected. Thus, the assessment of RC bridge columns through representative testing load protocols would play a key role in the future establishment of limit states, failure modes and acceptance criteria to be applied in the design of bridge columns and should be considered when megathrust subduction earthquake hazard affects the design criteria.

The design implementation of buckling-restrained braces as a transverse direction retrofit and repair measure for reinforced concrete multi-column bridge bents was presented through 4 main steps, which are assessment of the as-built bent, BRB design, design of 
connections and capacity check. The limit states for the connection were also outlined. The design concept aims to concentrate all the induced energy during an earthquake event in disposable elements, while the reinforced concrete bent behavior is essentially in the elastic range. Based on this retrofit strategy, case studies of two representative bridges found in Oregon were discussed. The numerical results showed that by implementing bucklingrestrained braces, the retrofitted and repaired bent was significantly stiffer than the as-built bent and yet provided for ductile response without significant damage to the concrete elements and could be a suitable retrofit measure for successfully achieving performance based dual-level design approaches.

The experimental results of seismic performances of seismically deficient bridge bents retrofitted and repaired using buckling-restrained braces in a diagonal configuration were presented. Retrofitted, repaired and as-built cases were tested using cyclic loading protocols representative of the displacement demands in $\mathrm{RC}$ bridge bents subjected to subduction zone earthquakes. The retrofitted RC bridge bent was designed to perform elastically or with minor inelastic excursions within the original bent throughout the different seismic hazard design levels. Two BRB designs were considered in the retrofitted study in an effort to assess the influence of BRB stiffness on the overall structural performance. One $\mathrm{BRB}$ design was considered in the repaired condition. A novel gusset plate to $\mathrm{RC}$ bent connection was used, in which the gusset plates were directly connected to the horizontal $\mathrm{RC}$ elements without interfering with the columns. The results of these large-scale experiments successfully demonstrated the effectiveness of utilizing buckling-restrained braces for achieving high displacement ductility of the retrofitted and repaired structure, while also controlling the damage of the existing vulnerable reinforced concrete bent up to 
the design performance levels. Further, the experimental results showed that the BRB retrofit measure can achieve operational performance levels under both design levels, and repairing $\mathrm{RC}$ bents with $\mathrm{BRBs}$ proved to be an effective measure to restore the stiffness and strength of a damaged bent and provide high levels of energy dissipation.

The incorporation of the BRB also led to increasing forces in components of the RC bent. While this is not expected to significantly affect the column capacity for bridges, which often experience low axial demand/capacity ratio, additional care is recommended in the assessment of the footings due to the increase loading conditions. No damage was observed in the connection regions of the brace throughout the loading history, leaving the potential for replaceability of the sacrificial BRB element. The potential for improving the overall seismic behavior and the design performance levels with BRBs offers bridge design professionals a viable method for performance driven retrofit of multi-column reinforced concrete bridge bents.

A modified equation to calculate the stiffness degradation rate for BRB retrofitted bents was proposed by adding an exponential factor to the equation used for $\mathrm{RC}$ bents in order to account for the reduced rate in stiffness degradation.

The experimental results also indicated that despite the detailing deficiencies of the multi-column RC bridge bent built before 1970 in the Pacific Northwest, the cyclic response of the bent exhibited moderately ductile performance. The moderately ductile response was a result of a relatively long lap splice length and low axial column loads.

Subassemblage tests of three buckling-restrained braces were conducted in an effort to study the response of these elements. Results from those tests agreed with the BRB 
responses and failures observed during the retrofitted and repaired bent tests and demonstrated the high level of energy dissipation that BRBs can attain.

Numerical models were developed in SAP2000 and OpenSees in order to characterize the nonlinear response of buckling-restrained braces and $\mathrm{RC}$ bridge bents in their as-built, retrofitted and repaired conditions. The numerical results showed that the models could provide a reasonable approximation of the nonlinear behavior of the BRBs and bents, including stiffness and strength degradation. A numerical case study of a retrofitted bent showed that when soil effects are expected to amplify the demands for medium and long period structures, retrofitting them with a stiff and ductile hysteretic damper can be an effective retrofit option.

Fragility curves that were developed using nonlinear analysis showed that the representative as-built bridge bent is more susceptible to subduction zone ground motions as compared to the response of the RC bent retrofitted with buckling-restrained braces. As a result, the analyses of the fragility curves revealed the effectiveness of the proposed retrofit measure in mitigating probable damage undergo by the structure during a major seismic event. The fragility curves and the retrofit measure as presented in this study aim to improve the criteria for retrofitting prioritization, estimation of potential losses and help with the decision of selecting a suitable retrofit measure in areas prone to subduction zone mega earthquakes.

\subsection{FUTURE WORK}

The implementation and experimental validation of buckling-restrained braces as retrofit and repair measure for RC bridges was the primary outcome of this study. Future research on the implementation of buckling-restrained braces as seismic retrofitting and 
repairing of multi-column bridge bents on the field of retrofit and repair measures considering subduction zone earthquakes would be very beneficial and is summarized below. Quasi-static loading protocols were utilized in the experimental part of this project. Dynamic or Pseudo-dynamic tests should be conducted to further validate the proposed loading protocols and the proposed retrofit measure.

More case studies with different configurations should be analyzed in order to broad the applicability of the BRB system, and to assess the effect of the retrofit measure by using different BRB configurations.

Impact of the implementation of BRB on landscape, issues with debris collection and long term effects, such as corrosion, should be investigated.

$\square$ Different connections between gusset plate and concrete should be analytically and experimentally studied to ensure satisfactory seismic performance.

$\square \quad$ Comparisons between the retrofit measure presented in this report and others currently available in the market should be performed. Cost effectiveness should be included in such analysis.

$\square$ Experimental studies are needed to validate other structural fuses such as the hysteretic dampers described in Chapter 3.

$\square \quad$ In this study, foundations were modeled using either fixed supports or springs. Pilefooting interaction was not considered. Soil and liquefaction effects should be incorporated into the models.

Fragility curves can be improved by further studying the use of different bridge bent models with variable geometry, material properties and BRB designs. 


\section{REFERENCES}

AASHTO, 2009. Guide Specifications for LRFD Seismic Bridge Design, s.l.: American Association of State Highway and Transportation Officials.

ACI 318-11, 2011. Building Code Requirements for Structural Concrete and Commentary. s.l.:American Concrete Institute.

ACI 374.2R-13, 2013. Guide for Testing Reinforced Concrete Structural Elements under Slowly Applied Simulated Seismic Loads, Farmington Hills, MI: American Concrete Institute.

Aiken, I., Nims, D. \& Kelly, J., 1992. Comparative study of four passive energy dissipation systems. Bull. New Zealand Nat. Soc. For Earthquake Engrg, 25(3), pp. 175-192.

AISC 341-10, 2010. Seismic Provisions for Structural Steel Buildings. Chicago, Illinois: American Institute of Steel Construction.

AISC, 2011. Steel Construction Manual. American Institute of Steel Construction.

Andrawes, B., Shin, M. \& Wierschem, N., 2010. Active Confinement of Reinforced Concrete Bridge Columns Using Shape Memory Alloys. Journal of Bridge Engineering, 15(1), pp. 81-89.

ANSI/AISC 360-10, 2010. Specification for Structural Steel Buildings. Chicago, Illinois: American Institute of Steel Construction, Inc..

Ariyaratana, C. \& Fahnestock, L., 2011. Evaluation of buckling-restrained braced frame seismic performance considering reserve strength. Engineering Structures, 33(1), pp. 77 89.

ASCE/SEI 41-06, 2007. ASCE/SEI 41-06: Seismic Rehabilitation of Existing Buildings, Reston, Virginia: American Society of Civil Engineers. 
ASTM E1049-85, 2005. Standard Practices for Cycle Counting in Fatigue Analysis. West

Conshohocken, PA: American Society for Testing and Materials.

ATC, 1991. Seismic vulnerability and impact of dismption of lifelines in the conterminous United States,

Redwood City, CA: ATC.

ATC-24, 1992. Guidelines for Cyclic Seismic Testing of Components of Steel Structures for Buildings, Redwood City, CA: Applied Technology Council.

Atwater, B. et al., 2005. The orphan tsunami of 1700-Japanese clues to a parent earthquake in North America. U.S. Geological Survey Professional Paper 1707, p. 135.

Aviram, A., Mackie, K. \& Stojadinovic, B., 2008. Guidelines for Nonlinear Analysis of Bridge Structures in California, Berkeley, CA: Pacific Earthquake Engineering Research Center (PEER).

Badoux, M. \& Jirsa, J., 1990. Steel bracing of RC frames for seismic retrofitting. Journal of Structural Engineering, 116(1), pp. 55-74.

Bagheru, S., Barghian, M., Saieri, F. \& Farzinfar, A., 2015. U-shaped metallic-yielding damper in building structures: Seismic behavior and comparison with a friction damper. Structures, Volume 3, pp. 163-171.

Baker, J., 2015. Efficient analytical fragility function fitting using dynamic structural analysis. Earthquake Spectra, 31(1), pp. 579-599.

Basoz, N. \& Kiremidjian, A., 1998. Evaluation of Bridge Damage Data from the Loma Prieta and Northridge, CA Earthquakes, Buffalo, NY.: Multidisciplinary Center for Earthquake Engineering Research. 
Berry, M. \& Eberhard, M., 2008. Performance Modeling Strategies for Modern Reinforced Concrete Bridge Columns, University of California, Berkeley: Pacific Earthquake Engineering Research Center, PEER Report 2007/07.

Billah, A. \& Alam, M., 2014. Seismic performance evaluation of multi-column bridge bents retrofitted with different alternatives using incremental dynamic analysis. Engineering Structures, Volume 62, pp. 105-117.

Biskinis, D. \& Fardis, M., 2009. Deformations of Concrete Members at Yielding and Ultimate under Monotonic or Cyclic Loading (Including Repaired and Retrofitted Members), Patras, Greece: Report No. SEE 2009-0, Department of Civil Engineering, University of Patras.

Bolt, B., 1969. Duration of strong motion. Santiago, Chile, Proceedings of the 4th World Conference on Earthquake Engineering, pp. 1304-1315.

Borg, R., Rossetto, T. \& Varum, H., 2012. The Effect of the Number of Response Cycles on the Behaviour of Reinforced Concrete Elements Subject to Cyclic Loading. Lisboa, 15th World Conference on Earthquake Engineering.

Bracci, J. M., Reinhorn, A. M. \& \& Mander, J. B., 1995. Bracci, J. M., Reinhorn, A. M., \& Mander, J. B. (1995). Seismic retrofit of reinforced concrete buildings designed for gravity loads: performance of structural model. ACI Structural Journal, 92(6).

BSSC, B. S. S. C., 2003. The 2003 NEHRP recommended provisions for new buildings and other structures Part 1: Provisions, FEMA 450.

Caltrans, 1996. Earthquake Retrofit Guidelines for Bridges, SAcramento, California: California Department of Transportation.

Caltrans, 2013. Seismic Design Criteria, Sacramento, California: California Department of Transportation. 
Cameron, J., Makris, N. \& Aiken, I., 2004. Component Testing, Seismic Evaluation and Characterization of Buckling-Restrained Braces. Journal of Structural Engineering, ASCE, 130(6), pp. 880-894.

Carden, L., Itani, A. \& Buckle, I., 2006. Seismic Performance of Steel Girder Bridges with Ductile Cross Frames Using Buckling-Restrained Braces. Journal of Structural Engineering, 132(3), pp. 338-345.

Chai, Y. H., Priestley, M. J. N. \& Seible, F., 1991. Retrofit of Bridge Columns for Enhanced Seismic Performance. Seismic Assessment and Retrofit of Bridges, Issue SSRP 91/03, pp. 177-196.

Chai, Y., Priestley, M. \& Seible, F., 1991. Flexural Retrofit of Circular Reinforced Concrete Bridge Columns by Steel Jackets, University of California, San Diego: Department of Applied Mechanics and Engineering Sciences.

Chai, Y., Priestley, M. \& Seible, F., 1992. Retrofit of Bridge Columns for Enhanced Seismic Performance, University of California, San Diego: Department of Applied Mechanics and Engineering Sciences.

Chandramohan, R., Baker, J. \& Deierlein, G., 2015. Quantifying the influence of ground motion duration on structural collapse capacity using spectrally equivalent records. Earthquake Spectra.

Chandramohan, R., Lin, T., Baker, J. \& Deierlein, G., 2013. Influence of ground motion spectral shape and duration on seismic collapse risk. Tokyo, Japan, 10CUEE CONFERENCE PROCEEDINGS, 10th International Conference on Urban Earthquake Engineering. 
Chang, S., Li, Y. \& Loh, C., 2004. Experimental Study of Seismic Behaviors of As-Built and Carbon Fiber Reinforced Plastics Repaired Reinforced Concrete Bridge Columns. Journal of Bridge Engineering, 9(4), pp. 391-402.

Cheng, C., Yang, J., Yeh, Y. \& Chen, S., 2003. Seismic performance of repaired hollowbridge piers. Construction and Building Materials, 17(5), pp. 339-351.

Cheung, P., Pauley, T. \& Park, R., 1991. New Zealand Tests on Full-Scale Reinforced Concrete Beam-Column-Slab Sub-assemblages Designed for Earthquake Resistance, Detroit, MI: ACI Special Publication SP 123-1, American Concrete Institute.

Choi, E., 2002. Seismic Analysis and Retrofit of Mid-America Bridges, Atlanta, GA: Georgia Institute of Technology.

Clark, P. et al., 1999. Design Procedures for Buildings Incorporating Hysteretic Damping Devices. Santa Barbara, CA, s.n., pp. 355-371.

Clough, R. \& Jonhnston, S., 1966. Effect of stiffness degradation on earthquake ductility requirements. Tokyo, Japan, Proceedings of Japan Earthquake Engineering Symposium.

Computers and Structures Inc., C., 2011. SAP2000 Integrated Software for Structural Analysis and Design. Berkeley, CA.

Connor, J., Wada, A., Iwata, M. \& Huang, Y., 1997. Damage-Controlled Structures. I: Preliminary Design Methodology for Seimically Active Regions. Journal of Structural Engineering, April, 123(4), pp. 423-431.

Constantinou, M. C. \& Symans, M. D., 1993. Seismic response of structures with supplemental damping. The Structural Design of Tall Buildings, 2(2), pp. 77-92.

Constantinou, M., Kalpakidis, I., Filiatrault, A. \& Ecker, R., 2011. LRFD-Based Analysis and Design Procedure for Bridge Bearings and Seismic Isolators, s.l.: MCEER. 
Cosenza, C., Manfredi, G. \& and Ramasco, R., 1993. The use of damage functionals in earthquake engineering: a comparison between different methods.. Earthquake Engineering \& Structural Dynamics, 22(10), p. 855-868.

COSMOS, 2012. Consortium of Organizations for Strong Motion Observation Systems. [Online] Available at: http://www.cosmos-eq.org/

DesRoches, R., Nielson, B. \& Choi, E., 2003. Seismic fragilityof typical bridges in moderate seismic zones. Engineering Structures, p. 187-199.

Di Ludovico, M. et al., 2013. A proposal for plastic hinges modification factors for damaged RC columns. Engineering Structures, Volume 51, pp. 99-112.

Dobry, R. e. a., 1978. Duration characteristics of horizontal components of strong motion earthquake records. Bulletin of the Seismological Society of America, 68(5), pp. 1487-1520.

Dolce, M., Cardone, D., Ponzo, F. \& Valente, C., 2005. Shaking table tests on reinforced concrete frames without and with passive control systems. Earthquake Engng Struct. Dyn., Volume 34, pp. 1687-1717.

Dusicka, P. \& Knoles, S., 2012. Subduction Megathrust Earthquake Demands on Ductile Bridge Columns. Oakland, CA, (7NSC), Seventh National Seismic Conference on Bridges and Highways.

Dusicka, P. \& Tinker, J., 2013. Global Restraint in Ultra-Lightweight Buckling-Restrained Braces. Journal of Composites for Construction, 17(1), pp. 139-150.

Dyngeland, T., 1998. Retroffiting of bridges and building structures - a literature survey, s.l.: European Laboratory for Structural Assessment, JRC-ISIS.

Eberhard, M. \& Marsh, L., 1997. Lateral-Load Response of Two Reinforced Concrete Bents. Journal of Structural Engineering, 123(4), pp. 461-468. 
El-Bahey, S. \& Bruneau, M., 2011. Buckling-restrained braces as structural fuses for the seismic retrofit of reinforced concrete bridge bents. Engineering Structures, 33(3), pp. 1052-1061.

ElGawady, M., Endeshaw, M., McLean, D. \& Sack, R., 2010. Retrofitting of Rectangular Columns with Deficient Lap Splices. Journal of Composites for Construction, 14(1), pp. 22 35.

Elwood, K. \& Eberhard, M., 2009. Effective Stiffness of Reinforced Concrete Columns. ACI Structural Journal, 106(4), pp. 476-484.

Fakharifar, M. et al., 2016. Rapid Repair of Earthquake-Damaged RC Columns with Prestressed Steel Jackets. Journal of Bridge Engineering, 21(4), p. 04015075.

FEMA 356, 2000. Prestandard and commentary for the seismic rehabilitation of buildings, Washington, DC: Report No. FEMA 356. Prepared by American Society of Civil Engineers. Prepared for Federal Emergency Management Agency.

FEMA 461, 2007. Interim Protocols for Determining Seismic Performance Characteristics of Structural and Nonstructural Components Through Laboratory Testing, s.l.: FEMA 461, Federal Emergency Managment Agency.

FEMA P440A, 2009. Effects of Strength and Stiffness Degradation on Seismic Response, Washington, D.C. : Federal Emergency Managment Agency.

FEMA P695, 2009. Quantification of Building Seismic Performance Factors, Washington, D.C.: Federal Emergency Managment Agency.

FEMA306, 1998. Evaluation of Earthquake Damaged Concrete and Masonry Wall Buildings, Applied Technology Council (ATC-43 Project), The Partnership for Response and Recovery. 
FEMA307, 1998. Evaluation of Earthquake Damaged Concrete and Masonry Wall Buildings -

Technical Resources, s.l.: Federal Emergency Management Agency.

FHWA, 2006. Seismic Retrofitting Manual for Highway Structures: Part 1-Bridges, Department of Transportation, McLean, Virginia: Federal Highway Administration. Publication No. FHWA-HRT-06-032.

Ghannoum, W. M., 2007. Experimental and Analytical Dynamic Collapse Study of a Reinforced Concrete Frame with Light Transverse Reinforcement. s.l.:PhD Dissertation, Department of Civil and Environmental Engineering, University of California, Berkeley.

Ghobarah, A., Abou-Elfath, H. \& A., a. B., 1999. Response-Based damage assessment of structures. Earthquake Engineering and Sructural Dynamics, 28(1), pp. 79-104.

Goel, R. \& Chopra, A., 1997. Evaluation of bridge abutment capacity and stiffness during earthquakes. Earthquake Spectra, 13(1), pp. 1-23.

Goldfinger, C. et al., 2008. Late Holocene Rupture of the Northern SAn Andreas Fault and Possible Stress Linkage to the Cascadia Subduction Zone. Bulletin of the Seismological Society of America, 98(2), pp. 861-889.

Goldfinger, C., Nelson, C. \& Johnson, J., 2003. Holocene earthquake records from the Cascadia subduction zone and northern San Andreas Fault based on precise dating of offshore turbidites. Annual Review of Earth and Planetary Sciences, Volume 31, pp. $555-577$.

Goodnight, J., Kowalsky, M. \& Nau, J., 2013. Effect of Load History on Performance Limit States of Circular Bridge Columns. Journal of Bridge Engineering, 18(12), pp. 1383-1396.

Grigorian, C., Yang, T. \& Popov, E., 1993. Slotted-bolted connection energy dissipators. Earthquake Spectra, 9(3), pp. 491-504. 
Grossi, P., 2000. Earthquake Damage Assessment From Expert Opinion to Fragility Curves. Notre Dame, IN.

Gulkan, P. \& Sozen, M., 1974. Inelastic responses of reinforced concrete structures to earthquake motions. Journal of the American Concrete Institute, 71(12), pp. 604-610.

Haroun, M. A. \& Elsanadedy, H. M., 2005. Fiber-Reinforced Plastic Jackets for Ductility Enhancement of Reinforced Concrete Bridge Columns with Poor Lap-Splice Detailing. Journal of Bridge Engineering, 10(6), pp. 749-757.

Haselton, C., Liel, A., Taylor, S. \& Deierlein, G., 2008. Beam-Column Element Model Calibrated for Predicting Flexural Response Leading to Global Collapse of RC Frame Buildings, Berkeley, California: PEER Report 2007/03, Pacific Earthquake Engineering Research Center.

Hayashi, T., H., N. \& Fukuhara, M., 1980. Strengthening methods of the existing reinforced concrete buildings. s.l., In Proceedings of the seventh world conference on earthquake engineering (Vol. 4, pp. 89-97)., pp. 89-97.

Heaton, T. \& Hartzell, S., 1986. Source Characteristics of Hypothetical Subduction Earthquakes in the Northwestern United States. Bulletin of the Seismological Society of America, 76(3), pp. 675-708.

Heaton, T. \& Kanamori, H., 1984. Seismic Potential Associated with Subduction in the Northwestern United States. Bulletin of the Seismological Society of America, 74(3), pp. 933-941.

He, R., Grelle, S., Sneed, L. \& Belarbi, A., 2013. Rapid repair of a severely damaged RC column having fractured bars using externally bonded CFRP. Composite Structures, Volume 101, pp. 225-242. 
Hose, Y. \& Seible, F., 1999. Performance Evaluation Database for Concrete Bridge Components and Systems under Simulated Seismic Loads, Berkeley, California: PEER Report 1999/11, Pacific Earthquake Engineering Research Center.

Hoshikuma, J. \& Guangfeng, Z., 2013. Performance of Seismic Retrofitted Highway Bridges Based on Observation of Damage due to the 2011 Great East Japan Earthquake. Journal of JSCE, Volume 1, pp. 343-352.

Hutchinson, T. \& Wood, R., 2013. Cyclic Load Protocol for Anchored Nonstructural Components and Systems. Earthquake Spectra, 29(3), pp. 817-842.

Hwang, H., Jernigan, J. B. \& Lin, Y., 1999. Expected Seismic Damage to Memphis Highway Systems. Seattle, WA, American Association of Civil Engineers, pp. 1-10.

Hwang, H., Jernigan, J. B. \& Lin, Y., 2000. Evaluation of seismic damage to memphis bridges and highway systems. Journal of Brdge Engineering, pp. 322-330.

Hwang, H., Liu, J. B. \& Chiu, Y.-H., 2001. Seismic Fragility Analysis of Highway Bridges, Memphis: Center for Earthquake Research and Information.

Ibarra, L. \& Krawinkler, H., 2005. Global Collapse of Frame Structures under Seismic Excitations (PEER 2005/06), Berkeley, CA: Pacific Earthquake Engineering Research Center.

Ibarra, L., Medina, R. \& Krawinkler, H., 2005a. Hysteretic models that incorporate strenght and stiffness deterioration. Earthquake Engineering and Structural Dynamics, Volume 34, pp. 1489-1511.

Imbsen, R., 2006. Recommended LRFD Guidelines for the Seismic Design of Highway Bridges, Sacramento, CA: NCHRP 20-07/Task 193, Addendum for Task 6.

Imbsen, R. \& Nutt, R., 1981. Increased seismic resistance of highway bridges using improved bearing design concepts. Atlanta, ASCE, pp. 416-430. 
Kawashima, K. et al., 2011. Damages of Bridges during 2011 Great East Japan Earthquake. Tsukuba Science City, Japan, UJNR.

K-Net, n.d. Kyoshin Network Database, National Research Institute for Earth Science and Disaster Prevention. [Online] Available at: http://www.k-net.bosai.go.jp/

Korzekwa, A. \& Tremblay, R., 2009. Numerical simulation of the cyclic inelastic behaviour of buckling restrained braces. s.l., Proceedings of STESSA conference 2009.

Kotsoglou, A. \& Pantazopoulou, S., 2009. Assessment and modeling of embankment participation in the seismic response of integral abutment bridges. Bull. Earthquake Eng., pp. 343-361.

Kowalsky, M., 2000. Deformation limit states for circular reinforced concrete bridge columns. Journal of Structural Engineering, 126(8), pp. 869-878.

Krawinkler, H., 1996. Cyclic Loading Histories for Seimic Experimentation on Structural Components. Earthquake Spectra, 12(1), pp. 1-12.

Krawinkler, H., Gupta, A., Medina, R. \& Luco, N., 2000b. Loading Histories for Seismic Performance Testing of SMRF Components and Assemblies, s.l.: SAC Joint Venture.

Krawinkler, H. et al., 2000a. Development of a Testing Protocol for Woodframe Structures, Richmond, CA: CUREE Publication No. W-02.

Krawinkler, H. et al., 1983. Recommendations for Experimental Studies on the Seismic Behavior of Steel Components and Materials, s.l.: John A. Blumel Center, Department of Civil Engineering, Stanford University.

Kunnath, S., El-Bahy, A., Taylor, A. \& Stone, W., 1997. Cumulative Seismic Damage of Reinforced Concrete Damage Piers, Gaithersburg, MD: Building and Fire Research Laboratory, National Institute of Standards and Technology. 
Lanning, J., Benzoni, G. \& Uang, C., 2011. The Feasibility of Using Buckling-Restrained Braces for Long-Span Bridges. A Case Study, La Jolla, California: State of California. Department of Transportation. CA12-2149.

Lehman, D., Gookin, S., Nacamuli, A. \& and Moehle, J., 2001. Repair of EarthquakeDamaged Bridge Columns. ACI Structural Journal, 98(2), pp. 233-242.

Lehman, D. \& Moehle, J., 2000. Seismic Performance of Well-Confined Concrete Bridge Columns, University of California, Berkeley: Pacific Earthquake Engineering Research Center, Report No. PEER 1998/01.

Liao, W.-I. \& Loh, C.-H., 2004. Priliminary Study on the Fragility Curves for Highway Bridges in Taiwan. Journal of the Chinese Institute of Engineers, 27(3), pp. 367-375.

Lignos, D. \& Krawinkler, H., 2012. Sidesway Collapse of Deteriorating Structural Systems Under Seismic Excitations, Stanford, CA: Report No. 177, The John A. Blume Earthquake Engineering Center.

Lopez, W. \& Sabelli, R., 2004. Seismic Design of Buckling-Restrained Braced Frames, s.l.: Steel Tips. Luco, N. \& Bazzurro, P., 2007. Does amplitude scaling of ground motion records result in biased nonlinear structural drift responses?. Earthquake Engineering and Structural Dynamics, Volume 36, pp. 1813-1835.

Luco, N. \& Cornell, C., 1998. Effects of random connection fractures on the demands and reliability for a three-story pre-Northridge (SMRP) structure. Oakland, CA, Proceedings of the sixth US national conference on earthquake engineering.

Mackie, K. \& Stojadinovic, B., 2005. Fragility basis for california bighway overpass bridge seismic decision making, Berkeley, CA: Pacific Earthquake Engineering Research Center. University of California. 
Mackie, K. \& Stojadinovic, B., 2006. Seismic Vulnerability of Typical Multi-Span California Highway Bridges. San Francisco, s.n.

Mahin, S. et al., 2004. Seismic Performance of Buckling Restrained Braced Frame Systems. Vancouver, B.C., Canada.

Mander, J. \& Basöz, N., 1999. Enhancement of the Highway Transportation Lifeline Module in HAZUS, NIBS.

Mander, J., Priestley, M. \& Park, R., 1988. Theoretical Stress-Strain Model for Confined Concrete. Journal of Structural Engineering, 114(3), pp. 1804-1826.

Maroney, B. et al., 1994. Interpretation of Large Scale Bridge Abutment Test Results. Sacramento, CA, Caltrans.

Marsh, M., Buckle, I. \& Kavazanjian, E., 2014. LRFD Seismic Analysis and Design of Bridges, s.l.: Publication No. FHWA-NHI-15-004.

Mazzolani, F., Della Corte, G. \& D'Aniello, M., 2009. Experimental analysis of steel dissipative bracing systems for seismic upgrading. Journal of Civil Engineering and Management, 15(1), pp. 7-19.

McDaniel, C., 2006. Seismic Assessment and Retrofit of Existing Multi-Column Bent Bridges, Olympia, WA: Washington State Department of Transportation (WSDOT).

McDaniel, C., Cofer, W., D., M. \& Rodriguez-Merek, A., 2006. Performance of pre-1975 Concrete Bridges in Cascadia Subduction-Zone Earthquakes, Pullman, WA.

McLean, D., Kuebler, S. \& Mealy, T., 1998. Seismic Performance and Retrofit of Multi-Column Bridge Bents, Pullman, Washington: Washington State Transportation Center (TRAC). 
Mehary, S. \& Dusicka, P., 2015. Seismic Retrofit Benefit Considering Statewide Transportation Assessment, Portland, OR: Transportation Research and Education Center (TREC). Report OR-RD-15-15. .

Mehary, S., Dusicka, P. \& Bazaez, R., 2014. Cyclic Behavior of As-built and CFRP Wrap Retrofitted Square Reinforced Concrete Bridge Columns. s.l., Network for Earthquake Engineering Simulation (distributor), Paper, DOI:10.4231/D3B27PR73.

Midorikawa, S., Miura, H. \& Si, H., 2012. Preliminary Analysis for Characteristics of Strong Ground Motion from Gigantic Earthquakes. Paper No.0941, Proceedings of 15th World Conference on Earthquake Engineering.

Miller, D., Fahnestock, L. \& Eatherton, M., 2012. Development and experimental validation of a nickel-titanium shape memory alloy self-centering buckling-restrained brace. Engineering Structures, Volume 40, pp. 288-298.

Moehle, J. \& Deierlein, G., 2004. A framework methodology for performance-based earthquake engineering. Vancouver, B.C., Canada, 13th World Conference on Earthquake Engineering .

Mualla, I. \& Belev, B., 2002. Performance of steel frames with a new friction damper device under earthquake excitation. Engineering Structures, 24(3), pp. 365-371.

Muntasir Billah, A., Alam, M. \& Bhuiyan, M. R., 2012. Fragility Analysis of Retrofitted Multicolumn Bridge Bent Subjected to Near-Fault and Far-Field Ground Motion. Journal of Bridge Engineering, 18(10), pp. 992-1004.

Muntasir Billah, A. \& Shahria Alam, M., 2014. Seismic fragility assessment of highway bridges: a state-of-the-art review. Structure and Infrastructure Engineering, pp. 1-29. 
Nako, A. et al., 2009. Seismic Vulnerability of Oregon State Highway Bridges: Mitigation Strategies to Reduce Major Mobility Risks, Oregon: Oregon Department of Transportation, Bridge Engineering Section.

NCHRP, N. C. H. R. P., 2013. Performance-Based Seismic Bridge Design, Synthesis 440, Washington, D.C.: Transportation Research Board, National Academy of Sciences.

Nielson, B., 2005. Analytical Fragility Curves for Highway Bridges in Moderate Seismic Zones, Atlanta, GA: Georgia Institute of Technology.

Nielson, B. G. \& DesRoches, R., 2007. Analytical Seismic Fragility Curves for Typical Bridges in the Central and Southeastern United States. Earthquake Spectra, August, 23(3), p. 615-633.

ODOT, 2015. Bridge Design and Drafting Manual. Section 1 - Design, Oregon: Oregon Department of Transportation.

OpenSees, 2013. Open System for Earthquake Engineering Simulation, Pacific Earthquake Engineering Research Center, University of California, Berkeley V. 2.5.0. [Online] Available at: http://opensees.berkeley.edu

Ou, Y. et al., 2013. Ground Motion Duration Effects on Hysteretic Behavior of Reinforced Concrete Bridge Columns. Journal of Structural Engineering, 140(3), p. 04013065.

Padgett, J., 2005. Retrofitted Bridge Fragility Curves for Assessing the Consequences of an Earthquake Event. [Online] Available at: http://mae.cee.uiuc.edu/documents/slc online magazine 2005 july padgett.pdf [Accessed 11 December 2010].

Padgett, J., 2007. Seismic V ulnerability Assessment of Retrofitted Bridges using Probabilistic Methods, s.l.: Georgia Institute of Technology, $\mathrm{PhD}$ Thesis. 
Padgett, J. \& DesRoches, R., 2008. Methodology for the development of analytical fragility curves for retrofitted bridges. Earthquake Engineering and Structural Dynamics, 11 April, Volume 37, p. 1157-1174.

Padgett, J. \& DesRoches, R., 2009. Retrofitted Bridge Fragility Analysis for Typical Classes of Multispan Bridges. Earthquake Spectra, p. 117-141.

Padgett, J. E. \& DesRoches, R., 2007. Sensitivity of Seismic Response and Fragility to Parameter Uncertainty. Journal of Structural Engineering, December.pp. 1710-1718.

Padgett, J., Nielson, B. \& DesRoches, R., 2008. Selection of optimal intensity measures in probabilistic seismic demand models of highway bridge portfolios. Earthquake Engineering \& Structural Dynamics, 37(5), pp. 711-725.

Pall, A. \& Marsh, C., 1982. Response of friction damped braced frames. ASCE, Journal of the Structural Division, pp. 1313-1323.

Pantelides, C. \& Gergely, J., 2008. Seismic Retrofit of Reinforced Concrete Beam-Column T-Joints in Bridge Piers with FRP Composite Jackets. ACI Special Publication, SP 258(1), pp. 1-18.

Pan, Y., 2007. Seismic Fragility and Risk Management of Highway Bridges in New York State, New York: The City University of New York.

Park, R., 1989. Evaluation of Ductility of Structures and Structural Assemblages from Laboratory Testing. Bulletin of the New Zealand National Society for Earthquake Engineering, 22(3), pp. 155-166.

Paulay, T. \& Priestley, M., 1992. Seismic Design of Reinforced Concrete and Masonry Structures. s.l.:John Wiley \& Sons, Inc. 
PEER/ATC-72-1, 2010. Modeling and acceptance criteria for seismic design and analysis of tall buildings, Redwood City, California: Prepared by Applied Technology Council. Prepared for Pacific Earthquake Engineering Research Center (PEER).

PEER, 2006. NGA Database, Pacific Earthquake Engineering Research Center, University of California, Berkeley. [Online] Available at: http://peer.berkeley.edu/nga/

Petersen, M. et al., 2014. Documentation for the 2014 Update of the United States National Seismic Hazard Maps, Open-File Report 2014-1091, s.l.: U.S. Geological Survey.

Priestley, M. J. N., Calvi, G. M. \& Kowalsky, M. J., 2007. Displacement-based seismic design of structures. s.l.:IUSS Press, Italy..

Priestley, M. J. N., Seible, F. \& Calvi, G. M., 1996. Seismic Design and Retrofit of Bridges. New York: Wiley.

Priestley, M., Seible, F. \& Hines, E., 2002. Seismic Performance of Hollow Rectangular Reinforced Concrete Piers with Highly-Confined Boundary Elements, La Jolla, California: Department of Structural Engineering, University of California, San Diego. Report No. SSRP-99/15.

Priestley, M., Seible, F. \& Uang, C., 1994. The Northridge Earthquake of January 17, 1994:

Damage Analysis of Selected Freeway Bridges, University of California, San Diego: Structural Systems Research Project, Report SSRP-94/06.

Pujol, S., Sozen, M. \& Ramirez, J., 2006. Displacement History Effects on Drift Capacity of Reinforced Concrete Columns. ACI Structural Journal, 103(2), pp. 253-262.

Pulido, C. et al., 2004. Seismic Performance of Two-Column Bents-Part II: Retrofit with Infill Walls. ACI Structural Journal, pp. 642-649.

Raghunandan, M. \& Liel, B., 2013. Effect of ground motion duration on earthquake-induced structural collapse. Structural Safety, Volume 41, pp. 119-133. 
Ranf, R., Eberhard, M. \& Stanton, J., 2006. Effects of Displacement History on Failure of Lightly Confined Bridge Columns. ACI Special Publication, 236(2), pp. 23-42.

Reno, M. \& Pohll, M., 2010. Seismic Retrofit of California's Auburn-Foresthill Bridge. Transportation Research Record: Journal of the Transportation Research Board, 2201(1), pp. 83-94.

Richards, P. \& Uang, C., 2006. Testing Protocol for Short Links in Eccentrically Braced Frames. Journal of Structural Engineering. ASCE, pp. 1183-1191.

Ridell, R. \& Newmark, N., 1979. Statistical analysis of the response of nonlinear systems subjected to earthquakes, Urbana: Struct. Res. Series No. 468, University of Illinois.

Robinson, W., 1982. Lead-rubber hysteretic bearings suitable for protecting structures during earthquakes. Earthquake Engineering \& Structural Dynamics, 10(4), pp. 593-604.

Rodriguez, M. \& Park, R., 1994. Seismic Load Test on Reinforced Concrete Columns Strengthened by Jacketing. ACI Structural Journal, Mar-Apr, 91(2), pp. 150-159.

Saadatmanesh, H., Ehsani, M. \& Jin, L., 1997. Repair of Earthquake-Damaged RC Columns with FRP Wraps. ACI Structural Journal, 94(2), pp. 206-214.

Saatcioglu, M. \& Yalcin, C., 2003. External Prestressing Concrete Columns for Improved Seismic Shear Resistance. Journal of Structural Engineering, p. 1057-1070.

Schrage, I., 1981. Anchoring of bearings by friction. In Joint Sealing and Bearing Systems for Concrete Structures, World Congress on Joints and Bearings, Volume Vol. 1.

Scott, M. \& Fenves, G., 2006. Plastic Hinge Integration Methods for Force-Based BeamColumn Elements. Journal of Structural Engineering, 132(2), pp. 244-252. 
Seible, F. et al., 1995. Carbon Fiber Jacket Retrofit Test of Rectangular Flexural Column with Lap Spliced Reinforcement, University of California, San Diego: Advanced Composites Technology Transfer Consortium Report No. ACT'T-95/04.

Sezen, H., 2002. Seismic Response and Modeling of Reinforced Concrete Building Columns, University of California, Berkeley: PhD. Dissertation, Department of Civil and Environmental Engineering, .

Sezen, H. \& Setzler, E., 2008. Reinforcement Slip in Reinforced Concrete Columns. ACI Structural Journal, 105(3), pp. 280-289.

Shamsabadi, A., Khalili-Tehrani, P., Stewart, J. \& Taciroglu, E., 2010. Validated Simulation Models for Lateral Response of Bridge Abutments with Typical Backfills. Journal of Bridge Engineering, pp. 302-311.

Shamsabadi, A., Rollins, K. \& Kapuskar, M., 2007. Nonlinear Soil-Abutment-Bridge Structure Interaction for Seismic Performance-Based Design. Journal of Geotechnical and Geoenvironmental Engineering, pp. 707-720.

Sheikh, M. \& Légeron, F., 2014. Performance based seismic assessment of bridges designed according to Canadian Highway Bridge Design Code. Canadian Journal of Civil Engineering, 41(9), pp. 777-787.

Shin, M. \& Andrawes, B., 2011. Emergency repair of severely damaged reinforced concrete columns using active confinement with shape memory alloys. Smart Materials and Structures, 20(6), p. 065018.

Shinozuka, M., Feng, M. Q., Kim, H. \& Kim, S., 2000. Nonlinear Static Procedure for Fragility Curve Development. Journal or Engineering Mechanics, December.pp. 12871295. 
Shinozuka, M., Feng, M. Q., Lee, J. \& Naganuma, T., 2000. Statistical Analysis of Fragility Curves. Journal of Engineering Mechanics, December.pp. 1224-1231.

Shinozuka, M., Kim, S., Kushiyama, S. \& Yi, J., 2002. Fragility Curves of Concrete Bridges Retrofitted By Column Jacketing. Earthquake Engineering and Engineering Vibration, December, 1(2), pp. 195-202.

Shinozuka, M., Saxena, V., Deodatis, G. \& Feng, M. Q., 2001. Development of Fragility Curves for Multi-Span Reinforced Concrete Bridges. [Online] Available at: http://www.civil.columbia.edu/iassar/downloadfiles/SC1 texts/ [Accessed 30 September 2010].

Simon, J., Bracci, J. M. \& Gardoni, P., 2010. Seismic Response and Fragility of Deteriorated Reinforced Concrete Bridges. Journal of Structural Engineering, October.pp. 1273-1281.

Skinner, R., Robinson, H. \& McVerry, G., 1993. An Introduction to Seismic Isolation. Chichester: Wiley.

Sozen, M., Monteiro, P., Moehle, J. \& Tang, H., 1992. Effects of Cracking and Age on Stiffness of Reinforced Concrete Walls Resisting In-Plane Shear. Orlando, FL, Proceedings, Fourth Symposium on Current Issues Related to Nuclear Power Plant Structures, Equipment and Piping.

Stephens, J. \& Yao, J., 1987. "Damage Assessment Using Response Measurements". J. Struct. Eng., 113(4), pp. 787-801.

Stojadinović, B. \& Mackie, K., 2007. R-Factor Parameterized Bridge Damage Fragility Curves. Journal of Bridge Engineering, July/August.pp. 500-510.

Sugano, 1981. Seismic Strengthening of Existing Reinforced Concrete Buildings in Japan. Bulletin of the New Zealand National Society for Earthquake Engineering, 14(4), pp. 209-222. 
Takeda, T. \& Sozen, M. N. N., 1970. Reinforced concrete response to simulated earthquakes. ASCE Journal of the Structural Division, Volume 96, pp. 2557-2573.

Takemura, H. \& Kawashima, K., 1997. Effect of loading hysteresis on ductility capacity of reinforced concrete bridge piers. Journal of Structural Engineering, Volume 43A, pp. 849-858 (in Japanese).

Taucer, F., Spacone, E. \& Filippou, F., 1991. A fiber beam-column element for seismic response analysis of reinforced concrete structures, Berkeley, CA: Earthquake Engineering Research Center, College of Engineering, University of California. Report 91-17.

Teng, J., Chen, J., Smith, S. \& Lam, L., 2001. FRP: Strengthened RC Structures. s.l.:Wiley, Frontiers in Physics, 1.

Teng, T.-L., 1994. Southern California Earthquake Data Center. [Online] Available at: http://scedc.caltech.edu/significant/northridge1994.html\#e [Accessed 2015].

Teran, A. \& Ruiz, J., 1992. Reinforced concrete jacketing of existing structures. Balkema, Rotterdam, pp. 5107-5113.

Tremblay, R., Bolduc, P., Neville, R. \& DeVall, R., 2006. Seismic testing and performance of buckling-restrained bracing systems. Canadian Journal of Civil Engineering, 33(2), pp. 183-198.

U. Chile, 2010. University of Chile, Earthquakes of Chile. [Online] Available at: http://terremotos.ing.uchile.cl/registros/164

USGS \& Wilshire, H., 1989. USGS page-Oakland. [Online] Available at: http://pubs.usgs.gov/dds/dds-29/screens/022sr.jpeg [Accessed 2015]. 
Vamvatsikos, D. \& Cornell, C., 2002. Incremental dynamic analysis. Earthquake Engineering \& Structural Dynamics, 31(3), pp. 491-514.

Vargas, R. \& Bruneau, M., 2006. Analytical investigation of the structural fuse concept, Buffalo, NY: Multidisciplinary Center for Earthquake Engineering Research. MCEER-06-0004.

Vargas, R. \& Bruneau, M., 2009. Analytical Response and Design of Buildings with Metallic Structural Fuses. I. Journal of Structural Engineering, 135(4), pp. 386-393.

Vosooghi, A. \& Saiid Saiidi, M., 2012. Experimental Fragility Curves for Seismic Response of Reinforced Concrete Bridge Columns. ACI Structural Journal, 109(6), pp. 825-834.

Vosooghi, A. \& Saiid Saiidi, M., 2013. Design Guidelines for Rapid Repair of EarthquakeDamaged Circular RC Bridge Columns Using CFRP. Journal of Bridge Engineering, 18(9), pp. 827-836.

Wilson, J. \& Tan, B., 1990. Bridge abutments: formulation of a simple model for earthquake response analysis. Journal of Engineering Mechanics, 116(8), pp. 1828-1837.

Wolf, J., 1985. Dynamic Soil-Structure Interaction. Englewood Cliffs, NJ: Prentice-Hall, Inc..

Wright, T., DesRosches, R. \& Padgett, J., 2011. Bridge Seismic Retrofitting Practices in the Central and Southeastern United States. Journal of Bridge Engineering, 16(1), pp. 82-92. WSDOT, 2006. Bridge Design Manual M 23-50.

Xiao, Y., Wu, H. \& Martin, G., 1999. Prefabricated Composite Jacketing of RC Columns for Enhanced Shear Strength. Journal of Structural Engineering, 125(3), pp. 255-264.

Yamamoto, Y. \& Umemura, H., 1992. Analysis of reinforced concrete frames retrofitted with steel brace. Balkema, Rotterdam, s.n., pp. 5187-5192.

Yamazaki, F., Ohnishi, J. \& Tayama, S., 1999. Earthquake Damage Assessment of Expressway Structures in Japan. Taipei, Taiwan, s.n., pp. 205-214. 
Zhang, J. \& Huo, Y., 2009. Evaluating effectiveness and optimum design of isolation devices for highway bridges using the fragility function method. Engineering Structures, 31(8), pp. $1648-1660$.

Zsarnoczay, A., 2013. Experimental and Numerical Investigation of Buckling Restrained Braced Frames for Eurocode Conform Design Procedure Development, Budapest: (Phd. Dissertation) Budapest University of Technology and Economics. 


\section{APPENDIX}

This appendix shows the loading protocols that were developed considering all the inelastic excursions in case experimentalists decide those would be more appropriate for their application.

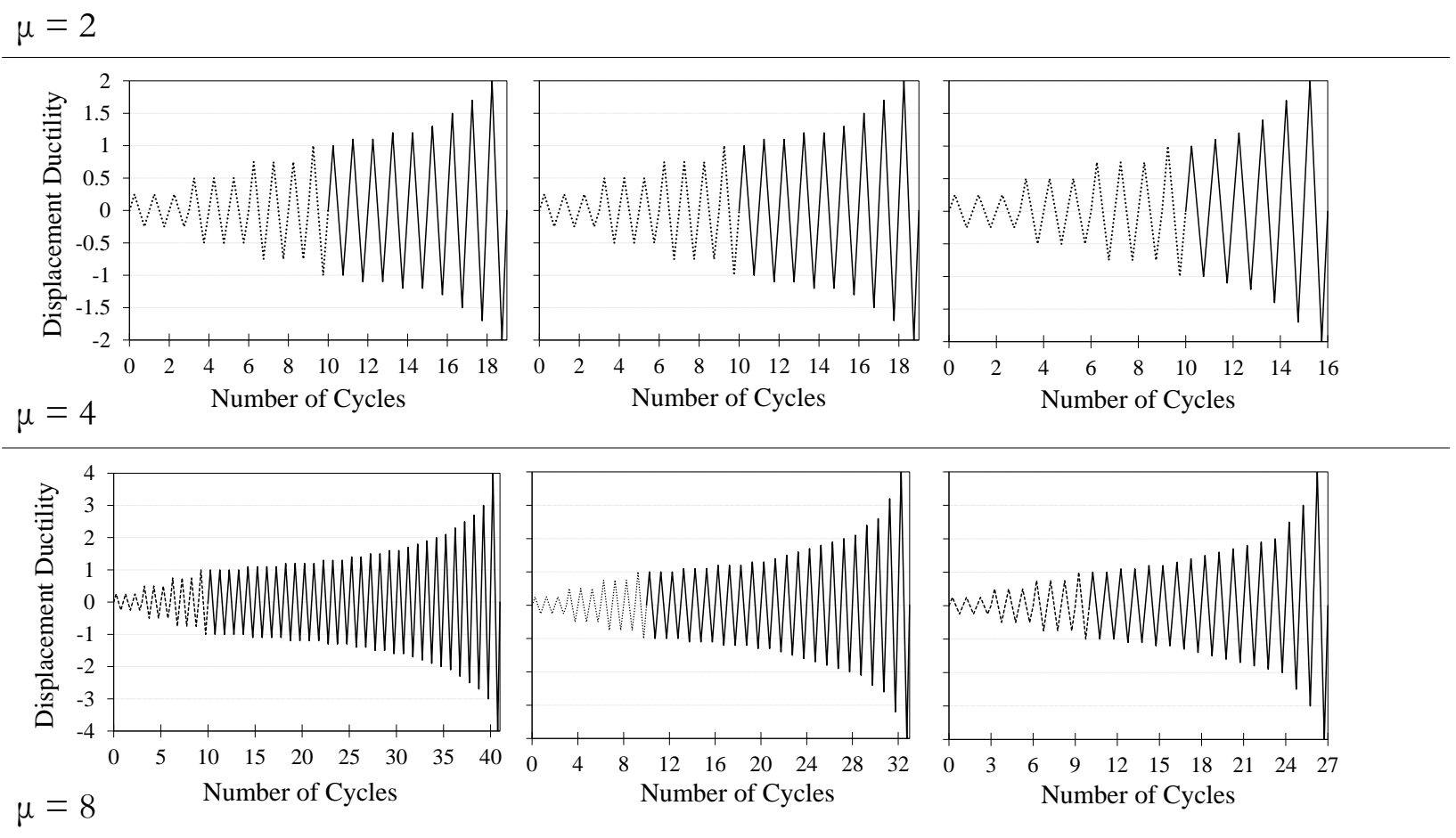

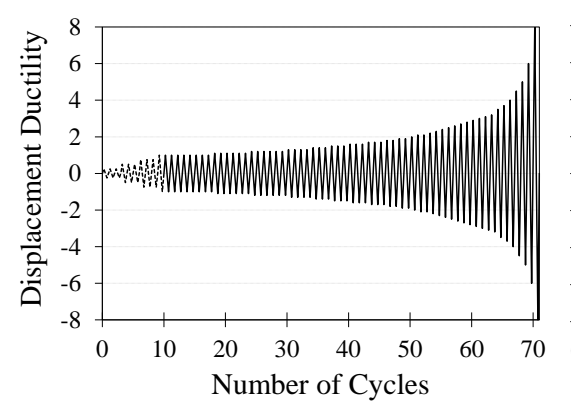

(a)

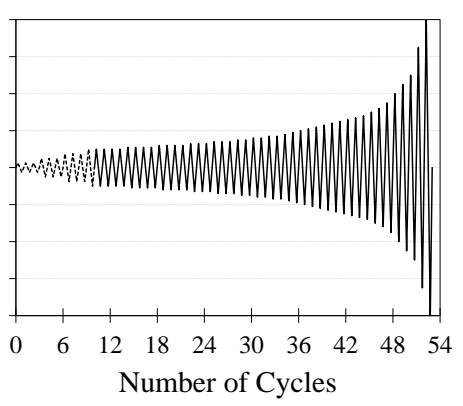

(b)

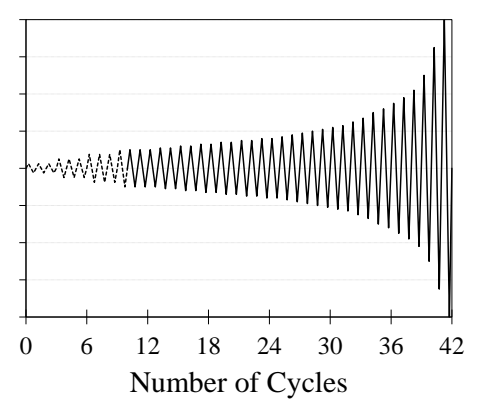

(c)

Figure 0.1 : Loading protocols considering all excursions for component ductilities $(\mu)=2,4$ and 8 . (a) $\mathrm{T}=0.5 \mathrm{sec}$, (b) $\mathrm{T}=1.0 \mathrm{sec}$, (c) $\mathrm{T}=2.0 \mathrm{sec}$.

Table 0.1 Loading protocols utilizing all excursions 


\begin{tabular}{c|c|c|c|c|c|c}
\hline \multirow{2}{*}{$\begin{array}{c}\text { Cycle } \\
\text { Amplitude } \\
\mathbf{x} \boldsymbol{\delta}_{\text {yield }}\end{array}$} & \multicolumn{6}{|c}{ Number of Inelastic Cycles } \\
\cline { 2 - 7 } & $\mathrm{T}=0.5 \mathrm{~s}$ & $\mathrm{~T}=1.0 \mathrm{~s}$ & $\mathrm{~T}=2.0 \mathrm{~s}$ & $\mathrm{~T}=0.5 \mathrm{~s}$ & $\mathrm{~T}=1.0 \mathrm{~s}$ & $\mathrm{~T}=2.0 \mathrm{~s}$ \\
\hline $\mathbf{1 . 0}$ & 4 & 3 & 2 & 8 & 4 & 3 \\
\hline $\mathbf{1 . 1}$ & 4 & 3 & 2 & 6 & 4 & 3 \\
\hline $\mathbf{1 . 2}$ & 4 & 3 & 2 & 6 & 4 & 2 \\
\hline $\mathbf{1 . 3}$ & 3 & 2 & 2 & 4 & 3 & 2 \\
\hline $\mathbf{1 . 4}$ & 2 & 1 & 1 & 3 & 3 & 2 \\
\hline $\mathbf{1 . 5}$ & 2 & 1 & 1 & 3 & 2 & 1 \\
\hline $\mathbf{1 . 6}$ & 2 & 1 & 1 & 3 & 2 & 1 \\
\hline $\mathbf{1 . 7}$ & 1 & 1 & - & 3 & 2 & 1 \\
\hline $\mathbf{1 . 8}$ & 1 & 1 & 1 & 2 & 2 & 1 \\
\hline $\mathbf{1 . 9}$ & 1 & 1 & - & 2 & 1 & 1 \\
\hline $\mathbf{2 . 0}$ & 1 & 1 & 1 & 2 & 1 & 1 \\
\hline $\mathbf{2 . 1}$ & 1 & - & - & 2 & 1 & 1 \\
\hline $\mathbf{2 . 2}$ & - & 1 & - & 1 & 1 & 1 \\
\hline $\mathbf{2 . 3}$ & 1 & - & - & 1 & 1 & - \\
\hline $\mathbf{2 . 4}$ & - & - & - & 1 & 1 & 1 \\
\hline $\mathbf{2 . 5}$ & 1 & 1 & 1 & 1 & 1 & - \\
\hline $\mathbf{2 . 6}$ & - & - & - & 1 & 1 & 1 \\
\hline $\mathbf{2 . 7}$ & - & - & - & 1 & - & - \\
\hline $\mathbf{2 . 8}$ & - & - & - & 1 & 1 & - \\
\hline $\mathbf{2 . 9}$ & - & - & - & 1 & - & - \\
\hline $\mathbf{3 . 0}$ & 1 & 1 & 1 & 1 & 1 & 1 \\
\hline $\mathbf{3 . 2}$ & - & - & - & 1 & 1 & - \\
\hline $\mathbf{3 . 5}$ & - & - & - & 1 & 1 & 1 \\
\hline $\mathbf{4 . 0}$ & 1 & 1 & 1 & 1 & 1 & 1 \\
\hline $\mathbf{4 . 5}$ & & & & 1 & - & 1 \\
\hline $\mathbf{5 . 0}$ & & & & 1 & 1 & 1 \\
\hline $\mathbf{5 . 5}$ & & & & - & - & - \\
\hline $\mathbf{6 . 0}$ & & & & 1 & 1 & 1 \\
\hline $\mathbf{6 . 5}$ & & & & - & - & - \\
\hline $\mathbf{7 . 0}$ & & & & - & - & 1 \\
\hline $\mathbf{8 . 0}$ & & & & 1 & 1 & 1 \\
\hline & & & & & & \\
\hline
\end{tabular}

